\title{
Report to The American Physical Society of the study group on science and technology of directed energy weapons
}

\section{APS Study Group Participants}

N. Bloembergen, Co-chair

Harvard University, Cambridge, Massachusetts 02138

C. K. N. Patel, Co-chair

AT\&T Bell Laboratories, Murray Hill, New Jersey 07974

P. Avizonis

Air Force Weapons Laboratory, Kirtland Air Force Base,

Albuquerque, New Mexico 87117

\section{R. G. Clem}

Sandia National Laboratory, Albuquerque, New Mexico 87185

\author{
A. Hertzberg \\ University of Washington, Seattle, Washington 98195 \\ T. H. Johnson \\ U.S. Military Academy, West Point, New York 10996
}

T. Marshall

Columbia University, New York, New York 10027

R. B. Miller

Sandia National Laboratory, Albuquerque, New Mexico 87185

W. E. Morrow

Massachusetts Institute of Technology,

Lexington, Massachusetts 02139

\section{APS Council Review Committee}

G. E. Pake, Chair

Xerox PARC, Palo Alto, California 94304

M. M. May

Lawrence Livermore Laboratory, Livermore, California 94550

W. K. Panofsky

Stanford University, Stanford, California 94305

\author{
E. E. Salpeter \\ Cornell University, Ithaca, New York 14853
}

\author{
A. M. Sessler \\ Lawrence Berkeley Laboratory, Berkeley, California 94720 \\ J. D. Sullivan \\ University of Illinois, Urbana, Illinois 61801 \\ J. C. Wyant \\ University of Arizona, Tucson, Arizona 85721
}

\section{A. Yariv}

California Institute of Technology, Pasadena, California 91125

R. N. Zare

Stanford University, Stanford, California 94305

A. J. Glass (Principal Consultant)

KMS Fusion, Ann Arbor, Michigan 48106

L. C. Hebel, Executive Officer

Xerox PARC, Palo Alto, California 94304 
Table of contents of the full report of the APS Study Group:

THE SCIENCE AND TECHNOLOGY OF

\section{DIRECTED ENERGY WEAPONS}

EXECUTIVE SUMMARY AND MAJOR CONCLUSIONS ....................................................................... S9

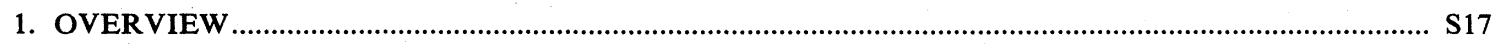

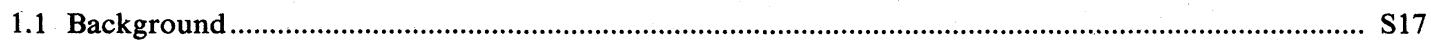

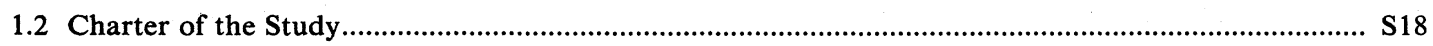

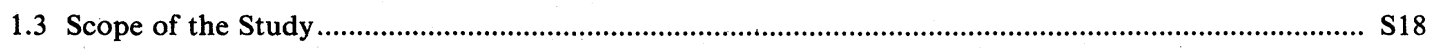

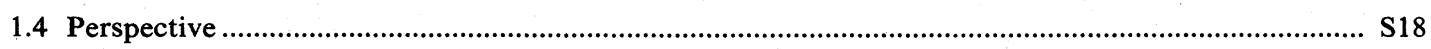

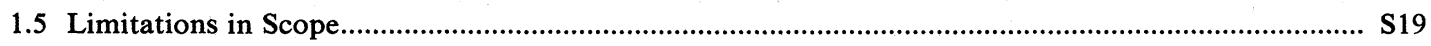

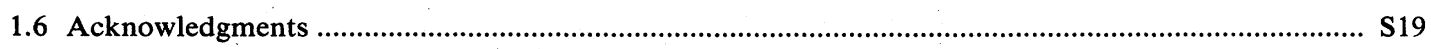

2. SOVIET BALLISTIC MISSILE THREAT: CURRENT AND RESPONSIVE ………………….......... S21

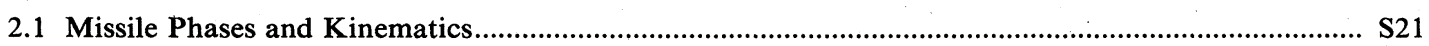

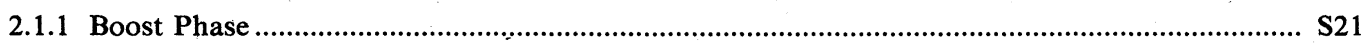

2.1.2 Post-Boost Phase

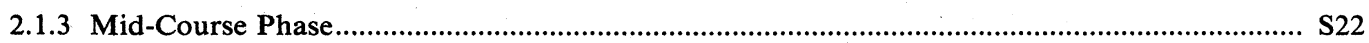

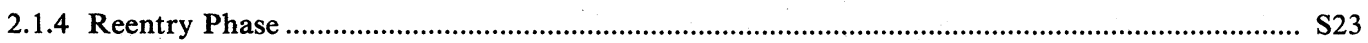

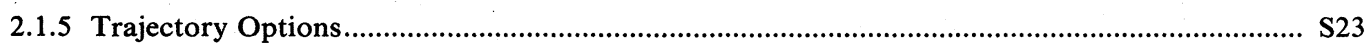

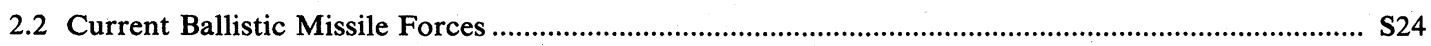

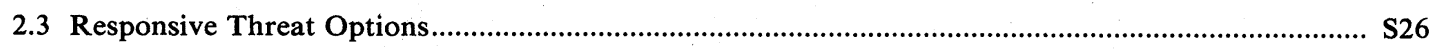

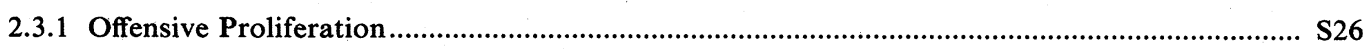

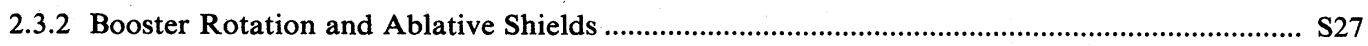

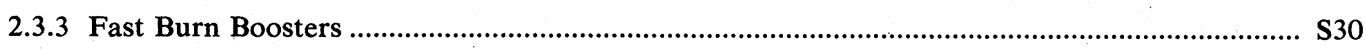

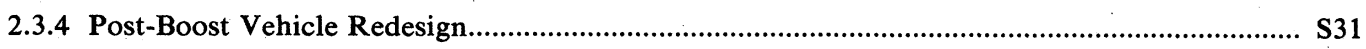

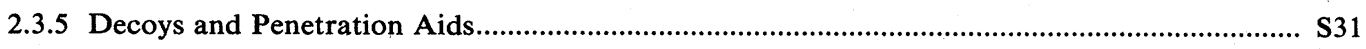

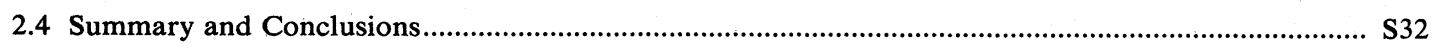

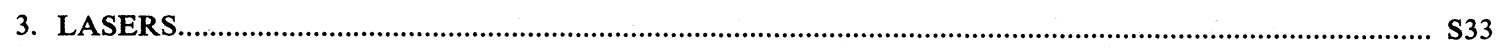

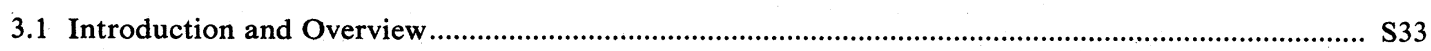

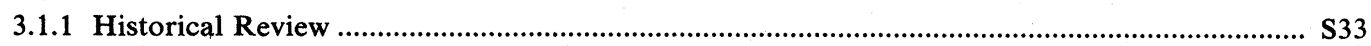

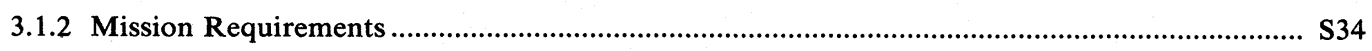

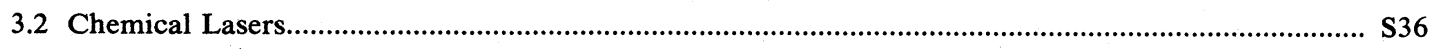

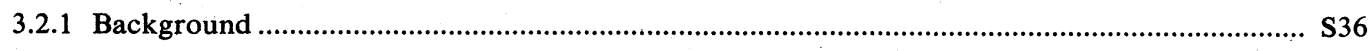

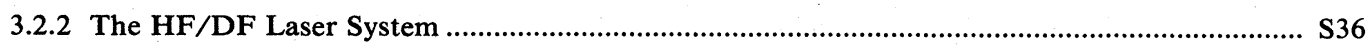

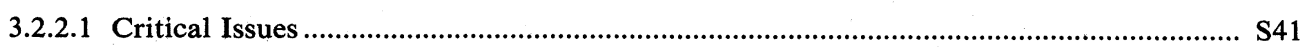

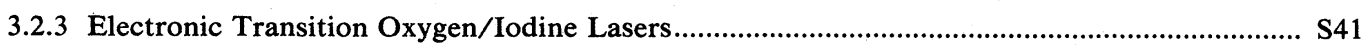

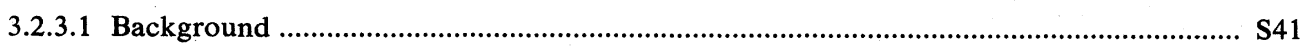

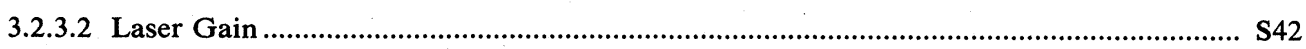

3.2.3.3 Chemical Generation of Excited Molecular Oxygen .............................................. S43

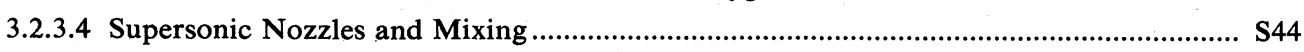

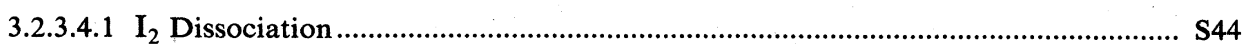


3.2.3.5 Scaling Parameters and Efficiencies for Large-Scale $\mathrm{O}_{2}$-I Lasers............................... S44

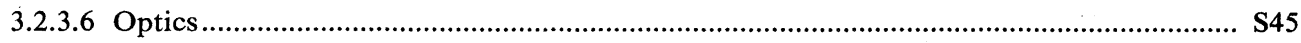

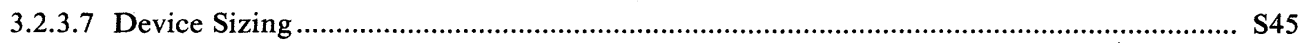

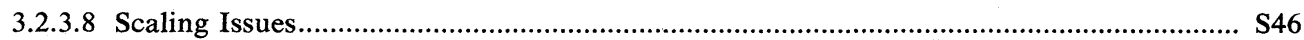

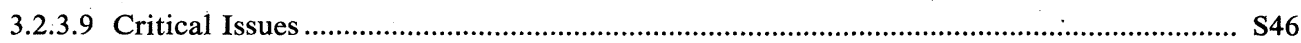

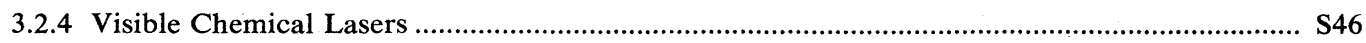

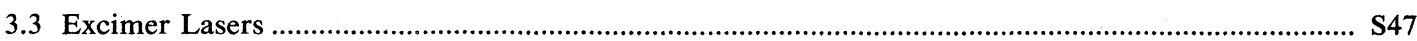

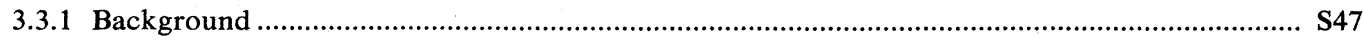

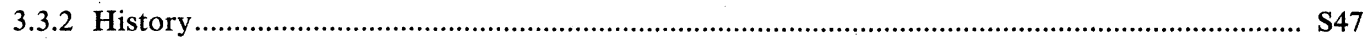

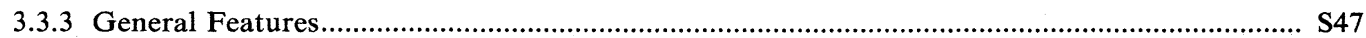

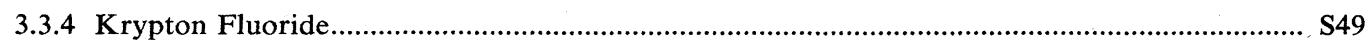

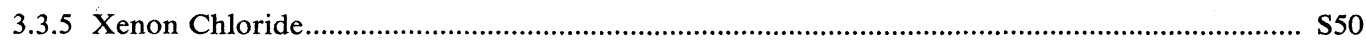

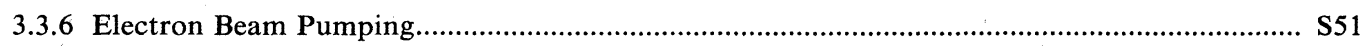

3.3.7 Raman Conversion and Beam Combination ................................................................... S52

3.3.8 Beam Cleanup Using Stimulated Brillouin Scattering ................................................... S54

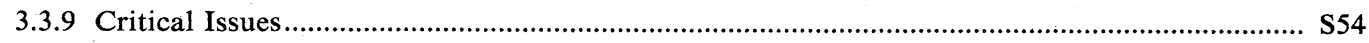

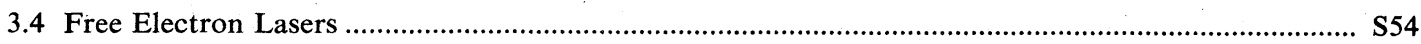

3.4.1 Principles of Operation and Recent Results ................................................................. S54

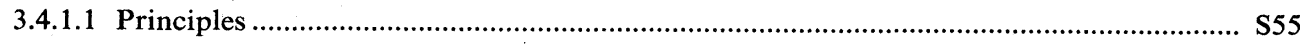

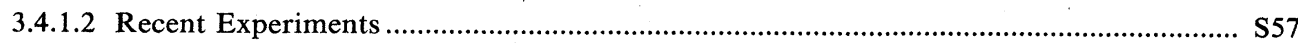

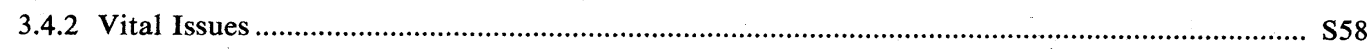

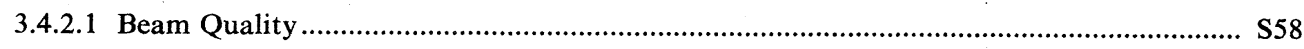

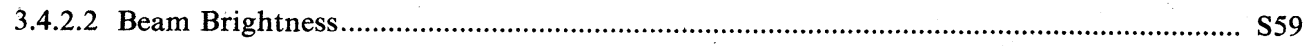

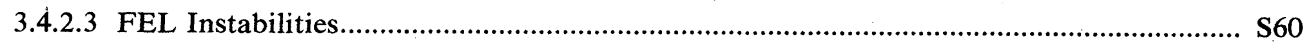

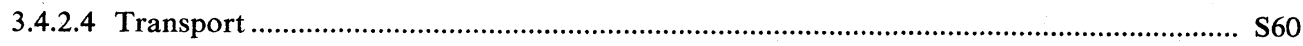

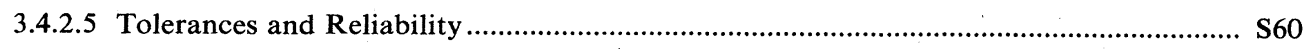

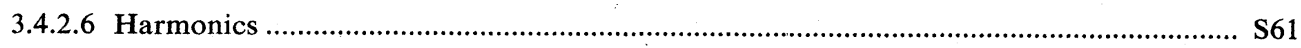

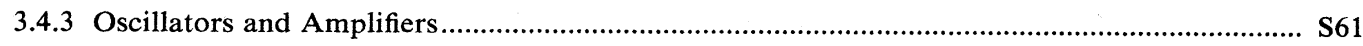

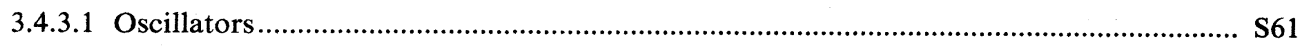

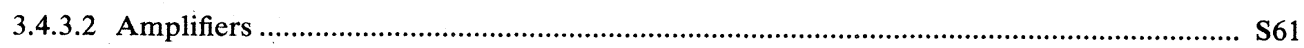

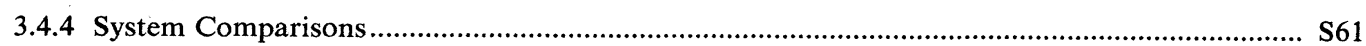

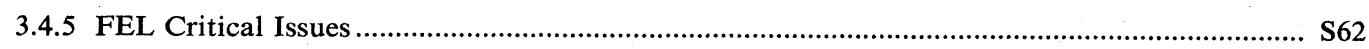

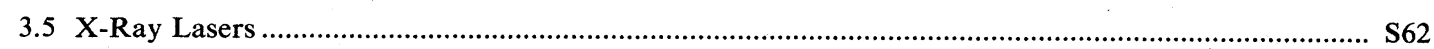

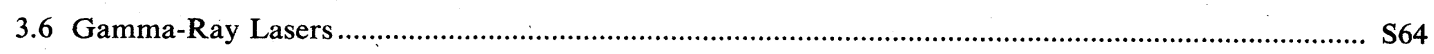

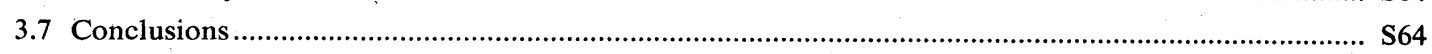

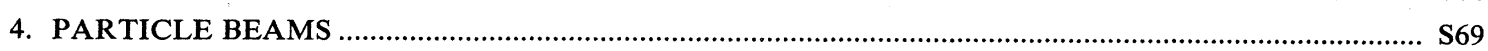

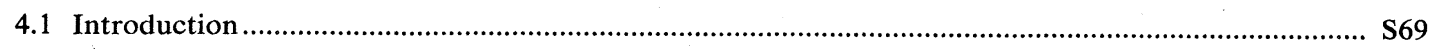

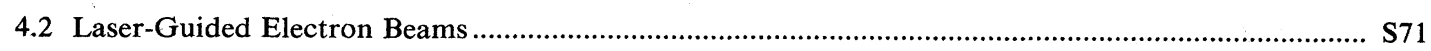

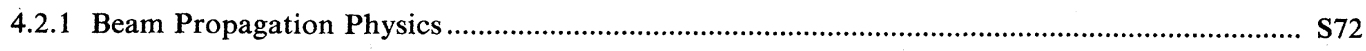

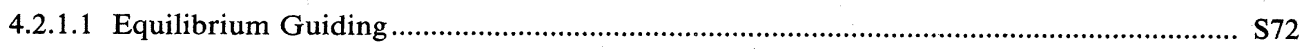

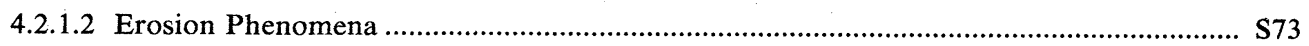

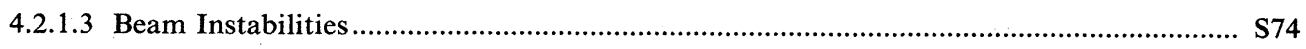

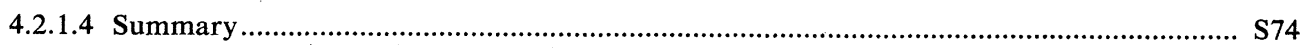

4.2.2 Laser Technology and Ionization Requirements ….......................................................... S75

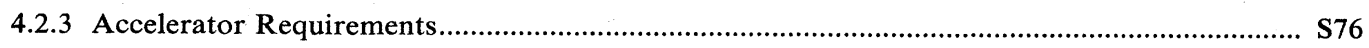

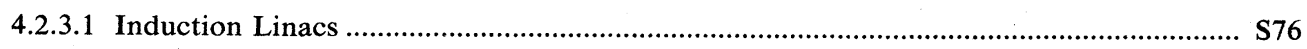

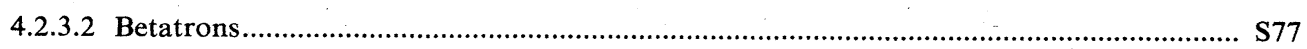

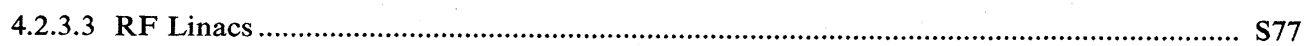

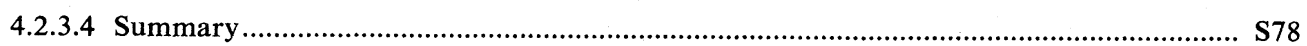

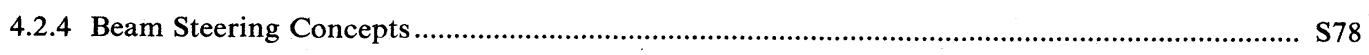




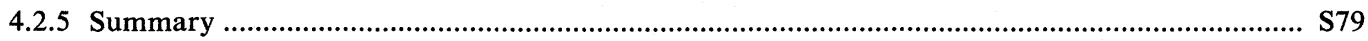

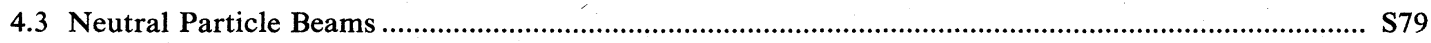

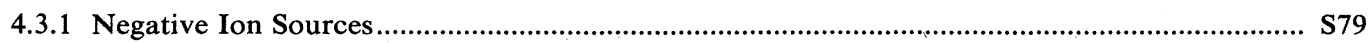

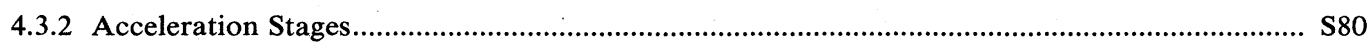

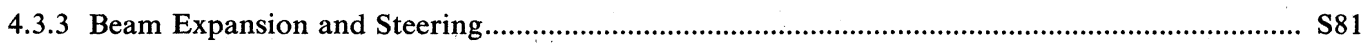

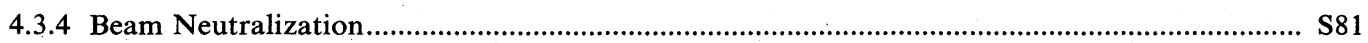

4.3.5 Sensing Direction for Neutral Particle Beams................................................................ S83

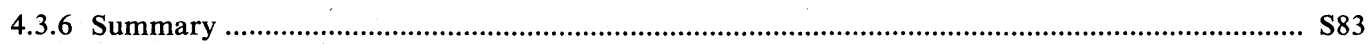

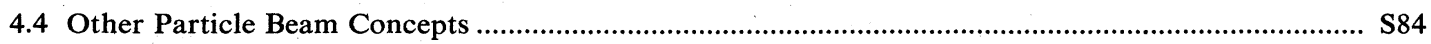

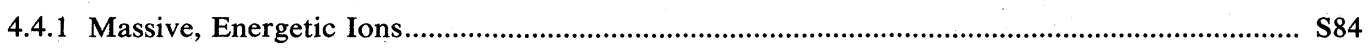

4.4.2 Charge- and Current-Neutralized Ion Beams (Plasmoids) ................................................. S84

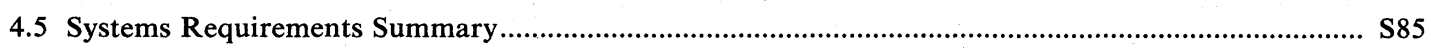

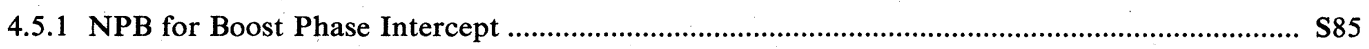

4.5.2 Laser-Guided Electron Beam for Mid-Course Discrimination.............................................. S86

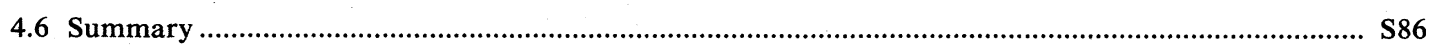

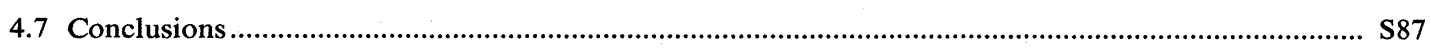

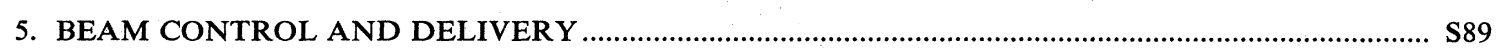

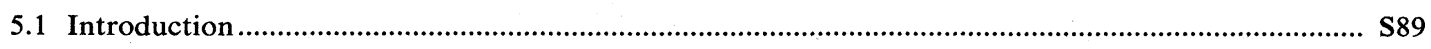

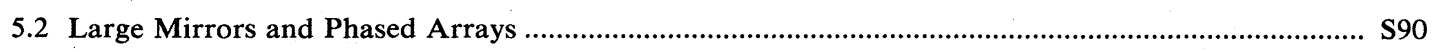

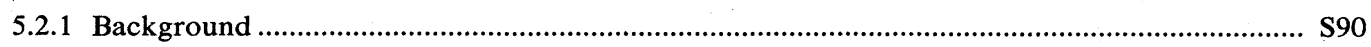

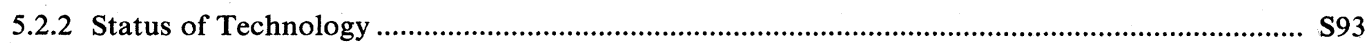

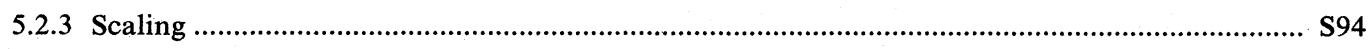

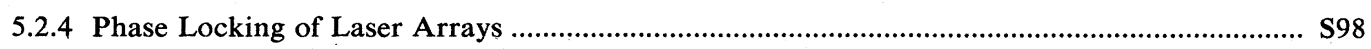

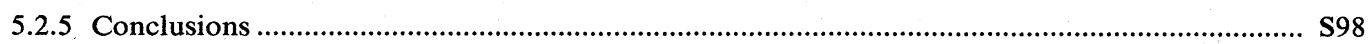

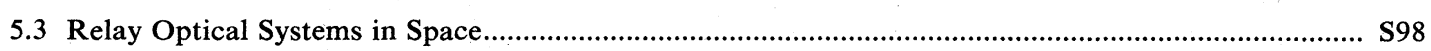

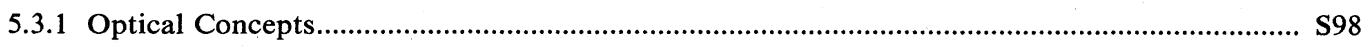

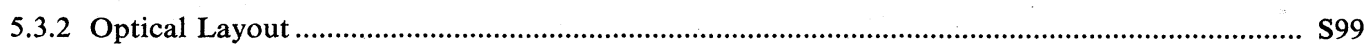

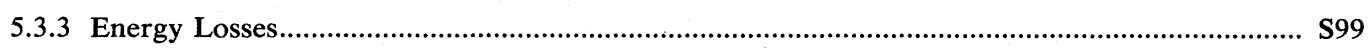

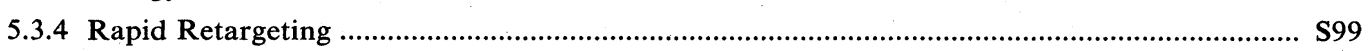

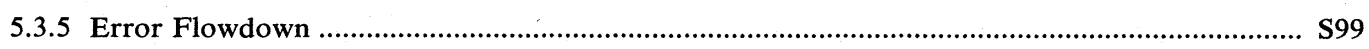

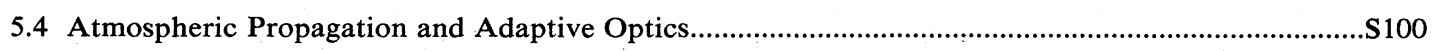

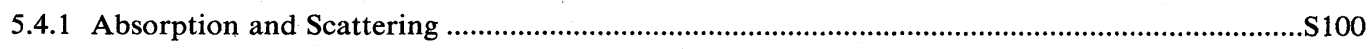

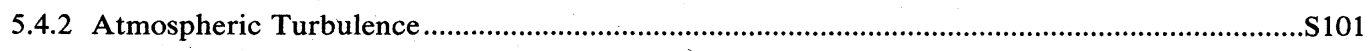

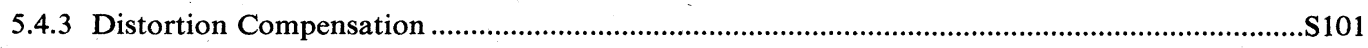

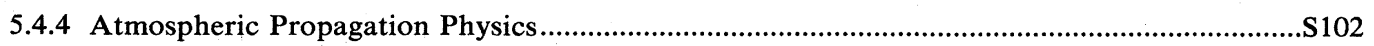

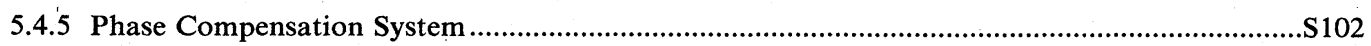

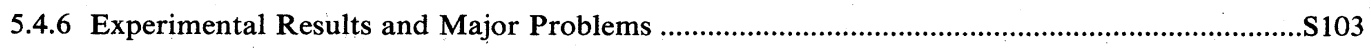

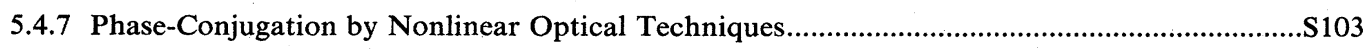

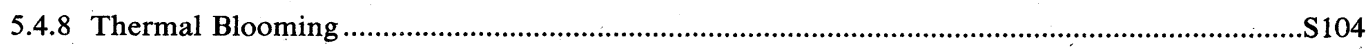

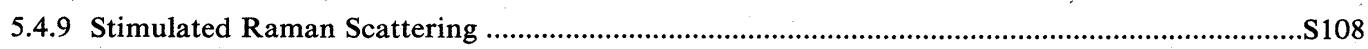

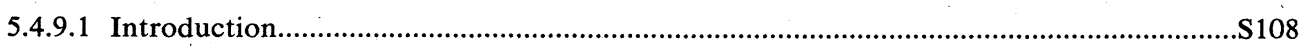

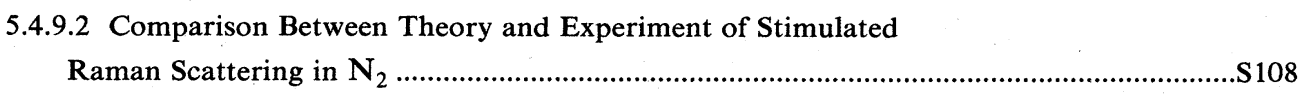

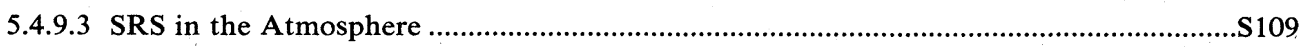

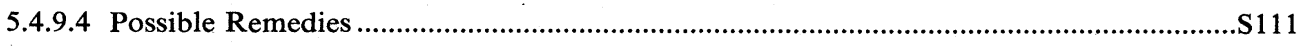

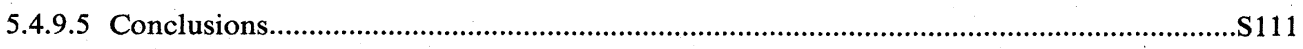

5.4.10 Atmospheric Propagation of High-Intensity X-Ray Pulses ................................................S111

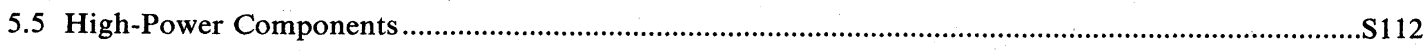

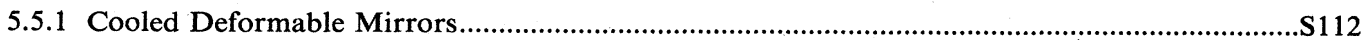




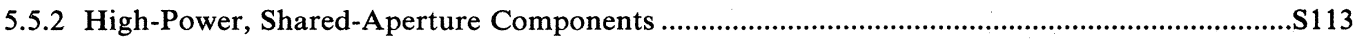

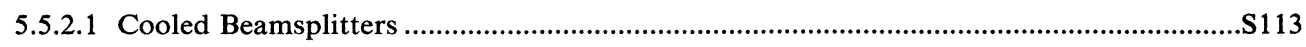

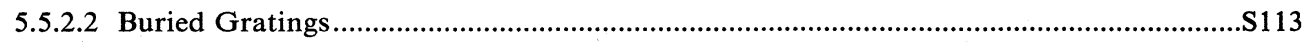

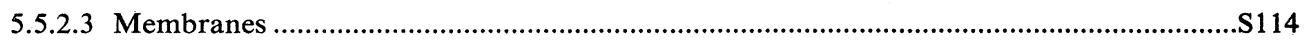

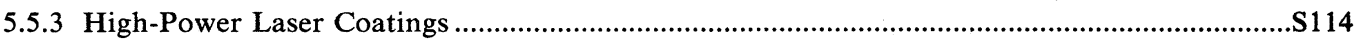

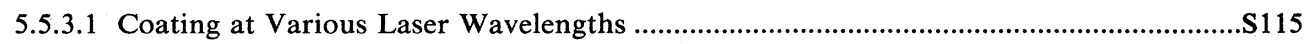

5.5.3.2 Advanced Deposition Processes.....................................................................................115

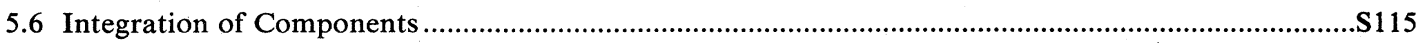

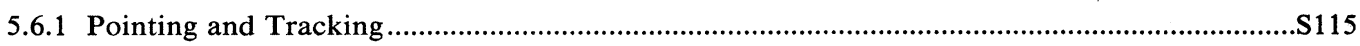

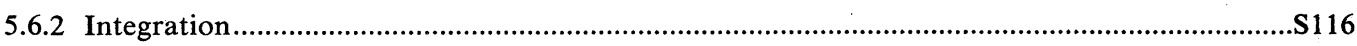

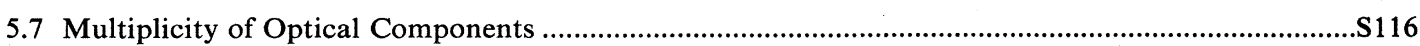

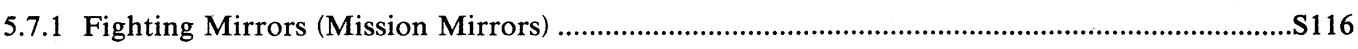

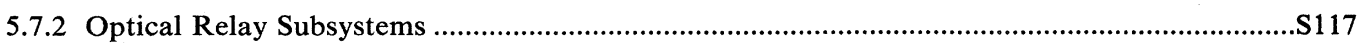

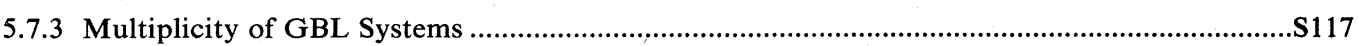

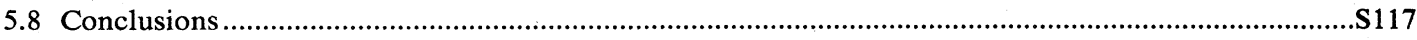

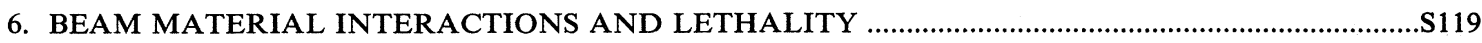

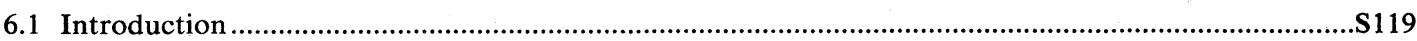

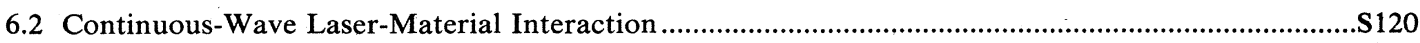

6.2.1 Melt-Through of a Metal Plate.............................................................................................

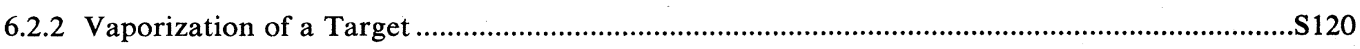

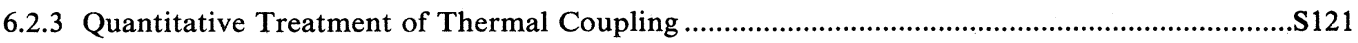

6.2.4 Heating and Plasma Formation by Repetitively Pulsed Lasers..............................................S122

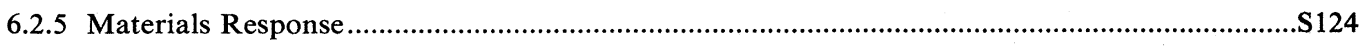

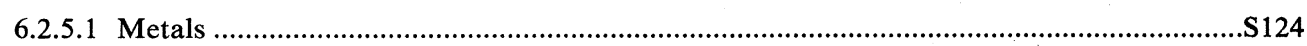

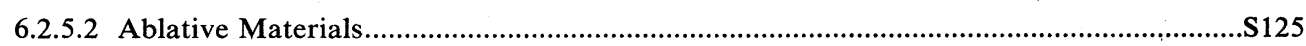

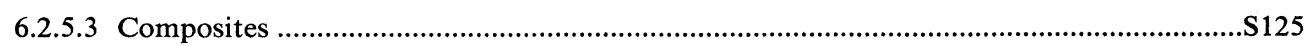

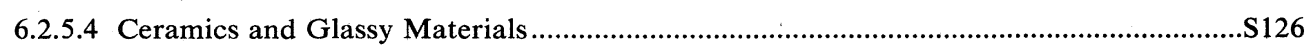

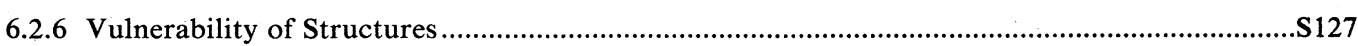

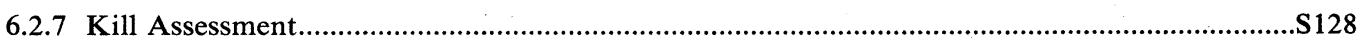

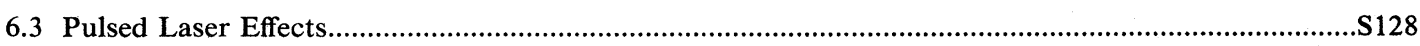

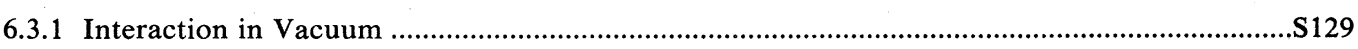

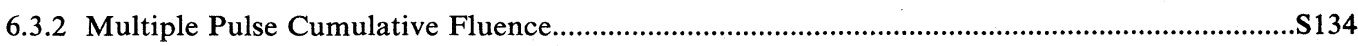

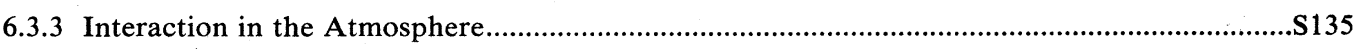

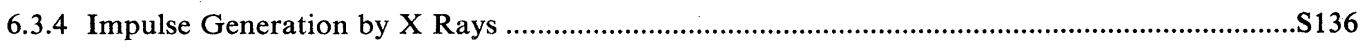

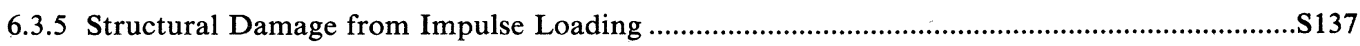

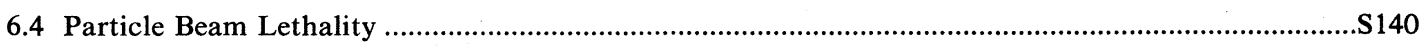

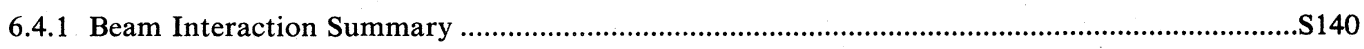

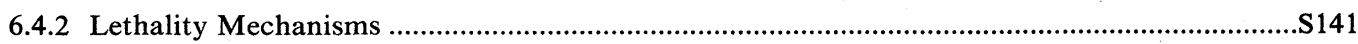

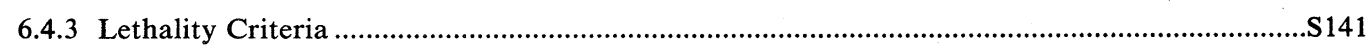

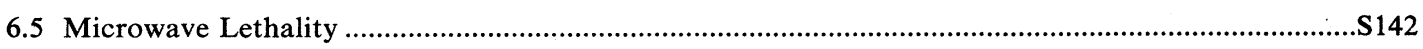

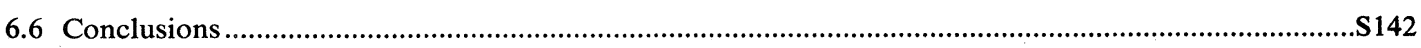

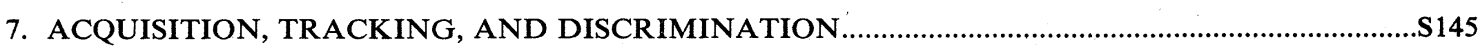

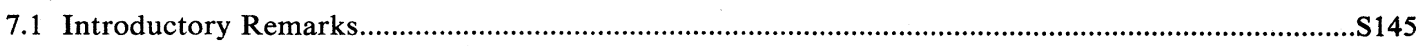

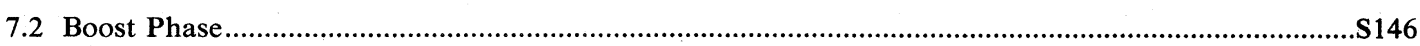

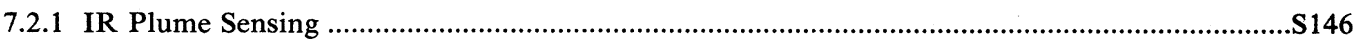

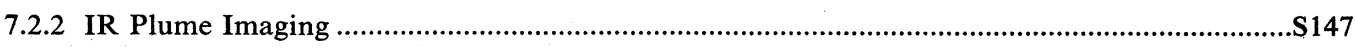

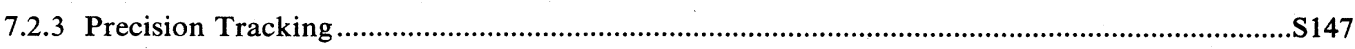

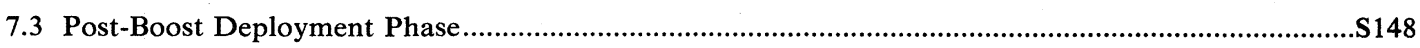

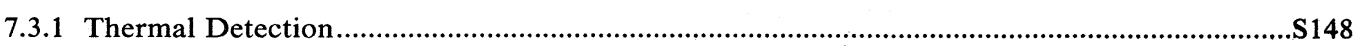

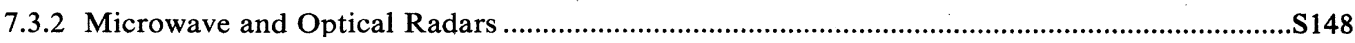




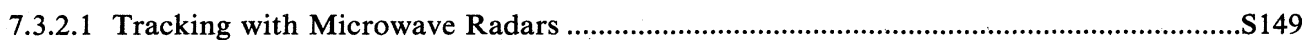

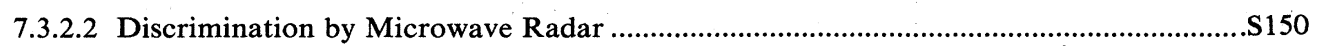

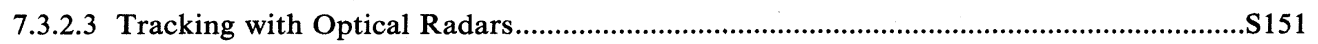

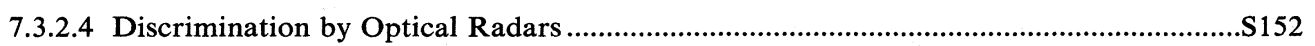

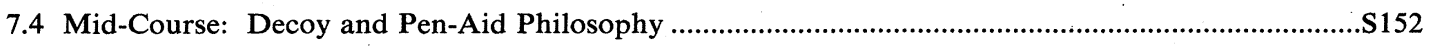

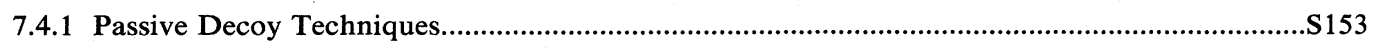

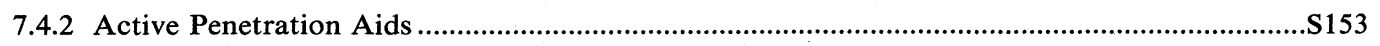

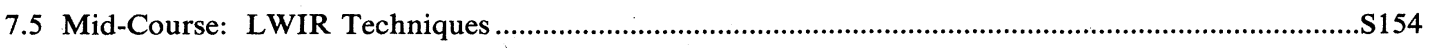

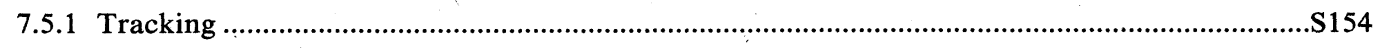

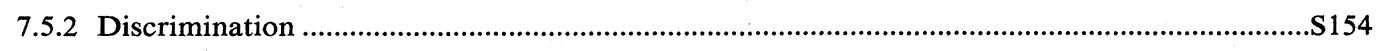

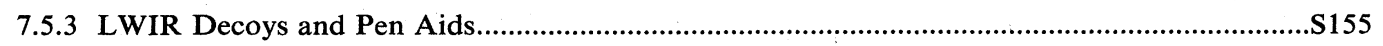

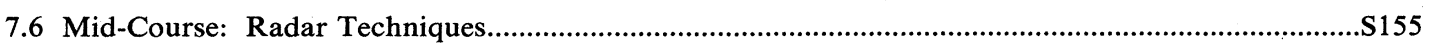

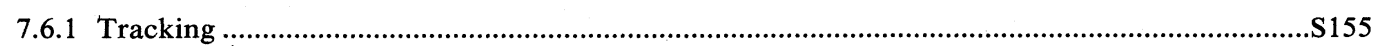

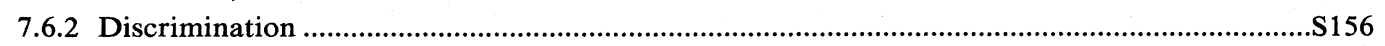

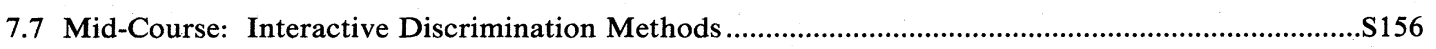

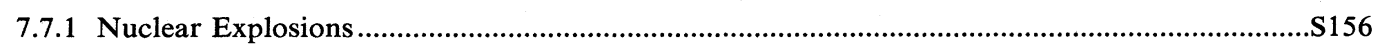

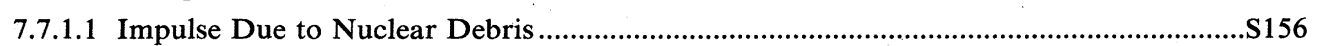

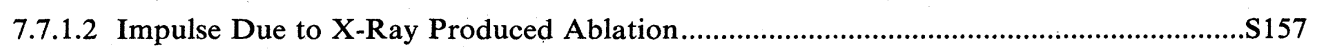

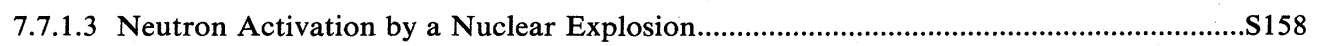

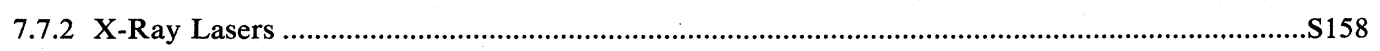

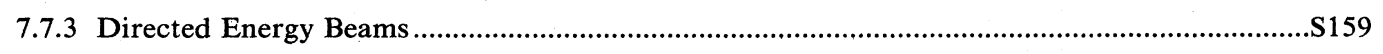

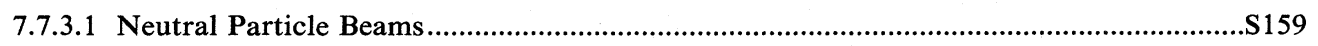

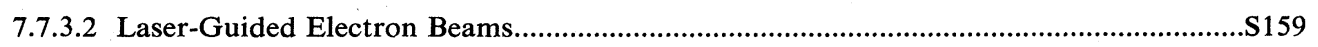

7.7.3.3 Laser-Induced Impulse and Target Destruction ..........................................................S160

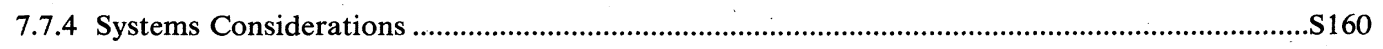

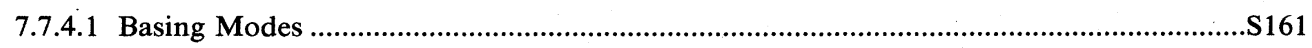

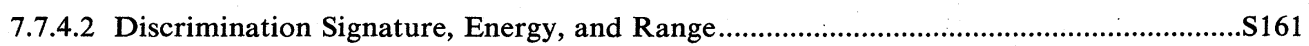

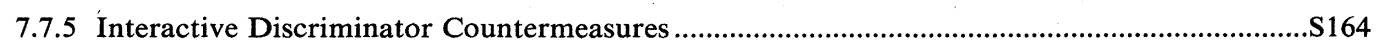

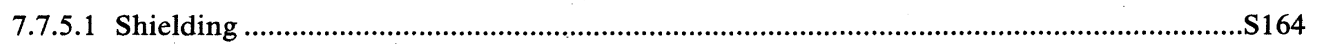

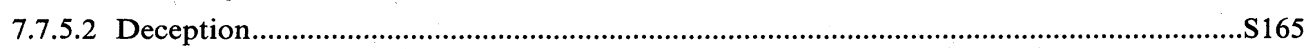

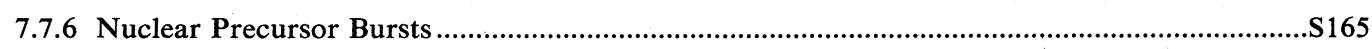

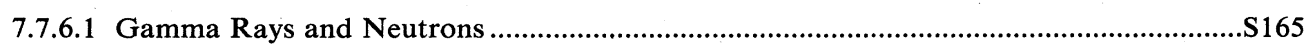

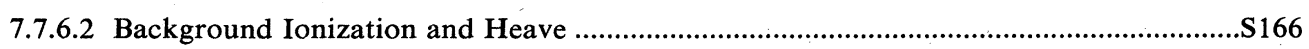

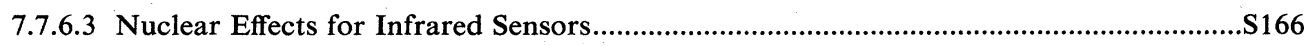

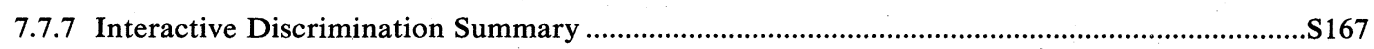

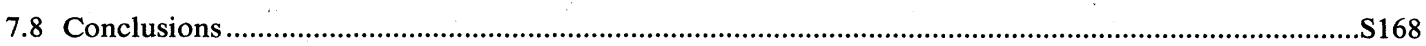

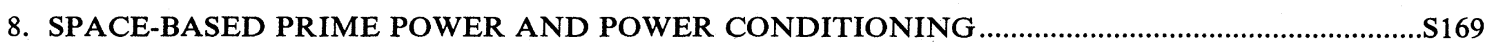

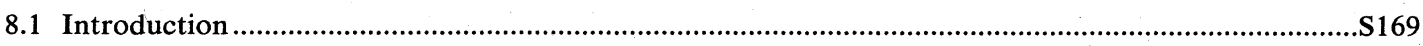

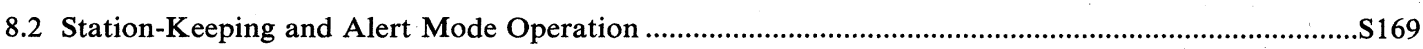

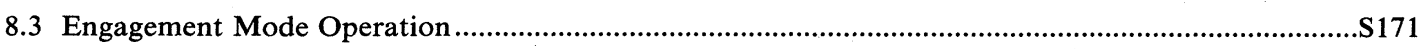

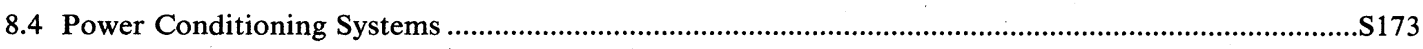

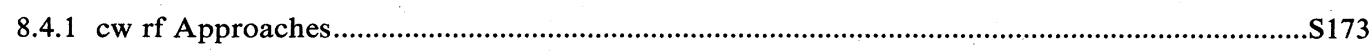

8.4.2 Pulsed Power Induction Linac Approaches......................................................................... 174

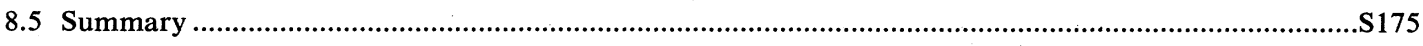

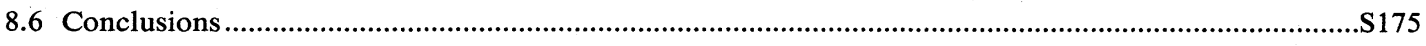

APPENDIX 8.A: NEW DEVELOPMENT IN BATTERY TECHNOLOGY ….......................................

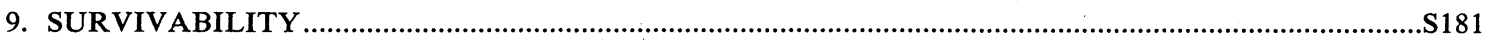

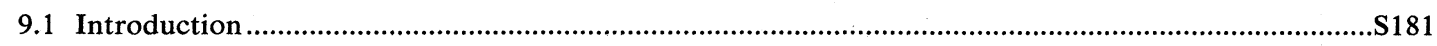

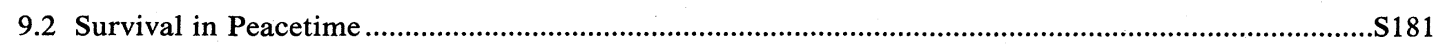

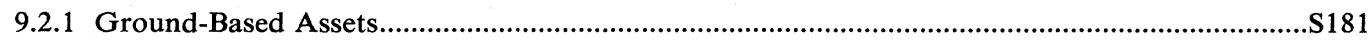




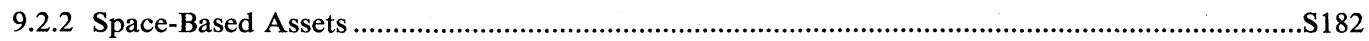

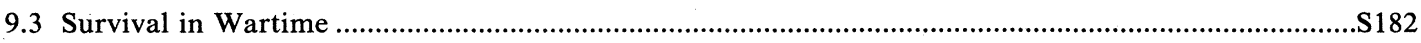

9.3.1 Survivability of Space-Based Components ..........................................................................S183

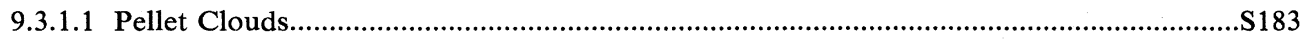

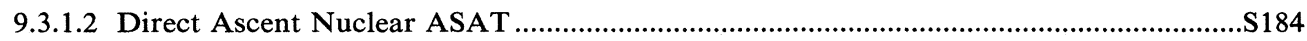

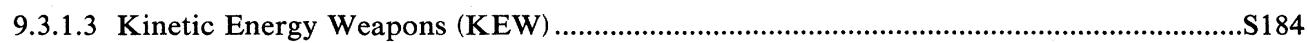

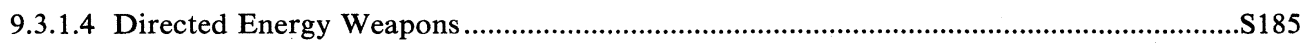

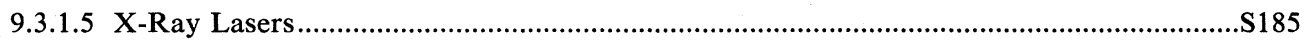

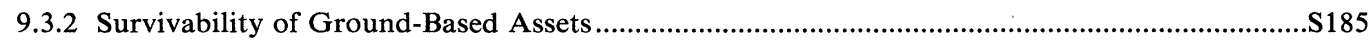

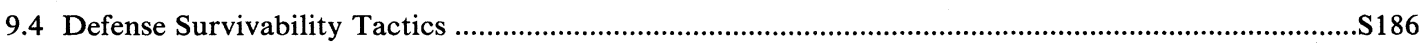

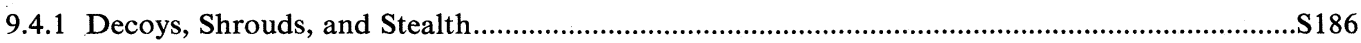

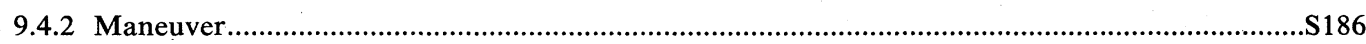

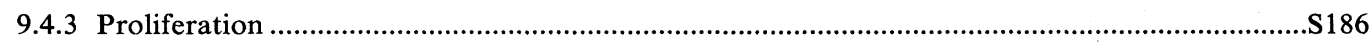

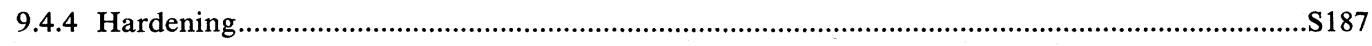

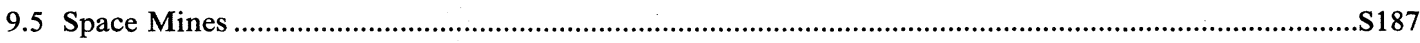

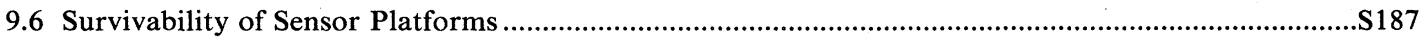

9.6.1 Infrared, Longwave Infrared, and Optical Sensor Platforms.................................................S187

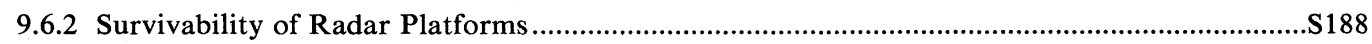

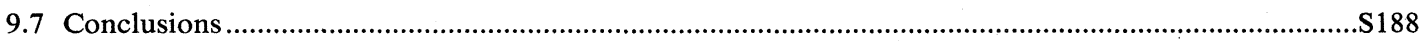

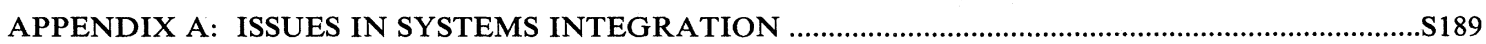

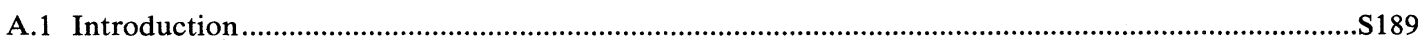

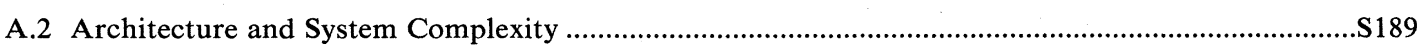

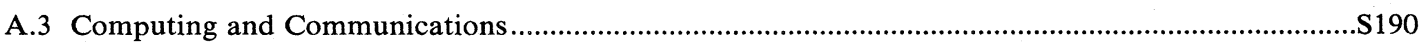

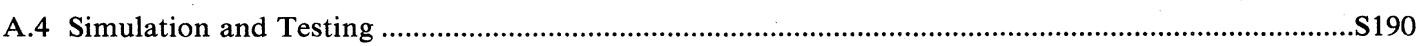

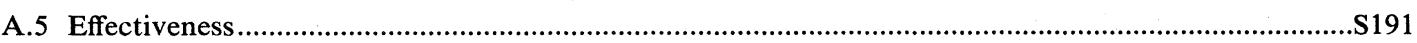

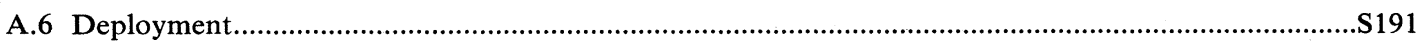

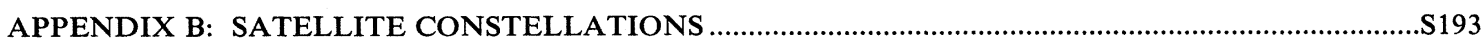

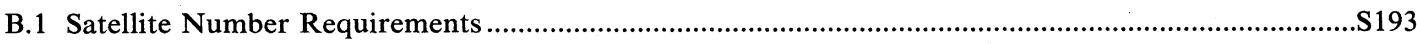

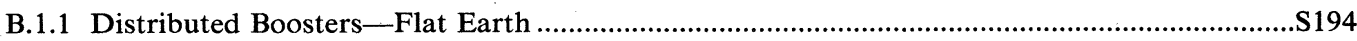

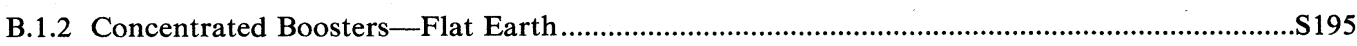

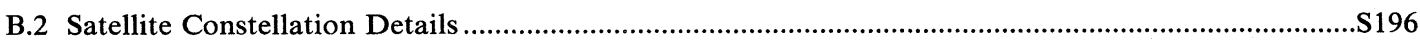

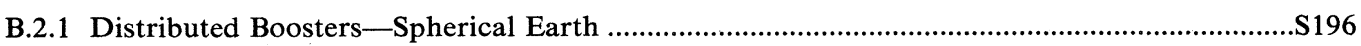

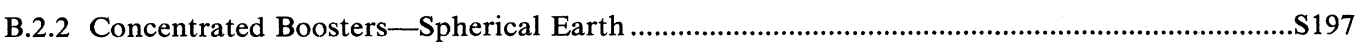

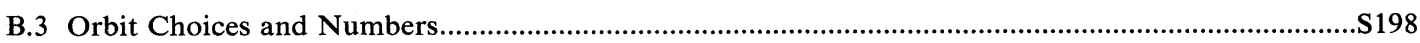

APPENDIX C: APS COMMITTEE ON THE STUDY

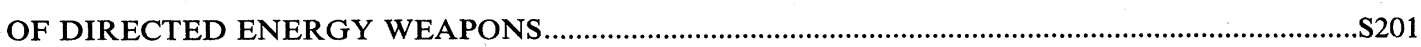




\section{EXECUTIVE SUMMARY AND MAJOR CONCLUSIONS}

The American Physical Society (APS) convened this Study Group to evaluate the status of the science and technology of directed energy weapons (DEWs). The evaluation focuses on a variety of lasers and energetic particle beam technologies for their potential applications to the defense against a ballistic missile attack. This action by the APS was motivated by the divergence of views within the scientific community in the wake of President Reagan's speech on March 23, 1983 in which he called on the U.S. scientific community to develop a system that "...could intercept and destroy strategic ballistic missiles before they reach our soil... ." Directed energy weapons were expected to play a crucial role in the ballistic missile defense (BMD).

The APS charged the Study Group to produce an unclassified report, which would provide the membership of the Society, other scientists and engineers, as well as a wider interested audience, with basic technological information about DEWs. It is hoped that this report, detailing the current state of the art and the future potential of DEWs for strategic defense purposes, will serve as a technical reference point for better-informed public discussions on issues relating to the Strategic Defense Initiative.

The study concentrated on the physical basis of high intensity lasers and energetic particle beams as well as beam control and propagation. Further, the issues of target acquisition, discrimination, beam-material interactions, lethality, power sources, and survivability were studied.

The technology of kinetic energy weapons (KEWs) is not explicitly reviewed, but the role of space-based KEWs in support of DEW systems is considered in the report where appropriate. Further, many important issues concerning command, control, communication, and intelligence $\left(\mathrm{C}^{3} \mathrm{I}\right)$, computing hardware, software creation and reliability for battle management, and overall system complexity have been identified but not discussed in detail. Other issues, which were recognized but not addressed, include manpower requirements, costs and cost-effectiveness, arms control and strategic stability, and international and domestic policy implications.

DEW technology is considered in BMD applications both for mid-course discrimination between decoys and reentry vehicles, and for kill in the boost phase, postboost phase, and mid-course phase of ICBMs. Such consideration has become serious because of numerous technological advances during the past decade in DEW technologies. Although the achievement of an effective defense of the entire nation may require a substantial boost phase intercept component, other strategic defense scenarios, including discrimination for hard point defense purposes, would place less demanding requirements on DEW systems. The Study Group deemed it important to describe the current state of the art in DEW technology, and to evaluate it with respect to substantial boost phase intercept and mid-course discrimination roles.

\begin{abstract}
Although substantial progress has been made in many technologies of DEWs over the last two decades, the Study Group finds significant gaps in the scientific and engineering understanding of many issues associated with the development of these technologies. Successful resolution of these issues is critical for the extrapolation to performance levels that would be required in an effective ballistic missile defense system. At present, there is insufficient information to decide whether the required extrapolations can or cannot be achieved. Most crucial elements required for a DEW system need improvements of several orders of magnitude. Because the elements are inter-related, the improvements must be achieved in a mutually consistent manner. We estimate that even in the best of circumstances, a decade or more of intensive research would be required to provide the technical knowledge needed for an informed decision about the potential effectiveness and survivability of directed energy weapon systems. In addition, the important issues of overall system integration and effectiveness depend critically upon information that, to our knowledge, does not yet exist.
\end{abstract}

The following observations elaborate on the above finding.

We estimate that all existing candidates for directed energy weapons (DEWs) require one or more orders of magnitude (powers of 10) improvements in power output and beam quality before they may be seriously considered for application in ballistic missile defense systems. In addition, many supporting technologies such as space power, beam control and delivery, sensing, tracking, and discrimination need similar improvements over current performance levels before DEWs could be considered for use against ballistic missiles.

Directed energy weapon candidates are currently in varied states of development. Among the many possibilities, infrared chemical lasers have been under study for the longest period and several high power laboratory models have been built. However, because of their long wavelengths and other technical features, these lasers are perceived to be less attractive candidates for BMD weapons even though they are closest to the 
required performance levels in a relative sense. Free electron lasers and excimer lasers are currently perceived as more attractive candidates for BMD missions; but few high power laboratory models have been operated, and the scaling required to reach relevant power levels is estimated to be greater than that for chemical lasers. Nuclear-explosion-pumped $\mathrm{x}$-ray lasers, although the subject of much public discussion, are currently under study at the research level. In our opinion their BMD potential is uncertain. ${ }^{1}$ Charged and neutral particle beam devices build on an existing base of accelerator technology but require considerable extrapolations beyond current performance levels.

Supporting technologies are also in varied states of development. In many areas, research is progressing at a rapid pace; for example, schemes for rapid steering of optical beams, and active systems for tracking to microradian class or better. ${ }^{2}$ Other critical technologies, such as the techniques for interactive discrimination, are being conceived and addressed. The same caution described above for DEWs applies here, namely, proposed supporting technologies need to be systematically studied before their performance at parameter levels appropriate to BMD applications can be realistically evaluated.

Like any defensive system an effective DEW defensive system must be able to handle an evolving and unpredictable missile threat. In addition to retrofit and redesign of the missiles themselves, decoys and other effective penetration aids can be developed by the offense over the long times required to develop and deploy ballistic missile defenses. In contrast to the technical problems faced in developing DEWs capable of boost phase kill for defense systems, the options available to the offense, including direct attacks on DEW platforms, may be less difficult and costly to develop and may require fewer orders-of-magnitude performance improvements.

A successful BMD system must survive, but survival of high value space-based assets is problematic. Ground-based assets of DEW systems are also subject to threats. Architectures which address the responsive threat are still in their infancy. As an overall BMD system employing directed energy weapons becomes more complex, the currently unresolved issues of computability, testability, and predictability become increasingly critical.

For directed energy weapons to have an important role as a kill mechanism in a strategic defense system designed to defend the entire nation against a ballistic missile attack the following requirements need to be met.

\section{For operations in the boost and post-boost phases:}

A. Sufficient power/energy from the directed energy weapons to kill the ballistic missile in

\footnotetext{
1"X-ray Lasers for Missile Defense," Defense Science and Engineering, November 1986, pp. 17-19.

${ }^{2}$ U.S. Congress, Office of Technology Assessment, Ballistic Missile Defense Technologies, OTA-ISC-254 (U.S. Government Printing Office, Washington, D.C., September 1985).
}

the boost phase, or to kill the post-boost vehicle during the deployment phase.

B. Sufficient beam quality, pointing accuracy, and agility (retargetability) to deliver lethal powers or energies to targets within the available engagement time provided by the system.

C. For lasers, optical systems for transmitting beams from sources to targets.

D. Accurate detection, location of the booster in its plume, and precision tracking from detection until kill is accomplished.

E. Reliable kill verification.

\section{For operations during the mid-course:}

A. Reliable means of discrimination between reentry vehicles and decoys unless all objects can be destroyed.

B. Accurate detection, tracking of a very large number of objects in mid-course flight, and kill verification.

C. Rapid retargeting and sufficient delivered power/energy from the DEW to destroy the reentry vehicles.

\section{For terminal phase:}

We do not expect DEWs to play an important role in the terminal phase of the trajectory of ballistic missiles.

\section{For space-based elements:}

A. Nuclear reactors or other means to supply adequate electrical power for housekeeping functions.

B. Adequate burst power for operation of DEWs during engagements.

C. Space qualified reliability of all components and subsystems on the platform notwithstanding long periods of dormancy.

\section{For system survivability:}

A. DEW must be able to operate in a hostile environment during a conflict.

B. DEW must be integrated in an overall system that includes a survivable command, control, communication, and intelligence $\left(\mathrm{C}^{3} \mathrm{I}\right)$ system.

We have examined most of these issues in some detail, except for items III, IV.C and V.B. The following major conclusions are based on detailed considerations in the main body of the report indicated by relevant section numbers in parentheses.

1. We estimate that chemical laser output powers at acceptable beam quality need to be increased by at least one order of magnitude for HF/DF lasers for use as an effective kill weapon in the boost phase. Similarly for atomic iodine lasers, at least five ders of magnitude improvement is necessary. 
The HF/DF cw chemical lasers have been stated to yield power levels exceeding $1 \mathrm{MW}$ with acceptable beam quality. ${ }^{3}$ Based on these data, we estimate that even the least demanding strategic defense applications require power levels to be increased further by at least a factor of twenty while retaining beam quality. However, the laser geometry which achieved the above demonstration will have scaling problems to higher power levels; thus, the combination of power scaling and adequate beam quality must be explored for some different chemical laser design, yet to be demonstrated. A chemically pumped atomic iodine laser at $1.3 \mu \mathrm{m}$ has been developed, although at this point only $5 \mathrm{~kW}$ of continuous wave power has been demonstrated. Because of atmospheric absorption, the HF laser $(\lambda=2.8 \mu \mathrm{m})$ would have to be deployed on space platforms, while the DF laser $(\lambda=3.8 \mu \mathrm{m})$ and the atomic iodine laser $(\lambda=1.3 \mu \mathrm{m})$ could also operate on the ground. When based in space, chemical lasers face a special set of problems arising from vibrations and the exhaust of the burnt fuel (Section 3.2).

2. We estimate that the pulse energy from excimer lasers for strategic defense applications needs improvement by at least four orders of magnitude over that currently achieved. Many advances are needed to achieve the required repetititve pulsing of these lasers at full scale.

The pulsed excimer lasers have demonstrated single pulse energies of about $10 \mathrm{~kJ}$ in $1 \mu$ s pulses from a single module 4 (Section 3.3). This laser currently uses krypton fluoride $(\lambda=249 \mathrm{~nm})$; the other principal contender excimer species is xenon chloride $(\lambda=308 \mathrm{~nm})$. From our estimates, assuming an overall propagation loss factor of four (relay mirror losses, Rayleigh scattering losses, and atmospheric losses), ground-based excimer lasers for strategic defense applications must produce at least $100 \mathrm{MJ}$ of energy in a single pulse or pulse train with a total duration between several and several hundred microseconds (Section 6.3). To kill multiple targets a firing rate of ten per second would be desirable. For thermal kill $1 \mathrm{GW}$ of average power would be required (Section 6.2). The gap of four orders of magnitude might be bridged by first combining lasers into modules at the hundreds of kilowatt level, then combining many modules optically. To produce high optical quality beams from the modules, the output from low optical quality amplifier apertures may be combined using stimulated Raman scattering or other means (Section 3.3). We estimate that the techniques for Raman beam combination must be scaled up by two orders of magnitude or more in combined laser power and efficiency from that which has been demonstrated in the laboratory. The technology for phase locking a large number of modules is not yet demonstrated (Section 5.4).

\footnotetext{
${ }^{3}$ See Reference 19 of Chapter 3.

${ }^{4}$ See Reference 39 of Chapter 3.
}

3. Free electron lasers suitable for strategic defense applications, operating near $1 \mu \mathrm{m}$, require validation of several physical concepts.

The free electron laser (FEL) is one of the newest laser technologies to be demonstrated. Peak powers of approximately $1 \mathrm{MW}$ have been produced at a wavelength of $1 \mu \mathrm{m}$; peak powers of approximately $1 \mathrm{GW}$ have been produced at a wavelength of $8 \mathrm{~mm}$, demonstrating high gain and high efficiency at that wavelength. ${ }^{5}$ Scaling to short wavelengths at high powers is a more difficult technical problem than simply increasing average power. Obtaining high efficiency, high power free electron laser operation at $1 \mu \mathrm{m}$ requires experimental verification of physical concepts which thus far are only theoretically developed, e.g., optical guiding and transverse sextupole focusing for the amplifier configuration, and sideband and harmonic control for the oscillator configuration. ${ }^{6}$ We estimate that for strategic defense applications, a groundbased free electron laser should produce an average power level of at least $1 \mathrm{GW}$ at $1 \mu \mathrm{m}$ wavelength, corresponding to peak powers of 0.1-1.0 TW (Sections 3.4 and 6.3).

\section{Nuclear-explosion-pumped $x$-ray lasers require validation of many of the physical concepts before their application to strategic defense can be evaluated. $^{7}$}

A subcommittee of the Study Group reviewed the progress in $\mathrm{x}$-ray lasers. A nuclear-explosion-pumped $\mathrm{x}$ ray laser has been demonstrated. This is a research program where numerous physics and engineering issues are still being examined. What has not been proven is whether it will be possible to make a militarily useful $\mathrm{x}$ ray $\operatorname{laser}^{7}$ (Section 3.5). Atmospheric interaction limits the use of nuclear-explosion-pumped $\mathrm{x}$-ray lasers to altitudes greater than about $80 \mathrm{~km}$ (Section 5.10). The high energy-to-weight ratio of the nuclear explosives makes it possible for these devices to be considered for “pop-up" deployment (Section 9.3).

5. We estimate that neutral particle beam (NPB) accelerators operating at the necessary beam current levels ( $\geq 100 \mathrm{~mA}$ ) must be scaled up by two orders of magnitude in voltage and duty cycle with no increase in normalized beam emittance. The required pointing accuracy and retargeting rates remain to be achieved. These devices must be based in space to avoid beam loss via atmospheric interactions.

\footnotetext{
${ }^{5}$ T. J. Orzchowski et al., Phys. Rev. Lett. 57, 2172-2174 (1986). ${ }^{6}$ See Reference 74 in Chapter 3.

${ }^{7}$ E. Walbridge, "Angle Constraint for Nuclear Pumped X-ray Laser Weapons," Nature 310, 180-182 (1984), and references cited therein; George Miller (Associate Director, Lawrence Livermore National Laboratory) quoted in "Experts Cast Doubt on X-ray Lasers," Science 230, 647 (1985).
} 
Structural kills with NPB devices require an equivalent charge of about 1 Coulomb (e.g., $100 \mathrm{~mA}$ for $10 \mathrm{~s}$ ) delivered at a few hundred $\mathrm{MeV}$, with a beam divergence of $0.75-1.5 \mu \mathrm{rad}$ (as discussed and calculated in Sections 4.3 and 6.4). Disruption of electronic function because of radiation dose could occur at significantly lower beam parameters, although this kill mechanism is system dependent, and kill assessment may be difficult (Chapter 4).

Existing radio frequency ( $r f$ ion accelerators have achieved particle kinetic energies of several hundred $\mathrm{MeV}$, but at beam current levels two orders of magnitude below the required levels (Section 4.3). New negative ion sources have achieved the necessary peak currents and low beam emittances, but such sources have not been reported to operate continuously. Additional issues are emittance growth of the high current beams in the first part of the accelerator, and the development of large bore magnetic optics. Power requirements and weight are also significant issues (Chapter 8).

Ionization of the neutral beam atoms via atmospheric collision (and subsequent ion deflection in earth's magnetic field) establishes a minimum operating altitude of about $120 \mathrm{~km}$ for beam kinetic energies of a few hundred $\mathrm{MeV}$ (Section 4.1).

NPB devices have been suggested for use in an interactive mid-course discrimination mode (identifying massive reentry vehicles in a postulated threat cloud which includes light weight decoys). In this case the beam power requirements will not change significantly, but the target dwell times may be reduced by a factor of 10-1000 compared to boost phase kill requirements, and retargeting rates of $>10 \mathrm{~s}^{-1}$ may be necessary. Hence, device issues which will require new ideas and further exploration for this mission are development of rapid retargeting mechanisms using magnetic beam steering and fast accurate methods for beam direction sensing (Section 7.7).

6. Energetic electron beams require propagation in laser-created plasma channels in order to avoid beam deflection in the earth's magnetic field; this restricts the operational altitude at the low end by beam instability and at the high end by ion density starvation. We estimate that booster kill applications require a scale-up in accelerator voltage by at least one order of magnitude, in pulse duration by at least two orders of magnitude, and in average powers by at least three orders of magnitude. Active discrimination applications require scale-up in pulse duration by at least two orders of magnitude, and in average power by at least two orders of magnitude. The lasers needed for the creation of plasma channels require development. We estimate that propagation distances must be increased by several orders of magnitude.

Propagation through a laser-created plasma channel is necessary to prevent beam space-charge blow-up and beam bending in the earth's magnetic field. This implies both a lower and an upper altitude operational limitations. The lower bound arises from beam stability considerations, while the upper bound results from ion density starvation. This mechanism for beam guiding has been successfully demonstrated in the laboratory, but over distances of only $95 \mathrm{~m}^{8}$ (Section 4.2 ). For optimum beam currents of a few kiloamperes, delivering lethal pulses to distances in excess of $1000 \mathrm{~km}$ will require beam kinetic energies of several hundred $\mathrm{MeV}$. Useful ranges for some suggested interactive discrimination applications could be as small as a few hundred kilometers, in which case the particle energy requirement would decrease by an order of magnitude (Section 7.7). Existing linear induction accelerators have demonstrated the necessary peak power capability (tens of $\mathrm{MeV}$ at peak currents of tens of kiloamperes and pulse repetition rates of a few hertz), although not for required pulse lengths of microseconds (Section 4.2). Although several approaches have been suggested, the laser technologies required for creating the plasma channel have not been demonstrated. Because of the limited engagement space, rapid retargeting $(\sim 0.1 \mathrm{sec})$ and high repetition rates $(>10$ $\mathrm{Hz}$ ) are essential.

7. Phase correction techniques are required for obtaining near diffraction limited performance of most types of laser weapon devices. Further, phase control techniques are required for coherently combining outputs from different modules in a multiple laser system into a single diffraction limited beam. These techniques, demonstrated at low powers, must be scaled up by many orders of magnitude in power.

High power laser systems are likely to require active control and correction of the optical phase of the output beam to reach the nearly diffraction limited performance desired for strategic defense applications. Several techniques are available for these purposes. These include correction of slowly varying phase errors with low spatial frequencies through use of adaptive optics and selfcorrection of phase errors using nonlinear phase conjugation techniques, such as stimulated Brillouin scattering, or four-wave mixing; and combining beams from multiple apertures by phase locking of multiple laser modules, or through stimulated Raman scattering. Each of the laser technologies under development may use different types of phase corrections. All of these approaches for phase correction have been demonstrated

${ }^{8}$ G. J. Caporaso, F. Rainer, W. E. Martin, D. S. Prono, and A. G. Cole, "Laser Guiding of Electron Beams in Advanced Test Acceleration," Phys. Rev. Lett. 57, 1591-1594.(1986). 
on a laboratory scale, but extensions to high power systems and large apertures remain to be demonstrated (Section 5.4).

8. Dynamic phasing of arrays of telescopes requires extensive development in order to obtain large effective aperture optical systems. As calculations indicate (Section 5.4.5), the number of phase correcting elements must be increased by at least two orders of magnitude over currently demonstrated values.

Optical laser systems will require large effective optical apertures in order to achieve the necessary beam intensity on target. Such radiating apertures have to provide near diffraction limited beams which can be rapidly retargeted. The state of the art for ground-based monolithic telescope primaries for astronomical applications is about $8 \mathrm{~m} .^{9}$ Torque requirements for rapid steering of large telescopes limit monolithic telescopes to approximately $8 \mathrm{~m}$ aperture; the larger "effective aperture" primaries have to be synthesized by dynamically phasing a number of smaller telescopes. Such phasing of a number of telescopes has been accomplished ${ }^{10}$ by dynamically controlling the wavefront "piston," tilt, and focus of the laser beams feeding each telescope of the array. This adds complexity to the system but allows beam pointing in terms of target tracking without requiring slewing of telescopes (Section 5.2).

The phase front of the outgoing wave is monitored in such phasing schemes, and corrections are applied via electrically driven actuators. Components for control of about several hundred such actuators are commercially available. For the large apertures contemplated for BMD applications the number of actuators needed lies between ten thousand and one hundred thousand, a substantial extrapolation. The technology of phase-controlling an array of primary mirrors is in an early stage of development. Scaling of such arrays to high power has not been accomplished (Section 5.4).

An alternative approach is to use telescopes where the primaries are made out of single large flexible membranes which are appropriately distorted by many actuators. The concept has been demonstrated only for small flexible primaries at low powers. Extensions to larger mirrors at higher powers remains to be shown (Section 5.4).

9. The optical coatings of large primary mirrors are particularly vulnerable in space-based optical systems.

The large primary mirror, which directs the laser beam towards the target, is particularly vulnerable to radiation from other lasers (from any direction) (Section 5.6). Based on dicussions with commercial vendors, we find that the $\mathrm{cw}$ power loading threshold for reflective coatings

\footnotetext{
${ }^{9} \mathrm{C}$. H. Townes (private communication).
}

${ }^{10}$ See References 2 and 3 of Chapter 5 . is about $100 \mathrm{~kW} \mathrm{~cm}^{-2}$. For laser pulses of a few microseconds or less, the damage threshold will be about $8 \mathrm{~J} \mathrm{~cm}^{-2}$ of absorbed energies, corresponding to peak powers of $10 \mathrm{MW} \mathrm{cm} \mathrm{cm}^{-2}$. These damage thresholds are for operation at a nominal laser wavelength of $3 \mu \mathrm{m}$ (Section 6.2). If attacked by lasers at other wavelengths in the visible, near ultraviolet (UV), or x-ray region, the damage threshold may be significantly lower. Further, there is a possibility of damage to the high reflectivity coatings from energetic particles in the ambient background, i.e., $\mathrm{MeV}$ protons and electrons, during long term residence of the high reflectivity mirrors in space.

10. Small secondary mirrors in the optical trains of high power lasers will need very low absorptivity coatings and will have to be cooled.

The requisite power levels for ballistic missile defense lethality will necessitate cooling of the small mirrors in the optical train of high power lasers to prevent damage. A beam power of $1 \mathrm{GW}$ on a mirror of $100 \mathrm{~cm}^{2}$ area implies an incident power of $10^{7} \mathrm{~W} \mathrm{~cm}^{-2}$. High reflectivity coatings with less than $10^{-4}$ absorptivity are needed. Such mirrors have been demonstrated, and lead to an absorbed power of $1 \mathrm{~kW} \mathrm{~cm}^{-2}$. Cooled silicon or silicon carbide mirrors show promise for raising this threshold (Section 5.5).

11. Ground-based laser systems for BMD applications need geographical multiplicity to deal with adverse weather conditions.

For each ground-based laser system which must be available in battle, a number of geographically separated laser sites are needed to provide availability of at least one site in the system when the others are obscured by adverse climatic conditions. These locations must be separated by distances greater than the coherence length scale for weather patterns. Based on weather statistics, a multiplicity of five independent ground-based lasers could provide a $99.7 \%$ availability. By going to seven climatically isolated locations in the continental U.S., availability of $99.97 \%$ is possible. At each of these sites, local cloud cover conditions require further multiplicity of the large ground telescopes, separated by few $\mathrm{km}$ (Section 5.4).

12. Ground-based laser systems require techniques for correcting atmospheric propagation aberrations. We estimate that these techniques must be extended by at least two orders of magnitude in resolution (number of actuators) than presently demonstrated. Phase correction techniques must be demonstrated at high powers.

Ground-based laser systems will require either linear or nonlinear adaptive optics of a very sophisticated nature in order to precompensate the laser beam for 
atmospheric aberrations caused by atmospheric turbulence and by thermal blooming induced by the laser beam itself. A retroreflector or a low power laser located at an appropriate point-ahead position in front of a space-based relay mirror would provide a reference source for transmission through the atmosphere to the ground telescope, where the wavefront would be analyzed for acquired aberrations due to the atmosphere. This information would be used to control adaptive optics of high resolution $(\geq 10000$ actuators per aperture) at high bandwidths $(\approx 1.0 \mathrm{kHz})$. This technique requires an extensive computational capability. Such atmospheric compensation experiments have been successfully demonstrated at low powers (no thermal blooming in the atmosphere) and at average atmospheric viewing conditions for Mt. Haleakala, Maui (moderate turbulence) with a small number of actuators $(<100)$. At high power levels, the turbulence may be high enough to cause a beam intensity redistribution which could be uncorrectable (Sections 5.2 and 5.4).

The incorporation of phase correction schemes in pulsed induction linac FEL amplifier is particularly stressing because the atmospheric compensation must be carried at high power levels. Atmospheric compensation techniques are needed for point-ahead angles which are large and for targets which may be noncooperative.

13. Uplink in a ground-based laser system faces transmission losses in the atmosphere.

The uplink of high power output from a ground-based laser system faces natural atmospheric losses such as Rayleigh scattering, which stress the short wavelength systems, and atmospheric absorption losses, primarily from water vapor, which stress the longer wavelength systems. The optimum wavelength region is $0.4-1.0 \mu \mathrm{m}$. Even in this region, nonlinear effects such as stimulated Raman scattering and thermal blooming force the use of large final transmitting optics on ground (Section 5.4).

14. Nonlinear scattering processes in the atmosphere impose a lower limit on the altitude at which targets can be attacked with a laser beam from space.

Power delivery downward through the atmosphere to rising targets may be limited by stimulated Raman scattering and thermal blooming by ozone absorption. These phenomena limit the minimum attack altitude to $80 \mathrm{~km}$ for very short pulses, or require a longer pulselength (1-10 ms), because the laser beam must be focused to a small, $\sim 1 \mathrm{~m}^{2}$, spot size on the target. At the required high laser intensities, nonlinear effects may throw the optical power out of the focused beam before reaching the target (Section 5.4).

15. Detection and acquisition of ICBM launches will pose stringent requirements for high detection probability and low false alarm rates.
The achievement of boost phase kill probabilities of $90 \%$ implies booster detection and acquisition probabilities of better than $90 \%$. In addition, successful operation of a mid-course system depends importantly on being given good booster trajectory information. Of even greater importance, low false alarm rates are required so that a BMD system is not activated in peace time because of the false alarms (Section 7.2).

16. For boost phase, infrared tracking of missile plumes will have to be supplemented by other means to support sub-microradian aiming requirements of DEWs.

Tracking of missiles by detecting the intense short wavelength infrared (SWIR) radiation from booster plumes is a technology which has been pursued for some time. The plume brightness greatly exceeds that of the missile, and the position of the missile within the plume depends in a complex manner on altitude, missile type, rocket motor, fuel characteristics, etc., and is susceptible to variation by the offense in a manner which cannot be predicted by the defense. Other passive means of accurately locating and tracking missiles in boost phase are in early stages of study (Section 7.5).

Active means of tracking may be required. Of the likely candidates, microwave radars are the most developed although electronic countermeasures for them are also well developed. Optical radars may be more promising, if the illuminating beam can be rapidly retargeted, and if an imaging capability can be achieved (either range-Doppler or angle-angle systems would be sufficient). If rapid retargeting cannot be developed and if power-aperture requirements for microwave radars become too severe hundreds to thousands of space platforms will be needed (Section 7.6).

17. For post-boost and mid-course, precision tracking will require active sensor systems.

Observation of PBVs and RVs (at $300 \mathrm{~K}$ ) will require detection of weak thermal signatures since these signatures vary as $T^{4}$. Similar signatures are associated with objects in mid-course. Thermal detectors in the long wavelength infrared (LWIR) can be used only above the earth's limb against a cold space background. Low noise LWIR detector assemblies having the appropriate resolution, i.e., large element arrays, are being developed. Because of the long wavelengths involved $(8-12 \mu \mathrm{m})$, sub-microradian tracking accuracy is not feasible in LWIR without using telescopes with apertures in excess of $10 \mathrm{~m}$ (Section 7.2). Thus, thermal detectors will have to be supplemented by some active means such as microwave or optical radars. A large number of spacebased platforms will be required. These might be the same platforms that are performing similar duties in the boost phase (Section 7.3). 
18. For mid-course, when the RVs are interspersed with penetration aids, interactive discrimination may be required. At present the application of DEW technologies to this task is in the conceptual and early experimental stage.

Missiles which survive the boost phase can deploy large numbers of decoys and other penetration aids. Since LWIR and radar signatures depend largely on surface phenomena, there are many options available to the offense desiring to confuse or saturate the defense (use of balloons, for example). Directed energy technologies may offer the possibility of "mass" discrimination by interactive, perturbing means, e.g., detection of particlebeam-induced secondary emissions or velocity changes caused by laser-ablation-induced impulse. DEW platforms absent from the boost phase intercept theater might be useful in this function. Such interactive discrimination is in a conceptual and early experimental stage, and would require large numbers of additional sensor/detector platforms, plus the ability to function in nuclear-disturbed backgrounds (Section 7.7).

19. The development of an effective boost phase defense is highly desirable, perhaps essential for limiting the number of objects with which the mid-course and terminal defense elements must cope.

Given the present number of Soviet boosters and their capability, the offense can deploy half a million or more threat objects (reentry vehicles and decoys). Boost phase attrition is required if mid-course discrimination systems can deal with only a limited number of threat objects. Even an $80 \%$ effective boost phase defense would leave 100000 or more objects entering the mid-course phase. If further increases in the offensive threat or degraded performance of the boost phase tier overload the tracking and discrimination capabilities of later tiers, then the overall performance of the defensive system would degrade catastrophically rather than linearly when saturation is approached. The tracking and discrimination of tens to hundreds of thousands of objects during the mid-course phase poses formidable challenges to sensors and battle management computers. If discrimination requires birth-to-death tracking of all threat objects, these problems become even more demanding (Section 2.3).

20. Housekeeping power requirements for operational maintenance of many space platforms for strategic defense applications necessitate nuclear reactor driven power plants on each of these platforms.

The power requirements for "housekeeping," i.e., the requirements for a space platform to control attitude, to cool mirrors, to receive and transmit information, to operate radars, etc., is estimated to be in the range of 100 $\mathrm{kW}-700 \mathrm{~kW}$ of continuous power. This would require a nuclear reactor driven power plant for each platform, necessitating perhaps a hundred or more of these nuclear reactors in space. These foregoing needs require solving many challenging engineering problems not yet explored. Cooling of large space-based power plants is a very difficult task (Chapter 8).

\section{During engagements prime power requirements for electrically driven space-based DEW present significant technical obstacles.}

The prime power required for electrically driven DEW, e.g., electron accelerator for a space-based free electron laser, is estimated to be $1 \mathrm{GW}$. For a space-based neutral particle beam weapon, the electrical power requirements range from $100 \mathrm{MW}$ (minimum) to $1 \mathrm{GW}$ depending on the desired range and retargeting rates. This power could be provided by large chemical or nuclear rocket engines and generators, deployed at considerable distances or otherwise decoupled from the DEW platforms in order to avoid mechanical disturbances and effects of exhaust gases. This may require complex power transfer systems comprising cables, microwave systems, etc. Correspondingly, chemical fuel consumption would be more than five tons per minute of operation per platform (Section 8.3).

22. Survivability is an essential requirement of any BMD system employing space-based assets; such survivability is highly questionable at present. Evaluation of this issue requires a systems approach that includes hardening, active defense, and operational tactics. During the deployment phase, the space-based assets are especially vulnerable.

The space platforms carry sensors, optical mirrors, or radar dishes, many of which have considerably lower damage thresholds than do the hardened boosters, postboost buses, and RVs. While sensors and optical mirrors on satellite platforms may be shielded during long periods of inactivity, they would be exposed when put on alert prior to an impending ICBM attack. Such an attack could be preceded by an attack on these platforms by space-based and ground-based DEW, space-based kinetic energy weapons (KEWs), space mines, or direct ascent nuclear and non-nuclear antisatellite (ASAT) weapons of the offense. Moreover, the system must be developed by a process of accumulation of space assets; during this period of accumulation the system is less capable of defending itself (Sections 9.3 and 9.4).

The ground-based laser systems for strategic defense applications require a substantial number of space-based optical elements and space-based sensors. The spacebased optical elements include telescopes with large primary mirrors, the size and numbers of which will depend on the basing modes for the relay and the fighting mirrors. These space-based elements entail the same 
vulnerability as any other space-based components (Section 9.3).

23. Survivability of ground-based facilities also raises serious issues. The relatively small number of large facilities associated with ground-based laser sites makes these facilities high-value targets.

The ground-based laser BMD facilities must be successfully protected from direct attack from many threats (e.g., cruise missiles, sabotage, etc.), in addition to ballistic missiles. Thus, any strategic defense system depending on ground-based lasers, or on other groundbased facilities which cannot be extensively proliferated, must be effective in defending against more threats than just ballistic missiles (Section 9.3).

24. Directed energy weapons with capabilities below those needed for many ballistic missile defense applications can threaten space-based assets of a defensive system.

If a DEW falls short of ballistic missile defense requirements, it may still be a credible threat to spacebased assets. Space-based platforms move in known orbits and can therefore be targeted over much longer time spans than ballistic missile boosters, post-boost buses, or reentry vehicles. The defense platforms may have key components that are more vulnerable than the boosters and the reentry vehicles. Furthermore, spacebased platforms in low earth orbits can be attacked from shorter ranges than those required for boost phase intercepts (Sections 9.3 and 9.6).

25. X-ray lasers driven by nuclear explosions would constitute a special threat to space-based sensors, electronics, and optics.

The high energy-to-weight ratio of nuclear explosive devices driving the directed energy beam weapons permits their use as "pop-up" devices. For this reason the x-ray laser, if successfully developed, would constitute a particularly serious threat against spacebased assets of a BMD (Sections 3.5 and 9.3).

26. Since a long time will be required to develop and deploy an effective ballistic missile defense, it follows that a considerable time will be available for responses by the offense. Any defense will have to be designed to handle a variety of responses since a specific threat cannot be predicted accurately in advance of deployment.

A thorough understanding of practical responses, such as attacks on the defensive assets, hardening of offensive systems, and rapid deployment of large number of decoys, must be established before conclusions about the technical feasibility and cost-effectiveness of a defensive system can be made. A DEW system designed for today's threat is likely to be inadequate for the threat that it will face when deployed (Section 2.3 and Chapter 9). 


\section{Chapter 1}

\section{OVERVIEW}

\author{
CONTENTS \\ 1.1 Background \\ 1.2 Charter of the Study \\ 1.3 Scope of the Study \\ 1.4 Perspective \\ 1.5 Limitations in Scope \\ 1.6 Acknowledgments \\ References
}

\subsection{BACKGROUND}

On March 23, 1983, President Reagan called upon the nation and its technological community to make a major intellectual and physical effort to find an alternative to the current policy of assuring national security through the threat of retaliation to deter a ballistic missile attack. After that speech the President ordered studies to explore further the promise of ballistic missile defense (BMD), and in 1984 the Department of Defense established an organization to expand and accelerate research in ballistic missile defense technologies. This program is now called the "Strategic Defense Initiative" (SDI).

The study of defense against ballistic missiles is not new; vigorous research efforts to develop antiballistic missile (ABM) technologies were begun in the late 1950s. However, by the late 1960 s it had become evident that ABM defenses would not be sufficiently effective to protect cities or other large, vulnerable targets, and the emphasis shifted to defense of hard military targets, such as ICBM silos. By 1972 it became apparent that the existing technology could not satisfy this mission objective either. In this case the critical weakness of the system lay not in the performance of the interceptor rockets or the nuclear weapons they carried. Rather, it lay in the acquisition, tracking, discrimination, and battle management functions, and especially in their vulnerability to direct attack.

During the next 10 years there were significant advances in several potentially relevant ABM technologies. For example, computers became smaller, cheaper, and more capable; higher frequency, higher power radars became available and overall radar systems became more compact, durable, and cheaper; and various directed energy technologies (lasers and particle beams) experienced rapid development. A virtually continuous series of government-sponsored studies of advanced strategic defense technologies were performed by organizations such as the Defense Science Board, the White House Science Council, and various private contractors during the period of 1979-83.

Following the President's speech the Department of Defense was instructed to reexamine the state of knowledge and policy relevant to the BMD problem. Three separate studies were commissioned and these worked through the summer and early autumn of 1983 . Two of these dealt with policy issues; the third, the Defense Technologies Study Team (DTST, popularly known as the Fletcher Panel), reexamined the readiness and potential of technologies to deal with interception of ICBMs in all phases of their trajectories. Based on the results of separate study subgroups dealing with the major technical aspects of the BMD problem - directed energy weapons (DEWs), kinetic energy weapons (KEWs), surveillance, acquisition, tracking and kill assessment, and battle management and system integration - the Fletcher Panel reported that it found many possibilities for dealing with these aspects. It further concluded that since none of the problems could be solved with existing technology, major development would be needed over an extended period of time. The recommendations of the Fletcher Panel resulted in the creation of the Strategic Defense Initiative Organization, which consolidated virtually all the BMD-relevant research in the government.

The ensuing intense debate unleashed by the Strategic Defense Initiative has largely focused on philosophical and political considerations, while technological options and limitations have not been analyzed in sufficient detail, or details may only be found in classified documents. ${ }^{1}$ Some technical issues are discussed in reports by the Office of Technology Assessment, ${ }^{2}$ by the Union of Concerned Scientists, ${ }^{3}$ by the Center for International Security and Arms Control of Stanford University, ${ }^{4}$ by the American Academy of Arts and Sciences, ${ }^{5}$ by the Brookings Institution, ${ }^{6}$ and other articles in professional journals, ${ }^{7-9}$ and others. ${ }^{10}$ In many reports the main thrust deals with implications for domestic and foreign policy. These reports are generally addressed to a broad audience and the scientific and technological analyses are necessarily abridged. The cited reports cover a broad range of complex questions raised by the SDI program, including its impact on arms control negotiations as well as existing international treaties, on stabilizing and destabilizing factors in the current offensive balance, on economic impact, and broad systems considerations. 


\subsection{CHARTER OF THE STUDY}

The American Physical Society recognized that there were considerable uncertainties and differences of opinion among its members concerning the present state of the art of directed energy technologies, as well as the requirements for satisfying various ballistic missile defense missions (boost phase defense, mid-course discrimination, etc.). It, therefore, commissioned a study of the science and technology of directed energy weapons through its Council action on November 20, 1983. By November 1984, a Study Group comprising scientists and engineers from federal laboratories, industrial organizations, and universities had been constituted. Some members of the Group were (and are) actively involved in directed energy research. The Group was specifically chartered to examine the status of, and requirements for, directed energy weapon technologies, and to document its findings in an unclassified report.

Responding to its charter, the Group has focused on the following central theme: perform an in-depth review of the several directed energy weapon technologies and estimate the parameter requirements necessary for accomplishing various future BMD missions. In light of this focus, we do not discuss KEW technologies nor do we address the complex issues associated with battle management and $\mathrm{C}^{3} \mathrm{I}$ (command, communications, control, and intelligence) including testability and reliability of the software. Also, this report does not address the related issues of arms control and strategic stability. Each of these issues is, however, sufficiently important to merit a separate study.

This study specifically does not evaluate the current SDI program, but rather establishes a framework which may be helpful to others interested in the evaluation of the DEW component of this program. The Group hopes that the report which follows will serve as a useful technical reference for members of the APS, and for other scientists and engineers, as well as for a wider audience in order that discussions of the issues related to the Strategic Defense Initiative be better informed.

\subsection{SCOPE OF THE STUDY}

Following this brief overview, the report first describes the targets at which the DEWs would be aimed. Thus, Chapter 2 deals with both the current and responsive missile threat. Next, all major candidates for laser DEWs are discussed in Chapter 3. Detailed technical information is presented for chemical lasers, excimer lasers, and free electron lasers, while only the principles of $\mathrm{x}$-ray lasers are described because of classification restrictions. The state of the art of each and the requirements for DEW devices intended for BMD applications are given. The other category of DEW devices, the relativistic particle beams, is described in Chapter 4 along with their propagation characteristics.
Characteristics of photon beam propagation are described in Chapter 5 which includes the technology of beam control, delivery, and atmospheric beam propagation effects.

The basic physical mechanisms by which photon beams and relativistic particle beams can damage targets are described in Chapter 6. The requirement of lethality, that the target be either destroyed or made inoperative, demands that a sufficient amount of energy and/or power must be delivered to the target.

The beams from DEWs must be directed at the targets, i.e., they must intercept hostile ballistic missiles and/or their payloads. Acquisition, tracking, and discrimination of objects require sensor platforms, radars, and possibly laser and particle beam tracking and discriminating devices in space. These problems are discussed in Chapter 7. The power requirements for space-based platforms present special problems which are examined in Chapter 8.

The important issue of survivability of DEWs is discussed in Chapter 9. It depends sensitively on both device parameters and system architecture. The overall architecture of a defensive system depends heavily on considerations of many factors. These include command, control, communication, and intelligence $\left(\mathrm{C}^{3} \mathrm{I}\right)$, hardware and software development and reliability for battle management, the possible inclusion of kinetic energy weapons, etc. The integration of all these components and systems into an overall system presents extremely challenging problems, some of which are enumerated in Appendix A. A discussion of satellite constellations is presented in Appendix B.

The combination of lethality, propagation, and range requirements determines the brightness required for directed energy weapons. For defense of the entire nation, including protection of population centers, via boost phase kill, the brightness requirements exceed by orders of magnitude the present state of the art of various types of lasers, particle beam devices, optical delivery systems, acquisition platforms, power supplies, etc. This is the main thrust of the detailed conclusions of this study which are presented in the Executive Summary.

\subsection{PERSPECTIVE}

The Group notes that predicting the course of technological progress can be particularly difficult. Very optimistic predictions are often made for technologies or schemes which are at very early stages of development. Whenever orders of magnitude of improvement are necessary in operating parameters, it is likely that many new discoveries and inventions will have to be made. The discrepancy between the present state of the art of DEWs and the ultimate requirements is so large that major gaps in technical understanding must be closed before engineering technology verification could be productive. 
Forcing immature technology to this verification phase can have two undesirable consequences. First, it tends to freeze technology at levels inadequate for its ultimate goals. And second, it tends to absorb resources which could otherwise be used for research on more promising ideas.

Past experience with the progression from theoretical concept, via proof of principle, understanding of details, technical development, engineering, to eventual deployment in a very large system, shows that technology typically is frozen several years before deployment, and basic science more than a decade before that. ${ }^{11}$ Because of the extensive development needed in many technological areas important to the systems, we judge that the deployment of a substantial DEW component in a BMD system cannot be foreseen before the year 2000 .

The offense can use the long development test and deployment time to respond with similar, or dissimilar, technological developments. The Group did not review classified intelligence information about the likely technological responses from the Soviet Union, but rather relied primarily on general scientific and engineering principles in considering potential countermeasures. The uncertainty about the responsive threat, in turn, may raise the requirements for lethality and will make survivability more uncertain. A deployed DEW defensive system may have to face the threat of DEWs on the offensive side, in addition to other conventional threats. If a DEW system is capable of disabling a ballistic missile in the boost or post-boost phase, it is likely that it also meets the lethality requirements for damaging a space platform.

Because achieving the ultimate goal of population defense appears so difficult from a technological point of view, many people have advocated more limited missions for DEWs, including antisatellite (ASAT) weapons, and target discrimination. DEW requirements can be considerably lower for these reduced objectives. The role of DEWs could be minor, if not negligible, in the case only hard-point defense of land-based silos is contemplated.

\subsection{LIMITATIONS IN SCOPE}

The sheer size of the technological development of DEWs, let alone deployment, is such that it raises questions about manpower and economic cost. Engineering manpower requirements are likely to be high. The Group believes that these are very important issues, and should be studied because of their possible impact on the civilian economy, international competitiveness, the armed services, and technical manpower. The Group notes the existence of these issues but does not address them and it refrains from conclusions about them.

Another important issue which has not been dealt with in detail is launch costs. Deployment of any BMD system with extensive space-based components will require that the cost of placing mass in orbit be significantly reduced. We have not evaluated the prospects for success in this endeavor. However, it is worth noting that such major cost reductions would also produce major changes in the nature and capabilities of the offensive threat.

Finally, this study does not deal with the very important issue of cost effectiveness of directed energy weapons in their use in ballistic missile defense. Cost effectiveness is variously defined, but the most cogent definition is contained in one of the Nitze criteria ${ }^{12}$ which requires the incremental cost of providing a ballistic missile defense to be less than the incremental cost incurred by the enemy for overcoming the defensive actions. For example, a recent paper by Field and Sperge ${ }^{13}$ has outlined a methodology for one aspect of DEW which may be used for such semiquantitative but exceedingly important evaluations of specific technologies. Cost estimates for the whole system are necessary, but are likely to be much more complex. Blechman and Utgoff $^{14}$ have described a heuristic approach to economic implications of strategic defense. Other limitations in scope have already been mentioned in Sections 1.2 and 1.3 .

\subsection{ACKNOWLEDGMENTS}

Financial support was received from Carnegie Corporation of New York and the MacArthur Foundation. The study also received full cooperation from the Strategic Defense Initiative Organization and from the Office of Science and Technology Policy. We are grateful to all officials and individuals who helped us with extensive briefings. We also wish to thank Dr. $R$. Cunningham, Dr. P. L. Kelley, and Dr. R. Kerber for assistance in drafting portions of this report. This report and its conclusions are, however, solely the responsibility of the Study Group. The names of the members of the Study Group appear in Appendix C.

\section{REFERENCES}

${ }^{1}$ For the unclassified summary of the Fletcher Panel report, see The Strategic Defense Initiative: Defense Technologies Study (Department of Defense, Washington, D.C., March 1984).

${ }^{2}$ U.S. Congress, Office of Technology Assessment, Ballistic Missile Defense Technologies, OTA-ISC-254 (U.S. Government Printing Office, Washington, D.C., September 1985).

${ }^{3}$ H. A. Bethe, R. L. Garwin, K. Gottfried, and H. W. Kendall, "Space-based ballistic missile defense," Sci. Am., October 1984, p. 46.

${ }^{4}$ S. D. Drell, P. J. Farley, and D. Holloway, The Reagan Strategic Defense Initiative: A Technical, Political and Arms Control Assessment (Center for International Security and Arms Control, Stanford University, 1984).

5"Weapons in Space," Daedalus, Spring-Summer 1985 (American Academy of Arts and Sciences, Cambridge, Mass., 1985).

${ }^{6}$ Ballistic Missile Defense, edited by A. B. Carter and D. N. Schwartz (The Brookings Institution, Washington, D.C., 1984).

${ }^{7}$ See, for example, J. A. Adam, M. A. Fischetti, J. Horgan, and P. Wallich, "SDI: The Grand Experiment," IEEE Spectrum, September 1985, pp. 34-64. 
${ }^{8}$ Gerold Yonas, "Strategic Defense Initiative: The politics and science of weapons in space," Phys. Today 38 (6), 24-32 (1985); "Materials for SDI," MRS Bulletin, May/June, 1986, pp. 17-28.

${ }^{9}$ Wolfgang K. H. Panofsky, "The Strategic Defense Initiative: Perception vs Reality,” Phys. Today 38 (6), 34-45 (1985).

${ }^{10}$ Robert Jastrow, How To Make Nuclear Weapons Obsolete (Little, Brown \& Co., Boston, 1985).

${ }^{11}$ Harold Brown, "Is SDI Technically Feasible," Foreign Affairs, 435 (1985).
${ }^{12}$ P. H. Nitze, "On the Road to a More Stable Peace," speech to the Philadelphia World Affairs Council, February 20, 1985; National Security Decision Directive 172 (May 30, 1985).

${ }^{13}$ George Field and David Spergel, "Cost of Space-Based Laser Ballistic Missile Defense," Science 231, 1387-1393 (1986).

${ }^{14}$ Barry M. Blechman and Victor Utgoff, Economic and Fiscal Implications of Strategic Defense (Westwood Press, 1986); "Macroeconomics of Strategic Defense," International Security, Fall 1986. 


\section{Chapter 2}

\section{SOVIET BALLISTIC MISSILE THREAT: CURRENT AND RESPONSIVE}

\section{CONTENTS}

2.1 Missile Phases and Kinematics

2.1.1 Boost Phase

2.1.2 Post-Boost Phase

2.1.3 Mid-Course Phase

2.1.4 Reentry Phase

2.1.5 Trajectory Options

2.2 Current Ballistic Missile Forces

2.3 Responsive Threat Options

2.3.1 Offensive Proliferation

2.3.2 Booster Rotation and Ablative Shields

2.3.3 Fast Burn Boosters

2.3.4 Post-Boost Vehicle Redesign

2.3.5 Decoys and Penetration Aids

2.4 Summary and Conclusions

References

This chapter presents a review of the phases and kinematics of missile flight, a summary of existing Soviet long range ballistic missile systems, and a discussion of some of the ways the Soviets might redesign their missile forces in response to the deployment of a U.S. ballistic missile defense system. It should be kept in mind that although current Soviet missile deployments provide a useful guide for the baseline capabilities which any U.S. ballistic missile defense must achieve, current Soviet deployments are a very uncertain guide for the future given the long time scale for U.S. defensive deployments in even the most optimistic of circumstances.

\subsection{MISSILE PHASES AND KINEMATICS}

The flight of a ballistic missile may be divided into four phases: boost, post-boost, mid-course, and reentry. This division is natural for the designer of missile systems and equally so for the designer of BMD. For single warhead missiles the post-boost phase is absent, but as we discuss below, in an era of strategic defenses it is likely that even single warhead missiles will employ decoys or other penetration aids and so the equivalent of a postboost phase will then be present. (Some authors use the term boost phase in a collective sense to include both boost and post-boost phases. For our purposes this is not convenient.)
Figure 2.1(a) shows the trajectory and four phases for a missile with the characteristics of the U.S. MX/Peacekeeper. Figure 2.1(b) shows the same for the SLBM. For an intermediate range ballistic missile like the Soviet SS-20 or the U.S. Pershing II the phases are illustrated in Figure 2.1(c).

\subsubsection{Boost Phase}

Boost phase begins when the missile leaves its launcher (typically an underground silo for an ICBM and an underwater missile launch tube for a SLBM) and ends when the propulsion motor of the last stage of the booster has shut down and the payload separates from the lifting vehicle. The fundamental idea of staging is to discard empty fuel tanks, large motors, etc. in order to avoid the fuel cost of accelerating parasitic mass to intercontinental range velocities. For ICBMs two or three stages are typically used; for SLBMs two is the norm. In all current Soviet and U.S. strategic (intercontinental range) missile designs, booster burnout occurs well above the sensible atmosphere, but this is not a fundamental requirement. Two examples of existing systems are MX/Peacekeeper (solid fuel) - total boost time $180 \mathrm{~s}$; SS-18 (liquid fuel), the largest of the current generation Soviet ICBMs-total boost time $350 \mathrm{~s}$.

The range of a ballistic missile is determined

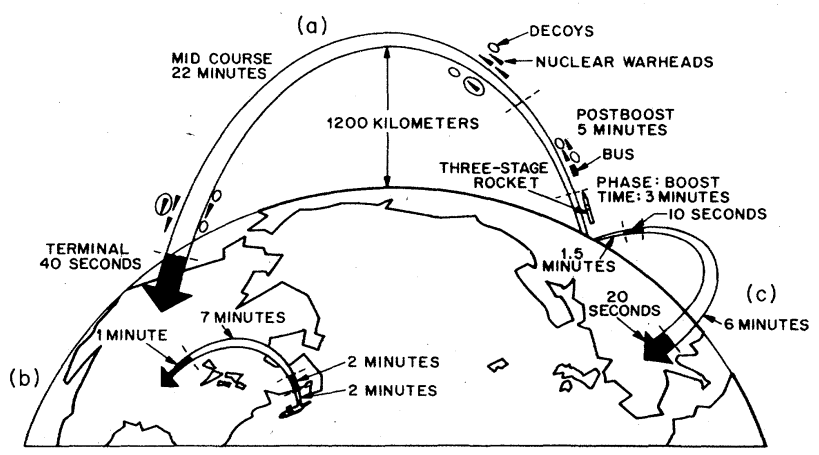

Figure 2.1 (a) Trajectory phases for an ICBM with the characteristics of the U.S. MX/Peacekeeper; (b) trajectory phases for a typical SLBM; (c) trajectory phases for a IRBM. (Illustration reprinted from the September 1985 issue of IEEE Spectrum.) 
primarily by the velocity achieved at final booster stage burnout and the angle between the trajectory and the horizontal at burnout. (This angle is called the inclination or reentry angle.) Similarly, total flight time is determined essentially by these same two quantities. The burnout altitude is of secondary importance for either range or total flight time. ${ }^{1,2}$

For a range of $10000 \mathrm{~km}$, flight times can vary from $28 \mathrm{~min}$ to $42 \mathrm{~min}$ as the inclination angle is varied from $15^{\circ}$ to $35^{\circ}$, respectively. For a solid propellant ICBM flying a $10000 \mathrm{~km}$ range a typical boost phase trajectory sequence is as follows:

\begin{tabular}{cccc}
$\begin{array}{c}\text { Booster } \\
\text { component }\end{array}$ & $\begin{array}{c}\text { Burnout } \\
\text { time (s) } \\
\text { (from launch) }\end{array}$ & $\begin{array}{c}\text { Burnout } \\
\text { altitude }(\mathrm{km})\end{array}$ & $\begin{array}{c}\text { Burnout } \\
\text { vel }(\mathrm{km} / \mathrm{s})\end{array}$ \\
\hline Stage 1 & 60 & 25 & 2.5 \\
Stage 2 & 120 & 95 & 4.5 \\
Stage 3 & 180 & 250 & 6.5 \\
PBV & 600 & $\geq 800$ & 7.1
\end{tabular}

This information represents a composite of typical threestage ICBM systems with a post-boost vehicle (PBV) capability. Actual performance depends upon the target set and number and weight of deployed objects.

During boost phase the most prominent observable is the infrared (IR) emission from the rocket plume of the missile. This may be readily observed from a satellite at geosynchronous orbit and currently provides the first sign to the U.S. of missile attack. Since the luminosity from the missile plume is so intense, it is impossible to hide the plume in any practical sense. Other signatures which might be exploited by boost phase defenses are the large radar cross section of boosters, visible and ultraviolet emissions from the plume, solar reflection from the missile body (daytime), and, during the atmospheric portion of flight, radiation from shock heated air.

\subsubsection{Post-Boost Phase}

At final stage thrust termination, the booster has given its payload sufficient velocity to reach the desired range. Elements of the payload are now separated from the lifting vehicle and left to fall in ballistic trajectories to impact. In multiple, independently targeted reentry vehicle (MIRV) systems small velocity increments are given to each reentry vehicle $(\mathrm{RV})$ to direct them to individually designated targets.

Although details differ, the Soviets have adopted the basic approach to independent targeting that was pioneered by the United States. Namely, an additional missile stage, called the bus or post-boost vehicle, is employed. The bus needs to have an inertial guidance system, thrusters (rockets), and a thrust control system; it carries RVs, and, if defenses are present the bus can be used also to carry and dispense decoys and other penetration aids. As its name implies, the bus releases RVs singly as preprogrammed velocities (and positions) are reached. Thrusters on the bus may burn continuously or intermittently. In addition to permitting independent targeting, the presence of a post-boost stage enables corrections to be made for errors accumulated in boost phase and boost thrust cutoff, thus improving overall accuracy.

Although conceptually the post-boost vehicle is just another rocket stage, in design and observable characteristics it is quite distinct. Whereas booster stages produce a net velocity appropriate to intercontinental range $(6-7 \mathrm{~km} / \mathrm{s})$, the bus stage typically imparts much smaller velocity changes per RV on the order of $0.5 \mathrm{~km} / \mathrm{s}$ or smaller. The PBV may carry enough propulsion fuel to give a total velocity change $\Delta \mathrm{V}=2-3 \mathrm{~km} / \mathrm{s}$. Typically this will be expended in transverse and longitudinal maneuvers.

The key advantage of current bus designs over other possibilities for independent targeting of multiple warhead missiles is that only a single inertial guidance and thruster system is required on each bus and within the limitations set by fuel, space, and missile throw-weight, any number of RVs can be accommodated. It is clear that the bus concept is ideal in many ways also for the release of decoys and other penetration aids in a world of missile defenses.

Observables in post-boost phase are generally much weaker than in boost phase. Sizes, masses, and radar cross sections of the objects of interest are smaller; IR emissions from the PBV thruster plume are orders of magnitude smaller than for the final booster stage (cold gas thrusters on the PBV can reduce this even further); and the number of potential targets to be tracked and designated by the defense grows steadily throughout the PBV phase. At the beginning of the post-boost phase, the bus is a high value target equal to the booster itself. As deployments ensue, the value of the bus steadily diminishes until the release of the last $R V$ when the value of the bus goes to zero.

\subsubsection{Mid-Course Phase}

For all but tactical missiles, mid-course is the longest of the trajectory phases. Throughout mid-course all the RVs and decoys, as well as bus and booster remnants (the "threat cloud") from a given missile move along nearby ballistic trajectories (the "threat tube") under the influence of gravity; light and heavy objects move alike. The mid-course phase ends at reentry when objects in the threat cloud experience drag forces in the upper atmosphere sufficient to cause observable deviations from ideal ballistic trajectories.

The relatively long length of the mid-course phase ( $\approx 20 \mathrm{~min}$ for intercontinental range) can be advantageous to the defender, since several minutes can be devoted to establishing track files as well as performing a discrimination function. Moreover, there is sufficient time for the defender to choose when to attack, to allow 
additional satellites to come into the battle space, and to revisit objects for follow-up attack or kill assessment. On the other hand, there are many disadvantages to the defender in mid-course. The number of objects is greatly increased over that of boost phase and early stages of the post-boost phase, and the high leverage of boost phase and post-boost phase kill of MIRVed missiles is lost. Once deployed RVs tend to be much harder targets than are boosters and post-boost vehicles. Special discrimination opportunities provided by observing postboost vehicle maneuvering and releases are no longer present, and mid-course signatures are generally few and weak relative to those present in all other phases of missile flight. Most significantly, because atmosphere drag is totally absent in mid-course, the offense can employ lightweight decoys which match the rigid body dynamics of massive RVs and the external observables of RVs as well.

\subsubsection{Reentry Phase}

Many of the taxing discrimination problems associated with mid-course defenses disappear or are greatly relaxed once reentry $(\approx 130 \mathrm{~km}$ altitude $)$ has occurred. Atmospheric drag not only produces changes in trajectory, it also increases the optical signature of reentering bodies through frictional heating. All these effects provide tracking and discrimination opportunities. However, these opportunities are offset by the short times (typically less than $60 \mathrm{~s}$ ) available to a terminal defense for tracking and for committing interceptors, and by the opportunity the offense has to perturb vast portions of the defenders field of view by nuclear precursor bursts. For these reasons terminal defenses are most attractive for hard sites (missile silos, underground command and control centers, etc.) and least attractive for city and population defense. Directed energy weapons are not currently viewed as playing a significant role in terminal defenses. Instead nuclear or kinetic energy weapons are favored; for these reasons we do not discuss the use of DEWs in terminal defenses in this report.

\subsubsection{Trajectory Options}

The trajectories shown in Figures 2.1(a)-2.1(c) are so-called minimum energy trajectories. ${ }^{1,2}$ For given launch and target points, they are the paths which maximize payload to the target for a given missile type, or equivalently, maximize the range to which a given missile can deliver a fixed payload. In a flat earth approximation (uniform gravity) with no atmospheric drag, minimum energy trajectories would be parabolic and have a $45^{\circ}$ elevation above the horizon.

When the effects of the curvature of the earth and the $1 / \mathrm{r}^{2}$ decrease of the gravitational acceleration with distance from the earth's center are included but thrust and drag are neglected, a ballistic trajectory is a portion of an ellipse making an angle $\gamma$ with the tangent plane to the earth at the launch and target points. As remarked above, $\gamma$ is called the inclination reentry angle. For the minimum energy trajectory,

$$
\gamma_{\mathrm{m}}=(\pi-\Phi) / 4
$$

where $\Phi$ is the range angle, i.e., the angle subtended at the earth's center by rays through the launch and target points; see Figure 2.2. For a typical intercontinental range, $R=10000 \mathrm{~km}, \Phi=90^{\circ}$, and so $\gamma_{\mathrm{m}}=22.5^{\circ}$. Atmospheric drag causes departures from an elliptic trajectory in the reentry phase; drag and thrust do the same during boost phase. By sacrificing range and/or payload one can employ lofted trajectories $\left(\gamma>\gamma_{\mathrm{m}}\right)$ or depressed trajectories $\left(\gamma<\gamma_{\mathrm{m}}\right)$. Figure 2.3 shows some examples for an intercontinental missile.

Lofted trajectories have increased flight time over the minimum energy trajectories and have greater velocities at burnout and impact. Except perhaps against terminal defenses which would be stressed by increased reentry velocities, lofted trajectories, or defense evasion techniques such as maneuvering RVs which exploit the presence of an atmosphere, do not appear to be a likely offense choice in the face of multitier missile defenses. However, since during boost phase they exit the atmosphere earlier than a minimum energy trajectory, lofted trajectories might conceivably have an advantage in that decoy release could be effected earlier; this is not likely to be significant.

Depressed trajectories, on the other hand, offer some attractive possibilities to the offense. A depressed trajectory shortens total flight time; it also increases the time a missile spends within the atmosphere and is therefore unreachable by weapons for which the

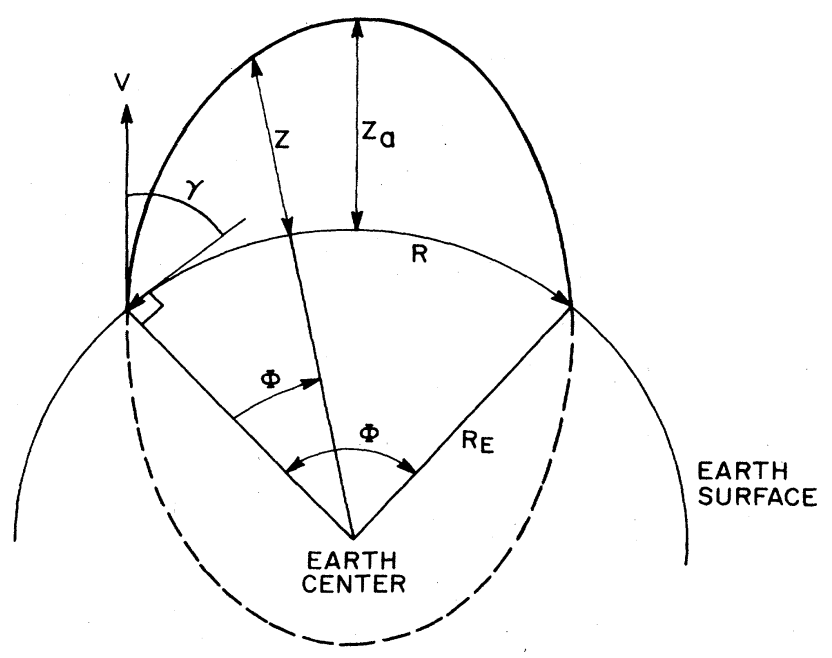

Figure 2.2 The elliptical trajectory of a missile flight in the approximation in which boosting is impulsive and atmospheric drag is neglected. The range angle is $\Phi$ and the reentry angle $\gamma$. 


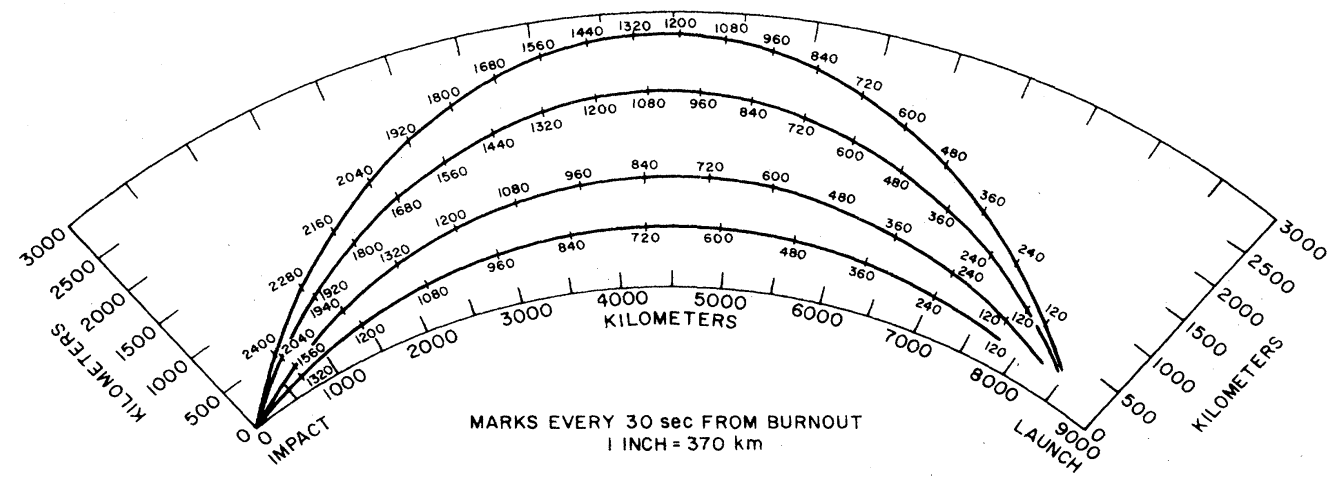

Figure 2.3 Minimum energy, lofted, and depressed trajectories for an ICBM.

atmosphere is opaque, and increases the amount of time the missile is below the earth's horizon as seen by a given satellite of the defensive system. Nevertheless, there are drawbacks to depressed trajectories. They result in decreased range, cause some loss in ballistic accuracy because of unpredictable atmospheric perturbations, and impose a delayed release of decoys compared to minimum energy trajectories. In addition, because of higher reentry velocities, RV heating is greater for depressed trajectories, a limitation on this option.

Another major drawback of depressed trajectories, reduced range at fixed payload, is probably least serious for SLBMs, since submarines have the option of moving closer to a target before launch. Also, since SLBMs currently are less accurate than ICBMs, small additions to missile inaccuracies are less significant. While neither the United States nor the Soviet Union has shown much interest in depressed trajectories to date, the situation could change rapidly in an era of ballistic missile defenses.

\subsection{CURRENT BALLISTIC MISSILE FORCES}

Figure 2.4 gives current American and Soviet ICBM deployments. ${ }^{3,4}$ The Soviets have approximately 1400 land-based strategic ballistic missiles, carrying a total of 6200 RVs. The bulk of these are of the SS-17, SS-18, and SS-19 types which together represent virtually all of the high accuracy ICBM RVs in the current Soviet inventory. All of these Soviet ICBMs are deployed in hardened underground silos, many of which have been upgraded since 1972. While these missiles are not invulnerable to attack by high accuracy nuclear weapons, it is noteworthy when thinking about future developments that the rate of upgrading of the ICBMs and silos has been more rapid than in the U:S. program. One should also note that in spite of the difference in modernization rates, the qualitative status of U.S. and Soviet ICBM systems is similar. The Soviets are thought to have a slight lead in silo hardness; the U.S. is ahead in solid propellant technology and in accuracy.

As indicated by the "Mod" numbers in Figure 2.4, a given Soviet missile type (especially its post-boost stage) typically goes through evolutionary changes. About 580 of the current Soviet ICBMs are members of the older SS11 and SS-13 classes. The SS-16 shown in Figure 2.4 is an early design solid-fuel missile which the Soviets agreed not to deploy under the terms of the SALT II agreement. The upper two stages of the SS-16 constitute the basis of the SS-20 missile currently deployed in various parts of the Soviet Union.

Soviet ICBM research and deployment is a dynamic, ongoing process carried out ${ }^{5}$ in several design bureaus under the Ministry of General Machine Building (GMB). At least two new generation ICBMs are in early deployment phases and others are reported under development. ${ }^{3}$ One of the new systems, designated in the West as the SS-24, is similar to the U.S. MX/Peacekeeper: 10 RVs, three stages, solid fuel. It is speculated ${ }^{5}$ that initial SS-24 deployments will be in silos and later deployments rail-mobile. The second newly deployed land mobile Soviet missile, the SS-25, is about the size of the U.S. Minuteman and appears to be the Soviet version of the single warhead, land-mobile missile which is in the early stages of development in the U.S. (unofficially, Midgetman). The SS-24 and SS-25 missiles and subsequent follow-on Soviet ICBMs can be expected to have improved accuracy and improved survivability. The move to increase use of solid propellants by the Soviets is likely to continue. ${ }^{3}$

From the European perspective, several other missile types are relevant. Some of these, the so-called longer range, intermediate range ballistic missiles (LRIRBMs), ${ }^{3,4}$ are shown in Figure 2.5. All U.S. and Soviet Union intermediate range missiles have unhardened basing; most are land-mobile. Not illustrated in Figure 2.5 are French land-based missiles, numerous shorter range U.S., Soviet, and People's Republic of China missiles, and tactical nuclear weapons. 


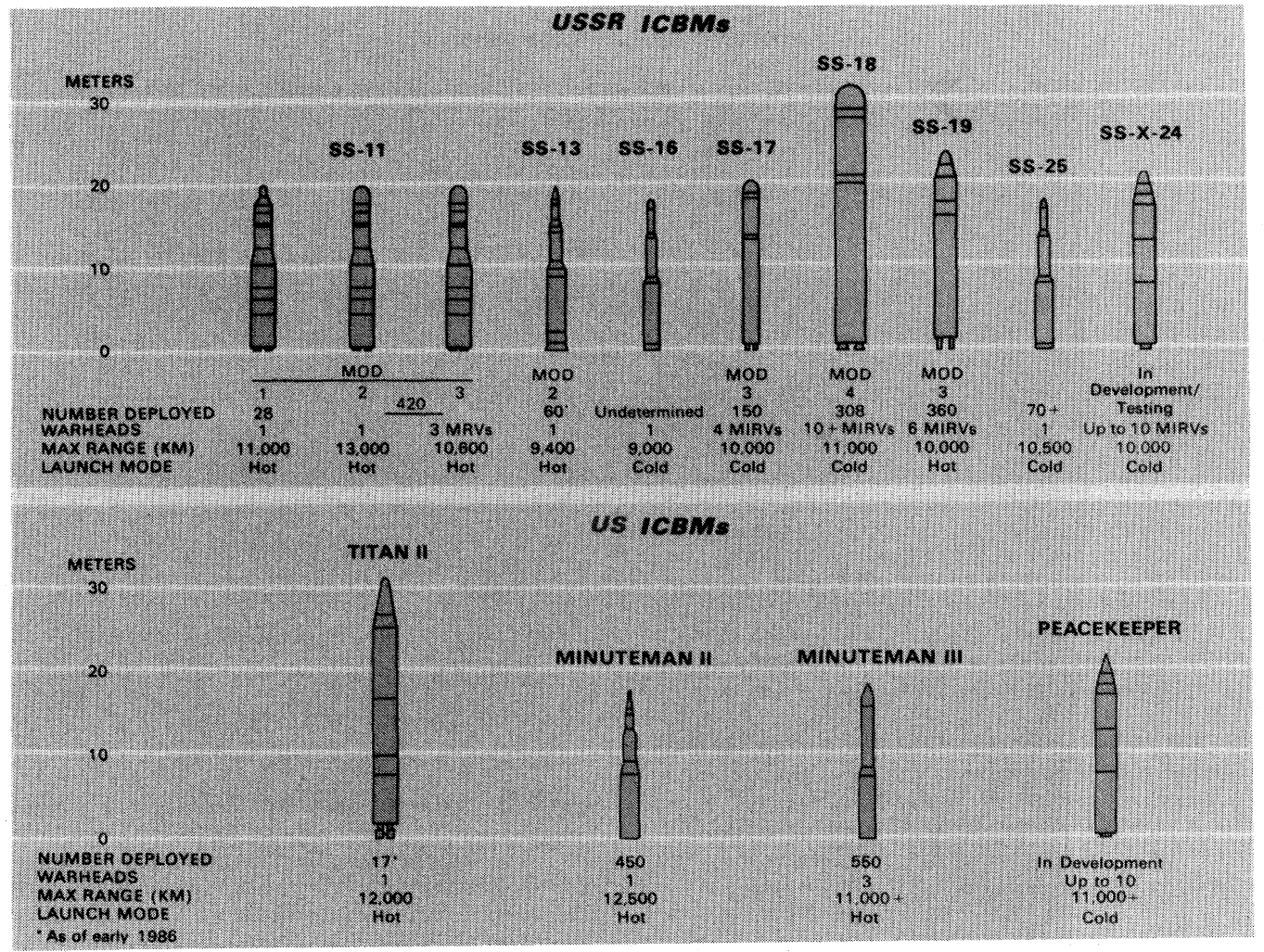

Figure 2.4 U.S. and Soviet ICBM deployments.

Soviet and American SLBM deployments ${ }^{3,4}$ are shown in Figure 2.6. Not shown on the U.S. side is the Trident II (D-5) missile currently under development. It will have a range comparable to the Trident I (C-4) missile but will have greatly enhanced accuracy. Also not shown in Figure 2.6 are the considerable British and French SLBM deployments.

Soviet SLBM deployments currently number about 928 strategic missiles aboard 62 nuclear powered submarines, many of recent vintage. Eighteen of these submarines (carrying 300 launchers) are fitted with
MIRVed missiles. These latter constitute about 1400 RVs out of a total of 2100 Soviet submarine-based RVs. The Soviets also currently maintain 13 diesel powered submarines capable of firing nuclear missiles.

As with ICBMs, Soviet SLBM and submarine development is carried out ${ }^{5}$ by specialized, ongoing design bureaus under the Ministry of GMB. It is anticipated ${ }^{3}$ that the Soviets will be testing versions of the SS-N-20 and SS-NX-23 in the near future. One can expect new types of Soviet SLBMs as well, and expect them to have greater accuracy and perhaps greater throw-weight than current

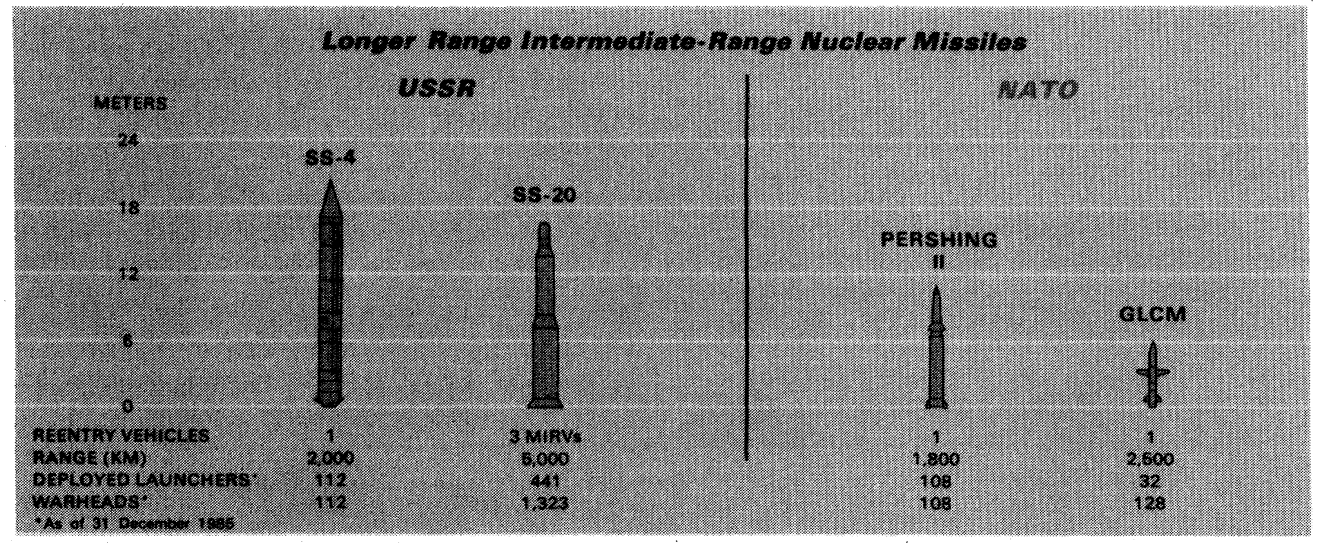

Figure 2.5 U.S. and Soviet LR-IRBM deployments. 


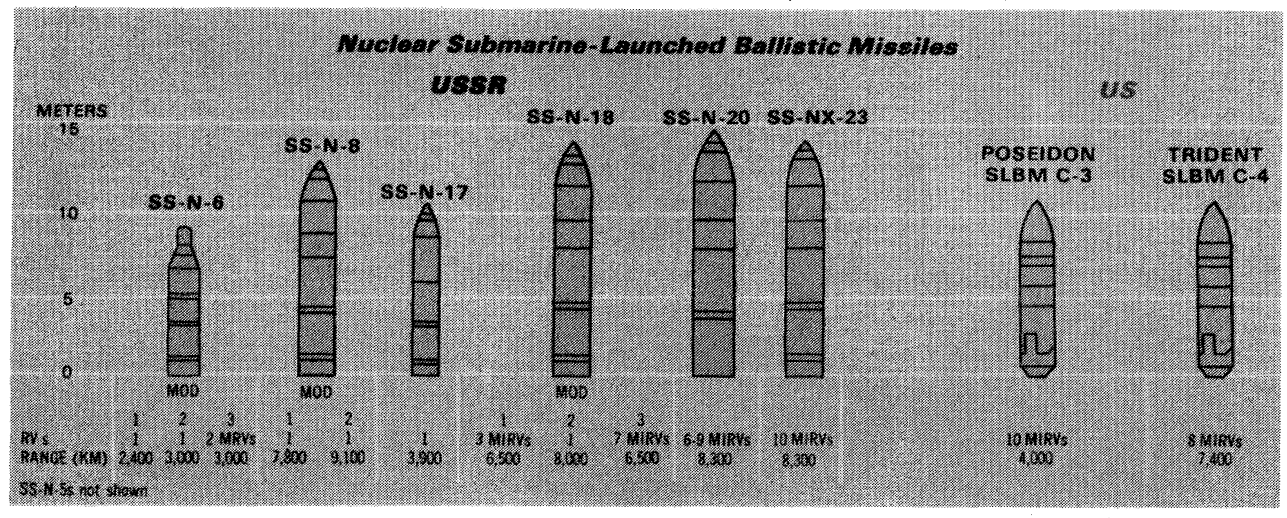

Figure 2.6 U.S. and Soviet SLBM deployments.

designs. Since the Soviets have historically depended more extensively on their land-based ICBMs and since Soviet ballistic missile submarines spend less time "on station" than their U.S. counterparts, it is likely that any shifts in Soviet deployment percentages, land-based versus sea-based, will occur only slowly.

\subsection{RESPONSIVE THREAT OPTIONS}

In thinking about how the Soviets might respond to a U.S. deployment of multilayered ballistic missile defenses several points are worth keeping in mind.

(i) A large number of years will be required to develop and deploy defenses; hence considerable time will be available to the offense to plan and execute responses. As a base for response, the Soviets have large, ongoing missile programs.

(ii) A complete defense architecture has not yet been defined by the U.S.; therefore, it is not possible (even for the Soviets) to make specific predictions regarding responses.

(iii) It is nevertheless important to analyze possible responses now since such analyses can aid in identifying those defensive technologies and architectures which are most and least attractive. It is necessary, moreover, to do such studies in order to gain an understanding of the relative difficulty (technology level, effectiveness, cost, lead time) of deploying defenses versus deploying offensive responses. A single response to U.S. defensive deployments would lack the high probability of success and reliability which the Soviets seek in their deployments. Thus, it is most likely that they will react in many ways simultaneously, by spreading their responses across the range of forces on which they currently rely, and also by introducing new systems as well.

Some possible Soviet responses are the following:

- Capability for direct attack of defense components
- Offensive proliferation

- Booster rotation and ablative shields

- Fast burn boosters

- Post-boost vehicle redesign

- Decoys and penetration aids

The above list of possible Soviet responses is illustrative and by no means inclusive. The first item above, direct attack (also called defense suppression), is so important that we treat it as the subject of a separate chapter (Survivability, Chapter 9). The importance of this subject is further underscored by the fact that survivability is the first of the "Nitze Criteria"6 for deployment of a U.S. defensive system. The remaining responses in the above list are treated in the subsections which follow. Clearly, a thorough understanding of the feasibility of these latter responses and an analysis of the financial cost and performance penalties these would extract from the offense is necessary before a judgment can be made on the efficacy of any proposed U.S. missile defense system. A succinct statement of this is the other of the "Nitze Criteria," 6 namely defensive deployments must be "cost-effective at the margin."

\subsubsection{Offensive Proliferation}

A common prediction for the response of the Soviets is that they will simply build more offensive boosters and RVs. That is the approach the U.S. took in the 1960 s and 70s partly in response to a prospective Soviet ABM. The U.S. fractionated both ICBM and SLBM missile payloads and deployed RVs in far greater numbers than were predicted for Soviet interceptors. This approach, defense exhaustion, has to date been judged to be cost effective when dealing with a defense whose number of potential intercepts is known. This response is also consistent with past Soviet responses, in which they have demonstrated an inclination toward continuing to rely on existing military forces, and on improving them incrementally.

For some but not all DEW-based defenses, the 
requirements for exhaustion may not be easily determined by the offense. In the case of chemical lasers the total kill potential of a satellite laser battle station is quantifiable by viewing fuel supplies. For ground-based FELs and for $\mathrm{x}$ ray lasers this is not so easily done. Whatever the case, if there is no boost phase intercept capability, offensive proliferation will be highly attractive.

Indicative of the missile production capability of the Soviets is 1985 testimony before the Senate Armed Services Committee by Gates and Gershwin," "By the mid-1990s, nearly all of the Soviets' currently deployed intercontinental nuclear attack forces-land- and seabased ballistic missiles and heavy bombers-will be replaced by new and improved systems. New mobile intercontinental ballistic missiles (ICBMs) and a variety of cruise missiles are about to enter the force. The number of deployed strategic force warheads will increase by a few thousand over the next five years, with the potential for greater expansion in the 1990s." In considering what might happen in the absence of arms control constraints these analysts went on to say, "While the Soviets would not necessarily expand their intercontinental attack forces beyond from 12,000 to 13,000 warheads in the absence of arms control constraints, they clearly have the capability for significant further expansion, to between 16,000 and 21,000 deployed warheads by the mid-1990s. The lower figure represents a continuation of recent trends in deployment rates; the upper figure is not a maximum effort but would require a substantially greater commitment of resources."

Similarly, the Department of Defense Publication Soviet Military Power 1986 indicates $^{3}$ that by the mid1990s, many of the current Soviet ICBMs will be retired and the deployed mix will consist of SS-19s, SS-24s, SS$25 \mathrm{~s}$, and a set of new heavy missiles (yet unnamed) as replacements for the SS-18s. Less dramatic but substantial changes are also predicted for Soviet SLBM forces. These Soviet modernization programs represent changes undertaken before any stimulus of possible U.S. missile defenses. There is ample evidence that the targets of tomorrow's U.S. strategic defenses are not today's Soviet offensive forces; instead the U.S. will face a responsive threat from the very beginning.

\subsubsection{Booster Rotation and Ablative Shields}

If the boost phase intercept employs thermal kill lasers which require long kill times (tenths of seconds or longer), a low technology countermeasure is booster rotation. Rotation of missiles at angular rates of the order of 1 rps have been studied and shown to extract little or no penalty to the offense. It is likely that missile rotation could be accomplished on a retrofit basis.

Rotation increases kill times by spreading the laser energy over an increased booster surface area. Although any amount of rotation works against the defense, ${ }^{*}$ to get

*Assuming a minimum spot size criterion has been met. the maximum effect from this countermeasure the offense needs to make the rotation period less than the kill time. We call this optimal rotation. Consider the situation of optimal rotation and a normally incident laser beam with no aiming error. Let the radius of the laser spot at the booster be a and the booster be a cylinder of radius $R$. There are two interesting cases: (1) $a>R$ and (2) $a<R$. In analyzing either case it is important to keep in mind that while the laser beam illuminates a large area on the booster, typically $1 \mathrm{~m}^{2}$ or more, the lethal fluence needs only to be achieved over a smaller area, say $30 \mathrm{~cm}$ in diameter, for kill to be accomplished. Failure occurs at the spot at which the integrated power first reaches lethal fluence. Under our assumptions of zero aiming and tracking error, this will occur along the missile centerline.

First consider the large beam case (1) and examine a vertical slice of the booster of height $\Delta z$ taken at the center of the laser spot. It intercepts a transverse area of the beam

$$
\mathrm{A}_{1}=2 \mathrm{R} \Delta \mathrm{z} \text {. }
$$

With optimal rotation the intercepted laser energy in the slice is spread over a booster area of

$$
\mathrm{A}_{\mathrm{B}}=2 \pi \mathrm{R} \Delta \mathrm{z}
$$

and kill time is increased by the ratio

$$
\frac{t_{k}(\text { rot })}{t_{k}(0)}=\frac{A}{A_{\perp}}=\pi
$$

Next consider the small beam case (2). The beam spot on the booster has area $\pi \mathrm{a}^{2}$. Assuming the defense keeps this spot fixed on the booster centerline and that optimal rotation is employed by offense, the energy in the laser spot is spread over a total booster area of $4 \pi \mathrm{Ra}$ (thermal conduction and reradiation are ignored). Hence kill time is increased according to

$$
\frac{\mathrm{t}_{\mathrm{k}}^{\prime}(\mathrm{rot})}{\mathrm{t}_{\mathrm{k}}(0)}=\frac{4 \pi \mathrm{Ra}}{\pi \mathrm{a}^{2}}=4\left[\frac{\mathrm{R}}{\mathrm{a}}\right]>4 .
$$

Hot spot tracking has been suggested as a means for the defense to counter booster rotation. It should be noted that hot spot tracking is applicable only to case (2) and that even in this case booster rotation increases kill time since the beam will not remain normal to the booster surface and eclipsing will occur if missile failure is not achieved before the hot spot rotates to the back side of the missile. For a sufficiently high rate of rotation eclipsing will always occur and kill time is increased by a factor of $\pi$ (thermal conduction and reradiation are ignored) as in case (1). Hot spot tracking places heavy burdens on the defense since it must achieve beam spot sizes small compared to missile diameters and in addition have the capability to sense and track the hot spot in a dynamic environment; in contrast, the offense must only achieve a certain rate of booster rotation. We see that booster rotation must be assumed if thermal kill lasers are used, since the offense can always enforce an increase in kill time by at least a factor of $\pi$. Booster rotation has no 
effect on impulse kill directed energy weapons.

Less discussed is rotation of post-boost vehicles. Here the attractiveness to the offense of rotation is less clear since the PBV must make precision deployments of RVs and decoys during its lifetime. These tasks are likely to be complicated by $\mathrm{PBV}$ rotation. For RVs there can be no doubts. To achieve stability in atmospheric reentry, all modern missile systems employ some means of spinning up RVs before or after release from the PBV; in short, RVs already spin.

In addition to rotation, boosters can be further hardened against lasers by the addition of a layer of ablative material on exterior surfaces. This, too, is a relatively simple countermeasure and can probably be done on a retrofit basis; a throw-weight penalty is involved. Alternatively, if the offense chooses to introduce a totally new missile design, either in response to a ballistic missile defense or in the regular course of a modernization program, it can select a slightly larger missile than the one being replaced and retain the previous throw-weight while including ablative coatings.

Since there has been controversy ${ }^{8}$ concerning the penalties for retrofitted ablative shielding, we present here a detailed discussion with emphasis on the basic physical principles involved. Our numbers are not intended to reflect serious engineering design. The reader is referred to the discussion of heats of ablation in Chapter 6 to learn what level of protection a given mass per unit area of ablator provides against thermal laser attack.

We note first that the missiles of interest are all multistage so the question of what stages need hardening must be discussed first. If we take the three-stage missile described in the table above which shows first stage burnout at $25 \mathrm{~km}$, it is clear that the offense would have no need to harden the first stage if the defense was using weapons unable to penetrate to this depth in the atmosphere, e.g., X-ray lasers, HF chemical lasers, short wavelength impulse kill lasers (having short pulse duration), and neutral particle beams. Even if the defense could penetrate below first-stage burnout altitudes, the offense could still forego first-stage hardening if defense response times exceed first-stage burnout time $(60 \mathrm{~s}$ in the example above but much less for the fast burn booster designs discussed below). Similarly, one may or may not need to harden the second stage of a multistage booster depending on burnout time and defensive weapon characteristics and response time.

For algebraic simplicity we consider a two-stage missile with a post-boost vehicle which we will refer to as the payload. This payload consists of PBV structural components and equipment, PBV fuel, RVs, and decoys. The formulas presented below are easily generalized to the three-stage case. We begin with the case of no shielding and write the mass of the first stage as $m_{1}=m_{s 1}+m_{p 1}$ and the mass of the second stage as $m_{2}=m_{s 2}+m_{p 2}$ where $m_{p 1}$ and $\mathrm{m}_{\mathrm{p} 2}$ denote propellant masses and $\mathrm{m}_{\mathrm{s} 1}$ and $\mathrm{m}_{\mathrm{s} 2}$ denote "dry" stage masses (i.e., shell, empty propellant tanks, rocket motors, etc.). Except for rocket engines and a few small components, it is a good approximation to assume that structure masses scale with propellant masses so we may write $\mathrm{m}_{\mathrm{s} 1}=\alpha_{1} \mathrm{~m}_{\mathrm{p} 1}$ and $\mathrm{m}_{\mathrm{s} 2}=\alpha_{2} \mathrm{~m}_{\mathrm{p} 2}$ where $\alpha_{1}$ and $\alpha_{2}$ are "tankage" factors. Typically for solid fuel rockets $\alpha=0.10$, whereas for liquid fuel missiles $\alpha=0.15$.

The rocket equation with the gravity term ignored gives for the total velocity increment,

$$
\begin{aligned}
\Delta \mathrm{v}_{\mathrm{tot}}= & \mathrm{gI}_{\mathrm{sp} 1} \ln \left\{\frac{\mathrm{m}_{1}+\mathrm{m}_{2}+\mathrm{m}_{\mathrm{p} 0}}{\mathrm{~m}_{\mathrm{s} 1}+\mathrm{m}_{2}+\mathrm{m}_{\mathrm{p} 0}}\right\} \\
& +\mathrm{gI}_{\mathrm{sp} 2} \ln \left\{\frac{\mathrm{m}_{2}+\mathrm{m}_{\mathrm{p} 0}}{\mathrm{~m}_{\mathrm{s} 2}+\mathrm{m}_{\mathrm{p} 0}}\right\} \\
= & \mathrm{gI}_{\mathrm{sp} 1} \ln \left\{\frac{\left(1+\alpha_{1}\right) \mathrm{m}_{\mathrm{p} 1}+\left(1+\alpha_{2}\right) \mathrm{m}_{\mathrm{p} 2}+\mathrm{m}_{\mathrm{p} 0}}{\alpha_{1} \mathrm{~m}_{\mathrm{p} 1}+\left(1+\alpha_{2}\right) \mathrm{m}_{\mathrm{p} 2}+\mathrm{m}_{\mathrm{p} 0}}\right\} \\
& +\mathrm{gI}_{\mathrm{sp} 2} \ln \left\{\frac{\left(1+\alpha_{2}\right) \mathrm{m}_{\mathrm{p} 2}+\mathrm{m}_{\mathrm{p} 0}}{\alpha_{2} \mathrm{~m}_{\mathrm{p} 2}+\mathrm{m}_{\mathrm{p} 0}}\right\}
\end{aligned}
$$

where the first logarithm on the right-hand side of Eq. (2.5) represents the velocity contribution from stage 1 and the second logarithm that from stage 2. The quantities $I_{\mathrm{sp1,2}}$ are the specific impulses of the rocket fuel; they are related to the exhaust velocities of the two stages according to $\mathrm{v}_{\mathrm{e}}=\mathrm{gI}_{\mathrm{sp}}$ where $\mathrm{g}$ is the acceleration of gravity at sea level. In Eq. (2.5) $\mathrm{m}_{\mathrm{p} 0}$ is the payload (without shielding).

If the tankage factors and specific impulses of the two stages are equal, optimal staging (minimum propellant to deliver the payload to a given range) occurs when the velocity increments of the two stages are equal. It follows that

$$
\begin{aligned}
& \frac{m_{\mathrm{p} 2}}{m_{\mathrm{p} 0}}=\frac{E-1}{1-\alpha(E-1)}, \\
& \frac{m_{\mathrm{p} 1}}{m_{\mathrm{p} 2}}=\frac{E}{1-\alpha(E-1)},
\end{aligned}
$$

where

$$
E \equiv \exp \left[\frac{\Delta v_{\text {tot }}}{2 \mathrm{gI}_{\mathrm{sp}}}\right) .
$$

As a numerical example consider a "nominal" SS-18 with $\mathrm{m}_{\mathrm{p} 0}=8000 \mathrm{~kg}$ (i.e., 8 tonnes), $\alpha=0.15, \Delta \mathrm{v}_{\text {tot }}=7 \mathrm{~km} / \mathrm{s}$, $\mathrm{I}_{\mathrm{sp}}=306 \mathrm{~s}$. The above equations give stage masses $\mathrm{m}_{1}$ $=146.2$ tonnes, $\mathrm{m}_{2}=30.4$ tonnes, a gross (liftoff) mass $\mathrm{M}_{0}=\mathrm{m}_{1}+\mathrm{m}_{2}+\mathrm{m}_{\mathrm{p} 0}=184.6$ tonnes, and an exhaust velocity $\mathrm{v}_{\mathrm{e}}=3 \mathrm{~km} / \mathrm{s}$.

Now add shielding as a retrofit. The propellant and structure masses of the two stages remain fixed and so also does $\Delta v_{\text {tot }}$ since we are supposing the same range. To achieve this same final velocity after adding shielding, the payload is reduced to $m_{p}$ and is the quantity we wish to calculate. It is obvious that any shielding added to the PBV will subtract from the useful payload on a kilogram-for-kilogram basis. Recognizing this, it is 
technically convenient to discuss the case of no PBV shielding and then subtract by hand the effect of such shielding.

Denoting the mass of the ablative shields as $m_{a 1}$ and $\mathrm{m}_{\mathrm{a} 2}$ on stages 1 and 2 , respectively, the corresponding rocket equation follows by making the substitutions $\mathrm{m}_{\mathrm{s} 1} \rightarrow \mathrm{m}_{\mathrm{s} 1}+\mathrm{m}_{\mathrm{a} 1}, \mathrm{~m}_{\mathrm{s} 2} \rightarrow \mathrm{m}_{\mathrm{s} 2}+\mathrm{m}_{\mathrm{a} 2}$, and $\mathrm{m}_{\mathrm{p} 0} \rightarrow \mathrm{m}_{\mathrm{p}}$ in Eq. (2.5). Because the payload and mass of the second stage shield enters only in the combination $m_{a 2}+m_{p}$ we see immediately that the second stage shielding also subtracts from payload on a kilogram-for-kilogram basis. Physically this is obvious since the second stage shield is carried to the final payload velocity before being discarded. We may express this as

$$
-\left[\frac{\partial \mathrm{m}_{\mathrm{p}}}{\partial \mathrm{m}_{\mathrm{a} 2}}\right]_{\mathrm{m}_{\mathrm{a} 1}}=1
$$

Rather than treating the general case explicitly which is algebraically tedious, it is convenient to consider the case where the ablative shield masses scale according to $\mathrm{m}_{\mathrm{a} 1}=\sigma \mathrm{m}_{\mathrm{p} 1}, \mathrm{~m}_{\mathrm{a} 2}=\sigma \mathrm{m}_{\mathrm{p} 2}$. With the answer to this case in hand and the answer for second stage shielding alone one can readily calculate the payload reduction for any mix of first and second stage shielding.

The appropriate rocket equation for retrofitted shielding with first and second stage shielding and equal tankage factors may be obtained from Eq. (2.5) by the substitution $\alpha \rightarrow \alpha+\sigma$ and $\mathrm{m}_{\mathrm{p} 0} \rightarrow \mathrm{m}_{\mathrm{p}}$. Note, however, that one cannot make the same substitutions and use Eqs. (2.6) and (2.7) since these apply to optimal staging in the absence of shielding. After adding shielding on a retrofit basis, staging will not remain optimal-the two stages will give unequal increments to the net velocity. Only if one considers a new missile with shielding and optimal staging can Eqs. (2.6) and (2.7) be used.

For a retrofit shielding, the equation which determines $m_{p}$ as a function of $\sigma$ is

$$
\frac{\left[(1+\alpha+\sigma) \mathrm{m}_{\mathrm{p} 1}+(1+\alpha+\sigma) \mathrm{m}_{\mathrm{p} 2}+\mathrm{m}_{\mathrm{p}}\right]}{\left[(\alpha+\sigma) \mathrm{m}_{\mathrm{p} 1}+(1+\alpha+\sigma) \mathrm{m}_{\mathrm{p} 2}+\mathrm{m}_{\mathrm{p}}\right]} \frac{\left[(1+\alpha+\sigma) \mathrm{m}_{\mathrm{p} 2}+\mathrm{m}_{\mathrm{p}}\right]}{\left[(\alpha+\sigma) \mathrm{m}_{\mathrm{p} 2}+\mathrm{m}_{\mathrm{p}}\right]}=\mathrm{E}^{2}
$$

along with Eq. (2.8). The quantity $\sigma$ is in turn specified in terms of total shielding mass according to

$$
\sigma=\mathrm{m}_{\mathrm{a}} /\left(\mathrm{m}_{\mathrm{p} 1}+\mathrm{m}_{\mathrm{p} 2}\right) \text {. }
$$

Although Eq. (2.10) could be solved algebraically for $\mathrm{m}_{\mathrm{p}}$, the results are not particularly illuminating. However, the derivative of the payload versus shielding at the origin has a simple form

$$
-\left[\frac{\partial \mathrm{m}_{\mathrm{p}}}{\partial \mathrm{m}_{\mathrm{a}}}\right]_{\mathrm{m}_{\mathrm{a}}=0}=\left\{1-\frac{1}{\left[1+\frac{1-\sigma(\mathrm{E}-1)}{\mathrm{E}}\right]} \frac{1}{\left[1+\frac{1-\sigma(\mathrm{E}-1)}{1+\sigma(\mathrm{E}-1)^{2}}\right]}\right\}<1 .
$$

After solving Eq. (2.10) and substituting numerical parameters appropriate to the nominal SS-18 introduced above, we obtain the curves shown in Figure 2.7.

Physically the finding that a given mass of shielding distributed over both stages reduces the payload differentially by less than a factor of one reflects the fact that first stage shielding is not carried to the final payload velocity.

If we assume the total surface area of our nominal SS-18 is $300 \mathrm{~m}^{2}$ and that $2 \mathrm{~g} / \mathrm{cm}^{2}$ of shielding is added, the net shielding mass will be 6 tonnes. Proportioning this according to stage masses, we have 4.75 tonnes on the first stage, 0.99 on the second, and 0.26 on the PBV. From Figure 2.7 the payload reduction corresponding to a first and second stage shield of 5.74 tonnes is 1.74 tonnes. To this we must add the 0.26 tonne PBV shield; the net payload reduction is 2 tonnes.

It is incorrect to assume this payload reduction must be met by RV offload alone. The PBV carries fuel as well as RVs (and perhaps decoys). With fewer RVs on board, less RV fuel is required to achieve the same footprint. Furthermore, when faced with the challenge of penetrating a missile defense, the offense may well be willing to adjust its targeting to accommodate smaller footprints, sacrificing PBV fuel in favor of an increased number of RVs and decoys.

Going back to our example, suppose that the original 8 tonne PBV mass consisted of $10 \mathrm{RVs}$ each of about 300 $\mathrm{kg}$, an equal mass of fuel, and $2000 \mathrm{~kg}$ of PBV structure.

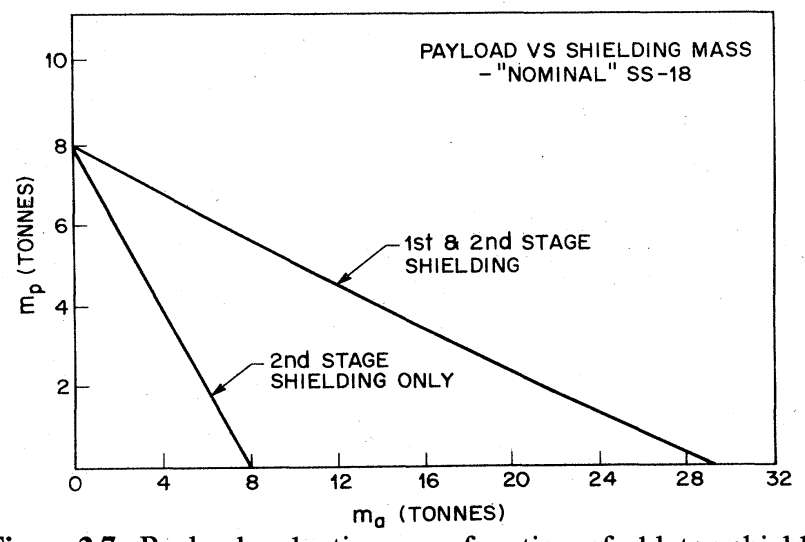

Figure 2.7 Payload reduction as a function of ablator shield mass for a "nominal" SS-18. See text for the assumptions which apply to the various cases. 
For a 2 tonne payload reduction dictated by the addition of 6 tonnes of ablator, equipartition between RV and fuel offloads results in a net reduction of $3 \mathrm{RVs}$. Not included in any of our considerations thus far is another option. The offense could accept a reduced second stage burnout velocity and use PBV fuel to make this up. Whether or not this permits a greater number of RVs to be carried to range depends on details such as the specific impulses of the booster and PBV fuels, missile tankage factors, etc. Additionally, the offense might redesign just the PBV stage (four models of the SS-18 PBV have already seen service) to reduce structure weight in response to the fact that the PBV will be carrying a reduced number of RVs. This might permit the retention of an additional RV which would otherwise have to be offloaded. In any case, it is clear that calculations of $\mathrm{RV}$ reductions required by retrofitting ablative shielding often have hidden assumptions. The offense has many options all of which must be explored before final conclusions can be drawn.

\subsubsection{Fast Burn Boosters}

Because of the high leverage of boost and post-boost phase defenses and because of the greater opportunity to employ decoys once these phases are passed, it is likely that the Soviet response will be strongly conditioned by its perceived capability of U.S. defenses in these first two phases. Current ICBMs have not been designed to cope with boost phase defenses. As a result the boost phase is quite long-typically 3 to 6 minutes, and the burnout altitude is high - typically 200 to $300 \mathrm{~km}$. By the same token, the pace of typical post-boost phases is leisurely and may take a minute or so to place each reentry vehicle (and penetration aid) on its proper trajectory. The SS-18, for example, has a total time from liftoff to completion of post-boost phase of $10 \mathrm{~min}$. Studies ${ }^{9}$ conducted during the past few years have concluded that boost and postboost phases need not be so time consuming. Through the use of modern solid rocket propellants with grain configurations designed for rapid burn, ICBM-range boosters that complete their burn in less than one minute at altitudes of 80 to $100 \mathrm{~km}$ appear feasible. There appear to be no physical barriers to such performance; the only issues are one of engineering tradeoffs.

Although an intercontinental range fast burn booster (FBB) would represent a significant new development, the offensive penalty in terms of throw-weight appears to be small. Studies ${ }^{9}$ performed by McDonnell Douglas Corporation and Martin Marietta in 1983 in support of the Fletcher (DTST) Study indicated that a solid propellant ICBM capable of burning out in $60 \mathrm{~s}$ at an altitude of $80 \mathrm{~km}$ was feasible. The associated payload reduction was found to be approximately $20 \%$ assuming the same launch weight for the FBB as for a conventional solid booster. More recent and comprehensive work performed at Lockheed ${ }^{10}$ which included an analysis of staging, exit heating, interstage structures, and controllability gives similar indications. Figure 2.8, adapted from the Lockheed study, shows throw-weight as a function of booster burntime. The 1983 analyses cited in Reference 9 also concluded that there need be no payload reduction at all associated with fast burn boosters if the overall launch weight is allowed to grow by $15-20 \%$.

Two things are noteworthy at this point. First, an important step toward a fast burn booster is that of using a solid propellant; that step is one already being taken by the Soviets in their SS-20, SS-24, and SS-25 systems. The Soviets are judged to be behind the U.S. in solid propellant technology, but it is not clear that this will be a permanent state of affairs. Second, it is important to note that the term "fast burn booster" can be misleading. What is required for intercontinental velocities at a burnout altitude of $80-100 \mathrm{~km}$ is a peak acceleration of 30-40 $\mathrm{g}$ versus the 8-15 g levels typical of current boosters. This difference does not represent a drastic change. In particular it does not call for technologies associated with very high acceleration boosters such as the 1960 s vintage SPRINT or Spartan interceptors, or for that matter, with modern Soviet ABM interceptors. Of course, fast burn boosters alone are not a fully responsive offensive countermeasure. The offense would want to minimize the total time to completion of RV deployment as well. We discuss rapid post-boost deployments below (Section 2.3.4).

The consequences of a fast burn booster (FBB) response are far reaching:

(i) Space-based $\mathrm{x}$-ray lasers (XRL) cannot penetrate into the atmosphere to altitudes below about $80 \mathrm{~km}$. Hence, FBBs remove booster intercept from XRL missions.

(ii) Space-based neutral particle-beam (NPB) weapons also cannot penetrate into the atmosphere to altitudes below about $120 \mathrm{~km}$. Hence, FBB removes booster intercept from NPB missions.

(iii) The FBBs short burn time also taxes weapons that are capable of penetrating into the atmosphere

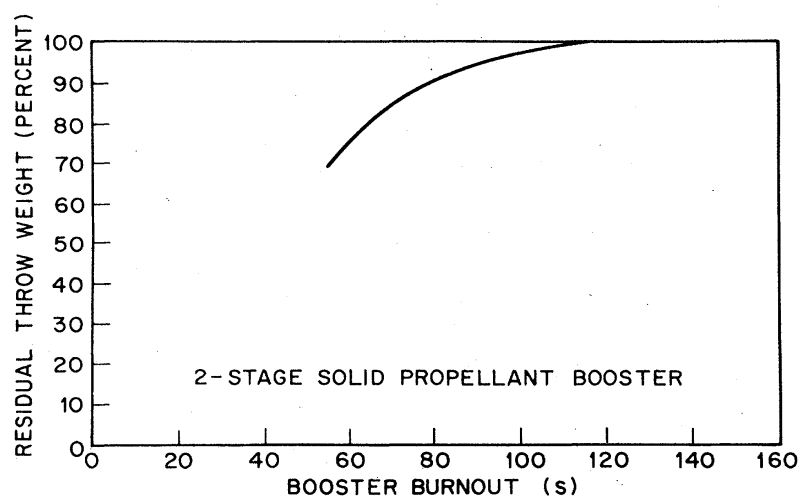

Figure 2.8 Fast burn booster performance: throw-weight penalty vs booster burn time. (Adapted from Lockheed, Ref. 10.) 
(space-based and ground-based lasers, and kinetic energy weapons) simply because the engagement of simultaneously launched boosters must be completed in a few tens of seconds. This places extreme demands for short retarget time, kill time, and/or increase the number of battle stations required to cope with a given size attack.

(iv) A FBB response would almost certainly make popup defense against the boost phase unreasonable due to the short time available for the battle.

\subsubsection{Post-Boost Vehicle Redesign}

While fast burn boosters pose severe problems for a defense seeking to kill the booster itself, the offense is not necessarily "home free" against DEWs or other defenses employed in the post-boost phase.

With a FBB burnout altitude of approximately $80 \mathrm{~km}$, the offense is faced with problems if it plans to deploy lightweight decoys. There is enough atmosphere at such altitudes to result in differential deceleration of heavy reentry vehicles and lightweight decoys. This difference could allow the defense elements to discriminate RVs from decoys, something the offense would wish to avoid. Figure 2.9 illustrates this problem and shows that if, for example, the defense is given credit for acceleration measurements of $10^{-2} \mathrm{~g}$, the offense would need to delay deployment from the PBV until an altitude exceeding $120 \mathrm{~km}$ was reached. This implies the need for a coast phase in the interval between booster burnout and the time $\mathrm{RV} / \mathrm{decoy}$ releases are initiated. Further, it implies that if faced by a defensive threat, the PBV designer will work to complete the release of all RVs and the deployment of all decoys and penetration aids in

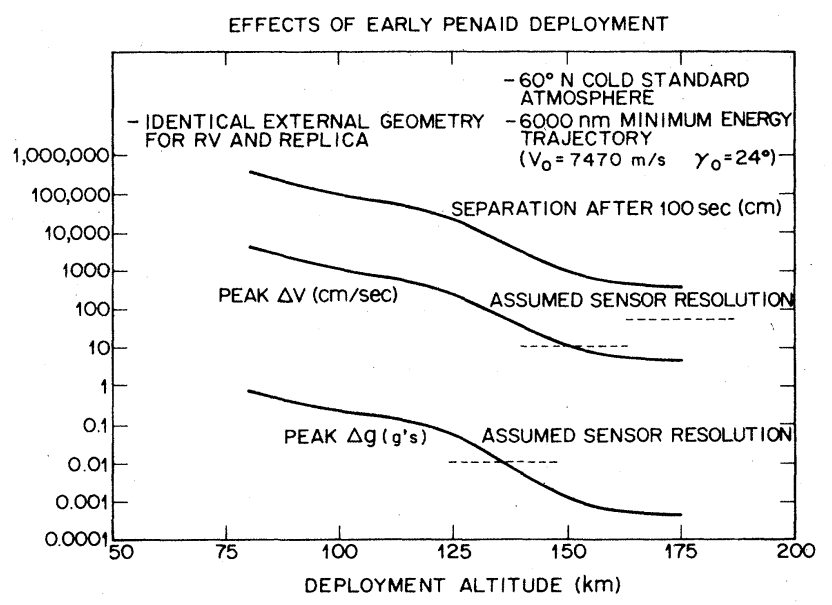

Figure 2.9 Atmospheric deceleration as a function of altitude for decoy weighing $1 \%$ that of an RV and of identical shape and size. Units of deceleration are g's $\left(9.8 \mathrm{~m} / \mathrm{g}^{2}\right)$; for other quantities as indicated in parenthesis. the shortest possible time once altitude is reached since the PBV represents a high value target until its operation is complete. Alternatively, one could contemplate adding small thrusters to RVs and decoys alike to make up the residual drag caused by the atmosphere, and thus permit release within the atmosphere. The thrust required is independent of the mass of the decoy or the RV provided the decoys have the same shape and size as RVs. Thrust would have to be programmed with altitude for optimum performance, however. It is not clear if this complexity would be an attractive option for the offense.

To date there has been no pressure to complete PBV operations rapidly since neither the Soviet Union nor the U.S. has been faced with a defensive threat in the postboost phase. Only cursory studies have been conducted in the area to date. Nevertheless, a substantial reduction in the time of the PBV phase could be achieved through changes in PBV operations (faster response time controls, improved guidance, software, etc.) while keeping unchanged the basic PBV concept.

An offense, faced with a perceived threat to its missiles in post-boost phase, might also make more drastic changes such as using multiple PBVs (mini-buses) on each booster with each dispensing an RV and one or more decoys against a given target. Such an approach would multiply the number of PBV targets and force the defense to shorter retarget times, shorter kill times, and/or a proliferation of battle stations. Such mini-buses could be released immediately after booster burnout reducing the high leverage enjoyed by post-boost phase defenses. After reaching $120 \mathrm{~km}$ altitude, each mini-bus could release its $\mathrm{RV}$ and decoys. It is worth noting that the multiple PBV concept will likely become more affordable in the future as electronics, and guidance systems are available at lower weight and volume - trends already present, and believed likely to continue.

In considering the possibilities for rapid deployment from a single PBV or changes to multiple PBV designs it is important to remember that very little work has been done in the area in the U.S. The data needed for the determination of offense/defense cost benefit exchange ratios can only be obtained if more attention (experimental as well as analytical) is given to this important problem.

\subsubsection{Decoys and Penetration Aids}

Once elements of the offense get through a defensive boost and post-boost phase, the battle and thus the offensive response can take on a very different nature. The offense will seek to deploy decoys and other penetration aids in large numbers. The key task in midcourse becomes one of discriminating lightweight decoys from heavy RVs and doing this in a high traffic environment. In thinking about mid-course countermeasures, it is important to remember that there is no atmospheric drag, and so all objects move in ballistic 
orbits. This permits countermeasures which are extremely lightweight.

We defer most of our discussion of decoys and penetration aids to Chapter 7 (Acquisition, Tracking, and Discrimination). Here we simply note that preliminary designs suggest that effective decoys (i.e., having the same shape and size as a $200 \mathrm{kt} \mathrm{RV)} \mathrm{can} \mathrm{be} \mathrm{constructed} \mathrm{with} \mathrm{a}$ mass of $1-2 \mathrm{~kg}$. Considering that a $200 \mathrm{kt} \mathrm{RV} \mathrm{might}$ weigh approximately $200 \mathrm{~kg}$, this suggests that for each RV offloaded one might be able to substitute approximately 100 to 200 replica decoys. For example, using the $2 \mathrm{~kg}$ figure for the replica mass a single $4000 \mathrm{~kg}$ payload booster, therefore, might deliver into the mid-course battle $10 \mathrm{RVs}$ and 1000 decoys (actually from $20 \mathrm{RVs}$ and 0 decoys to $0 \mathrm{RVs}$ and 4000 decoys depending on the offensive missile load-out).

For the nominal case, one sees that if 100 PBVs survive the boost and post-boost phases, the mid-course is faced with $1000 \mathrm{RVs}$ and 100000 decoys. Or if things go astray for the defense during the first two phase and 1000 PBVs survive, the mid-course defense could be faced with $10000 \mathrm{RVs}$ and 1000000 decoys. The mid-course defense's task is thus critically dependent on the success of the defense's boost phase. This cannot be overemphasized.

It is clear that, unless the boost and post-boost defenses are very successful, the mid-course defense faces a massive traffic and discrimination problem.

\subsection{SUMMARY AND CONCLUSIONS}

- One can confidently expect that there will be a strong response from the Soviets to the deployment of any type of missile defense by the U.S.

- Because the precise nature of the Soviet response is unpredictable, the job of designing effective defenses is especially difficult.

- The analysis of responsive threats needs focused analytical and experimental study in order to gain an understanding of the relative technology difficulty, effectiveness, cost, and lead time of defensive and offensive moves.

- It is reasonable that the Soviets will be able to deploy responses even before the U.S. can deploy a DEW defense. Hence U.S. defenses will be pitted against a responsive Soviet threat from the beginning.

- A key problem for boost phase defenses, and even more so for mid-course and terminal defenses, is potentially very high traffic rates. Boost phase is further complicated by potentially very short total battle time. Retarget and kill times are likely to be critical parameters in system architecture choices.
- Preliminary studies suggest that boost phase times can be reduced to less than $60 \mathrm{~s}$. These conclusions need to be explored in detail since such threats would greatly increase the difficulty of building defenses.

- Even after achieving a fast burn booster capability, the offense would still be faced with the need to develop means to deploy RVs and decoys quickly once above the sensible atmosphere. This area needs detailed study to clarify possible limitations and penalties.

- Key issues in the mid-course phase are the potentially very large number of objects with overlapping signatures, the fact that objects in midcourse have small signatures, and the requirement that the defense have large traffic handling capabilities and short, retarget times.

- The combined performance of the boost and postboost defensive layers is particularly critical since without a reasonably efficient boost/post-boost phase defense, the offense will find proliferation of large boosters an attractive and straightforward response.

\section{REFERENCES}

${ }^{1}$ A. D. Wheelon, "Free Flight of a Ballistic Missile," Journal of the American Rocket Society VVV, 915 (1959).

${ }^{2}$ J. J. Martin, Atmospheric Reentry (Prentice Hall, New York), Chapter 1.

${ }^{3}$ Soviet Military Power, 5th Edition (U.S. Department of Defense, 1986).

${ }^{4}$ U.S.-Soviet Military Balance 1980-1985, J. M. Collins (Pergamon-Brassey, Washington, 1985).

${ }^{5}$ R. P. Berman and J. C. Baker, Soviet Strategic Forces (The Bookings Institution, 1982).

${ }^{6}$ P. H. Nitze, "On the Road to a More Stable Peace," Speech to the Philadelphia World Affairs Council, Feb. 20, 1985, National Security Decision Directive 172 (May 30, 1985).

${ }^{7}$ Soviet Strategic Force Developments, Testimony Before a Joint Session of the Subcommittee on Strategic and Theater Nuclear Forces of the Senate Armed Services Committee and The Defense Subcommittee of the Senate Committee on Appropriations, June 26, 1985, by Robert M. Gates, Chairman, National Intelligence Council, and Deputy Director for Intelligence, Central Intelligence Agency; and Lawrence $\mathbf{K}$. Gershwin, National Intelligence Officer for Strategic Programs, National Intelligence Council.

${ }^{8}$ Letters to the Editor, Phys. Today, March 1986, July 1986.

${ }^{9}$ Studies done in support of the DTST; Short Burn Time ICBM Characteristics and Considerations, Martin Marietta (1983); Costs of Small Missiles, McDonnell Douglas Corp. (1983); see also U.S. Congress, Office of Technology Assessment, Ballistic Missile Defense Technologies, OTA-ISC-254 (U.S. Government Printing Office, Washington, D.C., September 1985).

${ }^{10}$ Fast-Burn Missile Requirement Analysis, Robert Dietz, Lockheed Missiles, and Space Co., Inc. (private communication, 1986). 


\section{Chapter 3}

\section{LASERS}

\section{CONTENTS}

3.1 Introduction and Overview

3.1.1 Historical Review

3.1.2 Mission Requirements

3.2 Chemical Lasers

3.2.1 Background

3.2.2 The HF/DF Laser System

3.2.2.1 Critical Issues

3.2.3 Electronic Transition Oxygen/Iodine Lasers

3.2.3.1 Background

3.2.3.2 Laser Gain

3.2.3.3 Chemical Generation of Excited Molecular Oxygen

3.2.3.4 Supersonic Nozzles and Mixing 3.2.3.4.1 $\mathrm{I}_{2}$ Dissociation

3.2.3.5 Scaling Parameters and Efficiencies for LargeScale $\mathrm{O}_{2}$-I Lasers

3.2.3.6 Optics

3.2.3.7 Device Sizing

3.2.3.8 Scaling Issues

3.2.3.9 Critical Issues

3.2.4 Visible Chemical Lasers

3.3 Excimer Lasers

3.3.1 Background

3.3.2 History

3.3.3 General Features

3.3.4 Krypton Fluoride

3.3.5 Xenon Chloride

3.3.6 Electron Beam Pumping

3.3.7 Raman Conversion and Beam Combination

3.3.8 Beam Cleanup Using Stimulated Brillouin Scattering

3.3.9 Critical Issues

3.4 Free Electron Lasers

3.4.1 Principles of Operation and Recent Results

3.4.1.1 Principles

3.4.1.2 Recent Experiments

3.4.2 Vital Issues

3.4.2.1 Beam Quality

3.4.2.2 Beam Brightness

3.4.2.3 FEL Instabilities

3.4.2.4 Transport

3.4.2.5 Tolerances and Reliability

3.4.2.6 Harmonics

3.4.3 Oscillators and Amplifiers

3.4.3.1 Oscillators

3.4.3.2 Amplifiers

3.4.4 System Comparisons

3.4.5 FEL Critical Issues
3.5 X-Ray Lasers

3.6 Gamma-Ray Lasers

3.7 Conclusions

References

\subsection{INTRODUCTION AND OVERVIEW}

High power lasers are considered potentially attractive as directed energy weapons because of their ability to deliver destructive energy at the speed of light to a distant target. Their promise for high rate of fire as well as agility coupled with aiming could permit tracking of a highly maneuvering target and shifting from target to target on command. The weapons potential of the laser was recognized soon after demonstration of the first lasers in the early sixties, and a broad program of weaponsoriented laser research and development has been conducted by various federal agencies for the last twenty years.

\subsubsection{Historical Review}

It is possible to gather a historical perspective on the realizability of technology goals from the experience of previous or currently more mature laser device development activities. Three classes of device technologies may be considered for such perspectives: the $\mathrm{CO}_{2}$ laser, ${ }^{1}$ the HF/DF chemical laser, ${ }^{2}$ and the Nd-glass solid state laser. ${ }^{3}$

The $\mathrm{CO}_{2}$ combustion driven gas dynamic laser (GDL) was developed ${ }^{4}$ in the latter half of the sixties and a major commitment to build a $1 \mathrm{MW}$ class GDL was made in 1969. This was estimated to be a two-year program. It actually took three years and achieved a substantially reduced level of performance with very poor beam quality. A second generation GDL technology device was built at the several hundred kilowatt level, again with poor beam quality, and a third generation GDL was started in 1974 for the Airborne Laser Laboratory. This device was conceived to be $0.5 \mathrm{MW}$ of power and 1.3 times diffraction limited. Such performance goals were realized, albeit two years later than planned.

In 1976 a commitment was made to develop a HF/DF chemical laser with a near diffraction limited power output in the megawatt class within five years. ${ }^{5}$ 
However, it was only in 1984 that megawatt class power output was realized. Near diffraction limited operation has been achieved with DF laser technology, but at lower power levels.

The Nd-glass laser was one of the first lasers developed, dating from the early 1960s. The first highpower rod system, developed at CGE in France, in the late $1960 \mathrm{~s}$, did achieve an output energy of order $100 \mathrm{~J}$ in $1 \mathrm{~ns}$. The addition of disk amplifiers made it possible to build $1 \mathrm{~kJ}$ class systems, which were operated in 1974. The first large, multibeam system, which incorporated sophisticated beam controls, spatial filtering and optical relaying, was the 20 -beam, $10 \mathrm{~kJ}$ Shiva system at LLNL, operational in 1977. The development of high energy storage glass and large aperture laser amplifiers led to the design of the Nova system, which became operational at the $\sim 60 \mathrm{~kJ}$ level in 1985. At each stage, a factor of ten improvement in performance was obtained, but each factor of ten required major technological advances. Each system met its design objectives, close to schedule, but the required funding exceeded the original budget by a significant factor.

While the evolution of high-power lasers has been rapid, it has occasionally taken completely unexpected turns, both in the physics (e.g., the introduction of raregas halide media) and the technology (e.g., the impressive rise and fall of gas dynamic lasers as weapon prospects). All the lasers currently under intense development seem to have promise of meeting the minimum device criteria for strategic defense weapons utility; yet performance of each of them is several orders of magnitude away from those criteria. Thus it is impossible now to predict which, if any, of these candidates will eventually meet the criteria or whether another, superior candidate will appear.

\subsubsection{Mission Requirements}

The common measure, both of laser effectiveness and systems requirement, is the quantity "brightness," measured in $\mathrm{W} / \mathrm{sr}$ (or $\mathrm{J} / \mathrm{sr}$ for a pulsed laser). This useful parameter is derived quite simply. The fluence, $F$ (in $\mathrm{J} / \mathrm{cm}^{2}$ ), delivered to a target by a laser is calculated by conservation of energy. For a laser of output power $\mathbf{P}$ (W), the fluence is the product of the power and the pulse length, $\Delta \mathrm{t}(\mathrm{s})$, divided by the area, $\mathrm{A}\left(\mathrm{cm}^{2}\right)$, of the laser spot on the target and a dimensionless number, $L$, to account for transmission losses between the laser and the target:

$$
F=\frac{P \Delta t}{L A}
$$

Here the pulse shape is assumed, for simplicity, to be rectangular.

It has become customary to leave the loss term $\mathrm{L}$ out of the brightness calculation, and to estimate the effects of such losses elsewhere. For the moment, then, $\mathbf{L}$ will be considered to be unity.

The radius of the spot on the target is the product of the range $\mathrm{R}$ and the far-field beam divergence $\sigma$ which, for a diffraction-limited beam is roughly the wavelength $\lambda$ divided by the laser aperture diameter $D$. Thus, the area of the laser spot is

$$
\mathrm{A}=\frac{\pi \mathrm{R}^{2} \lambda^{2}}{\mathrm{D}^{2}} .
$$

Combining this with the previous expression,

$$
\mathbf{F}=\frac{\mathrm{P} \Delta t \mathrm{D}^{2}}{\pi \mathrm{R}^{2} \lambda^{2}}
$$

Note now that if we multiply both sides by $\mathrm{R}^{2}$ and divide both by $\Delta t$, we will have one expression (on the left) defined entirely in terms of the laser's mission-the fluence on the target, the dwell time, and the range. At the same time, the terms on the right will be defined entirely in terms of the laser itself - its power, wavelength, and aperture size. There are then two equivalent expressions of brightness:

$$
\mathrm{B} \equiv \frac{\mathrm{FR}^{2}}{\Delta \mathrm{t}}=\frac{\mathrm{PD}^{2}}{\pi \lambda^{2}}
$$

It is instructive to demonstrate the ranges of interest of these parameters. In Figure 3.1 the required brightness-time product is plotted for various values of

BRIGHTNESS-TIME PRODUCT VS FLUENCE AND RANGE (TIME IN SEC)

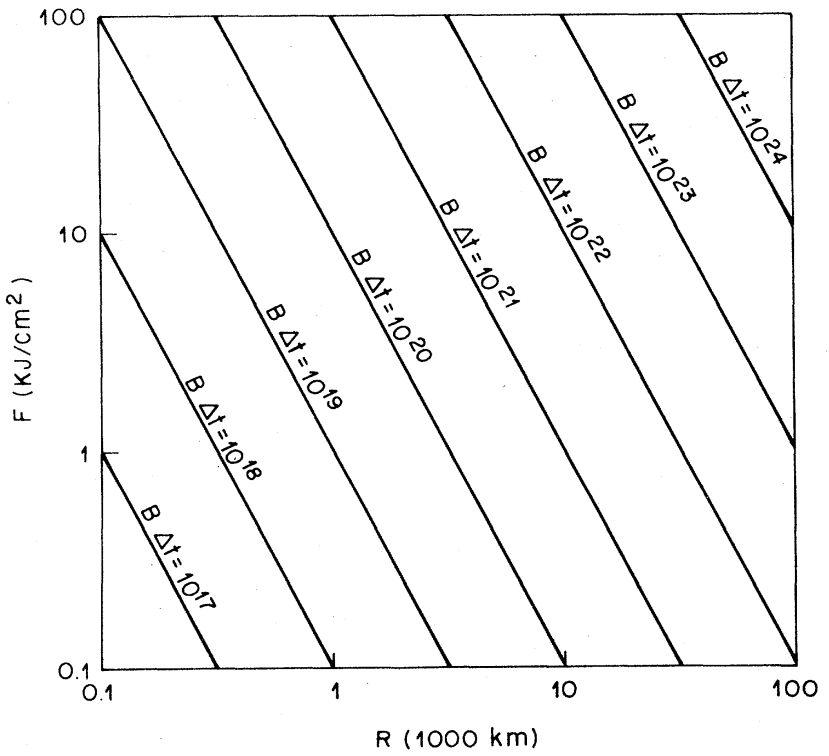

Figure 3.1. Brightness-time product required as a function of target hardness and range. 
target hardness, in $\mathrm{kJ} / \mathrm{cm}^{2}$, and target range. Hardness values range from $1 \mathrm{~kJ} / \mathrm{cm}^{2}$ (soft targets) to $100 \mathrm{~kJ} / \mathrm{cm}^{2}$ (hardened targets). For a nominal booster hardness ${ }^{6}$ of $10 \mathrm{~kJ} / \mathrm{cm}^{2}$, at a range of $1000 \mathrm{~km}$, the brightness-time product needed is $10^{20} \mathrm{~J} / \mathrm{sr}$. Assuming that the engagement time is $0.1 \mathrm{~s}$ (as noted in calculations which follow later in this chapter), a brightness of $10^{21} \mathrm{~W} / \mathrm{sr}$ is required. In contrast, to kill even a soft target from synchronous orbit $(38500 \mathrm{~km}), 10^{22}<\mathrm{B} \Delta \mathrm{t}<10^{23}$ is required.

To translate brightness requirements into laser performance, examine Figure 3.2. Here the product $\mathrm{B} \lambda^{2}$, where $\lambda$ is the operating wavelength, is plotted as a function of the laser power (MW) and mirror diameter (m). The advantages of shorter wavelength are apparent, assuming that all systems are diffraction limited. Diffraction-limited operation at $0.3 \mu \mathrm{m}$, with a $10 \mathrm{~m}$ mirror, requires a pointing accuracy and optical stability of better than 30 nrad.

If the mission requires a brightness of $10^{21} \mathrm{~W} / \mathrm{sr}$, a laser at $1 \mu \mathrm{m}$, operating with a $10 \mathrm{~m}$ mirror, will need to generate about $30 \mathrm{MW}$ (assuming no transmission losses). At $0.3 \mu \mathrm{m}$ wavelength, with the same mirror, only $3 \mathrm{MW}$ would be needed; at $3 \mu \mathrm{m}, 300 \mathrm{MW}$ would be required.

For reasonably hard targets, at ranges of a few thousand $\mathrm{km}, \mathrm{B} \Delta \mathrm{t}$ will fall in the range from $10^{20}-10^{21}$. With engagement times of 0.1 to $1 \mathrm{~s}$, and mirror diameters of $10-20 \mathrm{~m}$, it follows that the requirements will lie between $50 \mathrm{MW}$ and $500 \mathrm{MW}$, depending on the wavelength, basing mode, and optical quality, again assuming no propagation losses.
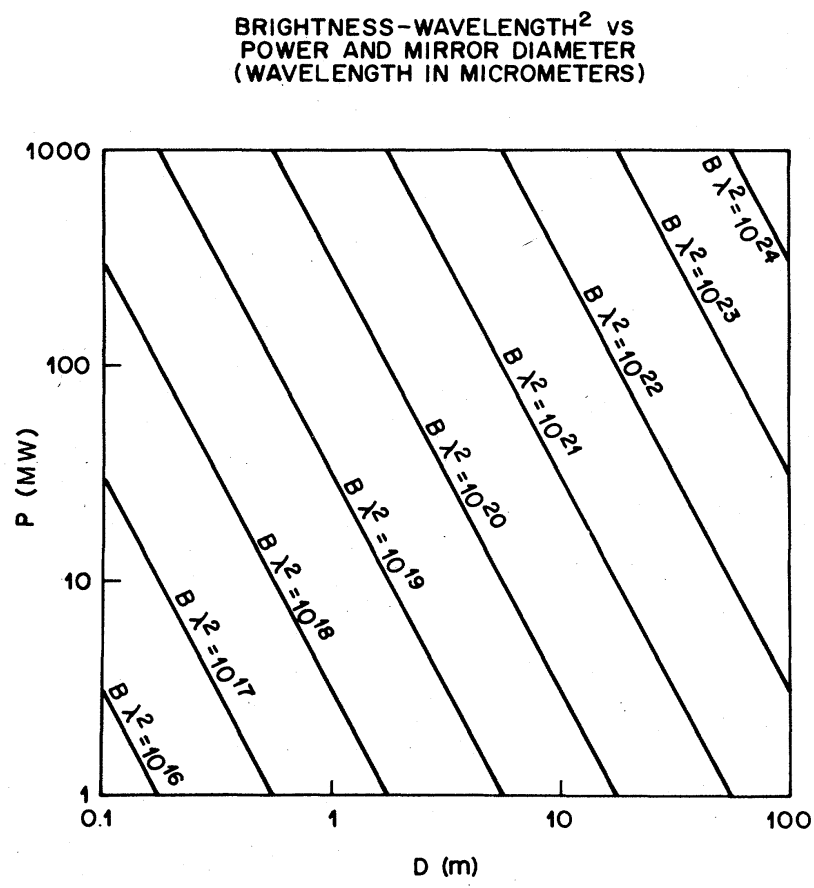

Figure 3.2. Brightness requirements.
Thus, our first-order estimates are clear. For strategic defense missions, we need lasers with brightnesses in the range of

$$
10^{20} \lesssim B \lesssim 5 \times 10^{23}
$$

These requirements imply that space-based lasers must have powers of tens of MW or more, ground-based excimer lasers must have energies exceeding $100 \mathrm{MJ}$, and ground-based FELs must have powers in the $\mathrm{GW}$ range or more.

It is necessary now to recall the two important idealizations made in the derivation. Losses, particularly arising from atmospheric scattering and absorption and from beam jitter, are certain to be significant, raising all the power/energy requirements to meet the mission requirements. Because of Rayleigh scattering of the soft UV light, it is probable that ground-based excimer laser will have to meet $\mathrm{a} \gtrsim 100 \mathrm{MJ}$ requirement. Propagation limitations are discussed in detail in Chapter 5.

The other idealization was the assumption of diffraction-limited beams. If the laser produces a beam which is $\mathrm{n}$ times diffraction limited (as measured by the radius of the first Airy ring), its brightness is reduced by a factor $\mathrm{n}^{2}$. Thus, there is a very strong incentive to achieve good beam quality in all these high-brightness lasers.

Three optical laser systems are candidates for a directed energy weapons system. These are the chemical laser, ${ }^{7}$ the excimer laser, ${ }^{8}$ and the free electron laser. ${ }^{9}$ While other high-power lasers such as the glass laser or the $\mathrm{CO}_{2}$ laser are perhaps more familiar types available, they are not suitable for meeting the military requirements of ballistic missile defense applications. For example, the poor thermal conductivity of glass or even crystal lasers, when combined with their relatively low efficiency, does not allow a feasible firing rate at very high powers. The $\mathrm{CO}_{2}$ laser was the first of the class of molecular vibrational-rotational transition lasers. While it does lend itself readily to high powers and indeed was one of the first of the high-power laser systems to be considered to have weapons potential, the $\mathrm{CO}_{2}$ laser is effectively ruled out in the U.S. in the present context due to its long wavelength (between $9 \mu \mathrm{m}$ and $11 \mu \mathrm{m}$ ) which dictates large optics.

A fourth class of laser has recently emerged. This is the x-ray laser, which uses a thermonuclear explosion to achieve population inversion and produce lasing action. Because of atmospheric absorption, the nuclearexplosion-pumped x-ray laser must be used as either a space-based or a "pop-up" device; in the latter case, the entire lasing system would be mounted on a rapid ascent rocket and lofted out of the atmosphere.

There are important trade-offs between the choice of wavelength and choice of basing mode. Very short wavelength $(\lambda<200 \mathrm{~nm})$ radiation penetrates the atmosphere so poorly that such lasers would have to be based in space (or popped up). On the other hand, laser 
wavelength in the mid- and long-wave infrared $(\lambda>2 \mu \mathrm{m})$ used for booster intercept with ground-based systems, would not be practical because the long distances between relay optics would lead to requirements for optical components that are too large. Hence, such lasers are generally discussed for space basing. In the intermediate wavelength regime (200 $\mathrm{nm}<\lambda<2 \mu \mathrm{m})$, the known efficiencies of the excimer lasers (typically several percent) impose thermal management difficulties, and necessitate large, bulky power supplies; as a result such lasers should be ground based. For the free electron lasers overall efficiencies may be tens of percent, and both ground-based and space-based systems are envisioned.

Of course, either basing mode has associated problems of cost and complexity in system design. Space-based components require many redundant elements to account for the fact that the majority of the space platforms will be out of range at any given time. This so-called absentee ratio is discussed in Appendix B, and the impact of the absentee ratio is discussed in Section 6.5.7.

Ground-based laser systems may require a severalfold increase in power or pulse energy because of atmospheric transmission losses, and require redundancy through geographic dispersion to assure availability in adverse weather conditions (see Section 5.7.3). Moreover, ground-based operations require a system of orbiting relay mirrors and ground optics which would compensate for atmospheric aberration, and direct the laser beam on target (Section 5.7.3). Hence, many factors must be considered before concluding which technology has the highest likelihood of meeting DEW system requirements.

In the following sections of this chapter we give detailed discussions of the technology status of these devices, their proposed performance level, and problems of physics and engineering.

\subsection{CHEMICAL LASERS}

\subsubsection{Background}

The term "chemical laser" refers to a generic class of laser systems in which the population inversion of the active medium is produced in the course of an exothermic chemical reaction. ${ }^{10}$ For this purpose it is necessary that some reaction selectively channels part of the energy liberated into one or more excited product states to create a population inversion. This can happen when some dynamical constraint in the course of the reaction prevents a purely statistical distribution of the excess energy of reaction. A molecule can store energy in the electronic, vibrational, rotational, and translational degrees of freedom, although the last alternative will be of little interest in what follows. The case of achieving laser action varies strongly with the degree of freedom involved and is a complex trade-off between gain and loss mechanisms. For electronic excitation, large Einstein A factors that raise the gain also limit the growth of population inversion because spontaneous emission drains off the population from the upper laser level. On the other hand, vibrationally excited molecules can typically undergo thousands of collisions without loss of vibrational excitation, whereas rotationally excited molecules are readily thermalized by collisions with other species. To date, molecular-transition chemical lasers with appreciable power output have only been achieved based on vibration-rotation transitions in the infrared portion of the spectrum, although efforts to extend this to shorter wavelengths using electronic transitions are being actively pursued. The only alternative demonstrated so far has been an atomic-transition laser using energy transfer from electronically metastable molecules generated in a chemical reaction.

Chemical lasers may be initiated in a number of ways, perhaps the easiest being by electron beam pumping, electrical discharge, and flash photolysis. In some cases, purely chemical initiation can be achieved, offering the possibility of making chemical laser systems the most powerful optical laser source by weight, an important consideration in airborne or space-based use. In what follows, two purely chemical laser systems are discussed, the HF/DF laser and the chemical oxygeniodine laser (COIL), the first of which has matured to the point where it can be considered to be a serious candidate system for DEW use.

\subsubsection{The HF/DF Laser System}

The discovery in 1965 by Kasper and Pimentel ${ }^{10}$ of the first chemical laser stimulated the search for a laser in which the population inversion could be produced by a chain reaction. A short time later, Kompa and Pimentel $^{2}$ reported laser action from the $\mathrm{HF}$ molecule when a mixture of $\mathrm{UF}_{6}$ and $\mathbf{H}_{2}$ was flash photolyzed, while at about the same time, Deutsch ${ }^{11}$ reported laser action from $\mathrm{HF}$ initiated by an electrical discharge of a mixture of $\mathbf{H}_{2}$ and various freons (fluorinated hydrocarbons). However, it was not until 1969 that two teams of Soviet scientists ${ }^{12}$ were able to demonstrate HF laser action from the $\mathrm{H}_{2} / \mathrm{F}_{2}$ chain reaction, which could be initiated by electrical discharge in a mixture of $\mathbf{H}_{2}$ and various freons.

Continuous-wave (cw) operation of the HF/DF chemical laser was demonstrated ${ }^{13}$ in 1969. Here separate oxidizer and fuel streams are supersonically mixed inside an optical cavity. Since the efficiency depends strongly on the rate of mixing, a large number of small nozzles are generally used. Collisional deactivation by vibration-to-translation $(\mathrm{V}-\mathrm{T})$ and vibration-tovibration $(\mathrm{V}-\mathrm{V})$ energy transfer competes with stimulated emission. Consequently, the optical, kinetic, and gasdynamic processes are coupled and much effort has been spent on modeling the behavior of this type of laser system. 
The cw HF/DF chemical lasers of interest are combustion-driven supersonic-mixing lasers. A combustor is used to produce atomic fluorine $(\mathrm{F})$ which subsequently reacts with molecular hydrogen $\left(\mathrm{H}_{2}\right)$ or deuterium $\left(\mathrm{D}_{2}\right)$ :

$$
\begin{aligned}
& \mathrm{F}+\mathrm{H}_{2} \rightarrow \mathrm{HF}(\mathrm{v}, \mathrm{J})+\mathrm{H}, \\
& \mathrm{F}+\mathrm{D}_{2} \rightarrow \mathrm{DF}(\mathrm{v}, \mathrm{J})+\mathrm{D} .
\end{aligned}
$$

This reaction is exothermic and it has the property that the HF or DF is formed almost exclusively in excited vibrational states, thus providing an inherent population inversion. For example, for the reaction in Equation (3.6) which liberates $31.7 \mathrm{kcal} / \mathrm{mole}$ of energy, the relative vibrational level populations, $N_{v}$, have been measured ${ }^{14}$ to be $\mathrm{N}_{1}: \mathrm{N}_{2}: \mathrm{N}_{3}=0.31: 1.00: 0.47$, with negligible production $^{15}$ of $\mathrm{N}_{0}$. The remainder of the excess energy of reaction appears primarily in product translation. This laser system has a good chemical efficiency $(\sim 15 \%$ of the energy of reaction is channeled to excitation) ${ }^{16}$ and appears to be scalable to very high laser power levels by straightforward increases in the reactant flow rates and unit reactor configurations. In particular, this technology has reached the state of maturity in which it has been demonstrated that (1) unit configurations have efficiencies approaching hundreds of joules of photon energy per gram of total reactant flow, and (2) sustained operation is possible at megawatt power levels in a single laser cavity, ${ }^{5}$ and (3) beam qualities approaching the diffraction limit at power levels of hundreds of kilowatts can be achieved.

A typical arrangement for an HF laser is shown in Figure 3.3, wherein the alternative chemical species to form a DF laser are shown in parentheses. A combustor is used to generate the atomic fluorine by thermal dissociation of an appropriate fluorine compound, typically $\mathrm{F}_{2}$ or $\mathrm{NF}_{3}$, "burned" in excess of stoichiometry at pressures of a few hundred torr or above and at temperatures around $1600 \mathrm{~K}$. Deuterium $\left(\mathrm{D}_{2}\right)$ is commonly used as the combustor fuel in an HF laser so that no HF is formed in the combustor which might interfere with the subsequent cavity laser process. Helium $(\mathrm{He})$ is also introduced as a diluent. The supersonic, reacting flow field in the cavity is established by a fine array of nozzles, alternately injecting the combustorderived $\mathrm{F}$ and the $\mathrm{H}_{2}$-bearing $\left(\mathrm{D}_{2}\right.$-bearing) streams. The heat capacity of the diluent and further flow expansion in the cavity help to maintain low temperature $(\sim 300 \mathrm{~K})$ and low pressure (a few torr) conditions as the cavity reactions proceed, an environment that favors efficient operation. The supersonic flow field also provides for rapid and continuous removal of the deexcited species and the excess thermal energy following laser action.

Gain occurs within the first few centimeters of the cavity flow. An optical resonator transverse to the flow direction extracts the laser beam. Figure 3.3 shows a central feedback, confocal, spherical, positive branch unstable resonator. Unstable resonators ${ }^{17}$ are commonly used because they can provide fundamental mode extractions from large volume gains. Figure 3.4 illustrates conditions representative of high efficiency space-based laser design. Lasers intended for groundbased applications typically have higher entrance pressures, more diluent, less temperature rise, and higher exit pressures and Mach numbers.

In Figure 3.3 the convex and concave spherical mirrors have a common focal point. This type of structure is called an unstable resonator because a ray of light reflected back and forth walks from the center off the edge of the convex mirror. When properly designed, the resonator sets up a field consisting of a spherical wave that expands as it travels from the convex mirror to the concave mirror, and a plane wave that is reflected from the concave mirror. A so-called "scraper mirror" deflects part of the expanded portion of the plane wave; this constitutes the laser output coupler in Figure 3.3. The plane wave interior region provides the required feedback for the resonator as it bounces off the convex mirror and is reflected back again as an expanding spherical wave. The near-field output of an unstable resonator is doughnut shaped, i.e., it has a hole in the center. The unstable resonator design provides the best practical method for obtaining high energy extraction from the laser medium in an output beam of good optical quality. Moreover, it uses mirror shapes that are fairly easily cooled for high-power applications. The near-field beam shape is commonly rectangular because of the rectangular feedback hole in the scraper mirror.

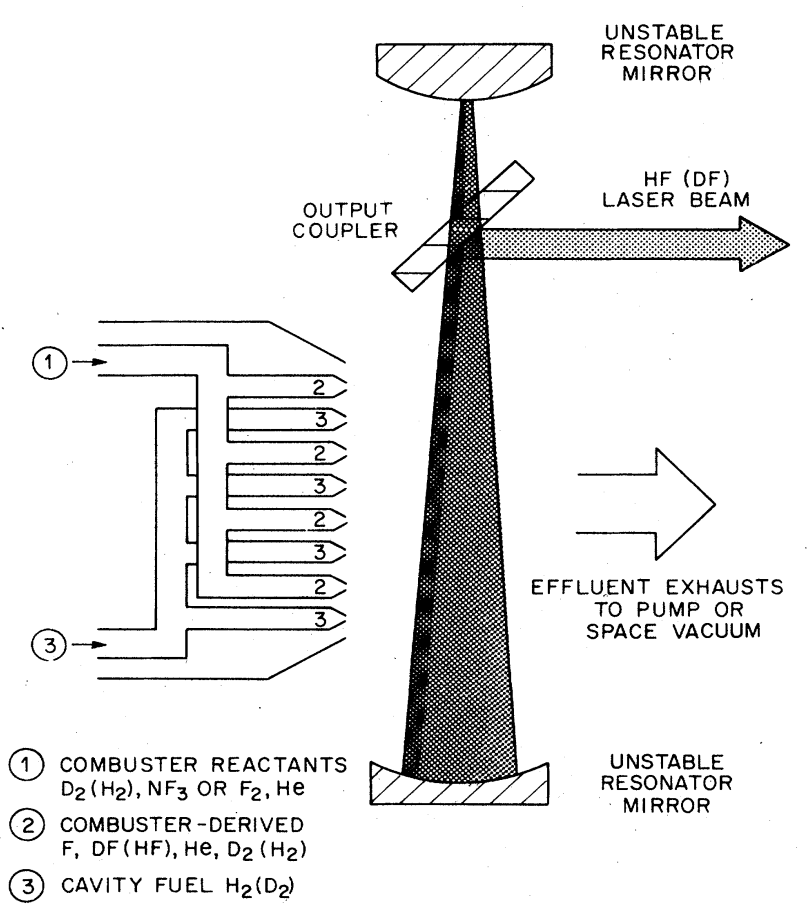

Figure 3.3. Schematic diagram of $\mathrm{cw}$ HF (DF) chemical laser. 


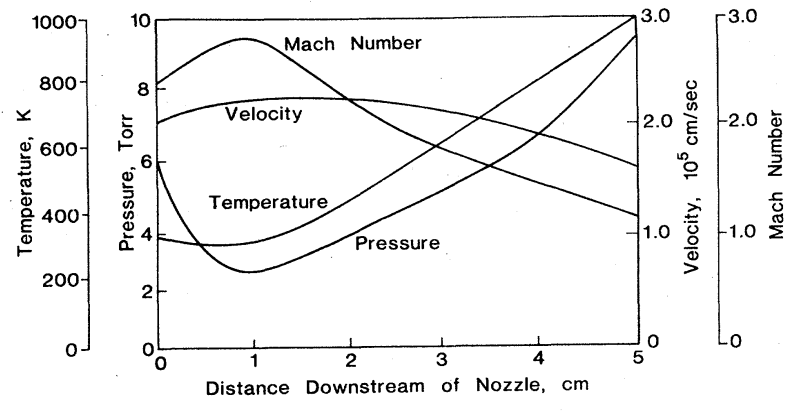

Figure 3.4. Typical flow conditions for an HF laser (cavity molar flow composition: $0.1 \mathrm{HF}, 0.1 \mathrm{DF}, 0.3 \mathrm{He}$, $0.5 \mathrm{H}_{2}$ ).

Figure 3.5 shows the spectral distribution measured in typical HF and DF laser beams and Figure 3.6 presents the transmission properties of the atmosphere for propagation of HF and DF laser beams for typical conditions. It is clear that the DF laser wavelengths have superior atmospheric transmission, but because the focused intensity of a plane-wave, coherent, diffractionlimited beam is proportional to the inverse square of the wavelength, the shorter wavelength HF laser is favored for applications where atmospheric transmission is not an issue. Emission is on several transitions because population inversion exists between several upper and lower state vibrational-rotational $(v, J)$ levels. The transitions follow the selection rules $\Delta v=1$, $\Delta \mathbf{J}= \pm 1$. This situation is illustrated in Figure 3.7. The three groups of horizontal lines represent vibrational states, $\mathbf{v}=0,1$, and 2 . Each line represents a rotational level, $\mathrm{J}=0,1,2, \ldots$ within a vibrational state. The length of a line is proportional to the population. The transitions are designated by standard spectroscopic notation. For example, consider the transition $\mathbf{P}_{1}(4)$. Here $\mathbf{P}$ designates a $\mathbf{P}$-branch transition, i.e., one in which the rotational quantum number of the lower state is one unit greater than the upper state. The subscript 1 on $P_{1}$ designates the vibrational quantum number of the upper level, i.e., the transition originates from $\mathbf{v}=1$. The quantity in parentheses, (4), designates the rotational quantum number of the lower level, i.e., the transition terminates on the rotational level $J=4$. Thus, $P_{1}(4)$ is a convenient short-hand for the $\left(v^{\prime}, J^{\prime}\right) \rightarrow\left(v^{\prime \prime}, J^{\prime \prime}\right)$ transition, $(1,3) \rightarrow(0,4)$. As can be seen from Figure 3.5 for a given upper state $\left(v^{\prime}, J^{\prime}\right)$ level, a Pbranch transition $\left(\mathrm{J}^{\prime}-\mathbf{J}^{\prime \prime}=-1\right)$ has in general more gain (larger population inversion) than the corresponding $\mathrm{R}$-branch transition $\left(\mathrm{J}^{\prime}-\mathrm{J}^{\prime \prime}=+1\right)$, so that laser action occurs exclusively on P-branch lines. ${ }^{1,18}$ In the example shown, the populations are such that there is actually no population inversion between vibrational levels, i.e., the total populations in the different vibrational levels are equal, as indicated by the lengths of the horizontal lines. However, even for this "noninverted" vibrational distribution, there is gain for the P-branch transitions shown. This situation exists when the rotational state populations are at a lower temperature than the vibrational state populations, and is referred to as partial inversion.

The nascent HF molecules are born with an inherent population inversion. Soon, collision processes redistribute the internal energy such that chemical lasers frequently operate in the partial inversion mode. As a consequence, the transitions showing largest gain vary as a function of distance downstream from the nozzle array.
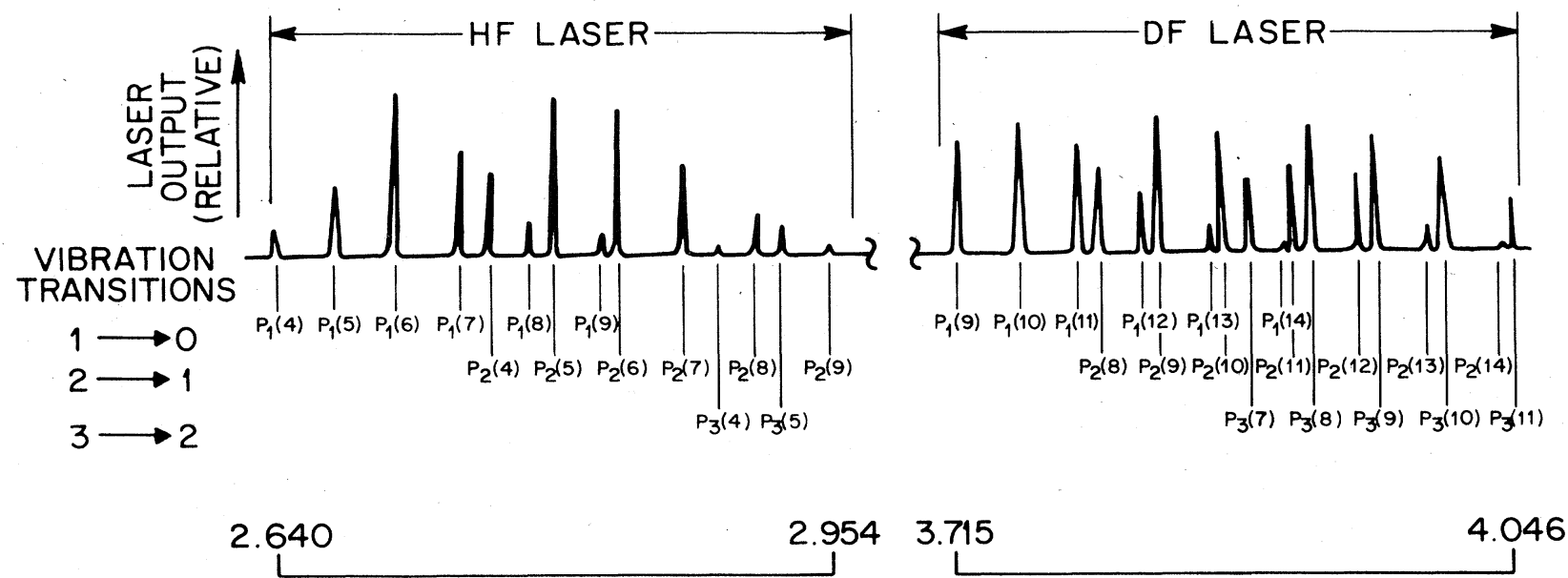

WAVELENGTH $(\mu \mathrm{m})$

Figure 3.5. Spectral distribution of output from HF and DF lasers. 


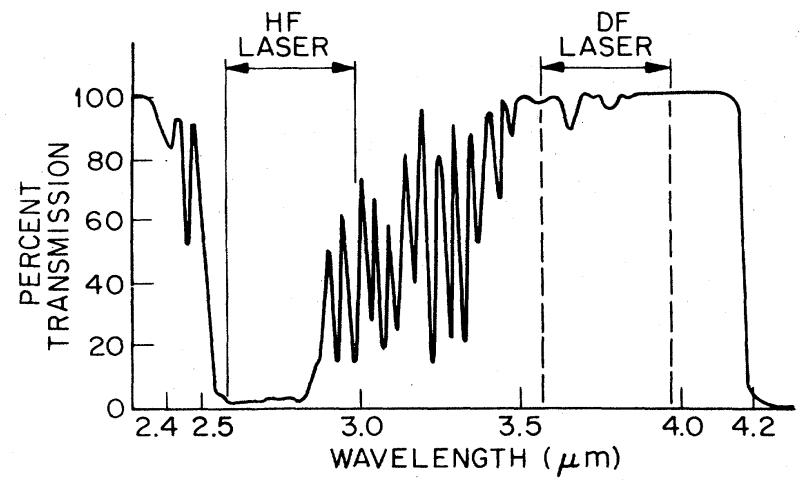

Figure 3.6. Atmospheric transmission characteristics as a function of wavelength.

This complicates the design of the HF laser system since a single optical system must extract energy in controlled fundamental modes from a number of spatial distributions of gain. This is a complex problem requiring the simultaneous treatment of the fluid mechanics of a supersonic, mixing, reacting flow field, the chemistry and collisional energy transfer properties of a multilevel vibrational-rotational system, and the stimulated emission and physical optics processes

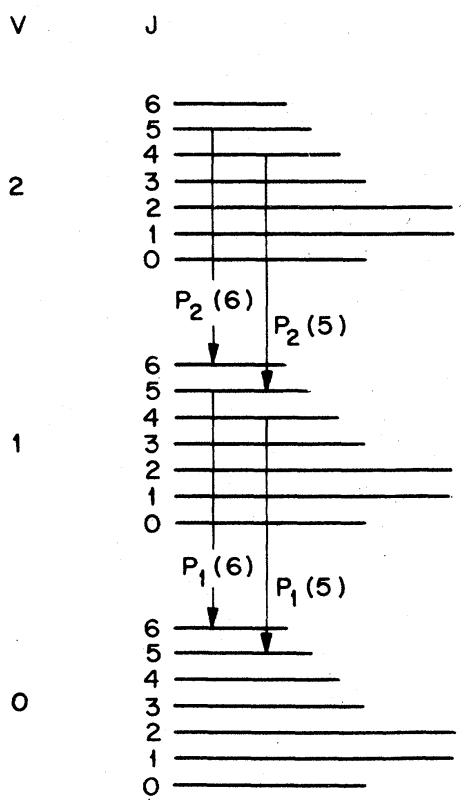

Figure 3.7. Vibrational-rotational transitions. $\mathrm{HF}$ and DF chemical lasers emit light on several spectral lines because of population inversion between several $(v, J)$ levels. P-branch transitions are those which occur when a molecular level $(\mathrm{v}, \mathrm{J})$ changes to a lower vibrational level $(\Delta \mathrm{v}=-1)$ simultaneously with a change to a higher rotational level $(\Delta \mathbf{J}=-1)$. The lengths of the horizontal lines represent relative population densities. occurring in large mode, unstable resonators of difficult geometries. To date no computer code exists that is able to treat all phenomena simultaneously at the required level of detail. Nevertheless, considerable progress has been made such that highly efficient devices have been constructed, many of their optical properties (power extraction, integral beam quality) correctly predicted, and a basis exists for scaling this technology to yet higher power levels.

Much of the engineering experience for a space-based version of laser (project ALPHA) has been obtained from a device called MIRACL (Mid-Infrared Advanced Chemical Laser), a high-power cw DF chemical laser with a linear configuration shown in Figure 3.3. MIRACL has produced an outcoupled $14 \mathrm{~cm} \times 14 \mathrm{~cm}$ beam with a measured power in excess of $1 \mathrm{MW} .{ }^{19}$ In tests made at $80 \%$ flow, a beam quality of approximately two times the diffraction limit, in the vertical plane, was measured with an average power in excess of $1 \mathrm{MW}$ as determined with a calorimeter. The possible production of "hot spots" in the beam profile is a major concern in assessing whether this power can be readily brought to focus on a target in the far field.

The MIRACL device closely approaches the limit of scaling for this linear configuration. In energy extraction, the important parameter is nozzle exit area which is easily related to laser medium volume. There are two choices: (1) to increase the length of the optical axis and (2) to increase the height of the nozzle array. Additive optical path disturbances impose a limit in the extension of the length of the optical axis which is close to that in the present length of the MIRACL device. As the height of the nozzle array is increased, scaling is complicated by the problem of structural support and the optics problem of unfavorable beam aspect ratio. The latter arises because the gain in the HF/DF chemical laser is established as the reagents react just downstream of the cavity injection, i.e., high gain is limited to just a few centimeters downstream of the nozzle exit. Consequently, for extremely high-power applications, as being attempted in Project ALPHA, the resonator structure is cylindrical (see Figure 3.8), because annular designs have the maximum nominal nozzle exit area per unit length and a superior support structure.

To extract the energy of this laser medium configuration with acceptable beam quality, TRW is proposing to use a high extraction, decentered, annular ring resonator HEX-DARR, whose geometric configuration is pictured in Figure 3.9. This is a traveling wave resonator which has two distinct counterpropagating but unidirectional modes. Diamond turning methods make it possible to fabricate the aspheric surfaces shown. For high-power operation, only a single propagation direction can be accommodated and the reverse mode must be suppressed. Because the total volume of the gain region that the reverse mode can access is a small portion of the forward wave, the laboratory simulation experiment has excellent rejection (better than 50:1) of the unwanted reverse mode. Even so, a reverse wave 
suppression mirror may be used to enhance the rejection.

The reaction products exit at the resonator structure radial to the optic axis in order to balance thrust and to minimize mirror contamination by the effluent. As the exhaust fuel gases exit radially, the underexpanded jet plumes and there is a subsonic boundary region which causes some of the exhaust gases to creep along the outside of the spacecraft. The extent of this problem varies with altitude. At $150 \mathrm{~km}$, atmospheric drag helps to prevent this flow, while at $1000 \mathrm{~km}$ the problem is more serious. Monte Carlo calculations show that the density of $\mathrm{HF}$ in front of the output optics will be bleached and hence will not contribute to significant degradation of the laser output or cause thermal blooming. Perhaps a more serious concern is contamination of the output coupler and other optics. A slow flow of the diluent $(\mathrm{He})$ may be used to purge the external mirror surfaces and it may be necessary to keep the optical surfaces hot compared to their surroundings.

As the power level of the cw HF laser system is increased in scale, the mirrors may become an important limitation. Present designs use either silicon (Si), silicon carbide (SiC), or molybdenum (Mo). The superior microcreep characteristics of $\mathrm{SiC}$ are expected to translate into better figure retention over a longer period of time in orbit. SiC has physical properties that give 3 times the performance of Mo in terms of figure stability, i.e., thermal expansion per heat conductivity. Mirror heating/damage occurs in the optical coating rather than the mirror itself. For HF wavelength $(2.7-2.9 \mu \mathrm{m})$ coating damage limit on uncooled mirrors for a 4 pair dielectric stack on gold substrate $(0.995$ reflectivity) has been measured ${ }^{20}$ to be $\sim 50 \mathrm{~kW} / \mathrm{cm}^{2}$. Liquid ammonia rather than water has been selected as the optical coolant because of its low freezing point (196 K), and has improved distortion performance in mirrors. Nevertheless, the damage threshold for the mirrors in laser operation in far excess of the $\mathrm{MW} / \mathrm{cm}^{2}$ level is not known.

Unwanted vibrations can impair the ability of this laser system to point accurately. The chemical combustion process responsible for vibrationally excited HF generation burns smoothly without "hiccupping," and the radial exhaust of the spent gases should also be relatively free of mechanical vibrations. Presently it is thought that the circulation of the resonator optics

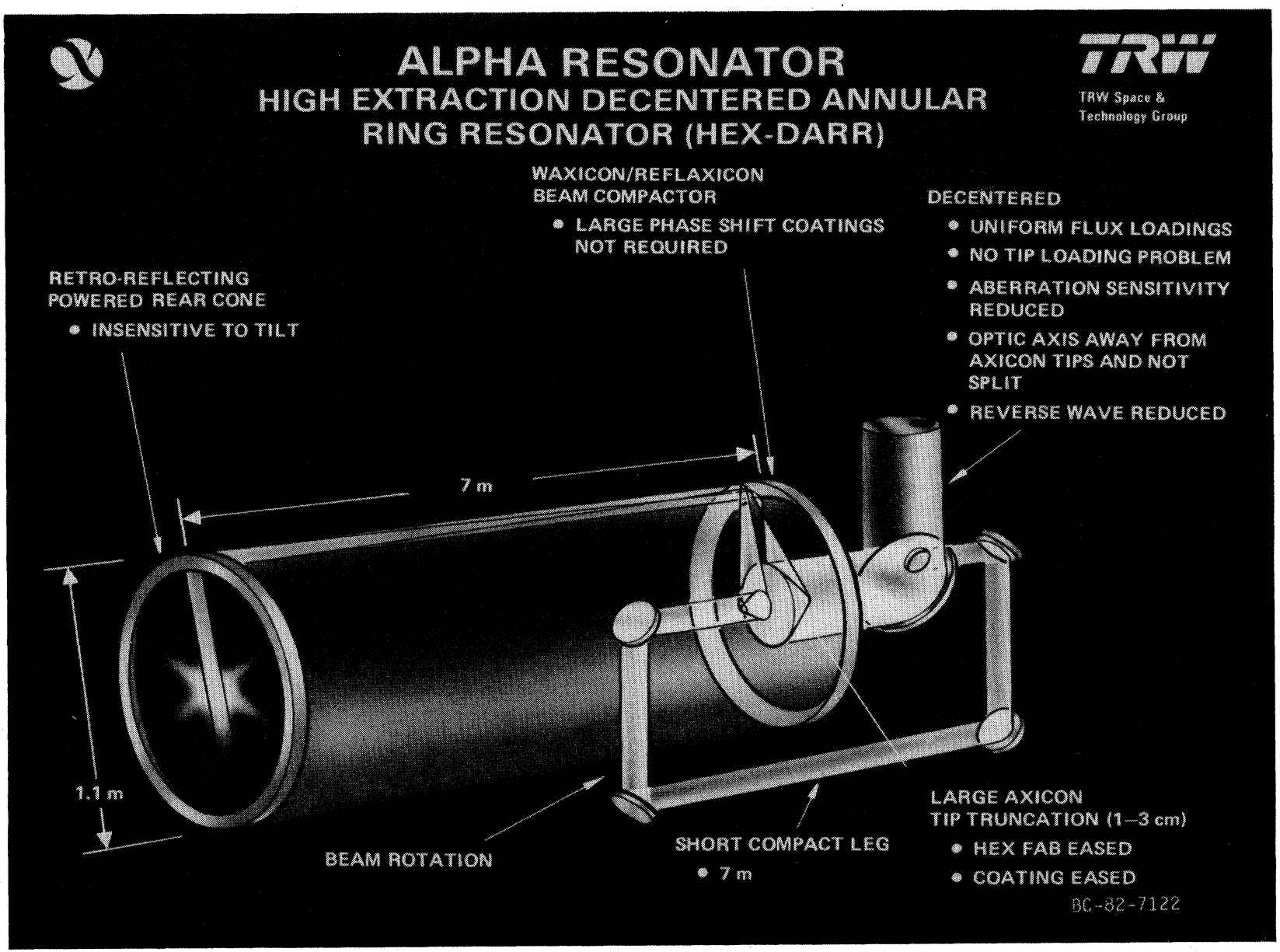

Figure 3.8. High extraction decentered annular ring resonator (HEX-DARR) design. 


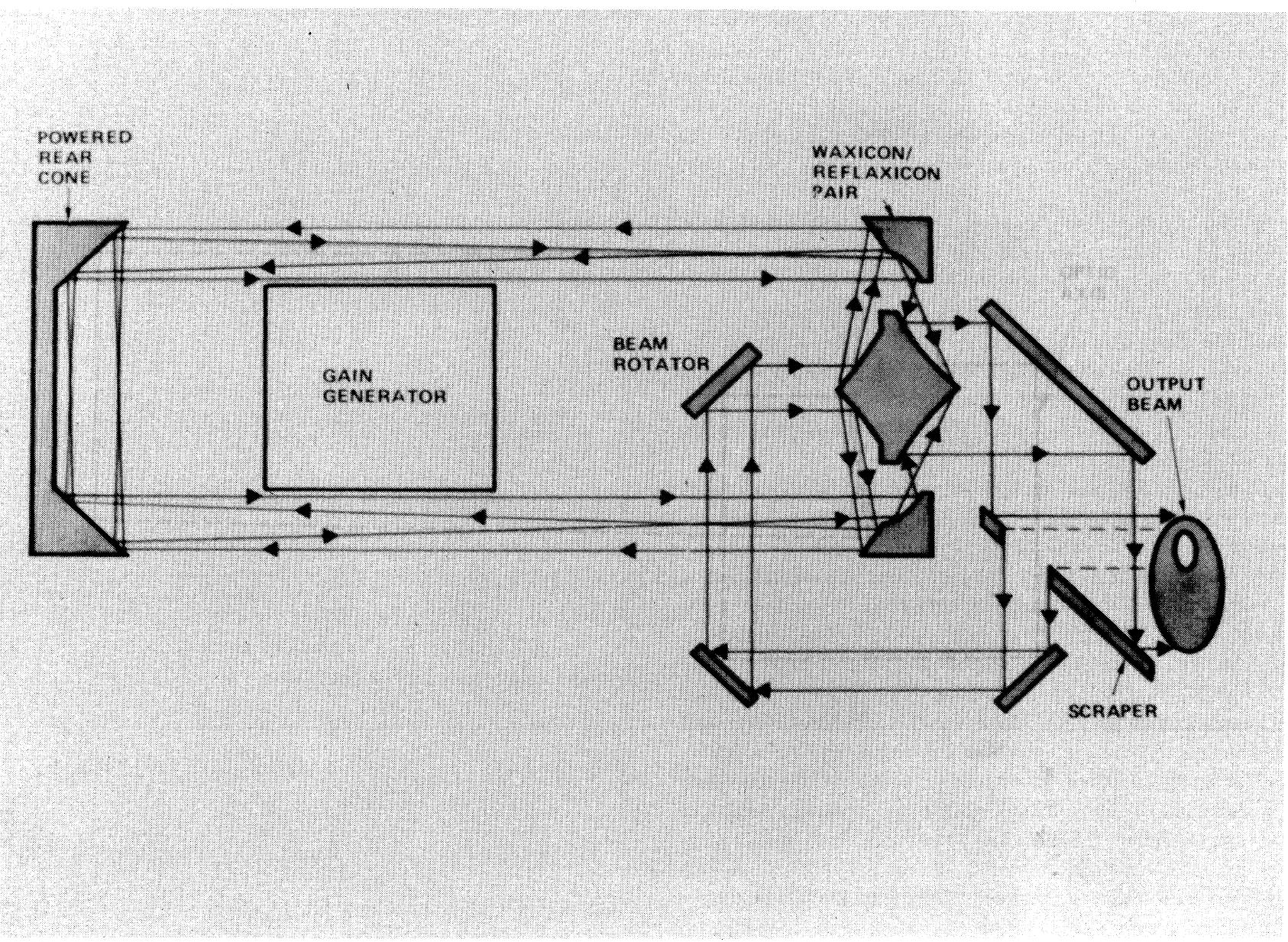

Figure 3.9. Optical ray trace of HEX-DARR design shown in Figure 3.8.

coolant, particularly during operation, may be the largest source of short-time mechanical instabilities. Nevertheless, none of these expectations have been validated by actual tests.

Concepts are currently being investigated for combining several lasers into phased arrays to provide yet more potent space-based high brightness systems. The problems inherent in phase locking and beam aperture combination of large numbers of high-power lasers are discussed in Chapter 5.

\subsubsection{Critical Issues}

Continuous wave HF/DF chemical lasers have advanced to a point that further significant scaling cannot be accomplished by simply extending the length. An annular resonator design has been proposed requiring complex optics. This is intended for space-basing with the shorter wavelength $(\lambda=2.8 \mu \mathrm{m}) \mathrm{HF}$ laser. Highpower operation of space-based HF laser using an annular ring resonator has not yet been demonstrated. Mode quality is a major concern because of the sensitivity of the annular resonator to misalignment through vibration and thermal expansion of the optical elements. Other issues involving balancing of the thrusts from the spent exhaust products and possible contamination from these gases of the optical elements also remain to be solved.

\subsubsection{Electronic Transition Oxygen/lodine Lasers}

\subsubsection{Background}

The chemical oxygen-iodine laser (COIL) is an electronic transition high energy chemical laser first demonstrated $^{21}$ at the Air Force Weapons Laboratory in 1978. It was a natural outgrowth of the photolytic iodine laser first reported by Kasper and Pimental. ${ }^{22}$ It is the shortest wavelength high energy chemical laser in existence today and is the first chemical laser to operate on an electronic rather than a rotational or vibrational 
transition. Laser output at $1.315 \mu \mathrm{m}$ is achieved by stimulated emission on the ${ }^{2} \mathbf{P}_{1 / 2} \rightarrow \mathrm{I}^{2} \mathbf{P}_{3 / 2}$ magnetic dipole transition in atomic iodine. The population inversion on this transition is maintained by resonant collisional energy transfer from metastable excited $\mathrm{O}_{2}\left({ }^{1} \Delta\right)$ molecules produced by a chemical reaction of $\mathrm{KOH}$, $\mathrm{H}_{2} \mathrm{O}_{2}$, and $\mathrm{Cl}_{2}$.

In the oxygen-iodine laser, the chemical reaction of $\mathrm{Cl}_{2}$ and $\mathrm{H}_{2} \mathrm{O}_{2}$ that produces oxygen molecules is very exothermic, and because of spin conservation considerations, channels its energy directly into the metastable electronically excited singlet delta state of the $\mathrm{O}_{2}$ molecule. The chemical reaction producing $\mathrm{O}_{2}$ yields nearly $100 \%$ of the oxygen in the excited state, although collisional deactivation process limits the realizable yields to about $80 \%$.

Since the $\mathrm{O}_{2}^{*}\left({ }^{1} \Delta\right)$ has a 45 minute lifetime ${ }^{23}$ and consequently an extremely small gain coefficient, it cannot be lased directly. Lasing can be achieved, however, if this energy is transferred to an atom or molecule which has a reasonable transition moment between its excited and ground state. The iodine $5^{2} \mathbf{P}_{1 / 2} \rightarrow 5^{2} \mathbf{P}_{3 / 2}$ magnetic dipole transition has an acceptable transition moment (the A coefficient is $\left.5 \mathrm{~s}^{-1}\right)^{23}$ and is nearly resonant with the $\mathrm{O}_{2}\left({ }^{1} \Delta\right)$ state in oxygen. The overall spectroscopy and resonant energy transfer may be seen from Figure 3.10.

The oxygen-iodine laser can in principle be operated in a pulsed or $\mathrm{cw}$ mode, offering several potential advantages, and preliminary studies are underway. Coupling to the target could be improved in pulsed operation. Further, a pulsed output can be efficiently converted to shorter wavelength by frequency doubling or tripling, using either nonlinear crystals or resonantly enhanced atomic vapors (with lowered overall efficiency, however). The issue of heat extraction from a highaverage-power frequency doubler remains unresolved. Absorption losses in the nonlinear crystals must be reduced below current levels to make this approach practical.

\subsubsection{Laser Gain}

The I atoms are excited by collisional energy transfer from electronically excited metastable $\mathrm{O}_{2}$ by the resonant energy transfer reaction: ${ }^{24}$

$$
\mathrm{O}_{2}\left({ }^{1} \Delta\right)+\mathrm{I}\left({ }^{2} \mathrm{P}_{3 / 2}\right) \underset{\mathrm{k}_{\mathrm{d}}}{\stackrel{\mathrm{k}_{1}}{\rightleftarrows}} \mathrm{O}_{2}\left({ }^{3} \Sigma\right)+\mathrm{I}^{*}\left({ }^{2} \mathrm{P}_{1 / 2}\right)
$$

The relevant energy levels are shown in Figure 3.10. The reaction is $279 \mathrm{~cm}^{-1}$ exothermic. Iodine has an inhomogeneously broadened line and lasing occurs only on the highest gain ${ }^{23}$ transition. Gain is calculated for this transition only. The degeneracies of the upper and lower laser levels are 7 and 9, respectively. Assuming the hyperfine levels to be populated according to their

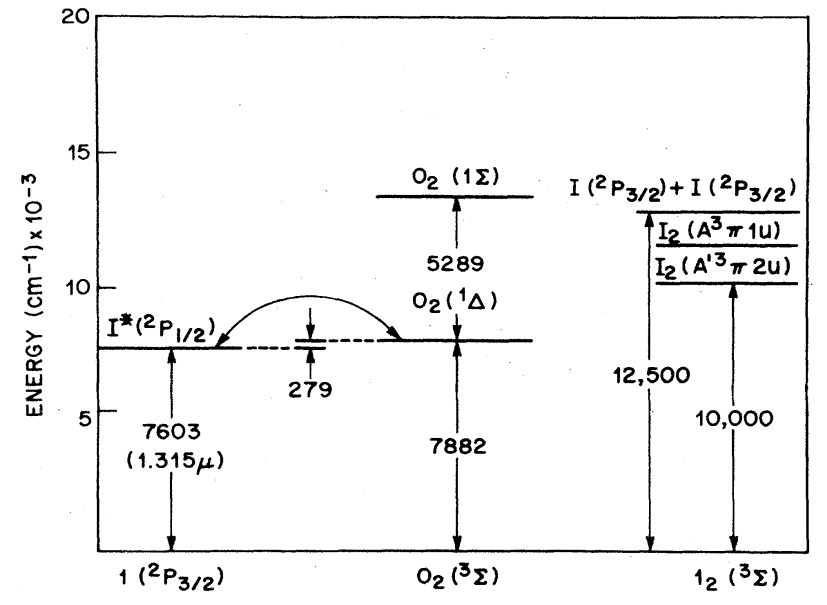

Figure 3.10. Oxygen-iodine energy level diagram.

statistical weights we obtain $\mathbf{N}_{\mathrm{u}}=\frac{7}{12}\left[\mathbf{I}\left({ }^{2} \mathbf{P}_{1 / 2}\right)\right]$ and $\mathrm{N}_{\mathrm{L}}=\frac{9}{24}\left[\mathrm{I}\left({ }^{2} \mathrm{P}_{3 / 2}\right)\right]$. Combining these results yields the following expression for the gain:

$$
\begin{aligned}
\gamma & =\frac{7}{12} \frac{\lambda^{3} \mathrm{~A}}{8 \pi}\left[\frac{\mathrm{m}}{2 \pi \mathrm{KT}}\right]^{1 / 2}\left(\left[\mathrm{I}^{*}\left({ }^{2} \mathbf{P}_{1 / 2}\right)\right]-0.5\left[\mathrm{I}\left({ }^{2} \mathbf{P}_{3 / 2}\right)\right]\right) \\
& =\sigma\left(\left[\mathrm{I}^{*}\left({ }^{2} \mathbf{P}_{1 / 2}\right)\right]-0.5\left[\mathbf{I}\left({ }^{2} \mathbf{P}_{3 / 2}\right)\right]\right)
\end{aligned}
$$

where the second expression serves to define $\sigma$, the stimulated emission cross section. At room temperature $\sigma$ has a value of $7.4 \times 10^{-18} \mathrm{~cm}^{2}$.

The ratio of upper and lower state population densities is determined by the amount of $\mathrm{O}_{2}\left({ }^{1} \Delta\right)$ present. Since reaction in Equation (3.8) is very fast, it is reasonable to assume an equilibrium situation exists.

$$
\mathrm{k}_{1}\left[\mathrm{O}_{2}\left({ }^{1} \Delta\right)\right][\mathrm{I}]=\mathrm{k}_{2}\left[\mathrm{O}_{2}\left({ }^{3} \Sigma\right)\right]\left[\mathrm{I}^{*}\right]
$$

The ratio of forward and backward rates can be calculated thermodynamically in terms of degeneracies, the exothermicity of the reaction and the temperature, $T$ :

$$
\frac{\mathrm{k}_{1}}{\mathrm{k}_{2}}=0.75 \exp (402 / \mathrm{T})
$$

Therefore

$$
\left[\mathrm{I}^{*}\right] /[\mathrm{I}]=0.75 \exp (402 / \mathrm{T})\left[\mathrm{O}_{2}\left({ }^{1} \Delta\right)\right] /\left[\mathrm{O}_{2}\left({ }^{3} \Sigma\right)\right] .
$$

Using Equations (3.10) and (3.13) a final expression can be derived for the gain in terms of excited oxygen, 
ground state oxygen, and the total initial added $\left[\mathrm{I}_{2}\right]$ where full dissociation is assumed $\left(2\left[\mathrm{I}_{2}\right]=\left[\mathrm{I}^{*}\right]+[\mathrm{I}]\right)$; combining these expressions yields

$$
\gamma=\sigma\left[\mathrm{I}_{2}\right] \frac{1.5}{0.75} \frac{[\exp (402 / \mathrm{T})] \mathrm{f}-1}{[\exp (402 / \mathrm{T})] \mathrm{f}+1}
$$

where $\mathrm{f}=\left[\mathrm{O}_{2}\left({ }^{1} \Delta\right)\right] /\left[\mathrm{O}_{2}\left({ }^{3} \Sigma\right)\right]$. The equation shows that the gain depends directly on the concentration of $\left[\mathrm{I}_{2}\right]$. Gains of $\geq 1 \% / \mathrm{cm}$ have been achieved.

\subsubsection{Chemical Generation of Excited Molecular Oxygen}

Singlet molecular oxygen is generated in the reactor by the overall reaction of chlorine, hydrogen peroxide, and an alkali hydroxide:

$$
\begin{aligned}
\mathrm{Cl}_{2}(\mathrm{~g})+\mathrm{H}_{2} \mathrm{O}_{2}(\ell)+2 \mathrm{MOH}(\ell) \rightarrow & \mathrm{O}_{2}^{*}\left({ }^{1} \Delta\right) \\
& +2 \mathrm{MCl}+2 \mathrm{~h} 2 \mathrm{O}
\end{aligned}
$$

where $\mathrm{M}=\mathrm{Li}, \mathrm{Na}, \mathrm{K}$. The alkali base is a solid which is typically dissolved in water prior to use in an $\mathrm{O}_{2}$ chemical generator. The hydrogen peroxide is used in either a $90 \%$ or $35 \%$ solution by weight with $\mathrm{H}_{2} \mathrm{O}$.

The addition of a base such as potassium hydroxide to hydrogen peroxide provides $\mathrm{OH}^{-}$, which interacts with the peroxide producing $\mathrm{HO}_{2}^{-}$by the reaction

$$
\mathrm{K}^{+}+\mathrm{OH}^{-}+\mathrm{H}_{2} \mathrm{O}_{2} \rightarrow \mathrm{H}_{2} \mathrm{O}+\mathrm{HO}_{2}^{-}+\mathrm{K}^{+} \text {. }
$$

When $\mathrm{Cl}_{2}$ is reacted with the basic peroxide solution hypoclorite is formed by

$$
\mathrm{Cl}_{2}+\mathrm{OH}^{-} \rightarrow \mathrm{Cl}^{-}+\mathrm{HOCl}
$$

Subsequent reaction of the hypoclorite plus $\mathrm{HO}_{2}^{-}$yields the excited molecular oxygen by the reaction

$$
\mathrm{HO}_{2}^{-}+\mathrm{HOCl} \rightarrow \mathrm{O}_{2}\left({ }^{1} \Delta\right)+\mathrm{H}_{2} \mathrm{O}+\mathrm{Cl}^{-},
$$

where spin conservation requirements dictate the formation of the oxygen in an excited singlet state. These reactions are strongly exothermic. Such a process for generating excited oxygen was first embodied in a reactor $^{21}$ as seen in Figure 3.11a.

Wetted wall reactors provide a large reaction surface area per unit volume while minimizing gas resonance time. An example of a wetted wall reactor is shown in Figure $3.11 \mathrm{~b}$. In this concept a pool of basic hydrogen peroxide $(\mathrm{BHP})$ is introduced into the reactor base. As

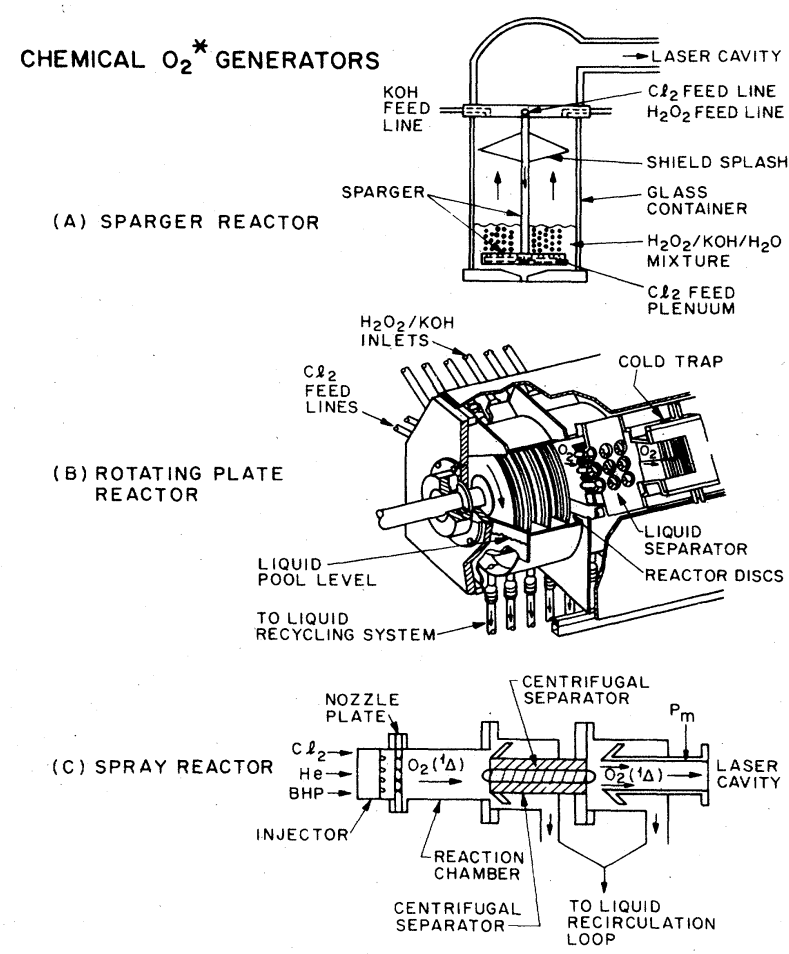

Figure 3.11. Chemical $\mathrm{O}_{2}^{*}$ generators.

the disks or "walls" are rotated through the liquid pool, the depleted reactant film is replaced by a fresh BHP film. Generation of $\mathrm{O}_{2}\left({ }^{1} \Delta\right)$ is obtained by introducing $\mathrm{Cl}_{2}$ into the reactor chamber and flowing it across the wetted rotating disks. Depleted reactants are replaced and reactant by-products removed by a chemical recycling system. Reactor thermal control is maintained by reactant recycling through a heat exchanger. Low film and disk temperatures are maintained by thermal contact with the BHP pool. Once the singlet delta oxygen is generated in the reactor vessel it is flowed into a liquid separator, where entrained particles down to $5 \mu \mathrm{m}$ diameters are removed by centrifugal separation. Next, the gas is flowed into a cold trap where the water vapor level is reduced to the required $2-3 \%$ of the total gas flow and the gas temperature is brought down to a nominal $300 \mathrm{~K}$.

An improved solution to the problem of maximizing reaction surface area per unit reactor volume while minimizing weight is to generate a fine spray of BHP in a reactor chamber containing $\mathrm{Cl}_{2}$. An example of this type of reactor is shown in Figure 3.11c. The nozzle is designed to produce $<20 \mu \mathrm{m}$ diameter droplets of BHP and to control the spray expansion angle such that the spray does not coagulate on the walls and reduce available reaction surface area. Thermal control in the system is maintained by running large excesses of the BHP to provide sufficient thermal mass to control the temperature rise. The major problem with the spray reactor is that of disengaging the spent liquid reactants 
from the flow. While in a wetted wall reactor only $2 \%$ of the liquids are entrained in the flow, in a spray reactor, $100 \%$ of the liquid is entrained in the flow. The requirement for efficient liquid disengagement stresses the centrifugal separator design, leading to several separation sections and large pressure drops.

\subsubsection{Supersonic Nozzles and Mixing}

The long lifetime of the lasing species allows the iodine to be injected either subsonically or supersonically. Generally, supersonic flow is required to remove waste heat. Therefore, the options exist in $\mathrm{O}_{2}$-I lasers for either mixing and dissociating the $I_{2}$ in a subsonic region, expanding through a supersonic nozzle into a laser cavity, or attempting to mix directly in the supersonic regions. Results show that subsonic injection appears to provide enhanced mixing over supersonic injection. A general schematic of such a nozzle may be seen in Figure 3.12.

\subsubsection{4. $1 \mathrm{I}_{2}$ Dissociation}

During the mixing of the molecular $I_{2}$ into the $\mathrm{O}_{2}\left({ }^{1} \Delta\right)$ flow phase reactions are initiated which result in the rapid dissociation of ground state molecular iodine $\left(I_{2}\right)$ into ground state iodine atoms $\left(I^{2} P_{3 / 2}\right)$. Since the only energy carrying species in the flow are $\mathrm{O}_{2}\left({ }^{1} \Delta\right)$ and $\mathrm{I}^{*}$ these species must be involved in the dissociation. Dissociation by $\mathrm{O}_{2}\left({ }^{1} \Delta\right)$ requires a multistep process since it carries inadequate energy to dissociate the $I_{2}$ on a single collision. The primary mechanism for dissociation must then be of the form

$$
\mathrm{nO}_{2}\left({ }^{1} \Delta\right)+\mathrm{I}_{2}(\mathrm{X}) \rightarrow \mathrm{nO}_{2}\left({ }^{3} \Sigma\right)+2 \mathrm{I}\left({ }^{2} \mathrm{P}_{3 / 2}\right)
$$

where $\mathrm{n}$ measures the number of excited singlet oxygen molecules needed, i.e., the energy expended in the process. Knowledge of the rate and efficiency of this process is critical to assessing the ultimate efficiencies of high-power chemical iodine lasers. High-power iodine

\section{$\mathrm{O}_{2} / I$ MIXING NOZZLES}
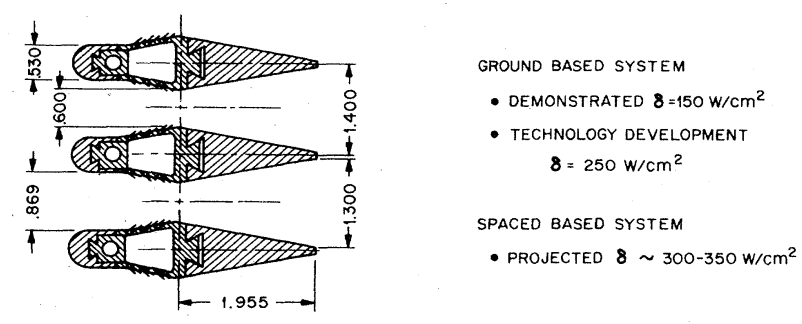

Figure 3.12. Oxygen-iodine mixing nozzle. lasers with a gain of $1 \% / \mathrm{cm}$ operate with $\mathrm{I}_{2} / \mathrm{O}_{2}\left({ }^{1} \Delta\right)$ ratios of 1:30. Experimental data for such mixtures indicate that it takes between 2 and 5 excited state $\mathrm{O}_{2}$ molecules to dissociate a single $I_{2}$ molecule by collision into iodine atoms. Because of the ratio of $\mathrm{I}_{2}$ to $\mathrm{O}_{2}\left({ }^{1} \Delta\right)$, this does not represent a significant loss of excited oxygen molecules, in terms of what remains available for pumping I atoms.

\subsubsection{Scaling Parameters and Efficiencies for Large- Scale $\mathrm{O}_{2}-\mathrm{I}$ Lasers}

Based on the current understanding of the chemistry and physics of $\mathrm{O}_{2}$-I lasers, scaling laws can be developed and efficiencies determined for large-scale laser devices. There are four principal scaling parameters which determine the power of a large-scale $\mathrm{O}_{2}-\mathrm{I}^{*}$ chemical laser. These are the fuel efficiency, nozzle flux, laser gain, and resonator extraction efficiency. The laser fuel efficiency, $\phi(\mathrm{kJ} / \mathrm{kg})$, is a parameter that is primarily driven by the chemistry and chemical engineering of the reactor. Efficiencies can be calculated for the chemical reactions. This result is shown in Table 3.1 for the three bases $\mathrm{LiOH}, \mathrm{NaOH}$, and $\mathrm{KOH}$, and for $\mathrm{K}_{2} \mathrm{O}_{2}$.

The nozzle flux $\delta\left(\mathrm{W} / \mathrm{cm}^{2}\right)$ is closely tied to the generator performance. Generators operating at the efficiencies described above will yield 10-20 torr of $\mathrm{O}_{2}$, $90 \%$ in the excited state. These generators are therefore capable of driving a Mach 2.2 nozzle with a resultant 1-2 torr of $\mathrm{O}_{2}$ in the laser cavity. Given the flow velocity, the $\mathrm{O}_{2}$ pressure, the $\mathrm{O}_{2}\left({ }^{1} \Delta\right)$ percentage, and assuming a nominal cavity temperature of $250 \mathrm{~K}$ the extracted nozzle flux can be calculated from the following expression:

$$
\delta\left(\mathrm{W} / \mathrm{cm}^{2}\right)=1.5 \times 10^{-19}[\mathrm{O} 2]_{\mathrm{cav}} \mathrm{V}\left(\mathrm{f}_{1}-\mathrm{f}_{2}\right) \mathrm{f}_{3}
$$

where the $1.5 \times 10^{-19} \mathrm{~J}$ is the extractable energy carried by one excited oxygen molecule, the $\left[\mathrm{O}_{2}\right]_{\mathrm{cav}}$ is the number

\begin{tabular}{|c|c|c|}
\hline Base & $\begin{array}{l}\text { Thermodynamic maximum } \\
(\mathrm{kJ} / \mathrm{kg}) 90 \% \mathrm{O}_{2}\left({ }^{1} \Delta\right) \text { yield }\end{array}$ & $\begin{array}{c}\text { Realizable (based } \\
\text { on kinetics) } \sigma(\mathrm{kJ} / \mathrm{kg}) \\
60 \%\left({ }^{1} \Delta\right) \text { yield }\end{array}$ \\
\hline $\mathrm{LiOH}$ & 594 & 344 \\
\hline $\mathrm{NaOH}$ & 491 & 285 \\
\hline $\mathrm{KOH}$ & 418 & 242 \\
\hline $\mathrm{K}_{2} \mathrm{O}_{2}$ & 423 & 305 \\
\hline
\end{tabular}

TABLE 3.1. Coil laser fuel efficiency. 
density of $\mathrm{O}_{2}, \mathrm{~V}(\mathrm{~cm} / \mathrm{s})$ is the velocity, $\left(\mathrm{f}_{1}-\mathrm{f}_{2}\right)$ is the fractional energy available which nominally equals 0.8 and $f_{3}$ is the optical extraction efficiency. Therefore 1.5 torr of $\mathrm{O}_{2}, 90 \% \mathrm{O}_{2}\left({ }^{1} \Delta\right)$ at $250 \mathrm{~K}, \mathrm{M}=2.2, \mathrm{f}_{3}=0.9$ (optical efficiency) yields an upper value of extracted $\delta$ of $450 \mathrm{~W} / \mathrm{cm}^{2}$. For an $\mathrm{O}_{2}^{*}$ yield of $60 \%$, a more realistic value would be $\delta=280 \mathrm{~W} / \mathrm{cm}^{2}$. A reasonable fraction of the thermodynamic value has been achieved.

\subsubsection{Optics}

The laser beam quality is degraded from the diffraction limit by phase aberrations introduced by refractive index gradients, mirror surface roughness, and mirror figure deformations due to power loading.

Several factors determine the magnitude and order of the refractive index gradients in the flow. The uniformity of the species concentrations in the flow is one principal cause of index gradients. A major contribution to index gradients could come from a poorly mixed flow.

Uniform mixing has been achieved, but heat release from chemical reactions in the flow can cause density gradients and index of refraction variations in the flow direction. The first and second order variations (Zernicke polynomials ${ }^{25}$ ) in refractive index to tilt and focus can be corrected in the resonator. Higher order corrections would require adaptive optics. The present understanding of the device operation suggests that these corrections may not be required. However, beam quality assessment is presently lacking, and this information is of key significance in judging the COIL system as to its promise for a DEW device.

\subsubsection{Device Sizing}

An overall block diagram of a device configuration is given in Figure 3.13. Using the described technologies and the general configuration of Figure 3.13 a $5 \mathrm{~kW}$ subsonic and several $4 \mathrm{~kW}$ supersonic flow lasers at 1.315 $\mu \mathrm{m}$ have been built at AFWL and other laboratories. Currently a $25 \mathrm{~kW}$ laser is being built at AFWL using a $1 \mathrm{~mole} / \mathrm{s} \mathrm{O}_{2}$ generator.

A one megawatt average power device could be built using a linear addition (in length) of 30 to 40 of the modules used for the $25 \mathrm{~kW}$ device. This is shown in Figure 3.14. Because $\mathrm{O}_{2}-\mathrm{I}$ is a low gain laser, we foresee no limits in theory for such scaling in length and nozzle height for devices of the order of up to $10 \mathrm{MW}$; beyond this level linear gain may become sufficiently high to cause parasitic oscillations and super fluorescent losses.

Scaling beyond such power levels might proceed via the "coupled resonator" approach as seen in Figure 3.15. Here $10 \mathrm{MW}$ individual laser devices have their optical resonators coupled through mutual feedback so that in essence one has a distributed optical resonator. Such optical resonators can produce highly discriminated

\section{SUPERSONIC OXYGEN-IODINE CHEMICAL LASER}

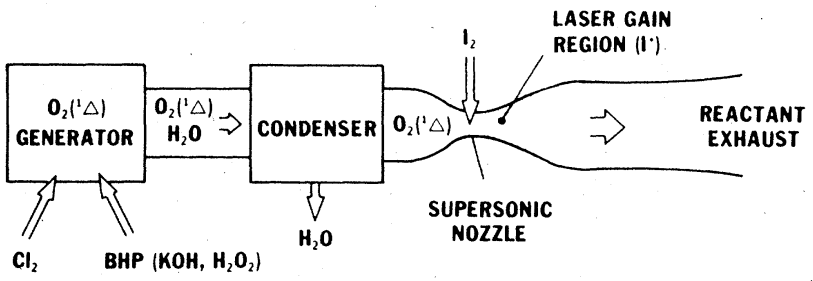

Figure 3.13. Supersonic oxygen-iodine chemical laser.

supermodes, producing multiple parallel beams that are totally phased. However, there has been no demonstration to date that modules at such high powers could be phase coupled. Such device (oscillator) phasing has been demonstrated ${ }^{26}$ for six $\mathrm{CO}_{2}$ oscillators at $10.6 \mu \mathrm{m}$, two oscillators at $3.8 \mu \mathrm{m}$ (DF lasers) and two oscillators at $1.3 \mu \mathrm{m}$ (I lasers). Such experiments have only been carried out at lab scale, and no high average power data are available.

Emerging technology indicates that a minimum of four such devices should be coupled to provide appropriate mode differentiation between supermodes, and six such coupled oscillators have been demonstrated ${ }^{26}$ using $\mathrm{CO}_{2}$ laser media. While it is conceivable that ten or more such $10 \mathrm{MW}$ oscillators could be coupled for a $100 \mathrm{MW}$ class system at $1.315 \mu \mathrm{m}$, as stated above no high-power experiments have yet been demonstrated. However, a device of such size requires a scaling of at least a factor of $10^{3}$. In addition, the final device will essentially be operated as a single homogeneous $\mathrm{cw}$ oscillator. The multiplicity of components and the large physical dimensions, on the order of tens of meters, warrant caution in extrapolating the available data and analysis to the proposed system.

MEDIUM POWER FIELD DEMO

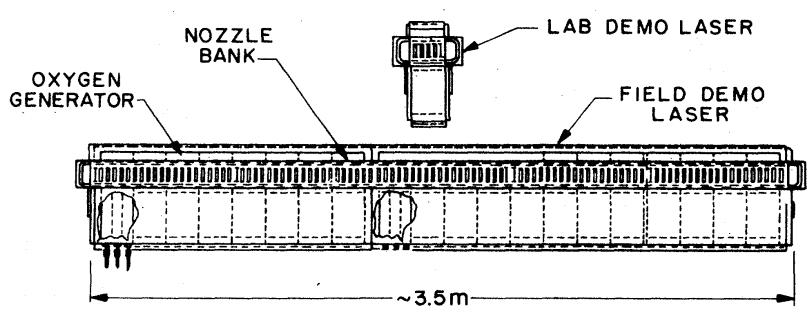

Figure 3.14. Medium power field demo. 


\section{HIGH POWER COUPLED IODINE LASERS}

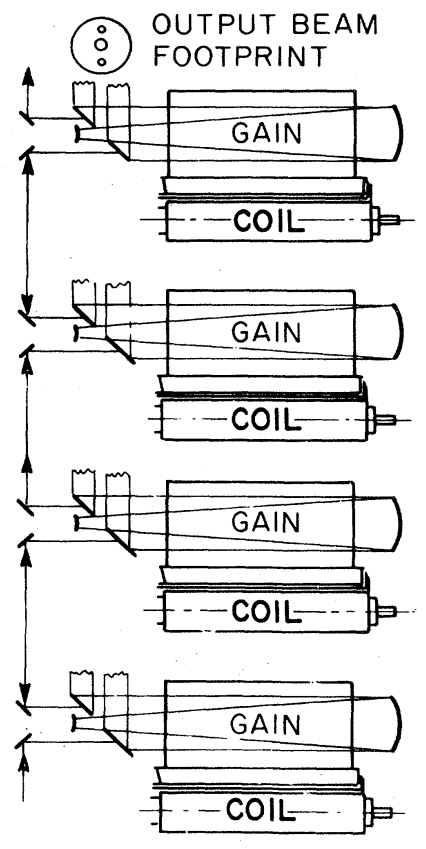

Figure 3.15. High power coupled iodine lasers.

\subsubsection{Scaling Issues}

Several technologies have been devised for the generation of $\mathrm{O}_{2}\left({ }^{1} \Delta\right)$. The clorox reaction in Equation (3.15) can yield up to $\sim 300 \mathrm{~kJ}$ of excited oxygen per $\mathrm{kg}$ of fuel. Assuming $50 \%$ efficiency in converting this energy to laser light, a $100 \mathrm{MW}$ laser will require a fuel flow of 0.7 tonne/s. For space-based operation, the oxygen generator technology requires significant development. Sparger technology is difficult to scale to the requisite size. The rotating plate reactor is scalable to large size, but it is unsuited for space-based use because of its incompatibility with a zero-g environment. The spray reactor, in which reactants form an aerosol spray, is in the early stage of development. It is adaptable to zero-g, but requires significant improvement in performance. It is desirable to generate excited oxygen by a system which does not use liquids. A solid-phase system based on endoperoxide chemistry ${ }^{27}$ has been developed, but has only been demonstrated at a laboratory scale.

At present, most systems use electrically heated $I_{2}$ generators. Although these are inefficient, the amount of energy required to create $I_{2}$ vapor is small, at the low concentrations of $I_{2}$ envisioned. The mixing of iodine and oxygen presents a more serious problem. The disparity in molecular weight limits the achievable mixing efficiency. Further, the mixing nozzles must be designed to reduce the formation of wakes, which will perturb the optical quality of the laser medium. The design of the iodine generator and the mixing nozzles is an engineering problem, but one which must be addressed as part of a scale-up of the laser technology.

\subsubsection{Critical Issues}

Chemical oxygen-iodine lasers are in an early stage of development; a $25 \mathrm{~kW}$ module is presently being built. Current technology must be extrapolated to output powers of $10 \mathrm{MW}$ per aperture; beyond that level, multiple optically-coupled apertures are necessary. An assessment of beam quality does not exist and this information is required to evaluate the COIL system for DEW applications.

For space-based use, liquid generation of excited oxygen may not be acceptable. Both solid-phase and aerosol spray generators have been proposed, but require significant development.

\subsubsection{Visible Chemical Lasers}

Chemical lasers operating at shorter wavelengths than the chemical oxygen iodine laser $(1.39 \mu \mathrm{m})$ presently have not been demonstrated, although such devices offer the hope of possibly being able to deliver more energy to a far-field target and/or of having more energy output per pound of total device. In the past there has been an intensive effort to develop visible chemical lasers primarily based on exothermic reactions of various metal atoms with powerful oxidizers. ${ }^{28}$ What became evident from such studies is that there is little reason to believe that any reaction of ground state reactants will yield an electronic population inversion in a fast radiator in a single reaction step. This is a general consequence of the separation of electronic and nuclear motions (the BornOppenheimer approximation) for low energy collisions implying that electronic ground state reagents yield dominantly electronic ground state products. This means that partial population inversion is possible between vibrational levels of the excited electronic state and unpopulated vibrational levels of the ground electronic state, but such a laser system is anticipated to have low efficiency. Nevertheless, the same arguments when combined with overall spin conservation in a chemical reaction indicates that it should be possible to create high yields of metastable electronically excited states-and indeed this has been confirmed in a number of experiments that generate $\mathrm{O}_{2}\left({ }^{1} \Delta_{\mathrm{g}}\right.$ ) (which is employed in the chemical oxygen iodine laser) and the isoelectronic $\mathrm{NF}\left({ }^{1} \Delta\right)$ and analog $\mathrm{NCl}\left({ }^{1} \Delta\right)$ and $\operatorname{NBr}\left({ }^{1} \Sigma\right)$ metastable species. For metastable state levels in excess of $2 \mathrm{eV}$, it appears that excited state spin conserving reactions must be invoked, such as those that produce $\mathrm{N}\left({ }^{2} \mathrm{D}\right)$ metastable states at $2.34 \mathrm{eV}$ through a reaction of $\mathrm{D}\left({ }^{2} \mathrm{~S}\right)+\mathrm{NF}\left({ }^{1} \Delta\right) \rightarrow \mathrm{N}\left({ }^{2} \mathrm{D}\right)+\mathrm{DF}\left({ }^{1} \Sigma^{+}\right)$. It is conceivable that collisions with such metastable species, in excess, i.e., $\mathrm{O}_{2}\left({ }^{1} \Delta_{\mathrm{g}}\right)$ or $\mathrm{N}\left({ }^{2} \mathrm{D}\right)$, may be used to populate substantial 
concentrations of the upper level of a visible chemical laser.

The successful development of visible chemical lasers must solve questions such as

- What excited energetic species can be generated by purely chemical means?

- What subsequent energy transfer process or chemistry is required to produce a candidate visible chemical laser system?

- Do reactants need to be premixed or can they be brought together in the right sequence and with sufficient mixing?

Even if a visible chemical laser can be demonstrated in the laboratory, experience suggests that development of a device that can meet DEW needs will be highly problematic.

\subsection{EXCIMER LASERS}

\subsubsection{Background}

Properly speaking, excimer lasers are those which operate on electronic transitions in molecules whose ground state potential energies are essentially repulsive; ${ }^{\dagger}$ in practice, the term is also applied to some related molecules with weakly-bound ground states. The transition energies involved make possible gas lasers of reasonable energies operating in the visible and soft UV wavelengths. One particular class of excimers, the rare gas-halogen excimers, has also proved to have high efficiency relative to previous UV gas lasers. Demonstrated overall efficiencies have been in the range $3-5 \%$, which includes about $50 \%$ for conversion of delivered electricity to energy deposited in the gas medium. Extremely high-power applications of excimers, particularly in inertial confinement fusion and strategic defense, have thus concentrated attention on the rare gashalogen excimers. In both communities of researchers, and in the public press discussions of strategic defense, the broader term of excimers has been used when in fact only the rare gas-halogens are being discussed. That shorthand has also been adopted in the present report, following a general background section.

\subsubsection{History}

Emission from various dimer excimers was first observed in the 1930s; the rare gas dimer excimers were extensively studied in the 1950s. Although obtaining gain from bound-free electronic transitions was proposed ${ }^{29}$ in

\footnotetext{
${ }^{\dagger}$ Even more strictly, excimers would only be molecules formed of identical atoms; heterogeneous molecules would be exciplexes.
}

1960, it was not until 1971 that the first excimer was demonstrated ${ }^{7}$ using $\mathrm{Xe}_{2}(170 \mathrm{~nm})$ in liquid phase; a laser in the gas phase was achieved the following year. ${ }^{8}$ Lasers using $\mathrm{Kr}_{2}(146 \mathrm{~nm})^{30}$ and $\mathrm{Ar}_{2}(126 \mathrm{~nm})^{31}$ were demonstrated shortly thereafter. Unfortunately, the efficiency of these rare gas dimer excimers was found to be very low because of excited state absorption in the gas medium. These lasers have proven to be very useful for low-power laboratory application where efficiency is not important.

In 1975, Velazco and Setser observed fluorescence from a number of molecules formed with xenon and halogen atoms. ${ }^{32}$ Quite rapidly, lasing action was reported on a number of rare gas-monohalide molecules; of particular importance were $\mathrm{XeF}$ at $351 \mathrm{~nm},{ }^{33} \mathrm{KrF}$ at 248 $\mathrm{nm},{ }^{34} \mathrm{ArF}$ at $193 \mathrm{~nm},{ }^{35}$ and $\mathrm{XeCl}$ at $308 \mathrm{~nm} \cdot{ }^{36}$ In the first experiments, gas mixtures near atmospheric pressure were excited in very small cavities by high current density ( $>100 \mathrm{~A} / \mathrm{cm}^{2}$ ) electron beams.

Over the next five years, two lines of development were followed to scale the rare gas-halides to larger total energies and higher efficiencies. Large volumes were excited by moderate-strength $\left(5-30 \mathrm{~A} / \mathrm{cm}^{2}\right)$ electron beams to get high energies and high volumetric energies; gas pressures in these cases were generally raised to the 2-3 atm range. These efforts quickly resulted in the extraction of energies of hundreds of joules at local (or "intrinsic") efficiencies ${ }^{37,38}$ (laser energy out/energy deposited in the gas) of about $10 \%$.

Concurrently, a major éffort was undertaken to increase this efficiency by pumping the medium gas (1-3 atm) with electric discharges, either e-beam substained $^{39}$ or UV-preionized. ${ }^{40}$ Unfortunately, it was found that $\mathrm{KrF}$ discharges are limited by multistep ionization of the excited rare gas atoms; analytic calculations $^{41}$ predicted that ionization instability will occur unless the electron attachment rate is larger than twice the field-driven ionization rate. These predictions were verified through experiments. Effectively, this problem places a limit on the number of $\mathrm{Kr}^{*}$ atoms, hence on the creation efficiency of $\mathrm{KrF}^{*}$, in both of the metastable regimes of discharge operation, ${ }^{42}$ keeping the discharges slightly less efficient than the direct-pumped cases.

Much of the work done since about 1980 on scaling these excimers to higher energies remains classified. An important exception is a single $1 \times 1 \mathrm{~m}^{2}$ aperture $\mathrm{KrF}$ device constructed at LANL in 1983-1985 for the inertial confinement fusion program. This laser reportedly delivered $10 \mathrm{~kJ} .{ }^{43}$ Many theoretical papers in the open literature have proposed designs for $\mathrm{MJ}$-scale $\mathrm{KrF}$ lasers. $^{43,44}$

\subsubsection{General Features}

The general structure of rare gas-monohalide molecules is shown in Figure 3.16. The covalent ground 


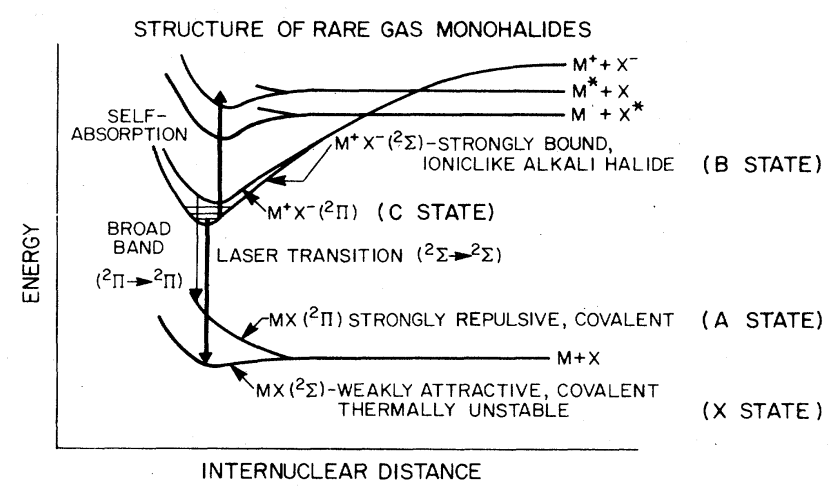

Figure 3.16. Schematic potential energy diagram illustrating the electronic structure of the rare gas monohalides.

state corresponds to ground state ${ }^{1} \mathrm{~S}$ rare gas atoms and ${ }^{2} \mathbf{P}$ halogen atoms at infinite internuclear separation. The ground state manifold consists of two states because the halogen atom has a net orbital angular momentum of one. The higher of these states, ${ }^{2} \mathrm{II}$, labeled the A state, is always repulsive, as shown in Figure 3.16. The lower, the ${ }^{2} \Sigma$, is the true ground state (hence known as $\mathrm{X}$ ), and has the singly-occupied halogen orbital directed toward the rare gas atom. The $\mathrm{X}$ state is generally also repulsive (e.g., KrF) or very weakly bound (as shown in Figure 3.16; $\mathrm{XeCl}$ is bound by only $255 \mathrm{~cm}^{-1}$ ). The sole significant exception to this is $\mathrm{XeF}$, which is bound by approximately $1065 \mathrm{~cm}^{-1}$.

The upper laser level is ionically bound, consisting asymptotically of a charge-transfer state corresponding to the ${ }^{2} \mathrm{P}$ rare gas positive. ion and the ${ }^{1} \mathrm{~S}$ halogen negative ion at infinite internuclear separation. At large internuclear separation, at an energy equal to the ionization potential of the rare gas less the electron affinity of the halogen, the ionic binding curve crosses the covalent curves corresponding to combinations of neutral rare gas and halogen atoms. This crossing is of central importance to the high efficiency of these excimers because it permits several entrance channels to the upper laser level.

The emission spectra of the rare gas-halides consists of several bands, as shown in Figure 3.17 for $\mathrm{KrF}$. The strongest emitter is the $\mathbf{B}\left({ }^{2} \Sigma\right) \rightarrow X\left({ }^{2} \Sigma\right)$ transition, which is the lasing transition in all instances of practical interest. A typical fluorescent bandwidth is (as here in $\mathrm{KrF}$ ) about $30 \AA$. The natural lifetimes of the excited upper states of rare gas-halides are on the order of $10 \mathrm{~ns}$, making them very difficult to measure directly because they are difficult to prepare. The lifetimes for $\mathrm{XeF}^{*}(15 \mathrm{~ns})^{45}$ and $\mathrm{KrF}^{*}$ $(9 \mathrm{~ns})^{46}$ have been measured, since they can be prepared directly from $\mathrm{XeF}_{2}$ and $\mathrm{KrF}_{2}$. Others are known generally from $a b$ initio computer calculations, ${ }^{47,48}$ which appear to be reasonably accurate $\left(12 \mathrm{~ns}\right.$ for $\mathrm{XeF}^{*}$ and $6.7 \mathrm{~ns}$ for $\left.\mathrm{KrF}^{*}\right)$.

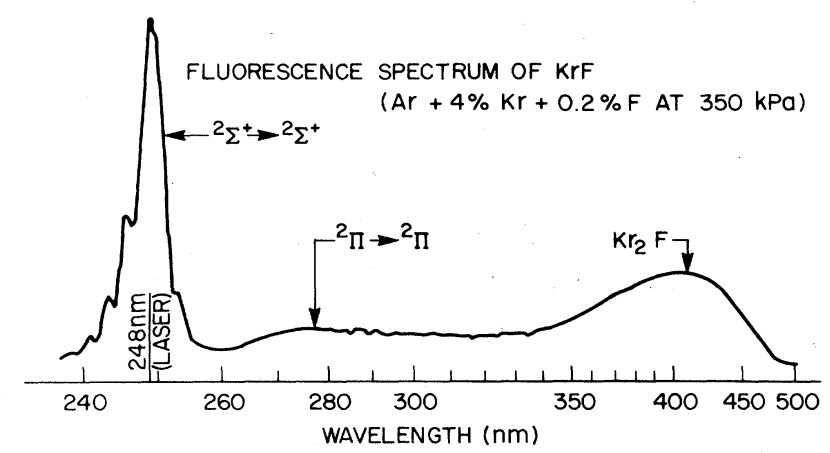

Figure 3.17. Fluorescence spectrum of $\mathrm{KrF} \ldots$

The dominant molecular parameter for laser performance is the stimulated emission cross section, which depends upon both the shape and width of the emission band. The product of the cross section and upper state lifetime has been calculated for $\mathrm{KrF}$ from detailed data on the $\mathrm{B} \rightarrow \mathrm{X}$ band; this product leads to a value of stimulated emission cross section for $\mathrm{KrF}$ of $\sigma=2.4 \times 10^{-16} \mathrm{~cm}^{2}$. Cross sections for the other rare gas halides, generally calculated assuming a roughly Gaussian line shape, tend to be roughly equivalent.

Laser performance is also complicated and limited by the presence in the gas medium of absorbing species, which may absorb by molecular dissociation, photoionization, or photodetachment. Cross sections for most of these processes have been measured. Key performance and scaling issues are related to whether the absorbers produced for a particular excimer are or are not saturable.

For e-beam pumping of excimer gases, the electron beams are usually oriented transverse to the lasing axis; the third dimension is available for flowing the gas in a repetitively pulsed laser. In single pulse applications, energy is usually delivered to the e-beam diodes from large capacitor banks through water-insulated transmission lines, with some form of pulse-shaping. For repetitively pulsed systems, pulse-forming networks, e.g., capacitive-inductive storage systems developed originally as radar power technology, have been maturing for the last twenty years. The gas is most efficiently pumped from both sides at once, to provide good spatial beam quality with uniform energy deposition. For the same reason, it is necessary in large volumes to guide the electrons with magnetic fields $(\sim 0.1-1.0 \mathrm{~T})$ in order to prevent beam pinching and consequent nonuniform deposition.

At these short wavelengths, partially transmissive mirrors are not practical, particularly for high energies: deposition in the mirror material will be destructive. In fact, UV energy deposition even in $99.9 \%$ reflective mirrors becomes an issue for lasers at high energies, and poses limits in total specific energy per pulse to prevent serious damage to reflective coatings. To minimize this problem, then, unstable resonators ${ }^{17}$ are chosen for all 
oscillators and multipass amplifiers, and fluxes on all surfaces must be kept below damage (a few $\mathrm{J} / \mathrm{cm}^{2}$ ), a clear constraint on weapon systems designs.

This potential of optical damage by the short wavelengths constitutes one of the principal disadvantages of the excimers. Another disadvantage is relatively poor atmospheric transmission (compared to visible or wellchosen IR); for excimers of most interest, this is not caused by any absorption problems, but by increased Rayleigh scattering $\left(\propto \lambda^{-4}\right)$. This problem can be somewhat ameliorated by Raman shifting the excimer output to longer wavelengths, which is also desirable to achieve high beam quality without expensive and vulnerable optics (see below). A final drawback of excimers is the fact that high energies must be obtained (see below also) by combining many individual apertures, and this poses problems of system complexity.

The advantages which make excimers attractive are effective target coupling with high energy photons and short, intense pulses; high efficiencies (relative to other existing lasers of similar wavelength); flexibility of laser design; beam combination and cleanup in Raman cells (which allows large amplifier modules to be individually of low optical quality, and which offers possibility of using low power, uncooled optics in the compensation portion of the beam train); and high specific energy yield.

Of the four rare gas-halides specifically mentioned above, one (ArF) has too short a wavelength to be compatible with optics damage criteria at high energies, and one $(\mathrm{XeF})$ is too limited in performance by its kinetics, particularly its bound ground state. Thus the remaining two are the excimers of primary importance as possible weapons. $\mathrm{KrF}$ has the difficulties of optics and transmission, relative to $\mathrm{XeCl}$, of its shorter wavelength; but it also has about twice the intrinsic efficiency. A few details of both lasers will thus be discussed, followed by more general description of the generic technologies.

Extensive reviews of rare gas-halides in general $^{49-51}$ and of $\mathrm{KrF}$ in particular, ${ }^{52}$ have been written. To date no comprehensive explanation of $\mathrm{XeCl}$ laser operation has appeared.

\subsubsection{Krypton Fluoride}

The upper levels of $\mathrm{KrF}^{*}$ are the $\mathrm{B}\left({ }^{2} \Sigma\right)$ state and two ${ }^{2}$ II states, split by spin-orbit coupling into the $\mathrm{C}\left({ }^{2} \mathrm{II}_{3 / 2}\right)$ and $\mathbf{D}\left({ }^{2} \mathrm{II}_{1 / 2}\right)$ states. Emissions from $\mathrm{C}$ and $\mathrm{D}$ bands in $\mathrm{KrF}$ are at significantly lower intensity than for the $\mathrm{B} \rightarrow \mathrm{X}$ transition, indicating a form of quenching from the $\mathbf{C}$ to the $\mathbf{B}$ state.

$\mathrm{KrF}^{*}$ can be formed from ions in the reaction

$$
\mathrm{Kr}^{+}+\mathbf{F}+\mathbf{M} \rightarrow \mathrm{KrF}^{*}+\mathbf{M}
$$

as well as from excited atomic states of krypton by the charge-transfer ("harpoon") reactions

$$
\mathrm{Kr}^{*}+\mathrm{F}_{2} \rightarrow \mathrm{KrF}^{*}+\mathrm{F}
$$

and

$$
\mathrm{Kr}^{* *}+\mathrm{F}_{2} \rightarrow \mathrm{KrF}^{*}+\mathrm{F} .
$$

In these reactions, $M$ represents a nonreactive collision partner and $\mathrm{Kr}^{*}$ and $\mathrm{Kr}{ }^{* *}$, respectively, represent the ${ }^{5} \mathrm{~S}$ and ${ }^{5} \mathrm{P}$ states of krypton. These excited states store energy effectively because their transitions are either metastable or optically trapped as resonance radiation.

Direct electron beam pumping produces an electronion pair with an investment of approximately $26 \mathrm{eV}$ in argon-rich mixtures. Partitioning of this energy into ionization, secondary production, and metastable excitation is estimated ${ }^{53}$ to produce a theoretical upper bound to intrinsic efficiency of $22 \%$. Argon is frequently used as a background gas in $\mathrm{KrF}$ laser mixtures because it stops energetic beam electrons well, is inexpensive compared with krypton, and collisionally transfers its energy to krypton effectively. The lower-lying argon states also store energy effectively, an additional practical advantage in using $\mathrm{Ar} / \mathrm{Kr}$ mixtures. Of course, $\mathrm{ArF}^{*}$ is formed by reactions similar to those above, but $\mathrm{KrF}^{*}$ is also formed by the displacement reaction,

$$
\mathrm{ArF}^{*}+\mathrm{Kr} \rightarrow \mathrm{KrF}^{*}+\mathrm{Ar} .
$$

The lifetime of $\mathrm{ArF}^{*}$ from displacement is generally short compared to the spontaneous radiative lifetime of the excimer, so $\mathrm{ArF}^{*}$ formation is part of a pumping channel rather than a loss mechanism.

Some attention has recently been drawn to unbuffered mixtures of $\mathrm{Kr}$ and $\mathrm{F}_{2}$, which must be run near atmospheric pressure because of 3-body quenching processes involving krypton. In fact, the first detailed calculations of "Kr-only" mixtures ${ }^{54}$ were performed to optimize a laser which had to be run below atmospheric pressure for other reasons. Whether such mixtures can in fact be run at higher electrical efficiencies than buffered mixtures remains a topic of some contention. But the differences of interpretation and prediction only cover a range of about $2 \%$-from $12 \%$ to $14 \%$ electrical efficiency-and no one maintains that any serious increase over $\mathrm{KrFs}$ demonstrated volumetric efficiency of $20-30 \mathrm{~J} / 1$ is reasonable to expect.

The most effective fluorine donor has been $F_{2}$, although $\mathrm{NF}_{3}$ has also been used successfully. Since $\mathrm{F}_{2}$ absorbs $\mathrm{KrF}$ radiation and $\mathrm{NF}_{3}$ does not, the latter would seem to have an advantage. But the charge transfer of $\mathrm{Kr}^{+}$to $\mathrm{NF}_{3}$ represents a loss to the ion channel of $\mathrm{KrF}^{*}$ formation. Consequently, mixtures of $\mathrm{Ar} / \mathrm{Kr} / \mathrm{F}_{2}$ have become standard for e-beam pumped $\mathrm{KrF}$ lasers.

The kinetics of pumping, quenching, radiation, and absorption of $\mathrm{KrF}$ is extremely complicated. A representative compilation of the kinetics ${ }^{55}$ employs 23 


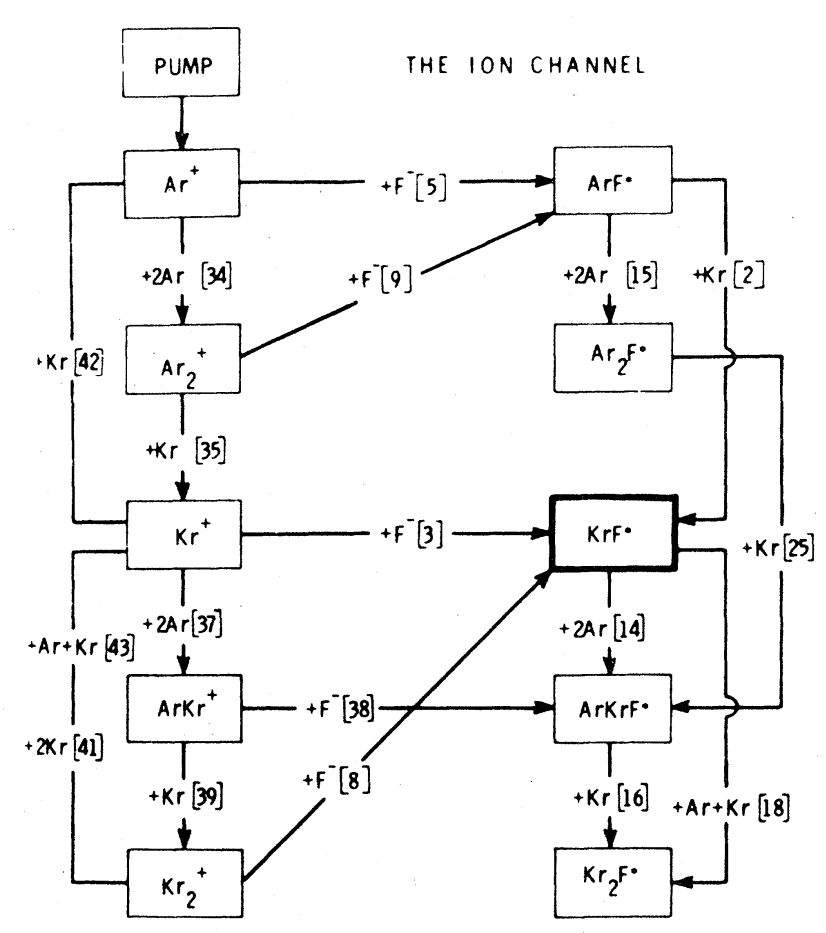

Formation of krypton fluoride through the ion channel.

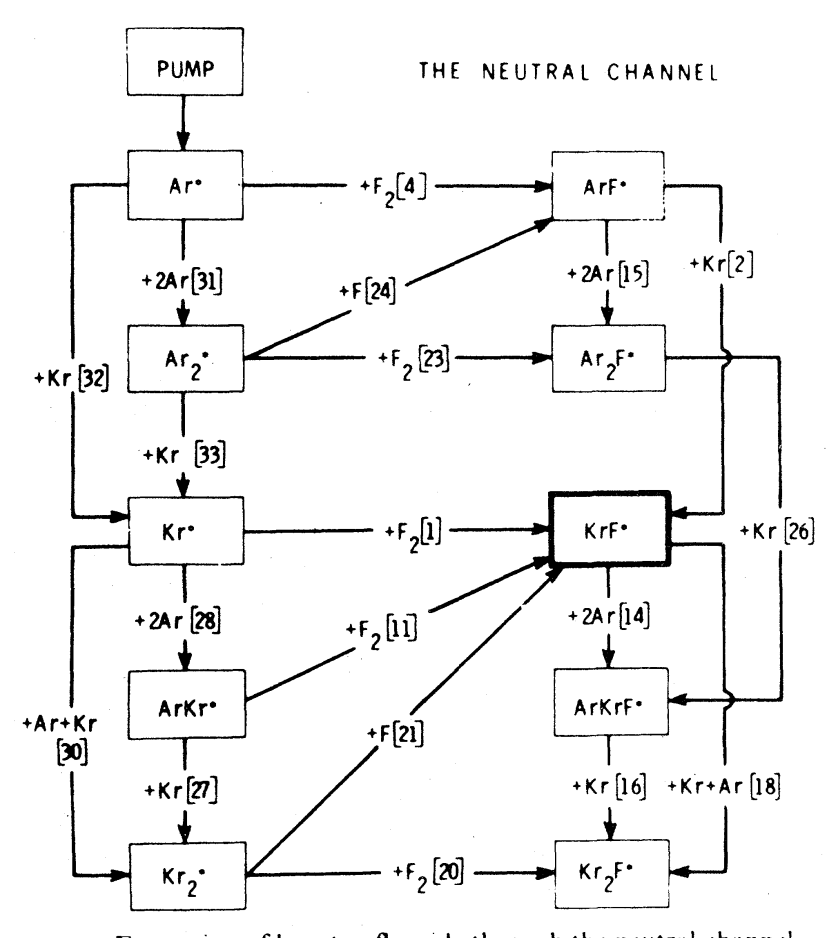

Formation of krypton fluoride through the neutral channel.

Figure 3.18. Ion and neutral kinetic paths in the formation of $\mathrm{KrF}$. chemical species and nearly a hundred reactions. Pumping is accomplished simultaneously in ion and neutral channels, with the branching between them determined by a combination of parameters, principally pump power. These channels are shown graphically in Figure 3.18, which also shows losses by quenching and interception (diversion of excitation energy before $\mathrm{KrF}^{*}$ is formed). Note that both the bottom products of quenching and interception-the triatomic $\mathrm{Kr}_{2} \mathrm{~F}^{*}$ and the molecular ion $\mathrm{Kr}_{2}{ }^{+}$, respectively - are strong absorbers. For e-beam pumped $\mathrm{KrF}$, the electron-dependent kinetics are important primarily because the rate constant for dissociative attachment to fluorine is a sensitive function of the shape and characteristic temperature of the electron distribution function. This distribution function will not be Maxwellian in steady state, because attachment removes electrons at very low energies and beam ionization creates secondaries at (typically) a few eV.

In general, for pumping by moderate density e-beams $\left(15 \mathrm{~A} / \mathrm{cm}^{2}\right.$ is equivalent to a pumping rate of $0.3 \mathrm{MW} / \mathrm{cm}^{3}$ for a $2 \mathrm{~atm}$ gas) quenching dominates over interception. This is salutary, since it means that the primary loss rate is saturable: it depends directly on the $\mathrm{KrF}^{*}$ concentration, which can be kept instantaneously low with a high intensity intracavity radiation field $\left(\sim 1-5 \mathrm{MW} / \mathrm{cm}^{2}\right)$. It also means that the molecular absorption will be dominated by $\mathrm{Kr}_{2} \mathrm{~F}^{*}$, which is saturable (for the same reason), rather than by the nonsaturable $\mathrm{Kr}_{2}{ }^{+}$.

At these pumping rates, the loaded net gains of $\mathrm{KrF}$ are on the order of $0.01 \mathrm{~cm}^{-1}$. Ratios of $\mathrm{g}_{0} / \alpha$ (small-signal gain to absorption) on the order of 3-5 are typical, and thus the laser can extract efficiently at twice the saturation flux or more. Extraction efficiency tends to be limited by integrated absorption.

Because the loaded gain has an e-fold in about a meter, losses to amplified spontaneous emission off the lasing axis prevent the successful operation of apertures in excess of about one meter in either transverse dimension. ${ }^{56}$ The remaining parameters-scaling with gain length, pump power, mix, and pressure-have been optimized parametrically. ${ }^{57}$ The result of these calculations indicates, in brief, that the largest single module which can be expected to perform efficiently is one which produces about $20 \mathrm{~kJ}$.

Pulse length of $\mathrm{KrF}$ lasers is also limited by the kinetics. At pump rates of a few tenths of a $\mathrm{MW} / \mathrm{cm}^{3}$, the natural pulse length is in the range $0.6-1.2 \mu \mathrm{s}$, determined by burn-up of the $F_{2}$; putting in more $F_{2}$ significantly lowers performance by absorption. Longer pulses $(1-5 \mu \mathrm{s})$ can be produced by pumping more slowly $\left(0.1 \mathrm{MW} / \mathrm{cm}^{3}\right.$ or less), but at a cost of $20-50 \%$ in electrical efficiency.

\subsubsection{Xenon Chloride}

Like $\mathrm{XeF}, \mathrm{XeCl}$ tends to operate at an intrinsic efficiency of about half that of $\mathrm{KrF}(5-6 \%$ as opposed to 
10-12\%) under optimum conditions. ${ }^{59-61}$ Also like $\mathrm{XeF}$, $\mathrm{XeCl}$ has a chain of kinetic processes much more complex than that of $\mathrm{KrF}$ : their kinetic models must include roughly twice as many processes as are required to simulate $\mathrm{KrF}$.

As previously mentioned, both $\mathrm{XeF}$ and $\mathrm{XeCl}$ have bound ground states, but there is a significant difference. The $\mathrm{XeCl}$ ground state is bound by slightly less than $\mathrm{kT}$, so bottlenecking of the laser can be easily avoided; XeF is bound by more than three times kT, which effectively imposes more limits on its scaling. Both molecules also pump significant fractions of energy into the $\mathrm{C}$ state. Since the $\mathrm{C} \rightarrow \mathrm{A}$ transition in $\mathrm{XeCl}$ has a lifetime well in excess of $100 \mathrm{~ns}$, and since the B and C states are effectively mixed, a reasonable approximation can be made that roughly $20 \%$ of the pump energy is lost in the C state.

The standard chlorine donor for $\mathrm{XeCl}$ is $\mathrm{HCl}$. The laser is operated (for high power) in mixtures of 30-60 torr $\mathrm{Xe}$ and 4-6 torr $\mathrm{HCl}$, in buffer gases of 2 atm argon or 4-5 atm neon. Higher pressures of neon are necessary in order to get equivalent energy deposition from the e-beam. $\mathrm{HCl}$ has a disadvantage as a chlorine donor: dissociative attachment only has significant rates for vibrationally excited $\mathrm{HCl}$ molecules. $\mathrm{CCl}_{4}$ has also been successfully used as a chlorine donor, but it leaves unacceptable chemical residues in the cavity. The ease of vibrational excitation of $\mathrm{HCl}$ by discharge electrons, coupled with the low metastable excitation potential of $\mathrm{Xe}$ (compared to $\mathrm{Kr}$ ), suggest that $\mathrm{XeCl}$ can be operated well in a discharge mode, and indeed high repetition rate $\mathrm{XeCl}$ lasers have been demonstrated effectively at the joule level. ${ }^{61}$

Another principal difference between $\mathrm{KrF}$ and $\mathrm{XeCl}$ is the absence of the "harpooning reaction" for the first excited state of rare gas atom. That is, where a $\mathrm{KrF}^{*}$ can be produced by a $\mathrm{Kr}^{*}$ and an $\mathrm{F}_{2}$ molecule, an $\mathrm{Xe}^{* *}$ $(\sim 9 \mathrm{eV})$ is required to produce an $\mathrm{XeCl}^{*}$ by harpooning $\mathrm{HCl}$. A further difference in the neutral channels is that the displacement reaction $\mathrm{Xe}+\mathrm{ArCl}^{*} \rightarrow \mathrm{XeCl}^{*}+\mathrm{Ar}$ works for the argon buffer, but not for neon, since $\mathrm{NeCl}^{*}$ is believed to predissociate.

$\mathrm{XeCl}$ has been lased at moderate and high powers with e-beam pumping by several groups. ${ }^{58-60}$ Several kinetic models have also been proposed, ${ }^{62-64}$ but none is yet adequate to explain the full range of performance data. From what is now understood, the $\mathrm{XeCl}$ scaling behavior in pulse length, gain length, pump power, etc., is similar to the scaling of $\mathrm{KrF}$, except that the intrinsic and volumetric efficiencies are roughly halved.

\subsubsection{Electron Beam Pumping}

Although excimer laser experiments have been conducted with beam currents up to the $1000 \mathrm{~A} / \mathrm{cm}^{2}$ range, high-power excimers are pumped in either of two regimes: low $\left(3-5 \mathrm{~A} / \mathrm{cm}^{2}\right)$ currents of multi-microsecond pulses; and moderate $\left(10-20 \mathrm{~A} / \mathrm{cm}^{2}\right)$ currents for pulses 600-1200 ns. These currents must be delivered uniformly over large areas roughly $2-5 \mathrm{~m}^{2}$ (Reference 44).

The technology which supports these experiments is relatively mature on a single-shot basis. Pulse-forming is handled by standard Marx generators, in which fasttriggering gas switches shift capacitor networks from parallel to series, delivering hundreds of $\mathrm{kV}$ potentials with rise times in the tens of ns.

Technology for repetitively pulsed gases and switches is also relatively mature. Two different approaches were evolved under the EMRLD program about two years ago for producing $100 \mathrm{~Hz}$ electron gun drivers. Both techniques are scalable to the megavolt level required to drive the high voltage e-beam at multicoulomb total delivered charges. A magnetic modulator using magnetic switches (similar to those developed at Sandia and Lawrence Livermore Laboratories) was designed and tested at Maxwell Laboratories. A blown spark-gap switched pulse forming network was developed by AVCO Research Laboratories. Both have operated at $100 \mathrm{~Hz}$; the former switch operates at $250 \mathrm{kV}$, the latter at $1 \mathrm{MV}$.

A typical one-sided single-shot pumping geometry is shown in Figure 3.19. The transverse pumping geometry is the most flexible; although several others have been tried, it is now almost universally used for these lasers. The cold cathode is pulse-charged, and the diode interior evacuated to $10^{-4}$ torr or less. The thin foil anode separates the low-pressure diode interior from the highpressure (1-5 atm) laser cavity. Titanium foil of a few tenths of a mm thickness is a typical choice for high tensile strength/specific density. Because of the pressure difference, the foil must be given structural support; this is usually done with a thin grid structure known as a "hibachi." Field-enchancing techniques are frequently used on anode surfaces. Although the anode foils are quite thin, they have been demonstrated in practice to

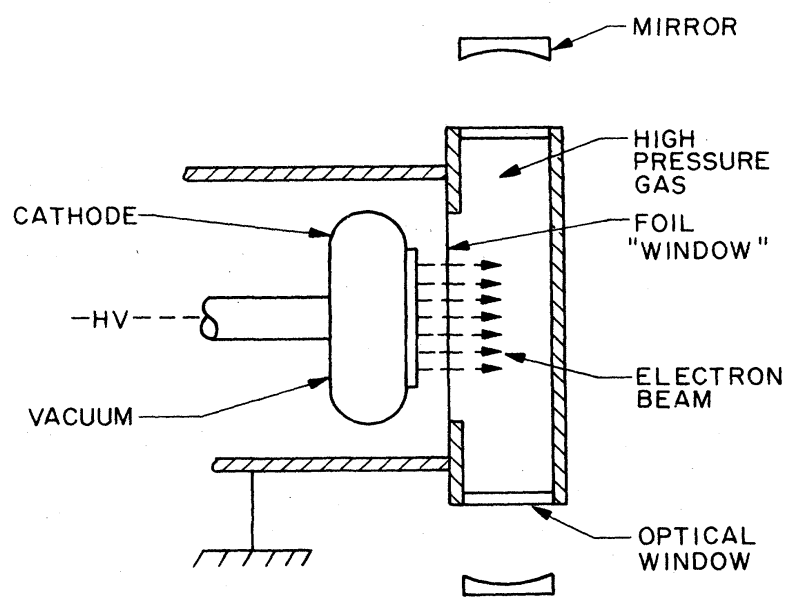

Figure 3.19. Schematic diagram of a laser pumped by high intensity electron beam. 
take hundreds of shots at full voltage without damage.

Although carbon felt cold cathodes are useful for single-pulse or low repetition rate devices (total shots of no more than 3000-5000), their lifetimes are limited for high rep-rate, long duration operation. The EMRLD technology program has developed actively cooled, hot cathode guns which appear to solve the lifetime problems. The cost of these guns for the large areas needed to pump large amplifiers may prove to be an issue.

The total current which can be drawn in the e-beam is limited by space charge considerations according to the Child-Langmuir Law,

$$
\mathrm{J}=2.3 \times 10^{3} \mathrm{~V}^{3 / 2} / \mathrm{d}^{2},
$$

where $\mathrm{V}$ is the voltage in megavolts and $\mathrm{d}$ is the diode gap in $\mathrm{cm}$. The hibachi foil support structures cause geometric losses by masking electrons. With other, smaller losses (such as scattering by the foil), it should be possible to design diodes which deliver $80-90 \%$ of the stored electric energy into the gas as a beam. Current designs are dominated more by cost and simplicity, and in most cases roughly $50 \%$ of the stored energy is actually deposited in the gas by the beam. Thus, overall or "wall-plug" efficiencies of excimer lasers are about half their local (intrinsic) efficiencies.

The pulse length of the e-beam is limited by diode closure, in which the plasma cloud created at the cathode drifts across the diode at $\sim 2 \times 10^{6} \mathrm{~cm} / \mathrm{s}$. The pulse duration permitted is a roughly linear function of the beam current. Beams of $15 \mathrm{~A} / \mathrm{cm}^{2}$ have been routinely sustained for pulses in excess of a microsecond before closure. Careful design of the diode and the pulseforming circuit is necessary to ensure that the e-beam current is reasonably constant in time, though it grows as the diode closes. A well-designed ballast circuit is also necessary to take the power load once the diode has closed.

Energy deposition by the beam must be spatially as well as temporally uniform, especially if efficient use is to be made of the laser medium. There are two important sources of spatial nonuniformities: nonlinear deposition of energy as the electrons travel through the gas, and selfpinching of the e-beam because of its high currents.

Nonlinearities along the beam can be effectively eliminated by two-sided pumping. For example, onesided pumping to a depth of $1 \mathrm{~m}$ by a $700 \mathrm{keV}$ electron beam in $2 \mathrm{~atm}$ of argon will leave most of the energy deposited in the $30-60 \mathrm{~cm}$ region, with deposition falling off sharply toward the far wall. But fixing this by raising the beam voltage will waste significant energy in electrons which travel completely across. Pumping at this voltage with beams from both sides produces a nearly flat profile in the longitudinal direction of the beam. ${ }^{44}$

Self-pinching arising from the beam's own magnetic field could introduce serious deposition nonuniformities. Experiments have demonstrated that applied magnetic fields in the range $0.1-1.0 \mathrm{~T}$ can prevent beam pinching and produce highly uniform energy depositions.

Foil heating may pose another limitation to excimer laser performance in the future. ${ }^{65}$ The single-shot e-beam pumped experiments thus far conducted are well below foil-heating limits. Similarly, e-beam sustained discharge lasers have operated at high rep-rates with low currents, well within heating limits. For high-average power excimers, foil heating requires active cooling techniques. While these are currently under test and development the techniques appear well within the state of engineering art.

Another historical difficulty in excimer DEW development has been the effects of flow and acoustics on output beam quality. Several years ago, it was believed necessary to incorporate massive flow systems into the laser to achieve values of $\Delta \rho / \rho$ of better than $10^{-4}$; otherwise beam quality from the amplifier would decrease markedly. This problem has essentially been solved by a completely different approach (see below).

The dominant device issues are of systems architecture: how to combine the single apertures efficiently into large beams.

\subsubsection{Raman Conversion and Beam Combination}

It has long been recognized that Raman scattering in appropriately chosen media could be useful for Stokes shifting the wavelength of UV lasers slightly to the red. In inertial confinement fusion, Raman cells have been extensively studied as a means of pulse length compression. $^{66}$ In directed energy weapons research, Raman scattering has been studied both as a means of shifting wavelength and as a means of combining many large-aperture, nonuniform beams into single, high-quality beams. A further important advantage of Raman conversion is that it provides an easy means of lengthening the pulse: The amplifiers pumping the Raman medium can be staged sequentially to provide a pulse length which is the sum of their individual pulse lengths.

A final significant advantage of Raman beam combination is that it fundamentally changes the character of the flow and acoustics problems. The extreme values of $\Delta \rho / \rho$ which had formerly applied to an e-beam pumped gas are no longer required when the beam quality is provided by the Raman cell rather than the excimer amplifier itself. The EMRLD program has demonstrated the clearing technology necessary to sustain $100 \mathrm{~Hz}$ operation in high-power excimers.

There are two disparate approaches to Raman beam combination: the collinear case, in which both Raman pump and Stokes waves are copropagating; and the cross-beam case, in which the pump beam enters the Raman medium at a relatively large angle (a few degrees or more) to the direction of propagation for the Stokes beam.

The collinear case, which was the first one extensively studied, has the advantages of using a high forward gain, and of being essentially independent of the 
linewidth of the pump beam. But, unfortunately, in this case a highly uniform pump intensity is required; even phase distortions of sufficiently short scale length can produce serious intensity distortions over the finite length of the amplifier. The broad-band pump decouples the phase of the pump beam and the intensity of the Stokes beam only if the pump and Stokes modes are spectrally correlated across the aperture. This occurs naturally in the case of Stokes buildup from spontaneous emission, but it is more difficult to satisfy this condition experimentally when the Raman cell is used not merely as a converter but as a Raman amplifier.

The basic problem with the collinear method is that intensity nonuniformities in the pump beam map directly onto the Stokes beam. Phase aberrations have also been observed to convert to intensity variations (and vice versa) during propagation, leading to Stokes nonuniformities after conversion. Finally, since the pump beams must be high quality, beam combination by the collinear method requires high-quality optics to be exposed to high fluences throughout the system. Experiments demonstrating onaxis conversion and beam cleanup have been performed by Northrop and the Naval Research Laboratory.

The off-axis or cross-beam case has the advantage that beam quality in the Stokes beam can be better than in the pump beams and a number of pump beams can be combined in a single Raman converter cell. ${ }^{62 a}$ Thus, phase and intensity noise can be disposed of by supplying a high-quality injected Stokes beam, and beam combination can be performed simultaneously with cleanup. The low optical quality pump beams can be transmitted into the Raman cell with low quality reflective surfaces and windows. The disadvantage of this case is that the linewidth of the pump lasers must be narrower than the linewidth of the Raman scattering medium. ${ }^{67}$ The standard Raman medium for excimers has become $\mathrm{H}_{2}$, which provides efficient Raman conversion, shifting $\mathrm{XeCl}$ from $308 \mathrm{~nm}$ to $353 \mathrm{~nm}$. For moderate pressure, room temperature $\mathrm{H}_{2}$, the bandwidth for Raman scattering is about $300 \mathrm{MHz}$; consequently, the pump beam(s) must be narrower than that.

Extensive experiments on cross-beam conversion have been conducted with both $\mathrm{KrF}$ and $\mathrm{XeCl}$. Experiments with $\mathrm{KrF}$ conducted at Western Research Corporation have been described in detail. ${ }^{67}$ The method used to produce the narrow band pump was injection locking. A flashlamp-pumped, doubled dye laser was used as the injection source to control the output bandwidth of the KrF pump laser, which is thus itself effectively an amplifier of the injected beam.

If the frequency of the injected radiation is not at the peak of the gain, there exists excess gain at frequencies outside the injected bandwidth, and the injection locking will last for only a brief period of time. If the lasing transition is inhomogeneously broadened, this is also true of the peak gain. $\mathrm{KrF}$ is the easiest candidate for injection locking because of its unbound ground state; the gain distribution for $\mathrm{KrF}$ is a true continuum and provides a medium with homogeneous broadening.

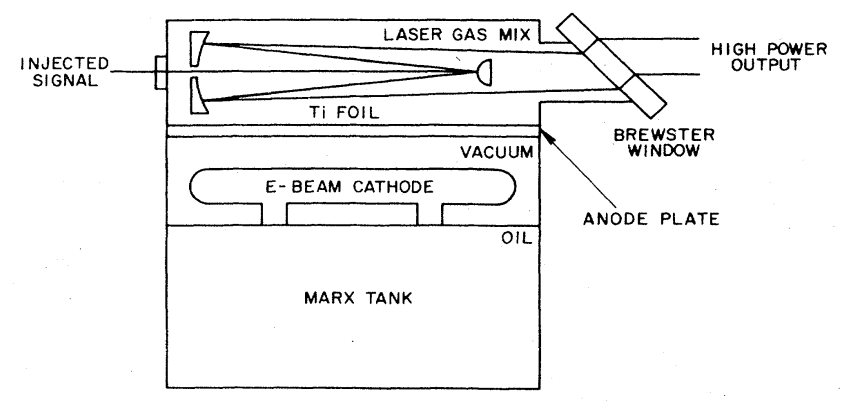

Figure 3.20. Schematic of injection-locked excimer laser.

Figure 3.20 shows a schematic of the injection-locked laser. The pulse length was varied from $0.5-1 \mu \mathrm{s}$. With no injected radiation, the $\mathrm{KrF}$ laser operated with approximately $3 \AA$ bandwidth and was unpolarized. The injected radiation was linearly polarized, and the degree of polarization directly related to the fractional control of the $\mathrm{KrF}$ laser by the injected frequency. All of the radiation which was narrow band and controlled by injection should be polarized parallel to the injected radiation; any broad band emission should be unpolarized. Measurements of the polarization demonstrated that about $80 \%$ of the radiation was locked with a bandwidth less than $50 \mathrm{GHz}$.

The injection-locked radiation was then used to demonstrate aperture combination; the pump beam was split into two equal beams and used to pump the Raman cell simultaneously from different directions. Multiple pump laser amplifiers can attain the necessary spectral correlation simply by injection locking all the pumps with a single master oscillator. Figure 3.21 shows a schematic of the experimental layout. The beams were directed into the $\mathrm{H}_{2}$ cell through Brewster windows at an included angle of 15 degrees, crossing in the center of the cavity formed by the unstable resonator. Threshold $\mathrm{H}_{2}$ pressure was 6 atm. Typical pulse lengths from the stimulated Raman laser were $200 \mathrm{~ns}$ for pump pulses of $300 \mathrm{~ns}$; no stimulated Raman lasing was detected without injection. Improvement in the beam quality by more than a factor of 100 was observed.

Further experiments have demonstrated efficient beam combination and cleanup with injection-locked $\mathrm{XeCl}$ near $\mathrm{kJ}$ pump level.

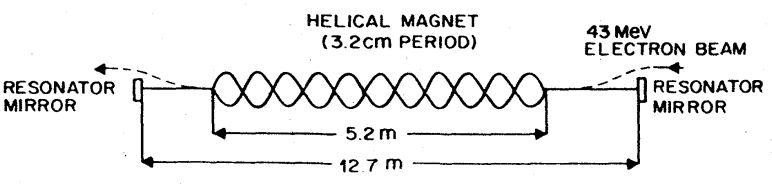

Figure 3.21. The layout of the Stanford $3.4 \mu \mathrm{m}$ FEL oscillator. 


\subsubsection{Beam Cleanup Using Stimulated Brillouin Scattering}

It is possible in principle to improve the mode structure of a high-power laser device by using a phaseconjugate mirror at one end of the optical resonator structure. As described in Section 5.4.7, stimulated backward Brillouin scattering in a fluid could conceivably be used for this purpose. A low-power diffraction-limited input signal enters the excimer laser gain medium from the left. The signal is amplified as it travels to the right, but also suffers phase distortion. The backward Brillouin scattered light travels to the left. On this second traversal, the beam is further amplified, but the phase distortion is compensated and a diffraction-limited high-power beam exits to the left. The principle of this scheme has been demonstrated in high-power Nd-glass lasers. It is an open question whether this scheme can be useful for high-power excimer lasers.

\subsubsection{Critical Issues}

The scaling and efficiency limitations of the excimers are reasonably well understood, and seem appropriate to the construction of devices on the scale required by BMD applications. The critical issues for large excimer devices (exclusive of requirements for adaptive optics, to be discussed later) are those alluded to above:

1. Further development of cost-effective e-beam technology capable of repetitive pulses, high efficiency, and durability.

2. Demonstration of beam combination and cleanup at full power and with high efficiency.

3. Efficient operation of an architecture which effectively combines the outputs from hundreds of individual pump amplifiers, while remaining below optical damage limits, to demonstrate multi-MJ energy levels.

\subsection{FREE ELECTRON LASERS}

\subsubsection{Principles of Operation and Recent Results}

The free electron laser (FEL) is a device which amplifies short wavelength radiation by stimulated emission, using the energy of an electron beam. The conversion of electron kinetic energy into radiation is caused by a magnetostatic device known as a "wiggler" or "undulator," wherein the electron is caused to oscillate periodically by a spatially periodic, transverse magnetic field. The undulator can be a helical field (period $1_{0}$ ) or on the other hand, it can be set up by an array of alternating-polarity magnets. The radiation, circularly polarized in the former, and linearly polarized in the latter, is twice Doppler shifted by the relativistic factor $\gamma_{\|}^{2}=\left(1-v_{\|}^{2} / \mathrm{c}^{2}\right)^{-1}$, where $\mathrm{v}_{\|}$is the component of electron velocity along the undulator axis; the wavelength of spontaneous radiation is

$$
\lambda_{\mathrm{s}} \approx 1_{0} / 2 \gamma_{\|}^{2}
$$

in the direction collinear with the electron momentum.

Spontaneous radiation from such a device was observed by Motz ${ }^{68}$ (1951) and indeed a slightlyrelativistic microwave version, the "Ubitron," was operated successfully by Phillips ${ }^{69}$ (1960).

In 1976-7, a group at Stanford under Madey (Elias et al., ${ }^{9}$ 1976; Deacon et al., ${ }^{70}$ 1977) successfully demonstrated amplification at $10.6 \mu \mathrm{m}$ and then configured the system as a laser oscillator (Figure 3.21) at $3.4 \mu \mathrm{m}$, using the Stanford superconducting accelerator. A period of theoretical understanding followed, in which it was appreciated that the FEL is essentially a classical device, and can be understood as a traveling-wave amplifier (Kroll and McMullin, ${ }^{71}$ 1978). Amplification proceeds by an electron bunching process, caused by an axial, nonlinear, ponderomotive force, which is set up by the interaction of the transverse component of electron motion (induced by the undulator) with the amplitude of the magnetic component of scattered field.

A limiting case-high $\gamma$, low current-was successfully modeled by Colson ${ }^{72}$ (1977); the electron dynamics were described by the pendulum equation coupled to the self-consistent EM field equations. Here amplification occurs from an interference effect made possible by the finite-length undulator. The gain-unlike an atomic laser-does not follow the spontaneous emission line function, but is rather related to the derivative of it (Figure 3.22). Electrons traveling slightly faster than the "resonant" energy $\gamma_{\mathrm{s}}$ will do work on the

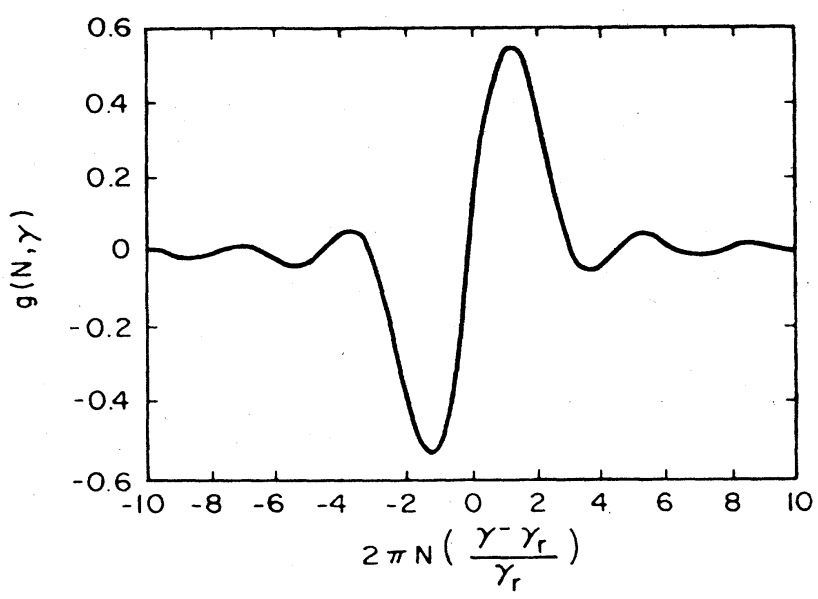

Figure 3.22. The linear gain curve for an FEL. 
growing ponderomotive wave and enhance the radiation field. Electrons moving slower than the resonance energy [defined by $\gamma_{\|}$in Equation (3.26)] will extract energy from the ponderomotive wave. Thus, by changing either $\lambda_{s}$ or $\gamma_{\|}$, the FEL can be either a laser or an electron accelerator. This model was adequate to describe the initial FEL device.

\subsubsection{Principles}

It was not until the early part of this decade that extensive experimentation with the FEL began (the reader wishing a systematic account of the FEL is referred to a recent book by Marshall ${ }^{73}$ ). Well before then, it was noted that the efficiency of a low gain FEL should be $\sim 1 / 2 \mathrm{~N}$, where $\mathrm{N}$ is the number of undulator periods. As the FEL gain increases with $\mathrm{N}$, it follows for practical reasons that $\sim 1 / 2 \%$ efficiency is to be expected. A notable feature of the FEL is tunability: e.g., (1) shows that $\lambda_{\mathrm{s}} \sim 1 \mu \mathrm{m}$ will result from a $100 \mathrm{MeV}$ beam passing down an $1_{0} \sim 3 \mathrm{~cm}$ period undulator. This also has an unpleasant side: the energy of the electron beam must be held to a close tolerance $(<1 / \mathrm{N})$ so that the wavelength to be amplified in the optical resonator always falls within the gain-bandwidth of the device. There also follows a requirement on electron beam "quality": an excessive spread of velocities $\left(\delta V_{\|}\right)$within the beam will degrade the FEL gain. Preservation of FEL gain requires that the fractional "Doppler" spread of electron parallel momenta in the beam:

$$
(\delta \gamma / \gamma)_{\|} \lesssim 1 / \mathrm{N}
$$

where $(\delta \gamma / \gamma)_{\|}=\gamma^{2}\left(\delta \mathrm{V}_{\|} / \mathrm{c}\right) ; \quad \mathrm{N}$ is the number of undulator periods, $\mathrm{N}=\mathrm{L} / 1_{0}$.

It was noted early in FEL theoretical work (see, for example, Kroll et al. ${ }^{74}$ ) that FEL efficiency could be changed by "tapering" the undulator, that is, by an adiabatic change of the undulator period and/or amplitude along the axis. This may be seen by recasting Equation (3.26) using the relation $\gamma^{2}=\left(1-\mathrm{v}_{\|}^{2} /\right.$ $\left.c^{2}-v^{2} / c^{2}\right)^{-1}$. The transverse equation of motion gives $\mathrm{v}_{\perp} / \mathrm{c} \approx \mathrm{a}_{\mathrm{w}} / \gamma$ where $\mathrm{a}_{\mathrm{w}}=\mathrm{eB} / \mathrm{k}_{0} \mathrm{mc}^{2}, \mathrm{k}_{0}=2 \pi / l_{0}$ in cgs units. Thus Equation (3.26) becomes

$$
\lambda_{\mathrm{s}} \approx \frac{1_{0}}{2 \gamma^{2}}\left(1+\overline{\mathrm{a}_{\mathrm{w}}^{2}}\right)
$$

As electron kinetic energy decreases along the undulator due to radiation, $\gamma$ must fall. We can maintain gain by requiring that $\lambda_{\mathrm{s}}$ be a "constant of the motion." Then it follows that $1_{0}$ and/or $a_{w}$ should change axially. A more thorough study shows that as appreciable energy is extracted from the beam, the amplitude of the ponderomotive wave grows large enough to entrain (or "trap") the beam electrons. Changing $1_{0}$ also changes the speed of the ponderomotive wave, so that it maintains synchronism with the electrons; in this way large amounts of energy may be extracted.

The dynamics of an electron in an FEL can be understood in terms of phase slippage between the electron motion and the optical wave $\left(\omega_{\mathrm{s}}=\mathrm{k}_{\mathrm{s}} \mathrm{c}\right)$ and the change in the electron energy due to the interaction. The phase slippage is

$$
\Psi=\left(\mathrm{k}_{0}+\mathrm{k}_{\mathrm{s}}\right) \mathrm{z}-\omega_{\mathrm{s}} \mathrm{t}+\phi
$$

where $\phi$ is the optical phase. Equation (3.29) can be put in the following form in which we relate the phase shift to the difference between the electron energy and the "resonant energy" $\gamma_{\mathrm{r}}, \Delta \gamma \equiv \gamma-\gamma_{\mathrm{r}}$ :

$$
\frac{\mathrm{d} \Psi}{\mathrm{dz}}=2 \mathrm{k}_{0}(\Delta \gamma / \gamma)
$$

where $\gamma_{\mathrm{r}}$ is the energy of a "resonant electron" specified by Equation (3.28) with $\gamma$ replaced by $\gamma_{\mathrm{r}}$. If the electron has nonresonant energy, it can exchange energy with the optical wave by means of $\mathbf{j} \cdot \mathbf{E}_{\mathrm{s}}$; one finds

$$
\frac{\mathrm{d}}{\mathrm{dz}}(\Delta \gamma)=-\left(\mathrm{k}_{\mathrm{s}} \mathrm{a}_{\mathrm{s}} \mathrm{a}_{\mathrm{w}} / \gamma_{\mathrm{r}}\right)\left[\sin \Psi-\sin \Psi_{\mathrm{r}}\right]
$$

where $\Psi_{\mathrm{r}}$ is the resonant phase and $\mathrm{a}_{\mathrm{s}}=\left(\mathrm{eE} \mathrm{E}_{\mathrm{s}} / \mathrm{k}_{\mathrm{s}} \mathrm{mc}^{2}\right)$. Combining Equations (3.30) and (3.31) one obtains a pendulum equation

$$
\frac{\mathrm{d}^{2} \Psi}{\mathrm{dz}^{2}}=-\Omega_{\mathrm{s}}^{2}\left[\sin \Psi-\sin \Psi_{\mathrm{r}}\right]
$$

In Equation (3.32), $\sin \Psi_{\mathrm{r}}$, the "constant torque" term, depends on the undulator taper $\Delta 1_{0} / 1_{0}$. The characteristic distance for small amplitude electron oscillations in the ponderomotive potential well is $L_{s}=2 \pi / \Omega_{s}=l_{0} / \sqrt{a_{s} a_{w}}$. Periodic motion of the electron in these wells is referred to as the "synchroton oscillation." One can study the dynamics of electrons in the FEL using Equation (3.32) by injecting an ensemble of randomly phased electrons into the undulator, and then calculating the ensemble-averaged energy at the end of the undulator as the electrons interact with a specified optical field. It is found that net gain results if the electron energy is greater than the resonant energy. If the current is small and the energy is high, the gain scales unfavorably as $\lambda_{\mathrm{s}}^{3 / 2}$, but in the near infrared and visible region, gain is adequate to sustain oscillation if the current is at least $\sim 1 \mathrm{~A}$.

A tapered undulator is designed by an appropriate choice of the "resonant phase," $\Psi_{\mathrm{r}}$. In practice, one tries to optimize both the fraction of electrons trapped and the energy extracted from this group; usually the efficiency can be enhanced an order of magnitude by this 
procedure. The design is usually best suited to the amplifier mode of FEL operation, i.e., the case in which the signal amplitude is specified as a boundary condition at the undulator input. However, tapering the undulator "smears out" the gain curve, causing a loss of small-signal gain. For this reason, undulators are usually "hybrids," consisting of a section with constant period, followed by a zone where $1_{0}$ or $a_{w}$ changes. In this manner, gain and efficiency are optimized.

The electron dynamics, described by the pendulum equation (3.32), must self-consistently include the energy loss or gain from the optical fields. The electron current, bunched by the dynamics given by Equation (3.32), interacts with the optical wave over many cycles, so that a slow variation of amplitude $a_{s}$ and phase $\phi$ along the undulator can be assumed:

$$
\dot{\mathrm{a}}_{\mathrm{s}}=\mathrm{A}\left\langle\frac{\sin \Psi}{\gamma}\right\rangle, \quad \mathrm{a}_{\mathrm{s}} \dot{\phi}=\mathrm{A}\left\langle\frac{\cos \Psi}{\gamma}\right\rangle,
$$

where the average is an ensemble average taken over all electrons, initially injected into the undulator with random phases; $\mathrm{A}$ is a constant involving the system parameters, and the overdot indicates $\mathrm{d} / \mathrm{d} \hat{\mathrm{t}}$ where $\hat{\mathrm{t}} \equiv \mathrm{t} /(\mathrm{L} / \beta \mathrm{c})$. In the course of bunching, the right-hand side of Equation (3.33) develops a nonzero average. Another way of looking at Equation (3.33) is to describe the electrodynamics in terms of a nonlinear, complex index of refraction: the imaginary part causes wave growth, while the real part is responsible for phase modification. A careful look at this formulation shows that it is possible for the real part of the nonlinear refractive index of the electron beam in the FEL to cause a mild focusing of the radiation being amplified. The FEL electron beam therefore resembles a fiber light guide. This has important consequences, as we shall see.

Another "limiting case" is that of high beam current and low $\gamma$, applicable to early "Raman" experiments. ${ }^{75}$ In this case the growth of the traveling optical wave proceeds in an exponential fashion. Unlike the case of high $\gamma$ and low density (customarily referred to as the "Compton" FEL), where the gain is linear in beam density or current, the Raman growth scales as $n^{1 / 4}$ or $I^{1 / 4}$. This shows the gain process is no longer "single-particle" in nature, but rather "collective." The high space charge of the beam permits the excitation of the beam space charge wave, and the FEL gain process can be understood as a convective three-wave parametric process. This applies so long as $\left(\omega_{\mathrm{p}} \mathrm{L} / \gamma \mathrm{c}\right) \gg 1$ [where $\omega_{\mathrm{p}^{2}}=\left(4 \pi \mathrm{n}_{\mathrm{e}}^{2}\right) /(\gamma \mathrm{m})$ is the plasma frequency], and indeed the Raman efficiency exceeds the Compton efficiency by this factor. This inequality shows that there is an upper limit on beam $\gamma$ (or a lower limit on $\lambda_{s}$ ) for the high gain Raman process: this is typically $\sim 100 \mu \mathrm{m}$. Thus, Raman FELs are microwave or millimeter/far infrared devices. The Raman process involves a "weak" undulator field.

Exponential gain has advantages, and it is fortunate that it can be recovered for conditions appropriate to short-wavelength operation. If the undulator field is very strong and the beam density is sufficiently high, exponential growth occurs again in the traveling wave theory in the "strong pump" limit. ${ }^{76}$ It has also been obtained in the context of the pendulum equation formulation, ${ }^{77}$ and further modified by Scharlemann et $a l .{ }^{78}$ to include both diffractive and self-focusing effects. This, together with $2 \mathrm{D}$ computations, has shown how small signals may grow to high amplitude along long undulators, many Rayleigh-ranges long (the focusing compensates for diffraction: Figure 3.23). This has important implications for FEL research, both at high power as well as at short wavelength (e.g., VUV). Once again, the small-signal gain is nonlinear in beam current, showing that the process is a "collective" instability (in this case, a beneficial one).

The FEL can be operated as an oscillator or an amplifier. In either case, the design must incorporate details which depend on the electron accelerator. Experimentation with short wavelength FEL oscillators has utilized the $\mathrm{rf}$ linac. As the electron current pulses are very short $(3-10 \mathrm{ps})$, the optical resonator length must be optimized very carefully with respect to the parameter $\mathrm{L}_{\mathrm{c}}-\mathrm{L}_{\mathrm{p}} / 2 \beta$, where $\mathrm{L}_{\mathrm{c}}$ is the cavity length and $L_{p}$ is the spacing of the current pulses. To optimize the FEL with respect to walkoff of the circulating optical pulse from the current pulse, an adjustment of the resonator length in dimension $\sim 10 \mu \mathrm{m}$ is required out of a total length of several meters. In the case of the highpower amplifier, the optical beam must be accurately centered on the electron beam over a distance of many meters.

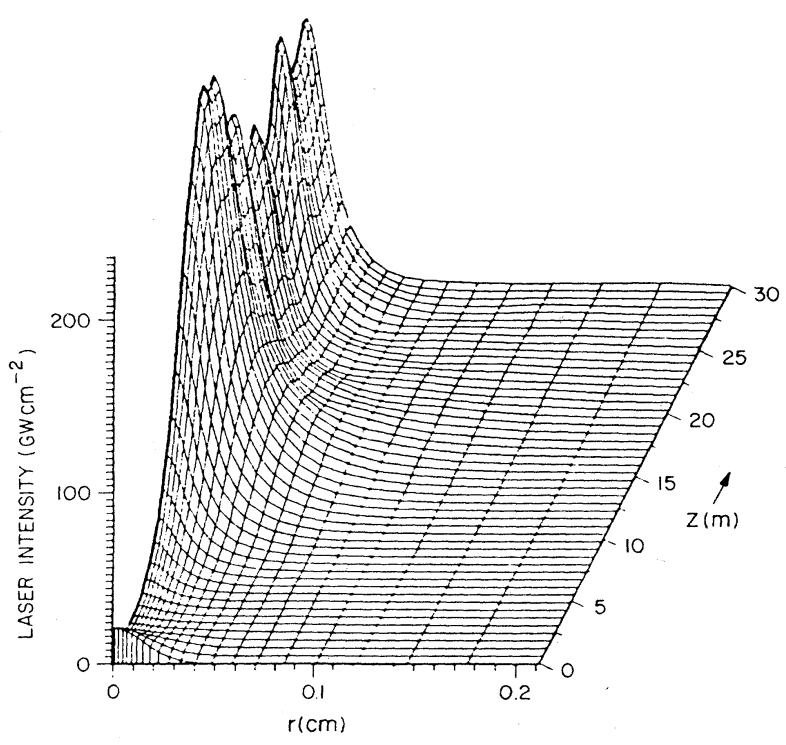

Figure 3.23. Optical guiding in an FEL: beam current is 270 $\mathrm{A}$, the beam radius is $0.01 \mathrm{~cm}$, the light is at $12.5 \mu \mathrm{m}$, and the initial laser intensity is 30 MW. The light remains in the beam for more than 60 Rayleigh lengths (from Reference 78). 
Oscillators must include mirrors for feedback, whereas an amplifier requires a coherent source (laser) for the input. The oscillator mirrors must accommodate high power without damage; the design is driven to a long resonator, complicated mirror system, and tight tolerances. However, an amplifier requires an optical telescope at the output, and to spread the optical beam diffractively from the millimeter-size diameter electron beam to an aperture of several meters requires an evacuated pipe several $\mathrm{km}$ in length.

FEL technology is a category of electron accelerator technology. This is familiar to most physicists, and it should not be surprising that FEL physics evolved rapidly once these connections were properly appreciated. The features of the traveling wave amplifier (convective instability, unidirectional growth) are also familiar to specialists in electron tube technology. Overall FEL efficiency may be improved by electron beam energy recovery using a variety of techniques after the beam has left the undulator. Considering the power of relativistic electron beam systems, it was inevitable that interest in powerful, efficient FELs would blossom.

\subsubsection{Recent Experiments}

While megawatt pulsed Raman FELs were demonstrated in 1978, high-power short wavelength FEL demonstrations have required sophisticated hardware and are a more recent phenomenon. Typical of these is the work of the group at LANL on a tapered-undulator FEL amplifier $^{79}$ at $10.6 \mu \mathrm{m}$ and an oscillator configuration. ${ }^{80}$ Figure 3.24 shows a graph of the extraction efficiency versus electron energy for the $10.6 \mu \mathrm{m}$ amplifier. Not only is the extraction efficiency high $(\sim 4 \%)$ by virtue of the tapered undulator, but the agreement with theory is very satisfactory. A notable result from the oscillator was

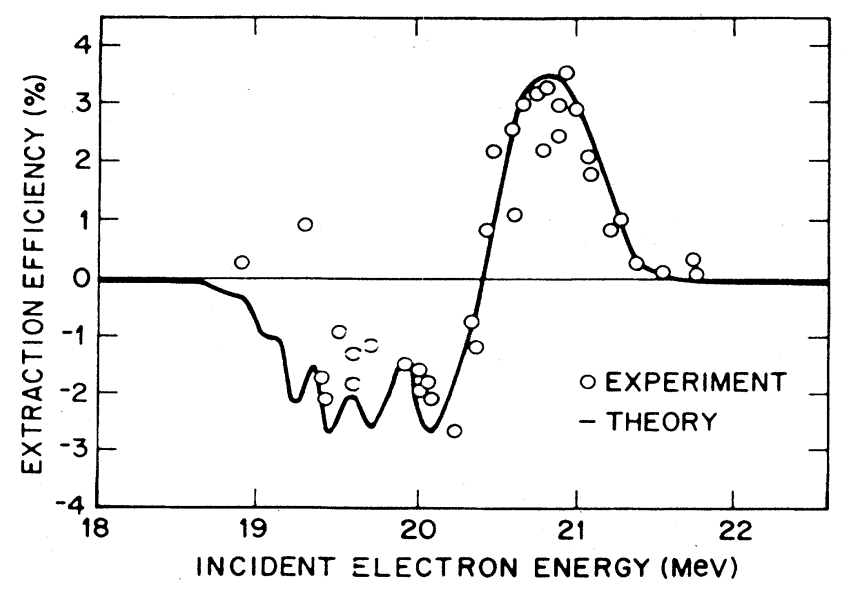

Figure 3.24. Extraction of electron beam energy in the LANL FEL at $10.6 \mu \mathrm{m}$ (from Reference 79).

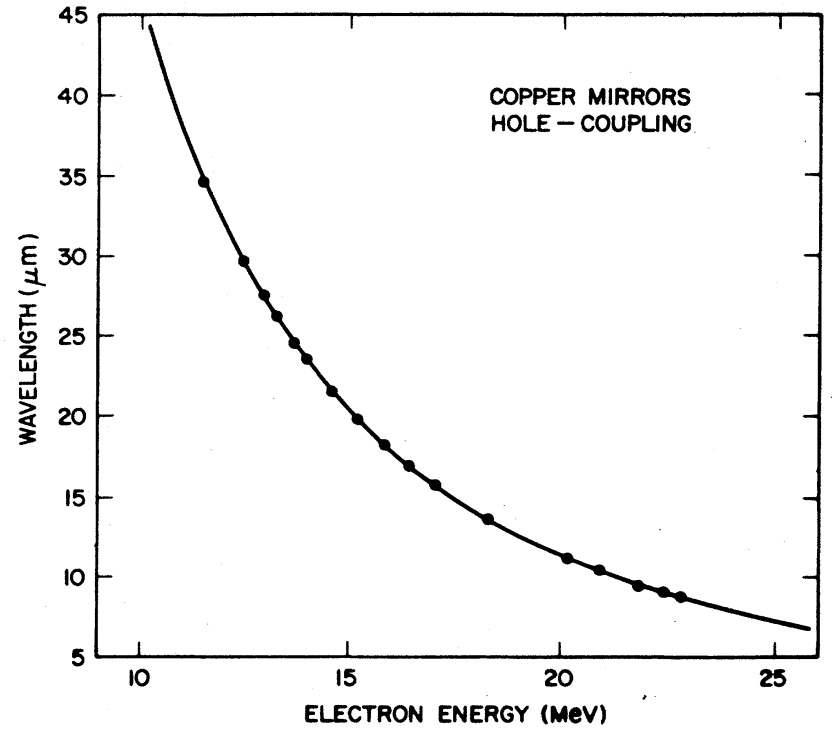

Figure 3.25. Tunability of LANL FEL oscillator.

$10 \mathrm{MW}$ peak power pulses at $7 \%$ output coupling, wideband tunability $(10-35 \mu \mathrm{m})$ (Figure 3.25) and excellent optical quality (Figure 3.26).

A recent experiment ${ }^{81}$ at LLNL has obtained promising results using the ETA facility (500 A, 3.3 MV). Whereas the LANL experiment used an improved rf linac, the Livermore project uses a few modules of a pulsed induction linac, the ETA accelerator. Although configured to amplify $\lambda_{\mathrm{s}} \sim 1 \mathrm{~cm}$ in an overmoded waveguide, the experiment was a test-bed for a much shorter wavelength project also in the strong-pump limit of exponential gain. Figure 3.27 shows the exponential growth of the microwave signal along the undulator, followed by saturation and synchrotron oscillation

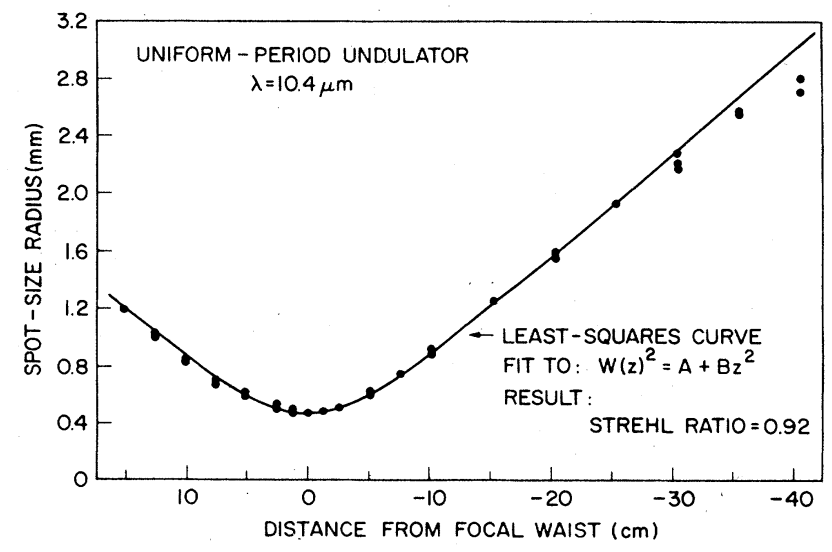

Figure 3.26. Optical beam quality from the LANL FEL oscillator. 


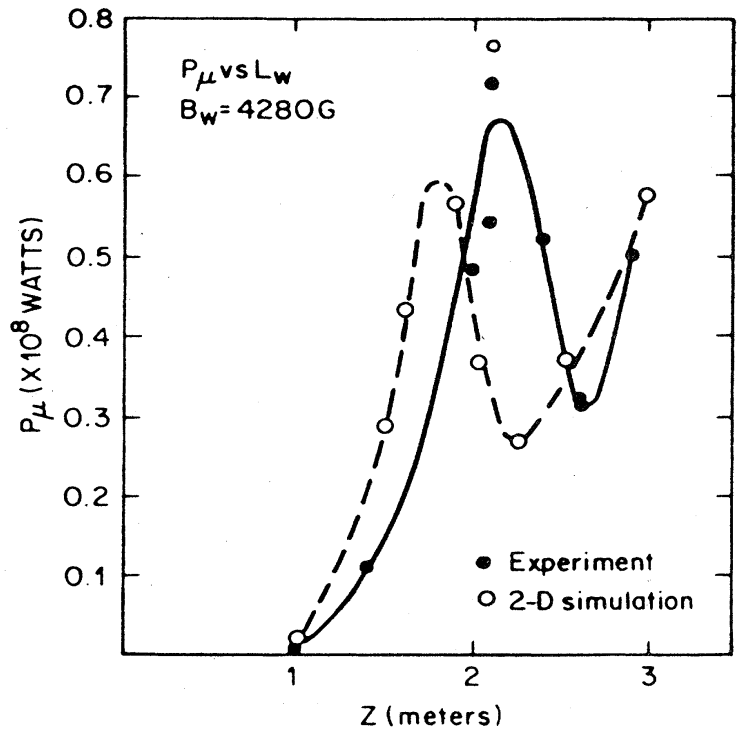

Figure 3.27. Output from the LBL/LLNL FEL at $8.6 \mathrm{~mm}$ as a function of wiggler length.

trapping effects at high power. Gain $\sim 13 \mathrm{db} / \mathrm{m}$ was obtained. More recently, optimization of the undulator has resulted in a high extraction efficiency.

This summary shows that the FEL, because of the desirable properties of the optical medium, i.e., free electrons in vacuo, is promising for ultrahigh power applications (e.g., $100 \mathrm{MW}$ average power, $\lambda_{\mathrm{s}}=1 \mu \mathrm{m}$ ). Several critical issues, involving possible instabilities and beam propagation problems, must still be resolved before the FEL approach can be considered as a well-established high-power laser option. These interesting issues involving both basic physics and technology, are discussed in the following section.

\subsubsection{Vital Issues}

\subsubsection{Beam Quality}

A monoenergetic electron beam is required for optimum FEL performance; as mentioned previously, gain and efficiency are degraded by a thermalized beam. Thus, it is not possible, for example, to recirculate the electrons repeatedly through the undulator in order to extract additional energy. A poor quality beam causes the gain line shape to be inhomogeneously broadened. At high energy, the principal contribution arises from the emittance of the beam. Before entering the undulator, the beam has an intrinsic transverse component of motion, $\mathrm{V}_{\mathrm{x}}$, due to finite cathode temperature, nonideal electron optics, etc. The divergence, $V_{x} / V_{\|}$, is expressed in terms of an "emittance," $\epsilon_{\mathrm{x}} / \mathrm{x}$, where $\mathrm{x}$ is the transverse dimension. Even if the electrons are monoenergetic, a spread of transverse velocities therefore results in a spread of longitudinal velocities. In an "ideal" accelerator, the normalized emittance

$$
\epsilon_{\mathrm{N}}=\gamma \beta \epsilon
$$

is a constant through the acceleration. The parallel momentum spread is then

$$
(\delta \gamma / \gamma)_{\|}=\frac{1}{2}\left(\epsilon_{\mathrm{N}} / \mathrm{r}_{\mathrm{b}}\right)^{2}\left(1+\mathrm{a}_{\mathrm{w}}^{2}\right)^{-1}
$$

The emittance determines the beam brightness:

$$
\mathrm{B}_{\epsilon} \equiv 2 \mathrm{I}_{\mathrm{b}} / \epsilon_{\mathrm{N}}^{2}
$$

"Bright" electron beams are directly related to the "bright" optical beams that emerge from the FEL. From Equation (3.36), it follows that a typical, "acceptable" emittance for FEL operation is $\epsilon_{\mathrm{N}}<30 \pi \mathrm{mm}$ mrad for the wavelengths in question. Figure 3.28 shows how the emittance affects the gain of the proposed $0.5 \mu \mathrm{m}$ FEL oscillator system under construction at Boeing/Spectra Technology (B/STI). If the gain drops too low, the oscillator may fail to start because of low accelerator duty cycle.

Figure 3.29 shows several systems for which brightness has been measured. There has been considerable effort devoted both to measuring as well as improving beam brightness, and the results have shown much improvement. Based on past experience and present numerical computations the FEL community believes that it is clear (but not easy or inexpensive) how to generate electron beams having the correct current, energy, and quality for FEL operation at visible wavelengths. This has required the design of new

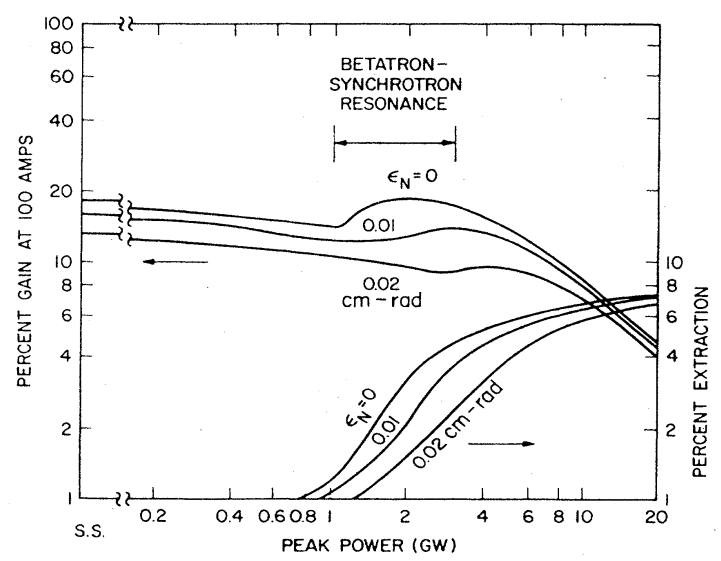

Figure 3.28. Gain and extraction dependence on the beam emittance for the Boeing/STI $0.5 \mu \mathrm{m}$ FEL (Reference 87). 


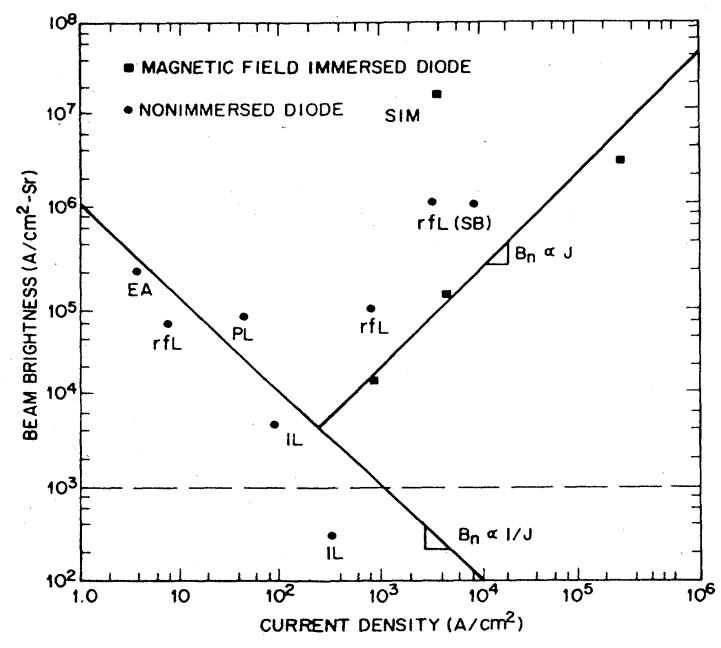

Figure 3.29. Achieved beam brightness versus beam current [C. W. Roberson, IEEE J. Quantum Electron. QE-21, 810 (1985)].

sources, electron guns, and beam transport systems suitable for beam current in the range of $100 \mathrm{~A}-1000 \mathrm{~A}$. The RF linacs have achieved close to required beam brightness. ${ }^{82}$ The induction linacs have yet to demonstrate the requisite brightness. However, an injector has achieved the needed brightness on the Livermore test stand but this has not yet been connected to an accelerator.

\subsubsection{Beam Brightness}

There has been, through the years, great effort on obtaining very bright electron beams. For FEL amplifiers the need is severe, and whether or not an FEL can be operated efficiently is a strong function of beam brightness. Motivated by this need Barletta et al. ${ }^{83}$ have made a careful study of sources of beam emittance.

Beam brightness is determined by design of the electron gun, choice of the cathode type, and by the matching out of the gun. More fundamentally, the emittance $\epsilon$ of relativistic electron gun is determined by (1) source temperature, (2) source uniformity, (3) magnetic field normal to the cathode, (4) beam filamentation, (5) nonlinear applied forces, (6) nonlinear space charge forces, and (7) multiplicity or motion of emissive surfaces.

Source Temperature: The normalized source brightness, $B$, is related to the temperature $T(e V)$ and source emissivity $\mathbf{J}\left(\mathrm{A} / \mathrm{cm}^{2}\right)$ by

$$
\mathrm{B} \simeq 1.6 \times 10^{5} \mathrm{~J} / \mathrm{T}
$$

In existing high current guns, this contribution is insignificant in comparison to other contributors to emittance. However, as we push brightnesses toward
$10^{6} \mathrm{~A} / \mathrm{cm}^{2} \mathrm{rad}^{2}$, maintaining nonemission limited sources with an effective temperature below $1 \mathrm{eV}$ will be critical for cathode types with emissivity less than $10 \mathrm{~A} / \mathrm{cm}^{2}$.

Source Uniformity: To the extent that emission is nonuniform, the beam transport through regions dominated by nonlinear radiation forces will entrain "phase space vacuum" via phase mixing. This dilution can become irreversible after one-quarter of a betatron wavelength. Maintaining source uniformity argues in favor of small area cathodes with high emissivity.

Normal Magnetic Field: In the presence of a magnetic field normal to the cathode surface, electrons are emitted with a finite canonical angular momentum $\mathbf{P}_{\theta}$. When the electrons leave the region of axial field, they acquire a kinetic momentum sufficient to keep their canonical angular momentum constant. This motion gives the beam an equivalent emittance in both transverse planes: $\epsilon=\mathrm{eB}_{\mathrm{z}} \mathrm{R}^{2} / 2 \gamma \beta \mathrm{mc}^{2}$, where $\mathrm{B}_{\mathrm{z}}$ is the mean normal field, $\mathbf{R}$ is the cathode radius, $\gamma \beta$ are the usual relativistic factors, $\mathrm{m}$ is the electron mass, and $\mathrm{c}$ is the speed of light. Reducing the cathode radius reduces this contribution.

Filamentation by a Grid: In gun designs with a grid, each hole in the grid can act as a focusing or defocusing lens with a focal length given by

$$
1 / \mathrm{f}=\mathrm{e}(\Delta \mathrm{E}) / 2 \gamma \beta^{2} \mathrm{mc}^{2}
$$

where $\Delta \mathrm{E}$ is the voltage difference across the grid. The phase space is distorted by the finite grid spacing, occurring via filamentation downstream of the grid. The most prudent choice in the design of a high brightness gun is to seek a design in which the grid has been omitted.

Nonlinear Applied Forces: The radial forces from the applied electric and magnetic fields in most injectors have significant anharmonic components (proportional to $\mathrm{R}^{3}$ ). In general, these effects can be made to compensate for each other by careful design.

Nonlinear Space-Charge Forces: The strong space-charge forces of an intense, low energy beam will distort the free space equipotentials to result in a defocusing of spherical aberration in the beam transport systems. Proper shaping of the potentials by a graded accelerating column with shaped electrodes (Pierce correlation) can eliminate this effect for a particular operating condition; that is, for a specified operating voltage and beam current. Self-forces can also lead to an increase in emittance whenever the beam cannot be matched into the transport.

Emission from Positions of Different Potentials: Even if the electrons are born with zero intrinsic temperature, the beam can acquire significant emittance in the extraction process if the electrons originate on different 
equipotentials. Such multiple source beams have an instantaneous energy spread which will phase-mix into macroscopic emittance as the beam is accelerated and transported through the space-charge dominated regime.

\subsubsection{FEL Instabilities}

These are of two types: the sideband and synchrotron/betatron instability.

The sideband instability occurs when the radiation field interacts with the longitudinal synchrotron motion of electrons trapped in the ponderomotive wells. This results in sidebands, separated from the FEL carrier by $\delta \omega / \omega \sim 1 / N$. Computer simulations have shown these can grow to high power, and indeed these sidebands have been observed in the LANL FEL oscillator ${ }^{79,80}$ (Figure 3.30). The spectrum involves a modulation of the radiation pulse into shorter pulses, as well as a modulation of the current pulses. The sideband instability appears to have little effect on beam quality experimentally, but it must be suppressed-usually by the introduction of dispersive or dissipative structures in the resonator. This problem is still to be resolved, particularly in the important case of the tapered undulator. Otherwise, powerful radiation may be generated outside the mirror reflectivity bandwidth, with consequent unacceptable damage of optical surfaces.

Betatron (viz., transverse) oscillation of the electrons occurs by virtue of finite emittance $\left(v_{x}\right)$ and the necessary transverse electron focusing. This is not bad, as long as this motion does not couple to the synchrotron oscillations. However, Rosenbluth ${ }^{84}$ has shown that betatron/synchrotron coupling can occur because of the

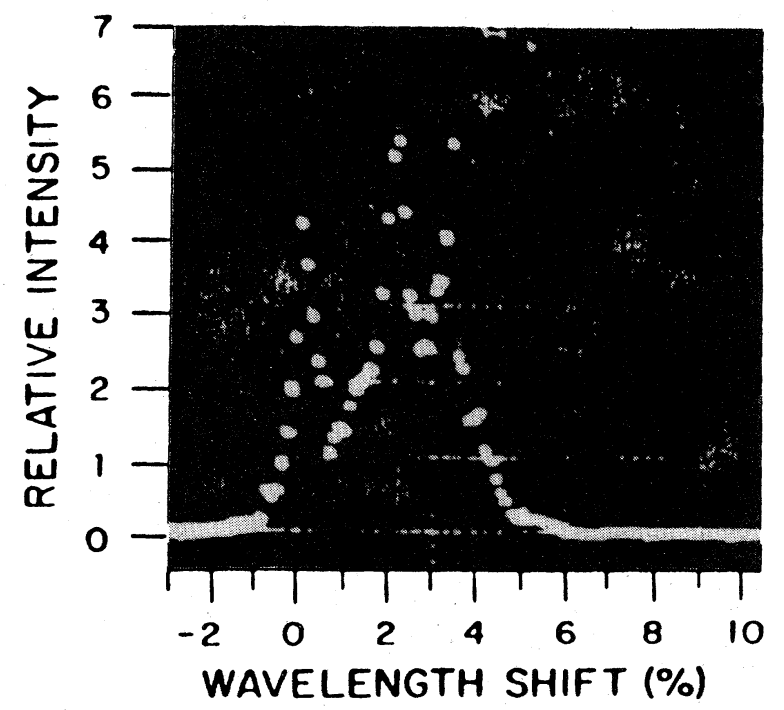

Figure 3.30. Sidebands in the LANL free-electron laser (Reference 80). optical field profile. Should this happen electrons can be detrapped and the FEL efficiency will decrease. However, theoretical calculations by Fawley et al. ${ }^{85}$ indicate this situation only occurs for a very limited range of parameters and, in general, is not a serious detrapping mechanism for high-power FEL amplifiers. Experimental confirmation of these theoretical studies still needs to be done.

\subsubsection{Transport}

The radial profile of the optical pulse, in the FEL, is an important problem in connection with the multipass FEL oscillator. However, for an amplifier such as that being built by LLNL, one needs a very long (several Rayleigh ranges) undulator, so optical guiding is required to occur. This concept is still to be demonstrated experimentally, and it is crucial to this mode of operation. This problem is worrisome because a minor perturbation in beam density or direction can cause a failure of axisymmetry. This may disrupt the system if the optical axis wanders off the beam axis (in the LLNL amplifier, toward the end of the undulator, the optical and electron beams are transporting comparable power). A 3D numerical simulation study of such nonaxisymmetries indicates that they do not occur for the LLNL proposed parameters. Optical quality may not be seriously degraded in this case. However, a full analytic treatment of this subject has not yet been developed, and as yet, there are no experimental results.

An issue relating to electron beam transport is that of "beam break-up." In the rf linac, this results when the beam interacts with an on-axis transverse magnetic field component associated with a natural resonance of the $\mathrm{rf}$ accelerator cavities. This requires detailed rf mode suppression. In the induction linac, heretofore there have been serious problems in transporting a high current density electron beam down a lengthy accelerating structure. This was "cured," through the use of a laser-produced ionized channel in a low pressure gas, which supplies a very strong focusing channel, but this approach spoils the beam quality and is not satisfactory for an FEL.

\subsubsection{Tolerances and Reliability}

In all high-power FELs, the characteristic dimensions and tolerances are pushed to technologically challenging levels. For example, the B/STI oscillator (at $0.5 \mu \mathrm{m}$ ) uses a $55 \mathrm{~m}$ cavity, $300 \mathrm{~kW} / \mathrm{cm}^{2}$ peak mirror loading, and very tight stability tolerance on the cavity $(\sim 500 \mathrm{nrad})$ which required an actively stabilized arrangement. The undulator is a sophisticated affair, having length $5 \mathrm{~m}$, peak field of $8.7 \mathrm{kG}$, and $0.0005^{\prime \prime}$ tolerances on the gap. (this raises the issue of temperature control inside the undulator under 
conditions of high average beam power). The B/STI program has the sensible objective of increasing the FEL average power by increasing the system duty cyclethus the peak micropulse output remains the same. ${ }^{87}$

More ambitious facilities proposed by LANL and LLNL involve either resonator or beam lengths (respectively) of several hundred meters. Operation of such systems is currently beyond state of the art, and require further development of mirror and accelerator technology.

The reliability of the FEL-that is assured operation with minimum maintenance-is an important subject. There has been no experience with totally automated accelerator systems, and the tolerances on many components (including resonator length) are exceedingly strict.

\subsubsection{Harmonics}

Harmonic radiation from the undulator is a feature of FEL operation, and indeed oscillation at harmonics has been observed. ${ }^{88}$ However, radiation of appreciable harmonic power has not been an important factor in recent experiments at LANL or LLNL. In fact, there is less intensity observed than would be theoretically expected and thus this is an effect which is not properly understood at present. In principle, harmonic radiation could be suppressed by dichroic optics and/or gas absorbers. Harmonic oscillation at high-power output would be disaster for high-power optical surfaces.

In summary, ultra-high-power FELs face several key issues. For the rf approach, we estimate that extraction efficiency must be increased from $1.3 \%$ to about $25 \%$, the average accelerated current in the rf linac needs to be increased from $0.3 \mathrm{~A}$ to $4.0 \mathrm{~A}$, and the peak current must be increased from $10 \mathrm{~A}$ to $300 \mathrm{~A}$. In the induction linac approach, brightness must be increased from $5 \times 10^{5} \mathrm{~A} /(\mathrm{rad} \mathrm{cm})^{2}$ to $2 \times 10^{6} \mathrm{~A} /(\mathrm{rad} \mathrm{cm})^{2}$, optical guiding ${ }^{78}$ (i.e., good extraction of beam power and optical quality) must be established, and the propagation through a very long $(100 \mathrm{~m})$ tapered wiggler is necessary. Both approaches could suffer from sideband instabilities and harmonic generation, but not much is known about the relative importance of these phenomena in oscillators and amplifiers.

\subsubsection{Oscillators and Amplifiers}

\subsubsection{Oscillators}

As has been described above, oscillators have been made to operate at a large number of places (Stanford, TRW, LANL, Orsay). Based upon this work, Boeing/STI has embarked upon constructing a highpower visible-range oscillator. The design issues of such a system are
Physical Characteristics: (1) Wiggler length implies a long Rayleigh length and low divergence beam, (2) $5 \%$ extraction will provide $10 \mathrm{~mJ} /$ micropulse, (3) low gain implies a large circulating power $(\sim 300 \mathrm{~kW})$;

Systems Implications: (1) Large circulating power combined with low divergence forces long cavity length, (2) long cavity has tight stability tolerance ( $\sim 500$ n rad) which forces actively stabilized cavity.

The group at LANL, ${ }^{89}$ which has achieved a $1.3 \%$ extraction, in an oscillator which is tunable from $9 \mu \mathrm{m}$ to $35 \mu \mathrm{m}$, and $7 \mathrm{~kW}$ of extracted average power, has proposed building a high-power $1 \mu \mathrm{m}$ oscillator. For an entry level weapons class, e.g., $10 \mathrm{MW}$, system one would need a $13 \mathrm{~m}$ wiggler, an electron beam energy of $90 \mathrm{MeV}$, an average beam current of $0.5 \mathrm{~A}$, a peak current of $300 \mathrm{~A}$, a resonator length of $300 \mathrm{~m}$, and a brightness of $1.5 \times 10^{8} \mathrm{~A} /(\mathrm{cm} \mathrm{rad})^{2}$. The predicted extraction efficiency is $20 \%$.

\subsubsection{Amplifiers}

As has been already noted, present achievements with high-power amplifiers is encouraging; at LLNL at $8.6 \mathrm{~mm}$ (42\% extraction) and at LANL at $10.6 \mu \mathrm{m}$. Based upon this, LLNL has undertaken a program (Paladin) employing the Advanced Technology Accelerator (ATA), (50 MeV, $10 \mathrm{kA}$ ) in a single-pass FEL. This FEL is $25 \mathrm{~m}$ long and will operate at $10.6 \mu \mathrm{m}$. Expected performance ${ }^{90}$ is based on a beam current of $3 \mathrm{kA}$, and a brightness of $5 \times 10^{5} \mathrm{~A} /(\operatorname{rad~cm})^{2}$. This experiment will explore optical guiding, sidebands, harmonic generation, beam propagation, and optical beam quality.

The FEL as a directed energy weapon also depends upon a high-rep rate, high-brightness, linear induction accelerator. Considerable progress has been made, in recent years, on such devices. ${ }^{91}$ The recent progress in increasing the average power of induction accelerators is based upon the use of saturable magnetic switches instead of spark gaps. ${ }^{91}$ (Spark gaps have a firing rate which is limited by hydrodynamics; i.e., the rate at which fresh gas can be blown into the gap.) A magnetic switch has been built and operated for millions of cycles.

\subsubsection{System Comparisons}

The FEL has developed very rapidly as a high power and potentially efficient laser. Its tunable feature may be helpful in optimizing atmospheric penetration. The FEL appears to be capable of demonstrating at $1 \mu \mathrm{m}$ an average power level $\sim 1 \mathrm{MW}$ in the future.

Two competing acceleration systems for the ultrahigh-power FEL are the rf linac and the induction linac. Up to this time, neither system has actually demonstrated 
FEL performance (in lower power experiments) that can be scaled beyond $\sim 1 \mathrm{MW}$ average power with adequate confidence. For example, the induction linac amplifier FEL has yet to establish optical guiding and exponential gain at $10.6 \mu \mathrm{m}$, let alone at $1 \mu \mathrm{m}$. On the other hand, the rf linac FEL oscillator still faces the necessity for improvement of efficiency and average beam current of roughly one order of magnitude each. Nevertheless, we shall "compare" the two based on the assumption that both systems reach their targeted performance.

The advantage of the rf linac system centers around its superior duty factor, which helps to reduce problems of single-pulse damage to mirrors and the problem of Raman Stokes conversion at high intensity in the atmosphere. However, there is an important problem about the optical resonator (actually a ring resonator with elements near grazing incidence): high circulating resonator power, and difficulty with output coupling. Recently, it has been shown that the electron beam energy can be recovered by recirculating the electron beam in the rf linac; this could improve the overall system efficiency. ${ }^{92}$

In the induction linac system, the optics appear tractable providing the self-focusing actually occurs, is stable, and yields a high-quality beam. It is proposed that the beam output can be diffractively expanded to fill a large aperture using an evacuated pipe several $\mathrm{km}$ long. On the other hand, the optics must handle very highpower single pulses, lasting $\sim 50 \mathrm{~ns}$. Expanding the beam so that atmospheric propagation is assured without the Raman complication may require an output telescope $\sim 10 \mathrm{~m}$ aperture, which is currently beyond technological capability. (Both systems as ground-based lasers require adaptive optics for atmospheric compensation.)

Optical control of the FEL beam involves the requirement of maintaining stability in beam pointing and control of beam aberrations over a time commensurate to the target engagement time. This problem is different for the oscillator versus the amplifier FEL. Whereas the oscillator cavity causes a mode to appear which represents a stable solution to such problems as thermal effects at the mirror, in the amplifier optical corrections can only be applied to the subsequent pulse. This means substantially improved mechanical and optical tolerances.

\subsubsection{FEL Critical Issues}

1. Optical guiding and sextupole focusing have yet to be demonstrated experimentally.

2. The required electron beam quality so as to allow electron beam propagation at adequate current needs to be demonstrated.

3. A high-power tapered amplifier or oscillator FEL at about $1 \mu \mathrm{m}$ remains to be demonstrated.

4. Sideband and synchrotron-betatron instabilities must be sufficiently suppressed to obtain high-power output from the FELs.

\subsection{X-RAY LASERS}

A subgroup of the panel was briefed on current aspects of $x$-ray laser technology at the Lawrence Livermore National Laboratory. Since such data are classified, this report will only review the physics background of $x$-ray lasers, which has been published in the open literature. ${ }^{93,94}$ Just as a ruby laser or a Nd-glass laser is pumped by the blackbody visible radiation from a flashlamp with $\mathrm{Xe}$ discharge, so the pumping by an $\mathrm{X}$-ray continuum from a nuclear explosion can create inversions between pairs of ionic levels in the dense hot plasma when such $x$-ray bursts are absorbed by atoms and ions. The hot plasma is, of course, at high pressure and will start to expand, and exponential gain may occur. Population inversion between a selected pair of energy levels in a particular ionic species is the result of many rate processes, including photoionization, electron-ion collisions, radiative decays, and recombination processes that occur between species, each with its own set of energy levels, in the expanding hot, dense plasma. Elaborate codes exist to calculate the behavior of such plasmas, which are created by sudden deposition of large amounts of energy in initially condensed matter.

Open literature describes an x-ray plasma created by the sudden absorption of energy from a short laser pulse by a thin metallic film. Rosen et al. ${ }^{95}$ at Lawrence Livermore National Laboratory describe the explodingfoil technique, in which radiation at $\lambda=530 \mathrm{~nm}$ from a frequency-doubled Nd-glass laser $(1 \mathrm{~kJ}, 450 \mathrm{ps}$, $\lambda=530 \mathrm{~nm}$ ) is focused onto a spot of $1.2 \times 0.02 \mathrm{~cm}^{2}$ of a selenium foil. The average intensity during a $20 \mathrm{ps}$ long pulse is about $5 \times 10^{13} \mathrm{~W} / \mathrm{cm}^{2}$. The selenium $(\mathrm{Z}=34)$ is transformed to a plasma with a temperature of about 90 eV. This plasma contains 24 times ionized selenium atoms. The $\mathrm{Ne}$-like ions exhibited a population inversion between some pairs of levels of the $3 p^{5} 3 p$ and $2 P^{5} 3 s$ configurations. Exponential gain was observed ${ }^{96}$ at the $\mathrm{x}$-ray wavelengths $\lambda=20.63 \mathrm{~nm}$ and $20.96 \mathrm{~nm}$.

Similar experiments were performed at Princeton by pumping a carbon disk with a pulse from a $\mathrm{CO}_{2}$ laser (1 $\mathrm{kJ}, 75 \mathrm{~ns}, \lambda=10.6 \mu \mathrm{m})$. In the plasma, gain was observed $^{97}$ at $\lambda=18.2 \mathrm{~nm}$ and $\lambda=13.5 \mathrm{~nm}$, corresponding to transitions between level pairs $(n=4 \rightarrow 2)$ in five-times ionized carbon.

Laser-induced plasmas may be used as sources of incoherent soft $x$ rays. When $0.26 \mu \mathrm{m}$ laser light is focused on a gold target, the conversion efficiency in the $0.1-1.6 \mathrm{keV}$ range can be as high as $80 \%$ at a laser intensity of $2.4 \times 10^{13} \mathrm{~W} / \mathrm{cm}^{2} .{ }^{98}$ Such "x-ray flashlamps" give promise of being able to pump various proposed $x$ ray laser sources. However, any scheme requiring coherent light to be converted to incoherent $x$ rays in order to pump a coherent $\mathrm{x}$-ray source is thought to have inherently poor efficiency.

Because of classification issues, we are not able to comment directly on the details of the physics of the nuclear explosion pumped x-ray lasers. However, a 
number of publications in the open literature ${ }^{99-108}$ (of which the subcommittee is aware) describe possible configurations for an $\mathrm{x}$-ray laser pumped by a nuclear explosion. The solid angle $\mathrm{d} \Omega_{\mathrm{L}}$ into which the laser radiation is emitted is set, in part, by geometrical aspect ratios, diffraction, and the optical properties of the lasing medium. The enhancement in x-ray brightness over that obtained from a nuclear explosion is given by $\eta / \mathrm{d} \Omega_{\mathrm{L}}$, where $\eta$ is the overall efficiency for converting the nuclear explosion energy into $x$-ray laser energy. The overall efficiency is a product of the fraction of the nuclear explosion energy that results in incoherent $\mathrm{x}$ radiation acting as the pump, the fraction of this incoherent $\mathrm{x}$-ray flux intercepted and absorbed by the lasing subsystem, and the fraction of the absorbed energy which is converted into the $x$-ray laser output. For convenience of the reader, this relationship is plotted in Figure 3.31.

The emission of the $x$-ray laser pulse must occur on a time scale of less than $1 \mu \mathrm{s}$, before the material is blown away. For times $t>D / v_{\text {at }}$, where $D$ is a typical linear dimension of the device and $v_{a t}$ is the velocity of the particles emanating from the explosion, i.e., weapon debris, there is nothing left of the original configuration.

It is shown in Chapter 6 that significant damage (to the target) is done by $x$-radiation pulses for a fluence of $3 \mathrm{~kJ} / \mathrm{cm}^{2}$, i.e., $3 \times 10^{7} \mathrm{~J} / \mathrm{m}^{2}$, on the target. Assume a target with a typical linear dimension of $1 \mathrm{~m}$ at a range of $1000 \mathrm{~km}$. To get all of the emitted $\mathrm{x}$ radiation on the target would require a solid angle $d \Omega=10^{-12}$ sr. The total pump energy $Q$ required in this case would be $3 \times 10^{7} \eta \mathrm{J}$. In general, the fluence on target will be $\eta \mathrm{Q} / \mathrm{R}^{2} \mathrm{~d} \Omega_{\mathrm{L}}$. (A 30 kiloton nuclear explosion releases $\sim 1.2 \times 10^{14} \mathrm{~J}$ of energy.) Figure 3.32 shows,

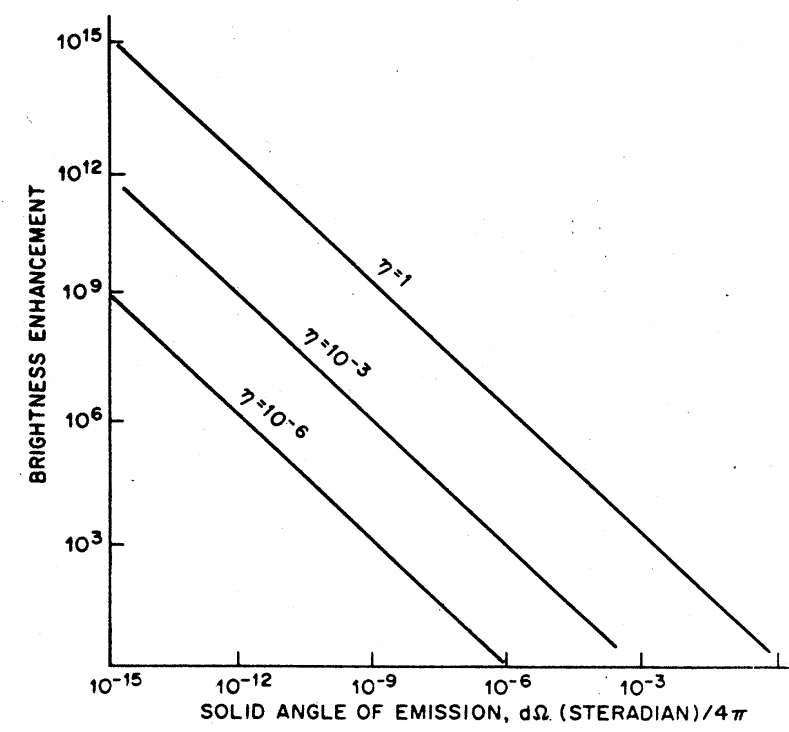

Figure 3.31. Calculated brightness enhancement for a nuclear explosion pumped $\mathrm{x}$-ray laser as a function of the solid angle of laser emission. Three typical variations are shown for the overall conversion efficiency (see Section 3.5) of $1,10^{-3}$, and $10^{-6}$.

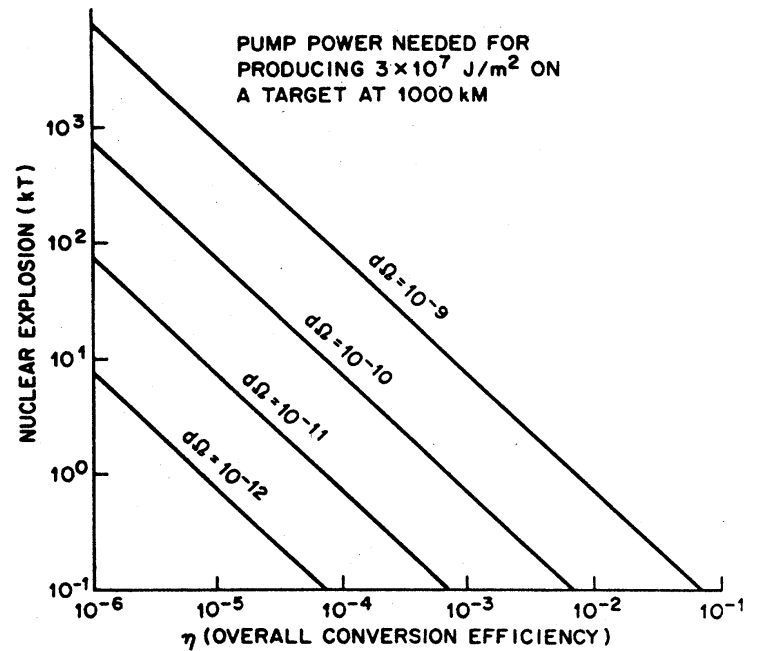

Figure 3.32. Calculated nuclear explosion pump power needed for the delivery of a fluence of $3 \mathrm{~kJ} / \mathrm{cm}^{2}$ on a target at a range of $1000 \mathrm{~km}$ as a function of the overall conversion efficiency (see Section 3.5 for the definition of overall conversion efficiency). Variations are shown for a number of solid angles of $\mathrm{x}$-ray laser emission.

parametrically, the nuclear explosion energy needed to produce an $\mathrm{x}$-ray fluence of $3 \times 10^{7} \mathrm{~J} / \mathrm{m}^{2}$ on a $1 \mathrm{~m}$ (linear dimension) target at a range of $1000 \mathrm{~km}$ as a function of the overall conversion efficiency.

The available gain is an extremely sensitive function of the plasma composition, its geometrical configuration, and the high number of rate processes that can occur, taking account of the large number of energy levels of the various ionic species occurring in the plasma. Very elaborate computational codes must be augmented by extensive test procedures, which are much more complex than those for lasers at optical wavelengths, because of the extreme environment caused by the nuclear explosion. As in all lasers, the extent to which diffraction limited operation can be obtained will be determined by the homogeneity of the lasing medium.

The very favorable energy-to-mass (weight) ratio of the nuclear explosion pumped device, at their asssumed efficiencies, gives such $\mathrm{x}$-ray lasers a special advantage over other directed energy weapons that can be used in a pop-up mode. Although $\mathrm{x}$ rays cannot propagate substantial distances in the atmosphere (Section 5.4.10) at an altitude of approximately $80 \mathrm{~km}$, the $\mathrm{x}$-ray laser beam may penetrate upwards through the remaining atmosphere with relatively little loss because it can bleach an ionized path through the upper atmosphere. There is an interesting offense-defense asymmetry, since a spacebased $x$-ray weapon of the same brightness shooting down, cannot penetrate down to the same altitude. Due to divergence of the beam, the degree of bleaching in the atmosphere is inferior to that of the same weapon shooting upward. Pop-up x-ray lasers would make the survivability of space platforms highly questionable. 


\subsection{GAMMA-RAY LASERS}

As a part of the Strategic Defense Innovative Research Program, the Office of Naval Research is conducting an investigation of gamma-ray laser technology. In a gamma-ray laser, energy is stored in a long-lived isomeric nuclear state. ${ }^{110,111}$ A very high density of energy can be stored in this form. If, for example, one percent of the nuclei of a solid could be excited to a $100 \mathrm{keV}$ energy level, the total energy storage would approach gigajoules per liter. This represents storage of a far higher energy than in any other proposed laser medium. Isomeric states can be excited by exposing the solid material to a neutron flux from a nuclear reactor. In the concept presented by the Office of Naval Research for a space-based gamma-ray laser, the storage medium would be pumped in a reactor on earth, and the medium would then be placed in space to be triggered upon command. The fundamental technical issues that need to be resolved are how to trigger the release of the gamma-ray energy, and provide as well collimated a gamma-ray beam as possible.

As presently envisioned, all gamma-ray lasers will rely on the Mössbauer effect to provide a narrow linewidth for the laser transition, and to bring the emission and the absorption frequencies into coincidence. Current gamma-ray laser proposals envision a long-lived "storage level" in the nucleus, a stable isomeric state. To initiate laser action, a transition to an upper laser level is induced by an optical pump field. Because the upper lasing level has a very short lifetime, the pump must act in a very short time. Both direct pumping schemes, in which a real intermediate state is involved, and Raman schemes, with virtual intermediate state, have been proposed.

A further property of all proposed gamma-ray laser schemes is the use of the Borrmann effect to provide lossless transmission of the laser energy out of the crystal. ${ }^{109}$ An unresolved issue is whether the host crystal can maintain its low temperature lattice properties in the presence of the necessary intense optical pump and the hoped-for gamma-ray laser action.

Only a few nuclei have been identified with the required level structure, consisting of long-lived isomer lying within a kilovolt or less of the level which can serve as an upper laser level. Of these, only six have been found which can decay to an excited state, allowing a four level laser action. These are ${ }^{86} \mathrm{Rb},{ }^{110} \mathrm{Ag},{ }^{179} \mathrm{Hf}$, ${ }^{183} \mathrm{~W},{ }^{152} \mathrm{Eu}$, and ${ }^{242} \mathrm{Am}$. None of these candidate nuclei exhibit the transition probabilities needed for a gammaray laser action, so the search for an appropriate nuclear level continues.

It is anticipated that the appropriate nucleus, once identified, will be doped into a suitable crystal lattice. As indicated above, even at a relatively low doping concentration which may be limited by the need to keep the host lattice properties unperturbed, a high energy storage density can be achieved. A possible limit on the photon energy which can be generated in a gamma-ray laser is the saturation fluence, given by $\mathrm{E} / \sigma$, where $\mathrm{E}$ is the photon energy, and $\sigma$ is the cross section for stimulated emission. An upper bound on the cross section is given by

$$
\sigma<\lambda^{2} / 2 \pi
$$

where $\lambda$ is the gamma-ray laser wavelength. Any process which competes with gamma-ray emission, such as internal conversion, will reduce the cross section. Further, the cross section will be reduced by the effect of the finite Debye temperature, which dictates that the recoilless Mössbauer transition has a probability of less than unity.

The photon wavelength can be written as hc/E, so that the saturation fluence is

$$
\mathbf{J}_{\mathrm{s}}<2 \pi \mathrm{E}^{3} /(\mathrm{hc})^{2}
$$

At $10 \mathrm{keV}$, the saturation fluence exceeds $64 \mathrm{~J} / \mathrm{cm}^{2}$, but at $100 \mathrm{keV}$, it exceeds $64 \mathrm{~kJ} / \mathrm{cm}^{2}$. At these fluences, even a small amount of absorption will prove destructive to the host material.

As with the $x$-ray laser, it may be possible to extract the energy from the gamma-ray laser in a time short compared to the disassembly time of the lattice. However, the effect of intense energy deposition on the Mössbauer effect and the Borrmann effect are issues which need to be addressed.

By far the greatest uncertainty relating to the gamma-ray laser feasibility is the "transfer" process, wherein the stable isomeric state is excited to the rapidly decaying upper laser level. This transfer process must be accomplished with an external pumping field, probably an intense optical laser. Current research centers around finding collective excitations of atomic electrons which can transfer kilovolt energies into the nucleus.

In conclusion, gamma-ray lasers represent an extemely high risk and long term approach to strategic defense. The attractive feature is the potential for storing very large energy densities in nuclear isomeric states. The disadvantages are the lack of a proven concept for extraction of the stored energy, either by direct lasing process or by a Raman process. Further concerns relate to the extremely high saturation fluence of the system which will prevent efficient extraction of the stored energy at manageable levels of power density and the absence of conventional optics to provide collimation and focusing of the beam.

\subsection{CONCLUSIONS}

1. We estimate that chemical laser output powers at acceptable beam quality need to be increased by at least one order of magnitude for HF/DF lasers for use as an effective kill weapon in the boost phase. 
Similarly for atomic iodine lasers, at least five orders of magnitude improvement is necessary.

2. We estimate that the pulse energy from excimer lasers for strategic defense applications needs improvement by at least four orders of magnitude over that currently achieved. Many advances are needed to achieve the required repetititve pulsing of these lasers at full scale.

3. Free electron lasers suitable for strategic defense applications, operating near $1 \mu \mathrm{m}$, require validation of several physical concepts.

4. Nuclear explosion pumped $\mathrm{X}$-ray lasers require validation of many of the physical concepts before their application to strategic defense can be evaluated.

\section{REFERENCES}

${ }^{1}$ C. K. N. Patel, "Interpretation of $\mathrm{CO}_{2}$ Optical Maser Experiments," Phys. Rev. Lett. 12, 588-591 (1964).

${ }^{2}$ K. L. Kompa and G. C. Pimentel, J. Chem. Phys. 47, 857 (1967).

${ }^{3}$ A. J. Glass, "Historic Overview of Inertial Confinement Fusion: What Have Learned," J. Vac. Sci. Technol. A4, 1098-1105 (1986).

${ }^{4}$ See, for example, J. D. Anderson, Jr., Gas Dynamic Lasers (Academic, New York, 1976).

${ }^{5}$ IEEE Spectrum, September 1985, pp. 36-64.

${ }^{6}$ U.S. Congress, Office of Technology Assessment, Ballistic Missile Defense Technologies, OTA-ISC-254 (U.S. Government Printing Office, Washington, D.C., September 1985).

${ }^{7}$ Handbook of Chemical Lasers, edited by R. W. F. Gross and J. F. Bott (Wiley, New York, 1976).

${ }^{8}$ N. G. Basov, V. A. Danilychev, and Y. M. Popov, Sov. J. Quant. Electron. 1, 18 (1971); H. A. Kohler et al., Appl. Phys. Lett. 21, 198 (1972).

${ }^{9}$ L. R. Elias et al., Phys. Rev. Lett. 36, 710 (1976).

${ }^{10}$ J. V. V. Kasper and G. C. Pimentel, Phys. Rev. Lett. 14, 352 (1965).

${ }^{11}$ T. F. Deutsch, Appl. Phys. Lett. 10, 234 (1967).

${ }^{12}$ O. M. Batovskii, G. K. Vasil'ev, E. F. Makarov, and V. L. Tal'rose, JETP Lett. 9, 200 (1969); N. G. Basov, L. V. Kulakov, E. P. Markin, A. I. Nikitin, and A. N. Oraevsky, ibid. 9, 375 (1969).

${ }^{13}$ D. J. Spencer, T. A. Jacobs, H. Mirels, and R. W. F. Gross, Int. J. Chem. Kin. 1, 493 (1969); T. A. Cool, R. R. Stephens, and T. J. Falk, ibid. 1, 495 (1969); J. R. Airey and S. F. McKay, Appl. Phys. Lett. 15, 401 (1969).

${ }^{14}$ J. C. Polanyi and K. B. Woodall, J. Chem. Phys. 57, 1574 (1972).

${ }^{15}$ D. M. Neumark, A. M. Wodtke, G. N. Robinson, C. C. Hayden, and Y. T. Lee, J. Chem. Phys. 82, 3045 (1985).

${ }^{16}$ H. Mirels and D. J. Spencer, IEEE J. Quant. Electron. QE-7, 501 (1971).

${ }^{17}$ A. E. Siegman, "Unstable Optical Resonators for Laser Allications," Proc. IEEE 53, 277-287 (1965).

${ }^{18}$ C. K. N. Patel, "Continuous-wave Laser Action on Vibrational-Rotational Transitions of $\mathrm{CO}_{2}$," Phys. Rev. 136A, 1187-1193 (1964); 136A, 588 (1964).
${ }^{19}$ Michael A. Dornheim, "TRW Builds Chemical Laser to Demonstrate Key Technologies," Aviat. Week Space Technol., August 4, 1986, p. 33.

${ }^{20}$ Micheal Feazel, "U.S. Researchers Mark Progress in Laser, Rail Gun Programs," Aviat. Week Space Technol., June 2, 1986, pp. 68-71.

${ }^{21}$ W. E. McDermott et al., Appl. Phys. Lett. 32, 469 (1978).

${ }^{22}$ J. V. V. Kasper and G. C. Pimentel, Appl. Phys. Lett. 5, 231 (1964).

${ }^{23}$ R. M. Badger, A. C. Wright, and R. F. Whitlock, J. Chem. Phys. 43, 4345 (1965).

${ }^{24}$ R. G. Dervent and B. A. Thrush, Chem. Phys. Lett. 9, 591 (1971); R. G. Dervent and B. A. Thrush, Trans. Faraday Soc. 53, 162 (1972).

${ }^{25}$ M. Born and E. Wolf, Principles of Optics, 5th ed. (Pergamon, New York, 1975), p. 459.

${ }^{26}$ W. Fader and G. Palma, Proc. SPIE 440, 153 (1983); W. Fader and G. Palma, Opt. Lett. 10, 381 (1985); S. Townsend, Proc. SPIE 642, 51 (1986); J. Bender, W. Fader, and G. Palma, ibid. 642, 42 (1986).

${ }^{27}$ G. Busch, R. A. Nolan, and A. P. Schapp, "Storage and Retrieval of Singlet Oxygen," U.S. Patent \#4,436,715 (13 March 1984).

${ }^{28}$ M. C. Lin, M. E. Umstead, and N. Djeu, Ann. Rev. Phys. Chem. 34, 557 (1983).

${ }^{29}$ F. G. Houtermans, Helv. Phys. Acta 33, 933 (1960).

${ }^{30}$ P. W. Hoff, J. C. Swingle, and C. K. Rhodes, Appl. Phys. Lett. 23, 245 (1973).

${ }^{31}$ W. M. Hughes, J. Shannon, and R. Hunter, Appl. Phys. Lett. 24, 488 (1974).

${ }^{32}$ J. E. Velazco and D. W. Setser, J. Chem. Phys. 62, 1990 (1975).

${ }^{33}$ C. A. Brau and J. J. Ewing, Appl. Phys. Lett. 27, 433 (1975).

${ }^{34}$ J. J. Ewing and C. A. Brau, Appl. Phys. Lett. 27, 350 (1975).

${ }^{35}$ J. M. Hoffman, A. K. Hayes, and G. C. Tisone, Appl. Phys. Lett. 28, 538 (1976).

${ }^{36}$ J. J. Ewing and C. A. Brau, op. cit.

${ }^{37}$ J. M. Hoffman et al., op. cit.

${ }^{38}$ R. O. Hunter, C. Howten, and J. Oldenettel, AIAA 15th Aerospace Science Meeting, Paper 77-26, 1977 (unpublished).

${ }^{39}$ J. A. Mangano and J. H. Jacobs, Appl. Phys. Lett. 28, 724 (1976).

${ }^{40}$ R. Burnham and N. Djeu, Appl. Phys. Lett. 29, 707 (1976).

${ }^{41}$ J. D. Daugherty, J. A. Mangano, and J. H. Jacob, Appl. Phys. Lett. 28, 581 (1976).

${ }^{42}$ A. M. Hunter and T. H. Johnson, Bull. Am. Phys. Soc. 22, 1041 (1977).

${ }^{43}$ R. J. Jensen, Conference on Lasers and Electro-optic, paper THLI (23 May 1985).

${ }^{44}$ A. M. Hunter, R. O. Hunter, and T. H. Johnson, IEEE J. Quant. Elec. QE-22, 386-404 (1986).

${ }^{45}$ R. Burnham and S. K. Searles, J. Chem. Phys. 66, 2742 (1977).

${ }^{46}$ R. Burnham and S. K. Searles, J. Chem. Phys. 67, 5967 (1977).

${ }^{47}$ P. J. Hay and T. H. Dunning, J. Chem. Phys. 66, 1306 (1977).

${ }^{48}$ T. H. Dunning and P. J. Hay, J. Chem. Phys. 69, 134 (1978).

${ }^{49}$ C. A. Brau, in Excimer Lasers, edited by C. K. Rhodes (Springer, New York, 1979).

${ }^{50}$ J. J. Ewing, in Laser Handbook, edited by M. L. Stitch (North-Holland, Amsterdam, 1979).

${ }^{51}$ M. Rokni, J. A. Mangano, J. M. Jacob, and J. C. Hsia, J. Quantum Electron. QE-14, 464 (1978).

${ }^{52}$ T. H. Johnson and A. M. Hunter, J. Appl. Phys. 51, 2406 
(1980).

${ }^{53}$ D. C. Lorents, Physica 82C, 19 (1976).

${ }^{54}$ A. Hunter and E. Salesky, "Amplifier Staging for Phase IB KrF," P-Division, Los Alamos National Laboratory report, 1983 (unpublished).

${ }^{55}$ T. H. Johnson and A. M. Hunter, op. cit.

${ }^{56}$ A. M. Hunter and R. O. Hunter, IEEE J. Quantum Electron. QE-17, 1879 (1981)

${ }^{57}$ A. M. Hunter, R. O. Hunter, and T. H. Johnson, op. cit.

${ }^{58}$ G. C. Tisone and J. M. Hoffman, IEEE J. Quantum Electron. QE-18, 1008 (1982)

${ }^{59}$ D. Rothe, J. B. West, and M. L. Bhaumik, IEEE J. Quantum Electron. QE-15, 314 (1979).

${ }^{60}$ L. F. Champagne, Appl. Phys. Lett. 33, 523 (1978).

${ }^{61} \mathrm{H}$. Komine et al., Conference on Lasers and Electro-optics, paper WG3 (22 May 1985).

${ }^{62}$ F. Kannari et al., IEEE J. Quantum Electron. QE-19, 1587 (1983).

${ }^{63}$ L. A. Levin et al., IEEE J. Quantum Electron. QE-17, 2282 (1981).

${ }^{64}$ T. C. Genoni and T. H. Johnson, " $\mathrm{XeCl}$ Modeling," Proceedings of the International Conf. on Lasers 1984 (November 1984).

${ }^{65}$ J. D. Dougherty, in Laser Plasmas, edited by G. Bekefi (Wiley, New York, 1976).

${ }^{66}$ J. Goldhar, J. R. Murray, and K. S. Jancaitisy, in Laser Program Annual Report 83, Lawrence Livermore National Laboratory UCRL-50021-83 (1984), p. 6-96.

${ }^{67}$ A. M. Hunter, NRL Nonlinear Optics Workshop, Navel Research Laboratory (4 December 1984).

${ }^{68}$ H. Motz, J. Appl. Phys. 22, 527 (1951).

${ }^{69}$ K. M. Phillips, Trans. IRE Elect. Dev. 7, 231 (1960).

${ }^{70}$ D. A. G. Deacon et al., Phys. Rev. Lett. 38, 892 (1977)

${ }^{71}$ N. M. Kroll and W. A. McMullin, Phys. Rev. Lett. 17A, 300 (1978).

${ }^{72}$ W. B. Colson, Phys. Quant. Elect. 5, 152 (1977) (Jacobs et al., editors, published by Addison-Wesley).

${ }^{73}$ T. C. Marshall, "Free Electron Lasers" (Macmillan, New York, 1985).

${ }^{74}$ N. M. Kroll et al., J. Quantum Electron. QE-17, 1496 (1981).

${ }^{75}$ D. B. McDermott et al., Phys. Rev. Lett. 41, 1368 (1978)

${ }^{76}$ P. Sprangle, R. A. Smith, and V. L. Granatstein, Infrared Millimeter Waves 1, 174 (1979).

${ }^{77}$ R. Bonifacio, C. Pelligrini, and L. M. Narducci, Opt. Commun. 50, 373 (1984).

${ }^{78}$ E. T. Scharlemann, A. M. Sessler, and J. S. Wurtele, Phys. Rev. Lett. 54, 1925 (1985).

${ }^{79}$ R. W. Warren et al., J. Quantum Electron. QE-19, 391 (1983).

${ }^{80}$ B. E. Newman et al., J. Quantum Electron. QE-21, 867 (1985).

${ }^{81}$ T. J. Orzechowski, B. Anderson, W. M. Fawley, D. Prosnitz, E. T. Scharlemann, S. Yarema, D. Hopkins, A. C. Paul, A. M. Sessler, and J. Wurtele, "Microwave Radiation from a HighGain Free Electron Laser Amplifier," Phys. Rev. Lett. 54, 889 (1985).

${ }^{82}$ W. B. Colson and A. M. Sessler, "The Free Electron Laser," Annu. Rev. Nucl. Particle Sci. 35, 25 (1985).

${ }^{83}$ W. A. Barletta et al., Nucl. Instrum. Methods Phys. Res. A239, 47 (1985).

${ }^{84}$ M. Rosenbluth, J. Quantum Electron. QE-21, 966 (1985).

${ }^{85}$ W. M. Fawley, D. Prosnitz, and E. T. Scharlemann, Phys. Rev. A 30, 2471 (1984).

${ }^{86}$ G. J. Caporaso, F. Rainer, W. E. Martin, D. S. Prono, and A.
G. Cole, Phys. Rev. Lett. 57, 1591 (1986)

${ }^{87}$ J. Slater et al., "Visible Wavelength Free Electron Laser Oscillator," Proc. 7th Intl. Conf. on Free Electron Lasers, Tahoe City, September 1985, p. 228 (1986); D. M. Shumwell et al., "Optical Cavities for Visible Free Electron Laser Experiments," Proc. 8th Intl. Conf. on Free Electron Lasers, Glasgow, Scotland, September 1986 (to be published in J. Nucl. Instrum. Methods, 1987); K. E. Robinson et al., "Field Certification of a High Srength Tapered Hybrid Undulator," Proc. 8th Intl. Conf. on Free Electron Lasers, Glasgow, Scotland, September 1986 (to be published in J. Nucl. Instrum. Methods, 1987).

${ }^{88}$ J. A. Edighoffer et al., Phys. Rev. Lett. 52, 344 (1984).

${ }^{89} \mathrm{~J}$. Goldstein et al., "Comparison of the Results of Theoretical Calculations with Experimental Measurements for the Los Alamos Free Electron Laser Oscillator Experiment," Proc. 7th Intl. Conf. on Free Electron Lasers, Tahoe City, September 1985, p. 4 (1986).

${ }^{90}$ D. Prosnitz, Invited Talk, "An Overview of the LLNL Free Electron Laser Program," Proc. 8th Intl. Conf. on Free Electron Lasers, Glasgow, Scotland, September 1986 (to be published in J. Nucl. Instrum. Methods, 1987); LLNL Energy and Technology Review, December, 1986.

${ }^{91}$ D. L. Birx et al., "The High Brightness Test Stand," Proc. of the 7th Intl. Conf. on Free Electron Lasers (to be published) (1986); D. L. Birx et al. "Technology of Magnetically Driven Accelerators," Proc. 1985 Particle Accelerator Conference (to be published), 1985; L. L. Reginato et al., IEEE Trans. Nucl. Sci. NS-30, 2970 (1983).

${ }^{92}$ J. M. Watson, "Status of the Los Alamos Free Electron Laser," Proc. 7th Intl. Conf. on Free Electron Lasers, Tahoe City, September 1985, p. 1 (1986); D. Feldman et al., "Energy Recovery in the Los Alamos Free Electron Laser," Proc. 8th Intl. Conf. on Free Electron Lasers, Glasgow, Scotland, September 1986 (to be published in J. Nucl. Instrum. Methods, 1987).

${ }^{93}$ G. Chapline and L. Wood, Phys. Today 6, 40 (1975).

${ }^{94}$ F. Bunkin, V. Derzhiev, and S. Yakovlenko, Sov. J. Quantum Electron. 11, 971 (1981).

${ }^{95}$ M. D. Rosen et al., Phys. Rev. Lett. 54, 106 (1985).

${ }^{96}$ D. L. Matthews et al., Phys. Rev. Lett. 54, 110 (1985).

${ }^{97}$ S. Suckewer et al., Phys. Rev. Lett. 55, 1753 (1985).

${ }^{98}$ R. Kodama, K. Okada, N. Ikada, M. Mineo, K. A. Tanaka, P. Mochizuki, and C. Yamanaka, "Soft X-ray Emission from $\Omega_{0}$, to $\Omega_{0}$, and for $\Omega_{0}$ Laser-Produced Plasmas," J. Appl Phys. 59, 3050-3052 (1986).

${ }^{99}$ A. B. Carter, Directed Energy Missile Defense in Space (U.S. Congress Office of Technology Assessment, Washington, D.C., 1984), pp. 24-28, OTA-BP-ISC.

${ }^{100}$ S. D. Drell, P. J. Fawley, and D. Holloway, The Reagan Strategic Defense Initiative (Stanford University Press, Stanford, 1984), pp 50-53.

${ }^{101}$ H. A. Bethe and R. L. Garwin, in Weapons in Space, Daedalus, Summer 1985, pp. 346-348.

${ }^{102}$ Space Based Missile Defense, a report by the Union of Concerned Scientists, pp. 102-104.

${ }^{103}$ R. S. Sagdeev and S. N. Rodinov, Space Based Antimissile System, a report of the Space Research Institute of the Academy of Sciences of the USSR, 1986, pp. 42ff.

${ }^{104}$ Weaponry in Space: The Dilemma of Security, edited by Y. P. Velikhov, A. Kokoshkin, and R. S. Sagdeev (Mir, Moscow, 1986), pp. 33-38.

${ }^{105}$ E. Walbridge, "Angle Constraint for Nuclear-Pumped X-Ray Laser Weapons," Nature 310, 180-182 (1984). 
${ }^{106}$ A. N. Robinson, "Experts Cast Doubt on X-Ray Laser," Science 230, 646-648 (1985).

${ }^{107}$ L. Wood, "Concerning Some Technical Aspects of Strategic Defense," Presented at the 4th Session of the International Seminar on Nuclear War, Erice, Italy, August 20-24, 1984 (conference transcript).

${ }^{108}$ D. M. Ritson, "The Nuclear Pumped X-Ray Laser" (unpublished).
${ }^{109}$ B. W. Batterman and H. Cole, "Dynamical Diffraction of Xrays by Perfect Crystals," Rev. Mod. Phys. 36, 681 (1964).

${ }^{110}$ G. C. Baldwin, J. C. Solem, and V. I. Gol'danskii, Rev. Mod. Phys. 53, 687 (1981).

${ }^{111} \mathrm{An}$ extensive discussion of gamma-ray lasers concepts, relevant data and fuller references can be found in Advances in Laser Science-I, edited by W. C. Stwalley and M. Lapp (AIP Conf. Proc. No. 146) (AIP, New York, 1986). 


\section{Chapter 4}

\section{PARTICLE BEAMS}

\section{CONTENTS}

4.1. Introduction

4.2 Laser-Guided Electron Beams

4.2.1 Beam Propagation Physics

4.2.1.1 Equilibrium Guiding

4.2.1.2 Erosion Phenomena

4.2.1.3 Beam Instabilities

4.2.1.4 Summary

4.2.2 Laser Technology and Ionization Requirements

4.2.3 Accelerator Requirements

4.2.3.1 Induction Linacs

4.2.3.2 Betatrons

4.2.3.3 RF Linacs

4.2.3.4 Summary

4.2.4 Beam Steering Concepts

4.2.5 Summary

4.3 Neutral Particle Beams

4.3.1 Negative Ion Sources

4.3.2 Acceleration Stages

4.3.3 Beam Expansion and Steering

4.3.4 Beam Neutralization

4.3.5 Sensing Direction for Neutral Particle Beams

4.3.6 Summary

4.4 Other Particle Beam Concepts

4.4.1 Massive, Energetic Ions

4.4.2 Charge- and Current-Neutralized Ion Beams (Plasmoids)

4.5 Systems Requirements Summary

4.5.1 NPB for Boost Phase Intercept

4.5.2 Laser-Guided Electron Beam for Mid-Course Discrimination

4.6 Summary

4.7 Conclusions

References

\subsection{INTRODUCTION}

Energetic neutral particle beams (NPBs), e.g., atomic hydrogen, deuterium, tritium, etc., and charged particle beams (CPBs), e.g., electrons, protons, heavy ions, etc., when accelerated to relativistic velocities, offer the potential for delivering significant amounts of energy to distant targets within fractions of seconds. Moreover, both NPBs and CPBs interact strongly with matter; therefore, they may have extremely high lethality. Before this potential could be realized, however, favorable answers must be obtained to several key questions involving the efficient generation, acceleration, and propagation of low divergence particle beams, as well as to several important systems questions. These issues will be discussed in some detail in this chapter.

Figures 4.1(a) and 4.1(b) indicate the basic elements of both NPB and CPB devices. In the NPB approach, a low divergence beam of negative ions $\left(\mathrm{H}^{-}\right.$, for example) is generated and accelerated to high energy. After the beam is focused and (magnetically) aimed, the weakly attached $(\sim 0.7 \mathrm{eV})$ extra electron is removed in a "stripping cell," by atomic collisions or photodetachment processes. The neutral atoms would then propagate to the target in straight-line trajectories, unaffected by the earth's magnetic field.

For the CPB approaches, electron or positive ion beams are accelerated to the desired energy, and magnetically aimed. In order to avoid deflection in the earth's magnetic field, the beam must either be guided by a laser-generated plasma channel (electron beams), have some mechanism for neutralizing the beam current and space charge (plasmoids), or must have sufficient momentum that the deflection of the beam particles in the earth's magnetic field is small for the ranges considered.

In contrast to lasers, which deposit energy in the surface layers of targets, energetic particle beams are extremely penetrating and the energy transfer mechanism is fully predictable. For example, $4 \mathrm{~cm}$ to $41 \mathrm{~cm}$ of aluminum would be required to shield the interior of a target from $100 \mathrm{MeV}$ to $400 \mathrm{MeV}$ hydrogen atoms, respectively. Consequently, the concept of beam brightness, defined as the beam power in watts divided by the square of the divergence angle, which is so useful in defining laser requirements, has limited utility when applied to particle beam approaches. Rather, it is more appropriate to discuss particle beam lethality criteria in terms of energy deposited per unit mass of target material, i.e., joules per gram or radiation dose $(10 \mathrm{~J} / \mathrm{g}$ is equivalent to one megarad). In addition to beam energy fluence, the energy deposition criterion will depend on the particle type and kinetic energy, and weakly on the density of the target material. For example, a beam of $250 \mathrm{MeV}$ hydrogen atoms with an incident energy fluence of $250 \mathrm{~J} / \mathrm{cm}^{2}$ will deliver about 0.5 megarad within the particle deposition volume; a $100 \mathrm{MeV}$ hydrogen beam with the same incident fluence $\left(250 \mathrm{~J} / \mathrm{cm}^{2}\right)$ will deliver about 2.5 megarads to the deposition volume because the penetration depth will be about five times shorter. Because of the in-depth penetration property, passive shielding is not an attractive countermeasure; also, the hardness of every interior component, as well as possible 


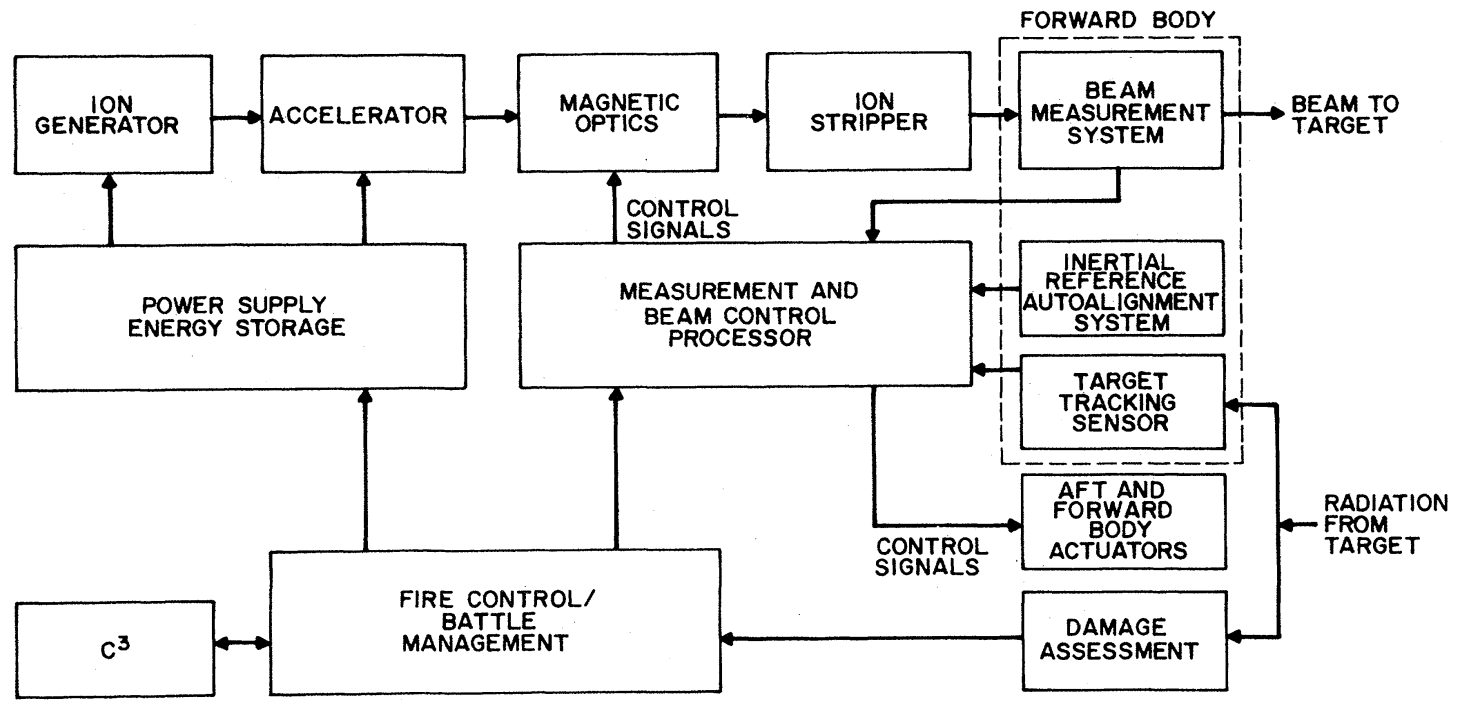

(b)

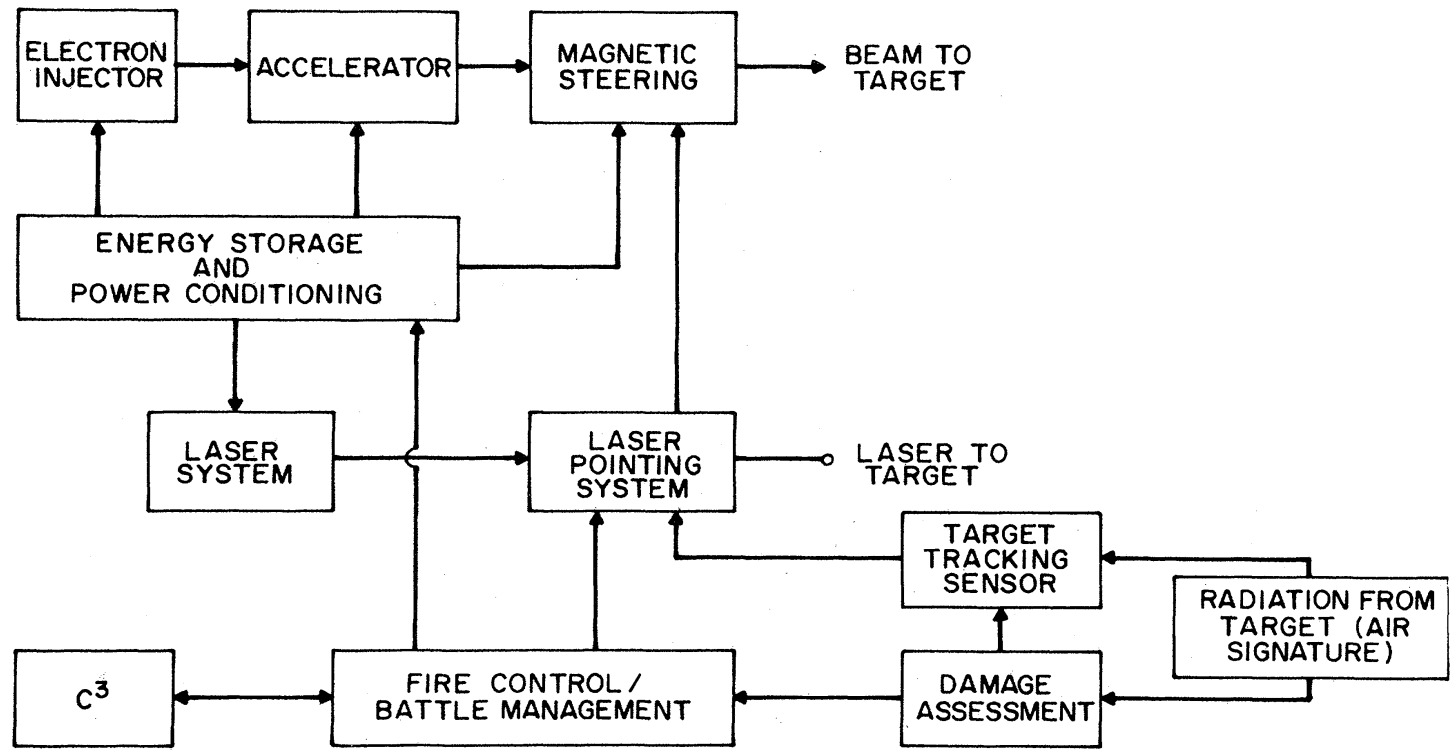

Figure 4.1. (a) The basic elements of a neutral particle beam device. (b) The basic elements of a laser-guided charged particle beam device.

synergistic radiation damage effects, must be taken into consideration.

There are important operational altitude restrictions for almost all particle beam approaches. These arise from the interaction of the various beams with the background gas density. In case of the neutral particle beams, the presence of gas is detrimental while in the case of the charged particle beams there is a need for certain minimum density of gas as described below. The results of the following discussions are summarized in Figure 4.2.

The neutral particle beams interact very strongly 


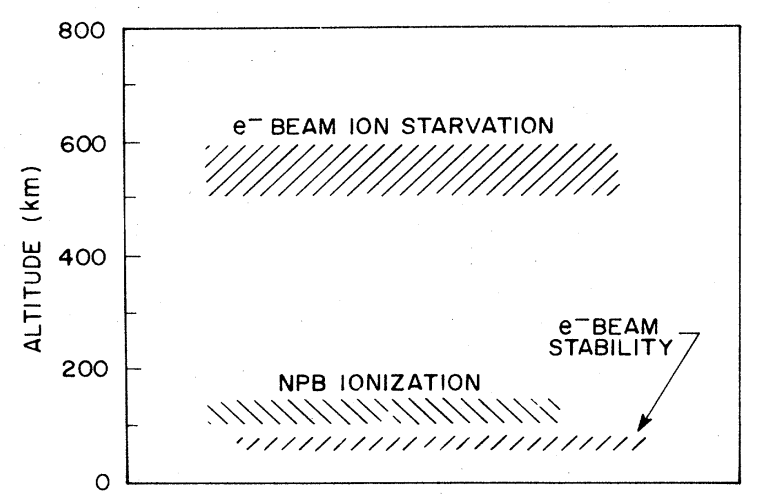

Figure 4.2. Approximate altitude limitations for neutral particle beams and laser-guided electron beams.

with matter, and therefore interactions with intervening gas makes the propagation of NPB through atmosphere impractical. Scattering interactions will strip the atomic electron and ionize the neutral particle beam. The resulting positive ions will be deflected in the earth's magnetic field. The decrease in the neutral particle beam intensity depends on the ionization cross section $\left(\sigma \sim 10^{-18} \mathrm{~cm}^{2}\right)$, and the integrated atmospheric density through which the beam propagates. Modeling the decrease in the atmospheric density with altitude $\mathrm{x}$ as an exponential, $\mathrm{e}^{-\mathrm{x} / \mathrm{h}}$, with an average scale height of $\mathrm{h} \approx 7 \mathrm{~km}$, and assuming that the beam is directed downward toward the center of the earth, the neutral particle beam intensity I will vary as

$$
\ln \left(\mathrm{I} / \mathrm{I}_{0}\right)=\sigma \mathrm{n}_{0} \mathrm{he}^{-\mathrm{x} / \mathrm{h}}
$$

where $I_{0}$ is the neutral particle beam intensity at the NPB platform altitude $(>500 \mathrm{~km})$, and $\mathrm{n}_{0}$ is the atomic number density at the earth's surface. This relation indicates that approximately $50 \%$ of a $250 \mathrm{MeV}$ neutral hydrogen beam injected vertically downward toward the earth (from an altitude $\gg 120 \mathrm{~km}$ ) will be lost by the time the beam reaches an altitude of $120 \mathrm{~km}$.

For laser-guided electron beams, an upper altitude limit of several hundred kilometers exists because the laser-generated ion density (which depends on the background neutral atom density) must be sufficiently large to counterbalance the deflecting force caused by the earth's magnetic field. It is believed that there is also a lower altitude limit for this approach because of the development of electron streaming instabilities at altitudes of several tens of kilometers.

Because of these lower altitude limits, particle beam weapon systems will have to be sited on exoatmospheric platforms, e.g., satellite constellations or rocket-borne pop-up platforms. These basing options impose stressing constraints on the weight, size, and primary power requirements, as well as forcing considerations of heat dissipation, cryogen storage, lifetime in space, maintenance and testing, etc.
There are at least two general applications of directed particle beam technologies. In the first, the particle beams could be used to kill booster/bus/reentry vehicles via structural damage, electronic degradation, propellant ignition, or detonation of high explosives. A second important proposed use is that of interactive discrimination between reentry vehicles and accompanying decoys in the mid-course. This application is based on the fact that all materials emit gamma rays, $x$ rays, and neutrons when irradiated with high energy particles. Since the secondary particle production will scale approximately with the target mass, a particle beam could be used to determine the target mass by monitoring the quantity (and perhaps spectra) of the induced secondary emissions.

In the following sections of this chapter we will first consider in some generality the physics associated with several particle beam weapon approaches, including laser-guided electron beams, neutral particle beams, and other particle beam concepts. We will then return to various system considerations by examining a few specific examples in some detail.

In particular, we will show that beam kinetic energies of hundreds of $\mathrm{MeV}$ are necessary for applications which require long propagation ranges $(\gtrsim 1000 \mathrm{~km})$. We conclude that the accelerator technology base for neutral particle beams is relatively mature. The important issues which require further study are the development of high brightness, continuously-operating, ion sources, the development of appropriate beam transport techniques, stripping cell technology, and precise beam pointing with adequate methods for sensing the beam direction. For laser-guided electron beams, the accelerator technology base is also reasonably mature, there are several laser approaches that appear feasible, and beam steering concepts have been suggested. However, the theoretical scaling laws believed to govern beam propagation (erosion and stability analyses) must be experimentally verified before this concept can be considered a serious option.

\subsection{LASER-GUIDED ELECTRON BEAMS}

Since energetic charged particle beams have great penetrating power and can be easily generated in high current bursts, the lethality of such beams should be very high provided they can be efficiently and stably propagated over useful ranges to militarily-significant targets. In the past, the exoatmospheric use of charged particle beams was dismissed because such beams would be deflected from straight trajectories by the earth's magnetic field (e.g., in $0.3 \mathrm{G}$ the radius of curvature of a $100 \mathrm{MeV}$ electron is approximately ten kilometers). However, recent reports ${ }^{1}$ have contained descriptions of several techniques which have successfully used laser-formed ionization channels to guide intense relativistic electron beams. In this section, we examine 
the requirements and prospects for developing this technology, including a discussion of beam propagation requirements, ionization channel formation and laser technology, electron accelerator technology, beam steering concepts, and target lethality.

\subsubsection{Beam Propagation Physics}

Electron beam propagation in laser-ionized guide channels has now been experimentally demonstrated over distances up to $95 \mathrm{~m}$ (Ref. 2), in a narrow pressure range and various analyses of this propagation mode have appeared. $^{2,3}$ In brief, a laser is used to photoionize a channel in a low-pressure gas background. The subsequent injection of an electron beam pulse into the channel causes rapid space-charge expulsion of the plasma electrons. The residual ion core then focuses and guides the electron beam pulse. As a result, this propagation mode is called the laser-assisted ion-focused regime, or laser-IFR.

This technique has been successfully used to suppress the beam break-up instability in the advanced test accelerator (ATA), permitting operation at the full design parameters. ${ }^{2}$ It has also been suggested as a beam-guiding mechanism for use in recirculating linacs. ${ }^{4,5}$ Here our interest is somewhat different; we will explore the possibilities of using this technique for possible defensive missions in the tenuous upper atmosphere of the earth.

For propagation over significant ranges, the laser-created ionization channel must perform two important functions: (1) prevent beam divergence caused by space charge repulsion and (2) eliminate beam deflection in the earth's magnetic field. Assuming these two basic criteria are satisfied, questions of beam losses from possible erosion processes and beam instabilities must be satisfactorily addressed. Finally, operational limits must be established in order to define various accelerator and laser requirements.

\subsubsection{Equilibrium Guiding}

For an electron beam propagating in vacuum in the absence of external electromagnetic fields, the important field quantities are the radial space-charge field $E_{r}$ and the azimuthal magnetic field $B_{\theta}$ of the beam current. These quantities depend on the beam density $n_{b}$ and velocity $v$ and can be calculated from Gauss's and Ampere's laws. Under the action of these self-fields, solutions of simple beam envelope equations ${ }^{6}$ (equations of motion for the beam radius) indicate that the beam radius will double in a propagation length $1_{d}$, which scales as

$$
1_{\mathbf{d}} \sim \mathbf{r}_{0} \gamma\left(\mathbf{I}_{\mathbf{b}}\right)^{-1 / 2}
$$

where $I_{b}$ is the beam current, $r_{0}$ is the initial beam radius, and $\gamma=\left(1-\beta^{2}\right)^{-1 / 2}$ with $\beta=\mathrm{v} / \mathrm{c}$, where $\mathrm{c}$ is the speed of light in vacuum. To appreciate the variation of the propagation length with beam current, we show in Figure 4.3 the propagation distance versus electron beam current for a number of beam energies assuming a beam radius of $10 \mathrm{~cm}$.

If the electron beam is injected into an ionized plasma channel, the resulting motion of the plasma charges tends to neutralize the beam self-fields, as indicated $^{2}$ in Figure 4.4. The beam space charge will become neutralized by the expulsion of plasma electrons in approximately one plasma oscillation period $\tau_{\mathrm{p}} \sim\left(4 \pi \mathrm{e}^{2} \mathrm{n}_{\mathrm{b}} / \mathrm{m}_{\mathrm{e}}\right)^{-1 / 2}=\left(\omega_{\mathrm{p}_{\mathrm{e}}}\right)^{-1}$, assuming that the beam density and the plasma electron density are approximately equal.

To avoid possible electron-electron streaming instabilities, ${ }^{3}$ one will generally be interested only in cases in which the plasma electron density does not exceed the beam density; however, there is a minimum plasma density necessary for a radial force balance equilibrium. To calculate this density, assume that all plasma electrons have been expelled so that the beam is propagating in a stationary ion background of density, $n_{i}$. The fractional beam electron space charge neutralization is denoted by $\mathrm{f}_{\mathrm{e}}=\mathrm{n}_{\mathrm{i}} / \mathrm{n}_{\mathrm{b}}$. Solving for the radial space charge electric field and the azimuthal magnetic field and substituting these quantities into the equation of motion, yields an expression for the equilibrium radius,

$$
r_{b}^{2}=\frac{\epsilon^{2}}{c^{4}\left(\gamma^{2}-1\right)} \frac{I_{A}}{2 I_{b}\left(f_{e}-1 / \gamma^{2}\right)}
$$

where $\mathbf{I}_{\mathrm{A}}=\beta \gamma \mathrm{mc}^{3} / \mathrm{e}$ is the Alfven current and $\mathrm{I}_{\mathrm{b}}=\pi \mathrm{r}_{\mathrm{b}}^{2} \mathrm{en}_{\mathrm{b}} \beta \mathrm{c}$ is the beam current. The beam emittance $\epsilon$ is a constant which describes the randomness

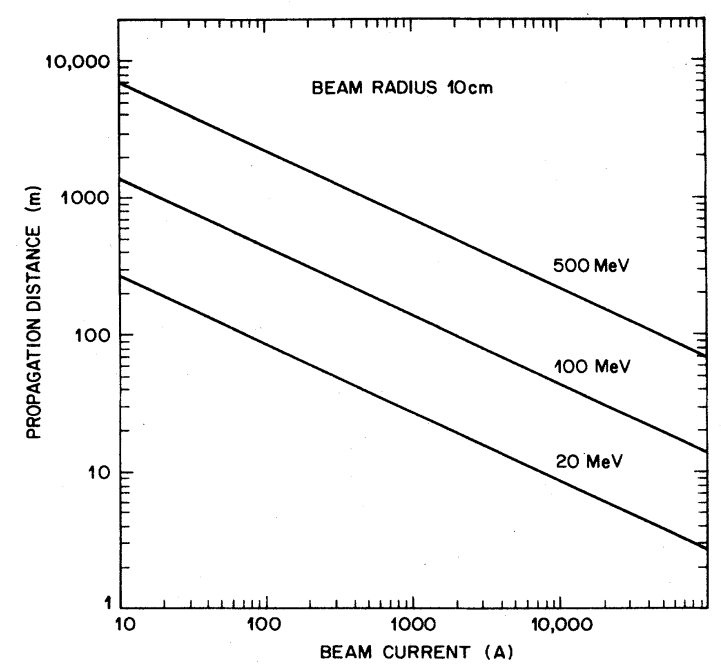

Figure 4.3. Propagation distance as a function of electron beam current for various beam energies assuming a beam radius of $10 \mathrm{~cm}$ [from $\mathrm{Eq}$. (4.2)]. 


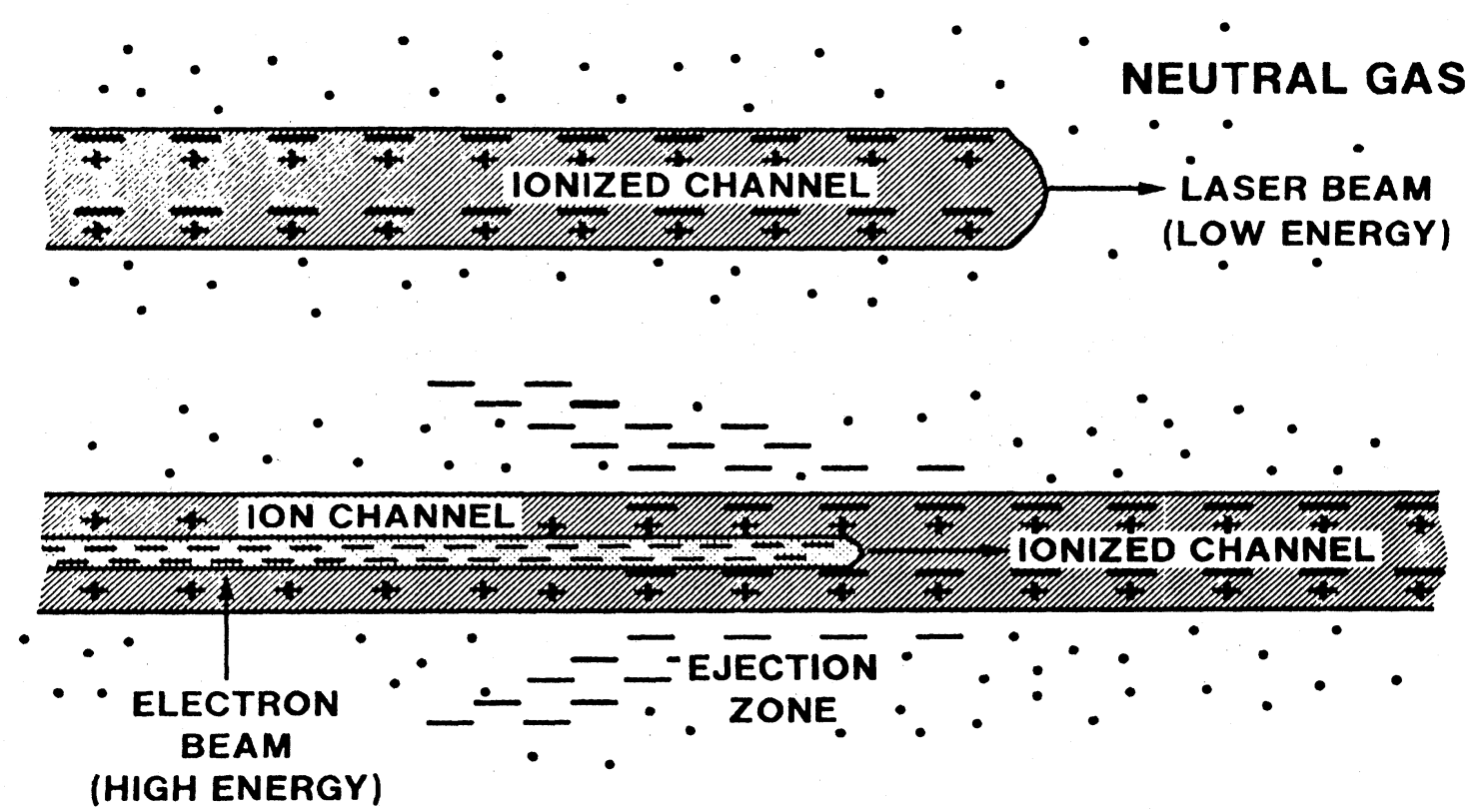

Figure 4.4. Injection of a relativistic electron beam into a plasma channel will cause rapid expulsion of the plasma electrons. The massive, positive ions will guide and focus the electron beam.

of the particle velocities perpendicular to the beam propagation direction. It is apparent that there is no equilibrium unless $f_{e} \geq 1 / \gamma^{2}$. In the limit of large beam kinetic energy, the beam self-fields essentially cancel, and the beam is radially confined by the electrostatic force of the ion channel. In this case, Eq. (4.3) becomes

$$
\mathbf{r}_{\mathrm{b}} \simeq \frac{\epsilon}{\gamma \mathrm{c}^{2}}\left(\frac{\mathrm{I}_{\mathrm{A}}}{2 \mathrm{f}_{\mathrm{e}} \mathrm{I}_{\mathrm{b}}}\right)^{1 / 2}
$$

In addition to preventing beam divergence, the ion channel must strongly reduce the amount of beam deflection in the earth's magnetic field. Assume now that the ion channel is of uniform density $n_{i}$ out to a radius $r_{c}$. In the limit of large $\gamma$, the only forces acting on the beam electrons are the electrostatic ion forces and the deflecting force due to the earth's magnetic field. The net deflecting force is thus

$$
\begin{aligned}
\mathrm{F}_{\mathrm{t}} & =-\mathrm{e}\left[\mathrm{E}_{\mathrm{i}}-\beta \mathrm{B}_{\mathrm{x}}\right] \\
& =-\mathrm{e}\left(2 \pi \mathrm{en}_{\mathrm{i}} \mathrm{y}-\beta \mathrm{B}_{\mathrm{x}}\right),
\end{aligned}
$$

where $B_{x}$ is the earth's magnetic field. Clearly, if $\mathrm{n}_{\mathrm{i}}<\beta \mathrm{B}_{\mathrm{x}} / 2 \pi \mathrm{er}_{\mathrm{c}}$, the electron beam will not be bound to the ion channel. Assuming $0.3 \mathrm{G}$ for the earth's magnetic field and a channel radius of $10 \mathrm{~cm}$, the minimum required density is $\sim 10^{7}$ ions per cubic centimeter. Note that in arriving at this condition, we have taken into account all the ions inside the channel radius. Hence, one may define a new neutralization fraction $f_{N}=n_{i} r_{c}^{2} / n_{b} r_{b}^{2}$, and restate the tracking criterion above:

$$
\mathrm{f}_{\mathrm{N}} \gtrsim \beta^{2} \mathrm{~B}_{\mathrm{x}} \mathrm{r}_{\mathrm{c}} \mathrm{c} / \mathrm{I}_{\mathrm{b}}
$$

\subsubsection{Erosion Phenomena}

Having calculated the zero-order beam equilibrium requirements, we now return to the details of how the positive ion channel becomes established and we examine the behavior of the electrons at the beam front during this process. (The erosion formulas presented in this section were derived in the course of this study. Similar expressions have been derived by other workers in the field. See for example the report by Godfrey et al. ${ }^{7}$ and by Buchanan. ${ }^{7}$ ) At least three significant effects are expected:

(1) emittance-driven beam expansion until $\mathrm{f}_{\mathrm{e}} \sim 1 / \gamma^{2}$

(2) magnetic deflection of the beam until $\mathrm{n}_{\mathrm{i}}>\beta \mathrm{B}_{\mathrm{x}} / 2 \pi \mathrm{er}_{\mathrm{c}}$, and

(3) a loss of energy from beam electrons to the plasma electrons which are expelled.

Each of these processes will cause a loss of beam particles/energy at the beam front (erosion), decreasing the effective pulse length as the beam propagates. We now estimate the magnitude of each of these effects, and derive appropriate "erosion" formulas.

In the case of emittance-driven erosion, the natural scale lengths are the length of beam pulse necessary to establish the force balance criterion and the axial 
propagation distance necessary for the beam to expand outside the channel. Roughly, the rate at which the beam is lost will be given by dividing the first of these scale lengths by the second. Simple estimates give

$$
\left.\frac{\mathrm{dx}}{\mathrm{dz}}\right|_{\text {emittance }} \simeq\left(\frac{2}{\gamma \mathrm{r}_{\mathrm{c}}}\right] \frac{\mathrm{f}_{\mathrm{e}}^{1 / 4} \epsilon}{\mathrm{r}_{\mathrm{b}}^{3 / 2}}
$$

Guiding within the ion channel does not completely remove the problem with bending in the earth's magnetic field until tracking is established. The Study Group considered this problem but is not able to provide any description because of security classification.

The erosion resulting from beam energy loss caused by expelling electrons from the channel can also be simply estimated by calculating the difference in electrostatic potential for an unneutralized beam compared with a beam that is neutralized by the channel ion space charge. Since this difference comes only from the ions, the average electric field acting to slow the beam electrons is just $\Delta \mathrm{V} / 1=\mathrm{E} \simeq\left(\mathrm{I}_{\mathrm{b}} \mathrm{f}_{\mathrm{N}} / \beta_{0} \mathrm{c}\right)(1$ $+2 \ln \mathrm{r}^{*} / \mathrm{r}_{\mathrm{c}}$ ), where $\mathrm{r}^{*}$ is some large radius on the order of tens to hundreds of meters. Consequently, the rate at which beam electrons will lose energy is just $\mathrm{eE} /(\gamma-1) \mathrm{mc}^{2}$, or

$$
\left.\frac{\mathrm{dx}}{\mathrm{dz}}\right|_{\text {energy loss }} \simeq \frac{v \mathrm{f}_{\mathrm{N}}}{(\gamma-1)}\left(1+2 \ln \mathrm{r}^{*} / \mathrm{r}_{\mathrm{c}}\right)
$$

where $v=\mathrm{I}_{\mathrm{b}} /\left(\beta \mathrm{mc}^{3} / \mathrm{e}\right)$ is the dimensionless beam current. The logarithm term is of order 5-10.

The trends exhibited by these erosion rate formulas are indicated in Figure 4.5. The overall erosion rate is minimized for an optimum beam current of about one kiloampere over a fairly wide range of beam kinetic energies. As an example, the erosion rate for a $100 \mathrm{MeV}$ beam is about $0.8 \mu \mathrm{s}$ per $100 \mathrm{~km}$ of propagation distance.

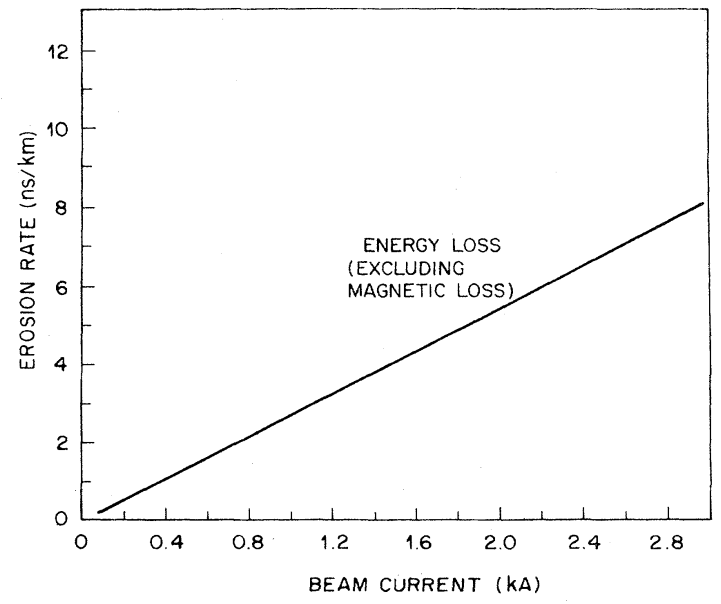

Figure 4.5. Estimates of the various beam erosion rates as a function of beam current for an electron kinetic energy of $100 \mathrm{MeV}$.

\subsubsection{Beam Instabilities}

The beam erosion equations suggest that long propagation ranges can be achieved by simply using long pulses. However, possible growth of various streaming instabilities between the beam electrons and the plasma species may limit the practical pulse length. If the beam itself does not generate significant ionization, then the instability most likely to occur is a transverse streaming instability between the beam electrons and the channel ions. Conventional instability analyses indicate that the characteristic growth time must scale as the ion bounce period, i.e., the time required for an ion to oscillate radially through the beam. In this case, the number of efoldings of (linear) instability growth $\mathrm{N}_{\mathrm{ef}}$ during the pulse length $t_{p}$ should scale approximately as

$$
\mathrm{n}_{\mathrm{ef}} \sim \mathrm{t}_{\mathrm{p}} / \mathrm{r}_{\mathrm{c}}\left(\mathrm{I}_{\mathrm{b}} / \mathrm{m}_{\mathrm{i}}\right)^{1 / 2}
$$

where $m_{i}$ is the ion mass. It is also expected, however, that this instability should saturate nonlinearly if the amplitude of the oscillation should become of the order of the beam radius. As for the cases of the various erosion models, experimental data are essential for the verification of these analytical estimates.

At the higher background neutral densities associated with operation at lower altitudes, it is possible that the beam itself can generate significant ionization. If the condition $f_{N} \gtrsim 1$ occurs late in the pulse, then not all the electrons will be expelled from the channel, and a fast-growing streaming instability between the beam and plasma electrons can rapidly deplete the beam kinetic energy. Straightforward estimates indicate that this phenomenon sets a lower limit for the operational range of this propagation mode.

As a final point, note that the creation of the ion channel does not cause the earth's magnetic field to vanish. What must occur is a small deflection of the electron beam centroid from the centroid of the ion channel. As a result, there will be a constant force leading to a net transverse motion of the ion channel as a whole. While not an instability, this effect should set an upper limit to the maximum allowable pulse length. Together with the erosion formulas, this limit effectively establishes the maximum propagation range.

\subsubsection{Summary}

From these simple analytic estimates, it is possible to set crude allowable parameter ranges for this propagation mode. First, the operational altitude must lie between certain limits because of electron-electron two-stream instability at the lower end, and at the upper end, lack of sufficient residual gas density which, when ionized, would give an ion density sufficient to provide a tracking force in the earth's magnetic field. Also, it is anticipated that 
channel neutralization fractions of order $0.05<\mathrm{f}_{\mathrm{N}}<1.0$ are optimal. Conflicting requirements indicate that minimum beam erosion should occur for beam currents of the order of one kiloampere. With these parameter ranges, the maximum pulse length criterion ( $\leqslant 10 \mu \mathrm{s})$ indicates that propagation ranges of the order of $1000 \mathrm{~km}$ can only be achieved with relatively high beam kinetic energies (hundreds of $\mathrm{MeV}$ ). Correspondingly, for ranges of the order of $100 \mathrm{~km}$, tens of $\mathrm{MeV}$ may be sufficient.

\subsubsection{Laser Technology and Ionization Requirements}

The partially ionized channel needed to guide the electron beam must be provided by laser induced photoionization. The densities of the various constituents of the upper atmosphere are presented in Figure 4.6. To obtain reasonable upper altitude limits, it will be necessary to photoionize atomic oxygen. A simplified energy level diagram of atomic oxygen is shown ${ }^{8}$ in Figure 4.7. The ground state is the spin-split multiplet $(2 \mathrm{p})^{4} 3 \mathrm{P}_{\mathrm{J}}$, at energies of $0,158.5$, and $226.5 \mathrm{~cm}^{-1}$, for $\mathbf{J}=2$, 1 , and 0 , respectively. The number density of atoms available for photoionization is proportional to $2 \mathrm{~J}+1$ times a Boltzmann factor, the product at $800 \mathrm{~K}$ being $0.632,0.284,0.084$ for $\mathrm{J}=2,1,0$. The cross section for direct one-photon ionization of $(2 p)^{4} 3 P_{J}$ at wavelengths less than about $90 \mathrm{~nm}$ is about ${ }^{9}$ $4 \times 10^{-17} \mathrm{~cm}^{2}$. Alternately, photoexcitation of an intermediate level followed by photoionization from that level could be used. One-photon excitation of $(2 p)^{4} 3 P_{\mathbf{J}}$ to the resonant level, $3 \mathrm{~s}^{2} \mathrm{~S}^{0}$, requires ${ }^{10} \lambda=130.22$, 130.48, and $130.60 \mathrm{~nm}$ for $\mathrm{J}=2,1,0$. Two-photon excitation of the ground level to $n p^{3} P_{J}$ or $n f^{3} F_{J}$ requires ${ }^{10}$ specific wavelengths between 225.6 and $185.8 \mathrm{~nm}$ for $3 \leqslant n \leqslant 7$.

Single photon ionization processes are attractive because the ion recombination time is long and the laser energy is relatively independent of laser pulse width. Also, the laser energy requirement is quite modest, with only a few joules being required to ionize a $10 \mathrm{~cm}$ radius

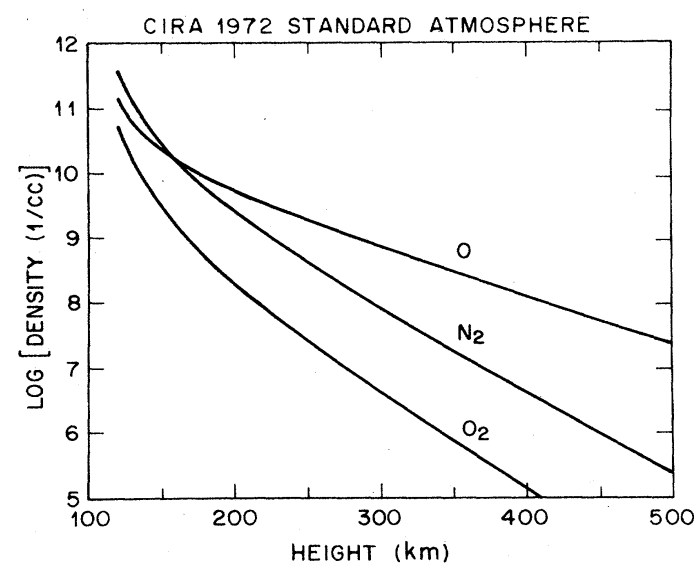

Figure 4.6. Densities of the various atmospheric constituents as a function of altitude.
IONIZATION SCHEMES FOR ATOMIC OXYGEN

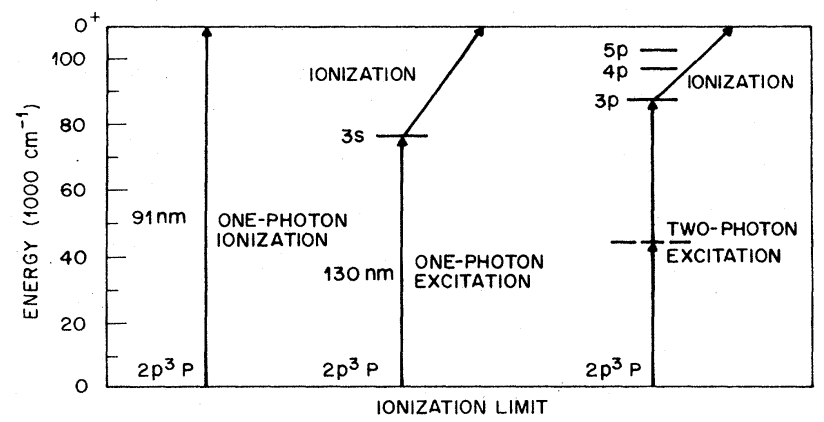

Figure 4.7. A simplified energy level diagram for atomic oxygen.

channel over hundreds of kilometers. However, at present there are no lasers which operate efficiently at these wavelengths, and there are no appropriate optics presently available. As a result, we will not consider this option further, simply noting that short wavelength laser research, if successful, could have benefit for this application.

The prospects for generating $130 \mathrm{~nm}$ light are somewhat better, and several optical sum frequency mixing approaches which might be capable of reasonable efficiencies $(5-10 \%)$ have been suggested. ${ }^{11}$ Because the transition in this case is single-photon allowed, spontaneous radiative decay to the ground state is rapid ( $\sim 2 \mathrm{~ns})$, necessitating short laser pulse widths to prevent depopulation of the excited state. The ionization cross section from the excited state is of the order of $10^{-18} \mathrm{~cm}^{2}$ for $248 \mathrm{~nm}$ light $(\mathrm{KrF})$, and the laser energy requirement for this scheme would probably not be severe $(\sim 100 \mathrm{~J})$. Although there may be problems because of the short wavelengths and optics, this scheme also offers high leverage, and research to evaluate it should be pursued.

The cross section for the two-photon excitation to the $3 \mathrm{P}$ level is of the order ${ }^{12}$ of $0.5-1.0 \times 10^{-17} \mathrm{~cm}^{4} / \mathrm{W}$. An estimate for the photoionization cross section from the $3 \mathbf{P}$ level is $5 \times 10^{-19} \mathrm{~cm}^{2}$. Under the condition of second step saturation, the ratio of the ion density to neutral density is given by

$$
\frac{\mathrm{n}_{\mathrm{i}}}{\mathrm{n}_{0}}=1-\mathrm{e}^{-\mathrm{kI}^{2} \tau}
$$

where $\mathrm{k}=1.25 \times 10^{-4} \mathrm{~W}^{-2} \mathrm{~s}^{-1} \mathrm{~cm}^{4}, \tau$ is the laser pulse width, and $I$ is the intensity of the laser at $226 \mathrm{~nm}$ in $W / \mathrm{cm}^{2}$. This result includes the effects of ground state depletion, but neglects spontaneous emission. For a beam area of roughly $1000 \mathrm{~cm}^{2}$, the required laser energy at few-hundred-kilometer altitudes will be on the order of one kilojoule.

There are a number of schemes for generating the necessary radiation for two-photon ionization. ${ }^{13}$ These processes are expected to be relatively efficient. For example, for a laser pulse width of $30 \mathrm{~ns}$ and an anti- 
Stokes Raman conversion efficiency of $10 \%$, the $\mathrm{KrF}$ laser energy for a range of $200 \mathrm{~km}$ at an altitude of $250 \mathrm{~km}$ is of the order of a few kilojoules. At present, ground-based $\mathrm{KrF}$ lasers with energies of a few tens of $\mathrm{kJ}$ are presently being developed for inertial confinement fusion research; defense research is also underway on high-power excimer lasers. However, a major research and development program would be necessary to define and engineer a laser system that would satisfy full-system constraints. Electron-beam pumped excimer lasers have demonstrated intrinsic efficiencies of $\sim 10 \%$. While it is generally believed that system efficiencies can be increased to the $10 \%$ level, substantial development is needed to accomplish this objective. Such an effort must also include the development of low weight, repetitively-pulsed electron-beam drivers. The above calculations also assume reasonably good beam quality (twice diffraction limited).

\subsubsection{Accelerator Requirements}

The electron accelerator must be capable of supplying a high voltage electron beam of sufficient current and pulse duration to propagate to the target, plus enough additional energy either to kill the target or to produce a large bremsstrahlung signature (in case the application is discrimination). In the case of the latter objective, the kinetic energy of individual beam electrons must be at least $10-20 \mathrm{MeV}$ in order to assure sufficient target penetration for a good target mass measurement. (See Section 7.7.3.)

For target kill, deep penetration into a warhead will require kinetic energies of order $100 \mathrm{MeV}$, and we will assume a nominal lethality criterion of one megajoule of delivered energy per pulse over a nominal $10 \mathrm{~cm}^{2}$ spot size. At $100 \mathrm{MeV}$, electrons will penetrate $30-50 \mathrm{~g} / \mathrm{cm}^{2}$ of almost any material, so the energy deposition per unit mass will be of the order of one kilojoule/gram. Deposition of this amount of energy over short times (microseconds) should be sufficient to cause target destruction by several mechanisms (for example, booster propellant ignition).

In addition to these requirements, the beam propagation phenomenology indicates that the accelerator must produce a beam current of one kiloampere for several microseconds $(\leqslant 10 \mathrm{mC}$ ) at kinetic energies of tens to hundreds of $\mathrm{MeV}$ depending on mission range. Consequently, the energy required per pulse varies from $\sim 200 \mathrm{~kJ}$ to $10 \mathrm{MJ}$. There are several accelerator candidates which might fulfill these requirements, including induction linear accelerators, high current betatrons, and high current rf linac concepts. We will briefly consider each approach.

\subsubsection{Induction Linacs}

Several high current induction linacs (Figure 4.8) have now been developed and successfully accelerate beam currents of nominally tens of kiloamperes to kinetic energies of tens of $\mathrm{MeV}$ for pulse durations of tens of nanoseconds. $^{2,4}$ If this technology is to be used, it is essential that the accelerator not produce a single beam pulse of many microseconds duration, or else the accelerating cavity dimensions become much too large, or the weight of magnetic cores in the accelerating cavities

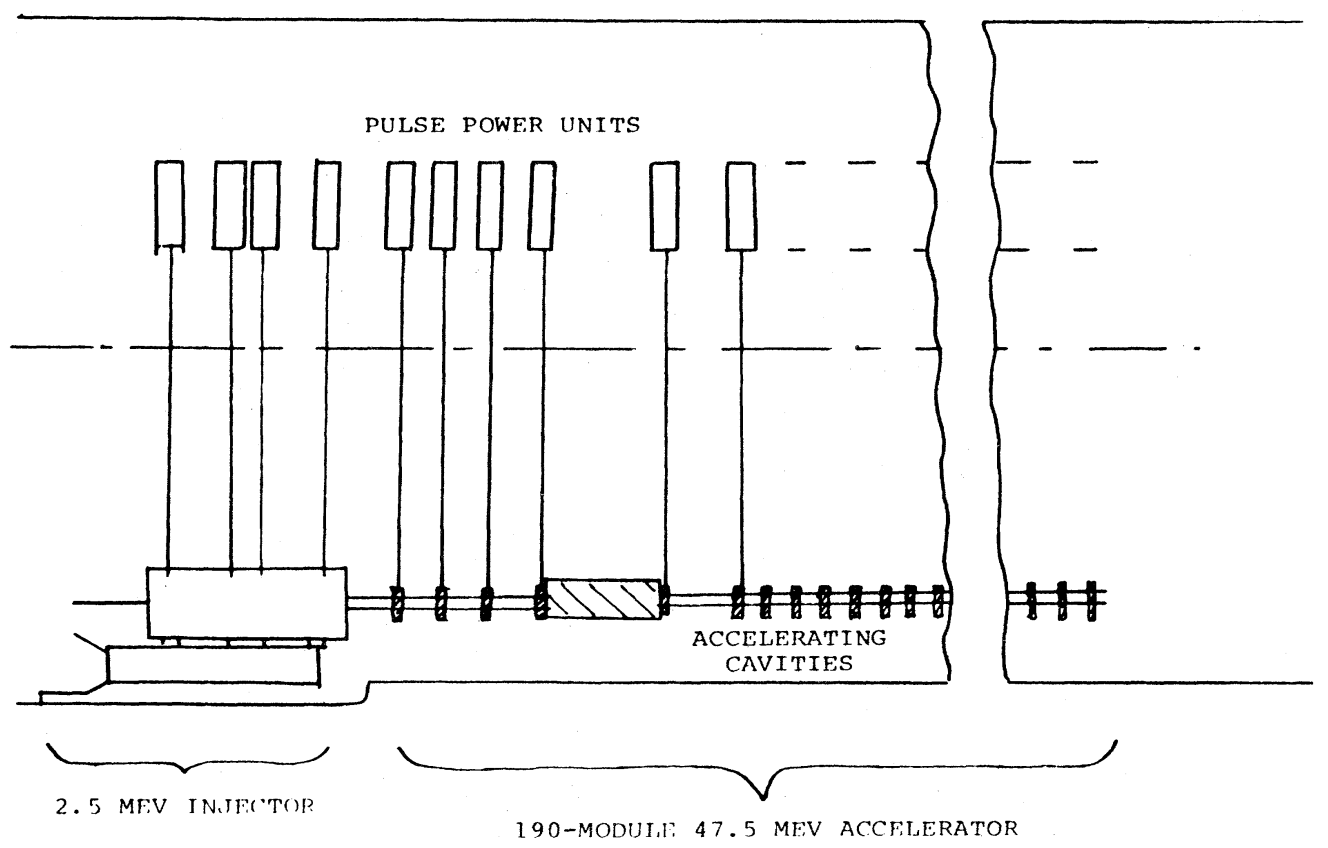

Figure 4.8. Schematic diagram of the Advanced Test Accelerator at Lawrence Livermore National Laboratory. This device delivers $50 \mathrm{MeV}, 10 \mathrm{kA}, 70 \mathrm{~ns}$ pulses of electrons using laser-guided beam transport. 
becomes much too high. To illustrate this point in the case of the latter approach, note that the induction principle requires that the product of the acceleration voltage $V_{a}$ and the acceleration pulse width $\tau$ be equal to the product of the cross-sectional area $\mathrm{A}$ of the magnetic core and the available flux swing $\Delta \mathrm{B}$ set by the hysteresis loop of the magnetic material

$$
\Delta \mathrm{B} \cdot \mathrm{A}=\mathrm{V}_{\mathrm{a}} \cdot \tau
$$

For $\mathrm{V}_{\mathrm{a}}=100 \mathrm{MeV}, \tau=5 \times 10^{-6} \mathrm{~s}$, and $\Delta \mathrm{B}=1 \mathrm{~T}$, the required area is $5 \times 10^{2} \mathrm{~m}^{2}$. Assuming an average gradient of $0.5 \mathrm{MeV} / \mathrm{m}$, the accelerator would be $200 \mathrm{~m}$ in length, and the average radial dimension of the cores would be $2.5 \mathrm{~m}$.

In this case, the mass of just the magnetic material alone would exceed 6000 metric tonnes! Consequently, more promising approaches would break the pulse train into many smaller pulses of a few tens of nanoseconds each with a duty cycle approaching 50\%. Another possibility is to use each accelerating cavity many times by providing a repeating voltage waveform at the accelerating gap and recirculating the beam through the accelerating structure. ${ }^{4}$ In these cases, suitable beam transport techniques, using either quadrupole or solenoidal magnetic field focusing, or perhaps IFR transport, which minimize the growth of accelerator beam instabilities such as transverse beam breakup, must be developed.

\subsubsection{Betatrons}

Betatrons (Figure 4.9) have the potential for development into light-weight accelerators with very high effective gradients because the beam acceleration occurs over very many passes through the same path. However, this fact may also drastically worsen the problem of instability growth, especially at the high beam currents required. Conventional betatron approaches are not well suited for high current applications because of space charge limitations during acceleration at low voltages. As a result, "modified" betatron approaches which use higher voltage injectors $(\mathrm{MeV})$, with special magnetic field configurations to enhance stability, have been suggested. ${ }^{14}$ The feasibility of these is now being investigated experimentally. In addition to the stability issues, methods of efficiently extracting the circulating current over many electron transit times have yet to be developed.

\subsubsection{RF Linacs}

Although accelerating gradients of tens of $\mathrm{MeV} / \mathrm{m}$ are routinely achieved with electron $\mathrm{rf}$ accelerators, on first examination it would appear that they are not wellsuited for high current applications because not enough energy can be stored in the cavities. However, several "direct injection" cavity designs have recently been suggested which might be capable of continuously

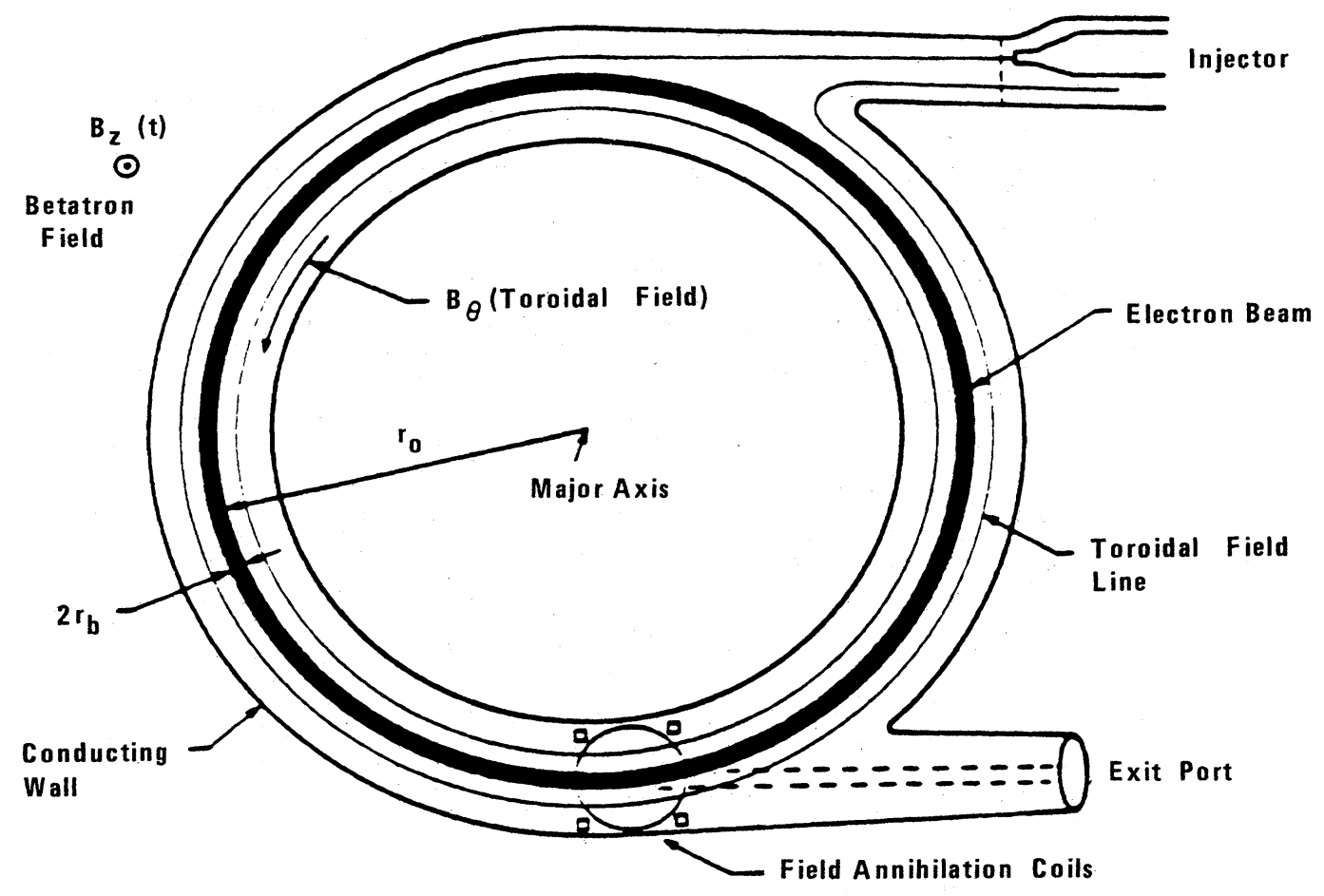

Figure 4.9. Schematic diagram of the modified, high current betatron at the Naval Research Laboratory. Beam confinement experiments on this device have just been initiated. 
replenishing the cavity energy depleted from acceleration of the high current beam. ${ }^{15}$ An important trade-off in this respect is imposed by a maximum rf duty cycle of order $20 \%$. Either the peak current in the bunches must be of the order of five kiloamperes to deliver an average current of one kiloampere, or else the beam kinetic energy must be increased sufficiently to slow the erosion rate by a factor of $\sim 5$ [as seen from Eqs. (4.7)-(4.8) and Reference 7]. (The particular approach depends on the frequency of the rf; at high frequencies the rf period is much less than the electron plasma frequency and the channel electrons will only respond to the average beam current.)

\subsubsection{Summary}

At present there are no accelerators which operate at the desired voltages, current, and pulse durations. Of the various options, only the induction linacs have sufficient operational experience to permit reasonably sound estimates of accelerator weights and volumes. Such analyses indicate that recirculation and/or trains of much shorter pulses are essential for this approach. Betatrons and direct drive rf accelerators offer the potential for more compact, lower weight, high power accelerators, but considerable efforts will be required before these technologies can be considered mature enough to permit reasonable size and weight estimates.

\subsubsection{Beam Steering Concepts}

Conceptually precise electron beam steering $(\leq 1$ $\mu \mathrm{rad})$ can be achieved by coarsely steering the electron beam magnetically into a precisely-aimed laser ionization channel. ${ }^{5}$ Schematically such a system might appear as described in Figure 4.10. The deflection angle $\theta_{\mathrm{d}}$ of an electron in a perpendicular magnetic field $\mathrm{B}$ is given by (mks)

$$
\theta_{\mathrm{d}}=\frac{\mathrm{elB}}{\gamma \mathrm{mc}},
$$

where 1 is the pathlength of the electron in the field. The required (1B) product for deflecting a $100 \mathrm{MeV}$ electron through a $10^{\circ}$ angle is only $0.06 \mathrm{~T} \mathrm{~m}$. The capture angle for the laser channel will be approximately determined by the ratio of the channel radius to the beam radius, multiplied by the channel strength parameter:

$$
\Delta \theta \approx \frac{\mathrm{r}_{\mathrm{c}}}{\mathrm{r}_{\mathrm{b}}}\left(\frac{2 \mathrm{If} \mathrm{f}_{\mathrm{e}}}{\mathrm{I}_{\mathrm{A}}}\right)^{1 / 2}
$$

For $f_{e}=0.1$ and $r_{c} / r_{b}=2$, the acceptance angle for a $100 \mathrm{~A}$ to $10 \mathrm{kA}, 100 \mathrm{MeV}$ electron beam varies from $3^{\circ}$ to $0.3^{\circ}$, respectively. Hence, for this particular example, the precision required of the deflecting magnetic field is only about $10 \%$. Deflection of a $2 \mathrm{kA}, 2 \mathrm{MeV}$ electron beam through a $45^{\circ}$ angle has been experimentally demonstrated using this technique.

While the magnetic field requirements are relatively modest, the problems associated with rapidly retargeting the laser light are stressing. These issues have been considered in detail in other sections and will not be repeated here. Nonetheless, it is important to note that the electron beam does not diverge as it propagates; rather, it erodes. The laser beam, on the other hand, does diverge, and minimizing this effect requires a large diameter mirror at the output telescope of the laser system, especially in the case of the two-photon excitation mechanism for long range applications.

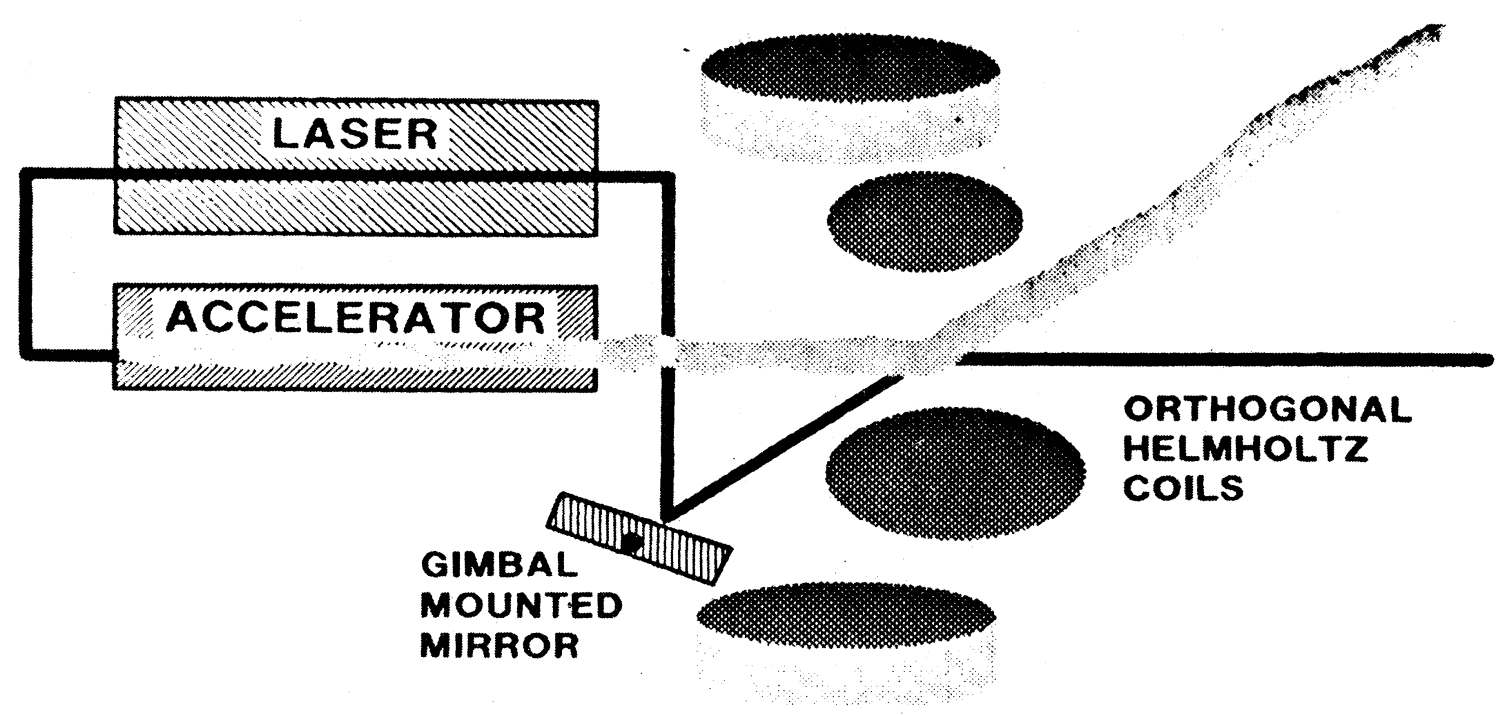

Figure 4.10. A schematic electron beam steering system using crossed laser channels and magnetic field bending. 


\subsubsection{Summary}

In this section we have analyzed the propagation physics of laser-guided electron beams in order to set requirements for the accelerator and laser technologies. It should be stressed that since much of the analysis has not been experimentally verified, there may be considerable uncertainty in these requirements. Nevertheless, at present it appears that substantial technology development must be pursued in order to make this concept viable for defensive missions. In order to warrant this development, this concept must make sense in a systems construct, and be robust against potential countermeasures. These issues are discussed in Chapters 7 and 9.

\subsection{NEUTRAL PARTICLE BEAMS}

Since neutral particle beams (NPBs) can propagate in straight lines across the earth's magnetic field, and still penetrate deeply into a target if the particles are sufficiently energetic, such beams may offer considerable promise for defensive missions provided that the necessary currents and low divergences can be obtained. In this section, we examine the requirements and prospects for developing the technology to produce very high power neutral beams, including a discussion of ion sources, acceleration techniques, beam transport approaches and beam steering, beam neutralization, and beam direction sensing. We will begin with an example using approximate lethality criteria in order to provide some background for discussing the technology requirements.

From Chapter 6, the penetration distance measured in units of $\mathrm{g} / \mathrm{cm}^{2}$ is given approximately by

$$
1\left[\frac{\mathrm{g}}{\mathrm{cm}^{2}}\right)=\frac{3.3 \times 10^{-3} \mathrm{E}^{1.74}}{\mathrm{Z}^{2} \mathrm{~A}^{0.74}}
$$

where $A$ is the mass number, $Z$ is the charge number, and $\mathrm{E}$ is the particle energy in $\mathrm{MeV}$. The power delivered per unit volume of target material is

$$
\mathbf{P}=\frac{\mathrm{IE}}{\pi(\mathrm{R} \Delta \theta)^{2} 1}
$$

where $I$ is the beam current, $E$ is the particle kinetic energy, and $R$ is the distance from the accelerator to the target. As will be shown later, the beam divergence $\Delta \theta$ is energy dependent and is expected to scale approximately as $(E)^{-1 / 2}$. Hence, the specific power divided by the beam power, $\mathrm{P} / \mathrm{IE}$, scales as $\mathrm{E}^{-0.74}$; i.e., lower particle energies are somewhat more efficient from a lethality viewpoint. However, the beam spot size at the target should be roughly comparable to or smaller than the target area to avoid wasting of energy. Also, the particle energy should be sufficiently high to penetrate through the heat shield into the interior of the target. For protons a few hundred $\mathrm{MeV}$ probably is a reasonable compromise between these conflicting requirements.

As discussed in Chapter 6 (compare, in particular, Figure 6.24), lethality criteria range from $1-10 \mathrm{~kJ} / \mathrm{g}$ for material vaporization to $0.01-1 \mathrm{~J} / \mathrm{g}$ for electronics upset. We choose as a numerical example a lethality criterion of $100 \mathrm{~J} / \mathrm{g}$ for massive electronics upset. A beam of 100 to $400 \mathrm{MeV}$ hydrogen atoms has a range ${ }^{16}$ of about 10 to $110 \mathrm{~g} / \mathrm{cm}^{2}$, respectively, as calculated from Equation (4.14) and shown in Figure 6.25. Thus the lethality criterion is equivalent to an incident beam energy fluence of $5 \mathrm{~kJ} / \mathrm{cm}^{2}$. Assuming a beam spread of about one meter at the target, which is comparable to the target size, one requires a total beam energy of

$$
\begin{aligned}
& \mathrm{IEt} \approx 5 \times 10^{7} \mathrm{~J}, \\
& \mathrm{It} \geq 10^{-1} \mathrm{C} .
\end{aligned}
$$

This charge can be delivered by a beam of $100 \mathrm{~mA}$ in one second. It is further noted that a spot size of one meter is obtained at the target range of $1000 \mathrm{~km}$ for a beam divergence $\Delta \theta=10^{-6} \mathrm{rad}$.

In addition to a lethal intercept, a neutral particle beam can also be used to determine the mass of a target, and thus discriminate whether it is a reentry vehicle or a lightweight decoy. At the beam energies suggested above, nuclear interactions between NPB particles and target nuclei are approximately $10 \%$ of the ionization losses in magnitude. Detection of the secondary particles emitted by the struck nuclei would provide the desired mass measurement. This interactive discrimination mechanism is discussed in considerable detail in a later chapter, and will not be discussed further here, except to note that for the particle beam parameters discussed above, the total beam energy (and hence the pulse duration) required to interrogate a single target may be a factor of 10-1000 less than that required for single-target kill.

\subsubsection{Negative Ion Sources}

From the foregoing, it is apparent that high-current, low-divergence negative ion sources will be necessary. It is expected that the minimum divergence attainable will be limited by two sources of beam emittance or transverse beam temperature. These are the emittance resulting from the ion source and the emittance growth during the subsequent beam transport sections (the acceleration, beams optics, and beam steering), and the emittance contribution resulting from the process of removing the extra attached electron (stripping or neutralization). We will estimate the latter effect in order to place a brightness 
constraint on the ion source.

We assume that removal of the additional electron will cause a recoil of the atom that is roughly given by the uncertainty principle, i.e., $\Delta \mathrm{p} \sim \mathrm{h} / \Delta \mathrm{x} \sim \mathrm{h} / 2 \mathrm{a}_{0}$, where $\mathrm{a}_{0}$ is the radius of the negative ion. Using a binding energy of $\sim 0.7 \mathrm{eV}, \mathrm{a}_{0} \sim 10^{-9} \mathrm{~m}$. Normalizing $\Delta \mathrm{p}$ by $\mathrm{p}_{0}=\left(2 \mathrm{~m}_{\mathrm{i}} \mathrm{E}\right)^{1 / 2}$, the forward momentum of the negative ion gives

$$
\Delta \theta=\frac{\Delta \mathrm{p}}{\mathrm{p}_{0}} \sim \frac{15}{(\mathrm{EA})^{1 / 2}} \mu \mathrm{rad}
$$

where $\mathrm{E}$ is the particle kinetic energy in $\mathrm{MeV}$, and $\mathrm{A}$ is the ion mass in atomic mass units. Experiments have been performed to determine the actual value of the numerical coefficient, and verify the energy scaling over the range of $4-800 \mathrm{MeV}$. This relation suggests that as we vary the hydrogen atom energy from $100 \mathrm{MeV}$ to $400 \mathrm{MeV}$ the divergence of the beam will vary from 1.5 $\mu \mathrm{rad}$ to $0.75 \mu \mathrm{rad}$, respectively, provided that very bright, low divergence ion sources, as well as low emittance growth beam transport techniques, can be developed.

It is useful to characterize an accelerated beam by the normalized beam emittance. There are many definitions of this quantity; for the purposes of this discussion, the normalized beam emittance is related to the divergence angle according to

$$
\epsilon=\beta \gamma \mathrm{r} \Delta \theta
$$

where $\beta$ and $\gamma$ are the usual relativistic factors, and $r$ is the beam radius. The utility of this emittance parameter is that it remains constant under ideal conditions for acceleration and beam expansion. Consequently, by assuming no emittance growth in the beam transport processes, the final beam parameters can be used to specify the ion source emittance requirement. For a beam radius of one meter, and $\Delta \theta=10^{-6}$, $\epsilon=0.02 \pi \mathrm{cm} \mathrm{mrad}$.

Penning-discharge negative ion sources ${ }^{17}$ have been extensively studied in the literature. One such source is shown in Figure 4.11; it has delivered 140-180 mA at the design voltage of $22-24 \mathrm{keV}$ for pulse times on the order $^{18}$ of $\sim 700 \mu \mathrm{s}$. The primary emittance limitation for these sources probably arises from the ion energy spread in the source plasma itself, although electrostatic extractor aberrations may also contribute. The measured emittance of this source at currents in excess of the required $100 \mathrm{~mA}$ is $0.024 \pi-0.027 \pi \mathrm{cm} \mathrm{mrad}$. For dc operation electrode heating could be an important consideration.

The negative ion beam must be extracted from the source and drifted to the first acceleration stage. It is well known that emittance can greatly increase in this low energy transport section, although the reasons are not fully understood. Potential problems may be due to the high negative space charge, beam current fluctuations,

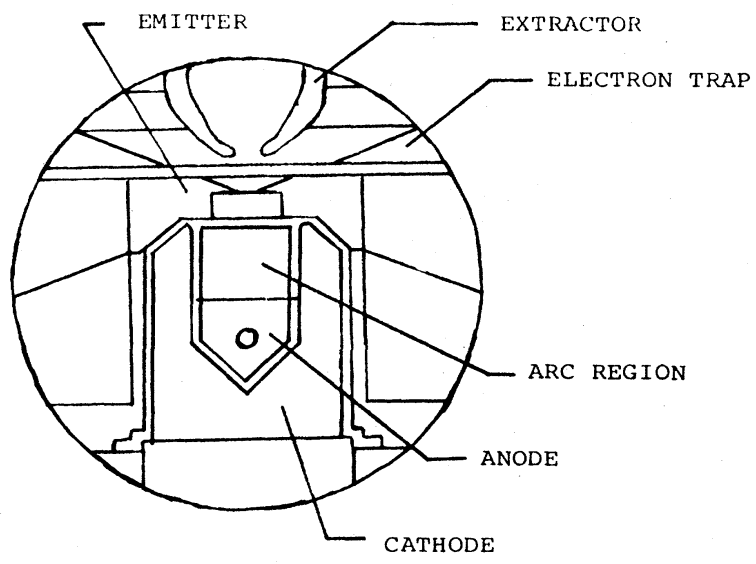

Figure 4.11. A schematic drawing of a Penning-discharge, negative ion source.

and ion acoustic beam plasma instabilities. These problems deserve continued study.

\subsubsection{Acceleration Stages}

Linear radio-frequency ( $\mathrm{rf}$ ) accelerators ${ }^{19}$ have been successfully developed, at ion beam currents of a few hundred milliamperes or less, for a variety of applications ranging from medical uses to high energy particle physics. In these devices, the particles are accelerated by their interaction with an axial electric field of a radio frequency wave which is carefully synchronized with the particle velocity.

In the past, ion linacs have used magnetic focusing in the early rf acceleration stages. However, since the required magnetic field strength varies inversely with particle velocity, it was difficult to package sufficient strength in the available space to account for the $\mathrm{rf}$ defocusing and space charge effects for proton energies less than about $0.5-1 \mathrm{MeV}$. As a result, a large dc Cockroft Walton column injector has been typically used up to that voltage. The abrupt insertion of the beam into the following $\mathrm{rf}$ linac invariably caused emittance growth.

A novel solution to this problem is a Soviet invention called a radio-frequency quadrupole ${ }^{20}$ (RFQ) accelerator. A diagram of this device is shown in Figure 4.12. By carefully designing the vanelike structure, application of $\mathrm{rf}$ power results in continuous, gentle, particle accelerations (much like a surfer riding a wave), plus strong electrostatic focusing, which is independent of particle velocity. This structure also bunches the particles adiabatically (in longitudinal phase space) with minimum degradation of emittance. Experimentation with RFQs is in progress at many accelerator laboratories around the world. At Los Alamos, a beam of $75 \mathrm{~mA}$ has been accelerated to $2 \mathrm{MeV}$ in an RFQ powered by a $413 \mathrm{MHz}$ klystron. The normalized emittance was observed to grow $^{21}$ by a factor of 1.5 . 

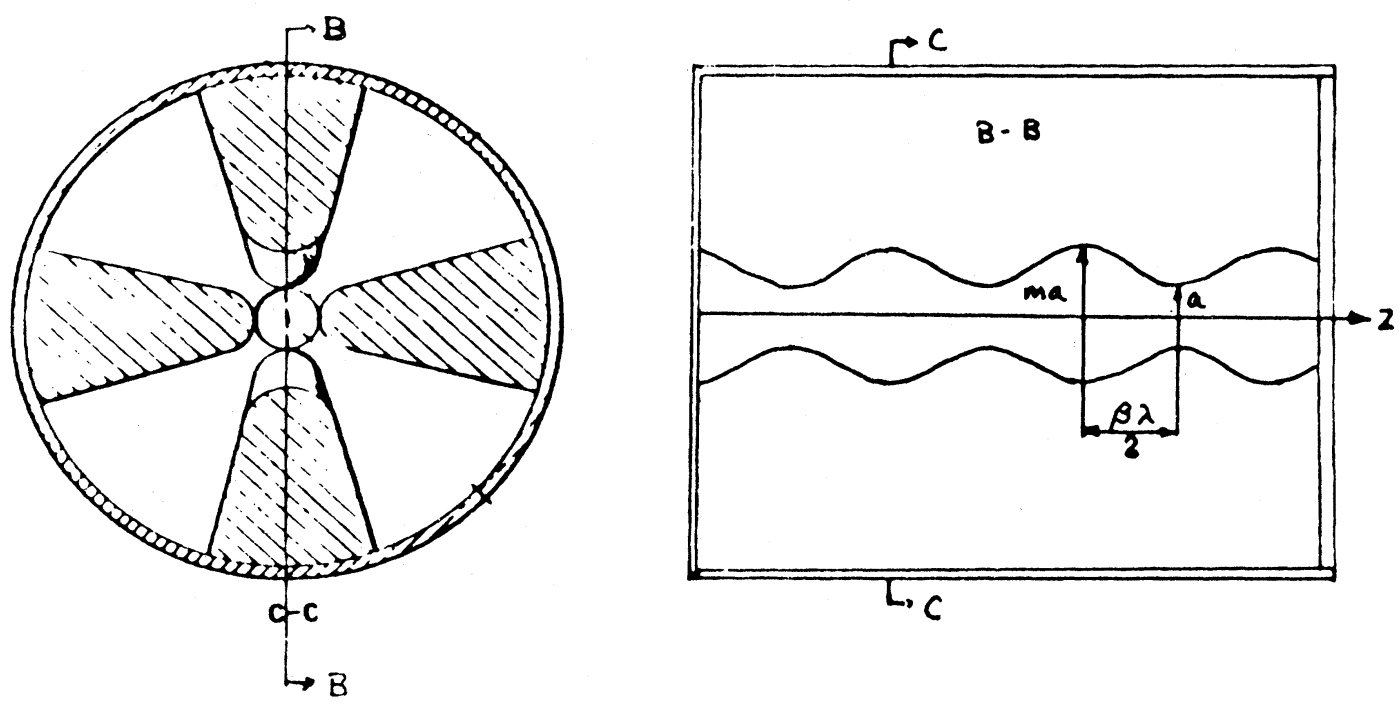

Figure 4.12. A schematic diagram of a radio-frequency quadrupole accelerator section.

After the ions reach energies of a few $\mathrm{MeV}$ in an RFQ, further acceleration to the desired endpoint kinetic energy could be accomplished with a standard drift tube linac $^{19}$ (DTL) (Figure 4.13). These structures consist of drift tubes separated by acceleration gap regions. Particles which arrive at the gaps at the proper phase on the $\mathrm{rf}$ wave are given acceleration impulses. As the electric field of the rf wave reverses, the particles are shielded from deceleration by passing through the drift tubes. They reemerge from the drift tube sections when the rf again reaches the proper phase. Transverse focusing of the beam is usually achieved with magnetic focusing inside the drift tubes themselves. Typical acceleration gradients for such ion linacs are of order $1.5-2 \mathrm{MeV} / \mathrm{m}$, usually for economic reasons. Electric field breakdown (sparking) limits may permit higher gradients.

There is an extensive U.S. technology base for such accelerators in major DOE laboratories (LANL, BNL, LBL, LLNL, and ANL) as well as at major accelerator centers (SLAC, FNAL). Also, there do not appear to be major physics issues associated with these accelerators; rather, the issues are more "engineering" in nature including accelerator weight in orbit, reliability, etc. A particularly key problem is the development of lightweight, compact, rf power sources. At present ${ }^{22}$ such sources are typically klystrons, weighing $1-5 \mathrm{~g} / \mathrm{W}$. For a $100 \mathrm{~mA}, 250 \mathrm{MeV}$ accelerator, the rf power conditioning alone would weigh $\sim 100$ metric tonnes. The use of an emerging solid state rf power technology ${ }^{23}(250 \mathrm{~W}$ transistors with a $40 \mathrm{~V}$ dc input) might reduce the weight to $\sim 0.25 \mathrm{~g} / \mathrm{W}$. Solid state sources have been operated up to $250 \mathrm{~kW}$, but these have not been engineered to be light weight. [The general subject of power sources and conditioning is treated in more detail (in Chapter 8).]

\subsubsection{Beam Expansion and Steering}

In order to keep the magnetic focusing elements in the DTL to reasonable sizes and weights, the transverse dimensions of the beam in the accelerator should be relatively small $(\leq 1 \mathrm{~cm})$. As a result, the beam divergence angles in the accelerator will be very much greater than $1 \mu \mathrm{rad}$, implying a certain amount of tolerance to guide field errors. In order to achieve final beam divergences of the order of 0.75 to $1.75 \mu \mathrm{rad}$, however, the beam will have to be expanded considerably (perhaps a factor of $\geq 100$ ) outside of the accelerator. This expansion must be achieved with very linear beam handling elements in order to minimize chromatic and spherical aberrations (and, hence, emittance growth). While there do not appear to be any serious physics limitations to designing such large-bore magnetic optics, their construction and testing remain open issues.

After expansion, the beam must be directed to the target, again using magnetic optics. The beam pointing accuracy must be at least as accurate as the beam divergence, viz., $0.75-1.75 \mu \mathrm{rad}$. These bending magnets must also have large apertures, although the slewing can be done electrically (rapidly). As for the beam expansion optics, extreme care must be taken to avoid spherical or chromatic aberrations which could cause emittance growth or aiming errors. Also, very precise control of the current sources used to deflect the beam must be maintained.

\subsubsection{Beam Neutralization}

It was previously indicated that removal of the extra electron could be achieved with a theoretical divergence 


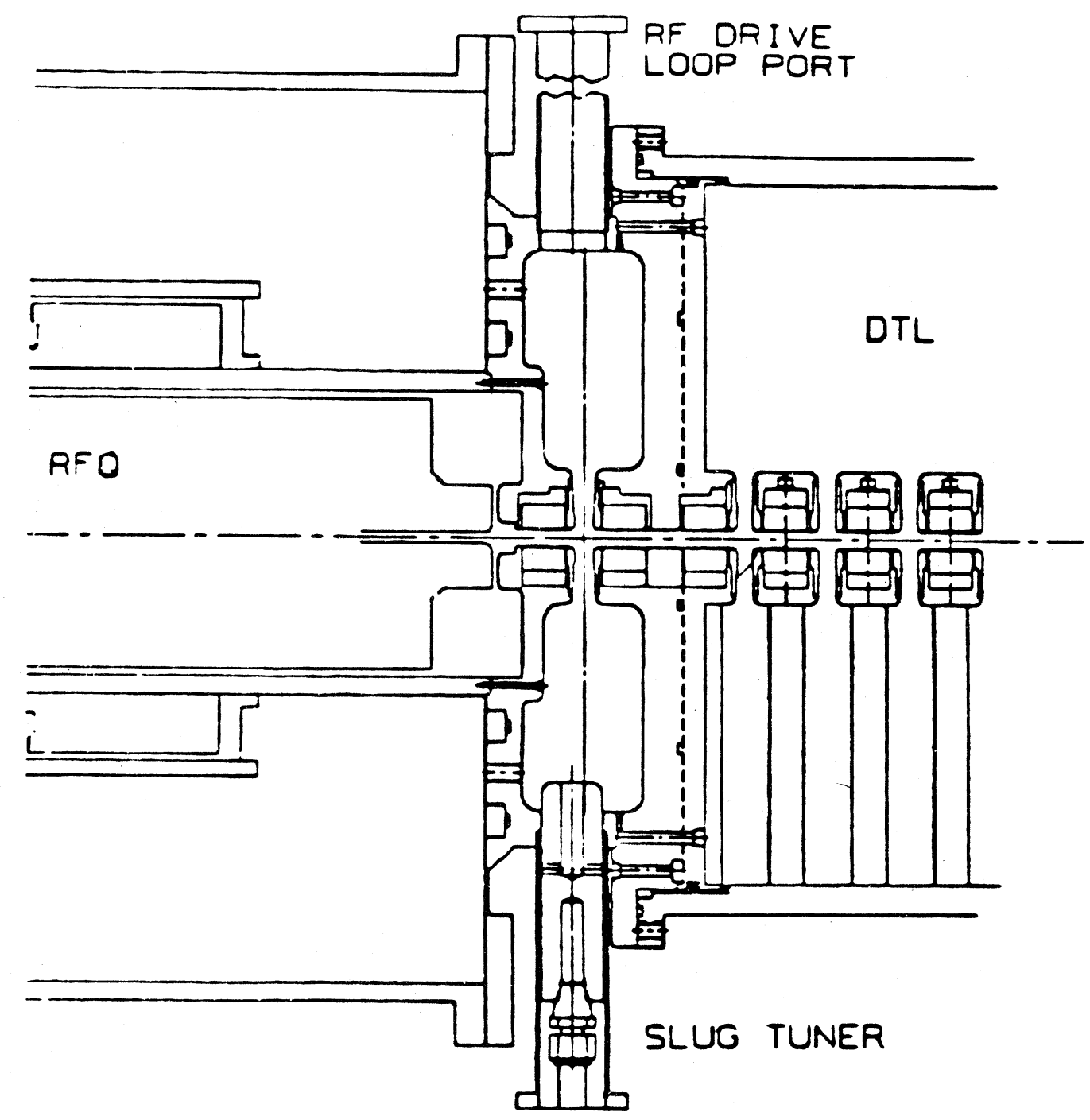

Figure 4.13. The interface connections for a standard drift tube linac (DTL) accelerator section matched to an RFQ section.

increment of the order of $1 \mu \mathrm{rad}$. There are at least three techniques that might be used for this stripping process: very thin foils, gas cells, and photodetachment. In the first technique, passage of the negative hydrogen ion through a thin foil would result in stripping both electrons unless the foil were extremely thin $(\sim 100 \mathrm{~nm}$ for $\mathrm{H}^{-}$at $250 \mathrm{MeV}$ is the optimal thickness of a graphite foil).

Stripping in a gas neutralization cell will undoubtedly work, with efficiencies perhaps exceeding $50 \%$, i.e., $>50 \%$ of the injected negative ions will emerge as atoms, with the remainder of the particles either being stripped twice to positive ions, or not being stripped at all. For $\mathbf{H}^{-}$beams of the order of $100 \mathrm{MeV}$, gas pressures of $\sim 1$ torr over cell lengths of a few meters are probably reasonable. However, it will not be an easy task to engineer such a system for a space-based platform. Since the gas flow cannot be confined by foils, the gas flow must be directed across the negative ion flow. Also, it may be necessary to collect and condense the gas at temperatures $\lesssim 20 \mathrm{~K}$, in order to avoid high-voltage breakdowns on the weapon platform, and inadvertent ionization of the neutral atom beam. The latter will become important when the integrated line density approaches $10^{18}-10^{19} / \mathrm{cm}^{2}$, for example, a $100 \mathrm{~m}$ radius cloud of $10^{14} / \mathrm{cm}^{3}$ gas density. (This problem may be a very serious consideration for effluents produced by chemical combustion power sources.)

Since the photodetachment cross section has a broad maximum $^{24}$ centered around $800 \mathrm{~nm}$, a Nd:YAG laser (4\% efficient) should be a suitable light source. Because of the small cross section $\left(\sim 4 \times 10^{-17} \mathrm{~cm}^{2}\right)$, a high power optical beam in a resonator configuration (Figure 4.14 ) is probably required. The axial mean free path for an ion to undergo a photon collision that will detach the electron should be roughly the beam diameter, 


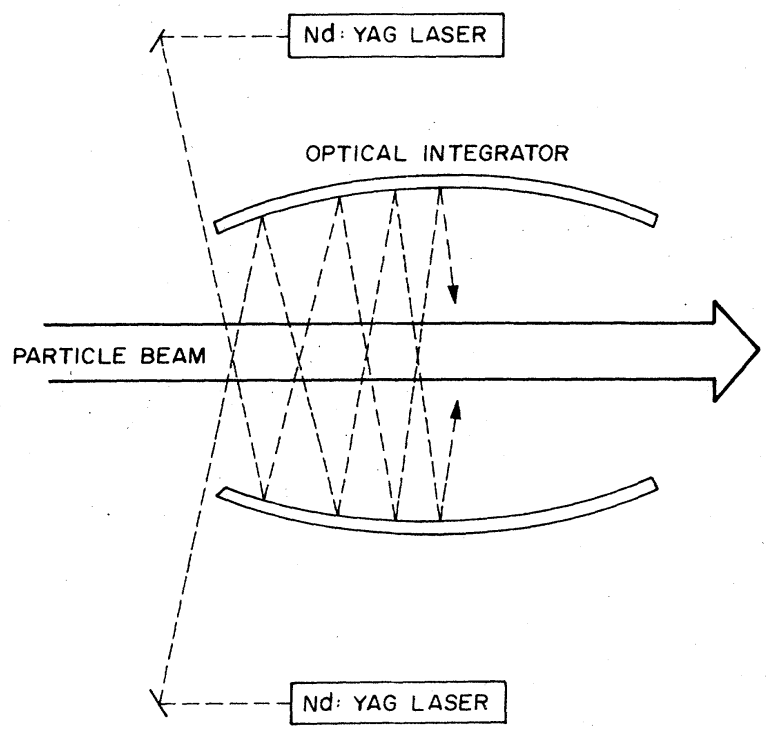

Figure 4.14. Resonator cavity for a high-power, laser stripping cell for a neutral particle beam.

$(\mathrm{r} \approx 1 \mathrm{~m})$. From this we obtain a photon density $\sim 2.5 \times 10^{14} \mathrm{~cm}^{-3}$, or an intensity (inside the resonator) of $\approx 1 \mathrm{MW} / \mathrm{cm}^{2}$, which is roughly the injected laser power. As very little power $(\sim 1 \mathrm{~W})$ is removed from the optical beam by photoabsorption, the mirror losses must be very small. The actual requirement depends on the resonator geometry and the ratio $c / v_{i}$. A mirror absorption of $10^{-4}$ to $10^{-5}$ appears necessary, which is a very demanding requirement! Hence, while it may be possible to achieve $\sim 100 \%$ neutralization by photodetachment, the demands on the optical surfaces are severe, and a high power laser system $(\sim 25 \mathrm{MW})$ is required. The actual technology has not been demonstrated. Photodetatchment schemes appear to be more appropriate for space-based high power (killer) applications. Finally, the laser stripper technique does not produce a significant population of hydrogen atoms in the excited $2 S$ state. Consequently, the beam sensing techniques which rely on laser induced $2 \mathrm{~S} \rightarrow 3 \mathrm{P}$ transitions will not work (see next subsection).

\subsubsection{Sensing Direction for Neutral Particle Beams}

It is necessary to sense the direction of the beam of neutrals, in order to develop a control system to steer and align the neutral particle beam accurately with an acquisition, tracking, and pointing (ATP) optical system. One technique that appears promising is based on the fact that approximately $7 \%$ of the neutral atoms emerging from a gas stripper cell are expected to be in the metastable $2 \mathrm{~S}$ state. ${ }^{25}$ By using a laser to induce $2 \mathrm{~S} \rightarrow 3 \mathrm{P}$ transitions and observing the subsequent rate of decay to the ground state, the beam direction can be determined. For example, the frequency of the laser light in the beam frame will be Doppler-shifted to

$$
v_{0}=\nu_{\mathrm{L}} \gamma(1-\beta \cos \theta),
$$

where $v_{\mathrm{L}}$ is the laser frequency and $\theta$ is the angle between the neutral beam and the laser beam. $\beta$ and $\gamma$ are the usual relativistic factors for the neutral beam. For a fixed $v_{\mathrm{L}}$, the variation of the ground state transition fluorescence can be directly related to $\theta$. By varying $v_{\mathrm{L}}$ to give the maximum transition rate (and decay rate), the angle $\theta$ can be determined accurately. The sensitivity to variations in the beam kinetic energy can be minimized by operating near the angle $\theta_{0}=\cos ^{-1} \beta$, while the sensitivity of the angular measurement, $\mathrm{d} \theta / \mathrm{d} v$, varies as $\left(\beta \gamma v_{\mathrm{L}} \sin \theta\right)^{-1}$.

The $2 \mathrm{~S} \rightarrow 3 \mathrm{P}$ transition involves the fine structure $\left(3 \mathrm{P}_{1 / 2,3 / 2}\right)$ of the upper state, $\Delta v_{1 / 2,3 / 2}$. Measurements show that the fine structure is well resolved for $\Delta \theta \sim 10 \mu \mathrm{rad}$. Some measurements ${ }^{26}$ which have achieved an accuracy of $\pm 250 \mu \mathrm{rad}$ are shown in Figure 4.15; experiments to demonstrate higher accuracies are reportedly in progress.

\subsubsection{Summary}

In this section we have analyzed the various components of a neutral particle beam system, beginning with a discussion of beam interaction phenomena, in order to establish kinetic energy, beam current, divergence requirements, etc. The results of these analyses indicate that particle beam weapon platforms

COMPARISON OF BEAM SENSING THEORY WITH VAN DE GRAAFF EXPERIMENTAL DATA

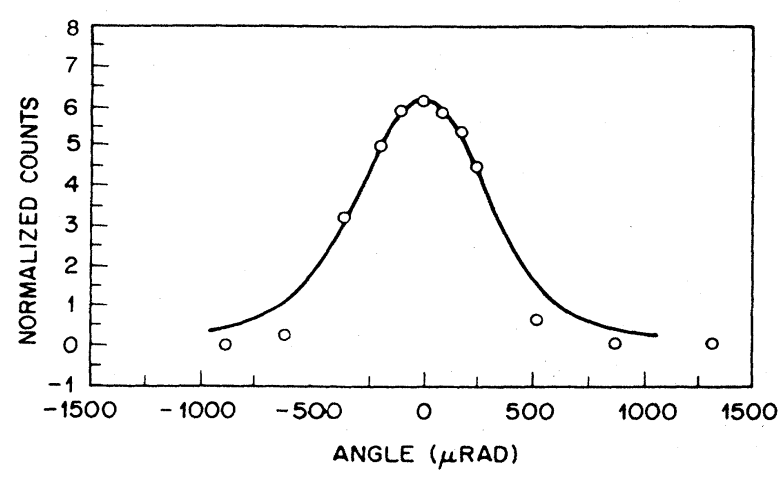

BACKGROUND SUBTRACTED DATA (POINTS) BEAM4 CALCULATION (SOLID LINE)

Figure 4.15. Experimental data from beam sensing measurements indicating the achievement of $\pm 250 \mu \mathrm{rad}$ pointing accuracy. 
must be space based, and must have particle kinetic energies of a few hundred $\mathrm{MeV}$ to deliver lethal fluence to ranges in excess of $1000 \mathrm{~km}$.

In support of the neutral particle beam approach, much progress has been made in recent years in the development of high brightness negative ion sources and the low energy acceleration section (the radio-frequency quadrupole), although further improvements will be necessary to minimize emittance growth. Use of a drifttube linac as the major acceleration section appears relatively straightforward. To meet the microradian class divergence requirement substantial beam expansion will be necessary; development of large bore magnetic optics (for focusing and steering) with the necessary tolerances are designed, but have not been demonstrated experimentally. From a physics viewpoint, beam neutralization with minimal divergence $(0.75-1.75 \mu \mathrm{rad})$ appears to be feasible, although practical stripping cells for use in the space environment must be developed. Finally, a promising approach for sensing the direction of beam propagation has been developed, although the required accuracy has yet to be demonstrated. Further, this beam sensing technique does not work if the photodetachment process is used for beam neutralization. In summary, the beam voltage and duty cycle must each be increased by two orders of magnitude, while keeping the normalized emittance at the lowest level currently attained. Required pointing accuracy to microradian class or better $(1 \mathrm{~m}$ target at a range of $1000 \mathrm{~km})$ remains to be established in combination with rapid retargeting rates. Furthermore, NPB weapons require space-based operations, with concomitant stressing requirements on weight, space power supplies and power conditioning. These issues are further discussed in Chapter 8 .

\subsection{OTHER PARTICLE BEAM CONCEPTS}

\subsubsection{Massive, Energetic Ions}

The radius of curvature of a charged particle in a transverse magnetic field is given by

$$
\mathrm{r}=\frac{\gamma \mathrm{mv}}{\mathrm{eB}}
$$

Hence, massive ions could cross the earth's magnetic field without large deflections if they were sufficiently energetic. A deflection of one meter over a distance of $1000 \mathrm{~km}$ corresponds to a radius of curvature of about $5 \times 10^{11} \mathrm{~m}$. Assuming $0.3 \mathrm{G}$ for the earth's magnetic field indicates a necessary particle momentum of $\mathrm{p}=\gamma \mathrm{mv}=2.4 \times 10^{-12} \mathrm{~kg} \mathrm{~m} / \mathrm{s}$. For singly ionized $\mathrm{U}^{238}$, a relativistic $\gamma$ of $2 \times 10^{4}$ is required!

An alternate approach is the use of charged macroparticles. For a velocity of $100 \mathrm{~km} / \mathrm{s}$, a particle mass of greater than $2.4 \times 10^{-17} \mathrm{~kg}$ is necessary.
Schemes which would use magnetically-insulated electrostatic accelerators for such macroparticles have been suggested; ${ }^{27}$ however, these ideas are very new and will require much study (of the magnetic insulation process, of accurate pointing methods, etc.) before a serious evaluation is possible.

It has also been suggested that energetic positive ion beams might be guided by laser ionization channels. This seems somewhat improbable; ion channel guiding of electron beams occurs because the massive ions of the channel are relatively immobile and can easily attract the lighter beam electrons.

\subsubsection{Charge- and Current-Neutralized Ion Beams (Plasmoids)}

It has also been suggested that it might be possible to create a charge-neutral beam by combining separately accelerated high current electron and ion beams of equal densities to form an intense beam of neutral atoms. Recombination can be described by

$$
\frac{\mathrm{dn}}{\mathrm{dt}}=-\alpha \mathrm{n}_{\mathrm{i}} \mathrm{n}_{\mathrm{e}}
$$

with $\alpha$ the total recombination coefficient. For equal initial densities, $n_{i}=n_{e}=n_{i 0}$, the free charge will decay with time as

$$
\mathrm{n}_{\mathrm{i}}=\frac{\mathrm{n}_{\mathrm{i} 0}}{1+\alpha \mathrm{n}_{\mathrm{i} 0} \mathrm{t}}
$$

Hence, the rapid formation (few microseconds) of such beams requires a large recombination coefficient and high plasma densities. Very low temperatures are also required since the divergence angle of the neutral atom beam will be approximately $\Delta \theta \sim\left(\mathrm{kT} / \mathrm{E}_{\mathrm{k}}\right)^{1 / 2}$. (For $100 \mathrm{MeV}$ ions with a transverse temperature of $1 \mathrm{eV}$, $\Delta \theta \gtrsim 10^{-4}$.) For a proton/electron plasma, $\left(\alpha \mathrm{n}_{\mathrm{i} 0}\right)^{-1}$ is plotted in Figure 4.16 as a function of density for several different temperatures, assuming radiative and collisional (three-body) recombination processes. $^{28}$ To reach the necessary neutralization time requires very high densities $\left(>10^{14} / \mathrm{cm}^{3}\right)$ and very low temperatures $(\lesssim 0.1 \mathrm{eV})$. Since the current state of the art is about $10^{12} / \mathrm{cm}^{3}$ at tens of electron volts, this concept does not appear attractive.

The existence of astrophysical jets which can be extremely long (thousands of light years) and stable, ${ }^{29}$ suggests that it might be possible to produce clouds of neutralized plasma which would propagate across the earth's magnetic field without deflection in a self-pinched propagation mode. A basic limitation is due to the plasma virial theorem, ${ }^{30}$ however, which states that any finite collection of electromagnetic fields and particles cannot be in stress balance in steady state equilibrium; i.e., the cloud must expand. Hence, novel ideals involving radial confinement of a central plasma core at the expense 


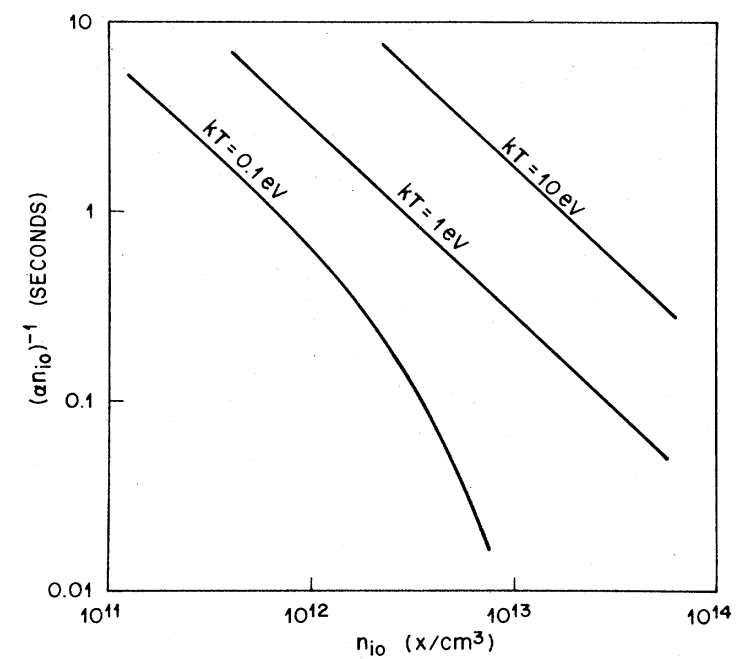

Figure 4.16. Recombination times for a proton/electron plasma as a function of initial density for several different temperatures.

of axial erosion, or expansion of some outer cylinder have been suggested. ${ }^{31}$ At present, however, these ideas are somewhat speculative, and we will not consider them further.

\subsection{SYSTEMS REQUIREMENTS SUMMARY}

In addition to the basic technology issues discussed in the preceding sections, there are several systems level issues that must be examined in some detail, including weight, size, and primary power requirements. Further, for systems consisting of orbiting satellite constellations, additional issues including heat dissipation and cryogen storage, component lifetime in the space environment, and maintenance and testing in orbit, must be considered. We will now examine some of these issues in the context of specific examples, including a neutral particle beam used for boost phase intercept, and a laser-guided electron beam used as a pop-up discriminator in late mid-course.

\subsubsection{NPB For Boost Phase Intercept}

As before, we will assume that deposition of $100 \mathrm{~J} / \mathrm{g}$ will ensure a hard, verifiable, booster kill. The resultant beam divergence $\Delta \theta_{\mathrm{r}}$ will have two important contributions, including source and accelerator emittance $\left(\Delta \theta_{\mathrm{a}}\right)$ and collisional stripping to the atomic state $\left(\Delta \theta_{\mathrm{s}}\right)$. An estimate of the latter was given as Equation (4.18).

Since, $\Delta \theta_{\mathrm{r}} \approx\left(\Delta \theta_{\mathrm{a}}^{2}+\Delta \theta_{\mathrm{s}}^{2}\right)^{1 / 2}$, large efforts to reduce $\Delta \theta_{\mathrm{a}}$ below the minimum $\Delta \theta_{\mathrm{s}}$ will not have large benefits, and for our scoping purposes, we will assume that $\Delta \theta_{\mathrm{a}} \approx \Delta \theta_{\mathrm{s}}$. In this case,

$$
\Delta \theta_{\mathrm{r}} \approx \frac{20}{(\mathrm{EA})^{1 / 2}} \mu \mathrm{rad}
$$

Hence, a $250 \mathrm{MeV} \mathrm{H}^{-}$beam will have a divergence angle of $\sim 1.3 \mu \mathrm{rad}$, while $335 \mathrm{MeV} \mathrm{D}^{-}$(higher energies are required to achieve the same in-target range) will have a divergence of $\sim 0.8 \mu \mathrm{rad}$. Assuming a stripping cell efficiency of $\sim 50 \%$, the total charge and total energy of the beam emerging from the accelerator required for a single target kill (at $100 \mathrm{~J} / \mathrm{g}$ ) is presented in Figure 4.17 as a function of range. For ranges of the order of a few thousand kilometers, the accelerator must deliver a charge of $\geq 1 \mathrm{C}$, and total beam energies of the order of a few hundred megajoules per target. If the nominal beam current is $\sim 100 \mathrm{~mA}$, then the required pulse time will be approximately $10 \mathrm{~s}$, and the average power requirement will be approximately $100 \mathrm{MW}$, assuming conversion efficiencies of $50 \%$. Issues associated with providing these power and energy levels are discussed in Chapter 8.

Since the NPB will be sited on a space platform, dissipation of the waste heat from the accelerator ( $\sim 50 \mathrm{MW}_{\mathrm{t}}$ during engagements) will be necessary. Because passive dissipation would require enormous radiators, it has been suggested that liquid hydrogen (which would also be used with liquid oxygen as the power source) could be used for removal of the heat. Fifty $\mathbf{M W}_{\mathrm{t}}$ corresponds to the evaporation of hundreds of liters per second of liquid hydrogen $(450 \mathrm{~J} / \mathrm{g})$, which is 1-2 orders of magnitude higher than the amount of fuel necessary for the generation of electrical power $\left(10^{4} \mathrm{~J} / \mathrm{g}\right)$. Also, careful control of any effluents will be necessary since only $\sim 10 \mu \mathrm{g} / \mathrm{cm}^{2}$ of gaseous medium will ionize the neutral particle beams.

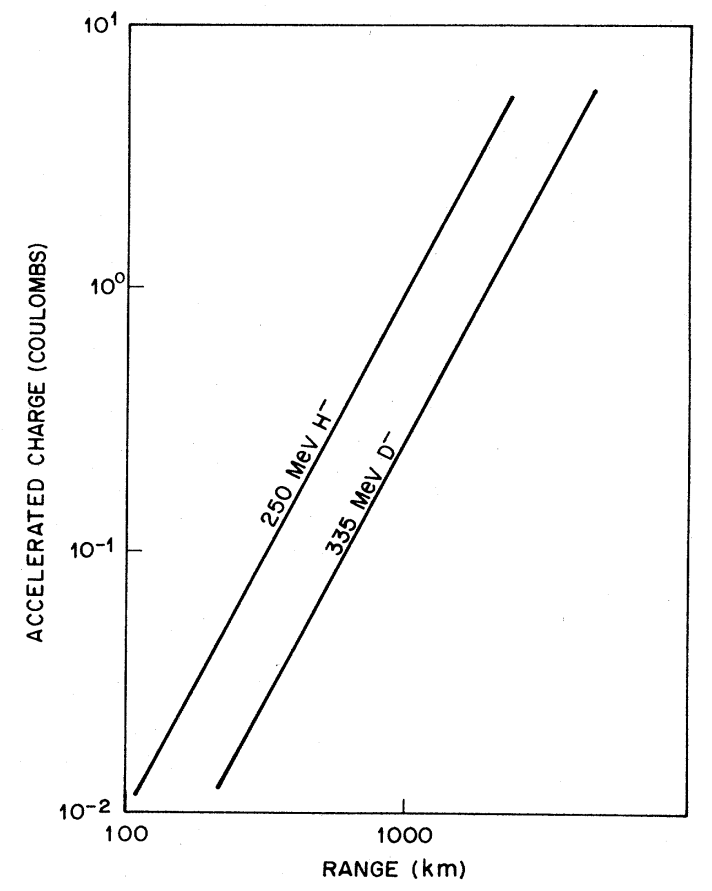

Figure 4.17. Total accelerated charge and neutral particle beam energy required for a single target kill (at $100 \mathrm{~J} / \mathrm{g}$ ) as a function of target range. 
On the basis of the constellation sizing estimates of Appendix B.1, for ranges of a few thousand kilometers, the number of NPB satellites required for full-earth coverage will be of the order of 100. Assuming reasonable advances in $\mathrm{rf}$ power generation technology, platform weights of about 100 tonnes are probably achievable, implying total constellation weights of $\sim 10^{4}$ tonnes. For comparison purposes, the present space shuttle payloads are limited to about 20 tonnes; the shuttle's solid rocket boosters, however, are capable of launching about 100 tonnes into low earth orbit.

The NPB accelerator approach is founded on a good technology base. Nevertheless, these rf ion accelerators are ground based and generally require a lengthy start-up period followed by occasional tuning by highly trained personnel. Thus, the reliability of a space-based NPB platform in an untended environment must be an important design criterion from the outset; space qualifying such hardware elements will surely increase costs. Moreover, years of in-orbit placement will stress system maintainability and may necessitate periodic refueling if $\mathrm{LH}_{2} / \mathrm{LO}_{2}$ is used, for example.

As a final point, the NPB accelerator platform is only one element of a system which must include an acquisition subsystem which accomplishes hand-over from the surveillance system and verifies the assigned target, a coarse tracker to establish track files, and a fine tracker which may have to illuminate and image the target. All of these functions, as well as the accelerator platform controls, may have to operate in a severe nuclear-disturbed environment, implying the necessity for radiation-hardened electronics and sensors. The issues associated with the incorporation of a NPB accelerator platform into a strategic defense system are discussed further in Chapters 7-9.

\subsubsection{Laser-Guided Electron Beam for Mid-Course Discrimination}

As a second example, we will consider a rocketborne pop-up discriminator for late mid-course which uses laser-guided electron beam technology. In general, pop-up platforms probably have some advantages in terms of survivability and maintenance because they do not have to be in orbit for many years. However, they necessarily will have time-line restrictions and more limited battle/engagement space. (The general subject of particle beam discrimination is treated in more detail in Chapter 7.)

Assuming ranges of a few hundred kilometers, and the desire for penetrating at least $10 \mathrm{~g} / \mathrm{cm}^{2}$, beam kinetic energies of a few tens of $\mathrm{MeV}$ at a few $\mathrm{kA}$ for pulse durations of several microseconds are probably required. Hence, several tens of kilojoules of beam energy will be necessary for each target interrogation. Assuming that one such rocket platform must be capable of discriminating all the objects (100-1000) carried by a single heavy booster, the total stored energy is approximately 100-1000 MJ. Assuming battery storage, for example, the total battery weight would be about $\leq 100 \mathrm{~kg}$. Because the discriminator is not space based, its engagement time will be limited to a few hundred seconds, implying an average power capability of about a megawatt with retarget times of $0.1-1.0 \mathrm{~s}$. The weight of the power conditioning alone for this approach will probably be of the order of one metric tonne. Assuming accelerator gradients of a few $\mathrm{MV} / \mathrm{m}$, the accelerator will be about ten meters in length. Depending on the approach, the laser used to form the guide channel could also weigh $1-2$ tonnes. Hence, a total platform weight of 5-10 metric tonnes could be anticipated.

\subsection{SUMMARY}

In this chapter, we have examined the physics issues associated with the use of energetic particle beams for defensive missions in the space environment. At present, there are two primary approaches including a space-based neutral particle beam and a laser-guided intense electron beam. Both types of particles interact strongly with matter, depositing their energy deeply within the target; if sufficiently intense, such beams can be quite lethal.

In the NPB approach, a low divergence beam of negative ions is generated and accelerated in a radiofrequency acceleration structure to energies $\geq 100 \mathrm{MeV}$. The beam is then magnetically expanded, aimed, and focused at a distant target. Finally, the extra attached electron is stripped, and the beam of neutral atoms propagates in straight-line trajectories to the target. The minimum beam divergence is expected to scale as $(\mathrm{EA})^{-1 / 2}$, where $\mathrm{E}$ is the particle kinetic energy, and $\mathrm{A}$ is the atomic mass number. For $\mathrm{E}=100$ to $400 \mathrm{MeV}$ and $\mathrm{A}=2$, a final beam divergence of approximately 1.75 to $0.75 \mu \mathrm{rad}$ may be achievable assuming minimal emittance growth in the low energy acceleration and transport sections. The accelerator technology base for NPB devices is firmly established and relatively mature, and can meet the necessary beam parameter requirements on a pulsed basis at low duty cycles in the laboratory. Consequently, the important engineering issues are the development of high brightness ion sources that operate continuously (not pulsed), the development of lowemittance-growth beam transport techniques at relatively high currents (100 mA), stripping cell technology, and precise beam pointing with adequate methods for sensing the beam direction. A program to investigate these issues is in progress, led by the Los Alamos National Laboratory and several defense contractors. Of course, as with all space-based approaches, there are many system level issues that must be resolved before deploying a weapon system based on NPB technology. These include the development of lightweight, efficient, rf power sources, methods of heat dissipation, effluent control, 
communications with surveillance elements, and routine maintenance functions on orbiting platforms. Programs which address these issues are being planned. Assuming reasonable technical progress, especially in rf power source development, NPB platform weights of 100 tonnes are probably achievable.

In the laser-guided electron beam approach, the electrons are accelerated to the desired energy and magnetically aimed. To prevent deflection by the earth's magnetic field, a laser is used to create a plasma channel which guides and focuses the beam as it propagates to the target. In contrast with the neutral particle beam approach, the development of laser-guided beam concepts is relatively new. While these ideas have been successfully used to transport beams in high current induction linacs, as well as in beam recirculation experiments, there is scant experimental data available to confirm simple theoretical scaling laws governing the propagation physics.

\subsection{CONCLUSIONS}

1. Neutral particle beam (NPB) accelerators operating at the necessary current levels $(\geq 100 \mathrm{~mA}$ ) must be scaled up by two orders of magnitude in voltage and duty cycle with no increase in normalized beam emittance. The required pointing accuracy and retargeting rate remain to be achieved. These devices must be based in space to avoid beam loss via atmospheric interactions.

2. Energetic electron beams require propagation in laser-created plasma channels in order to avoid beam deflection in the earth's magnetic field; this restricts the operational altitude at the low end by beam instability and at the high end by ion density starvation. We estimate that booster kill applications require a scale-up in accelerator voltage by at least one order of magnitude, in pulse duration by at least two orders of magnitude, and in average powers by at least three orders of magnitude. Active discrimination applications require scale-up in pulse duration by at least two orders of magnitude, and in average power by at least two orders of magnitude. The lasers required for the creation of plasma channels must be developed. We estimate that the propagation distances must be increased by at least four orders of magnitude.

\section{REFERENCES}

${ }^{1}$ W. E. Martin, G. J. Caporaso, W. M. Fawley, D. Prosnitz, and A. G. Cole, Phys. Rev. Lett. 54, 685 (1985); G. J. Caporaso, F. Rainer, W. E. Martin, D. S. Prono, and A. G. Cole, ibid. 57, 1591 (1986).

${ }^{2}$ D. S. Prono et al., IEEE Trans. Nucl. Sci. NS-32, 3144 (1985).
${ }^{3}$ S. L. Shope, C. A. Frost, G. T. Leifeste, C. E. Crist, P. D. Kiekel, J. W. Poukey, and B. B. Godfrey, IEEE Trans. Nucl. Sci. NS-32, 3092 (1985).

${ }^{4}$ R. B. Miller, IEEE Trans. Nucl. Sci. NS-32, 3149 (1985).

${ }^{5}$ C. A. Frost, S. L. Shope, R. B. Miller, G. T. Leifeste, and C. E. Crist, IEEE Trans. Nucl. Sci. NS-32, 2754 (1985).

${ }^{6}$ R. B. Miller, An Introduction to the Physics of Intense Charged Particle Beams (Plenum, New York, 1982).

${ }^{7}$ B. B. Godfrey, B. S. Newburger, L. A. Wright, and M. M. Campbell, Mission Research Corporation Report AMRC-R741 (November 1985); H. Lee Buchanan, "Electron Beam Propagation in the Ion-Focused Regime," Phys. Fluids 30, 221-231 (1987).

${ }^{8}$ S. Bashkin and J. O. Stoner, Atomic Energy Levels and Grotian Diagrams 1 (North-Holland, Amsterdam, 1975).

${ }^{9}$ A. K. Pradham and H. E. Saraph, J. Phys. B 10, 3365 (1977).

${ }^{10}$ W. L. Weiss, M. W. Smith, and B. M. Glennon, At. Trans. Prob. 1, 178 (1969).

${ }^{11}$ D. Reilly, private communication, AVCO-Everett Research Laboratory, 1984.

${ }^{12}$ W. K. Bischel, B. E. Perry, and D. R. Crosley, Appl. Opt. 21, 1419 (1982).

${ }^{13}$ T. R. Lorie, R. C. Sze, and D. L. Barker, Appl. Phys. Lett. 31, 37 (1977).

${ }^{14}$ P. Sprangle, C. A. Kapetanakos, and S. J. Marsh, in Proc. Fourth Intl. Conf. High Power Electron and Ion Beams (Palaiseau, 1981).

${ }^{15} \mathrm{~J}$. Bayless, private communication, Pulsed Science Inc. (1985).

${ }^{16}$ See, for example, P. Marmier and E. Sheldon, Physics of Nuclei and Particles (Academic, New York, 1969), p. 165.

${ }^{17} \mathrm{P}$. Allison and J. D. Sherman, in Production and Neutralization of Negative Ions and Beams, Brookhaven, 1983, edited by K. Prelec (AIP Conf. Proc. No. 111) (AIP, New York, 1984), pp. 511-518.

${ }^{18}$ H. V. Smith, Jr., P. Allison, and J. D. Sherman, IEEE Trans. Nucl. Sci. NS-32, 1797 (1985).

${ }^{19}$ M. S. Livingston and J. P. Blewitt, Particle Accelerators (McGraw-Hill, New York, 1962).

${ }^{20}$ I. M. Kapchinskii and V. A. Teplyakov, Pub. Tekh. Eksp. 2, 19 (1970).

${ }^{21}$ O. R. Sander, F. O. Purser, and D. P. Rusthoi, Proc. 1984 Linac Conf., Gesellschaft fur Schwerimenforschung GSI-84-11, 54 (1985).

${ }^{22}$ G. Faillon, IEEE Trans. Nucl. Sci. NS-32, 2945 (1985).

${ }^{23}$ D. R. Vaughan, G. E. Mols, D. W. Reid, and J. M. Potter, IEEE. Trans. Nucl. Sci. NS-32, 2857 (1985).

${ }^{24}$ S. J. Smith and L. M. Branscomb, J. Res. Nat. Bur. Stand. 55, 165 (1955).

${ }^{25}$ G. Rohringer, General Research Corp. Rept. CR-1-773, (1977).

${ }^{26}$ D. B. Holtkamp, R. S. Biddle, D. D. Chamberlain, J. D. King, R. J. Martinez, C. R. Quick, R. K. Sander, S. L. Wilson, and V. Yuan, Los Alamos Natl. Lab. Rept. LAUR 85-2925, (1985).

${ }^{27}$ J. P. Van Devender, private communication, Sandia National Laboratories (1985).

${ }^{28}$ D. R. Bates and A. Dalgano, Atomic and Molecular Processes, edited by D. R. Bates (Academic, New York, 1962), p. 245.

${ }^{29}$ J. O. Burns and R. Marcus, Sci. Am. 249, 56-66 (1983).

${ }^{30}$ C. L. Longmire, Elementary Plasma Physics (Interscience, New York, 1963), p. 68.

${ }^{31}$ T. R. Lockner, R. J. Lipinski, and R. B. Miller, SAND 850917 (December, 1985). 


\section{Chapter 5}

\section{BEAM CONTROL AND DELIVERY}

\section{CONTENTS}

5.1 Introduction

5.2 Large Mirrors and Phased Arrays

5.2.1 Background

5.2.2 Status of Technology

5.2.3 Scaling

5.2.4 Phase Locking of Laser Arrays

5.2.5 Conclusions

5.3 Relay Optical Systems in Space

5.3.1 Optical Concepts

5.3.2 Optical Layout

5.3.3 Energy Losses

5.3.4 Rapid Retargeting

5.3.5 Error Flowdown

5.4 Atmospheric Propagation and Adaptive Optics

5.4.1 Absorption and Scattering

5.4.2 Atmospheric Turbulence

5.4.3 Distortion Compensation

5.4.4 Atmospheric Propagation Physics

5.4.5 Phase Compensation System

5.4.6 Experimental Results and Major Problems

5.4.7 Phase-Conjugation by Nonlinear Optical Techniques

5.4.8 Thermal Blooming

5.4.9 Stimulated Raman Scattering

5.4.9.1 Introduction

5.4.9.2 Comparison Between Theory and Experiment of Stimulated Raman Scattering in $\mathbf{N}_{2}$

5.4.9.3 SRS in the Atmosphere

5.4.9.4 Possible Remedies

5.4.9.5 Conclusions

5.4.10 Atmospheric Propagation of High-Intensity X-Ray Pulses

5.5 High-Power Components

5.5.1 Cooled Deformable Mirrors

5.5.2 High-Power, Shared-Aperture Components

5.5.2.1 Cooled Beamsplitters

5.5.2.2 Buried Gratings

5.5.2.3 Membranes

5.5.3 High-Power Laser Coatings

5.5.3.1 Coating at Various Laser Wavelengths

5.5.3.2 Advanced Deposition Processes

5.6 Integration of Components

5.6.1 Pointing and Tracking

5.6.2. Integration

5.7 Multiplicity of Optical Components

5.7.1 Fighting Mirrors (Mission Mirrors)

5.7.2 Optical Relay Subsystems

5.7.3 Multiplicity of GBL Systems

5.8 Conclusions

References

\subsection{INTRODUCTION}

The laser weapon delivery system is a complicated optical system consisting of many elements, the main ones being the laser source, beam expanding and launching optics, relay optics, and mission (fighting) optics. The basic configuration of the system is shown, simplistically, in Figure 5.1. Each main component is in itself a composite system. For example, the launching optics consist of the primary aperture (single or segmented), the wavefront sensor and deformable mirror (adaptive optics) for correction of atmospheric distortions, and the tracking sensors using radiation that is transmitted back toward the laser.

The configuration of Figure 5.1 is general in the sense that it applies to both ground-based systems with space-based relay optics and space-based systems with/without relay optics. Naturally, in the space-based mode, there is no intervening atmosphere, and therefore the necessity to compensate for its optical aberrations does not exist. On the other hand, as in the ground-based case, the system's fine-control (pointing and tracking) is accomplished by the optical return from targets that are illuminated with lasers dedicated to fine target tracking, or by the weapon device itself.

For the system as a whole to perform satisfactorily, each of the components must operate well. In this review, we discuss, one at a time, the basic system components and their associated technologies, and assess the progress to date in comparison with the goals and requirements for ballistic missile defense (BMD). We also assess, where possible, the promise of established solutions as well as of new technical and scientific developments for bridging the gap between the present level of development and the necessary goals.

For example, in that regard one very challenging aspect of pointing and tracking of large effective aperture optical systems is the requirement for very rapid retargeting over large angles. Based on brightness

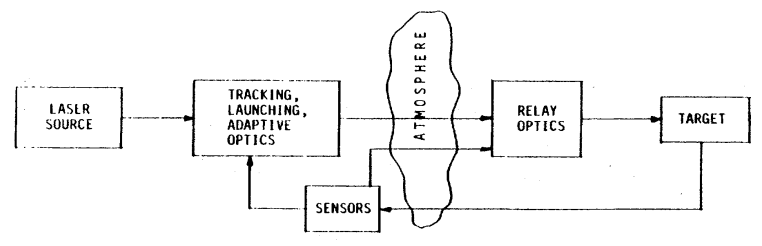

Figure 5.1. Schematic of a laser weapon delivery system. 
calculations in Chapter 3, the output optical system typically may have a total effective aperture of 10-40 m. Such a dimension requires a collection (array) of individual telescopes whose primaries might be as large as 4-8 m. It becomes extremely challenging to steer such large telescopes rapidly between targets with retargeting times of $<1 \mathrm{~s}$ over angles that may be as large as $20^{\circ}$ (for a typical target distance of $1000 \mathrm{kM}$ and size of the launch field). If the larger numbers above apply the solution to such a problem is achieved not through order of magnitude (or more) improvements of gimbals, bearings, torquers, and structures, but by invoking arrays of substantially smaller telescopes all dynamically phased to provide a coherent output wavefront. Rapid beam pointing/retargeting is then achieved not by swinging the whole array, but by slewing each of the telescopes in the array as in a venetian blind while maintaining overall aperture phasing.

It has been suggested that relay optics required by ground-based laser (GBL) systems may be "popped up." The low earth orbit relay mirrors have the advantage of smaller sizes and therefore may in principle have survivability advantages. Proposals for various means for popping up and developing such mirrors remain at conceptual stage.

Once the elements and components are optimized, it is necessary to consider system configuration and integration. Any optical system consists of optical components of several sizes. Individual telescope primaries might be as large as $4-8 \mathrm{~m}$ in diameter, and because the optical power density on such large optics is sufficiently low, they do not have to be cooled. On the other hand, the device and transfer optics are usually substantially smaller, typically $20-60 \mathrm{~cm}$ in dimension. Such optics, because of the power density that they have to handle, have to be cooled for the dissipation of the absorbed energy so that thermally driven optical aberrations are minimized. There may also be intermediate size optics $(\sim 1 \mathrm{~m})$ such that might be needed as tertiaries in wide field of view telescopes. They may or may not need to be cooled, depending upon the details of design and configuration.

Finally, we also discuss the integration of the various subsystems into the final system.

\subsection{LARGE MIRRORS AND PHASED ARRAYS}

\subsubsection{Background}

Space-based laser (HF/DF laser) systems for BMD missions require aperture sizes of $10-40 \mathrm{~m}$ in diameter. Such sizes pose challenges, particularly in fabrication, that are best met by phased arrays.

A monolithic primary mirror, which is the classic approach, is limited in size by the characteristics and quality of optical materials used, by fabrication techniques and equipment, by problems of coating over very large surfaces, and by cost. Current mirror fabrication facilities in this country generally reach limitations at the 4-m size for high-quality, lightweight mirrors. In the near future, this may be extended to 8 m. The largest high-quality space-weight mirror that has been fabricated in this country to date is the 2.4-m Hubbel Space Telescope primary mirror, which took six years to fabricate at a total system cost of approximately $\$ 1.2$ billion $^{1}$ in 1984 dollars. Not including development costs, the primary mirror itself cost approximately $\$ 5.5$ M. The largest mirror to be coated with a multilayer dielectric stack to withstand high-power loading was a 1.8-m-diameter primary mirror.

System design must also consider the effects of direct sunlight and differential thermal loading. A 20-m diameter mirror, with a focal length of $2500 \mathrm{~km}$, deviates from perfect flatness by only $20 \mu \mathrm{m}$. Differential expansion from solar heating can cause much greater distortion. Again, this is a matter of system design, but the remedy will increase system weight, complexity, and cost.

One alternative is to assemble a primary mirror made up of a number of panels (segments), which are phased into a single representative surface through active techniques. A 4-m-diameter segmented telescope mirror, ${ }^{2}$ shown in Figure 5.2, is presently being assembled. Torque considerations limit such approaches to diameters of $8-10 \mathrm{~m}$.

The next, and most promising, approach is to use phased arrays of smaller telescopes, analogous to microwave radars except that phasing tolerances are not millimeters but angstroms. A phased array is a group of telescopes designed to function as a single, coherent transmitting and/or receiving aperture. Figure 5.3 shows two possible configurations of phased arrays. Each telescope in the array has independent optical capabilities and produces a far-field intensity $\mathrm{I}$. When $\mathrm{N}$ telescopes are phased, the resulting far-field axial intensity is $N^{2} I$. Arrays can be operated in a totally phased mode for highest brightness, or subsets of telescopes within the array can be independently pointed and phased for simultaneous operation of a number of different object field locations. The former mode is most useful for longrange target negation, whereas the latter mode is attractive for the simultaneous negation of multiple targets at a closer range.

As implied above, the time and cost of fabricating a phased array is less than that of fabricating an equivalent monolithic mirror. An empirically derived equation for the time required to fabricate a high-quality spherical mirror from a suitable ultra-low-expansion material is (based on experience with several spherical telescope mirrors in the $0.5 \mathrm{~m}$ to $2.4 \mathrm{~m}$ diameter sizes and for $f$ numbers from 1.5 to 3.0 )

$$
\mathrm{t}(\text { months })=10 \mathrm{D}^{3.2} /\left(\mathrm{f}^{2} \sigma\right),
$$

where

$$
\mathrm{D}=\text { aperture diameter }(\mathrm{m})
$$




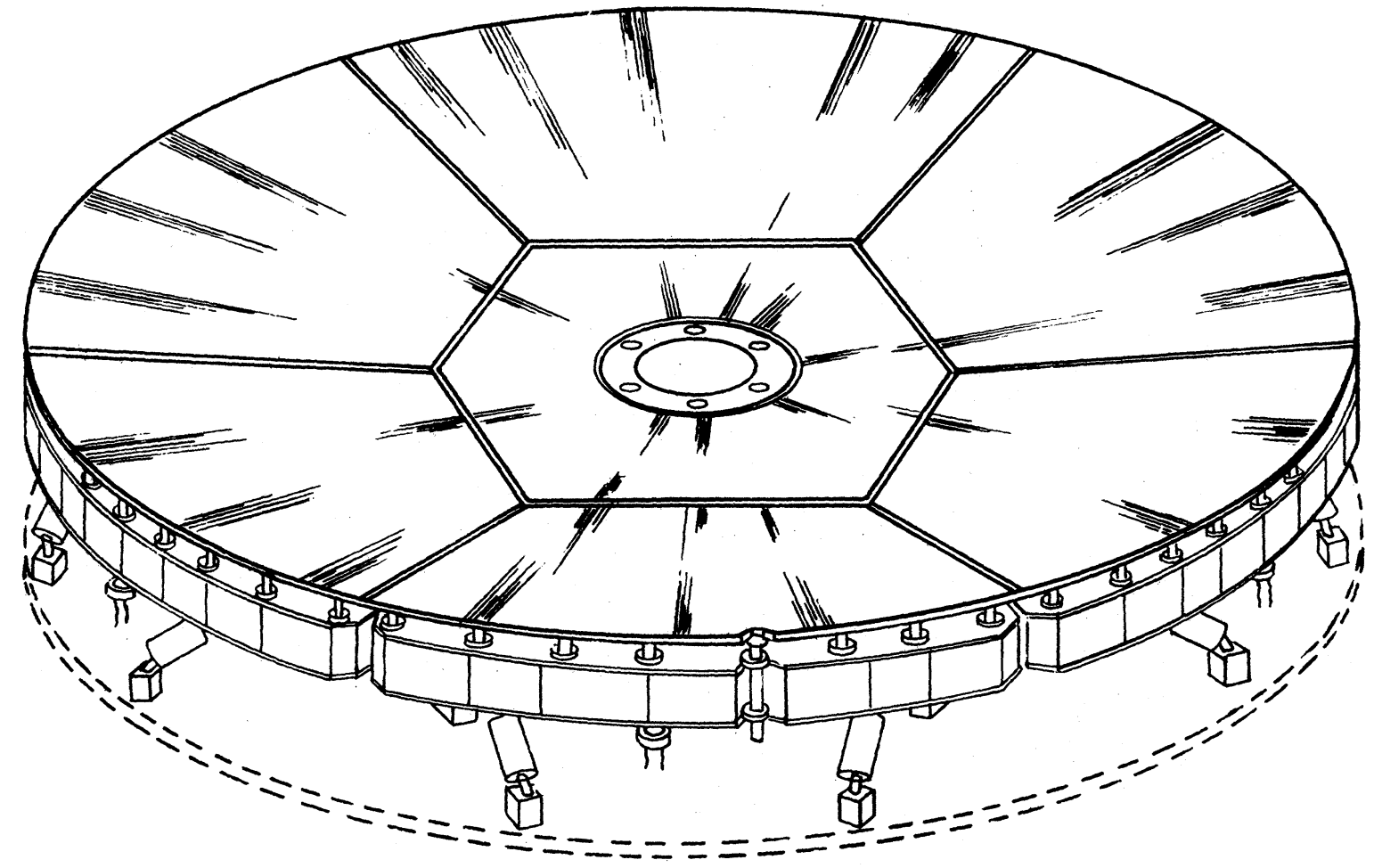

Figure 5.2. A 4-m-diameter segmented telescope mirror under construction. Segments are phased into a single representative surface through active techniques.

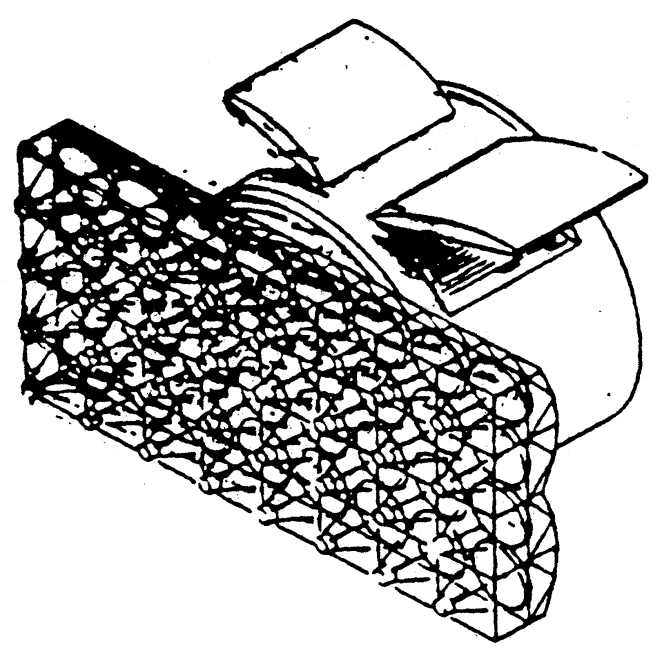

CONCEPT 1

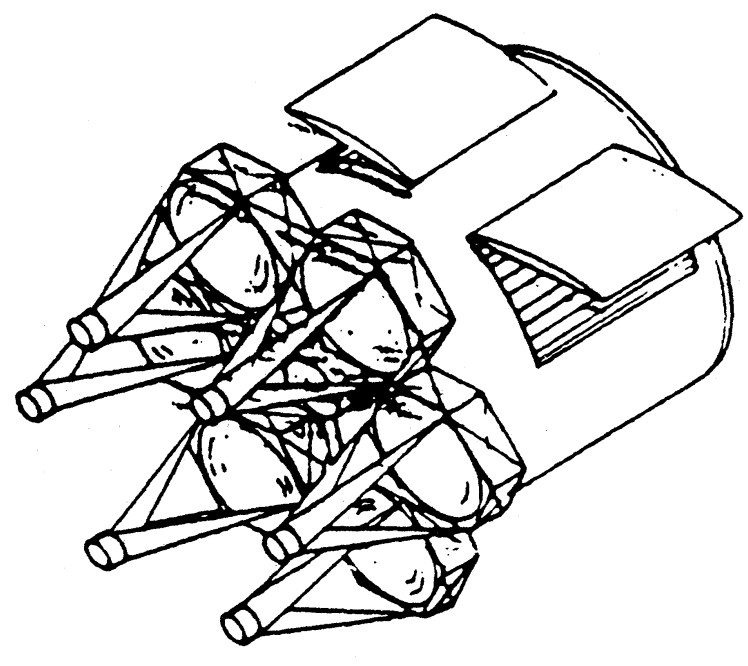

CONCEPT 2

Figure 5.3. Two possible configurations of phased arrays. (a) Many small individual telescopes, (b) few large diameter individual telescopes. 
$f=f$ number the mirror defined as the focal length of the mirror divided by its diameter,

$\sigma=$ rms surface quality $(\mu \mathrm{m})$.

The cost is generally proportional to $\mathrm{D}^{3}$. Therefore, fabricating $\mathbf{N}$ mirrors of diameter $\mathrm{d}=\mathrm{D} / \sqrt{\mathrm{N}}$ produces a cost saving of $\sqrt{\mathrm{N}}$ for an optical surface in an array versus that of a monolithic primary.

Aspherizing adds time to the fabrication process. How much time depends on, among other factors, the amount of aspheric departure from the parent sphere. For large optics, the maximum departure from a spherical surface demonstrated to date has been about $100 \lambda$.

A critical performance parameter for space-based laser systems is retargeting time. Such retargeting requirements are to slew from one target to another, which may be separated by as much as $20^{\circ}$, in time scales of $1 \mathrm{~s}$, with times of the order of $0.1 \mathrm{~s}$ preferred. Arrays have four methods of retargeting (Figure 5.4). In order of increasing angular reach, these are as follows.

(1) Rapid fine pointing, or aimpoint maintenance, which can be accomplished by relative phase adjustments across the array aperture. This is an optical corollary to radar beam steering. It is suitable for fine beam steering and has a useful maximum angular reach that is limited to about half the subaperture diffraction envelope. Thus, an array of smaller telescopes will produce a wider range for phase-shifted beam steering than the same total diameter array made of large-aperture telescopes. The advantage for monochromatic laser systems is one of bandwidth because it is accomplished through input phase manipulation with optical pathlength changes of less than one wavelength.

(2) Rapid retargeting by beam steering through the usable field of a stationary optical telescope. This is generally limited to less than $3 \mathrm{mrad}$ for two-mirror afocal configurations. For a typical dimension of a missile field of $20^{\prime} \mathrm{km}$ and a range of $1000 \mathrm{~km}$ from the fighting mirror, we have a ballistic missile defense requirement for beam steering of $\pm 20 \mathrm{mrad}$ in $0.1 \mathrm{~s}$. This requirement has led designers to prefer a three-mirror (tertiary) telescope systems with two, stages of magnification. Three-mirror designs have diffractionlimited performance over several degrees. Retargeting is accomplished by moving a tilt mirror in the beam train. Small beam steering mirrors $(\sim 3 \mathrm{~cm}$ diam $)$ have been
APPROACH
RAPID FINE POINTINC (AIMPOINT MAINTENANCE)

BEAM STEERINC (MIRROR POINTING)

SUBTELESCOPE SLEW ("VENETIAN BLINDING")

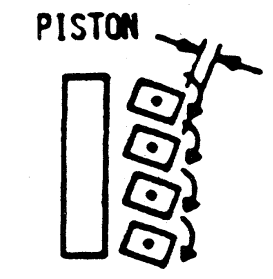

ARRAY SLEW

\section{FEATURES}

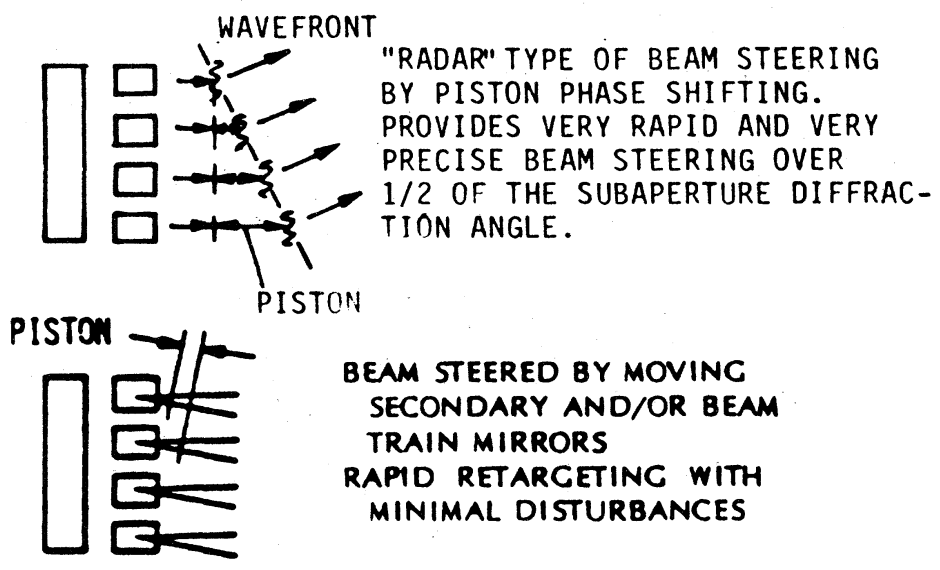

PISTON CORRECTED BY OPD ADIUSTERS

RAPID RETARGETINC BY MOVINC LOW MOMENT OF INERTIA TELESCOPE

\author{
SINGLE CIMBAL SLEW OF \\ LOW-MOMENT TELESCOPE
}

Figure 5.4. Retargeting options. (a) Rapid fine pointing “aimpoint maintenance," (b) beam steering "mini pointing” (secondary mirror not shown), (c) subtelescope slew "venetian blinding," and (d) array slew. 
demonstrated to operate at a bandwidth greater than $1 \mathrm{kHz}$ (such mirrors can be commercially obtained); system application might require cooled 20-60-cmdiameter mirrors.

(3) Mechanical slewing of individual gimballed telescopes "venetian blinding." In order to maintain a tightly packed array, "venetian blinding" is limited to angles of $10^{\circ}-15^{\circ}$. Path length delays across the mirrors must be corrected as telescopes are slewed. For slewing of such telescopes, a certain amount of torque is required to be applied to each telescope. The amount of torque required is established by considerations of the mass of the telescope (primarily determined by telescope diameter), and the angular displacement of the telescope that needs to be achieved over a given period of time (i.e., retargeting time). This sets the torque requirements on the actuators (control moment gyros, CMGs). Present designs of actuators can provide $4500 \mathrm{~N} \mathrm{~m}$ of torque (commercial configuration from Sperry Flight Systems). Based on above considerations, this would indicate that telescope having diameters of up to about $4 \mathrm{~m}$ can be slewed. It has been indicated that current CMG capabilities in torque might be scaled upward so that telescopes having diameters as large as 8-10 m might be slewed at the required performance level (private communication from Sperry Flight Systems). Beyond that point, the torque requirements exceed the capability of presently used materials. Short of developing new materials, larger telescopes cannot be slewed at the required performance levels.

(4) Finally, entire beam array slewing can be used in conjunction with any of the above-mentioned techniques.

\subsubsection{Status of Technology}

The key to achieving phased operation at infrared or visible wavelengths is sensing and controlling wavefronts. Radar system techniques of controlling large structures do not readily scale to optical tolerance levels. Phasing has to be dynamic and is accomplished by the measurement of outgoing phase difference between adjacent telescopes and appropriate manipulation of the input phase so as to implement coherent addition of the beams in the far field.

For optical phased arrays, controlling wavefronts rather than structures in phase is required for shortwavelength operation.

For beam-transmitting systems, four basic parameters must be sensed and controlled:

(1) Beam quality of each outgoing wavefront.

(2) Relative piston of all of the outgoing wavefronts.

(3) Pointing of each telescope to the same target point.

(4) Beam stabilization or jitter control of each outgoing wavefront. ${ }^{3}$

Beam quality is controlled by sensing each wavefront and correcting aberrations with an adaptive optic in the beam train. Portions of flat phase wavefronts from adjacent telescopes can be sampled as shown in Figure 5.5 to monitor piston. Pointing is accomplished by using modern tracking techniques or, for optimum resolution, by coherently combining multiple apertures to achieve the

\section{YOUNG'S EXPERIMENT APPLIED TO PHASE MATCHING}

\section{DIFFRACTING SCREEN}

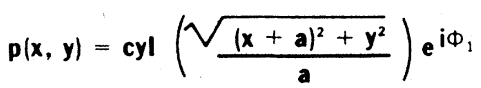

$+\operatorname{cyl}\left(\frac{\sqrt{(x a)^{2}+y^{2}}}{a}\right) e^{i \Phi_{2}}$

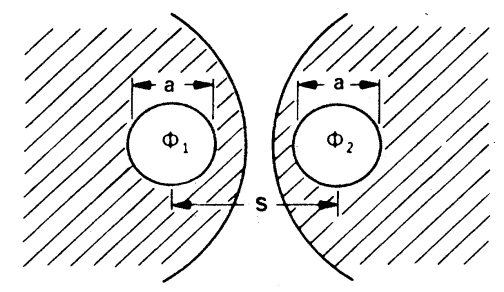

FAR FIELD IRRADIANCE

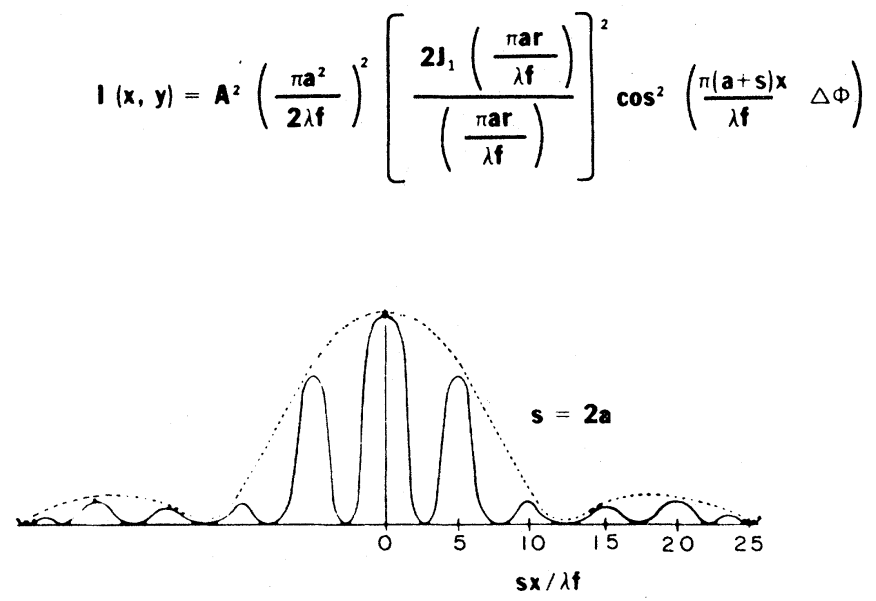

Figure 5.5. Young's experiment applied to phase matching. Phase difference between apertures cause shift of maximum irradiance in the far field irradiance distribution. 
resolution of the combined aperture diameters. Beam stabilization is maintained with high bandwidth tilt mirrors in each telescope beam train. Bandwidths of $500 \mathrm{~Hz}$ for $25-\mathrm{cm}$ cooled mirrors were demonstrated and described in connection with the Airborne Laser Laboratory in middle 1970s; however, these experiments were done at long wavelengths $(10 \mu \mathrm{m})$, with long coherence lengths and a rigid platform.

In order to use telescope arrays in a coherent fashion (have them act as a single aperture) one needs to perform certain phasing functions. These phasing functions, piston, tilt, and higher order wavefront aberrations can be seen in Figure 5.6. If one considers a wavefront as depicted in Figure 5.6, it consists of a spherical curvature corrupted by various kinds of phase ripple. If one samples such a wavefront with multiple telescopes, then each telescope will see a part of this wavefront consisting of both tilt and higher order aberrations. Furthermore, there is a displacement in "piston" of the sampled wavefront because of the telescope separation.

The "phasing" functions that are thus needed are the adaptive compensation of tilt and higher order aberrations within each telescope (subaperature) and then compensation for the piston displacement between telescopes. It is important to achieve phase compensation within each telescope before array piston phasing can be implemented, else such piston measurements will be corrupted by higher order aberrations.

The ability to sense and control, to optical precision, wavefronts from separate telescopes was demonstrated on the Phasar experiment at the Air Force Weapons Laboratory. Three laboratory telescopes were used as beam transmitters for an argon ion laser. Outgoing wavefronts were sensed in piston (average phase) to an accuracy of $\lambda / 134$ and controlled to better than $\lambda / 20(\bar{\lambda}=0.5 \mu \mathrm{m})$. All wavelengths were phased simultaneously using white light interferometry. Tilt was controlled to $1 \mu \mathrm{rad}$ over a range of $\pm 400 \mu \mathrm{rad}$ at a $500-$ $\mathrm{Hz}$ closed loop bandwidth. The piston control loop bandwidth was $100 \mathrm{~Hz}$. The measured far-field energy distribution from the Phasar telescopes is shown in Figures 5.7(a) and 5.7(b) and represents $\lambda / 20$ performance. (For a compilation of technical papers on optical phased arrays, see References 3 and 4.)

\subsubsection{Scaling}

The two primary design features that must be balanced in scaling optical phased arrays are the telescope size and the system complexity. The latter can be quantified in terms of wavefront sensing and control operations count. The former, telescope size, can be

\section{IMPACT OF ATMOSPHERE ON BEAM (WAVEFRONT) CONTROL}

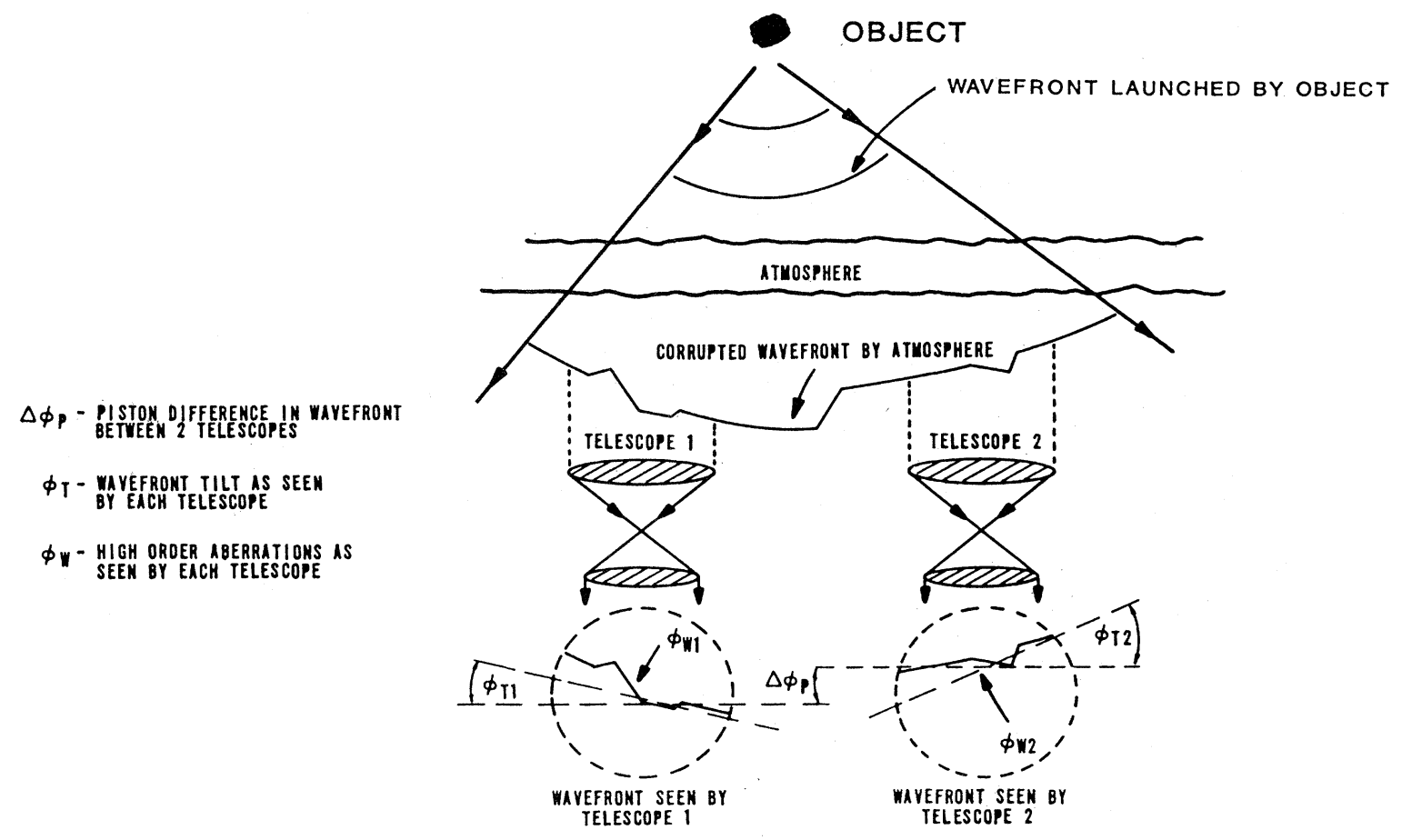

Figure 5.6. Impact of atmosphere on beam (wavefront) control. Atmosphere introduces (a) piston difference in wavefront between two telescopes, (b) wavefront tilt as seen by each telescope, and (c) high order aberrations as seen by each telescope. 
(a)

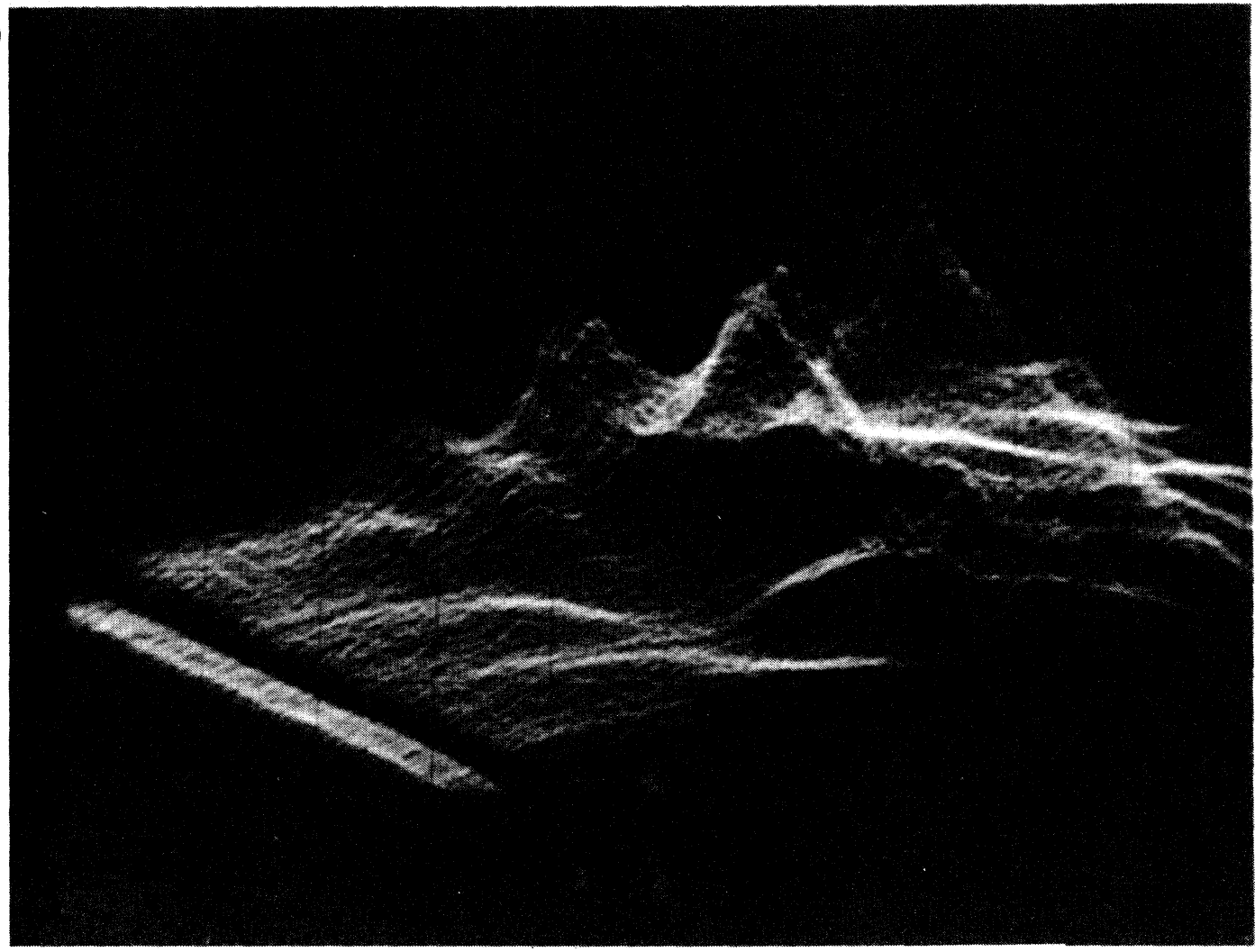

(b)

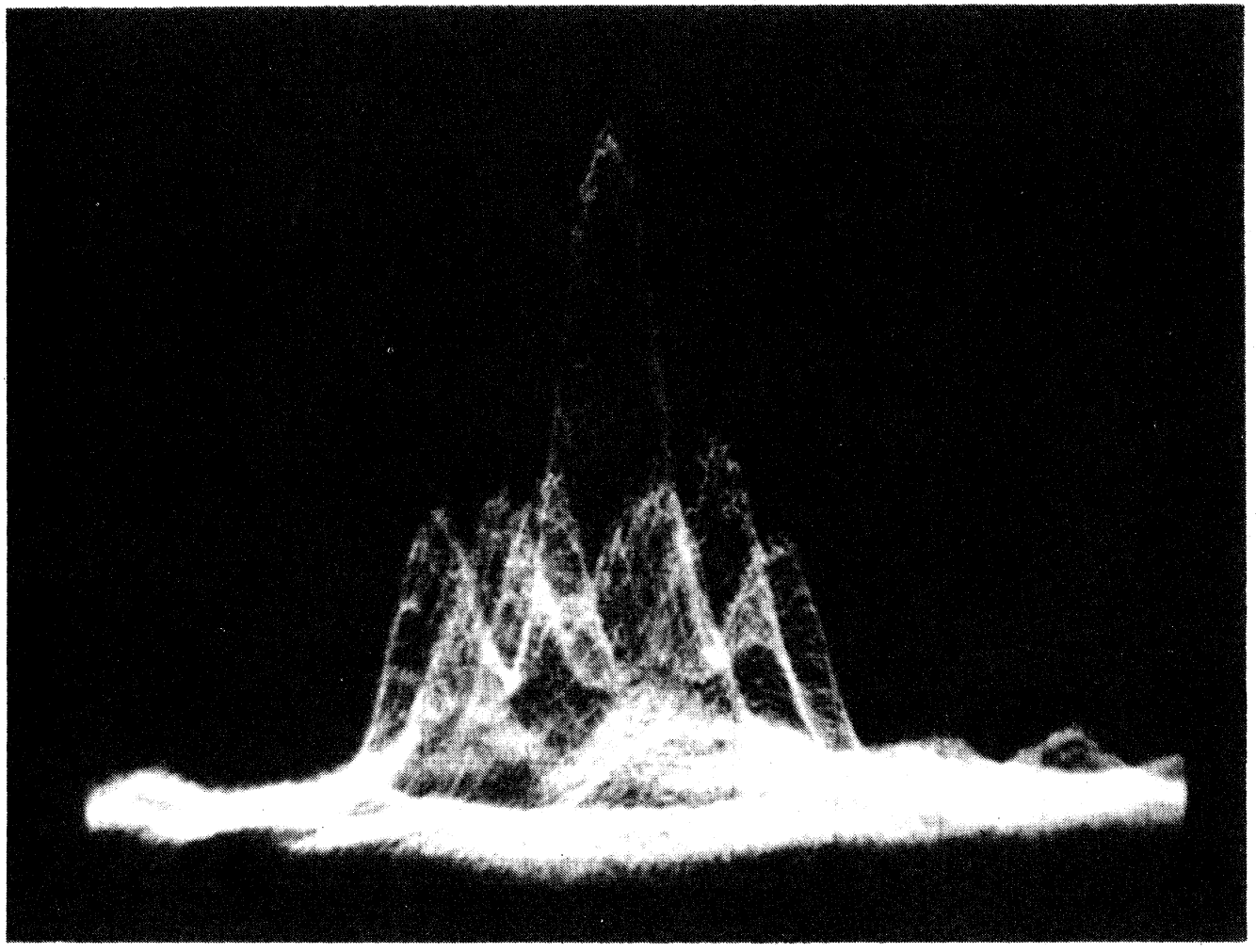

Figure 5.7. Measured far field intensity distribution of a three telescope array (from References 3 and 4). (a) Optically aligned but not phased (argon ion laser wavelength). (b) Dynamically phased for piston and tilt. Overall achieved phased error is $\lambda / 50$. (Argon ion laser wavelength.) 
optimized using total system performance of far-field irradiance.

For an aberration free optical system of diameter D, wavelength $\lambda$, and total power $\mathbf{P}_{\mathrm{T}}$ focusing light onto a target a distance $R$ away the peak irradiance $\left(W / \mathrm{m}^{2}\right), I_{0}$, of the focused beam is given by

$$
\mathbf{I}_{0}=\left(\pi \mathbf{P}_{\mathrm{T}} \mathbf{D}^{2} / 4 \lambda^{2} \mathbf{R}^{2}\right)
$$

If aberrations, misalignments, or other errors are present, the peak intensity is reduced by an amount, SR, called the Strehl Ratio.

If the optical system is made up of $\mathrm{N}$ individual apertures of diameter $d$ and a power $\mathbf{P}_{a}$ is transmitted through each individual aperture the peak irradiance is given by

$$
I_{0}=\left(\pi \mathbf{N}^{2} \mathbf{P}_{\mathrm{a}} \mathrm{d}^{2} / 4 \lambda^{2} \mathbf{R}^{2}\right)
$$

The effects of telescope piston and tilt errors on system performance have been examined by Butts. ${ }^{4}$ For uncorrelated errors, the exact Strehl ratio is ${ }^{4}$

$$
\begin{aligned}
\mathrm{SR}= & \left(2 / \mathrm{N} \pi^{2} \mathrm{p}^{2}\right)\left\{1-\exp \left(-\pi^{2} \mathrm{p}^{2}\right)\left[I_{0}\left(\pi^{2} \mathrm{p}^{2}\right)+I_{1}\left(\pi^{2} \mathrm{p}^{2}\right)\right]\right\} \\
+ & 4\left[(\mathrm{~N}-1) / \mathrm{N} \pi^{4} \mathrm{p}^{4}\right] \exp \left(-4 \pi^{2} \mathrm{p}_{0}^{2}\right) \\
& \times\left[1-\exp \left(-\pi^{2} \mathrm{p}^{2} / 2\right)\right]^{2},
\end{aligned}
$$

$\mathbf{N}=$ number of telescopes in the array, $\mathrm{p}=\sigma_{\mathrm{t}} \mathrm{d} / \lambda=\mathrm{rms}$ tilt error in units of $\lambda / \mathrm{d}$,

$\mathrm{d}=$ diameter of an individual telescope in the array, $\mathrm{p}_{0}=\sigma_{\mathrm{p}} / \lambda=\mathrm{rms}$ piston error in waves, $I_{0}, I_{1}=$ modified Bessel functions.

From Equation (5.4) it can be seen that the requirement for jitter control is much less stringent for a phased array than it is for a single aperture system with the same transmitting area. The wavefronts from a phased array can jitter $\sqrt{\mathrm{N}}$ times as much for a given equivalent level of performance, as compared to an equivalent area monolithic system. For the case where wavefront tilts are uncorrelated, this conclusion holds in an exact analysis. The physical argument for such a behavior is that each element (subaperture) of a phased array undergoes an averaging process over the "effective" or total system aperture if such disturbances or aberrations are uncorrelated. A monolithic aperture has to have its jitter controlled to one-half of its spot size. For example, the jitter (random phase tilt) that would be allowable for a $30-\mathrm{m}$ monolithic aperture at $3 \mu \mathrm{m}$ is of the order of $5 \times 10^{-8} \mathrm{rad}$. On the other hand, an array of 49 subapertures generating an effective $30-\mathrm{m}$ aperture can relax the requirement for phase tilt control per subaperture of $\mathrm{N}^{-1 / 2}$ monolithic value, or in this case $3.5 \times 10^{-7} \mathrm{rad}$. The latter value has been achieved in the laboratory. The former value has not.

Figure 5.8 shows the above-discussed effect on relative irradiance (compared to a monolithic aperture) as a function of subaperture number for fixed effective total system diameter, power, operating wavelength, range, and the same optical aberrations (tilt and wavefront ripple). We can readily see that for the parameters used a $15 \%$ increase in brightness of the array as compared to a monolithic equivalent aperture system.

Other significant errors affecting system performance are telescope polarization differences which manifest themselves as piston errors, focus, and higher order aberrations. These latter aberration terms are not included in Equation (5.4) for abbreviation and simplicity, but can be readily accomplished by addition of appropriate exponentials. Such effects are included in Figures 5.9 and 5.10 .

Constant parameters for Figures 5.9 and 5.10 are as below:

$$
\begin{aligned}
& D=\text { total effective diameter }=45 \mathrm{~m}, \\
& R=\text { focal range }=3500 \mathrm{~km}, \\
& P_{\text {total }}=\text { total power }=175 \mathrm{MW}
\end{aligned}
$$

Figure 5.9 shows peak irradiance as a function of operating wavelength for a family of systems $(\mathbf{N}=1$, $3,7,19,49$ ) with the previously listed parameters held constant. Increasing the number of mirrors in an array with fixed total system diameter, power, and performance tolerances (in absolute values of length and angle) produces an increased peak irradiance, as indicated. The brightness increases significantly as the aperture is divided into $\mathbf{N}=3,7$, or 19 beam trains, especially for shorter wavelengths. Relative performance improvement is less dramatic for larger numbers of mirrors $(N=49)$. The curves decay to the long wavelength side of the peak in brightness because of diffraction effects, and to the short

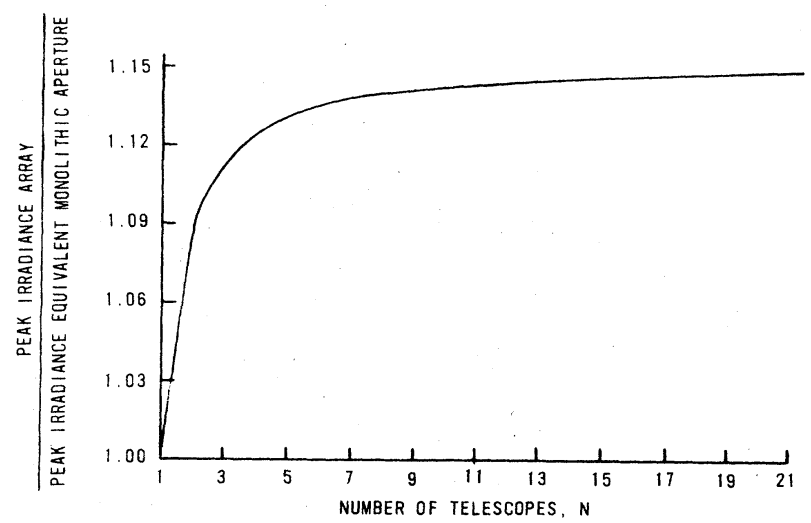

Figure 5.8. Peak (relative) irradiance produced by a constant area array of $\mathbf{N}$ mirrors. Total power, effective diameter, wavelength, range, and performance specifications are fixed. 


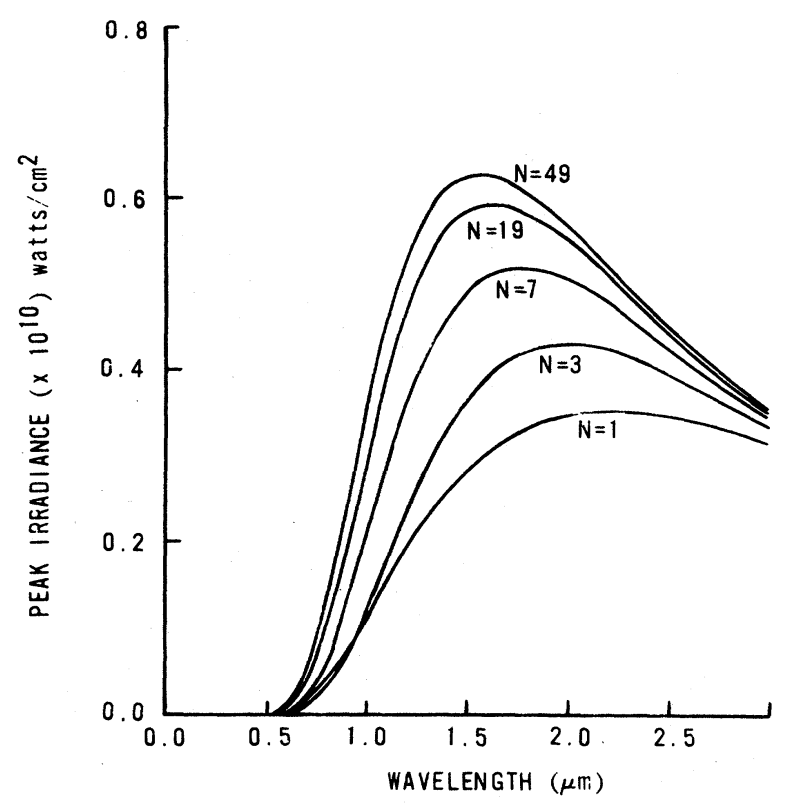

Figure 5.9. Peak irradiance as a function of operating wavelength for an $\mathrm{N}$-mirror array. Number of beam trains $\mathbf{N}=1,3,7,19,49$. Jitter $=25 \mathrm{nrad}$. Piston $=\lambda / 15$ at $3 \mu \mathrm{m}$. Aberrations $=\lambda / 15$ at 3 $\mu \mathrm{m}$. (Based on the model presented in Reference 4.)

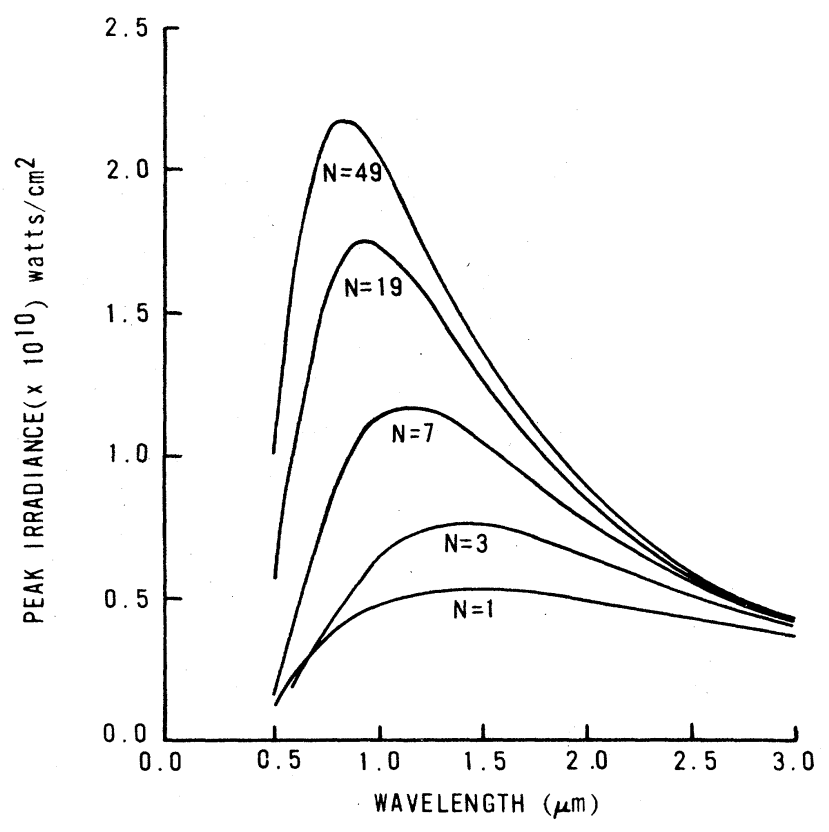

Figure 5.10. Peak irradiance as a function of operating wavelength for an $\mathrm{N}$-mirror array, with more stringently controlled piston and higher order aberrations than in Fig. 5.9. Number of beam trains $\mathbf{N}=1,3,7,19,49$. Jitter $=25 \mathrm{nrad}$. Piston $=\lambda / 30$ at $3 \mu \mathrm{m}$. Aberrations $=\lambda / 30$ at $3 \mu \mathrm{m}$. (Based on the model presented in Reference 4.) wavelength side because the wavelength becomes small compared to the scale size of the aberrations.

In Figure 5.9, piston and all higher order aberrations were constrained to be more stringently controlled, to $\lambda / 30$ at $3 \mu \mathrm{m}$. The predominant effects are increased far-field brightness and a shift of optimum operating wavelengths to smaller values. ${ }^{4}$ Although it is not shown in these figures, reduction of jitter errors solely will only increase brightness, but not shift the curves as a function of $\lambda$.

The optimum operating wavelength may be determined for each configuration. The diffraction contribution benefits from a reduced operating wavelength. On the other hand, aberration, misalignments, and piston errors are fixed and have an increasingly more deleterious effects on far-field performance as operating wavelength decreases. There is, therefore, an optimum operating wavelength, as indicated in Figures 5.9 and 5.10, where the effect of pure diffraction and error degradations balance to produce the

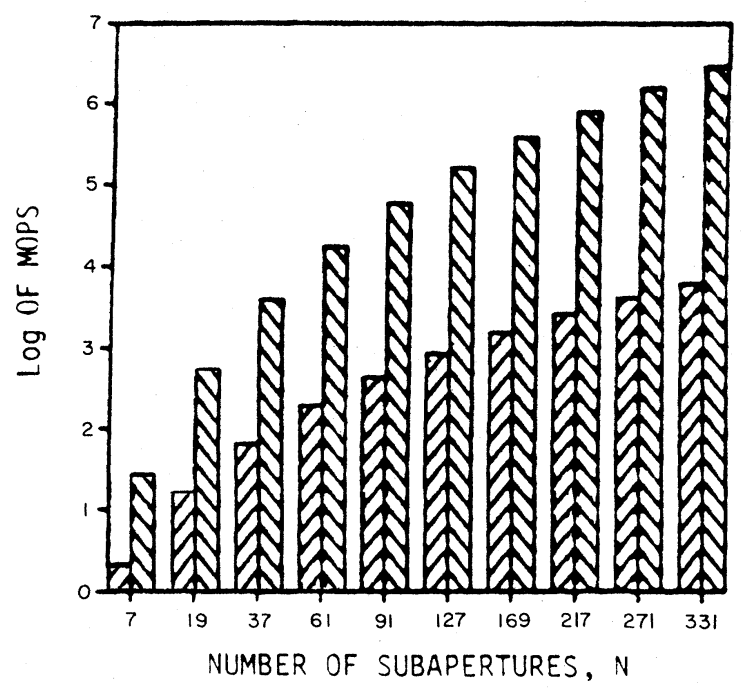

Figure 5.11. Array control requirements. Bandwidth $=1000$ Hz. Choice affects controller throughput, not loop count. For example: Full least-squares optimal piston controller throughput: MOPS $=\left[(6 \mathrm{~N})^{3} \times 10 \Delta \mathrm{F}\right] / 10^{6} ; \quad$ Decentralized, inde pendent piston control loops with maximum measure redundancy throughput per channel: MOPS $=(2 \mathrm{NM}+50) \times 10 \Delta \mathrm{F} / 10^{6} ; \quad$ MOPS $=$ Millions of operations per second, $\mathbf{N}$ =Number of subapertures, $\mathbf{M}=$ Maximum number of piston measurements, $\Delta F=$ Loop bandwidth. The higher set of values represents the number of operations required to control an array using only a single controller; that is, all measurements are sent to a single estimator. The lower set of values represents the number of operations required when arrays are controlled in a hierarchical fashion; that is, subsets of telescopes are controlled in parallel, and groups are phased with respect to each other. 
greatest far-field brightness.

System complexity is addressed in Figure 5.11, which is a histogram of operations required to control $\mathrm{N}$ subapertures or telescopes. The higher set of values represents the number of operations required to control an array using only a single controller; that is, all measurements are sent to a single estimator. The lower set of values represents the number of operations required when arrays are controlled in a hierarchical fashion; that is, subsets of telescopes are controlled in parallel, and groups are phased with respect to each other. Current computer technology approaches $10^{3}$ million operations per second (MOPS); therefore, from the viewpoint of complexity, arrays are limited to groups of less than 50 mirrors for decentralized processing or about 19 mirrors for agile controller implementation.

\subsubsection{Phase Locking of Laser Arrays}

Since HF chemical lasers and excimer lasers have a maximum size of module which falls short by one or more orders of magnitude of the estimated power output of $100 \mathrm{MW}$ or more, the laser weapon could be built only in a modular way comprising 10-100 modules. Here the problem of phasing active laser oscillators is encountered. They must oscillate coherently in a constructively reinforcing mode. As mentioned in Section 3.1.3.7, the phasing of a few low power modules of $\mathrm{HF}$ and $\mathrm{CO}_{2}$ lasers has been demonstrated. Large arrays of very low power semiconductor lasers have also been successfully locked for commercial applications. It remains an open question whether large arrays of high power HF lasers can be successfully phase locked. Their tendency to emit power in a broad spectral range and their complex mode geometry present severe obstacles. Different excimer laser modules could be used as incoherent pumps for a single Raman cell oscillator. This was discussed in Section 3.3.7 as the currently preferred method for beam combination of excimer lasers.

\subsubsection{Conclusions}

Phase controlling an array of mirrors to function as a single coherent transmitting or receiving aperture is a rapidly developing technology for achieving optical systems of extremely large effective diameters $(10-40 \mathrm{~m})$. Key functions for coherent beam transmission have been demonstrated on a laboratory scale, and significantly more sophisticated brassboard experiments are under way through various efforts. Challenges still remain in designing and implementing multiple mirror configurations to operate as coherent imaging optics across a wide field of view.

Calculations of array performance show that for a given size total aperture, it is more beneficial to use a large number of telescopes. Other advantages of using arrays with large $\mathrm{N}$ are reduction of fabrication complexity, cost saving, and relaxed error-sensing and control requirements. An upper bound on the number of array elements can be estimated on the basis of current computing capabilities and required actuators. Hierarchical control implementations allow very large numbers of mirrors to be simultaneously controlled, whereas controlling an array using a single estimator limits the array size.

\subsection{RELAY OPTICAL SYSTEMS IN SPACE}

Relay optical elements in space form an integral part of a ground-based laser (GBL) system for defense against ballistic missiles. A typical proposed method for using earth-based lasers to shoot down enemy missiles is illustrated in Figure 5.12. A nearly vertical path in the atmosphere must be used because a major system loss factor for ground-based lasers is atmospheric losses due to turbulence and blooming (see Section 5.4). Since the ground-based laser weapon and an attacking missile during the launch phase are not in a line of sight, the laser beam must be launched toward an orbiting optical transfer platform placed in high, near-geosynchronous orbit and thence to low-orbit fighting platforms that redirect and focus the laser energy onto the targets. Alternative architectures may place two or more transfer platforms in orbits at lower altitudes. The required mirror sizes for the relay stations are reduced in this case, but the required number of relay mirrors is increased because of absentee factors. This is discussed in Section 5.7.2.

\subsubsection{Optical Concepts}

Concepts for space optical elements fall generally into single-element flats (monocle) or dual telescopes (bifocal), shown in Figure 5.13, or variations of these, such as a coelostat using flat mirrors with some focusing capability but with no capability to expand the size of the laser beam. Present efforts have been directed at sorting out where these generic types fit the roles of relay and mission mirrors. To quantify the issues, optical system design studies are being conducted to estimate the size of

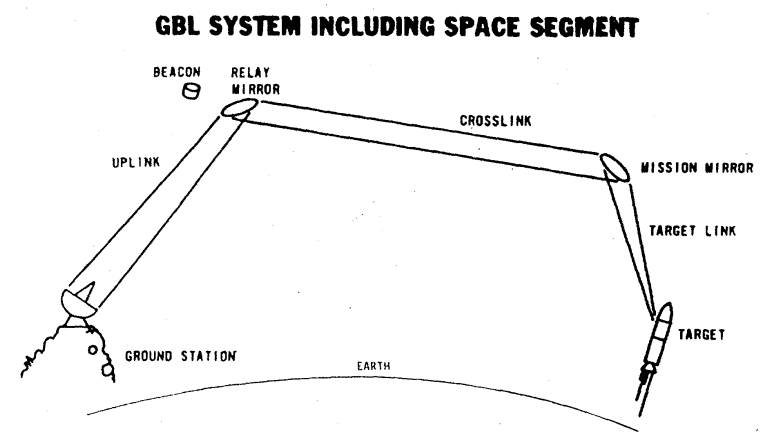

Figure 5.12. Ground-based laser/relay optical concept against boost phase missiles. 

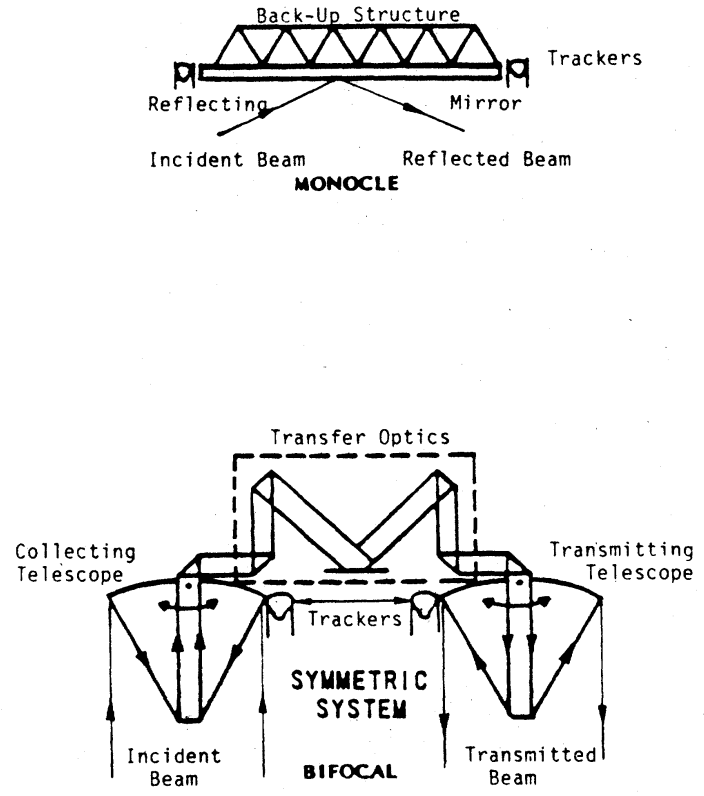

Figure 5.13. Relay mirror systems. (a) Single element flat (monocle), (b) dual telescopes (bifocal).

the space hardware fleet, including mirror clear-aperture requirements, total weight in orbit, and the flowdown of requirements for optical figure and jitter control.

In general, the monocle relay mirror concepts have fewer optical elements than the bifocal. They are also lighter and the optics do not require cooling. On the other hand, there is no effective way to measure and correct the arriving wavefront; one may only control the mirror surface through difficult glancing angle interferometry measurements. Also, the whole relay mirror has to be moved very precisely in order to track a target or to retarget. The bifocal relay uses a receiving telescope and a transmitting telescope with interconnecting transfer optics. Here actual wavefront (as opposed to mirror figure) control can be achieved by making wavefront measurements and adaptive manipulations with the transfer optics. If the telescopes are wide field of view or arrays, then beam tracking and retargeting could be done by steering the beam in the transfer optics, rather than moving large telescopes. The disadvantages are that of higher complexity and the requirement of cooled optics for the transfer functions.

\subsubsection{Optical Layout}

By placing optical focusing platforms both at the transfer and fighting locations, one reduces the diffraction spreading phenomena and the sensitivity to jitter. If the transfer optical subsystem is also an imaging device, then the ground telescope is imaged onto the fighting optical platform, matching the direction of the ground telescope to that of the fighting platform aperture. This latter dimension is determined by the distance between fighting platform and target and the desired spot size at the target. For a spot dimension of $30 \mathrm{~cm}$ and a range of $3000 \mathrm{~km}$, a $10 \mathrm{~m}$ aperture fighting platform would be required for a $1.0 \mu \mathrm{m}$ laser wavelength.

\subsubsection{Energy Losses}

Energy loss in the train can result from poor optical figure on the mirrors and from aberrations induced by the atmosphere. Because of the great distances between elements, aberrations of high spatial frequency will spread beyond the limits of any reasonably sized collecting mirror. Therefore, the intensity profile of the laser beam should not be allowed to have high spatial frequency content.

\subsubsection{Rapid Retargeting}

The inertial loading of the space mirrors will come from the dynamic line of sight (LOS) motions, which are most severe when the focus is moved from target to target. When systems with high brightness are developed that reduce the laser irradiation times, the time required for moving from target to target becomes the cost driver for system size and weight in orbit. Retargeting times required to be achieved are between 0.1 and $0.01 \mathrm{~s}$. Shortening the retarget times requires higher angular accelerations, which requires actuators beyond the present state of the art to move space elements, and also provides the forcing functions to excite structural resonances that distort the optics. Alternatives include steering with small segments on flat mirrors (similar to a venetian blind) and operating the telescopes off-axis using internal beam steering mirrors. For both, the added induced aberrations need to be quantified and the ability to compensate must be assessed. If retargeting times can be reduced to be substantially less than individual target engagement time, then the overall system performance becomes independent of retargeting time. Such retargeting times would, at best, be difficult to achieve.

\subsubsection{Error Flowdown}

Since system performance at the target is degraded by both jitter and optical distortions, it may be necessary to operate at shorter ranges and increase the number of platforms. A rule of thumb is that $86 \%$ of the time the jitter should be equivalent to less than half of the diffraction spreading, $\lambda / \mathrm{D}$. This is shown in Figure 5.14 where the relative intensity decrease due to jitter (jitter Strehl ratio) is plotted as a function of the ratio of overall rms jitter angle $\left(2 \sigma_{p}\right)$ to the diffraction angle $(\lambda / D)$. This allowable total error in turn must be allocated to the transmitter, relay(s), and mission mirror. For each platform, the contributions from its tracking and 


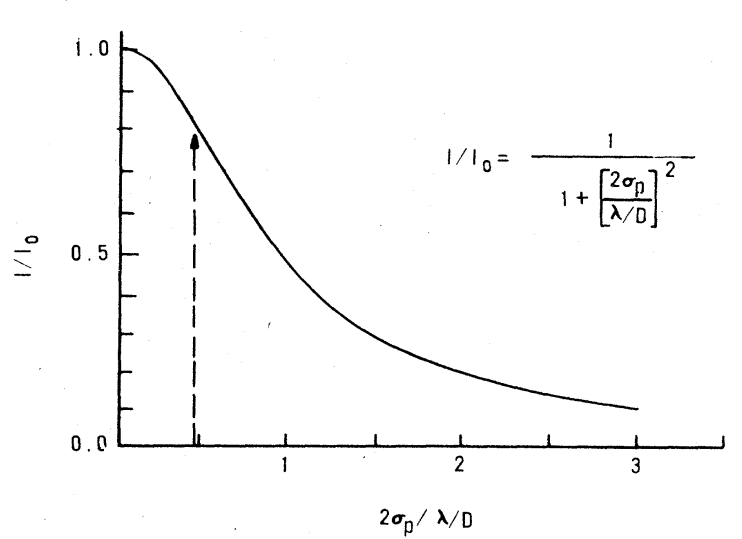

Figure 5.14. Relative intensity loss due to jitter $1 \sigma_{\mathrm{p}}$ of mirrors, as a function of the ratio of jitter angle to diffraction angle.

stabilization functions and mirror motions must be quantified, controlled, and allocated.

Each element needs to track and point the laser beam to the center of the next collecting optical element or, in the case of the mission mirror, to the target. The tracking control system will have noise inherent in its own sensor and will leave a residual jitter beyond its bandwidth, owing to motions of the next element. Track errors will be the least for the relay mirror, since the next element is cooperative and can provide a strong beacon for the sensor to track, and the kinematic motions of the missions mirror should be fairly benign. Track errors for the mission mirror would be the highest since the target will be uncooperative.

A flowdown of aberrations need be done only on the individual segments themselves, since the distances between mirrors is so great. The only aberrations that could be seen from one mirror to the next are low-order aberrations, such as defocus and astigmatism. The flowdown on a given segment is into the contribution of phasing of the individual segments of the large mirrors, and into the figure of an individual segment.

\subsection{ATMOSPHERIC PROPAGATION AND ADAPTIVE OPTICS}

This section examines the problems of propagating a laser beam through the earth's atmosphere, problems that must be solved if ground-based lasers (GBLs) are to be considered as an ABM weapon. Atmospheric propagation effects may also be of some importance in the "downlink" from the fighting mirror to the target, which has not completely emerged from the atmosphere. This section does not deal with the question of whether earthbased lasers are more effective or less effective than space-based ones. That decision will depend upon a complex set of factors and on future developments in laser technology.
Along the optical path from the weapon laser to the relay mirror, atmospheric conditions will cause severe degradation of the optical quality and, hence, of the amount of power delivered. There are several sources of atmospheric degradation of optical beams. These are (1) absorption and scattering by atmospheric aerosols, including rain and fog, (2) distortion by atmospheric turbulence, (3) distortion by thermal blooming, and (4) stimulated Raman scattering. For downlink propagation to targets in the atmosphere above $20-30 \mathrm{~km}$ only stimulated Raman scattering may be a significant degradation mechanism.

\subsubsection{Absorption and Scattering}

The total beam attenuation depends on the atmospheric conditions, wavelength of the radiation, and the total atmospheric pathlength. Laser beams cannot penetrate a curtain of rain and fog and the only solution in this case is to use a multiplicity of GBLs in widely dispersed geographical locations so that at least one GBL location is able to transmit the high power laser radiation to a relay mirror without significant attenuation. These locations have to separate from each other by typically at least $1000 \mathrm{~km}$ which is the coherence distance for weather patterns. Based on weather statistics, a multiplicity of five independent ground-based laser locations could provide $99.7 \%$ availability. By going to seven climatically isolated locations in the continental United States, availability of $99.9 \%$ is possible. ${ }^{5}$ Further, for each of the locations there will be a need to have multiples of transmitting telescopes, separated by perhaps $1000 \mathrm{~m}$, in order to get around the problem of local cloud cover. This need for redundancy is by no means a trivial issue, since it will significantly increase the number of the laser installations. It is, however, easily quantifiable and is addressed in Section 5.7.

In clear weather one needs to consider effects of scatter and absorption by the atmosphere on the optical beam. ${ }^{6,7}$ In general, the primary contributors to such losses in the visible/near infrared regions are water vapor and ozone absorption, and Rayleigh and aerosol scattering. This is shown in Figure 5.15 in terms of total atmospheric transmission as a function of wavelength. It is seen that water vapor absorption provides major transmission cutoff to the long wavelength side of $1.32 \mu \mathrm{m}$, while ozone provides the cutoff on the UV side for wavelengths shorter than $0.36 \mu \mathrm{m}$. Aerosol absorption might push this value to $0.5 \mu \mathrm{m}$ while Rayleigh and aerosol scattering become unimportant for wavelengths greater than $0.5 \mu \mathrm{m}$. Locating GBL systems at higher altitudes would tend to alleviate water absorption and particularly eliminate aerosol absorption, thus pushing the short wavelength limitations to those set by Rayleigh scatter and ozone absorption. The latter is particularly bothersome in that it is a very high altitude phenomenon that might lead to thermal blooming. 


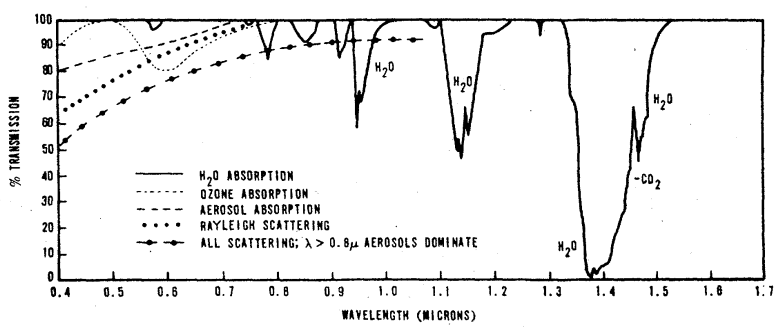

Figure 5.15. Atmospheric transmission of constituents as a function of wavelength. Water vapor transmission is based on Chesapeake Bay measurements [CRC Handbook of Lasers with Selected Data on Optical Technology, edited by R. J. Pressley (Chemical Rubber Co. Cleveland, Ohio)], and corrected by Bragg and Kelley (S. L. Bragg and J. D. Kelley, "Chemical Oxygen/Iodine Laser Propagation Studies," McDonnell Douglass Research Labs, AFWL TR 85-17, 1985), "Measurements of Water Absorption in the $1.3 \mu \mathrm{m}$ Region." Ozone transmission is from data of Griggs [M. Griggs, "Absorption Coefficients of Ozone in the Ultraviolet and Visible Regions," J. Chem. Phys. 49, 857 (1968)]. Scattering contributions are calculated estimates.

\subsubsection{Atmospheric Turbulence}

A second source of beam degradation is atmospheric turbulence. This is a very important source since we know of no way to avoid it (only minimize it by going to an "ideal" site) and it must have a reasonable solution if the system is to be viable.

The atmosphere is not a homogeneous optical medium. The temperature, humidity, and pressure are not spatially or temporally uniform, and this leads to random variations in the index of refraction $n(r, t)$ seen by a propagating laser beam. ${ }^{8,9}$ This variation in the index causes a collimated beam (diameter $=D$ ) to break up and spread at an angle that far exceeds the diffraction limit $\theta_{\mathrm{D}} \simeq \lambda / \mathrm{D}$. These distortions would normally cause a beam spread which is larger by two or three orders of magnitude than the diffraction limit and thus reduce the intensity of a laser beam which is launched through the atmosphere by such large factors ( $>10^{4}$ in a typical case) as to render it ineffective as far as booster burn-through applications are concerned. These aberrations must be corrected if GBL is to be used in BMD applications. In this section we will review the theoretical and experimental background in this field as well as outline some of the proposed solutions, progress and the remaining problems.

\subsubsection{Distortion Compensation}

If the distortions of the atmosphere were linear, i.e., independent of the optical beam intensity and slowly varying in time, they could be compensated nearly completely by adaptive optics technique. This technique is based on the observation that in a lossless linear medium, i.e., one where all the constitutive parameters (index of refraction, magnetic permeability losses) are not a function of the field amplitude, Maxwell's equations remain invariant under the substitution $t \rightarrow-t, E \rightarrow-E$. That is, if we reverse the direction of propagation of an electromagnetic field by, for example, letting $\mathrm{E} \rightarrow-\mathrm{E}$, otherwise keep the same instantaneous field values, the field's subsequent propagation will play itself back in reverse. It follows immediately that, if the propagation medium is distorting, the reverse propagating beam heals itself of the distortions it acquired during the forward propagation.

The principle of distortion correction of a monochromatic laser beam is illustrated in Figure 5.16. At site $A$ we launch a (nearly) plane wave $E=E_{0}^{i(\omega t-k \cdot r)}$. The initial effect of propagation through the random index of the turbulent atmosphere is to impose a random phase $\phi^{\prime}(\mathbf{r})$ so that after a short distance of propagation the field is given by

$$
\mathbf{E}=\mathbf{E}_{0} \mathrm{e}^{\mathrm{i}\left[\omega \mathrm{t}-\mathbf{k} \cdot \mathbf{r}-\phi^{\prime}(\mathbf{r})\right]}
$$

This variation leads, after additional propagation, through diffraction to intensity fluctuations

$$
\mathbf{E}_{1}=\mathbf{E}_{1}(\mathbf{r}) \mathrm{e}^{\mathrm{i}[\omega \mathrm{t}-\mathbf{k} \cdot \mathbf{r}-\phi(\mathbf{r})]},
$$

where $E_{1}(\mathbf{r})$ is real. If at the site of the received beam (B),

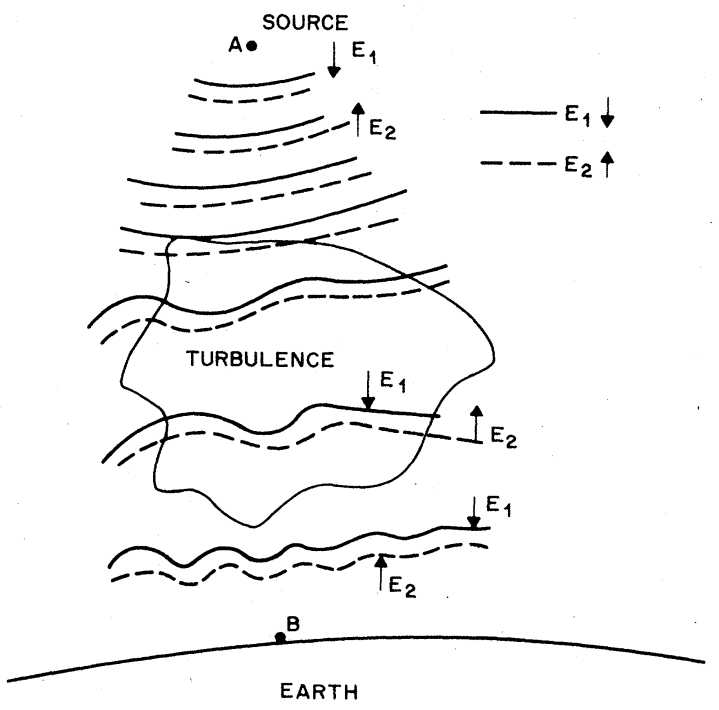

Figure 5.16. Principle of compensation of optical deformation due to atmospheric turbulence. A beacon at point A above the atmosphere sends down a light field $E_{1}$. If from a point $B$ on earth an upward light beam is launched with the same phase front, this field $E_{2}$ will converge on point $A$. 
we retrolaunch a new beam $E_{2}$, related to $E_{1}$ by phase reversal (conjugation),

$$
E_{2} \propto E_{1}^{*}(\mathbf{r}) e^{i[\omega t+k \cdot r+\phi(r)]},
$$

then the time-reversal symmetry of the electromagnetic wave equation in a linear lossless medium guarantees that $E_{2}$ will retrace, in reverse, the history of $E_{1}$, thus arriving at the original launch site (A) with spatial features identical to those of $\mathrm{E}_{1}$. The phase and intensity degradations due to the atmosphere are thus healed. We note that the field $E_{2}$ is related to $E_{1}$ by a reversal of the signs of the terms $\mathbf{k} \cdot \overline{\mathbf{r}}$ and $\phi(\mathbf{r})$ as well as in replacing $E_{1}(\mathbf{r})$ by $E_{1}^{*}(\mathbf{r})$. The sign of $\omega t$, however, was not changed. The practical significance of these relationships is that phase fronts of both fields coincide as shown in Figure 5.16.

This is the underlying principle behind the adaptive optics schemes for atmospheric compensation. ${ }^{10}$ To launch a beam from earth through the atmosphere, we first need a beacon beam to propagate through the atmosphere (emanating from point $\mathrm{A}$ in Figure 5.16). The distortion information thus acquired is then used to generate the conjugate high energy laser beam $\mathbf{E}_{2}$ which will be directed from the ground toward the target.

Two approaches exist today for correcting and compensating for atmospheric distortions. In the first of these two approaches, the phase variation $\phi(\mathbf{r})$ (due to atmospheric distortions) across the incoming beam is determined, in real time. The inverse of this variation, i.e., $-\phi(\mathbf{r})$ is imparted to an outgoing beam by bouncing it from a mirror whose reflecting surface is deformed at each point so as to impart the (conjugate) phase $-\phi(\mathbf{r})$ to the outgoing beam. ${ }^{10,11}$

The second approach relies on nonlinear optical techniques $^{12}$ to generate by mixing in some material medium an instantaneous phase conjugate replica $\mathrm{E}_{\text {conj }} \propto \mathrm{E}_{1}^{*}(\mathbf{r}) \mathrm{e}^{\mathrm{i}[\omega \mathrm{t}+\mathbf{k} \cdot \mathbf{r}+\phi(\mathbf{r})]}$ from an incoming wave $\mathrm{E}_{\mathrm{in}}=\mathrm{E}_{1}(\mathbf{r}) \mathrm{e}^{\mathrm{i}[\omega \mathrm{t}-\mathrm{k} \cdot \mathbf{r}-\phi(\mathbf{r})]}$. Both the linear and the nonlinear methods will be discussed in detail in what follows, but first we need to learn some more about the physics of atmospheric propagation and its terminology.

\subsubsection{Atmospheric Propagation Physics}

The two most important concepts with which we need to familiarize ourselves here are those of the coherence distance $r_{0}$ of the atmosphere and the concept of the isoplanatic angle, ${ }^{13} \theta_{0}$. The quantity $r_{0}$ is the coherence distance of the phase $\phi(\mathbf{r})$ across the aperture of a beam at some wavelength $\lambda$ that has propagated through a turbulent medium. In the absence of turbulence, $r_{0}$ is practically unlimited since the received phase variation $\phi(\mathbf{r})$ is determined uniquely by its initial distribution at the launching aperture. From a practical point of view, it follows that the maximum useful diameter of a transmitting mirror that still delivers a diffraction-limited spot size on target at a distance $\mathrm{L}$, or the maximum diameter of a receiving mirror that still allows for a diffraction-limited "seeing". is

$$
\mathrm{D}_{\max }=\mathrm{r}_{0}(\lambda, \mathrm{L})
$$

since portions of the beam launched from points whose separation exceeds $r_{0}$ are not coherent and thus will not interfere constructively at the target. If a coherent laser beam is launched from an aperture with a diameter D across an atmosphere characterized by a coherence length $r_{0}$, then its area at the target will increase from the diffraction limit of

$$
\mathrm{A}_{\text {ideal }} \simeq(\lambda \mathrm{L} / \mathrm{D})^{2}
$$

to

$$
\mathrm{A}=(\lambda \mathrm{L} / \mathrm{D})^{2}\left[1+\left(\mathrm{D} / \mathrm{r}_{0}\right)^{2}\right]
$$

The coherence length $r_{0}$ is a function of the path length $\mathrm{L}$, the atmospheric turbulence along it, and the wavelength of the beam. Typical magnitudes of $r_{0}$ in the visible for propagating through the atmosphere are in the range of $5-10 \mathrm{~cm}$, and $r_{0}$ scales as $\lambda^{6 / 5}$. This description does not take into account fluctuations in piston caused by turbulence. Both experimental and theoretical information regarding this effect are wanting.

The other quantity of importance is the isoplanatic angle $\theta_{0}$, which defines the cone in the atmosphere where turbulence is coherent in an angular sense. In particular, for turbulence-degraded phase fronts measured along two paths $\theta$ and $\theta+\theta_{0}$, the correlation coefficient between the two will be $e^{-1}$. Since adaptive optics depend on a reference wavefront, this reference cannot be displaced from the outgoing wavefront by more than $\theta_{0}$.

Typical values of $\theta_{0}$ are $3-10 \mu \mathrm{rad}$ in the visible, and it scales as $\lambda^{6 / 5}$. This is to be compared with the point ahead angle, $\theta_{\mathrm{pa}}$, required to track and point at fast moving objects (satellites in particular). At geostationary satellite altitudes $(40 \mathrm{Mm})$, the point ahead angle is 15-20 $\mu \mathrm{rad}$; hence a separate "beacon" source must be placed ahead of the laser aimpoint by $\theta_{\mathrm{pa}}$. In the mirror relay case this is certainly possible and would be a feature of such a satellite platform.

\subsubsection{Phase Compensation System}

To date, most of the progress to compensate phase distortion caused by atmospheric propagation has been achieved with a scheme using deformable mirrors. ${ }^{10,11}$ In this scheme the phase errors across the aperture of the incoming beacon beam are sensed by a detector array and used to deform the surface of a flexible mirror, as shown in Figure 5.17. The deformation is achieved by means of 


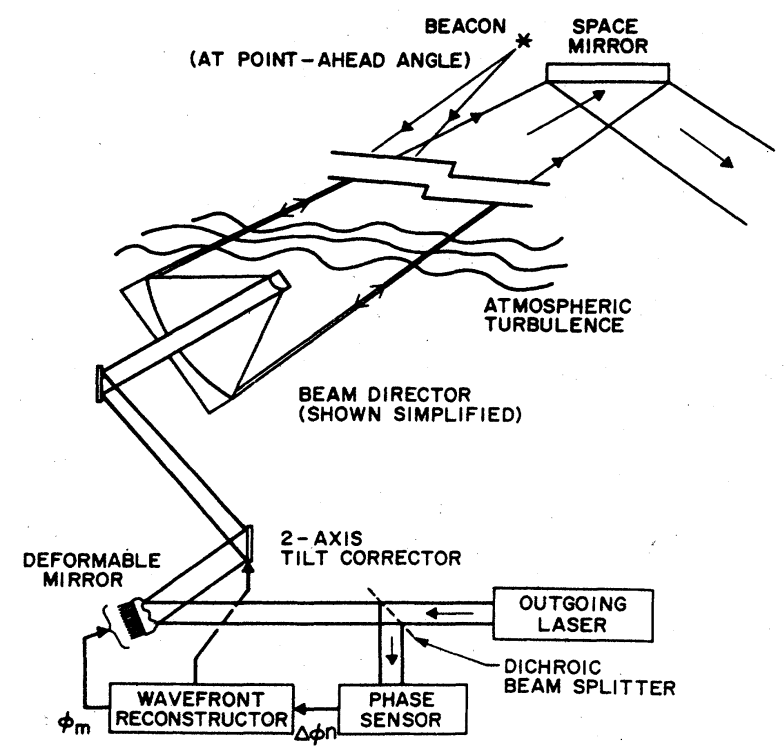

Figure 5.17. Schematic of adaptive optics to compensate for atmospheric wave front deformations.

actuators. Each actuator causes a local deformation in the reflecting (flexible) mirror membranes. The phase sensor is actually a phase gradient sensor. An array of wavefront tilts (roughly twice as many as there are actuators because of the two orthogonal directions of tilt) are measured in parallel. These are reconstructed using a network which solves Laplace's equation to generate the required phase values. The wave to be retransmitted through the atmosphere emerges as a plane or spherical wave from the transmitter laser and is incident on the deformed mirror, where it is imparted the compensatory phase, which, at each point $\mathbf{r}$ on the aperture, is opposite in sign and equal in magnitude to that of the incoming beam. The minimum number of phase sensors (also the number of actuators), $\mathrm{N}$, required for nearly full compensation is given by the area of the transmitting aperture $A_{t}$ divided by the coherence area $r_{0}^{2}$. In the numerical example used previously, where $\mathrm{D}=5 \mathrm{~m}$ and $\mathrm{r}_{0}=5 \mathrm{~cm}$, this is the number given approximately by

$$
\mathrm{N}=\left(5 \times 10^{2} / 5\right)^{2}=10^{4}
$$

If larger diameter apertures are to be employed, say D $>10 \mathrm{~m}$, the number of actuators and phase sensors in the compensation scheme described above will grow to the imposing value of $10^{5}$.

The phase sensors used in an adaptive optics system are designed in the following manner. The phase gradient sensor's total aperture is divided into subareas, each with a radius $\sim r_{0}$, the coherence length. In one approach, equivalent to the astronomer's Hartmann test, a lenslet in front of each subarea converts the angular tilt of the incident phase front to a unique location in the focal plane. A small two-dimensional integrated array of photodetector diodes is used to sense the beam location and thus extract the local phase gradients in the two transverse directions. This information is fed to a computer which integrates the gradients to come up with the phase distribution $\phi(\mathbf{r})$ across the aperture.

\subsubsection{Experimental Results and Major Problems}

A number of experiments conducted in 1984 and 1985 at Maui, Hawaii, involved the focusing and atmospheric correction of an optical beam shot through the atmosphere at moving aircraft, a space shuttle, or a sounding rocket. The experiments involved short wavelength laser beams $(0.4 \mu \mathrm{m})$ and an output aperture of $0.5 \mathrm{~m}$. They were successful in proving the validity of atmospheric compensation through the use of deformable mirrors for low intensity laser beams and relatively benign atmospheric turbulence. This is an important achievement, but crucial questions remain to be answered before the feasibility of actual systems can be contemplated.

The effective deformable mirror aperture diameter (about $0.5 \mathrm{~m}$ ) of these experiments is smaller by about an order of magnitude than those contemplated in the eventual real systems. The number of actuators will need to increase to more than $10^{4}$ in real systems. ${ }^{14} \mathrm{~A}$ similar increase will take place in the number of phase-sensing elements in the receiving aperture.

The reconstruction process (converting phase gradient to phase) is one of the least difficult technologies being addressed, in that it involves parallel processing of $\mathrm{N}_{\Delta \phi}$ into $\mathrm{N}_{\phi}$ actuator drives. If this were to be done serially, it would require computer rates of greater than $10^{9}$ operations per second; however, this approach is unnecessary. Parallel, special purpose (possibly analog) processing may help.

In addition to the need to scale up the number of phase sensors and actuators by two to three orders of magnitude the most serious problem confronting the feasibility of atmospheric compensation schemes is that of thermal blooming. This effect is due to the slight residual absorption of the propagating beam power by the atmosphere.

\subsubsection{Phase Conjugation by Nonlinear Optical Techniques}

An alternative to the use of deformable mirrors is the use of nonlinear optical techniques for phase conjugation. ${ }^{15}$ Two methods have been used: stimulated Brillouin scattering and degenerate frequency four-wave mixing. In the former method the distorted laser beam, i.e., the beacon signal after traversal from the space-based mirror through the atmosphere, is focused into a medium with a large electrostrictive coefficient suitable for Brillouin scattering, for example, methanol. A schematic geometry is shown in Figure 5.18. 

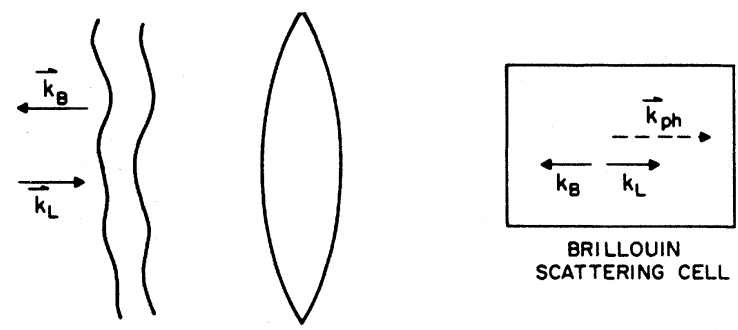

Figure 5.18. Phase-conjugation by Brillouin scattering. The phase front of the stimulated Brillouin scattered light in the backward direction $\mathbf{k}_{\mathrm{B}}$ is identical to that of the incoming beacon light $\mathbf{k}_{\mathrm{L}}$, which was distorted by passage through the atmosphere.

If the intensity in the focal region is high enough and the focal region is long enough, amplification of backscattered Brillouin light may lead to a backward stimulated Brillouin scattered wave at the frequency $\omega_{\mathrm{s}}=\omega_{\mathrm{L}}-\omega_{\mathrm{ph}}$, where $\omega_{\mathrm{ph}}$ is the frequency of the sound wave with wave vector $\mathbf{k}_{\mathrm{ph}}=\mathbf{k}_{\mathrm{L}}-\mathbf{k}_{\mathrm{s}}$. The wavelength of the sound wave is about half the light wavelength. If the threshold for stimulated Brillouin scattering is exceeded by a sufficient amount, the amplitude and phase distribution of the backward scattered mode $\mathbf{E}_{\mathrm{s}}$ will be essentially the phase-conjugate replica of the incident beam, $E_{L}^{*}$. The reason is that this particular mode has the highest exponential gain, and will be dominant over all other modes. Nearly all incident laser power may thus be reflected into a phase-conjugate replica. The slight frequency shift $\left(\omega_{\mathrm{ph}} / 2 \pi=\mathrm{n} \omega_{\mathrm{L}} \mathrm{v}_{\mathrm{ac}} / 4 \pi \mathrm{c} \sim 10^{9} \mathrm{~Hz}\right)$ is probably of little consequence, but the requirement that the intensity of the received beacon signal exceed the threshold for stimulated Brillouin scattering by a significant amount is a major disadvantage. The desired effect has been demonstrated in small-scale laboratory experiments.

Another nonlinear method for true phase conjugation without change in frequency is based on four-wave light mixing. In principle, any medium, including isotropic liquids and gases, has a nonlinear response with a polarization proportional to the cube of the electric field amplitude. Consider a field which is the superposition of three plane waves with the same frequency and the same linear polarization:

$$
E=\sum_{j=1,2,3} A_{i}^{i k_{j} \cdot r-i \omega t}+\text { c.c. }
$$

It is clear that the resulting nonlinear polarization will have a term, among others, at the original frequency proportional to

$$
\begin{aligned}
\mathbf{P}_{(\omega)}^{\mathrm{NL}}= & \chi^{(3)} \mathbf{A}_{1} \mathbf{A}_{2} \mathbf{A}_{3} \mathrm{e}^{\mathrm{i}\left(\mathbf{k}_{1}+\mathbf{k}_{2}-\mathbf{k}_{3}\right) \cdot \mathbf{r}+\mathrm{i}\left(\phi_{1}+\phi_{2}-\phi_{3}\right)-\mathrm{i} \omega \mathrm{t}} \\
& + \text { c.c. }
\end{aligned}
$$

Now take $\mathbf{k}_{1}=-\mathbf{k}_{2}$, and $\mathbf{A}_{1}=\mathbf{A}_{2}$, i.e., let beams 1 and 2 propagate exactly opposite to each other with equal intensities. These two waves thus constitute a standing wave "pump" field. Identify the third wave with the incoming beacon signal. It has a wave vector $\mathbf{k}_{\mathbf{3}}$ and a distorted phase front $\phi_{3}$. It is clear that the nonlinear polarization will radiate a backward phase conjugate wave,

$$
\mathrm{E}_{4} \propto \chi^{(3)} \mathrm{A}_{1}^{2} \mathrm{~A}_{3} \mathrm{e}^{-\mathrm{i} \mathbf{k}_{3} \cdot \mathrm{r}-\mathrm{i} \phi_{3}-\mathrm{i} \omega \mathrm{t}} \mathrm{e}^{\mathrm{i}\left(\phi_{1}+\phi_{2}\right)}+\text { c.c. }
$$

The experimental configuration is sketched in Figure 5.19. This four-wave mixing process does not possess a threshold, but it requires a standing wave pump field over a sufficiently large volume of interaction with the beacon beam, and the pump field should be coherent with the incident signal, i.e., the frequency should be the same. As in the case of stimulated Brillouin scattering, four-wave phase-conjugate mixing has only been reported in small-scale laboratory experiments.

\subsubsection{Thermal Blooming}

Intense laser beams propagating through the atmosphere can heat the medium due to absorption of the radiation by molecules and aerosols. This heating can cause the density to decrease in the region of the beam, producing an aberrated, typically negative lens, whose profile is related to the beam profile. The result is a deflection and defocusing of the beam in a complex fashion which will be discussed below. The effect has been given the colorful name "thermal blooming." Laser wavelengths for atmospheric propagation are chosen where absorption is weak; nevertheless, residual absorption together with the intense radiation can lead to blooming. The absorbed energy per unit volume which produces the heating is the product of the absorption constant, the beam intensity, and a time which can depend on beam motion through the air and heat

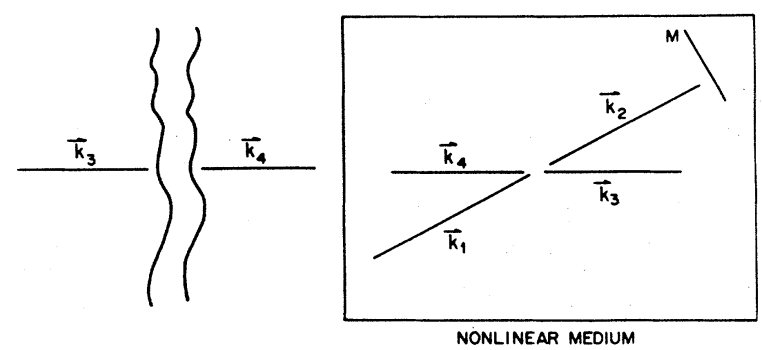

Figure 5.19. Phase conjugation by four-wave light mixing. The standing wave pump field is created by the incident pump $\mathbf{k}_{4}$, and the mirror JM. The beacon signal with a wave front distorted by passage through the atmosphere has a wave vector $\mathbf{k}_{3}$. In the nonlinear medium a backward phase conjugate wave with wave vector $\mathbf{k}_{4}=-\mathbf{k}_{3}$ is created. 
transport due to convection (wind) or conduction.

The phenomenon of thermal blooming was observed $^{16}$ in liquids not long after the invention of the laser and was subsequently found in solids and gases. The interest in propagation of intense laser beams in the atmosphere has led to extensive studies of the phenomenon (References 17-22 contain detailed discussions as well as extensive references to other literature). Because of the practical difficulty of full scale atmospheric tests, much of the work has concentrated on laboratory simulation and theory. Numerical models for beam blooming have been developed ${ }^{23}$ which incorporate scalar diffraction in the paraxial approximation as well as the relevant atmospheric processes. Both the nonlinear character of the beam propagation and influence of turbulence may require numerical calculations for accurate prediction of their effects. In order to reduce the effect of blooming, consideration has been given to shaping the output beam profile both in amplitude and phase using approximate predictive techniques, based on a knowledge of atmospheric parameters, and, additionally, iterative methods where the results of theoretical calculation or experimental measurement of blooming are used recursively to provide input beam parameters until convergence to an "optimal" solution is obtained.

It should be pointed out that the thermal blooming of finite beams is related to absorptively induced instabilities for infinite plane waves. ${ }^{24-26}$ Such instabilities, which build up from noise, may represent an ultimate limit on beam intensity for which no compensation can be made. It is also worth noting that absorptive heating of laser window materials ${ }^{27}$ and mirrors can also lead to thermal lensing.

The use of high-power lasers in strategic defense involves two situations where atmospheric propagation and hence thermal blooming must be considered. One involves propagating the output from a ground-based laser to an orbiting mirror, the other concerns directing a beam from an orbiting mirror toward a target in the atmosphere. All other things being equal, the effect of blooming in the first case is expected to be more serious as the process occurs close to the output where small deviations can have a large effect on the beam arriving at a distant, exoatmospheric point.

Only an elementary analytical and approximate model is presented here together with a discussion of the physics; readers can find more exact theory and experimental details in the references. To understand the basic thermal blooming process consider the very simple case where a stationary beam propagates through a macroscopically motionless medium where heat conduction is not significant during illumination by the beam. Due to absorptive heating the density and, therefore, the index of refraction decrease. If the thermal lens is thin in the sense that the beam intensity profile does not change while propagating through the absorptive medium, only the phase will be distorted as the beam exits the medium. This phase distortion will have a profile which follows the beam profile and which builds up linearly with time as the medium heats under laser illumination. If the beam profile is parabolic then the effect is that of a simple, nonaberrated negative lens. On the other hand, a Gaussian beam can produce a highly aberrated negative lens and a complex ring structure interference pattern can appear in the far field. A characteristic distance ${ }^{28}$ for beam distortion can be obtained from the eikonal equation ${ }^{29}$ of geometrical optics which describes the motion of a light ray in a medium with an inhomogeneous refractive index together with the equation for the change in refractive index due to absorptive heating. In the paraxial approximation, where it is assumed the rays do not deviate markedly from the propagation direction, the eikonal equation is

$$
\frac{\mathrm{d}^{2} \mathrm{r}}{\mathrm{dz}^{2}}=\frac{1}{\mathrm{n}} \nabla_{\perp} \mathrm{n},
$$

where $r$ is the position of the ray in the $x, y$ plane perpendicular to the propagation direction $z$, and $\perp$ indicates the index gradient is taken perpendicular to the propagation direction. For the case where only a small fraction of the beam power is absorbed, the index change is given by

$$
\mathrm{n}=\mathrm{n}_{0}+\frac{\mathrm{dn}}{\mathrm{dT}}\left(\frac{\alpha \mathrm{It}}{\rho_{0} \mathrm{C}_{\mathrm{p}}}\right)
$$

where $n_{0}$ is the unperturbed refractive index, $d n / d T$ is the change of refractive index with temperature, $\alpha$ is the absorption constant, $I$ is the laser intensity, $\rho_{0}$ is the density at sea level, $t$ is time, and $C_{p}$ is the specific heat. It is apparent from these equations that the rays deflect the most in the region where the radial intensity gradient is greatest. Combining Equations (5.15) and (5.16) and integrating with respect to $z$, assuming constant beam profile, a characteristic distance is obtained for an average ray to double its initial distance from the beam center:

$$
\mathrm{z}_{\mathrm{c}}=\frac{\rho_{0} \mathrm{C}_{\mathrm{p}} \mathrm{a}^{2}}{\left|\frac{\mathrm{dn}}{\mathrm{dT}}\right|\left(\alpha \mathrm{It} \mathrm{L_{ \textrm {a } } )},\right.}
$$

where $L_{a}$ is the propagation distance in the atmosphere and $a$ is the beam radius. In the case of laser transmission from ground to a distant exoatmospheric mirror, the size of the total optical system is minimized when $\mathrm{a}^{2}=\mathrm{R} \lambda$, where $\mathrm{R}$ is the range from the ground to the orbiting mirror and $\lambda$ is the laser wavelength; in other words, we assume equal mirror sizes and equate the range to the Rayleigh distance. Setting $z_{c}=R$, a critical intensity-time product is found from Equation (5.17):

$$
(\mathbf{I t})_{\mathrm{cr}}=\frac{\rho_{0} \mathrm{C}_{\mathrm{p}} \lambda}{\left|\frac{\mathrm{dn}}{\mathrm{dT}}\right| \alpha \mathrm{L}_{\mathrm{a}}}
$$


For the sea level atmosphere $\rho_{0}=1.2 \times 10^{-3} \mathrm{~g} / \mathrm{cm}^{3}$, $\mathrm{C}_{\mathrm{p}}=1.0 \mathrm{~J} / \mathrm{gK}$ and $\mathrm{dn} / \mathrm{dT}=-9.5 \times 10^{-7} \mathrm{~K}^{-1}$. The atmosphere can be crudely approximated using the sea level values and taking $\mathrm{L}_{\mathrm{a}}=10^{6} \mathrm{~cm}$, an approximate value for the scale height of the atmosphere. A "typical" value of $10^{-6} \mathrm{~cm}^{-1}$ is used for the absorption constant, $\alpha$. Values somewhat lower than $10^{-7} \mathrm{~cm}^{-1}$ have been predicted near $1 \mu \mathrm{m}$ using Air Force Geophysical Laboratory data and numerical models; predictions of such low absorption have great risks associated with the lack of knowledge of normally small effects such as dimerization, the presence of low concentration constituents, and aerosol variability. Improvement in the transmittable energy flux is to be expected, at least for this thermally transient case, if the ground-based beam projector produces larger beams in the atmosphere than that given by the Rayleigh distance criterion used above.

In the case where the beam diverges significantly in the atmosphere, and thus the intensity changes from the linear values, $(\mathrm{It})_{\mathrm{cr}}$ is found from Equation (5.18) by replacing $z_{c}$ by $L_{a} / 2$. In this case we have

$$
(\mathrm{It})_{\mathrm{cr}}^{\prime}=\frac{\rho_{0} \mathrm{C}_{\mathrm{p}} \lambda}{\left|\frac{\mathrm{dn}}{\mathrm{dT}}\right| \alpha \mathrm{L}_{\mathrm{a}}}\left(\frac{2 \mathrm{a}^{2}}{\lambda \mathrm{L}_{\mathrm{a}}}\right),
$$

where the prime is used to distinguish this thick lens result from the thin lens result. For a $3 \mathrm{~m}$ radius beam and the parameters used above, we find that $(\text { It })^{\prime}{ }_{\mathrm{cr}}=2.3 \times 10^{2} \mathrm{~J} / \mathrm{cm}^{2}$. The thick lens result is significant since it is known $^{30}$ that predictive adaptiveoptical phase compensation cannot correct for thermal blooming at substantially higher fluxes. The thin lens result is also important since it determines the limit above which compensation techniques must be employed.

Under some circumstances similar results may be found using a caustic approximation (ray crossing) criterion; it is expected that the caustic analysis has a wider range of applicability. ${ }^{31}$ The following topics should be considered in a more detailed analysis of thermal blooming.

1. The spatial and temporal characteristics of the initial beam.

2. The absorptive heating process including the kinetics of conversion from internal degrees of freedom to translation in molecules and the vaporization process for aerosols. An example where conversion delay can be significant occurs in the case of $\mathrm{CO}_{2}$ laser absorption by atmospheric $\mathrm{CO}_{2}$ molecules. ${ }^{19}$

3. Transient effects arising from the approach to isobaric conditions. Isobaric conditions are established in a time of the order of an acoustic transit time across the beam radius. For a $3 \mathrm{~m}$ radius beam this transit time is $10 \mathrm{~ms}$. During the time for establishing isobaric conditions the thermally induced refractive index grows as $\mathrm{t}^{3}$.
4. Convective (wind and buoyancy) and conductive heat flow. Wind and beam translation (slewing) have a profound effect on thermal blooming and will be discussed in detail below.

5. Diffraction.

6. Turbulence.

The more significant aspects of these problems are now discussed. Particularly noteworthy is the situation where wind or beam slewing removes heat from the region of the beam during laser illumination has the effect of converting a circular beam profile into an asymmetric beam with a principal crescent shaped region as shown in Figure 5.20. The heating effect builds up across the beam in the downwind direction causing the beam to both defocus and deflect upwind; the deflection is greatest in the center of the beam producing the crescent shape. The time constant for wind or slewing dominated blooming is $a / v$ where $a$ is the beam radius and $v$ is the wind or slewing speed. This time constant can replace the time in Equation (5.17) which gives the characteristic distance. Clearly, the higher the speed the larger the characteristic distance. For a $10 \mathrm{mph}(4.5 \mathrm{~m} / \mathrm{s})$ crosswind, the transit time constant is $0.67 \mathrm{~s}$ for a $3 \mathrm{~m}$ radius beam. Using the values of $0.13 \mathrm{~J} / \mathrm{cm}^{2}$ and $2.3 \times 10^{2} \mathrm{~J} / \mathrm{cm}^{2}$ obtained, in the ground to space case, for $(\mathrm{It})_{\mathrm{cr}}$ and $(\mathrm{It})^{\prime}{ }_{\mathrm{cr}}$, respectively, a value of $L_{c r}=0.19 \mathrm{~W} / \mathrm{cm}^{2}$ is found for the onset of significant thermal blooming and value of $\mathbf{I}_{\text {cr }}^{\prime}=3.4 \times 10^{2} \mathrm{~W} / \mathrm{cm}^{2}$ for the level above which predictive phase compensation cannot correct for blooming. Note that slewing is somewhat different from a uniform wind in that the slewing speed increases linearly with distance from the slewing mirror.

Natural wind or slewing cannot be counted on to remove heat from the region of the beam. Convection will also occur because of the buoyant force of heated air under the influence of gravity. ${ }^{32}$ The buoyant force depends on the beam heating, hence on the flow rate; also, the kinetic energy acquired in the flow must balance the buoyant potential energy. These two relations establish the steady flow speed in the absence of forced convection. In the absence of natural wind or slew, the time scale for heat deposition in the region of the beam is again the beam radius divided by the buoyant convective speed. The significance of blooming of this convection due to gravity and beam heating will naturally depend on the alignment of the beam with respect to the gravity vector; the convection effect should be most pronounced when the beam travels perpendicularly to gravity and should be weakest when the beam is vertical; the latter situation is closest to that found in propagation of beams from a ground-based laser to an orbiting mirror.

There appear to be no practical situations for which conduction determines the temperature rise in the beam. The thermal diffusivity of air is $0.22 \mathrm{~cm}^{2} / \mathrm{s}$ and, therefore, the time for conduction to reach a steady state is of the order of $10^{6} \mathrm{~s}$ for $3 \mathrm{~m}$ radius beam.

Change of gas density caused by absorptive heating is the primary cause for the change in refractive index of 
EXPERIMENT

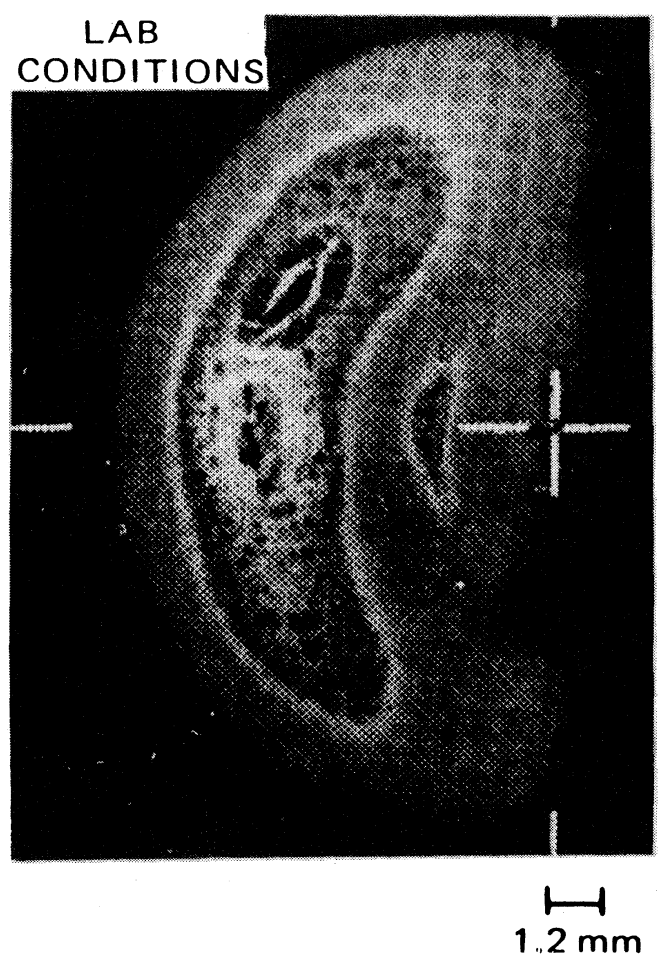

THEORY

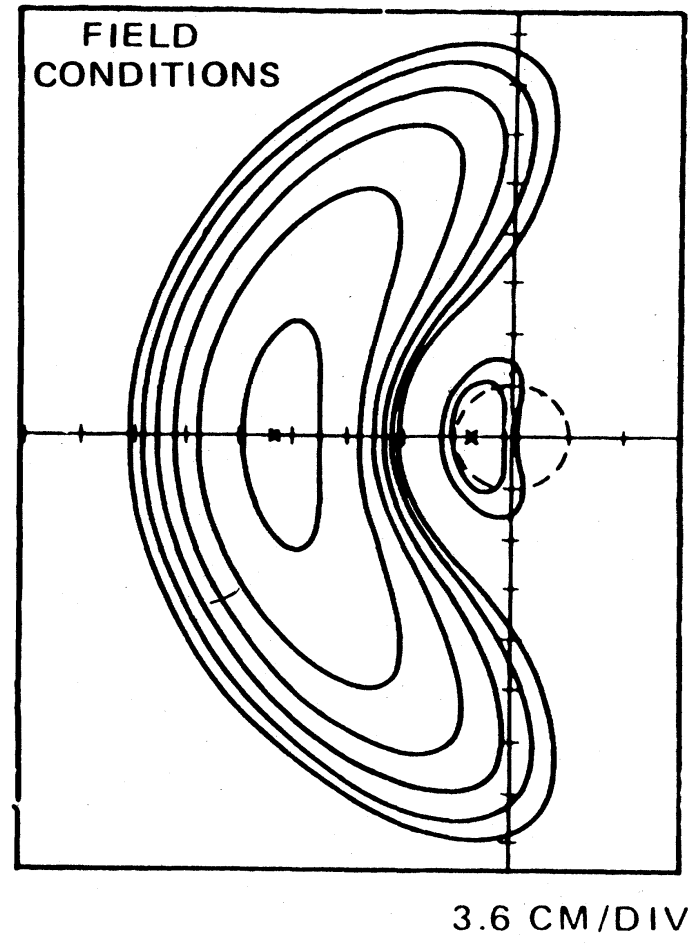

Figure 5.20. Photograph of $\mathrm{cw} \mathrm{CO}_{2}$ laser beam profile from laboratory simulation experiments of thermal blooming in the atmosphere and numerically calculated intensity contours from a theoretical simulation. Dimensionless parameters characterizing thermal blooming were the same in both cases; the results show good correlation and therefore understanding of cw thermal blooming. For further information see Reference 17.

gases. However, there is a delay in the density decrease; initially, on heating, the gas pressure rises and, after a time of the order of the time for an acoustic wave to propagate across the beam, the pressure equilibrates and the density drops. Prior to the establishment of isobaric conditions, the temporal change of index of refraction goes as $\left(v_{s} / a\right)^{2} t^{3}$ instead of $t$, where $v_{s}$ is the velocity of sound and $a$ is the beam radius. Pulses or pulse trains shorter than $a / v_{s}$ will have substantially less blooming than pulses or pulse trains whose duration is longer than $\mathrm{a} / \mathrm{v}_{\mathrm{s}} ;$ as noted previously, $\mathrm{a} / \mathrm{v}_{\mathrm{s}}=10^{-2} \mathrm{~s}$. Pulses or pulse trains will not experience blooming due to prior pulses or pulse trains if forced convection (wind) has removed the heated air away from the region of the beam in the time between pulses or pulse trains. The interpulse removal time for the case considered above, a $10 \mathrm{mph}$ wind and a $3 \mathrm{~m}$ beam radius is $0.67 \mathrm{~s}$. The problem of interpulse cooling has been studied ${ }^{33-35}$ and it has been found experimentally ${ }^{34}$ that pulse repetition rates of up to two or three times $\mathrm{v} / \mathrm{a}$ are possible before thermal blooming causes deleterious effects.

The interaction of turbulence with thermal blooming has been studied. ${ }^{36,37}$ It has been concluded $^{37}$ that turbulence does not effect blooming when $\sigma_{\mathrm{v}} \mathrm{l}_{0}<\mathbf{a v}_{0}$, where $\sigma_{\mathrm{v}}$ is the characteristic turbulent velocity, $1_{0}$ is the characteristic scale length of turbulence induced intensity fluctuations, $a$ is the beam radius, and $v_{0}$ is the convective (wind) speed; the inequality is satisfied in most practical situations.

Heating due to aerosol absorption has features which distinguish it from heating due to molecular absorption. A portion of the absorbed energy may be used in the vaporization of the aerosol particles. Furthermore, because of the low density of aerosols, the heating can have a nonuniform character for short irradiation times. The problem has been studied ${ }^{38}$ both experimentally and theoretically.

We conclude that theoretical and experimental investigations are needed before an intelligent assessment can be carried out of the effect of atmospheric thermal blooming on system performance. A significant experimental effort is underway at White Sands where the atmospheric distortion of a high power DF laser beam will be studied. A major effort under realistic field conditions is necessary before any substantial commitment to ground-based laser systems can be made. In addition, thermal blooming on the downlink to a target in the upper atmosphere appears to require further study. Careful evaluation must be made of the contribution to blooming of various atmospheric constituents such as 
ozone at short wavelengths and volcanic aerosols and of heating due to stimulated rotational Raman scattering in nitrogen (see next section).

Finally, it is not clear that atmospheric data can be obtained at required rates and employed in adaptive optical systems so as to achieve predictive and iterative blooming compensation at significant fluence levels in the $10-10^{2} \mathrm{~J} / \mathrm{cm}^{2}$ range. Thus, the ability to provide atmospheric compensation of high intensity laser beams is important, yet problematic.

\subsubsection{Stimulated Raman Scattering}

\subsubsection{Introduction}

In molecular Raman scattering an incident photon $\mathrm{h} v_{1}$ is inelastically scattered by a molecule to a photon $\mathrm{h} v_{2}$. The difference in photon energies, $\mathrm{h}\left(\boldsymbol{v}_{1}-v_{2}\right)$, is taken up by the molecule as vibrational and/or rotational excitation. In the absence of electronic resonant enhancement, i.e., if the incident light frequency is far removed from electronic absorption lines, the cross section is typically small, of the same magnitude as that for Rayleigh scattering:

$$
(\mathrm{d} \sigma / \mathrm{d} \Omega)=(2 \pi / \lambda)^{4}\left|\mathrm{~A}_{\mathrm{nm}}\right|^{2} \sin ^{2} \theta,
$$

where $\lambda$ is the wavelength of the scattered light, and $A_{n m}$ is the polarizability matrix element connecting the initial and final molecular states. For simple molecules, polarizability matrix elements are of order $10^{-25} \mathrm{~cm}^{3}$, so that for visible light, the Raman cross section (per molecule) is of order $10^{-30} \mathrm{~cm}^{2}$.

Like any other scattering process, Raman scattering can be stimulated. If two light waves are incident at frequencies separated by the frequency of the molecular excitation, the probability of the Raman scattering process becomes proportional to the product of the intensities in the two beams. If the number of photons per mode in the second laser beam is large compared to unity, the Raman scattering is greatly enhanced compared to the spontaneous process. The beam at $v_{2}$ will experience an exponential gain. This stimulated Raman process will start from the spontaneous scattering which produces the first photon in the direction of the beam to be amplified. The frequency shifted light at $v_{2}$ is referred to as the "Stokes" component.

The growth of the Stokes intensity $\left(I_{S}\right)$, and the attendant decrease of the laser intensity $\left(I_{L}\right)$, are described by the pair of equations

$$
\begin{aligned}
& \left(\mathrm{d} \mathbf{I}_{\mathrm{S}} / \mathrm{dz}\right)=\gamma \mathrm{I}_{\mathrm{L}} \mathrm{I}_{\mathrm{S}}, \\
& \left(\mathrm{d} \mathrm{I}_{\mathrm{L}} / \mathrm{dz}\right)=-\left(\lambda_{\mathrm{S}} / \lambda_{\mathrm{L}}\right) \gamma \mathrm{I}_{\mathrm{L}} \mathbf{I}_{\mathrm{S}} .
\end{aligned}
$$

Here $\lambda_{\mathrm{S}}$ and $\lambda_{\mathrm{L}}$ are, respectively, the Stokes and laser wavelengths, and $\gamma$ is the Raman gain coefficient. Equations (5.21) are strictly correct only in the absence of phase-matched four-wave interactions and transient phenomena, both of which reduce the gain coefficient from its steady-state value of

$$
\gamma(v)=\left(\lambda_{\mathrm{S}}^{3} \mathrm{~N} / \mathrm{hc}\right)(\mathrm{d} \sigma / \mathrm{d} \Omega) \mathrm{f}(v)
$$

Here $\mathrm{N}$ is the population difference between the initial and final molecular states, and $\mathrm{f}(v)$ is the molecular lineshape function. For atmospheric gases, several important features of the gain dependence are noted, as follows.

1. Since the cross section varies as $\lambda_{\mathrm{s}}^{-4}$, the Stokes gain is proportional to the scattered frequency.

2. Since the maximum value of the line-shape function is inversely proportional to the linewidth, the Stokes gain is independent of the atmospheric pressure, as long as the transition is pressure broadened.

3. The temperature dependence enters only through the population factor $\mathrm{N}$, as long as the transition is pressure broadened.

Stimulated Raman scattering builds up from ordinary (spontaneous) Raman scattering in the path of the laser beam. In the absence of any injected signal at the Stokes frequency, a total of approximately 25-e-fold gain is required, to amplify ordinary Raman scattering to $1 \%$ of the initial laser intensity. Once this level of Stokes signal is achieved, the laser beam is quickly depleted. Additionally, the Stokes beam becomes intense enough to start to generate significant Raman scattering itself. The onset of SRS is dramatic, and is often described in terms of a "threshold" for the process, corresponding to the exponential gain value of 25 . The transition with the largest gain will dominate in the stimulated gain regime.

\subsubsection{Comparison Between Theory and Experiment of Stimulated Raman Scattering in $\mathrm{N}_{2}$}

Stimulated Raman scattering (SRS) of both vibrational and rotational transitions in nitrogen gas has been observed. ${ }^{39,40}$ In the atmosphere which consists of about $80 \% \mathrm{~N}_{2}$ up to an altitude of $100 \mathrm{~km}$, Raman scattering from $\mathrm{N}_{2}$ molecules will be dominant. Extensive calculations on the Raman gain in the atmosphere for both $\mathrm{N}_{2}$ and $\mathrm{O}_{2}$ scattering processes have recently been published. ${ }^{41,42}$ At pressures of one atmosphere and below, rotational Raman scattering is dominant. The mode with the highest gain is the $S_{00}(8)$ rotational transition from the rotational state $J=8$ to $J=6$, while the molecule remains in the vibrational ground state. Adjacent rotational transitions, e.g., $J=10$ to $J=8$, may have comparable gain, which is largely determined by the population difference between the initial and final rotational state and 
thus depends on the temperature.

At pressures above three atmospheres, vibrational Raman scattering in the $\mathrm{Q}_{01}$ branch, with $\Delta \mathrm{J}=0$, becomes dominant, because the linewidth of this transition narrows with increasing pressure, as an average over all rotational states is taken for frequent collisions. The competition between vibrational and rotational scattering also depends on the state of polarization of the light beams. ${ }^{39,42}$ Incident circular polarization at $v_{1}$ favors rotational scattering, with $\Delta \mathrm{J}=2$, to a beam at $v_{2}$ with the opposite circular polarization.

The calculated Raman gain coefficient ${ }^{42}$ for $S_{00}(8)$ scattering at $\lambda=1.06 \mu \mathrm{m}$ in nitrogen is $2.6 \mathrm{~cm}^{-1}$ for an incident intensity of $10^{12} \mathrm{~W} / \mathrm{cm}^{2}$, or $\gamma=2.6 \mathrm{~cm} / \mathrm{TW}$.

This expression is valid for pressures from 1 atm down to 1 torr. In this regime the linewidth is collision dominated. These theoretical results agree rather well with experimental data obtained in nitrogen filled cells in laboratory experiments.

Averbakh et al. ${ }^{39}$ have measured the pressure dependence of the SRS threshold power in nitrogen for both rotational and vibrational scattering, with the results shown in Figure 5.21. At pressures up to about $3 \mathrm{~atm}$, the threshold for rotational SRS is essentially constant, while at high pressures, the rotational sublevels are unresolved for the vibrational transition, so the vibrational transition exhibits a lower threshold. At low pressures, the vibrational transition is observed only for linearly polarized light. The threshold was observed to increase with decreasing pressure down to 1 atm. The Soviet authors infer from this result that the rotational sublevels of the $\mathrm{Q}$ branch are not resolved, even at $1 \mathrm{~atm}$.

Henesian et al. ${ }^{40}$ observed rotational SRS on the $S_{00}(8)$ and $S_{00}(10)$ lines of nitrogen in the beam tubes of the Nova laser. They obtained a value for the gain

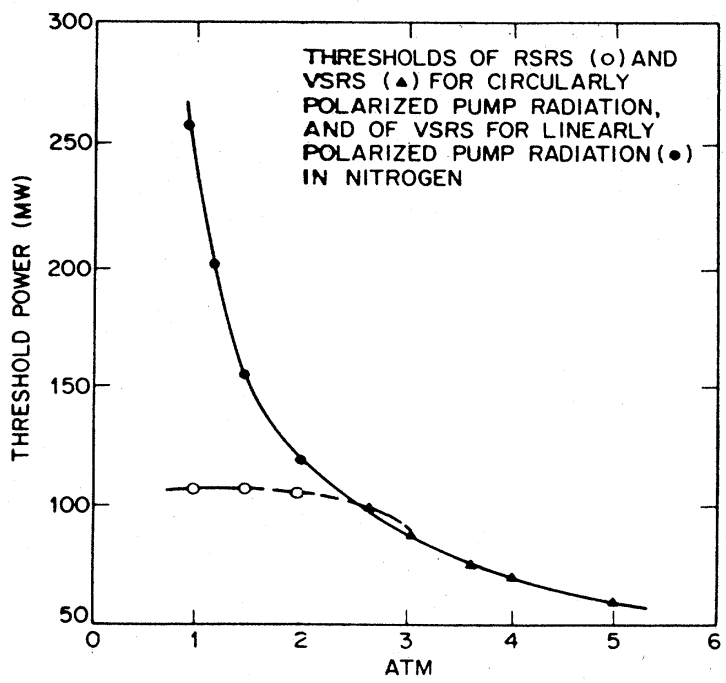

Figure 5.21. Competition between rotational and vibrational stimulated Raman scattering [from Averbach et al. (Reference 39)]. coefficient of $\gamma=2.5 \mathrm{~cm} / \mathrm{TW}$ for linearly polarized light at $1.05 \mu \mathrm{m}$, in $1 \mathrm{~atm}$ pressure air. They compare this result to the value of Averbakh et al. of $6.6 \mathrm{~cm} / \mathrm{TW}$, obtained with circularly polarized light at $0.53 \mu \mathrm{m}$, in pure nitrogen. The gain in the LLNL experiment is expected to be lower by a factor of 0.5 , due to the longer wavelength, and 0.67 , due to the use of linearly polarized light, and reduced by 0.8 , since the medium is air. Thus the measurement of Averbakh et al. would scale to $\gamma=1.8 \mathrm{~cm} / \mathrm{TW}$, under the LLNL conditions. The LLNL authors attribute the remaining difference to the use of a $2.5 \mathrm{~ns}$ pulse duration, compared to the pulse length of $1 \mathrm{~ns}$ used by the Soviet researchers. However, the Soviet authors present data showing that they have achieved steady-state conditions at pulse lengths slightly greater than $1 \mathrm{~ns}$.

For a pressure-broadened line, the requirement for steady-state gain is that the optical pulse duration $t_{p}$ exceed the collisional width. This condition may be written in the form

$$
\mathrm{t}_{\mathrm{p}}>2 \mathrm{~ns} / \mathrm{P}(\mathrm{atm})
$$

While the pulse durations envisioned for BMD applications satisfy this condition at lower altitudes, corrections for transient behavior are essential at higher altitudes. The linewidth of the $S_{00}(10)$ transition versus altitude is shown in Figure 5.22. As the disparity between the Soviet and LLNL results illustrates, transient effects must also be considered in interpreting laboratory experiments.

\subsubsection{SRS in the Atmosphere}

For pressures lower than 1 torr, i.e., for altitudes higher than $45 \mathrm{~km}$, the linewidth becomes constant, and is determined by the small Doppler broadening for

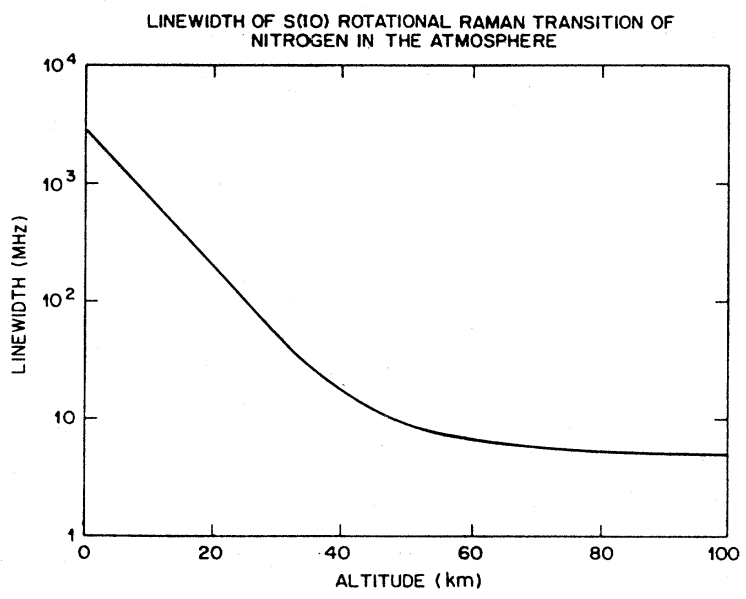

Figure 5.22. Linewidth of $S(10)$ rotational Raman line of nitrogen in the atmosphere [from Kurnit and Ackerhalt (Reference 41)]. 
forward Raman scattering, as shown in Figure 5.22. The gain constant then becomes proportional to the density of $\mathrm{N}_{2}$ and drops rapidly with increasing altitude. The gain constant is also dependent on the temperature profile because the population in the rotational states depends on the temperature. These two factors explain the gain profile shown in Figure 5.23. These data can be used to determine the "threshold" value for $1 \%$ conversion to Stokes light for propagation from the earth's surface straight up through the atmosphere.

The integrated gain along a zenith path is shown in Figure 5.24, for various pulse durations, for a parallel beam with an intensity of $1 \mathrm{MW} / \mathrm{cm}^{2}$. The steady-state limit is found to be about $13 \mathrm{~Np}$ at $1 \mathrm{MW} / \mathrm{cm}^{2}$. A threshold value of $25 \mathrm{~Np}$ implies that no more than $2 \mathrm{MW} / \mathrm{cm}^{2}$ can be propagated through the atmosphere, for a linearly polarized beam of $1 \mu \mathrm{m}$ light, in a long pulse, without significant beam degradation due to SRS. At an excimer laser wavelength of $353 \mathrm{~nm}$, the threshold reduces to less than $1 \mathrm{MW} / \mathrm{cm}^{2}$. The wavelength and polarization of dependence of the atmospheric gain of $25 \mathrm{~Np}$, or 1\% Stokes conversion is shown in Figure 5.25.

The nitrogen rotational Raman shift represents a small change in laser frequency. Shifts of $75 \mathrm{~cm}^{-1}$ and $91 \mathrm{~cm}^{-1}$ are observed at the $S_{00}(8)$ and $S_{00}(10)$ transitions, respectively. (The vibrational shift is much larger, $2360 \mathrm{~cm}^{-1}$.) Such small shifts are, by themselves, of little concern for BMD applications. The lethality of a DEW laser system would hardly be affected. The concern is, however, that the brightness of the beam will be seriously degraded, well above threshold, as Raman gain occurs not only in the exact forward direction, but in a solid angle which is roughly determined by the aperture D of the ground-based laser system and the effective height of the atmosphere, which was shown above to be about $40 \mathrm{~km}$. For $\mathrm{D}=10 \mathrm{~m}$, the converted Stokes radiation would roughly fill a cone with apex angle $\theta=2.5 \times 10^{-4}$.

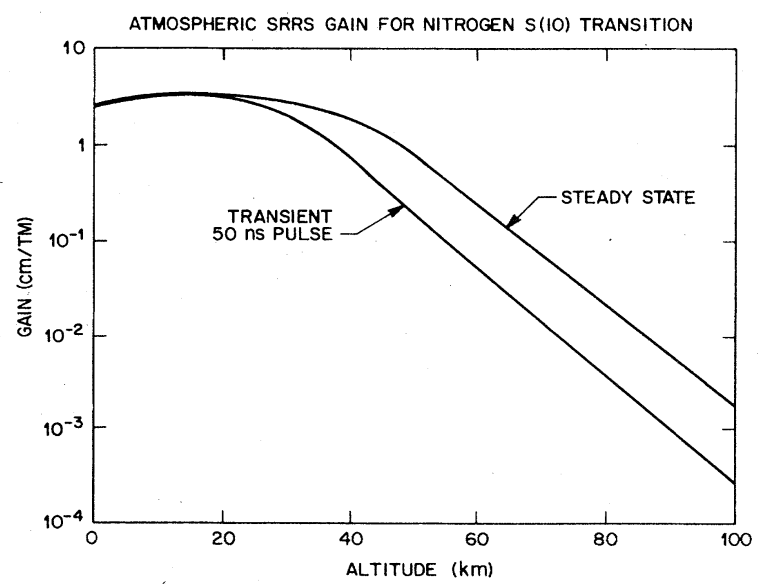

Figure 5.23. Gain coefficient of $\mathrm{S}(10)$ stimulated Raman scattering for nitrogen in the atmosphere [from Kurnit and Ackerhalt (Reference 41)].

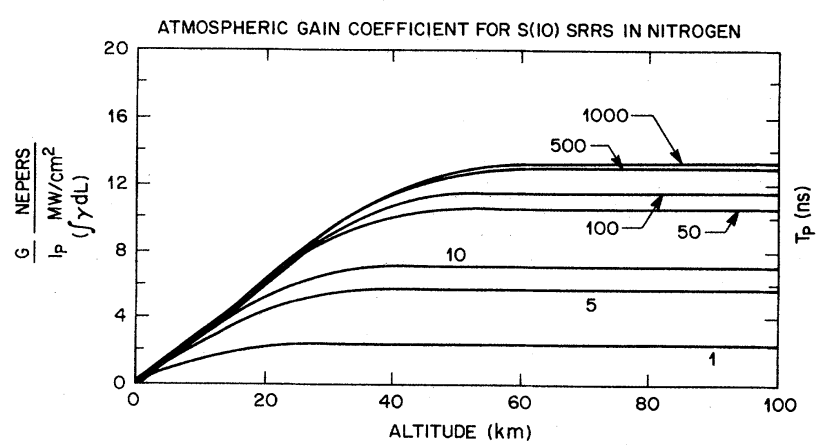

Figure 5.24. Integrated intensity of $\mathrm{S}(10)$ stimulated Raman scattering for nitrogen in the atmosphere for various pulse durations [from Kurnit and Ackerhalt (Reference 41)].

This should be compared with the diffraction spreading $\Delta \theta=\lambda / \mathrm{D} \sim 10^{-7}$ for $\lambda=1 \mu \mathrm{m}$ from the same aperture. This small diffraction limited angle must be maintained to keep an acceptable spot size at an optical relay platform, either in geosynchronous orbit or at an altitude of about $10^{3} \mathrm{~km}$. This simple geometrical argument shows that the beam brightness will be unacceptably degraded, if the threshold for SRS is exceeded by $50 \%$ or more. This limits the intensity to less than $2 \mathrm{MW} / \mathrm{cm}^{2}$ at the ground-based aperture. The maximum total power transmitted is about $2 \mathrm{TW}$ for $\mathrm{D}=10 \mathrm{~m}$.

In the LNLL experiments the Stokes beam divergence was increased by a factor of about three and showed a speckle pattern with a scale size of $2-3 \mathrm{~mm}$, even though the threshold was exceeded by less than a factor of two.

SRS is of even greater concern on the down link into the atmosphere. If targets are to be engaged below $40 \mathrm{~km}$ altitude, SRS can occur in the final focal volume. For either impulsive loading of a target, or thermal loading with a pulsed laser, intensities on target are estimated to be $10^{8} \mathrm{~W} / \mathrm{cm}^{2}$, as the beam is concentrated to a spot size

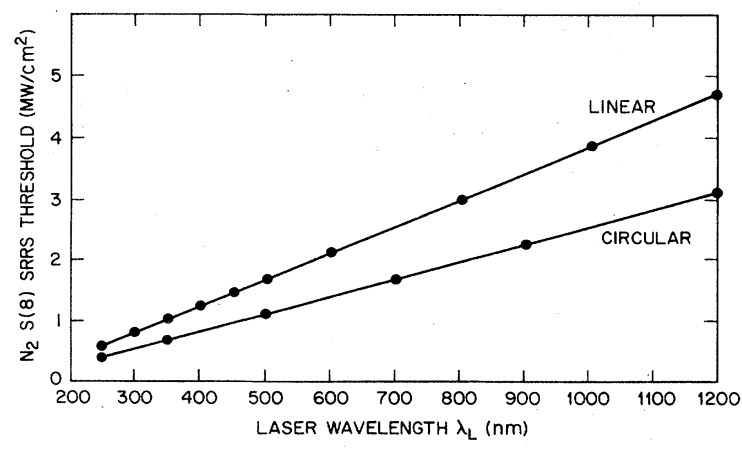

Figure 5.25. Threshold intensity for one percent conversion on the $S(8)$ transition in $N_{2}$ for vertical propagation through the atmosphere with a Fresnel number 5 [after Rokni and Flussberg (Reference 42)]. 
of $1 \mathrm{~m}^{2}$ or less. This intensity is present over the entire depth of focus, which will be about $10 \mathrm{~km}$. With a peak gain coefficient of $2.5 \mathrm{~cm} / \mathrm{TW}$, the net gain through the focal volume can be as high as $\mathrm{e}^{250}$, well above the nominal threshold. Again SRS Stokes radiation will escape from the focal region in a larger solid angle and significantly decrease the effective intensity on target. SRS appears to prevent significant engagement of targets below an altitude of $40 \mathrm{~km}$ for pulsed lasers.

\subsubsection{Possible Remedies}

To suppress SRS on the uplink, the simplest remedy is larger optics, which reduces the intensity in the beam. SRS in the final focal volume in downlink is more difficult to prevent, and is a major concern for pulsed lasers. It has been proposed to suppress SRS by expanding the bandwidth of the laser. If the laser energy can be distributed over a number of frequencies which cannot interact coherently, SRS will not be seen until each component exceeds threshold. In the atmosphere, however, the dispersion of the refractive index, which is the effect which dephases the various frequency components, is very weak. The frequency spectrum of the outgoing beam may have to be broader than the rotational Stokes shift.

An alternative proposal is to "seed" the Stokes frequency by injecting a coherent signal at the Stokes frequency as well as the laser frequency. If the broadband signals are injected at both frequencies, fluctuations in the two signals will be uncorrelated, and the gain will be reduced. As the beams copropagate, however, the frequency and phase distribution of the Stokes component will become reshaped, to provide maximum gain for SRS. The degree of suppression which can be achieved by this process is uncertain, but is not expected to be significant.

It has also been proposed to inject a strong coherent signal at the Stokes frequency to maintain good beam quality, even in the presence of SRS. The wide bandwidth of the FEL amplifier allows amplification of two signals separated by the rotational Raman shift in nitrogen. It is uncertain, however, whether this really provides a remedy for SRS. The strong signal at the Stokes wavelength becomes the generator of successive orders of Raman scattering, particularly on the downlink.

\subsubsection{Conclusions}

Although SRS in atmospheric nitrogen has been a concern for high-power laser systems for 20 years, there are essentially no observations of the effect in atmospheric paths. The fundamental parameters of the process, such as linewidth, frequency shifts, and gains, are well understood, and precisely measured. SRS in nitrogen has been observed and measured in the laboratory, and in the
Nova beam tubes. The interaction of SRS and other propagation effects, especially turbulent scattering, and its impact on SDI system design, are presently under investigation.

\subsubsection{Atmospheric Propagation of High-Intensity X-Ray Pulses}

The main energy loss mechanism in the atmosphere for propagation of $x$ rays with energies above $870 \mathrm{eV}$ is photoionization of nitrogen. ${ }^{43}$ (Resonant absorption for lower energies is beyond the scope of the present discussion.) The average photoionization cross section for atmospheric oxygen and nitrogen atoms is

$$
\sigma_{\mathrm{p}} \sim 8.6 \times 10^{-20} \mathrm{~cm}^{2} / \text { atom }(\mathrm{h} v / \mathrm{keV})^{-3} .
$$

This cross section changes little as the atoms become ionized. However, since the duration of an $\mathrm{x}$-ray laser pulse is typically much shorter than the recombination time for free electrons and ions in the upper atmosphere, the air will become completely ionized if the pulse is intense enough. About five photons must be absorbed by either nitrogen or oxygen atoms for complete ionization; in the process, nitrogen undergoes two, and oxygen three, Auger decays. Once the air becomes completely ionized, its cross section drops to that for scattering by free electrons:

$$
\sigma_{\mathrm{s}}=4.7 \times 10^{-24} \mathrm{~cm}^{2} / \text { atom }
$$

which is negligible except for very high fluences. Thus, if $F$ is the fluence (energy/area) of the $x$-ray beam, the energy loss $\epsilon$ per atom in the beam may be approximated as

$$
\epsilon \sim \operatorname{Min}\left(\mathrm{F} \sigma_{\mathrm{p}}, \eta \mathrm{h} v\right)
$$

where $\eta$ is the number of photons required to completely ionize an atom, i.e., about five. Equation (5.26) can provide the basis for a fairly accurate propagation model for photons above $870 \mathrm{eV}$; details ignored by Equation (5.26) may be shown to be unimportant in most cases. Rather than presenting such a model, however, we shall consider propagation in two limiting cases. Suppose that the atmospheric attenuation occurs in a fairly localized region. Let $F_{0}$ and $F_{1}$ be the fluences of the beam as it enters and exits the region, respectively. For a beam with an initial fluence $F_{0}$ less than

$$
\mathrm{F}_{\mathrm{p}} \equiv \eta \mathrm{h} v / \sigma_{\mathrm{p}} \sim 10 \mathrm{~kJ} / \mathrm{cm}^{2} /(\mathrm{h} v / \mathrm{keV})^{4}
$$

the air in the region will not be completely ionized and 


$$
\begin{aligned}
\mathrm{F}_{1} & =\mathrm{F}_{0} \exp \left[-\mathbf{A}(\mathrm{h} v / \mathrm{keV})^{-3}\right] \\
\mathbf{A} & =\sigma_{\mathrm{p}} \Sigma(\mathrm{h} v / \mathrm{keV})^{3} \\
& \sim \Sigma /\left(1.2 \times 10^{19} \text { atom } / \mathrm{cm}^{2}\right)
\end{aligned}
$$

where $\Sigma$ is the column density (atom/area) of the gas in the region and $\mathrm{A}$ is the number of mean free paths for absorption normalized for $1 \mathrm{keV}$ photons ( $\mathrm{h} v$ is denoted in keV). Thus, the beam is absorbed exponentially. For high fluence beams with $F_{1}>F_{p}$ the air is completely photoionized, and

$$
\mathrm{F}_{1}=\mathrm{F}_{0}-10 \mathrm{~kJ} / \mathrm{cm}^{2}(\mathrm{~h} v / \mathrm{keV}) \mathrm{A}
$$

Thus, the energy loss is linear with column density in this case. There is clearly a transition region for which $F_{1}>F_{p}>F_{0}$, which we ignore.

The dependence of the attenuation on photon energy is made explicit in Equations (5.28) and (5.29). We see that in the low fluence regime (incomplete photoionization) increasing photon energy sharply decreases the attenuation because of the decreased photoionization cross section; it also increases $F_{p}$, the fluence required for complete photoionization. However, if the air remains completely photoionized, increasing photon energy increases the energy loss because the number of photons absorbed remains constant.

For example, the number density of atoms at altitude $h$ in the atmosphere between $40 \mathrm{~km}$ and $140 \mathrm{~km}$ is well approximated by

$$
\mathrm{n} \sim 5.1 \times 10^{19} \mathrm{~cm}^{-3} \exp (-\mathrm{h} / 7 \mathrm{~km})
$$

Thus, the volume density for a beam propagating vertically down to a target at altitude $h$ from an overhead space platform has a column density

$$
\begin{aligned}
& \Sigma \sim 3.6 \times 10^{25} \exp (-\mathrm{h} / 7 \mathrm{~km}), \\
& A \sim \exp [(104 \mathrm{~km}-\mathrm{h}) / 7 \mathrm{~km}] .
\end{aligned}
$$

A beam of $1 \mathrm{keV}$ photons with a fluence of $20 \mathrm{~kJ} / \mathrm{cm}^{2}$ at the top of the atmosphere can deposit $10 \mathrm{~kJ} / \mathrm{cm}^{2}$ on a target at about $104 \mathrm{~km}$ [Equation (5.30)]; at $58 \mathrm{~km}$ for $10 \mathrm{keV}$ photons [Equation (5.28)]. Column densities are greater by a factor of $\sqrt{2 \pi R_{\oplus} / 7 \mathrm{~km}} \sim 76$ for a beam which propagates across the atmosphere at a low tangent altitude from a distant pop-up XRL source to a distant target, compared to a beam which propagates vertically down to the same low altitude from a space platform. This means that a beam with a fluence which drops from $20 \mathrm{~kJ} / \mathrm{cm}^{2}$ to $10 \mathrm{~kJ} / \mathrm{cm}^{2}$ across its tangent point must have a tangent altitude which is about $30 \mathrm{~km}$ higher than the corresponding target altitude of the previous example, i.e., $134 \mathrm{~km}$ for $1 \mathrm{keV}$; $88 \mathrm{~km}$ for $10 \mathrm{keV}$.

In Figure 5.26 the altitudes of targets receiving fluences of $10 \mathrm{~kJ} / \mathrm{cm}^{2}$ are shown as a function of photon energy for various fluences at the top of the atmosphere. These results are based on a more detailed atmospheric density model than the approximation in Equation (5.31) and on more exact calculations of beam loss than given in Equations (5.28) and (5.30). Nevertheless, they agree well with the above estimates. They show that a $200 \mathrm{~kJ} / \mathrm{cm}^{2}$ beam does not penetrate very much deeper than a $20 \mathrm{~kJ} / \mathrm{cm}^{2}$ beam $(20 \mathrm{~km}$ for $\mathrm{h} v \sim 1 \mathrm{keV}$ and only about $8 \mathrm{~km}$ for $\mathrm{h} v>2 \mathrm{keV}$ ). As above, tangent altitudes for propagation across the atmosphere must be about $30 \mathrm{~km}$ higher than target altitudes for downward propagation.

\subsection{HIGH-POWER COMPONENTS}

Two critical optical components required for high energy laser systems are the cooled deformable mirrors required for ground-based systems and shared-aperture components required for high-precision alignment and tracking.

\subsubsection{Cooled Deformable Mirrors}

Cooled deformable mirrors are required in groundbased high energy laser systems for removing wavefront phase variations introduced by atmospheric turbulence, and by aberrations in the optical system caused by misalignment, deformations caused by thermal problems, etc. They are also required in all high energy laser systems for removing laser-introduced phase variations.

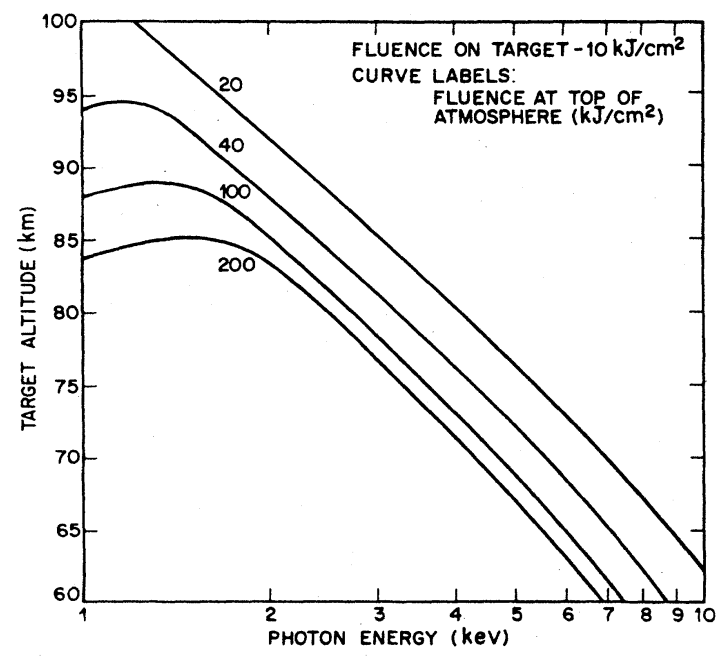

Figure 5.26. The altitude to which a downward propagating beam can deliver a given fluence as a function of photon energy. 
Two mirror materials that have been investigated are silicon and molybdenum. At present, silicon appears to be the leading candidate. The major problems with molybdenum are instabilities and difficulty of getting good optical polish.

Cooled silicon mirrors using silicon heat exchangers have been used at absorbed power densities of the order of $1 \mathrm{~kW} / \mathrm{cm}^{2}$. This would correspond to an incident irradiance of $10 \mathrm{MW} / \mathrm{cm}^{2}$ for an absorption of $10^{-4}$ as further discussed in Section 5.6. It is believed that they can be used at absorbed power densities up to at least $4 \mathrm{~kW} / \mathrm{cm}^{2}$ and possibly $10 \mathrm{~kW} / \mathrm{cm}^{2}$.

At present, the size of a cooled silicon faceplate for a deformable mirror is limited by the size of silicon boule that can be produced. Techniques for splicing pieces of silicon to form larger shapes, without degraded optical characteristics of the reflective surfaces, have not been developed. For size variation in atmospheric turbulence and aberration in optical systems from misalignments, deformation caused by thermal problems, etc., beyond the 35-cm-diameter boules that have been produced, it would be necessary to scale up a manufacturing process for the material or to develop a process for splicing and overcoating the spliced silicon wafers to achieve a surface suitable for optical finishing.

The making and handling of thin, large-diameter silicon wafers does not appear to be a serious limitation with regard to the size of deformable mirrors that can be produced. If silicon material of large diameter is available, it can be converted to the wafers required for large deformable mirrors.

The most significant aspect of the scale-up question is the bonding of the actuators to faceplate and base. Each actuator has two joints that must be leaktight (to coolant), one with the faceplate and one with the base. As the size of the mirror and the number of actuators increase, the statistics of the situation cause concern. Leaks must be avoided without blocking any of the coolant flow holes.

There appears to be no fundamental reason that very large deformable mirrors cannot be developed, but the efforts required certainly become more extensive as size increases.

\subsubsection{High-Power, Shared-Aperture Components}

Since high energy laser systems require highprecision alignment and tracking as well as concentration of the maximum energy density on targets, it is desirable to use an arrangement whereby the beam returning from the target and the alignment and tracker beams share the same optical path with the high energy laser beam. This is done by the use of high-power, shared-aperture components. A dichroic (or multichroic) beamsplitter operating in a high energy laser environment can be used to inject these optical paths into the high energy laser beam or split them from it.
A spectral shared aperture offers several advantages. First, since all beams share the same optical path, there is no difference in tracking, alignment, or beam control due to jitter, misalignment, or aberrations occurring within this optical path. Second, since there is no need for a separate set of alignment or pointing optics, the system size, weight, and complexity are reduced. Third, the LWIR radiation emitted from an uncooperative target can provide aberration information along the actual beam path and thereby allow detection of beam-induced aberrations as well as atmospheric aberrations for ground-based systems.

The performance requirements that a shared-aperture component must meet are formidable. The high energy laser beam should be reflected with high efficiency and sampled or combined with low efficiency. The sharedaperture component should handle very high, medium, and low-power beams simultaneously, while maintaining precise alignment among the beams and a good optical figure.

The types of shared aperture components being considered are (1) cooled beamsplitters, (2) buried gratings, and (3) membranes.

\subsubsection{Cooled Beamsplitters}

The cooling requirements determine the operational limits of both stationary and rotating dichroic beamsplitters. Rotating beamsplitters are preferable, but they require either gas coolants of cryogenic temperature or dichroic coating absorptivities that are lower than currently achievable. Aperture-sharing components that employ the well established operational parameters of fluid-cooled metal-mirror substrates appear much more promising.

\subsubsection{Buried Gratings}

A buried grating separates the outgoing laser beam from the incoming target-return beam in the longwavelength infrared (LWIR) region $(8-12 \mu \mathrm{m})$. The operation of this component in its simplest form is shown in Figure 5.27. A grating on a cooled substrate diffracts the incoming LWIR beam with high efficiency into the first order, as shown. The buried grating can be integrated with a complementary element to compensate for dispersion. Dispersion must be compensated for because each wavelength in the LWIR beam is diffracted in a different direction. These diffracted beams can be reassembled as one beam by use of a dual-grating rhomb. The grating is covered by a burying layer and a dichroic reflector coating that are transparent to the LWIR beam. The high energy laser beam is reflected by the dichroic coating, which is designed to be highly reflecting at the laser wavelength. In this way, the LWIR and high energy laser beams are spatially separated. This component can be designed to provide additional beam 


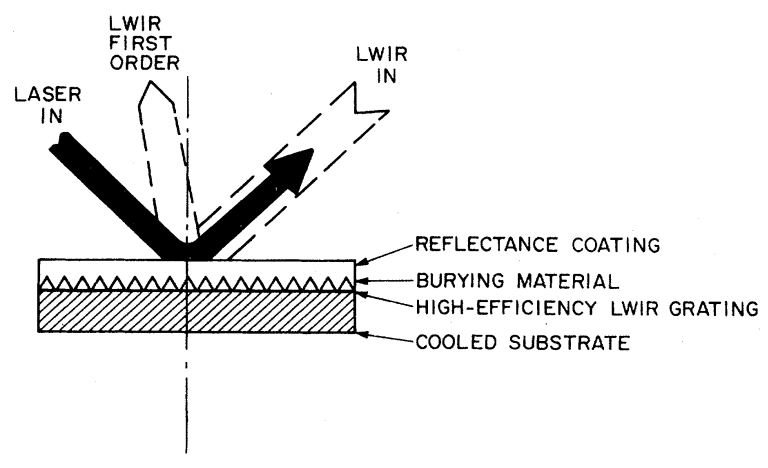

Figure 5.27. Operation of a buried-grating component.

separation for alignment beams and for low efficiency sampling of the high energy laser beam.

The construction of a buried grating is shown in Figure 5.28. A layer of gold is deposited on a cooled mirror substrate, and a diffraction grating is ruled in this layer using a conventional diamond ruling process. The material used for the burying layer depends upon the high energy laser wavelength. After deposition, this layer is polished flat to prevent "print through" of the grating profile, and a dichroic reflector is deposited on top of this layer. This filter is highly reflective in the high energy laser wavelength band and is typically formed from alternating layers of materials having high and low indices of refraction.

\subsubsection{Membranes}

Membrane, or "pellicle," aperture-sharing elements offer the potential of being transmissive rather than refractive/diffractive optical elements, and at the same time it is possible that they may not need to be cooled. The latter observation is based on measurements at several hundred kilowatts of power, in which no pellicle heating occurred. This implies that pellicles may have a near-zero optical depth owing to their thinness. The important parameter then becomes the absorption of the high energy laser reflective coating on the pellicle and the pellicle acoustical stability.

Although high-power shared-aperture components are difficult to make, and additional developmental work is required, we do not believe them to be the limiting problem in a high energy laser system.

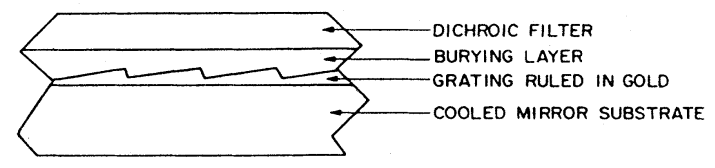

Figure 5.28. Construction of a buried-grating component.

\subsubsection{High-Power Laser Coatings}

There are three basic requirements in mirror coatings for high energy laser systems: (1) low absorption, (2) high damage threshold, and (3) uniformity over large diameters.

A common misconception is that the reflectance of a high energy laser mirror must lie in the range of 0.999-0.999 99. The truth is that the absorption must be less than 100 parts per million (ppm), and preferably $10 \mathrm{ppm}$. In high energy laser systems the amount of energy lost to scattering is not as important as the loss due to absorption. The film absorption is important because it thermomechanically maps the laser intensity on the face of mirrors, which then results in a phase aberration upon reflection of a laser beam from such a mirror. With present technology, absorption of $100 \mathrm{ppm}$ is reasonable, and $10 \mathrm{ppm}$ has been achieved on small optics. If actually required, it should be possible to obtain loss less than $10 \mathrm{ppm}$ on large optics. The major obstacle is the capability of performing the measurements on the large optics.

Absorption of high power radiation by the mirror coatings can lead to damage as well as to structural distortion of the mirror or the supporting structure through a temperature rise. Such distortion could lead to a reduction in the mirror quality. However, for small distortions, quality can be restored through active compensation using deformable secondaries (Section 5.5.1).

Only a small amount of data exists for damage threshold. A typical value achieved to date for microsecond pulses in the $1 \mu \mathrm{m}$ wavelength range is $7-8$ $\mathrm{J} / \mathrm{cm}^{2}$ incident fluence for large samples and approximately twice these values for small samples. For cw or quasi-cw beams, where the damage is primarily thermal due to average laser power, damage threshold of the order of $100-200 \mathrm{~kW} / \mathrm{cm}^{2}$ of absorbed energy has been achieved. Little, if any, experimental data exists for the damage threshold of high reflectivity multilayer dielectric coatings illuminated with radiation having a wavelength different from the wavelength for which the coating is designed. Unpublished theoretical calculations predict a drop in damage threshold of as much as $30 \%$ for wavelengths shifts of $10 \%$. Field stops can be placed in the optical system to protect small optics, but not the large primary mirror from radiation out of the normal field of view.

Optical coatings in space will have their performance degraded by the natural environment. This includes chemically active species such as excited atomic and molecular oxygen at low altitudes, charged particles in the ionosphere $(<500 \mathrm{~km})$, and Van Allen radiation belts in the protonosphere $(500-3000 \mathrm{~km})$. At higher altitudes, chemical deterioration is of less concern than the effects of charged particles. The damaging effects of the radiation belts at high altitudes can be further enhanced by detonation of nuclear weapons. 
Optical elements in space can be covered when not in use. Large optics could conceivably be covered with removable shutters, which would remain in place until the subsystem enters the battle. Additionally, certain vulnerable elements may face away from the earth when not in direct use. But surveillance sensors and other early warning elements cannot be shuttered and cannot face away from the earth. These exposed elements will degrade, but relatively slowly. Surveillance satellites have been in orbit at various altitudes for many years, but specific data on the degradation of their performance was not examined by the Study Group.

Coating mirrors up to $2.5 \mathrm{~m}$ in diameter is an engineering issue, not a scientific issue. The same applies for coating mirrors $4 \mathrm{~m}$ in diameter. It is less expensive by at least an order of magnitude to coat sixteen $1 \mathrm{~m}$ mirrors than to coat one $4 \mathrm{~m}$ mirror.

\subsubsection{Coating at Various Laser Wavelengths}

Choices for coating material are better characterized at 1.06 and $2.7 \mu \mathrm{m}$ than at $0.35,0.41$, and $1.315 \mu \mathrm{m}$. Table 5.1 gives some of the considerations for coating at the various wavelengths.

\subsubsection{Advanced Deposition Processes}

Deposition processes now being investigated that may be applicable to high energy laser systems include ion-assisted deposition, plasma-activated source, ion-beam sputtering, and ion plating. Some of the resulting coatings are superior to coatings produced by conventional processes with respect to stoichiometry, stress absorption, durability, and scatter. None, however, have shown higher resistance to damage. Indications from a very small data base are that very high vacuum deposition (rather than a particular deposition technique) may be more fruitful for low-absorptivity-high-damage coatings.

\subsection{INTEGRATION OF COMPONENTS}

In this section we consider the integration of the elements that control the high-power beam into a system. Such elements are telescopes (arrays of telescopes), transfer optics, adaptive optics, and wavefront sensors. These elements need to be integrated with target trackers and controls.

\subsubsection{Pointing and Tracking}

Two approaches to pointing and tracking are shown in Figure 5.29. In the first, the telescope(s) are placed on gimbals, and the gimbals are actuated in accordance with target information gathered by the tracker. Such a system

TABLE 5.1. Considerations for coating at various wavelengths.

\begin{tabular}{|c|c|}
\hline Wavelength & Comments \\
\hline $0.35 \mu \mathrm{m}$ & $\begin{array}{l}\text { Fluorine resistance required in some applications. UV, x-ray, and electron flux stabil- } \\
\text { ity required. Data base on materials and material combinations is limited. } \\
\text { Few high-index material choices. }\end{array}$ \\
\hline $0.41 \mu \mathrm{m}$ & $\begin{array}{l}\text { Little work done to date at this wavelength. } \\
\text { Little existing spectral measurement of laser damage test capability. } \\
\text { Most coating materials commonly used in the visible spectral region may be accept- } \\
\text { able. }\end{array}$ \\
\hline $1.06 \mu \mathrm{m}$ & $\begin{array}{l}\text { Most damage test data relate to single-shot, short-pulse conditions. } \\
\text { Several promising materials should be investigated for long repetitive pulse applica- } \\
\text { tions. }\end{array}$ \\
\hline $1.315 \mu \mathrm{m}$ & $\begin{array}{l}\text { Small, incomplete data base, but it should be possible to extrapolate from } 1.06 \mu \mathrm{m} \\
\text { results. } \\
\text { Coatings designed and built for } 1.315 \mu \mathrm{m} \text { based on } 1.06 \mu \mathrm{m} \text { technology appear to } \\
\text { work. }\end{array}$ \\
\hline $2.7 \mu \mathrm{m}$ & $\begin{array}{l}\text { Data base is being developed. } \\
2.7 \mu \mathrm{m} \text { water absorption band necessitates special facilities to measure coating spec- } \\
\text { tral performance. } \\
\text { Very high vacuum chambers are needed to control/eliminate such water vapor ab- } \\
\text { sorption. }\end{array}$ \\
\hline
\end{tabular}

${ }^{*}$ Data obtained from vendors. 
POINTING/TRACKING CONCEPTS

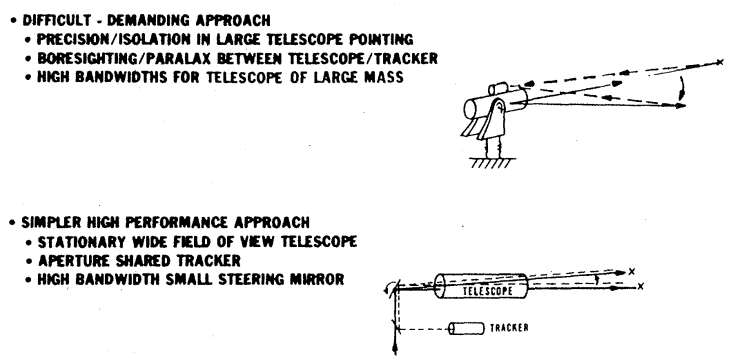

Figure 5.29. Two concepts in pointing, tracking, and beam control. (a) Telescope(s) placed on gimbals, and the gimbals are activated in accordance with target information gathered by tracker. (b) Telescope is stationary and beam is steered by small, high bandwidth mirror in optical train.

has been implemented in the Airborne Laser Laboratory and performed to a precision of $10 \mu \mathrm{rad}$ ( $\mathrm{rms}$ line of sight) in a very dynamic environment. ${ }^{44}$ The greatest problem in such a system is that one is actuating a large mass (telescope) and thus the bandwidths achievable are limited. In addition, base motion disturbances that enter the subsystem can be of very high frequency and the system has limited capability to reject them.

In the second approach, the telescope is stationary or has very low bandwidth response, but the beam is steered to the target by a small, high-bandwidth mirror in the optical train, again in accordance with target information obtained by the tracker. In this case one can achieve very large $(\mathrm{kHz})$ bandwidths, and thus can reject very highfrequency disturbance inputs. This approach requires telescopes that have very large fields of view and trackers that look through the optical system. This requires highpower aperture-sharing elements (beam splitters), which in themselves are a challenge. Such a subsystem has not yet been built, not even in brassboard.

\subsubsection{Integration}

One way the various optical elements might be integrated to provide overall "beam control" is shown in Figure 5.30. A high-power laser projects its beam through an adaptive optics element to grating No. 2, which acts as an aperture-sharing element. A sample from this grating is sent to a wavefront sensor, which drives the adaptive optics element to clean up the phase of the high-power laser beam and the distortions of the optical train to the right of the second aperture-sharing element.

The information from the target, which might be illuminated by an illuminating laser, returns and is collected by the telescope. It then propagates through the optical train to the first grating and associated return
INTEGRATED POINTING, TRACKING, AND BEAM CONTROL SCHEME

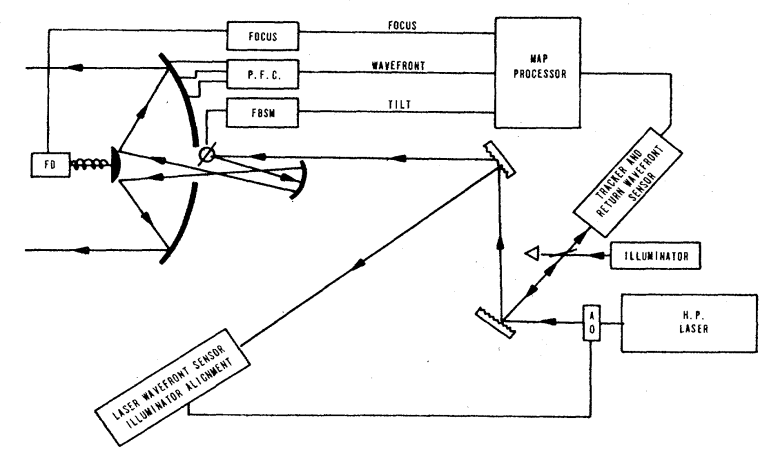

Figure 5.30. Schematic diagram for integration of pointing, tracking and beam control.

wavefront sensor. This information is used to determine (1) the necessary beam tilt correction, which is applied to the fast beam steering mirror (FBSM), (2) focus correction, which is applied to the secondary mirror, and (3) higher order wavefront aberrations applied to the primary focussing mirror (PFM). In such a manner an integrated outgoing and return wave beam control/adaptive optics system is implemented.

Several versions of such concepts have been built at the laboratory level aimed toward the necessary beam stabilization. However, it should be pointed out that such performance has been obtained at small beam-steering angles $(5-10 \mu \mathrm{rad})$ rather than the $\pm 1^{\circ}$ needed for ballistic missile defense applications. In other words, the mirrors had very narrow fields of view $(10 \mu \mathrm{rad})$ rather than the large fields of view required.

\subsection{MULTIPLICITY OF OPTICAL COMPONENTS}

There is a significant multiplicity effect on the required number of space-based telescopes/relay mirrors due to orbital absentee factors and on ground-based lasers and ground-based telescopes due to cloud cover and weather patterns.

\subsubsection{Fighting Mirrors (Mission Mirrors)}

Relay optical subsystems in space may be used by either ground-based lasers or space-based lasers in order to optimize the deployment of laser devices and associated telescopes and to reduce their vulnerability. The last optical element of such a relay system is what is commonly called the "fighting mirror" or "mission mirror" (see Figure 5.12), and its function is to receive the relayed optical beam, track the target, and focus the beam on the target. 
Since this "fighting mirror" performs a focusing function, it has to be located such that the distance between itself and the target it is engaging is shorter than the Rayleigh range. This requires it to be at relatively low altitudes $(1000-3000 \mathrm{~km})$, and the orbital mechanics limit its dwell time in the zone of engagement. Consequently, in order to be assured of energy delivery by a given relay optical systems at any time, one may need as many as 12 or more "fighting mirrors" to reduce to zero the absenteeism of energy delivery systems from the engagement. Tactics and effectiveness considerations might allow one to reduce the number of fighting mirrors per relay system and thus to accept some level of absenteeism.

Such considerations on the number of fighting mirrors in a given relay optical system generally do not depend on whether the laser system is on the ground or in space, and only on the absenteeism that one is willing to accept in the battle zone. See Appendix B for detailed calculations.

\subsubsection{Optical Relay Subsystems}

The relay mirror in an overall relay optical system is needed for a ground-based laser system to receive the laser beam from the ground and relay it to the fighting or mission mirror (see Figure 5.12). This relay mirror may be located either in geosynchronous orbit or at a lower earth orbit, depending on the system architecture.

Locating the relay mirror in a geosynchronous orbit requires a large optics at approximately $35000-40000 \mathrm{~km}$ above the ground laser. Only one such mirror is required per functioning ground laser (see Section 5.7.3 for the definition of such functionality); its size is set by diffraction physics. For example, a ground laser operating at a wavelength of $1 \mu \mathrm{m}$ would require a relay mirror of the order of $10 \mathrm{~m}$ diameter at geosynchronous altitude for a $10 \mathrm{~m}$ ground telescope. The other option is to place the relay mirror at a lower orbit of a few thousand kilometers. This would reduce the required mirror size, particularly for $1 \mu \mathrm{m}$ (FEL) GBLs. On the other hand, the consequence of lower orbit deployment is the requirement of additional relay mirrors because of absenteeism that comes into play for such lower orbit relay stations due to orbital mechanics. The laser beam, furthermore, may have to be directed to the fighting mirror using two or more relay stations. The total number of relay mirrors, therefore, depends sensitively on the details of the architectural configuration adopted.

\subsubsection{Multiplicity of GBL Systems}

For each ground-based laser system which must be available in battle, a number of geographically separated laser sites, are needed to provide availability of at least one site in the system when the others are obscured by adverse climatic conditions. These locations must be separated by distances greater than the coherence length scale for weather patterns. Based on weather statistics, a multiplicity of five independent ground-based lasers could provide a $99.7 \%$ availability. By going to seven climatically isolated locations in the continental U.S. availability of $99.97 \%$ is possible. At each of these sites, local cloud cover conditions require further multiplicity of the large ground telescopes, separated by a few $\mathrm{km}$ (Section 5.4).

One can investigate the limits of this problem with sample threat numbers. Presume a target set of 2000 fast-burn boosters, for which the engagable boost phase is only about $50 \mathrm{~s}$. Thus, the GBL system must kill about 40 missiles per second. For a quasi-cw GBL system (FEL or high rep-rate excimer), if an optimistic irradiation time of $0.1 \mathrm{~s}$ is assumed, and an additional 0.1 $\mathrm{s}$ is required for retargeting, then the kill rate is about 5 boosters per second per laser. Thus, one would need about $10 \mathrm{GBL}$ systems (devices, ground telescopes, and space relay optical platforms) in operation, neglecting any redundancy considerations. In order to overcome local cloud fluctuations at each ground laser complex, one may need as many as three ground telescopes per GBL system appropriately dispersed (1-2 km), each telescope being capable of being fed by the same laser device. We thus need, per GBL complex, on the order of 10 devices and 30 telescopes (or arrays), feeding 10 space relay systems. Including the required geographical dispersion discussed above, then, the total system size becomes of the order of 50-70 devices, 150-210 ground telescopes, and 10 space relay optical systems.

For a single-pulse excimer GBL, the numbers would be slightly different, since the kill time is negligible compared to the retarget time. Thus, one would need about four devices per site, with a total system size of 20-30 devices, 60-90 telescopes, and four space relay optical systems.

\subsection{CONCLUSIONS}

1. Phase correction techniques are required for obtaining near diffraction-limited performance of most types of laser weapon devices. Further, phase control techniques are required for coherently combining outputs from different modules in a multiple laser system into a single diffractionlimited beam. These techniques, demonstrated at low powers, must be scaled up by many orders of magnitude in power.

2. Dynamic phasing of arrays of telescopes requires extensive development in order to obtain large effective aperture optical systems. As calculations indicate, the number of phase correcting elements must be increased by at least two orders of magnitude over currently demonstrated values. 
3. The optical coatings of large primary mirrors are particularly vulnerable in space-based optical subsystems.

4. Small secondary mirrors in the optical trains of high-power lasers will need very low absorptivity coatings and will have to be cooled.

5. Ground-based laser systems for BMD applications need geographical multiplicity to deal with adverse weather conditions.

6. Ground-based laser systems require techniques for correcting atmospheric propagation aberrations. We estimate that these techniques must be extended by at least two orders of magnitude in resolution (number of actuators) than presently demonstrated. Phase correction techniques must be demonstrated at high powers.

7. Uplink in a ground-based laser system faces transmission losses in the atmosphere.

8. Nonlinear scattering processes in the atmosphere impose a lower limit on the altitude at which targets can be attacked with a laser beam from space.

\section{REFERENCES}

${ }^{1}$ G. Field and D. Spergel, "Cost of Space-Based Laser Ballistic Missile Defense," Science 231, 1387-1393 (1986).

${ }^{2}$ L. Marquet, Lasers ' 85 , Proceedings of the International Conference on Lasers '85, edited by C. P. Wang (STS Press, McLean, VA, 1986), pp.247-252.

3 J. S. Fender, "Synthetic Aperture Systems," SPIE Proceedings, Vol. 440, 1983.

${ }^{4}$ R. R. Butts, "The effects of piston and tilt error on the performance of multiple telescope," SPIE Proceedings, Vol. 293, 1981.

${ }^{5}$ John Bally and Catherine Pringent, "Optical Space to Earth Communications" (to be published).

${ }^{6}$ H. S. Stewart and R. F. Hopfield, Atmospheric Effects in Applied Optics and Optical Engineering, edited by R. Kingslake (Academic, New York, 1965), Vol. 1.

${ }^{7}$ R. A. McClatchey, R. W. Fenn, J. E. A. Selby, F. E. Volz, and J.S. Garing, "Optical Properties of the Atmosphere," IR Handbook of Optics, edited by W. G. Driscoll and W. Vaughn (McGraw-Hill, New York)

${ }^{8}$ R. J. Hill et al., "Refractive Index and Absorption Fluctuations in the Infrared Caused by Temperature, Humidity and Pressure Fluctuations," J. Opt. Soc. Am. 70, 1192-1205 (1987).

${ }^{9}$ D. L. Fried and H. T. Yura, "Telescope Performance Reciprocity for Propagation in a Turbulent Medium," J. Opt. Soc. Am. 62, 600-602 (1972).

${ }^{10}$ D. P. Greenwood, "Adaptive Compensation for Atmospheric Turbulence Effects on Optical Propagation," Proceeding AGARD Conference Proceedings, No. 345. North Atlantic Treaty Organization.

${ }^{11}$ J. E. Pearson, "Atmospheric Turbulence Compensation Using Coherent Optical Adaptive Techniques," Appl. Opt. 15, 622-631 (1976).
${ }^{12}$ A. Yariv, Optical Electronics, 3rd ed. (Holt, Rinehart and Winston, New York, 1985), p. 500.

${ }^{13}$ D. L. Fried, “Anisoplanatism in Adaptive Optics," J. Opt. Soc. Am. 72, 52-61 (1982).

${ }^{14} \mathrm{Jack}$ Hammond (private communication).

${ }^{15}$ Optical Phase Conjugation, edited by R. A. Fisher (Academic, New York, 1983).

${ }^{16}$ R. C. C. Leite, R. S. Moore, and J. R. Whinnery, Appl. Phys. Lett. 4, 141 (1964).

${ }^{17}$ P. M. Livingston, Appl. Opt. 10, 426 (1971).

${ }^{18}$ F. Gebhardt, Appl. Opt. 15, 1479 (1976).

${ }^{19}$ D. C. Smith, Proc. IEEE 65, 1679 (1977).

${ }^{20}$ Laser Beam Propagation in the Atmosphere, Vol. 25 of Topics in Applied Physics, edited by J. W. Strohbehn (SpringerVerlag, New York, 1978).

${ }^{21}$ V. E. Zuev, Laser Beams in the Atmosphere (Consultants Bureau, New York, 1982).

${ }^{22}$ V. E. Zuev, High Power Laser Radiation in Atmospheric Aerosols: Nonlinear Optics of Aerodispersede Media (D. Reidel, Dordrecht, 1985).

${ }^{23}$ L. C. Bradley and J. Hermann, JOSA 61, 668 (1971).

${ }^{24}$ K. A. Brueckner and S. Jorna, Phys. Rev. 164, 182 (1967).

${ }^{25}$ R. M. Herman and M. A. Gray, Phys. Rev. Lett. 19, 824 (1967).

${ }^{26}$ N. M. Kroll and P. L. Kelley, Phys. Rev. A 4, 763 (1971).

${ }^{27}$ See, for example, High-Power Infrared-Laser Windows, National Materials Advisory Board Publication NMAB-292 (1972).

${ }^{28}$ R. L. Carman and P. L. Kelley, Appl. Phys. Lett. 12, 241 (1968).

${ }^{29}$ See, for example, M. Born and E. Wolf, Principles of Optics (Pergamon, New York, 1969), p. 109.

${ }^{30}$ L. C. Bradley (private communication).

${ }^{31}$ L. C. Bradley and J. Hermann, Appl. Opt. 13, 990 (1974).

${ }^{32}$ A. D. Wood, M. Camac, and E. T. Gerry, Appl. Opt. 10, 1877 (1971).

${ }^{33}$ J. Wallace and J. Q. Lilly, JOSA 64, 1651 (1974).

${ }^{34}$ R. G. Buser, R. S. Rhode, P. J. Berger, F. G. Gebhardt, and D. C. Smith, Appl. Opt. 14, 1740 (1975).

${ }^{35}$ R. W. O'Neil, H. Kleiman, and H. R. Zwicker, Preprint No. 183, NATO AGARD Conference on Optical Propagation in the Atmosphere (Oct. 1975).

${ }^{36}$ F. G. Gebhardt, D. C. Smith, R. G. Buser, and R. S. Rhode, Appl. Opt. 12, 1794 (1973).

${ }^{37}$ B. S. Agrovskii, A. S. Gurvich, V. A. Myakinin, V. V. Vorob'ev, JOSA A2, 2304 (1985).

${ }^{38}$ See References 17 and 20 for discussions and further literature.

${ }^{39}$ V. S. Averbakh, A. I. Makarov, and V. I. Talanov, "Stimulated Raman Scattering on Rotational and Vibrational Transitions in Nitrogen Gas," Sov. J. Quantum Electron. 8, 472-476 (1978).

${ }^{40}$ M. A. Henesian, C. D. Swift, and J. R. Murray, "Stimulated Rotational Raman Scattering in Nitrogen in Long Air Paths," Opt. Lett. 10, 565-567 (1985).

${ }^{41}$ N. Kurnit and J. Ackerhalt, "Atmospheric Propagation," presented to the meeting on Applications of Nonlinear Optics to SDI, LAUR 84-3749, Los Alamos National Laboratory (1984).

${ }^{42}$ M. Rokni and A. Flussberg, IEEE J. Quantum Electron. QE-22, 1102 (1986).

${ }^{43}$ P. Marmier and E. Sheldon, Physics of Nuclei and Particles (Academic, New York, 1969), pp. 170-189.

${ }^{44} \mathrm{P}$. Avizonis (private communication). 


\section{Chapter 6}

\section{BEAM MATERIAL INTERACTIONS AND LETHALITY}

\section{CONTENTS}

6.1 Introduction

6.2 Continuous-Wave Laser-Material Interaction

6.2.1 Melt-Through of a Metal Plate

6.2.2 Vaporization of a Target

6.2.3 Quantitative Treatment of Thermal Coupling

6.2.4 Heating and Plasma Formation by Repetitively Pulsed Lasers

6.2.5 Materials Response

6.2.5.1 Metals

6.2.5.2 Ablative Materials

6.2.5.3 Composites

6.2.5.4 Ceramics and Glassy Materials

6.2.6 Vulnerability of Structures

6.2.7 Kill Assessment

6.3 Pulsed Laser Effects

6.3.1 Interaction in Vacuum

6.3.2 Multiple Pulse Cumulative Fluence

6.3.3 Interaction in the Atmosphere

6.3.4 Impulse Generation by $X$ Rays

6.3.5 Structural Damage from Impulse Loading

6.4 Particle Beam Lethality

6.4.1 Beam Interaction Summary

6.4.2 Lethality Mechanisms

6.4.3 Lethality Criteria

6.5 Microwave Lethality

6.6 Conclusions

References

\subsection{INTRODUCTION}

The effectiveness of a defensive weapons system is measured by its ability to deny the attacking system success in accomplishing its mission.

Lethality of a directed energy weapon is, in simplest terms, its capability to destroy a target. In the context of ballistic missile defense (BMD), it is appropriate to speak of lethality as the capability of a directed energy weapon to prevent a target from accomplishing a particular mission. Thus, there may be several measures of lethality for a given target set. For satellites, one may define lethality criteria relating to structural damage of the satellites, and other criteria relating to destruction or indefinite interruption of the sensors on which the satellite depends to accomplish its function. Similarly, actual destruction of a booster or reentry vehicle sets certain lethality criteria; but methods of destroying accurate weapons delivery, such as destruction of guidance electronics, may also generate acceptable lethality criteria for system designers. However, in the latter case, verification of a kill becomes problematical.

The ability of laser beams (pulsed, cw, and repetitively pulsed), at infrared, visible, ultraviolet, and $\mathrm{x}$ ray wavelengths, of neutral and charged particles, and of microwave radiation pulses to destroy various targets is analyzed. First, the physics of the interaction of various directed energy beams with materials is examined. This information is used to assess the effect of a given incident power or energy fluence on the target and the ability of the target to perform its mission after such an attack. These arguments, then, are used to size weapons systems to destroy enemy targets (lethality).

The fundamental kill mechanism of $\mathrm{cw}$ or quasi-cw repetitively pulsed laser beams is heating, with subsequent melting and/or evaporation of the wall of a liquid or solid booster rocket. Subsequently, ignition of booster fuel may take place, or mechanical failure of structures may occur before completion of burn-through. In a similar manner, the wall of the bus and components inside it may be damaged, so that the intended function of the missile is thwarted.

Particle beams can penetrate these targets, melting and vaporizing the walls of the missile or bus, which also results in a structural "kill" of the target. At much lower damage thresholds particle beams can penetrate guidance and control electronics possibly disabling them.

In addition to energy deposition, momentum is also transferred to the target by directed energy beams. Momentum transfer can damage targets through mechanical shearing or buckling. This damage mechanism has been demonstrated by pulsed laser beams for pulses less than or equal to $2 \mu \mathrm{s}$. Kill through repeated impulse damage has system-level advantages over thermal kill since the pointing requirements are far less severe. Momentum transfer may also be used as a discrimination tool for reentry vehicles and decoys in the mid-course.

In Section 6.2 the interaction of $\mathrm{cw}$ or quasi-cw laser radiation with targets is discussed. Lethality effects of pulsed electromagnetic radiation at various wavelengths are discussed in Section 6.3. The interaction of neutral and charged particle beams with generic targets is described in Section 6.4, and lethality criteria for this type of beams are derived. The possible effects of microwave radiation are treated briefly in Section 6.5. Finally, Section 6.6 summarizes the main conclusions, setting 
lower limits on power and energy that are imposed on a DEW system by the lethality requirements.

\subsection{CONTINUOUS-WAVE LASER-MATERIAL INTERACTION}

For cw laser beams of relatively low intensity, the only effect is heating from absorption of electromagnetic radiation. The temperature of the absorbing material is raised until a balance exists between the absorbed power and thermal losses from conduction, convection, and radiation.

It has been known since prehistory that sunlight can make absorbing surfaces hot, and in Greek antiquity it was known that concentrated sunlight, having traversed spherical water-filled flasks or convex pieces of glass, could kindle fires.* Archimedes proposed to concentrate the sun's rays by means of reflecting shields of soldiers standing on the hills surrounding the harbor of Syracuse. He hoped to ignite the ropes, sails, and spars of the vessels of the enemy's fleet.

The power flux density of the sun in zenith on the earth's surface is about $0.13 \mathrm{~W} / \mathrm{cm}^{2}$. Several thousandfold concentration to $1 \mathrm{~kW} / \mathrm{cm}^{2}$ leads to solar furnaces with temperatures near $3000^{\circ} \mathrm{C}$. If the irradiated area is large enough, lateral heat conduction may be ignored. The radiative loss of a black-body surface is given by Stephan-Boltzmann's law. The power flux density, $\sigma \mathrm{T}^{4}$, corresponds to $1 \mathrm{~kW} / \mathrm{cm}^{2}$ at $\mathrm{T}=3644 \mathrm{~K}$. Convective cooling by air-flow over a $3000 \mathrm{~K}$ surface at Mach number unity is only a few hundred $W / \mathrm{cm}^{2}$. At pressures prevailing at booster burn-out altitudes, $80-160 \mathrm{~km}$, convective cooling is completely negligible. From the foregoing, it is clear that the temperature of most materials may be raised above the melting temperature $T_{m}$ and the vaporization temperature $T_{v}$ for cw laser flux densities in the range of $1-100 \mathrm{~kW} / \mathrm{cm}^{2}$. In the early days of laser history, in 1961, when the pulsed ruby laser was the most powerful available, it was established that a focused ruby laser pulse of about $1 \mathrm{~J}$ energy could punch a hole in a razor blade. ${ }^{\dagger}$ Two very simple cases serve to establish the order of magnitude of fluxes and fluences on target required for lethality.

\subsubsection{Melt-Through of a Metal Plate}

Consider a normally incident power flux intensity, or irradiance, $I_{0}$ on a metal plate of thickness 1 . The reflectivity of the metal is $R$, the optical absorption coefficient is $\alpha\left(\alpha \mathrm{d}_{0} \gg 1\right)$, its specific heat $\mathrm{C}_{\mathrm{s}}(\mathrm{J} / \mathrm{g})$, its density $\rho\left(\mathrm{g} / \mathrm{cm}^{3}\right)$, and its thermal conductivity $\mathrm{K}=\rho \mathrm{C}_{\mathrm{s}} \kappa$, where $\kappa$ is the thermal diffusivity. The

\footnotetext{
* Aristophanes, Comedy of the Clouds, 434 B.C., English translation. The strength of those pulses was measured in the unofficial unit of "gillettes."
}

diameter of an incident laser spot is $d_{0} \gg 1_{0}$. For times $\sqrt{2 \kappa \mathrm{t}} \ll \mathrm{d}_{0}$, heat losses to radial conduction tangential to the surface may be ignored. For $I_{0} \gg 1 \mathrm{~kW} / \mathrm{cm}^{2}$, the radiative and convective cooling may also be neglected. All the absorbed power serves to heat and melt the metal. Assume that the melt is completely removed, either by gravity or by a convectional shear flow. This assumption is simplistic, as there is no shear in space and removal by gravity may be long compared to pulse duration/dwell time. The time for melt-through, $t_{m r}$, is then given by the simple heat balance equation,

$$
(1-\mathbf{R}) \mathbf{I}_{0} \mathrm{t}_{\mathrm{mr}} \geq \rho \mathrm{l}_{0}\left[\mathbf{C}\left(\mathbf{T}_{\mathbf{M}}-\mathbf{T}_{0}\right)+\mathbf{L}_{\mathrm{m}}\right],
$$

where $\mathrm{L}_{m}$ is the latent heat of melting at the melting point $T_{M}$. Substituting values for aluminum, with $1_{0}=0.5 \mathrm{~cm}$ at a wavelength for which $R=0.8$, one finds $t_{m r}=0.6 \mathrm{~s}$ for $I_{0}=10 \mathrm{~kW} / \mathrm{cm}^{2}$ and $t_{\mathrm{mr}}=0.06 \mathrm{~s}$ for $I_{0}=100$ $\mathrm{kW} / \mathrm{cm}^{2}$. This inequality may be severe if target vapor decouples the laser beam from the target, an effect not considered in this analysis.

\subsubsection{Vaporization of a Target}

When the melt is completely retained on the surface, the liquid will be further heated to the boiling point $T_{v}$. Make the simplification that all material properties are the same in the liquid and solid phase, and do not vary with temperatures. The time $t_{\mathrm{vr}}$ necessary to vaporize the metal plate is given by

$$
(1-R) \mathbf{I}_{0} t_{v r}=t_{m r}+\rho l_{0}\left[c\left(T_{v}-T_{m}\right)+L_{v}\right] .
$$

With a boiling temperature at one atmosphere $T_{v}$ $=2453{ }^{\circ} \mathrm{C}$ and a latent heat of boiling $\mathrm{L}_{\mathrm{v}}=10732 \mathrm{~J} / \mathrm{g}$ for aluminum, one finds $t_{\mathrm{vr}}=7 \mathrm{~s}$ at $\mathrm{I}_{0}=10 \mathrm{~kW} / \mathrm{cm}^{2}$ and $t_{\mathrm{vr}}=0.7 \mathrm{~s}$ at $\mathrm{I}_{0}=100 \mathrm{~kW} / \mathrm{cm}^{2}$. These times for removal by vaporization are an order of magnitude longer than for melt-through for two reasons. The boiling point for $\mathrm{Al}$ is considerably higher than the melting point, but more important is the fact that the latent heat of vaporization is usually an order of magnitude larger than for melting. This is true for nearly all materials of interest.

An aluminum structural element under high mechanical stress will, of course, fail well before complete melt-through has occurred. Failure by partial or complete melting of aluminum, titanium, or other metal structures may be averted by hardening them with heat shields. This technique was developed for preventing the burn-up of space craft and missiles on return through the atmosphere. Such a shield could consist of carbonphenolic or other carbon-containing materials. After pyrolysis the carbon vaporizes with an enthalpy of 
vaporization $\mathrm{H}_{\mathrm{c}} \approx 32000 \mathrm{~J} / \mathrm{g}$, as the gaseous phase consists predominantly of $\mathrm{C}_{3}$ and $\mathrm{C}_{2}$ molecules.

Consider the heat balance equation in a thin control volume at the surface of the carbon, ignoring again heat losses by convection and by reradiation. The various heat flow contributions are schematically indicated in Figure 6.1. A steady state results with

$$
(1-\mathbf{R}) \mathbf{I}_{0}=\dot{\mathrm{m}} \mathrm{H}_{\mathrm{c}}(\mathrm{g})
$$

and

$$
\mathrm{q}_{\mathrm{cond}}=\dot{\mathrm{m}} \mathrm{H}_{\mathrm{c}}(\mathrm{s}) \text {. }
$$

Here $\dot{m}$ is the mass removal rate per $\mathrm{cm}^{2} . H_{c}(g)$ is the enthalpy in the gas phase of carbon, while $\mathrm{H}_{c}(\mathrm{~s})$ is the enthalpy of the solid. Both quantities are taken at the surface temperature $T_{\mathrm{s}}$. The latter is fixed for given $I_{0}$ by Equation (6.3). The mass flow rate into vacuum may be estimated from

$$
\dot{\mathrm{m}}=\mathrm{N}\left(\mathrm{T}_{\mathrm{s}}\right)\left\langle\mathrm{v}_{\mathrm{z}}\right\rangle=\mathrm{p}\left(\mathrm{T}_{\mathrm{s}}\right) /\left(2 \pi \mathrm{k} \mathrm{T}_{\mathrm{s}} / \mathrm{M}_{\mathrm{c}}\right)^{1 / 2},
$$

where $\mathbf{N}\left(\mathrm{T}_{\mathrm{s}}\right)$ is the number density of carbon molecules in the vapor, $M_{c}$ the mass of the carbon molecules, and $\left\langle v_{z}\right\rangle$ the normally directed thermal velocity of a one-sided Maxwell-Boltzmann distribution at temperature $T_{s}$. The saturated vapor pressure $\mathrm{p}\left(\mathrm{T}_{\mathrm{s}}\right)$ may be obtained from the Clausius-Clapeyron equation. Thus, the surface temperature adjusts itself so that the mass removal rate required by Equation (6.3) is maintained. The temperature profile below the solid surface takes on the form required by the solution of Equation (6.4). With $\mathbf{H}_{\mathrm{c}} \sim 32 \mathrm{~kJ} / \mathrm{g}$, the burn-through time for a protective layer containing $1 \mathrm{~g} / \mathrm{cm}^{2}$ of carbon, will be $3.2 \mathrm{~s}$ at $10 \mathrm{~kW} / \mathrm{cm}^{2}$, assuming $\mathrm{R}<0.1$ for charred material. For $10 \mathrm{~g} / \mathrm{cm}^{2}$ of carbon shielding, $100 \mathrm{~kW} / \mathrm{cm}^{2}$ would be required to achieve burn-through in the same time. The burn-through time would be reduced to $0.32 \mathrm{~s}$ for $1 \mathrm{~g} / \mathrm{cm}^{2}$ with an absorbed flux density of $100 \mathrm{~kW} / \mathrm{cm}^{2}$.

These elementary considerations establish the fact

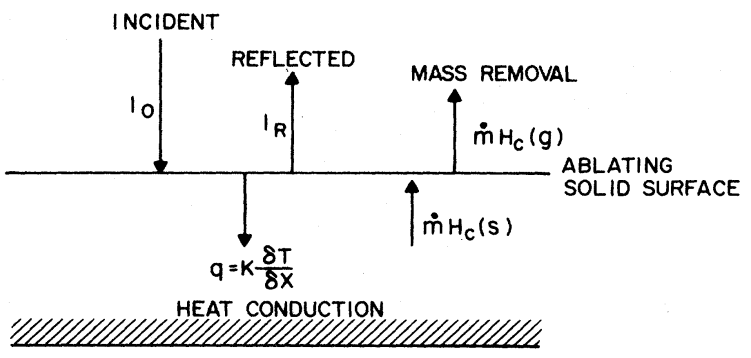

Figure 6.1. Surface heat balance schematic for an ablating material. that irradiances between 10 and $300 \mathrm{~kW} / \mathrm{cm}^{2}$ are required for laser-hardened target kill by heating with quasi-cw laser beams.

\subsubsection{Quantitative Treatment of Thermal Coupling}

One-dimensional models of heat diffusion are valid if the size of the incident laser spot $d_{0}$ satisfies the following inequalities:

$$
\mathrm{d}_{0} \gg \mathrm{l}_{0} \text { and } \mathrm{d}_{0} \gg(2 \kappa \mathrm{t})^{1 / 2} \text {. }
$$

The laser spot size should be large compared to the thickness $1_{0}$, and thermal diffusion tangential to the surface should be negligible during the time of the experiment. Note that thermal diffusivity, $\kappa=\mathrm{K} / \rho \mathrm{C}_{\mathrm{s}}$, is on the order of unity in good conductors (in $\mathrm{cm}^{2} / \mathrm{s}$ ), as it takes about $1 \mathrm{~s}$ for the heat to diffuse on $1 \mathrm{~cm}$ along the stem of a silver spoon stirring a cup of hot coffee. $K$ is the heat conductivity, $\rho$ is the density, and $\mathrm{C}_{\mathrm{s}}$ is the specific heat. Thus, for spot sizes $d_{0}>10 \mathrm{~cm}$, and irradiation times less than a few seconds, the inequalities (6.6) are satisfied. If we take the normal to the surface in the $\hat{x}$ direction, the one-dimensional heat diffusion equation is

$$
\begin{aligned}
\rho \mathrm{C}_{\mathrm{s}} \frac{\partial \mathrm{T}}{\partial \mathrm{t}}= & \frac{\partial}{\partial \mathrm{x}}\left[\mathrm{K} \frac{\partial \mathrm{T}}{\partial \mathrm{x}}\right] \\
& +\alpha(1-\mathrm{R}) \mathrm{I}_{0} \exp -\left(\int_{0}^{\mathrm{x}} \alpha \mathrm{dx}\right) .
\end{aligned}
$$

$\alpha$ is the absorption coefficient for light at the incident wavelength, so that $\mathrm{dI} / \mathrm{dx}=-\alpha \mathrm{I}$. In this equation all material constants are a function of the local temperature. The boundary condition at the front surface, $x=0$, is

$$
-\mathrm{K} \frac{\partial \mathrm{T}}{\partial \mathrm{x}}+\rho \dot{\mathrm{s}} \Delta \mathrm{H}_{\mathrm{v}}+\bar{\epsilon} \sigma \mathrm{T}^{4}+\mathrm{C}^{\prime}\left(\mathrm{T}-\mathrm{T}_{0}\right)=0
$$

Here $\epsilon$ is the average emissivity for "gray-body" radiation, $C^{\prime}\left(T-T_{0}\right)$ represents the convective heating coefficient at the front surface. $\Delta \mathbf{H}_{\mathrm{v}}$ is the enthalpy of vaporization. The surface recession rate $\dot{s}$ is a strong function of the surface temperature, $T(x=0)$ as discussed above.

Anisimov ${ }^{1}$ first discussed a more accurate treatment of the vaporization than given by Equation (6.5), taking into account the presence of gas-kinetic collisions in a Knudsen layer of a thickness of about three mean free paths in front of the surface. The recession rate into a vacuum is reduced by about $18 \%$ from that given by Equation (6.5), as a fraction of the vaporized molecules is 
returned to the surface by gas-kinetic collisions. If there is an atmosphere in front of the vaporizing surface, the corrections are, of course, more important. Knight ${ }^{2}$ has discussed theoretical modeling of rapid surface vaporization with back pressure. The initial temperature condition is $T(t=0)=T_{0}$, independent of $x$.

The temperature variation of all physical quantities $\kappa, \rho, \mathrm{C}_{\mathrm{s}}, \alpha, \mathrm{R}, \dot{\mathrm{s}}, \Delta \mathrm{H}_{\mathrm{v}}$, must be known. Then the equations can be solved by a computer code. If a phase transition, such as melting, occurs, this can be incorporated into the code by adding a delta function to the specific heat $\Delta H_{m} \delta\left(T-T_{m}\right)$ on the left-hand side of Equation (6.7), where $T_{m}$ is the melting temperature. For $T>T_{m}$, values for the physical quantities should be those appropriate for the liquid phase.

If a rear surface exists at $\mathrm{x}=1_{0}, \mathrm{a}$ boundary condition similar to Equation (6.8) has to be imposed at that surface. If radiation, vaporization, and convective losses may be ignored, the condition at the isolated surface is simply $\partial \mathrm{T} / \partial \mathrm{x}=0$ at $\mathrm{x}=1_{0}$.

If the absorption depth is very small, the heat deposition term may be omitted from the right-hand side of Equation (6.7), and added to the surface boundary condition (6.8). Analytic solutions for the set of equations

$$
\begin{aligned}
& \rho \mathrm{C}_{\mathrm{s}} \frac{\partial \mathrm{T}}{\partial \mathrm{t}}=\frac{\partial}{\partial \mathrm{x}}\left(\mathrm{K} \frac{\partial \mathrm{T}}{\partial \mathrm{x}}\right), \\
& -\mathrm{K} \frac{\partial \mathrm{T}}{\partial \mathrm{x}}=(1-\mathrm{R}) \mathrm{I}_{0} \text { at } \mathrm{x}=0, \\
& \frac{\partial \mathrm{T}}{\partial \mathrm{x}}=0 \text { at } \mathrm{x}=1_{0}, \\
& \mathrm{~T}=\mathrm{T}_{0} \text { at } \mathrm{t}=0,
\end{aligned}
$$

may be found in a standard text (Carslaw and Jeager ${ }^{3}$ ) on heat conduction when $\rho, \mathrm{C}_{\mathrm{s}}, \mathbf{K}, \mathbf{R}$, and $\mathrm{I}_{0}$ are all constant. For the short times, $\mathrm{Kt} / 1_{0}^{2}<1$, the heat transport has not yet reached the back surface, and the temperature distribution will be equivalent to that of a semi-infinite medium with a heat load at the surface. ${ }^{4}$

Two characteristic temperature profiles for the heating of an aluminum slab by an incident laser flux of $10^{7} \mathrm{~W} / \mathrm{cm}^{2}$ are shown in Figure 6.2. These profiles were calculated by Rosen et al.,$^{5}$ in connection with experiments performed by them on the interaction of $0.5 \mu \mathrm{s}$ excimer laser pulses at $\lambda=0.35 \mu \mathrm{m}$. The temperature has penetrated only a few microns into the metal during this time. The surface temperature continues to rise until steady state vaporization is reached.

Codes have been further developed to take into account additional features occurring in real situations. For carbon-phenolics, fiberglass epoxies, tungsten bearing resins and other materials of practical interest, pyrolytic

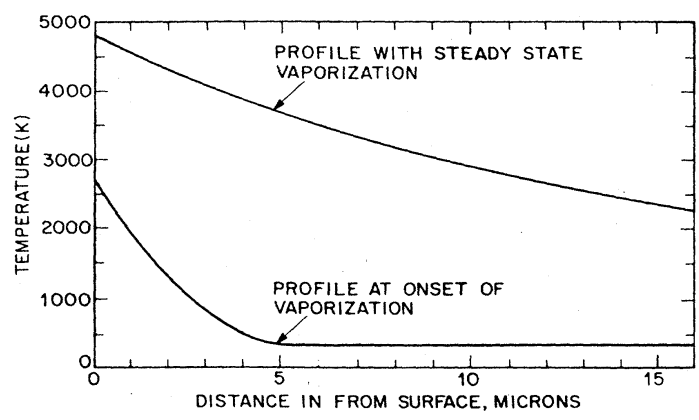

Figure 6.2. Temperature profiles of an aluminum target absorbing a laser flux of $10^{7} \mathrm{~W} / \mathrm{cm}^{2}$ (after Reference 5).

reactions take place. Production and energy convection by pyrolytic gases may be taken into account by adding the terms

$$
\Delta \mathrm{H}_{\mathrm{p}} \frac{\partial \dot{\mathrm{m}}_{\mathrm{g}}}{\partial \mathrm{x}}+\frac{\partial}{\partial \mathrm{x}}\left(\dot{\mathrm{m}}_{\mathrm{g}} \mathrm{h}_{\mathrm{g}}\right)
$$

to the right-hand side of Equation (6.7). Here $\dot{m}_{g}$ is the rate of pyrolytic gas flow toward the front surface, $h_{g}$ is the sensible enthalpy of pyrolytic gas, and $\Delta H_{p}$ is the endothermic heat of pyrolysis.

Codes have also been extended to two- or even three-dimensional cases, to account for anisotropy in heat conduction in layered materials such as fiberglass and carbon-carbon fiber composite, as well as to take account of radial heat conduction. The latter is important because in laboratory test geometries the beam diameter often does not satisfy the inequalities (6.6).

\subsubsection{Heating and Plasma Formation by Repetitively Pulsed Lasers}

Many lasers are operated in a pulsed mode, for example, the excimer lasers. Free electron lasers may be operated either in a cw or pulsed mode. When power is delivered at the same average rate as the rate for $\mathrm{cw}$ heating, one may expect that the same global energy balance equations used in the previous section are still applicable. While this is true in a first approximation, there are important differences. Consider again the example of an excimer laser which emits pulses of $1 \mu \mathrm{s}$ duration with a pulse repetition rate of $100 \mathrm{~s}^{-1}$ at an average irradiance on target of $10 \mathrm{~kW} / \mathrm{cm}^{2}$. The peak intensity during a single pulse is $10^{8} \mathrm{~W} / \mathrm{cm}^{2}$. For surface energy deposition in a metal or any target materials with a shorter absorption length, a layer of thickness $\left(2 \kappa \mathrm{t}_{\mathrm{p}}\right)^{1 / 2}$ will be heated up rapidly to a very high temperature. Thus, vaporization will occur at a high vapor pressure during pulsed operation. Between pulses the surface layer will rapidly cool off by thermal conduction to the 
underlying material and by radiative surface emission. In a certain regime of pulse duration and pulse peak power level, the vaporization rate may even be higher than in the $\mathrm{cw}$ regime with the same average power. The evaporation of material during the pulse will lead to an impulse loading of the surface which may be estimated as $\int_{0}^{t_{p}} \frac{1}{2} p_{v}(t) d t$, where $p_{v}(t)$ is the vapor pressure to be calculated from the surface temperature $T(t)$. At peak power levels below $10^{7} \mathrm{~W} / \mathrm{cm}^{2}$, this impulse loading is not so severe as to lead to a new failure mechanism. The impulse loading will become very important for larger rates of energy deposition by single pulses, e.g., from single-pulse excimers and from $x$-ray lasers. This situation will be discussed in Section 6.3.

Since the surface temperature may rise rapidly to over $5000 \mathrm{~K}$ during $1 \mu \mathrm{s}$ at the irradiation level of $10^{7} \mathrm{~W} / \mathrm{cm}^{2}$, resulting in vapor pressures of $1 \mathrm{~atm}$ or more, the vapor may be partially ionized. The electron and ion concentrations may be calculated from Saha's equation. In addition, there will also be neutral atoms in excited electronic states. The absorption by free electrons in the collisional plasma will lead to further heating. In addition, photoionization of excited neutral atoms may increase the density of charged particles. Thus, a dense plasma in front of the solid or liquid surface may develop. This plasma could absorb a large fraction of the incident light, and consequently, reduce the thermal and impulse coupling to the target surface. The coupling will, however, not vanish, as UV recombination radiation emitted by the plasma may be absorbed at the target surface, the effectiveness depending on the distance of the plasma from the surface. In addition, gas-dynamic processes following the expansion of the plasma may also produce an impulse coupling.

For initially highly reflecting surfaces, the existence of a plasma may even increase the thermal coupling. Consider the interaction of radiation from a $\mathrm{CO}_{2}$ laser at $10.6 \mu \mathrm{m}$ with a metallic surface, which may have a reflectivity $\mathbf{R}=0.995$ or higher at this wavelength. Very high flux densities are required to cause initial heating. There are, however, some absorbing impurities, for example, a flake of paint. Their evaporation leads to the creation of a small plasma blob. It emits UV radiation, which gets readily absorbed by the metal surface, which locally starts to evaporate. This leads to increased plasma formation and establishes the thermal coupling required. The process starts more readily at initial high laser flux densities. Afterwards the intensity can be reduced while the plasma is maintained. The details of plasma development depend sensitively on the atomic composition of the target, on the presence of readily ionized impurities, and on ambient air pressure.

There exists a very extensive literature on the energy coupling between lasers and plasmas. A good starting point to become familiar with these general questions of laser interaction physics is provided by two textbooks $\left(\right.$ Ready $^{6}$ and Hughes ${ }^{7}$ ), which also give many references to the early literature. The incident laser flux densities may be increased to well above $10^{14} \mathrm{~W} / \mathrm{cm}^{2}$, as is the case for laser targets for thermonuclear fusion with inertial confinement. A recent review of the pertinent physical processes in this limit has been given by Anisimov et al. ${ }^{8}$ Laser plasma formation and laser supported combustion (LSC) and laser supported detonation (LSD) waves are also described in the early Soviet literature. ${ }^{9-11}$ The influence of these plasma mechanisms on the coupling with a solid target has been described by Pirri et al. ${ }^{12}$

For the question of heating by repetitive pulsing, the interest is more narrowly focused on the initial stages of plasma formation at peak power flux densities less than $10^{8} \mathrm{~W} / \mathrm{cm}^{2}$ and pulse durations $>0.5 \mu \mathrm{s}$. This regime has been studied both theoretically and experimentally by Rosen et al. ${ }^{5,13}$ Some of their results of the interaction of an $\mathrm{XeF}$ excimer laser pulse at $\lambda=0.35 \mu \mathrm{m}$ wavelength with an $\mathrm{Al}$ and a $\mathrm{Ti}$ alloy target surface in vacuum are reproduced in Figures 6.2 and 6.3 to illustrate the magnitude of the physical parameters involved.

Figure 6.3 shows the surface temperature rise of a $\mathrm{Ti}$ target versus time at a relatively low intensity of $4 \times 10^{6} \mathrm{~W} / \mathrm{cm}^{2}$. Figure 6.4 shows the calculated density of $\mathrm{Al}$ atoms, excited $\mathrm{Al}$ atoms, and of electrons and ions in the vapor as a function of time. The observed thermal coupling coefficient is not changed ${ }^{5,13}$ by plasma formation up to irradiances of $10^{8} \mathrm{~W} / \mathrm{cm}^{2}$ in $0.5 \mu \mathrm{s}$ pulses, although the theory predicts some plasma shielding at this level especially for longer wavelengths where inverse bremsstrahlung absorption is stronger in the blow-out plasma than in the target. The impulse coupling shown in Figure 6.5 also follows the behavior of simple vaporization without apparent modification by plasma effects. Impulse coupling from multiple pulses of I $\geq 10^{8} \mathrm{~W} / \mathrm{cm}^{2}$ and pulse durations of microseconds is discussed in Section 6.3.

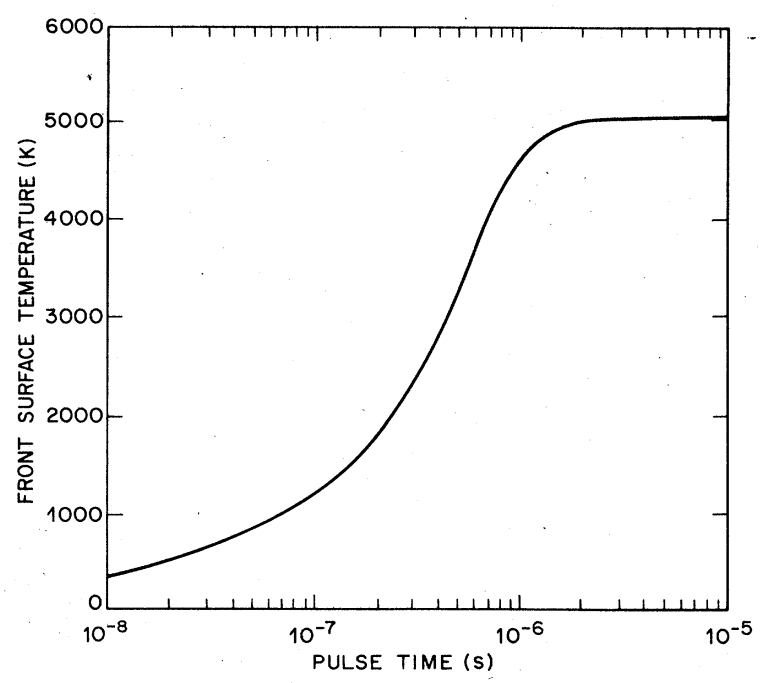

Figure 6.3. Surface temperature rise in a Ti-alloy target on irradiation with $I=4 \times 10^{6} \mathrm{~W} / \mathrm{cm}^{2}$ at $\lambda=1.35 \mu \mathrm{m}$ versus time (after Reference 13). 


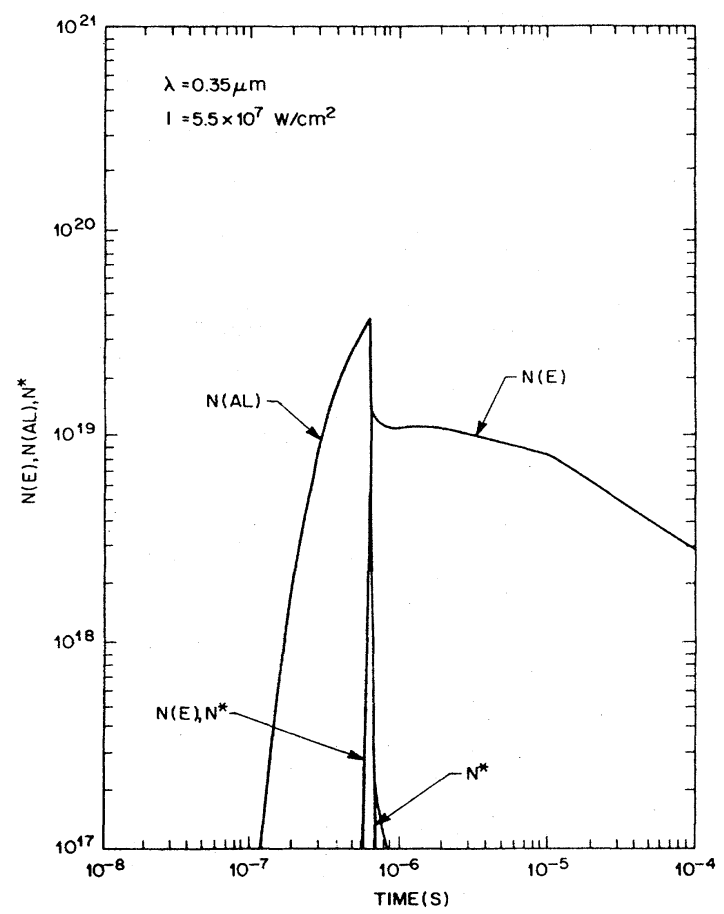

Figure 6.4. Calculated species densities in aluminum vapor versus time for an incident laser intensity of $5.5 \times 10^{7} \mathrm{~W} / \mathrm{cm}^{2}$. The reflection coefficient of the alloy at $\lambda=0.35 \mu \mathrm{m}$ is assumed to be $\mathrm{R}=0.75$ (after Reference 5).

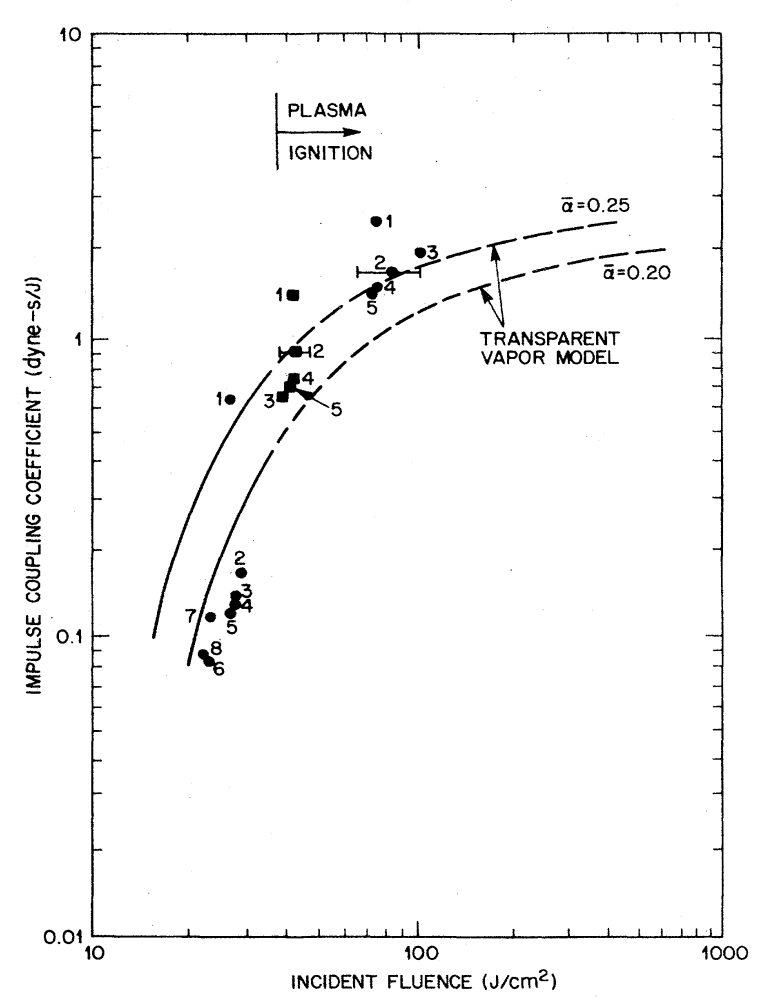

Figure 6.5. Specific impulse coupling on an A1-2024 target, irradiated with a $0.5 \mu \mathrm{s}$ pulse at $\lambda=0.35 \mu \mathrm{m}$ (after Reference 5).
This concludes the discussion of the basic physics of laser materials interaction by $\mathrm{cw}$ and quasi-cw lasers. The fundamentals of the interaction are well understood over a wide range of parameters. Now attention is turned to characteristic responses of specific target materials.

\subsubsection{Materials Response}

There are four broad classes of materials for which the response to $\mathrm{cw}$ laser radiation is of special interest.

1. Metals, including alloys of aluminum and titanium used in air frame and missile structures.

2. Ablative materials, including carbon-phenolics, carbon-carbon fiber composites, and tungsten bearing resins. These are used as heat shields and may also serve for thermal hardening, to raise the damage threshold against directed energy laser radiation.

3. Composites, consisting of a resin, or other carbon containing matrix materials with reinforcing fibers. In fiberglass the fibers consist of a glassy ceramic, in Kevlar epoxy of nylonlike polymer fibers. In carbon-carbon composites, the matrix consists of carbon in which carbon fibers are embedded.

4. Ceramics, including various glasses and other metal oxides, sulfides, and fluorides. Such materials are used in optical and infrared windows, as well as in optical coatings of mirrors.

The salient features of the response to laser radiation at different wavelengths will be briefly described for each of these classes.

\subsubsection{Metals}

The first cw high power lasers available were the $\mathrm{CO}_{2}$ lasers at $10.6 \mu \mathrm{m}$ and $9.6 \mu \mathrm{m}$ wavelength. Although these lasers are not at present actively considered for strategic defense purposes, because the long wavelength would require large dimensions of optical mirrors and because they rapidly decouple from the target through vapor absorption, their interaction with metals for welding and machining is of great interest. Very pure metals may have a very high reflectivity $R>0.999$ at this wavelength, which would drastically reduce the radiation coupling. In practice, actual metals in the skin of airplanes and missiles will consist of alloys, and the surface may have scratches, paint layers, or absorbing impurity flakes. All these factors will reduce the reflectivity. Paint layers or adsorbed flakes of impurities may start the vaporization process. For repetitive pulsing at levels $>5 \times 10^{6} \mathrm{~W} / \mathrm{cm}^{2}$, coupling is improved. The plasma can be formed early in each pulse and the metal surface has a lower reflectivity for the ultraviolet recombination radiation from the plasma. At very high 
irradiances for targets in air, the coupling decreases again, as a laser supported detonation wave rapidly propagates away from the target. ${ }^{9-12}$ Similar coupling effects occur for the interaction with $2.8 \mu \mathrm{m}$ radiation from $\mathrm{HF}$ lasers, although the initial reflectivity is not quite as high. As the factor 1- $\mathrm{R}$ increases significantly towards the visible, vaporization without plasma formation will occur in the cw regime. The penetration and burn-through of metal plates depends, therefore, rather sensitively on wavelength, surface preparation, and melt-removal by air flow, but the observed thresholds for damage by this mechanism in the near infrared, visible, and ultraviolet are always considerably lower than those of ablative materials, which must be penetrated by evaporation.

For the thermal coupling mechanism it is immaterial if the back side of metal is insulated or in contact with a liquid, as might be the case of liquid fuel booster. The back-face temperature will rapidly rise above the nucleate boiling point for the liquid, beyond which further thermal transport to the fluid may be ignored. The melting of the metal will proceed at the same rate or faster for repetitive pulses at the same average power of radiation. The thermal coupling may be enhanced significantly by plasma formation, especially at instantaneous power levels at infrared wavelengths approaching $10^{7} \mathrm{~W} / \mathrm{cm}^{2}$.

\subsubsection{Ablative Materials}

It is customary for all ablative targets to define an effective heat of ablation by

$$
\mathrm{Q}^{*}=\mathrm{I}_{0} / \dot{\mathrm{m}}
$$

It is equal to the heat of vaporization only if the reflectivity is zero and other heat losses are negligible, as is evident from Equation (6.3). This empirical quantity will be larger than $H_{c}(g)$ if such losses are important. It can be smaller than $\mathrm{H}_{\mathrm{c}}(\mathrm{g})$ if considerable mass loss occurs through spallation, or mass loss in the form of liquid or solid particles instead of vaporization.

The simplest material from the point of view of theoretical interpretation is presented by the carboncarbon composite samples. The all-carbon target is, indeed, penetrated by evaporation. The theoretical $\mathrm{Q}^{*}$ as a function of a cw laser flux density at $\lambda=2.8 \mu \mathrm{m}$ is shown in Figure 6.6. There is a threshold below which all absorbed heat is carried away by conduction, convection, and/or radiation. Below this threshold no vaporization occurs and the effective $Q^{*}$ is infinite. For a sufficiently large spot size, radial conduction losses are negligible, and $Q^{*}$ approaches the theoretical value determined by the heat of vaporization $\mathrm{H}_{\mathrm{c}}(\mathrm{g})$, corresponding to $32 \mathrm{~kJ} / \mathrm{g}$, as the reflectivity is close to zero. At flux densities above $10^{5} \mathrm{~W} / \mathrm{cm}^{2}$ experimental data are not sufficient to verify theoretical calculations. Hence, $Q^{*}$ may rise again, if shielding by vapor or plasma

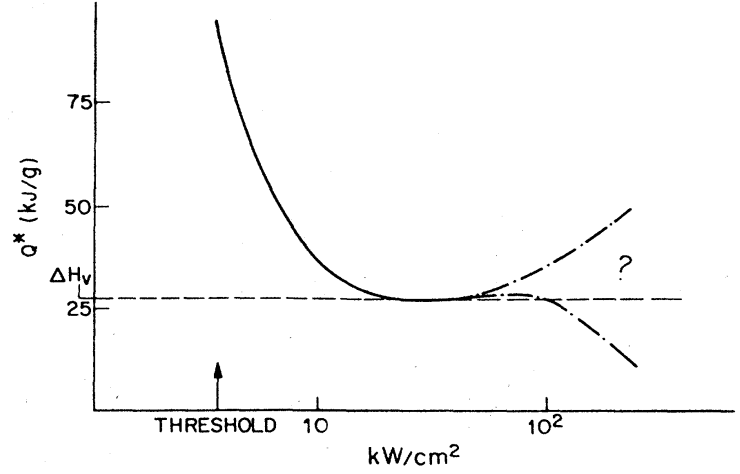

Figure 6.6. Theoretical behavior of the effective heat of ablation $Q^{*}$ for ablation of a carbon target.

formation occurs. It may also be significantly lower, if discrete mass removal takes place.

Turning to carbon-phenolic and other materials with a resin matrix, the first step in the thermal process will be pyrolysis of the bonding material. The heat transport balance will be changed by the gaseous products of pyrolysis.

It is important to note that tungsten bearing resins have $Q^{*}$ higher than that for pure carbon composites. The apparent reason is that the tungsten forms a reflective liquid metallic layer on the surfaces, through which carbon diffuses and evaporates. Similar behavior is found for tungsten carbide. The reflectivity of the high temperature metallic layer is about 0.5 at $\lambda=2.5 \mu \mathrm{m}$. It is possible that ablative materials with metallic coating may lead to higher reflectivities over a wide spectral range. An increase in reflectivity from 0.5 to 0.75 would lead to an increase of a factor of two in $\mathrm{Q}^{*}$.

An often asked question is, why can the reflectivity not be made higher? Obviously for a reflectivity of 0.99 , the damage threshold would be increased by almost two orders of magnitude. In fact, some mirrors in optics of high power laser trains are made to withstand flux densities of $20 \mathrm{MW} / \mathrm{cm}^{2}$. These mirrors have dielectric coatings with absorptivity of less than $10^{-4}$. The coatings are deposited on a substrate with efficient cooling channels and are made out of materials with high thermal conductivity, such as molybdenum or single crystal silicon, as discussed in Chapter 5. The outer skin of a booster cannot be fabricated in this manner. The skin must withstand rigors of launch and atmospheric drag. One needs a material that even when heated to high temperatures continues to exhibit relatively high reflectivity at all wavelengths. Thus, a reflectivity of 0.5 for booster surfaces is already a considerable achievement.

\subsubsection{Composites}

Consider, for example, fiberglass and Kevlar-epoxy materials. In some regime of operation, when the initial penetration depth of laser radiation is rather large, 
pyrolysis and vaporization of glass or polymer reinforcing fibers may occur to a considerable depth. ${ }^{14,15}$ Then delamination may take place and material will be removed at a more rapid rate, described by an anomalously low value of $\mathrm{Q}^{*}$. This behavior occurs in fiberglass at $10.6 \mu \mathrm{m}$ wavelength at power levels below $100 \mathrm{~W} / \mathrm{cm}^{2}$, and pulse fluences between 1 and $10 \mathrm{~J} / \mathrm{cm}^{2}$. At pulse repetition rates between $10^{2}$ and $10^{3} \mathrm{~s}^{-1}$, with peak power levels less than $1 \mathrm{~kW} / \mathrm{cm}^{2}$, the glass fibers melt and the molten material is removed by thermomechanical vibrations. Only at power levels of about $10 \mathrm{~kW} / \mathrm{cm}^{2}$, or total fluences above $10 \mathrm{~J} / \mathrm{cm}^{2}$, is the material rapidly charred during the early phases of radiation. One then observes a value for $Q^{*}$ $=13.5 \mathrm{~kJ} / \mathrm{g}$, corresponding closely to the average heat of vaporization of the constituents. This rather complex behavior is illustrated in Figures 6.7 and 6.8. In general the vulnerability of composites is difficult to predict from first principles. Extensive testing is required over a range of wavelengths, power flux densities, and fluence levels.

\subsubsection{Ceramics and Glassy Materials}

These materials are of special importance because of their use as windows in optical systems, in infrared and visible photodetectors, and as reflective or antireflective coatings.

Processes of melting and vaporization are again possible, provided an irradiation wavelength is chosen where the absorptivity is not too low. A new failure mechanism is, however, possible because of the brittle nature of the material. Thermal stresses may be set up at intensity levels of irradiation well below those required for evaporation. This may lead to the initiation of cracks, and eventually to fracture. After a temperature rise by a heating pulse takes the glass above the softening point, subsequent rapid cooling of the heated volume elements after the laser pulse is terminated will leave the glassy material with a lower specific density than the carefully

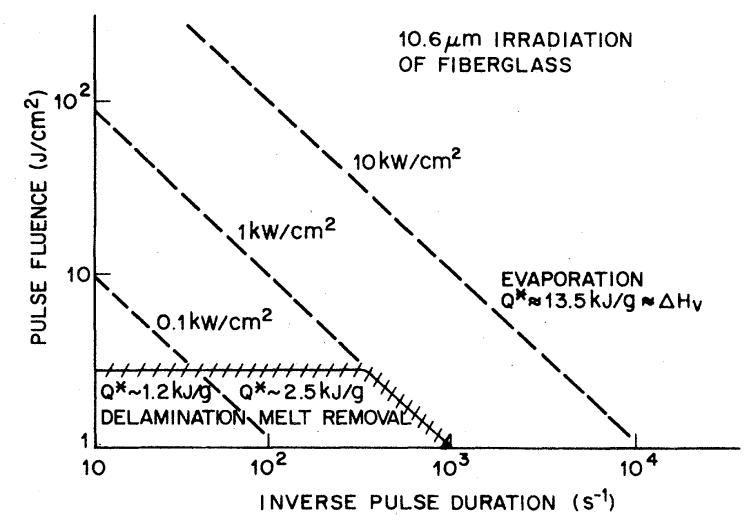

Figure 6.7. Regimes at mass removal for fiberglass of $\lambda=10.6 \mu \mathrm{m}$ (after Reference 15).

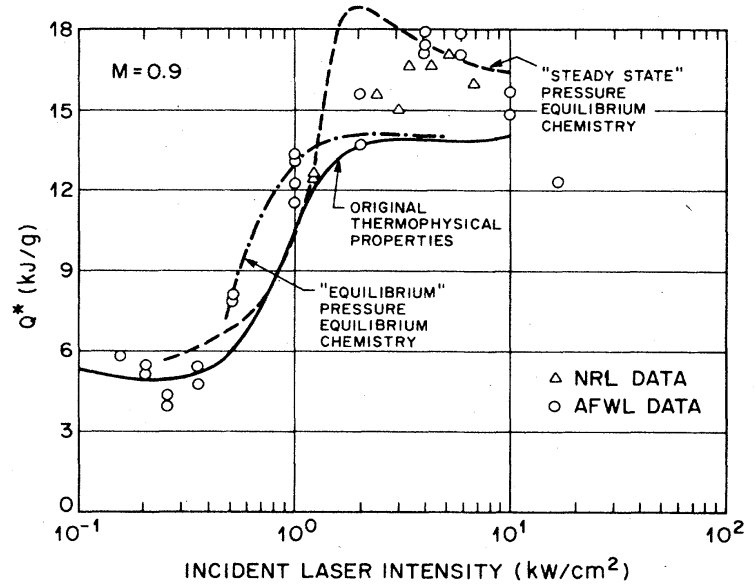

Figure 6.8. Comparison of fiberglass model predictions for three sets of thermophysical properties with six-ply penetration data for $\mathrm{cw} 10.6 \mu \mathrm{m}$ radiation (after Reference 15).

annealed original material. Thus, thermally-induced stresses remain in the material. These may be sufficient to degrade the optical quality, and they may lead to cracking under repetitive pulse loading.

The maximum induced thermal stress will occur for a pulse duration $t_{p^{\prime}}$ when the temperature of the rear surface is just starting to rise due to heat diffusion. For a thickness $1_{0}$, this time is given by $1_{0}^{2}=\kappa \mathrm{t}_{\mathrm{p}}$. The average heat balance in the plate leads to a temperature differential $\Delta \mathrm{T}$ between front and back, determined by

$$
(1-\mathbf{R}) \mathbf{I}-2 \mathrm{~K} \Delta \mathrm{T} / 1_{0}
$$

The thermal stress is given by

$$
\sigma=\operatorname{Ea} \Delta \mathrm{T}(1-v)^{-1}
$$

where $\mathrm{E}$ is Young's modulus, $\mathrm{a}$ is the coefficient of thermal expansion, and $v$ is Poisson's ratio. If $\sigma_{F}$ is the stress threshold for fracture, combination of the above three equations yields for the time of irradiation to failure, $t_{F}$, and the critical pulse fluence $I_{F}$ the relationship

$$
\mathrm{It}_{\mathrm{F}}=\frac{2 \mathrm{~K} 1_{0} \sigma_{\mathrm{F}}(1-v)}{\kappa a \mathrm{E}(1-\mathrm{R})}
$$

with $\mathrm{K} / \kappa=\rho \mathrm{C}_{\mathrm{s}}$.

A display of the various stages of optical damage to $\mathrm{BK} 7$ glass exposed to irradiation from $\mathrm{CO}_{2}$ laser pulses of a few $\mu$ s duration at $10.6 \mu \mathrm{m}$ wavelength as a function of pulse fluence is shown in Figure 6.9. The damage level has only qualitative significance. The regime of bubble formation occurs because of the presence in the glass of 


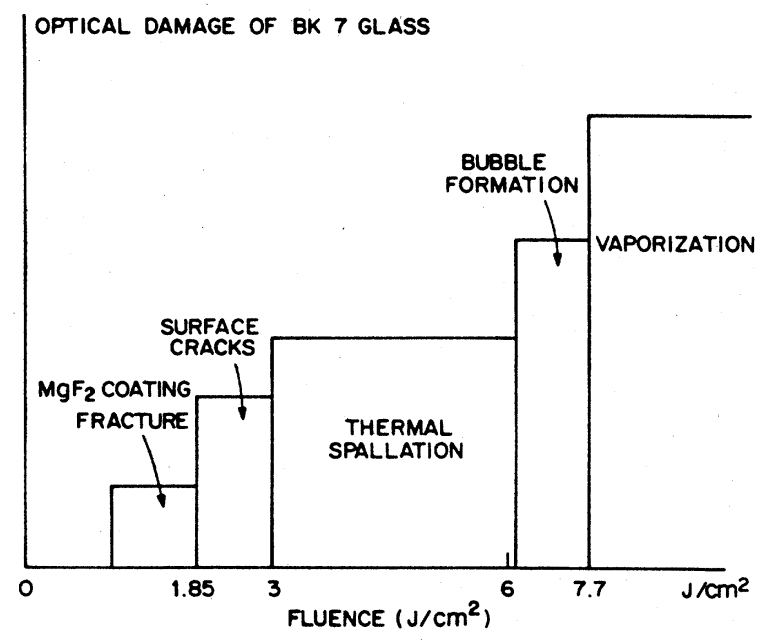

Figure 6.9. Types of optical damage of BK7 glass as a function of fluence at $\lambda=10.6 \mu \mathrm{m}$.

carbonates, which undergo chemical dissociation. (For example, $\mathrm{CO}_{2}$ lasers are commercially used for cutting glass.) Clearly many optical window materials and coatings must be tested at a variety of wavelengths and irradiation levels. In addition, prolonged exposure to ultraviolet light from the sun or from low level UV laser irradiation will produce color centers in many ceramic materials. This will, in turn, enhance absorption in the visible and near ultraviolet, leading to lower damage thresholds. The presence of atomic oxygen at altitudes between $100-500 \mathrm{~km}$ also leads to oxidation and deterioration of metal mirror surfaces. The presence of impurities on optical surfaces will also lead to a lowering of damage thresholds. Space-based lasers will require large amounts of power. If this power is obtained from chemical reactions, massive amounts of spent exhaust products must be prevented from contaminating optical surfaces.

Quite generally, the damage thresholds of optical windows and components lie at least an order of magnitude below those of other unhardened surfaces, and more than two orders of magnitude below those of hardened surfaces. Systematic testing of materials at $2.8 \mu \mathrm{m}$ up to fluence levels of $50 \mathrm{~kW} / \mathrm{cm}^{2}$ has been carried out. The testing of the various materials of potential interest should be extended to higher power flux densities, above $50 \mathrm{~kW} / \mathrm{cm}^{2}$. Facilities such as the MIRACL at the White Sands Proving Grounds in New Mexico are available to carry out such experiments at $2.8 \mu \mathrm{m}$ and $3.8 \mu \mathrm{m}$ wavelengths. Good beam quality and extensive instrumentation is essential for beam diagnostics, to monitor front and back surface temperature, surface reflectivity, surface recession rate, analysis of products of ablations, etc. Systematic testing at shorter wavelengths, corresponding to the operation of excimer lasers and free electron. lasers at $1 \mu \mathrm{m}$ or in the visible, is in progress over a wide range of power and fluence levels for all materials of potential interest. While mirror coatings are designed to be nonabsorbing at the wavelength of the operating laser they must reflect, the coatings may have substantially lower reflectivities and higher absorptivities at other wavelengths, and consequently the damage thresholds at wavelengths used in offensive threat may be much lower.

\subsubsection{Vulnerability of Structures}

So far, discussion has focused on optical damage thresholds of some materials. Here we delineate those intensity and fluence thresholds which influence the damage levels of structures composed of these materials.

An aluminum alloy casing for a liquid fuel booster will fail if melting has reduced the thickness of a loadbearing area of the wall or another structural component below a certain value, so that the stress in the remaining part exceeds a critical stress to failure. This critical value may itself depend on the temperature of the remaining material. Further, the mechanical properties of load bearing solid structure may be "softened" at elevated temperatures. Failure may then occur even before melting occurs. For solid fuel boosters Kevlar-epoxy materials are used. The hardness of boosters and postboost buses may be enhanced by ablative heat shields, which may increase the optical hardness by an order of magnitude for a weight penalty of a few $\mathrm{g} / \mathrm{cm}^{2}$ based on $\mathrm{Q}^{*}$ mentioned above.

It is desirable to provide thermal insulation between the ablative shield and the wall material with a much lower softening or melting temperature. Even if the protective material has not been completely ablated by irradiation from a directed energy weapon, damage could still result after the laser irradiation has stopped, as the temperature of the underlying structural material continues to rise as the heat stored in the ablated material is conducted to the cooler wall.

The damage threshold can be raised by rotating the booster and the bus. The detailed heat loading may be spread out over the entire circumference. The hot spot may be tracked, but the angle of incidence changes until the spot disappears at the limb. The factor to be gained depends, of course, on the ratio of the laser spot size to the diameter of the vehicle, on the angle of incidence, and on the ratio of time to burn-through to the period of revolution. Typical increases in hardening levels by factors of two to three may be obtainable for one to two revolutions per second, unless the burn-through time is $0.1 \mathrm{~s}$ or shorter.

If a thrusting booster is attacked, and its casing fails, termination of the mission ensues. If a bus in the postboost phase is attacked, damage during or after burnthrough will depend on details of the construction, of the deployment mechanism, and the arrangement of the reentry vehicles and decoys inside the bus. The laser beam may do little damage if it hits the heat shields of the reentry vehicles, but it may readily burn up the balloon 
material of the decoys.

Laser directed energy weapons have particular advantages for use in the boost phase. They can attack in the upper atmosphere at altitudes above the highest cloud cover. In the basic concept of a layered defense, they would attack before the deployment of MIRVed reentry vehicles and decoys. Although current liquid fuel boosters have burn times up to $300 \mathrm{~s}$ and burn-out altitudes of $200 \mathrm{~km}$, the technology of fast burn solid boosters is already available. Hardening of the boosters and post-boost buses is also readily accomplished on a shorter time scale than would be required to develop a credible laser weapon. Thus, a hardened solid booster with a burn-out time of $60 \mathrm{~s}$ and a burn-out altitude of $80 \mathrm{~km}$ is a realistic objective. The post-boost bus will have to travel to an altitude of about $150 \mathrm{~km}$ before effective deployment of reentry vehicles and decoys can take place. These considerations yield an available engagement time of about $20 \mathrm{~s}$ for the booster, following its acquisition and tracking above a cloud cover, and another 20-30 s for the bus phase.

Thus, the times for irradiation should be short if the same laser weapon has to engage a number of nearly simultaneously launched targets. According to considerations at the beginning of Chapter 3 , this requires irradiances exceeding $10 \mathrm{~kW} / \mathrm{cm}^{2}$, with spot sizes of $10^{3}$ to $10^{4} \mathrm{~cm}^{2}$. The total energy required is $10-100 \mathrm{MJ}$. For a distance of the laser to target of $3000 \mathrm{~km}$ and a spot diameter of $30 \mathrm{~cm}$, this would require a diffraction limited laser with an output aperture of $(\lambda / 30) \times 3 \times 10^{8}$ $=10^{3} \mathrm{~cm}$ at $1 \mu \mathrm{m}$. The power required for lethality against unhardened satellites, especially with unhardened optical windows, will be at least two orders of magnitude lower. In this case, there is also no limit on the engagement time, and the range at attack time may be chosen to be about equal to the altitude of the satellite orbit.

\subsubsection{Kill Assessment}

The verification that an enemy target has been effectively "killed" presents additional problems. Such information is obviously essential for effective command and control of battle management.

If a burning booster is disabled, the deviation from the normal extrapolated trajectory should be readily detectable by the same tracking equipment that was used to acquire the target in the first place. In this case, the situation is analogous to that of shooting down an airplane.

In the post-boost phase the assessment is much more difficult. The specific momentum transfer from laser irradiation is about 2 dyn $\mathrm{s} / \mathrm{J}$ (compare Figure 6.5). Even for $10^{8} \mathrm{~J}$ absorbed the momentum change of $10^{8}$ dyn $\mathrm{s}$ caused by irradiation is very small compared to the total momentum of the missile. This is nearly $10^{13}$ dyn s, assuming a weight of 10 tons at near-orbital velocity of
$7 \mathrm{~km} / \mathrm{s}$. Note that a directional deviation of about $10^{-5}$ rad could be expected, which might produce a deviation of $100 \mathrm{~m}$ from a target point at a distance of $10^{4} \mathrm{~km}$. Such small angular deviations can, of course, easily be corrected (or simulated) by small auxiliary thrusters. The proof of successful kill of the bus in the post-boost phase would, therefore, have to await the deployment of the reentry vehicles and decoys, or rather the absence or impairment of such deployment. This would require continued tracking throughout the post-boost phase. In particular, it would be very difficult to decide how long the laser beam should be kept on target. This would directly affect the number of missiles that each laser weapon could engage in combat, in case of a massive simultaneous launch.

Presumably, infrared tracking sensors could monitor the hot spot produced by laser radiation. This would also be essential for keeping the laser beam on target. A sudden discontinuity in radiative emission on burnthrough of the bus could be detected by the infrared detectors. Further analysis of this question clearly belongs to the field of command, control, communications, and intelligence.

Lethality assessment of enemy satellites poses similar problems. If the power generation of the satellite is affected, a change in its temperature may be monitored by infrared detectors. If only the sensors are blinded, perhaps a sudden change in its data transmission could be intercepted.

\subsection{PULSED LASER EFFECTS}

In this section, we discuss pulsed laser effects that result in the transfer of momentum to the material surface. This impulse loading causes the device or structure to fail. Damage may take the form of severe structural failure of a missile, post-boost vehicle, or possibly an RV. For the missile, this damage can destroy the structural integrity of the missile body or can result in rupture of the fuel tanks for liquid fuel boosters or failure of casings (unzipping) of solid fuel boosters. The physical phenomena controlling this interaction are strongly dependent upon the atmospheric environment of the surface, the laser spot size, the laser intensity, and the laser pulse length. The interaction is weakly dependent on the laser wavelength, the type of material, and the condition of the surface. Several excellent tutorial papers are available on this subject. ${ }^{11,16-19}$

Pulsed laser effects are easily divided into interactions in vacuum and interactions in the atmosphere. That format is used for the following discussion, which considers the interaction in the 0.25-10.6 $\mu \mathrm{m}$ wavelength range. Current development of excimer lasers would yield giant pulse systems operating in the $0.25-0.35 \mu \mathrm{m}$ region. The expanded wavelength region is included to incorporate some previous data from $\mathrm{CO}_{2}$ and chemical lasers. The effects 
of soft $x$-ray photons are also included to permit a lethality assessment of an $\mathrm{x}$-ray laser system.

\subsubsection{Interaction in Vacuum}

If the laser intensity upon a surface is above some minimum level, $10^{6}-10^{8} \mathrm{~W} / \mathrm{cm}^{2}$, then the thin surface layer of the material is rapidly (in $1 \mu \mathrm{s}$ or less) heated to its vaporization temperature. This vapor is ionized and the absorption of the beam takes place in the surface vapor and the plasma. As a result the absorption is relatively insensitive to the surface material. The resulting vapor leaves the surface at high velocity at translational temperatures in the range of 2000-4000 K. From conservation of momentum, an impulse is delivered to the surface. The efficiency of this process is represented by a coupling coefficient $\mathrm{C}$, defined by

$$
\mathrm{C}=\mathrm{I}_{\mathrm{p}} / \mathrm{E}=\mathrm{T} / \mathrm{F}_{0}
$$

where $I_{p}$ (dyns) is the impulse transferred to the surface and $E$ is the energy absorbed at the surface, and $T$ (dyn $\mathrm{s} / \mathrm{cm}^{2}=$ taps) is the specific impulse per unit area, and $F_{0}$ the laser fluence.

The time dependence of response of the surface to intense laser radiation may be described in several stages. First, the radiation is coupled directly to the surface until the absorption is sufficient to melt the surface. Normally the vapor emitted from the target during this period does not strongly absorb the radiation. Therefore, surface reflection is controlling the interaction during this phase. If the radiation is sufficient, the material reaches its vaporization temperature and the surface recedes as the vapor is emitted and the melt interface propagates into the surface. Equilibrium vaporization without plasma formation has been modeled by Anisimov. ${ }^{1}$ His model assumes that under strong laser radiation, the vaporization wave propagates into a solid at velocity

$$
\mathrm{v}_{\mathrm{s}}=\mathrm{c}_{\mathrm{s}} \exp \left(-\mathrm{H} / \mathrm{C}_{\mathrm{p}} \mathrm{T}\right)
$$

where $\mathrm{c}_{\mathrm{s}} \sim 5 \times 10^{5} \mathrm{~cm} / \mathrm{s}$ is the speed of sound in the solid, $\mathrm{H} \sim 10 \mathrm{~kJ} / \mathrm{cm}^{3}$ is the heat of vaporization of the material, $C_{p}$ is the heat capacity, and $T$ is the temperature at which vaporization takes place at the elevated pressure of the blow-off material. These constants are summarized in Table 6.1 for common metallic target materials. The energy invested in the ejected vapor flux per unit time is balanced by the laser irradiance $I_{0}$ at the target surface under equilibrium conditions.

$$
\mathrm{I}_{0}=\rho_{\mathrm{v}} \mathrm{v}_{\mathrm{v}}\left(\mathrm{H}+\mathrm{C}_{\mathrm{p}} \mathrm{T}\right) .
$$

Momentum conservation requires that

$$
\rho_{\mathrm{s}} \mathrm{v}_{\mathrm{s}}=\rho_{\mathrm{v}} \mathrm{v}_{\mathrm{v}}
$$

where $\rho_{\mathrm{s}}$ is the solid mass density, so $\mathbf{I}_{0}$ determines the vaporization temperature through the relation

$$
\mathrm{I}_{0}=\rho_{\mathrm{s}} \mathrm{c}_{\mathrm{s}}\left(\mathrm{H}+\mathrm{C}_{\mathrm{p}} \mathrm{T}\right) \exp \left(-\mathrm{H} / \mathrm{C}_{\mathrm{p}} \mathrm{T}\right)
$$

TABLE 6.1. Values of constants.

\begin{tabular}{lllll}
\hline Solid metal constants & \multicolumn{1}{c}{ Units } & Aluminum & Copper & Titanium \\
\hline Specific heat of sublimation & $\mathrm{H}(\mathrm{erg} / \mathrm{g})$ & $12.0 \times 10^{10}$ & $5.6 \times 10^{10}$ & $9.0 \times 10^{10}$ \\
Thermal diffusivity & $\mathrm{K}\left(\mathrm{cm}^{2} / \mathrm{s}\right)$ & 0.95 & 1.2 & 0.089 \\
Density & $\rho_{\mathrm{s}}\left(\mathrm{g} / \mathrm{cm}^{3}\right)$ & 2.7 & 8.9 & 4.5 \\
Heat capacity & $\mathrm{C}_{\mathrm{v}}\left(\mathrm{erg} / \mathrm{K} \mathrm{cm}^{3}\right)$ & $2.5 \times 10^{7}$ & $3.5 \times 10^{7}$ & $2.36 \times 10^{7}$ \\
Ionization potential & $\phi_{1}(\mathrm{eV})$ & 6.0 & 7.7 & 6.83 \\
Vaporization wave & & & & \\
\multicolumn{1}{c}{ Temperature scale } & $\mathrm{T}_{0}(\mathrm{~K})$ & $3.8 \times 10^{4}$ & $3.8 \times 10^{4}$ & $5.1 \times 10^{4}$ \\
\multicolumn{1}{c}{\begin{tabular}{l} 
Velocity scale \\
\hline \hline
\end{tabular}} & $\mathrm{c}_{\mathrm{s}}(\mathrm{cm} / \mathrm{s})$ & $5.2 \times 10^{4}$ & $5.1 \times 10^{4}$ & $5.6 \times 10^{6}$ \\
\hline
\end{tabular}

* F. D. Bennett, Phys. Fluids 8, 1425 (1965). 
Thus, for a given $I_{0}$ the vaporization temperature is determined by solving this equation numerically.

The velocity of the target vapor is assumed to be sonic. The vapor is further heated and accelerated away from the target. In the range of $T=1-30 \mathrm{eV}$, an approximate equation of state for both air and target materials is given by the SESAME tables from Los Alamos National Laboratories as

$$
\epsilon=\mathrm{bT}^{1.2} \rho^{-0.12},
$$

where $\epsilon$ is in $\mathrm{erg} / \mathrm{g}, \mathrm{T}$ is in $\mathrm{eV}$, and $\rho$ is in $\mathrm{g} / \mathrm{cm}^{3}$. The constant $b$ is a function of the material; for aluminum, $\mathrm{b}=1.44 \times 10^{11}$.

The initial absorption in the ionized vapor is caused by free electrons through inverse bremsstrahlung. The inverse absorption length $\alpha$ in $\mathrm{cm}^{-1}$ for aluminum ${ }^{18}$ is given by

$\alpha=\frac{2.432 \times 10^{-37}}{(\mathrm{~h} v)^{3}}[1-\exp (-\mathrm{h} v / \mathrm{kT})] \frac{\mathrm{z}^{2} \mathrm{n}_{\mathrm{e}} \mathrm{n}_{+}}{\sqrt{\mathrm{T}}}$

In this equation $\mathrm{z}$ is the ion charge number, $\mathrm{h} v$ is the photon energy in $\mathrm{eV}, \mathrm{n}_{\mathrm{e}}$ and $\mathrm{n}_{+}$are the electron and ion number densities per $\mathrm{cm}^{3}$, and $T$ is the gas temperature in eV.

A second important absorption mechanism involves photoionization of an excited state. This absorption is characterized by the optical cross section ${ }^{5,20}$

$$
\sigma=\left(7.9 \times 10^{-18}\right)\left(\frac{\mathrm{E}_{\mathrm{i}}}{\mathrm{h} v}\right)^{3}\left(\frac{\mathrm{I}_{\mathrm{H}}}{\mathrm{E}_{\mathrm{i}}}\right)^{1 / 2},
$$

where $\sigma$ is in $\mathrm{cm}^{2}, \mathbf{E}_{\mathrm{i}}$ is the energy of ionization of the excited state, $\mathrm{h} v$ is the photon energy of the laser light, and $I_{H}$ is the hydrogen ionization potential in the same units as $\mathrm{E}_{\mathrm{i}}$ and $\mathrm{h} \boldsymbol{v}$. For example, opacities for aluminum vapor including electron-neutral and electron-ion bremsstrahlung and photoionization have been calculated by several researchers. The results of computations by Rosen et al., ${ }^{5}$ and Gurtman, ${ }^{21}$ and Pirri $^{22}$ are shown in Figure 6.10. The absorption coefficient is equal to the opacity multiplied by the appropriate density.

A compilation of early experimental data is shown in Figure 6.11. The experiments with a pulsed ruby laser $(\lambda=0.69 \mu \mathrm{m})$ by Gregg and Thomas $^{23}$ have been a benchmark for subsequent investigations. Impulse coupling coefficients were measured for beryllium, graphite, aluminum, zinc, silver, and tungsten. This study, published in 1966, used a pulse $7.5 \mathrm{~ns}$ (full width at half maximum) in duration. A pendulum was used to determine the momentum transfer to the target. This

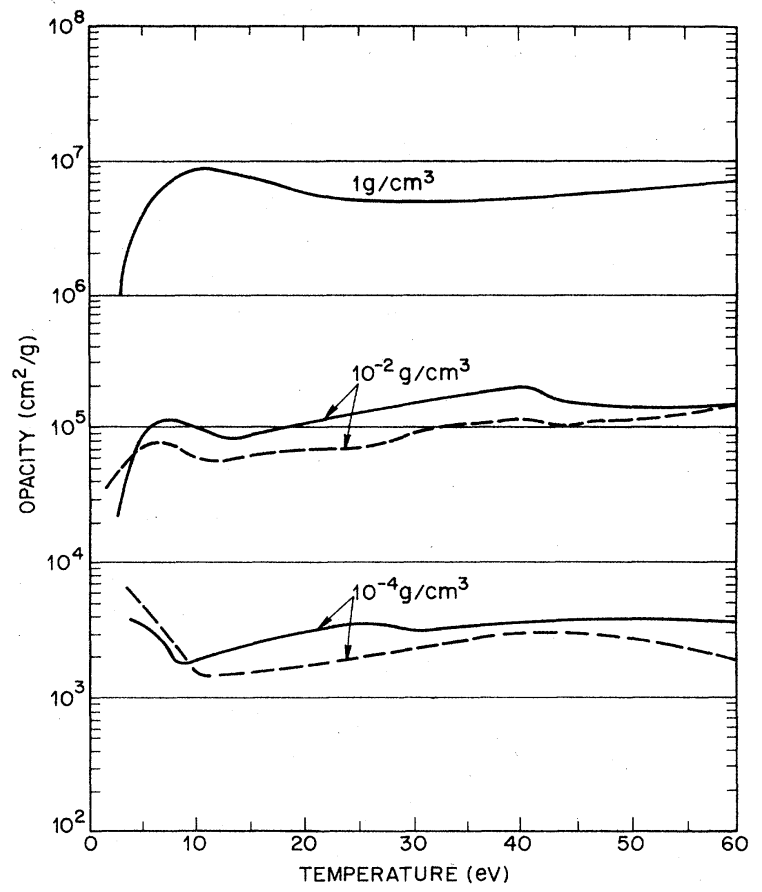

Figure 6.10. Opacity of aluminum plasma for ultraviolet radiation at $\mathrm{h} v=3.5 \mathrm{eV}$, as a function of plasma temperature for three nominal vapor densities. The solid lines are theoretical calculations by Pirri et al. (Reference 22); the dashed lines are calculated by Gurtman (Reference 21).

experiment showed that the momentum transfer resulted from material being ejected from the surface, and it was four orders of magnitude larger than the momentum associated with total reflection of the laser light. Shock pressures in the material can be estimated using the relation $P_{s}=I_{p} / A t_{p}$ where $I_{p}$ is the momentum

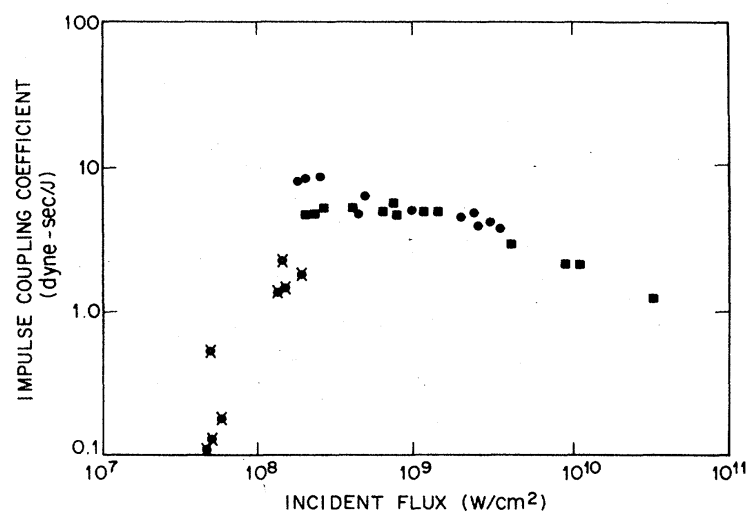

Figure 6.11. Experimental impulse coupling coefficients in aluminum. - Data from Reference 23 at $0.69 \mu \mathrm{m}, \mathrm{t}_{\mathrm{p}}=7.5 \mathrm{~ns}$. $\bullet$ - Data from Reference 24 at $0.308 \mu \mathrm{m}, \mathrm{t}_{\mathrm{p}}=5 \mu \mathrm{s}$. 2 - Data from Reference 5 at $0.35 \mu \mathrm{m}, \mathrm{t}_{\mathrm{p}}=0.5 \mu \mathrm{s}$. 
transferred to the target in time $t_{p}$, and $A$ is the spot area. Typical pressures are of the order of a few Mbars.

Recent measurements on momentum transfer of interest for ballistic missile defense targets include observations by Rosen et al. $(\lambda=0.35 \mu \mathrm{m})^{5,13}$ and Woods $(\lambda=0.308 \mu \mathrm{m}),{ }^{24}$ also shown in Figure 6.11. Other work in the area is included in References 25-31.

Very recent experiments, first reported in late 1985, have been performed on the SPRITE laser (at the Rutherford-Appleton Laboratory in England) by a group of Los Alamos National Laboratory personnel, and on the NOVA laser at Lawrence Livermore National Laboratory by Janeé. ${ }^{32-34}$ These lasers have characteristics that permit testing under conditions required for BMD applications. Generally speaking, this includes wavelengths in the range of $0.24-1.06 \mu \mathrm{m}$, pulse lengths of $1 \mu \mathrm{s}$ or longer, irradiances in the range of $10^{8}-10^{12} \mathrm{~W} / \mathrm{cm}^{2}$, and fluences in the range $10^{4}-10^{6} \mathrm{~J} / \mathrm{cm}^{2}$. Furthermore, the projected spot size on target should be such that the interactions will be one dimensional, i.e., the plasma expansion will be primarily normal to the surface and the distance of expansion during the pulse will be small compared to the lateral extent of the irradiated spot size. The earlier work at longer wavelengths, lower fluences and, most importantly, small spot sizes did not meet these requirements. If the pulse length is short (less than $1 \mu \mathrm{s}$ ) the important phenomenon of shielding of the target by blow-off plasma cannot be studied. The characteristics of the most recent experiments are as follows.

The SPRITE laser is a $100 \mathrm{~J} \mathrm{KrF}$ laser with an approximately $50 \mathrm{~ns}$ pulse length. The experiments were conducted at varying background pressures from vacuum to $1 \mathrm{~atm}$. A first series of experiments in vacuum were conducted in 1984 using a variety of materials. Figure 6.12 shows the impulse coupling coefficient, $\mathrm{C}=\mathrm{I}_{\mathrm{p}} / \mathrm{E}$, as a function of fluence both for metals (aluminum and titanium) and composites (Kevlar and graphite epoxy). For metals, the impulse coupling coefficient can reasonably be characterized by a straight power-law decline. For composites, the behavior of impulse coupling is more complex; the coupling is generally higher, especially at lower fluences. Data of mass loss in all these experiments show substantial scatter, suggesting some difficulties with the experimental procedures.

A second series of SPRITE experiments ${ }^{34}$ was conducted in 1985. These experiments showed that impulse coupling increases with background atmospheric pressure, and is roughly ten times the vacuum coupling at $1 \mathrm{~atm}$. The experiments also found impulse coupling to be a weak function of target material, in contrast with the earlier result. However, a problem with both SPRITE series is that (except for the very lowest fluence levels) the data are certainly two dimensional and do not directly address the actual case of large spot sizes. An effect of this problem is shown in the scatter of experimental data on Figure 6.13, which shows aluminum target experiments for both test series.
The NOVA laser results represent the first output of an ongoing research program. The laser can deliver more than $50 \mathrm{~kJ}$ of $1 \mu \mathrm{m}$ light per pulse (and is expected to increase to more than $100 \mathrm{~kJ}$ when new laser glass is installed). Pulse lengths are only in the range $0.5-5 \mathrm{~ns}$, although there are plans for ways to increase the pulse length to around $500 \mathrm{~ns}$. With this much laser power, it is possible to conduct impulse coupling experiments at $1 \mu \mathrm{m}$ that are unambiguously one dimensional up to fluences above $10^{5} \mathrm{~J} / \mathrm{cm}^{2}$ and fluxes above $10^{13} \mathrm{~W} / \mathrm{cm}^{2}$ using only one of the 5 beams of NOVA that can illuminate a flat target (there are 10 beams in all). NOVA

(a) COUPLING COEFICIENT FOR METALS SPRITE $\lambda=248 \mathrm{~nm} \quad \tau=50 \mathrm{nS}$ JULY 30,1984 LINEAR FIT

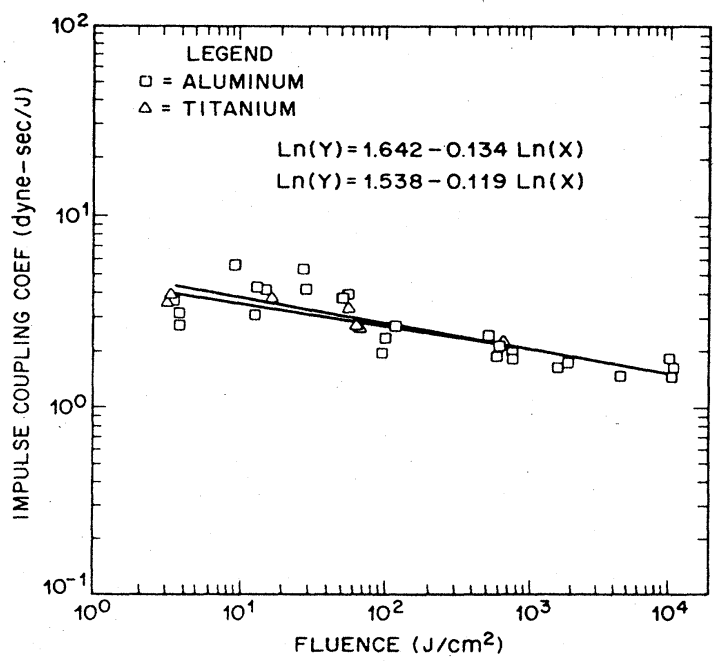

(b) COUPLING COEFFICIENT FOR KEVLAR AND GRAPHITE EPOXIE SPRITE $\lambda=248 \mathrm{~nm} \quad \tau=50 \mathrm{nS}$ JULY 30,1984 LINEAR FIT

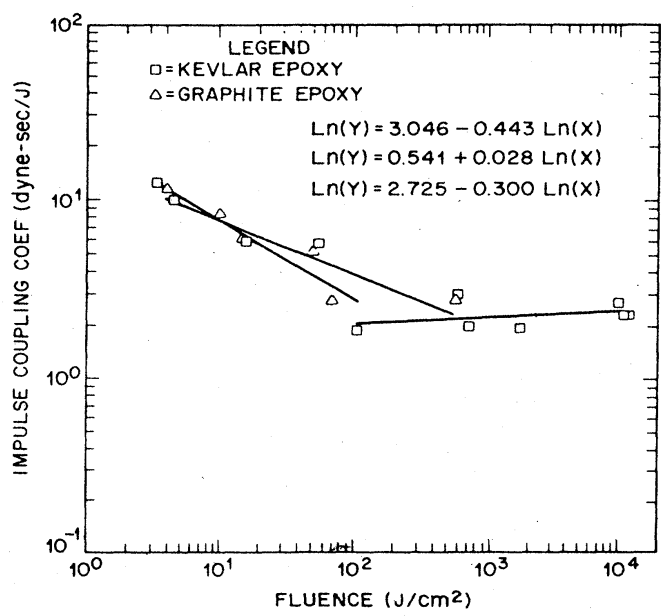

Figure 6.12. Impulse coupling to metals and composites (from AWRE/LANL SPRITE experiments, References 29 and 30 ). 


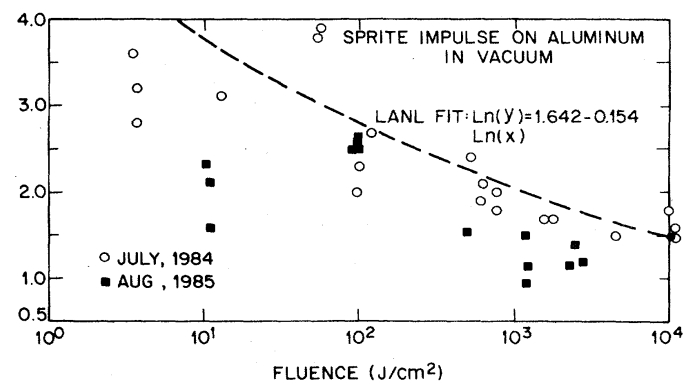

Figure 6.13. Comparison of impulse coupling to aluminum in two SPRITE test series (References 29 and 30).

can also produce reduced amounts of energy at $0.53 \mu \mathrm{m}$ (15 $\mathrm{kJ}$ in 5 beams) and $0.25 \mu \mathrm{m}(12.5 \mathrm{~kJ}$ in 5 beams). The preliminary results of the first NOVA test series on aluminum targets are shown in Figure 6.14, where $0.53 \mu \mathrm{m}$ NOVA data are compared with $1.06 \mu \mathrm{m}$ data taken on the earlier LLNL laser JANUS. These data appear to verify the general power-law scaling.

The possible shielding of the target by the vaporization plasma remains a dominant issue to be discussed. The critical question which must be settled experimentally is how the coupling coefficient scales for various intensity levels as a function of laser pulse length. Deposition of energies in the ranges of multiple $\mathrm{kJ} / \mathrm{cm}^{2}$ will require pulses of a few $\mathrm{GW} / \mathrm{cm}^{2}$ lasting tens of $\mu \mathrm{s}$. Detailed computer calculations of the processes of decoupling and shielding have been performed by numerous researchers, particularly Dingus ${ }^{35}$ and Hunter. ${ }^{36}$ The results of these calculations are summarized below.

For intensities above $1 \mathrm{GW} / \mathrm{cm}^{2}$, the vapor expands to a density of about $0.1 \mathrm{~g} / \mathrm{cm}^{3}$, drifts away from the target, and then expands again strongly about $1 \mathrm{~mm}$ from the target because of significant heating of the vapor by absorption of the laser beam. This produces vapor with a temperature of about $1 \mathrm{eV}$, which increases its absorption rapidly through a bootstrap mechanism to levels that

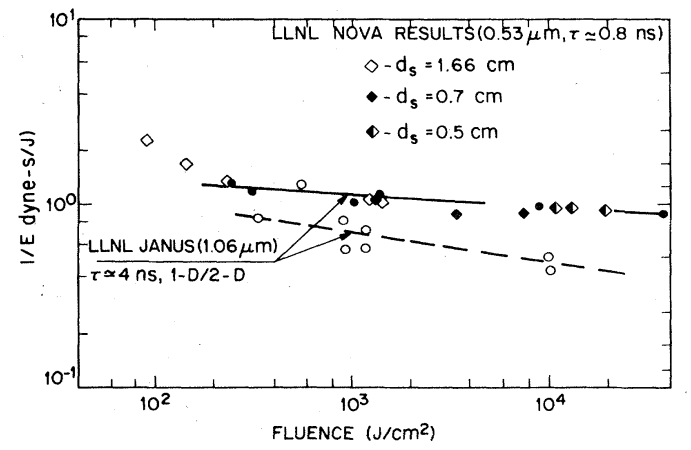

Figure 6.14. Impulse coupling to aluminum (6061) and gold in vacuum at $0.53 \mu \mathrm{m}$ and $10.6 \mu \mathrm{m}$, SAIC/LLNL NOVA experiments (References 29 and 30). produce a fully ionized plasma strongly attenuating the laser flux reaching the surface of the target. After this time, less energy is coupled into the target and little impulse is generated. The coupling coefficient of about 8 dyn $\mathbf{S} / \mathbf{J}$ is essentially the reciprocal of the vapor blow-off velocity prior to the time when self-shielding starts.

At intensities of about $3 \mathrm{GW} / \mathrm{cm}^{2}$, there is sufficient modification of the density profile. Near the plasmavacuum boundary, there is a region of large gradients where the density rapidly decreases and the temperature sharply increases. There is essentially a laser absorption wave standing at the point of maximum absorption; the wave intercepts a major fraction of the incident radiation and transmits only a few percent to the target surface. The absorption wave results in a greatly reduced vaporization rate at the surface, and, hence, a sharply reduced coupling coefficient. The pressure associated with this absorption peak is so great that it eventually reverses the velocity of the blow-off vapor and drives it back toward the target. The vapor stagnates against the target and contributes a post-pulse impulse after the laser pulse terminates.

Thermal radiation transport plays no significant role in the laser-target interaction. Because of rapid expansion of the vapor near the point of peak absorption, the vapor on the vacuum side of the peak is optically thin. The vapor on the target side of the peak is dense enough to be optically thick, but is too cold to radiate energy to the target. Thermal radiation can be ignored because of this separation of regions which are optically thick from those which are hot enough to radiate strongly.

The plasma phenomenology for a range of fluxes of $248 \mathrm{~nm}$ light is illustrated in Figure 6.15. The position of the expanding electron density front is plotted as a

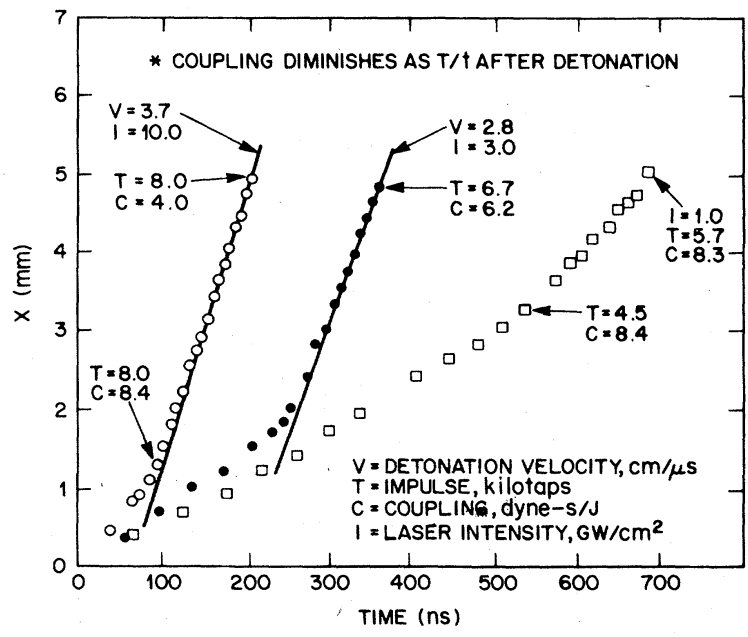

Figure 6.15. Distance of the electron density front in the plasma from the solid interface, as a function of irradiation time for several choices of impulse coupling and incident intensity at $\lambda=248 \mathrm{~nm}$ (after References 31 and 32). 
function of time. The front moves away from the target at an initial constant velocity that increases with increasing irradiance. After an interval of time (which decreases with increasing irradiance), the plasma front makes a transition to an abruptly increased velocity. The light on target terminates upon ignition of this detonation wave. The impulse delivered to the target ceases to increase and the coupling coefficient decreases as $t^{-1}$ for the remaining duration of the laser pulse. In short, the plasma begins to decouple the laser from the target when local absorption exceeds cooling. The adiabatic cooling rate of the expansion must be greater than the coupling constant times the irradiance,

$$
\alpha \mathrm{I}_{0}<-\rho \mathrm{C}_{\mathrm{p}} \mathrm{v}^{*} \frac{\mathrm{dT}}{\mathrm{dx}},
$$

where $\mathrm{v}^{*}$ is the blow-off velocity and $\alpha$ the absorption coefficient of the vapor for laser light. For inverse bremsstrahlung the absorption coefficient is given by ${ }^{18}$

$$
\alpha=\mathrm{A} \rho \mathrm{T} \exp \left(-\mathrm{E}_{\mathrm{i}} / \mathrm{T}\right)
$$

where $E_{i}$ the ionization potential and $T$ the temperature are measured in $\mathrm{eV}$. For aluminum $\mathrm{E}_{\mathrm{i}} \doteq 6 \mathrm{eV}$ and the constant $\mathrm{A}=3.2 \times 10^{7}(\mathrm{~h} v)^{-3}$. If one assumes that inverse bremsstrahlung is the dominant absorption mechanism and that the temperature profile follows from the Anisimov model with Saha equilibrium, the critical irradiance at which heating exceeds cooling is given by

$$
\mathrm{I}_{0} \approx(\mathrm{h} v)^{0.47} \mathrm{t}_{\mathrm{p}}^{-0.16} \mathrm{GW} / \mathrm{cm}^{2}
$$

where $\mathrm{h} v$ is the photon energy in $\mathrm{eV}$ and $\mathrm{t}_{\mathrm{p}}$ is the pulse duration in $\mu \mathrm{s}$. At irradiances above this value, energy continues to be coupled to the target, until a detonation wave is generated. The coupling constant $\mathrm{C}$ at these higher irradiances decreases from 4 or more to about 1 dyn $\mathrm{s} / \mathrm{J}$. It remains at this value up to irradiances of $10 \mathrm{GW} / \mathrm{cm}^{2}$ (at short wavelengths). This behavior has been confirmed by the experiments with the NOVA laser at LLNL. ${ }^{32}$

The same phenomenology occurs with longer wavelength radiation, as shown in Figure 6.16 for $3 \mu \mathrm{m}$ wavelength. However, since the inverse bremsstrahlung absorption coefficient scales as $\lambda^{3}$ for $T<$ few $e V$, plasma shielding occurs earlier in time than for short wavelength radiation.

The minimum diameter of a spot required for a onedimensional hydrocode can be determined from these figures by the relation $2 \mathrm{vt}_{\mathrm{p}}$. For $248 \mathrm{~nm}$ light at $0.5 \mu \mathrm{s}$, a 1-D spot has a $1-\mathrm{cm}$ diameter at $1 \mathrm{GW} / \mathrm{cm}^{2}$ and a $4-\mathrm{cm}$ diameter at $10 \mathrm{GW} / \mathrm{cm}^{2}$. For $3 \mu \mathrm{m}$ light at $2 \mu \mathrm{s}$, used in the experiments performed with the pulsed HF laser at the Air Force Weapons Laboratory, a spot of about

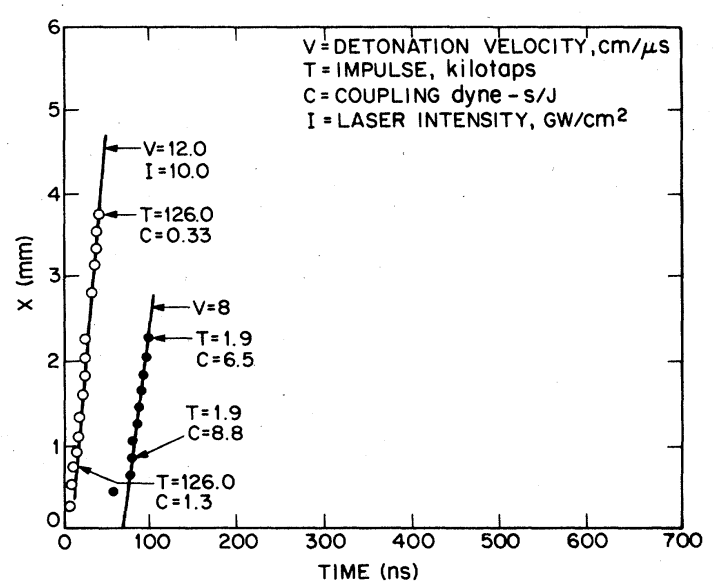

Figure 6.16. Distance of the electron density front in the plasma from the solid interface as a function of irradiation time for several choices of impulse coupling and incident intensity at $\lambda=3 \mu \mathrm{m}$ (after References 31 and 32).

$20 \mathrm{~cm}$ in diameter is large enough for a one-dimensional hydrocode to be applicable. Caution should be exercised that the intensity is sufficiently homogeneous, since the phenomena are highly nonlinear. One-dimensional experiments should be performed with intensities in the range $10^{8}-10^{10} \mathrm{~W} / \mathrm{cm}^{2}$ for various pulse durations. The scaling relations which fit both data and computer simulations are displayed in Table 6.2.

In summary, coupling coefficients for short wavelength lasers have recently been measured to be 4 to 5 dyn $\mathrm{s} / \mathrm{J}$ for irradiances $\mathrm{I}_{0}$ up to $4 \mathrm{GW} / \mathrm{cm}^{2}$ (and fluences up to $2 \mathrm{~kJ} / \mathrm{cm}^{2}$ ). At $2 \mathrm{~kJ} / \mathrm{cm}^{2}$ impulse loading is about 8000 dyn $\mathrm{s} / \mathrm{cm}^{2}$, or $8 \mathrm{ktap}$. The pressure generated at the target surfaces is $\mathrm{P}_{\mathrm{s}}=\mathrm{CI}_{0}$, or about $20 \mathrm{kbars}$. The parameters agree with theoretical calculations for one-dimensional spot sizes. The one-dimensional condition was fulfilled for at least one-half of the pulse duration.

Previous Soviet experiments ${ }^{1}$ at lower flux levels have shown deposition up to $50 \mathrm{~kJ} / \mathrm{cm}^{2}$ with a conservative coupling coefficient of 2 dyn s/J, which was experimentally verified by metal removal from the target. This corresponds to an impulse of $100 \mathrm{ktap}$.

The foregoing considerations, reinforced by experimental data, would favor relatively long pulses of short wavelength to fulfill lethality requirements. For a representative set of engagement parameters, $25 \mathrm{MJ}$ of laser energy deposited in $500 \mu \mathrm{s}$ on a spot area of $1 \mathrm{~m}^{2}$ corresponds to an irradiance of $5 \mathrm{MW} / \mathrm{cm}^{2}$, well below. the threshold for decoupling. With a coupling constant of 4 , the impulse would be $20 \mathrm{ktap}$.

There are several points for which more detailed experimental investigation is desirable:

(a) One-dimensional versus three-dimensional effects must be examined and compared with experiment 
TABLE 6.2. Scaling relations for laser decoupling from target.

\begin{tabular}{|c|c|}
\hline - Critical flux & $\phi_{\mathrm{C}}=\lambda^{-1 / 2} \tau^{-1 / 6}$ \\
\hline - Maximum fluence at $\phi_{\mathrm{C}}$ & $\mathrm{F}_{\max } \approx \tau \lambda^{-1 / 2}$ \\
\hline $\begin{array}{l}\text { - Energy required to deposit } \\
\mathrm{F}_{\max } \text { with 1-D spot }\end{array}$ & $\begin{array}{l}\mathrm{E}=\mathrm{F}_{\max } \pi \mathrm{d}^{2} / 4=\tau^{3} \lambda^{-1 / 2} \text { where } \mathrm{d}=2 \mathrm{v} \tau \\
\text { and } \mathrm{v}=\phi^{3 / 5} / 2\end{array}$ \\
\hline \multirow[t]{6}{*}{ - Units } & $\phi_{\mathrm{C}}\left(\mathrm{GW} / \mathrm{cm}^{2}\right)$ \\
\hline & $\tau(\mu \mathrm{s})$ \\
\hline & $\lambda(\mu \mathrm{m})$ \\
\hline & $\mathrm{v}(\mathrm{cm} / \mu \mathrm{s})$ \\
\hline & $\mathrm{d}(\mathrm{cm})$ \\
\hline & $\mathrm{F}\left(\mathrm{J} / \mathrm{cm}^{2}\right)$ \\
\hline
\end{tabular}

so that reasonable extrapolations to higher intensities can be made.

(b) Determination of the role of wavelength on the coupling coefficient must be made more precise.

(c) Systematic variation of the pulse duration must be investigated.

(d) Response of more complex target materials must be examined in more detail.

The NOVA experiments now underway will address some of these points. Current information permits the conclusion that the impulse coupling coefficient is known within a factor of 2-3 for most situations of interest.

\subsubsection{Multiple Pulse Cumulative Fluence}

One method for increasing the net impulse, and also for maximizing mechanical damage, is to hit the target with a sequence of pulses over a few microseconds. Serial pulsing with a few hundred nanoseconds interpulse separation allows the vapor to cool sufficiently between pulses to permit large cumulative impulse delivery.

The total impulse delivered and the coupling coefficient should be improved through sequentially generated laser pulses. As a target is irradiated with a few $\mathrm{GW} / \mathrm{cm}^{2}$, vapor is launched from the surface at about $0.1 \mathrm{~g} / \mathrm{cm}^{3}$. The vapor drifts away from the target at a velocity between 0.5 and $1 \mathrm{~cm} / \mu \mathrm{s}$. As described above, the vapor front becomes absorptive through increasing ionization by a bootstrap mechanism, its temperature increases to several electron volts; and the vapor detonates. The detonation wave separates from the target with a velocity of several $\mathrm{cm} / \mu \mathrm{s}$; absorption is sufficiently high at this time that no light reaches the target surface. Laser light absorption is divided between the detonation front and the vapor region between the front and the target.

The intervening vapor region decreases in density as the detonation front departs. The cool vapor eventually becomes sufficiently rarefied that it is no longer absorptive. Light once again irradiates the target surface causing additional vaporization and adds to the cumulative impulse. This process is oscillatory and is illustrated in Figure 6.17.

Since light is lost and the coupling coefficient

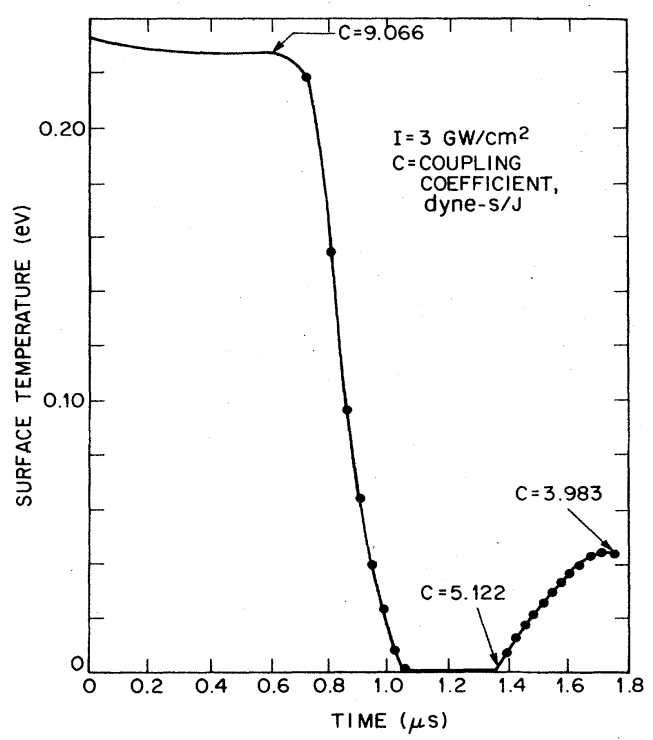

Figure 6.17. Variation of aluminum surface temperature due to plasma shielding for an incident pulse of $1.8 \mu \mathrm{s}$ duration with peak intensity of $3 \mathrm{GW} / \mathrm{cm}^{2}$ at $\lambda=308 \mathrm{~nm}$ (after Reference 31 ). 
TABLE 6.3. Laser-induced breakdown in air.

\begin{tabular}{|c|c|c|c|c|c|c|}
\hline Laser & $\lambda(\mu \mathrm{m})$ & $\mathrm{I}\left(\mathrm{W} / \mathrm{cm}^{2}\right)$ & $\mathbf{P}(\mathbf{T})$ & Spot size (mm) & $t_{p}(n s)$ & Ref. \\
\hline Ruby & 0.6943 & $2.2 \times 10^{11}$ & 760 & $43 \times 31$ ellipse & 40 & 1 \\
\hline Ruby & 0.6943 & $1.7 \times 10^{11}$ & 1000 & $D=70$ & 20 & 2 \\
\hline Nd:glass & 1.06 & $7.0 \times 10^{10}$ & 760 & $130 \times 34$ ellipse & 40 & 1 \\
\hline Nd:glass & 1.06 & $1.0 \times 10^{11}$ & 1000 & $D=70$ & 50 & 2 \\
\hline $\mathrm{CO}_{2}$ & 10.591 & $3.0 \times 10^{9}$ & 760 & $D=100$ & 200 & 3 \\
\hline
\end{tabular}

${ }^{1}$ R. G. Tomlinson, E. K. Damon, and H. T. Buscher, "The Breakdown of Noble and Atmospheric Gases by Ruby and Neodymium Laser Pulses," Physics of Quantum Electronics, edited by P. L. Kelly, M. Lax, P. E. Tannenwald (McGraw-Hill, New York, 1966).

${ }^{2}$ A. F. Haught, R. F. Meyerand, and D. C. Smith, "Electrical Breakdown of Gases by Optical Frequency Radiation," Physics of Quantum Electronics, edited by P. L. Kelly, M. Lax, P. E. Tannenwald (McGraw-Hill, New York, 1966).

${ }^{3}$ D. C. Smith, P. J. Berger, R. T. Brown, and M. C. Fowler, "Investigation of Gas Breakdown with 10.6 Micron Wavelength Radiation," United Technologies Research Center, Technical Report, February, AFWL-TR-72-182 (1972).

decreases following plasma detonation, the cumulative impulse may be increased by delivering a series of short pulses instead of a single long pulse. The analysis indicates that the individual pulses should each be a few hundred nanoseconds long.

\subsubsection{Interaction in the Atmosphere}

The coupling of pulsed laser energy to materials in an atmosphere is caused by the development of a blast wave generated by absorption. The intensity of laser energy delivered to the target in the atmosphere is limited by the atmospheric breakdown threshold. These limits are shown for various wavelengths at 1 atm in Tables 6.2 and 6.3 and as a function of pressure (altitude) in Figure 6.18. These limits indicate the upper bound of intensity of radiation that can be focused on a target in the atmosphere. The pressure delivered to the surface can be represented by a laser supported detonation wave at high intensities and a combustion wave just above the plasma ignition threshold. ${ }^{37,38}$ This interaction is shown schematically in Figure 6.19. Although the plasma ignition is a result of surface imperfections and is, therefore, wavelength and material sensitive, the resulting interaction with air and the impulse coupling coefficient is much less sensitive to these parameters. The pressuretime history is given by gas dynamics and the impulse is then the time integral of the pressure. For most engineering materials, the coupling in the atmosphere is

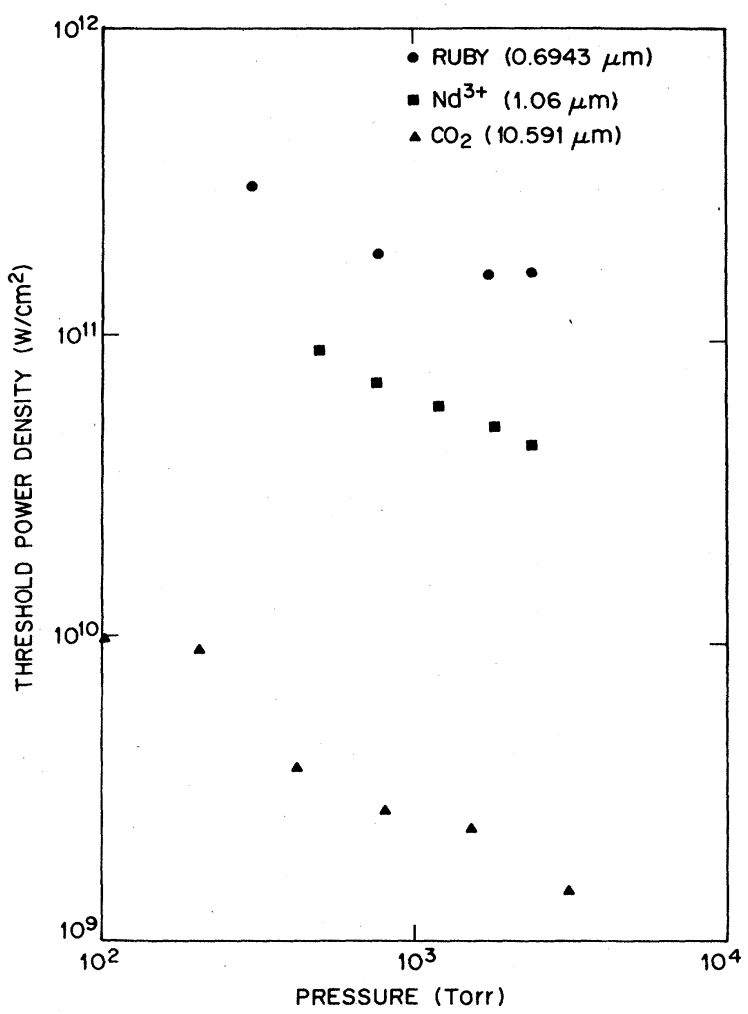

Figure 6.18. Power density threshold for air breakdown as a function of atmospheric pressure (from references listed in Table 6.3). 
- ATMOSPHERIC INTERACTION

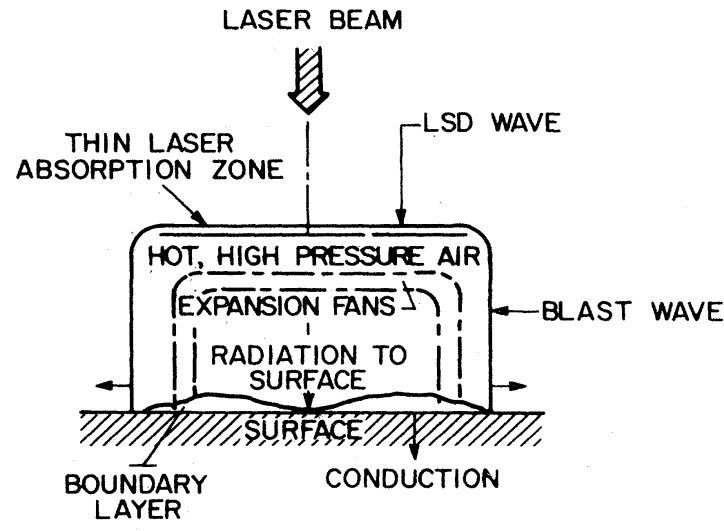

Figure 6.19. Schematic of the physical mechanism controlling the interaction of a laser pulse with a solid interface in the atmosphere (after Reference 12).

similar to that of aluminum which is shown in Figure 6.20. Existing data are available for the vacuum region and at $1 \mathrm{~atm}$. However, there is still significant uncertainty in the shape of the curve between $1 \mathrm{~atm}$ and vacuum. Gas dynamic coupling of the laser to the material increases the specific impulse to the surface. For the aluminum case shown, this increase is approximately a factor of four. As shown in Figure 6.21, gas dynamic models can predict impulse in the atmosphere. ${ }^{22}$

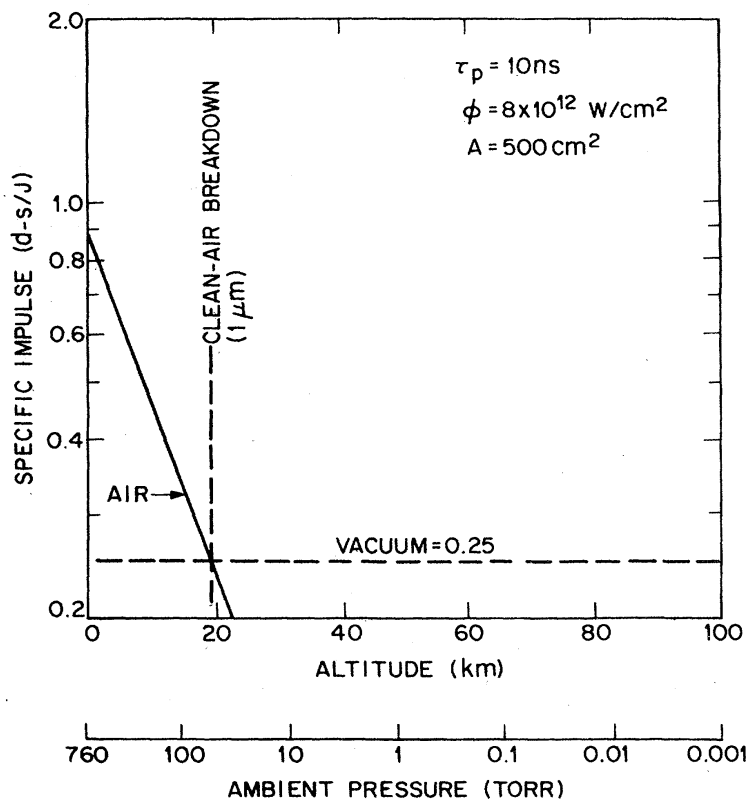

Figure 6.20. The effect of atmospheric pressure in the impulse coupling coefficient (after Reference 20).

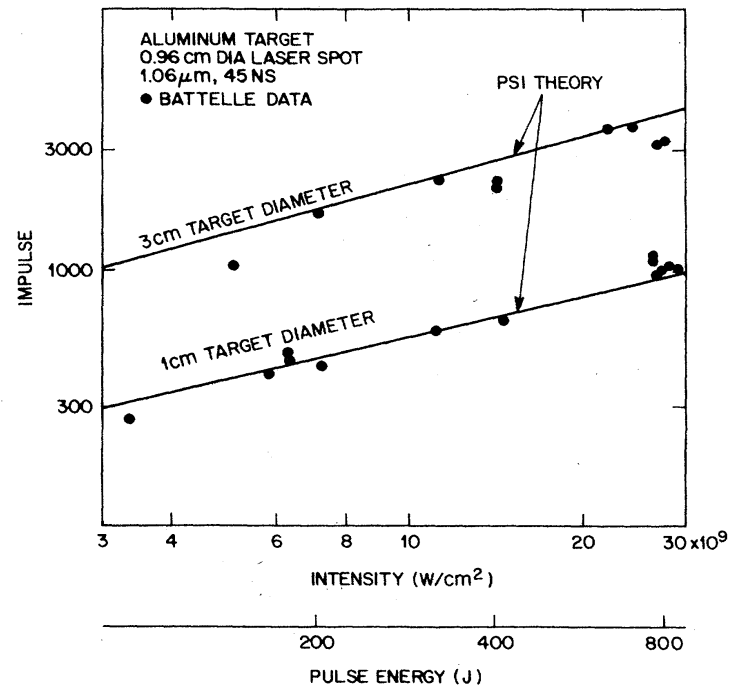

Figure 6.21. Comparison of theory and experiment for impulse coupling of an aluminum target in air as a function of incident intensity of a 45 ns pulse at $1.06 \mu \mathrm{m}$ wavelength (after Reference 20).

\subsubsection{Impulse Generation by X Rays}

A short pulse of $\mathrm{x}$ rays is absorbed in a layer of thickness a of material with a mass density $\rho$. There is no time for significant heat conduction into the interior. The layer is vaporized. Assume that the energy of vaporization is small compared to the energy deposited, and that the latter is equipartitioned into thermal energy and kinetic energy of the blown-off vapor. This kinetic energy per unit area is $\frac{1}{2} \rho a v^{2}=\frac{1}{2} F_{0}$, where $F_{0}$ is the fluence in the incident pulse. The corresponding impulse is $I_{p}=\rho \mathrm{av}=\left(\mathrm{F}_{0} \rho \mathrm{a}\right)^{1 / 2}=\left(\mathrm{F}_{0} / \mathrm{k}\right)^{1 / 2}$. Here we have introduced the opacity $\mathrm{k}=(\rho \mathrm{a})^{-1}$, in units of area/mass. This oversimplified picture has assumed that the energy deposition was uniform over the penetration depth a and zero elsewhere. It may be improved by taking account of the fact that the fluence inside the surface decays,

$$
\mathrm{F} \sim \mathrm{F}_{0} \mathrm{e}^{-\mathrm{k} \Sigma},
$$

where $\Sigma$ is the column density (mass/area) from the surface to an interior point. The impulse (momentum/area) generated may be approximated as arising from the energy deposited in each interior layer of the target

$$
\mathrm{I}_{\rho}=\int \mathrm{vd} \Sigma
$$

and

$$
\mathrm{v}^{2} \sim \mathrm{dF} / \mathrm{d} \Sigma,
$$


where $\mathrm{v}$ is the velocity of the expanding material from a particular layer. Equation (6.25) assumes an equipartition between thermal energy and the kinetic energy of the expansion of the gas and ignores the energy lost to vaporization and the transfer of energy between layers.

The integral indicated in Equation (6.24) may be performed explicitly, assuming that the opacity of the target is constant in Equation (6.23) (actually, it changes as the material is heated). We obtain for the impulse $\mathrm{e}^{39}$

$$
\begin{aligned}
\mathrm{I}_{\mathrm{p}} \lesssim 2\left(\frac{\mathrm{F}_{0}}{\mathrm{k}}\right)^{1 / 2} \sim & 2 \operatorname{ktap}\left(\frac{\mathrm{F}_{0}}{\mathrm{~kJ} / \mathrm{cm}^{2}}\right)^{1 / 2} \\
& \times\left(\frac{\mathrm{k}}{10^{4} \mathrm{~cm}^{2} / \mathrm{g}}\right)^{-1 / 2}
\end{aligned}
$$

$[\operatorname{tap} \equiv \mathrm{g} /(\mathrm{cm} \mathrm{s})]$. The baseline opacity of $10^{4} \mathrm{~cm}^{2} / \mathrm{g}$ is characteristic of many materials for soft $\mathrm{x}$-ray photon energies in the vicinity of $1 \mathrm{keV}$, as shown below. Thus, the primary determinants of the impulse generation are the fluence and the opacity, at least in regions of the target where the energy deposition greatly exceeds the vaporization energy $\epsilon$ (energy/mass) of the material. This condition is valid at the surface, if the incident fluence satisfies the inequality,

$\mathrm{F}_{0} \gg \epsilon / \mathrm{k} \sim \frac{1 \mathrm{~J}}{\mathrm{~cm}^{2}}\left[\frac{\epsilon}{10 \mathrm{~kJ} / \mathrm{g}}\right)\left(\frac{\mathrm{k}}{10^{4} \mathrm{~cm}^{2} / \mathrm{g}}\right)^{-1}$.

Thus, for structural damage to targets, typically requiring $1-10 \mathrm{~kJ} / \mathrm{cm}^{2}$, the assumption of negligible effects of vaporization energy is quite good. Indeed, detailed hydrodynamics calculations for $\mathrm{Al}$ and $\mathrm{Au}$ for fluences above $1 \mathrm{~kJ} / \mathrm{cm}^{2}$ are in good agreement $(\sim 10 \%)$ with Equation (6.26) which involves the approximations mentioned.

The opacities of a wide variety of elements as a function of photon energy are shown in Figure 6.22. As the figure shows, individual opacities change significantly at energies corresponding to $\mathrm{K}$-edges of the elements. However, if we make the defense-conservative assumption that the target has been protected against the $\mathrm{x}$-ray laser with a surface coating which has a high opacity at the $\mathrm{x}$ ray energies, the curves of Figure 6.22 show that

$$
\mathrm{k} \leqslant 10^{4} \mathrm{~cm}^{2} / \mathrm{g}(\mathrm{h} v / \mathrm{keV})^{-3 / 2}
$$

Thus, although $\mathrm{k} \propto v^{-3}$ is a familiar result for light elements between edges (e.g., $Z=6.220$ in Figure 6.22), the presence of edges and the assumption that the right elements will be chosen to counter the XRL imply a weaker dependence on photon energy.

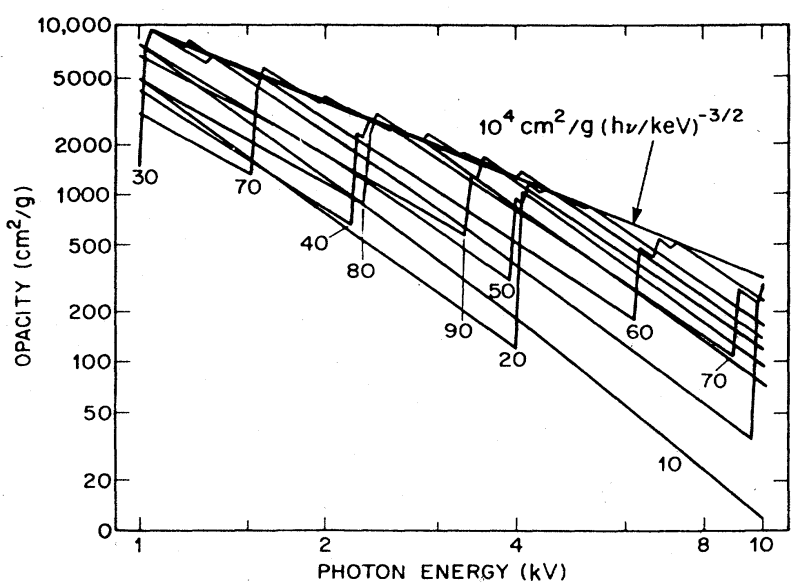

Figure 6.22. X-ray opacities for various elements (curve labels correspond to atomic number $\mathrm{Z}$ ) as a function of photon energy (courtesy: R. Cunningham).

With this approximation for the opacity of an impulse-reducing coating, Equation (6.26) may be solved for the fluence $F_{0}$ required for an impulse $I_{p}$

$\mathrm{F}_{0} \sim 0.25 \mathrm{~kJ} / \mathrm{cm}^{2}\left(\mathrm{I}_{\mathrm{p}} / \mathrm{ktap}\right)^{2}(\mathrm{~h} v / \mathrm{keV})^{+3 / 2}$

This equation demonstrates that higher energy photons are more effective in producing impulse.

If the target surface were not coated to reduce impulse generation, Figure 6.22 shows that its opacity might be lower than that predicted by Equation (6.28) by a factor of 5 or more, with a proportional decrease in the fluence required to produce a given impulse.

\subsubsection{Structural Damage from Impulse Loading}

Typical generic structural characteristics of the various strategic system components are shown in Figure 6.23. There are many potential damage modes to all targets. The criterion for a lethal attack is that the system is denied its mission. Most structures are complex with many different structural components composed of aluminum alloys, composite materials, and (potentially) hardening materials designed to disperse the impulsive load. The structure may be damaged through material failure, the ejection of spall fragments that result in interior damage, buckling of the cylinder, and blowout or bursting of fuel tanks. In addition to spall, the structure may be damaged by large deformation and strains resulting from the high impulsive load or the high strain rates. Boosters may also be subject to high flight loads, which add to the stress caused by the impulsive load. Many experiments simulating the effect of laser-induced impulse may be conducted using flyer plate, gas gun, or 
TARGET STRUCTURAL CHARACTERISTICS
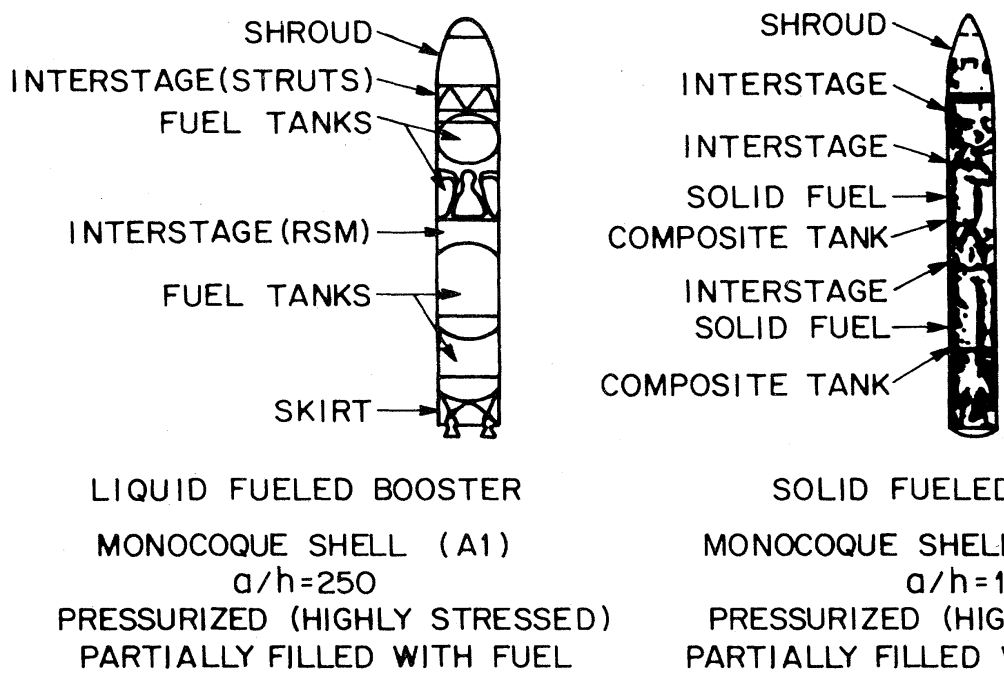

LIQUID FUELED BOOSTER

SOLID FUELED BOOSTER

MONOCOQUE SHELL (COMPOSITE)

$a / h=100$

PRESSURIZED (HIGHLY STRESSED)

PARTIALLY FILLED WITH SOLID FUEL
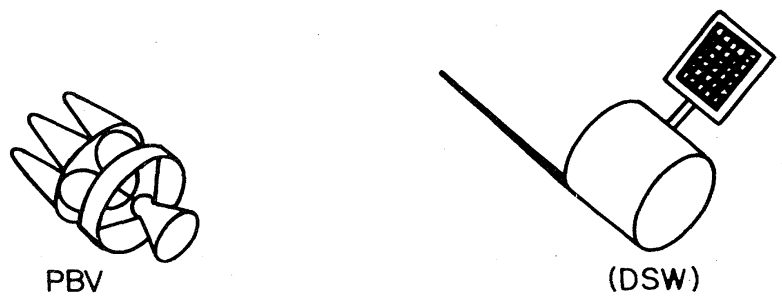

MANY STRUCTURAL OPTIONS BUT MUST HAVE COMPONENTS SUCH AS FUEL TANKS, ROCKET MOTOR, GUIDANCE SYSTEM, ełc.

THIN SHELL $a / h=100-300$

MAY BE PRESSURIZED

OTHER STRUCTURAL OPTIONS AND COMPONENTS POSSIBLE

Figure 6.23. Typical target structures encountered in strategic defense.

high explosive techniques. This is true since the major difference between these loadings occurs only at the coupling surface. The response of the structure is independent of the method of impulsive loading if the loading time is short compared to the structural response time and if there is no mass penetration.

Failure of the generic structures shown in Figure 6.23 typically requires impulse loading on the order of a few tens of ktap. As mentioned in Section 6.3.1, a loading of 20 kilotaps can be produced by a flux of $2.5 \mathrm{~kJ} \mathrm{~cm}^{-2}$, if the coupling constant $\mathrm{C}$ is 4 . This represents a reasonable lower limit. We take as a typical damage value 40 kilotaps, or $5 \mathrm{~kJ} \mathrm{~cm}^{-2}$. For a beam $1 \mathrm{~m}$ in diameter on the target, this will require about $40 \mathrm{MJ}$ of deposited energy.

Hardening and countermeasures for pulsed lasers are much more difficult than for cw lasers, since there is no way one can reduce the momentum imparted to the target. Some tricks have been proposed to use shields such as shown in Figure 6.24 to deflect the applied momentum from the missile surface. This hardening technique, however, is vulnerable to second shots which would then deliver impulse loads directly to the missile structure. Therefore, it is much more difficult to countermeasure a pulsed laser than a cw laser.

Most characteristic targets can be represented by hollow cylindrical structures. Rough estimates for the fluences required to damage thin plates through impulse loading are derived in this section. This treatment follows work done by Canavan. ${ }^{40} \mathrm{~A}$ fluence $\mathrm{F}$ delivered for a coupling coefficient $\mathrm{C}$ generates an impulse $\mathrm{CF}$, producing a plate velocity of

$$
\mathrm{V}=\mathrm{CF} / \rho_{0} \mathrm{~d}
$$

where $\rho_{0}$ is the density and $\mathrm{d}$ the thickness of the plate. This produces a kinetic energy $\mathrm{K}$ per unit area such that 

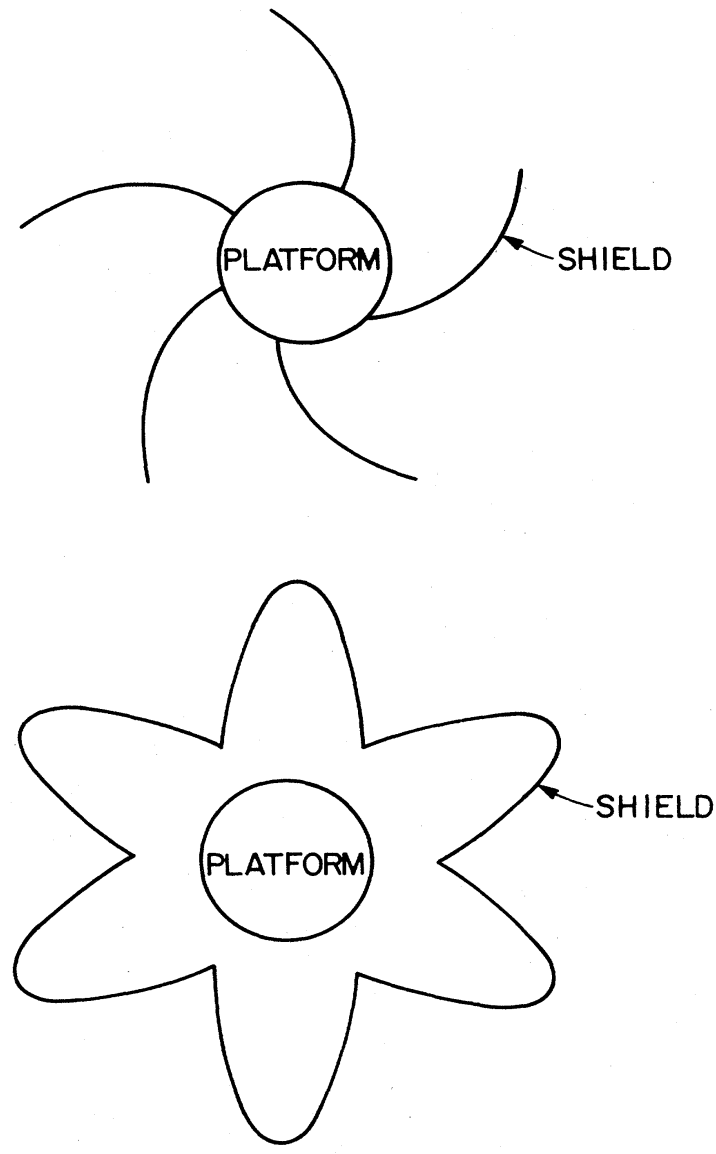

Figure 6.24. Schematic configurations of lightweight shields to protect target from momentum transfer by incident radiation pulse.

$$
\mathrm{K}=\frac{\left(\rho_{0} \mathrm{dV}\right)^{2}}{2 \rho_{0} \mathrm{~d}}=\frac{\mathrm{C}^{2} \mathrm{~F}^{2}}{2 \rho_{0} \mathrm{~d}}
$$

To strain the plate to failure, this kinetic energy must exceed the energy (per unit area) required to rupture it,

$$
\mathrm{dS}=\mathrm{d} \int_{0}^{\sigma_{\mathrm{m}}} \sigma \mathrm{d} \epsilon \approx \sigma_{\mathrm{m}} \epsilon_{\mathrm{m}}
$$

where $\sigma_{\mathrm{m}}$ and $\epsilon_{\mathrm{m}}$ are the stress and strain, respectively, at rupture. For example, $\sigma_{\mathrm{m}} \approx 3 \mathrm{kbars}$ and $\epsilon_{\mathrm{m}}=0.15$ for aluminum so $S \approx 50 \mathrm{~J} / \mathrm{cm}^{3}$. This is, perhaps, an overly conservative estimate, since the plate rupture will most likely require that $K$ exceed $S d$ near the edge of the spot and not over the full spot area. The requirement that the plate energy exceeds $\mathrm{Sd}$ means that

$$
\begin{aligned}
\mathrm{V} \geq \sqrt{2 \mathrm{~S} / \rho_{0}} & \approx \sqrt{(2)\left(5 \times 10^{8} \mathrm{erg} / \mathrm{cm}^{3}\right) /\left(3 \mathrm{~g} / \mathrm{cm}^{3}\right)} \\
& \approx 2 \times 10^{4} \mathrm{~cm} / \mathrm{s} .
\end{aligned}
$$

The corresponding fluence to rupture is

$$
\mathrm{F}=\left(\sqrt{\left.2 \rho_{0} \mathrm{dK}\right)} / \mathrm{C}=\mathrm{V} \rho_{0} \mathrm{~d} / \mathrm{C} \approx \mathrm{d}(\sqrt{2 \rho \mathrm{S})} / \mathrm{C} .\right.
$$

For a $0.3 \mathrm{~cm}$ thick plate $\left(1 \mathrm{~g} / \mathrm{cm}^{2}\right)$ and a coupling coefficient of $\mathrm{C}=3 \mathrm{dyn} \mathrm{s} / \mathrm{J}$,

$$
\begin{aligned}
\mathbf{F} & \geq(0.3)(2)\left(5 \times 10^{8} \mathrm{erg} / \mathrm{cm}^{3}\right)\left(3 \mathrm{~g} / \mathrm{cm}^{3}\right) / 3 \\
& \sim 5 \mathrm{~kJ} / \mathrm{cm}^{2}
\end{aligned}
$$

which is a modest fluence. It is smaller than that required for material removal, which determines the damage threshold in the cw regime.

For this one-dimensional estimate to be valid, the plate must be strained by at least its own thickness before the stress can relieve radially, or

$$
\frac{\mathrm{d}}{\mathrm{V}} \leq \frac{\mathrm{R}}{\mathrm{c}_{\mathrm{s}}}
$$

where $\mathrm{R}$ is the spot radius and $\mathrm{c}_{\mathrm{s}}$ is the speed of sound in the plate. The sound speed is given by

$$
\mathrm{c}_{\mathrm{s}}=\sqrt{\sigma_{0} / \rho_{0}}
$$

where $\sigma_{0} \approx 10^{12} \mathrm{erg} / \mathrm{cm}^{3}$ is the bulk modulus of the plate material. The required 1-D radius $R$ is

$$
\begin{aligned}
\mathbf{R} \geq \frac{\mathrm{dc}}{\mathrm{V}}=\mathrm{d} \frac{\sqrt{\sigma_{0} / \rho_{0}}}{\sqrt{2 \sigma_{\mathrm{m}} \epsilon_{\mathrm{m}} / \rho_{0}}} & =\mathrm{d} \sqrt{\sigma_{0} / 2_{\mathrm{m}} \sigma_{\mathrm{m}} \epsilon} \\
& \approx 10 \mathrm{~cm} .
\end{aligned}
$$

Thus, if enough fluence is delivered to rupture the plate in the first place, the spot sizes required for onedimensionality from the structural failure modeling point of view are modest.

It has been suggested that plates separated physically from the main target might be used to spread the pressure pulse in time. In fact, such plates would remain solid and be accelerated to velocities at which they would serve as useful flyer plates to shock or spall the main target. If wrinkled, the plates might generate random high velocity jets which could penetrate the target even more effectively. The applied momentum could perhaps be deflected by thin sheets in a configuration shown in Figure 6.24. The material tends to blow away normal to the surface. These theoretical conjectures also require experimental confirmation at $1-3 \mathrm{~cm}$ spot sizes in the laboratory. 
The required visible beam fluence for lethality varies roughly over a range from $2.5 \mathrm{~kJ} \mathrm{~cm}^{-2}(\mathrm{C}=4,20$ kilotaps $)$ to $20 \mathrm{~kJ} \mathrm{~cm}^{-2}(\mathrm{C}=1$, 40 kilotaps $)$. The required $\mathrm{x}$-ray fluence to produce the same specific impulse follows from Equation (6.29). For $1 \mathrm{keV} x$ rays $F>1.5 \mathrm{~kJ} / \mathrm{cm}^{2}$ is required. Thus, the required fluence for damage by an $x-$ ray pulse of photon energy somewhat lower than $1 \mathrm{keV}$ can be a factor of two to ten smaller than for a visible laser pulse.

Since for both pulses the energy is initially deposited in a thin layer, large temperature gradients and stress gradients are created in a very short time. This will lead to the creation of shock waves. This may cause damage by spallation at voids, internal cracks, or by reflection at the rear surface. Spallation in the NOVA experiments occurred at $10^{14} \mathrm{~W} / \mathrm{cm}^{2}$, or $10^{5} \mathrm{~J} / \mathrm{cm}^{2}$. Such type of damage may be minimized by inserting porous layers between the absorbing surface and the load-bearing shell. The stress due to shock waves can be attenuated, but the transfer of momentum cannot be eliminated.

\subsection{PARTICLE BEAM LETHALITY}

Energetic particle beams have great penetrating power, depositing energy deeply within a target. As a result, particle beam lethality criteria are usually expressed in terms of energy deposited per unit mass, rather than beam energy fluence at the target surface. Several kill mechanisms are possible ranging from thermal structural failure to dose and dose rate induced electronics damage. In this section we summarize the interactions of energetic charged particles with matter and present several lethality criteria associated with various damage mechanisms for energetic neutral particle beams and electron beams.

\subsubsection{Beam Interaction Summary}

A neutral particle beam (e.g., atoms of hydrogen or its isotopes) is almost instantaneously ionized after entering a target. The resulting charged nuclei $\left(\mathrm{p}^{+}, \mathrm{d}^{+}, \mathrm{t}^{+}\right)$lose energy through three important mechanisms: Coulomb interactions with the electrons of the target atoms, and nuclear inelastic and elastic collisions. The penetration range, ${ }^{41}$ measured in units of $\mathrm{g} / \mathrm{cm}^{2}$ in order to be roughly material independent, is given approximately by

$$
\mathrm{R}\left[\frac{\mathrm{g}}{\mathrm{cm}^{2}}\right]=\left(3.31 \times 10^{-3}\right) \frac{\mathrm{E}^{1.74}}{\mathrm{Z}^{2} \mathrm{~A}^{0.74}}
$$

where $\mathrm{A}$ is the mass number, $\mathrm{Z}$ is the charge number, and $\mathrm{E}$ is the particle energy in $\mathrm{MeV}$. Hence, the range of a
$100-400 \mathrm{MeV}$ proton varies from about $4 \mathrm{~cm}$ to $41 \mathrm{~cm}$, respectively, in aluminum and from about $1.3 \mathrm{~cm}$ to $14 \mathrm{~cm}$ in iron, respectively. The range of a $100 \mathrm{MeV}$ to $400 \mathrm{MeV}$ triton is about $1.6 \mathrm{~cm}$ to $18 \mathrm{~cm}$ in aluminum, respectively. The rate of energy loss (the stopping power) for protons in aluminum is shown in Figure 6.25 for several different proton kinetic energies. The effects of the enhancement in the energy deposition rate near the end of the particle range (the Bragg peak) will be significantly modified for exposure times greater than a few milliseconds because of thermal conduction processes.

The important energy loss mechanisms for energetic electrons are collisional excitation and ionization processes and radiative interactions (bremsstrahlung). The ionization energy loss per unit length scales linearly with the atomic density and atomic number of the target atoms, and weakly (logarithmically) with the beam kinetic energy. A useful approximate (empirical) expression is ${ }^{41}$

$$
\frac{1}{\rho}\left(\frac{\mathrm{dE}}{\mathrm{dx}}\right]_{\text {ion }} \simeq 1.5 \mathrm{MeV} \mathrm{\textrm {cm } ^ { 2 } / \mathrm { g }}
$$

where $\rho$ is the density of the target material. In contrast, the energy loss due to bremsstrahlung is proportional to

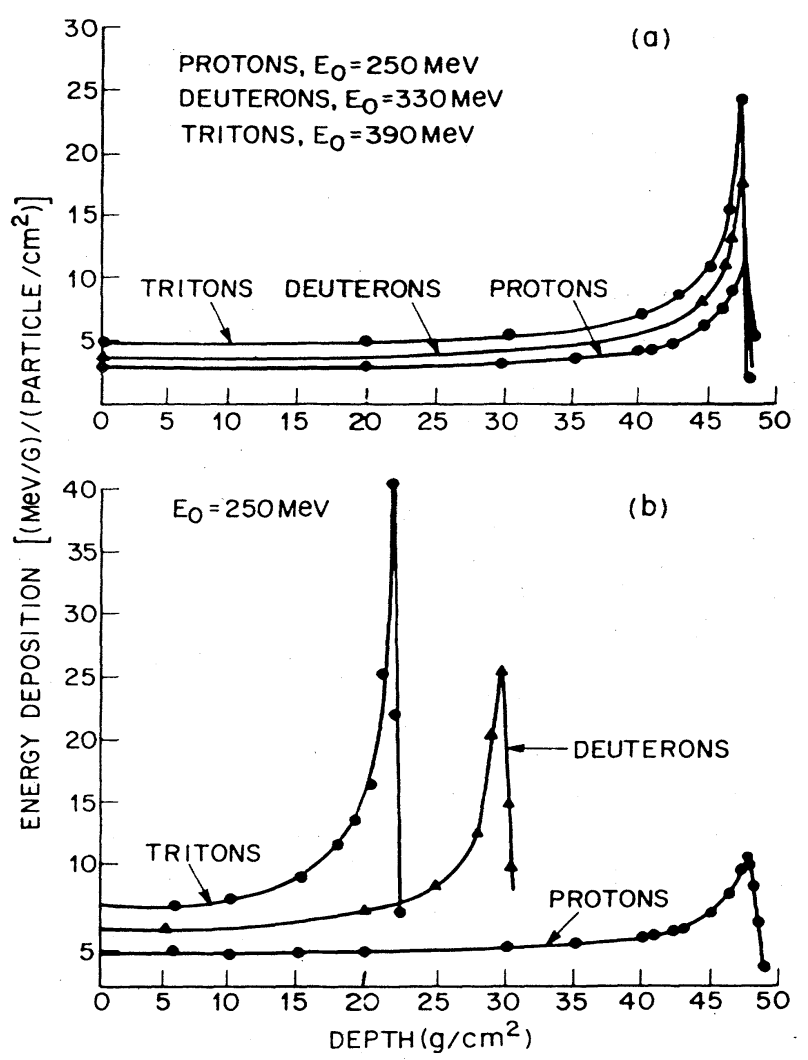

Figure 6.25. Typical energy deposition curves for hydrogenic particle beams. 
the square of the atomic number and increases nearly linearly with the kinetic energy of the incident electron. The ratio of the radiative loss to the ionization loss is given approximately by ${ }^{41}$

$$
\frac{(\mathrm{dE} / \mathrm{dx})_{\mathrm{rad}}}{(\mathrm{dE} / \mathrm{dx})_{\text {ion }}}=\frac{\mathrm{E}(\mathrm{MeV}) \mathrm{Z}}{800}
$$

Hence, the range of a $20 \mathrm{MeV}$ to $100 \mathrm{MeV}$ electron in aluminum is about $4 \mathrm{~cm}$ to $16 \mathrm{~cm}$, while in tungsten the range is about $0.5 \mathrm{~cm}$ to $2.5 \mathrm{~cm}$. In the first case at the low energy end bremsstrahlung is practically negligible and almost all the energy is dissipated locally; in the latter case at the high energy end, the ionization and radiative loss rates are initially equal and a substantial portion of the electron energy is not deposited locally.

\subsubsection{Lethality Mechanisms}

With in-depth energy deposition a variety of particle beam kill mechanisms are possible, including structural breakup, high explosive detonation, and propellant ignition, as well as dose and dose rate kill of electronic components and systems. In this section we discuss several of these mechanisms and summarize the various lethality criteria.

The energy deposition required to produce structural failure will depend on the heats of melting and vaporization of the various target materials. These are summarized for several important materials in Table 6.1. For example, deposition of approximately $500 \mathrm{~J} / \mathrm{g}$ will cause aluminum to melt, while only $200 \mathrm{~J} / \mathrm{g}$ will ensure melting of uranium. Ignition of solid rocket propellants ${ }^{42}$. and initiation of high explosives also occur in the range of 200-400 J/g, depending somewhat on the energy deposition time (and the effects of thermal conduction processes). If sufficient beam energy is deposited in times short compared with thermal relaxation times (typically tens of microseconds) the materials will explosively vaporize with the generation of strong shock waves. Because of the physics associated with the various particle beam approaches (Chapter 4) this process is likely to occur for the relatively high current electron beam approach (kiloampere beams with microsecond deposition times), but is unlikely to occur with the NPB approach (100 mA beams with $1 \mathrm{~s}$ deposition times). At somewhat lower deposition levels, differential thermal expansion between low specific heat, high $\mathrm{Z}$ metals in contact with insulating plastics and glasses is expected to produce significant shear stresses that might fracture seals or rupture bonds.

The radiation doses and dose rates resulting from particle beam irradiation can produce electronics damage by a variety of mechanisms, ranging from single event upset (SEU) to burn-out. As is known from commercial satellite programs, the total dose damage typically occurs at several megarads in silicon. Circuit failures can result from degradation of transistor gain, as well as high junction leakage currents and breakdown voltages. High dose rates $\left(10^{11}-10^{12} \mathrm{rad} / \mathrm{s}\right)$ can disrupt the thermal equilibrium in semiconductors by the generation of holeelectron pairs; the resulting currents can produce junction burn-out.

Other mechanisms, such as system-generated EMP (SGEMP), formation of parasitic pnpn structures (latchup), and logic upset resulting from recoiling atomic collisions (SEU) can occur at dose and dose rates orders of magnitude lower than the levels quoted above. However, system lethality levels will depend strongly on the design approach chosen by the offense, i.e., use of circumvention, redundant memories, etc. In addition, the effect produced by the electronics disruption mechanism may or may not be observable, depending on the particular time of irradiation during the missile flight. For example, erratic trajectories resulting from disruption of the booster guidance electronics or the bus sequencing computer could be observed; however, burn-out of an RV's electronic fire set cannot be verified during its flight.

\subsubsection{Lethality Criteria}

Energetic particle beams deposit energy deeply within a target; hence, lethality criteria are usually expressed in terms of energy deposited per unit target mass, or radiation dose. A variety of kill mechanisms are possible, ranging from single event upset to structural melt. The thresholds for these several mechanisms (the lethality criteria) can be represented as lines in the twodimensional space described by the total specific energy deposition and the deposition rate, as indicated in Figure 6.26 (see Rose $^{43}$ for details).

As a hypothetical example, consider a $100 \mathrm{MeV}$ to $400 \mathrm{MeV}, 0.1 \mathrm{~A}$ proton beam with a divergence angle of about $1 \mu \mathrm{rad}$ (see Section 4.3.1). At a distance of 1000 $\mathrm{km}$, the beam radius will be about $1 \mathrm{~m}$. Since the particle range in the target is about $10-111 \mathrm{~g} / \mathrm{cm}^{2}$, the specific energy deposition rate in the target is about 3 to $40 \mathrm{~J} / \mathrm{g} \mathrm{s}$.

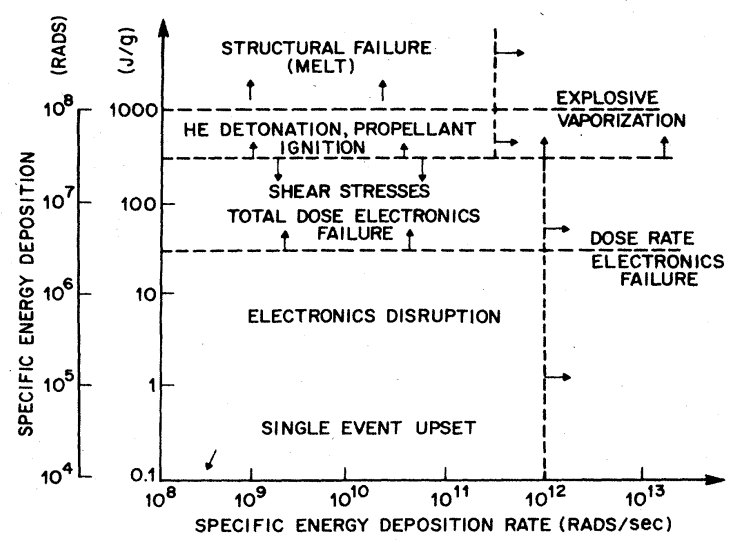

Figure 6.26. Lethality criteria for particle beams [see text and Rose (Reference 43)]. 
At these energy depositions, structural damage will not occur but electronic malfunction is likely. Hence, under these conditions, serious electronic malfunctions can be anticipated for irradiation times of tens of milliseconds leading to total electronics failures in a few seconds, while structural failures can be expected for irradiation times of several tens of seconds. At higher beam energies the times needed for the above failures will be appropriately reduced, and can be calculated in a straightforward manner.

As a second example, consider a $100 \mathrm{MeV}, 2 \mathrm{kA}$ electron beam. From Chapter 4, the equilibrium beam radius in the ionization channel can be quite small (a few centimeters), although the beam front will erode (see Section 4.2.1.2) at a rate of about $0.8 \mu \mathrm{s}$ per $100 \mathrm{~km}$ of propagation distance. Assuming a beam diameter of $5 \mathrm{~cm}$ and sufficient pulse width for the beam to reach the target, the specific energy deposition rate will be about $2 \times 10^{8}(\mathrm{~J} / \mathrm{g}) / \mathrm{s}$. Hence, a target could be catastrophically destroyed in a beam irradiation time of a few microseconds. This rapid, verifiable lethality mechanism is a potential advantage for the charged particle beam approach.

\subsection{MICROWAVE LETHALITY}

The offense's ICBM carries a moderate amount of electronics in its interior, especially for its MIRV deployment during the post-boost phase. Electromagnetic pulses from lightning strokes or nuclear explosions have had profound effects on some pieces of electronic equipment. This raises the question whether the purposeful generation of strong bursts of microwave radiation by some strategic defense asset could provide an indirect kill mechanism by upsetting the electronics of the PBV. The data on this subject are contradictory. Electronic equipment which has not been designed with this problem in mind can be upset by microwave radiation at intensity levels which are many orders of magnitude smaller than that required to destroy the entire apparatus. On the other hand, careful design of electronic equipment and the container in which it is encased (including filters, TR switches, etc.) can make the equipment remarkably immune (assuming radiation levels well below melting threshold). This careful design is likely to cost the offense an undetermined weight penalty. Weapons generating intense microwave radiation are likely to have poor directivity and are likely to be noncompetitive with DEWs for delivery of energy per unit area on target. If laboratory tests confirm that effective microwave shielding at all relevant wavelengths can be accomplished for electronic components in the bus, microwaves do not constitute an attractive kill mechanism.

\subsection{CONCLUSIONS}

The discussion of lethality requirements may be summarized in the following set of conclusions.
1. Thermal structural kill of hardened targets by $\mathrm{cw}$ laser beams may require the deposition of 30-60 kJ per gram of ablative material. For a spot size on target of $30 \times 30 \mathrm{~cm}^{2}$ and a hardening by $1 \mathrm{~g} / \mathrm{cm}^{2}$ of ablative material, the energy delivered on target should exceed $3 \times 10^{7} \mathrm{~J}$. The needed DEW laser power is kill time dependent, for example, for a kill time of $1 \mathrm{~s}$, a minimum power of the DEW laser of $30 \mathrm{MW}$ is required. A kill time of $0.1 \mathrm{~s}$ will necessitate a DEW laser power of $300 \mathrm{MW}$. This number must be scaled up to compensate for optical propagation losses in the optical systems and in the atmosphere. For spot size on target of $1 \mathrm{~m}^{2}$, the power requirement is increased by an order of magnitude. It is, however, reduced in the case of unhardened booster skins.

2. Structural impulse kill by pulsed lasers should require about $5 \mathrm{~kJ} / \mathrm{cm}^{2}$ delivered on target for expected values of the coupling coefficients, valid for most materials. For a spot size of $1 \mathrm{~m}$, this requires $40 \mathrm{MJ}$ delivered to the target. The peak powers in the pulse must exceed $10^{12} \mathrm{~W}$. The numbers are for structures with typical thickness of $1 \mathrm{~cm}$. The lethality requirement scales about linearly with this dimension.

3. Impulse structural kill by pulses from soft $x$-ray lasers may require between a factor of two to ten less fluence delivered on the target than that required for lasers at visible or near-infrared wavelengths.

4. The central problem with pulsed lasers in the near IR is the decoupling by the plasma, which limits the impulse delivered to the target.

5. The basic mechanisms of structural kill by thermal or impulse loading are partially understood. Lethality assessment in boost and post-boost phase is possible by tracking of the trajectory. Hardening against repeated impulse loading presents unsolved problems.

6. For NPB weapons the kill mechanism that requires the lowest amount of energy is that involving the disabling of electronic components. For existing silicon technology used in spacecrafts a deposition of energy of about $10^{-2} \mathrm{~J} / \mathrm{g}$ is necessary according to current estimates, or a fluence of roughly 0.1-1.1 $\mathrm{J} / \mathrm{cm}^{2}$ for $100 \mathrm{MeV}$ to $400 \mathrm{MeV}$ protons, respectively. Shielding of electronics against 100 $\mathrm{MeV}$ to $400 \mathrm{MeV}$ protons would require 10-110 $\mathrm{g} / \mathrm{cm}^{2}$, respectively. Electronics kill assessment presents difficulties.

7. Thermal kill by NPB weapons favors the use of heavier particles with lower kinetic energy. The requirements for thermal kill are then similar to those for laser kill in terms of energy delivered. The threshold amount of $1 \mathrm{~kJ} / \mathrm{g}$ stresses the size of the NPB weapon for this purpose. 
8. Lethality of microwave pulses is uncertain. Careful shielding of all devices against microwave leakage appears feasible.

9. A continuing research program on materials properties, structures, mirrors, and sensors for hardening against pulsed and quasicontinuous irradiation, at high power flux densities over a very wide range of the electromagnetic spectrum, will permit improved assessment of lethality (and survivability) questions.

\section{REFERENCES}

${ }^{1}$ S. I. Anisimov, "Vaporization of Metal Absorbing Laser Radiation," Sov. Phys. JETP 27, 182 (1968).

${ }^{2}$ C. J. Knight, "Theoretical Modeling of Rapid Surface Vaporization with Back Pressure," AIAA Journal 17, 519 (1979).

${ }^{3}$ H. S. Carslaw and J. C. Jaeger, Conduction of Heat in Solids (Oxford University Press, 1947). [See especially Eq. (2.93) on p. 104.]

${ }^{4}$ See Reference 3, Eq. (7) on p. 56.

${ }^{5}$ D. I. Rosen, J. Mitteldorf, G. Kothandaraman, A. N. Pirri, and E. R. Pugh, "Coupling of pulsed $0.35 \mu \mathrm{m}$ laser radiation to aluminum alloys," J. Appl. Phys. 53, 3190 (1982).

${ }^{6}$ J. F. Ready, Effects of High-Power Laser Radiation (Academic, New York, 1971).

${ }^{7}$ T. P. Hughes, Plasmas and Laser Light (Wiley, New York, 1975).

${ }^{8}$ S. I. Anisimov, A. M. Prokhorov, and V. E. Fortov, Sov. Phys. Usp. 27, 181 (1984).

${ }^{9}$ Yu.P. Raî̌er, Sov. Phys. Usp. 87, 29 (1965); Sov. Phys. JETP 58, 2127 (1970); JETP Lett. 11 (1970).

${ }^{10}$ O. N. Krokhin, in Physics of High Energy Density, edited by P. Caldirola and H. Knoepfel (Academic, New York, 1971), pp. 278-305.

${ }^{11}$ N. G. Basov, V. A. Gribkov, O. N. Krokhin, and G. V. Sklizkov, "High temperature effects of intense laser emission focused on a solid target," Sov. Phys. JETP 27, 575 (1968).

${ }^{12}$ A. N. Pirri, R. G. Root, and P. K. S. Wu, "Plasma Energy Transfer to Metal Surfaces Irradiated by Pulsed Lasers," AIAA Journal 16, 1296 (1978).

${ }^{13}$ D. I. Rosen, D. E. Hastings, and G. M. Weyl, "Coupling of pulsed $0.35 \mu \mathrm{m}$ laser radiation to titanium alloys," J. Appl. Phys. 53, 5882 (1982).

${ }^{14}$ P. K. S. Wu and R. G. Root, AIAA Journal 18, 1857 (1980).

${ }^{15}$ R. G. Root, P. K. S. Wu, and L. A. Popper, "Pulsed Theory and Modeling of Metals and Fiberglass," AFWL Technical Report TR 83-41 (April 1983).

${ }^{16}$ J. T. Schriempf, "Response of materials to laser radiation: A Short Course," Report No. 7728, Naval Research Laboratory, Washington, D.C., July, 1974.

${ }^{17}$ T. T. Pirri, "Theory for momentum transfer to a surface with a high-power laser," Phys. Fluids 16, 1435 (1973).

${ }^{18}$ J. E. Lowder, "Laser-Surface Impulse Delivery in Vacuum," 3rd AIAA \& ASME Joint Thermophysics, Fluids, Plasma, and Heat Transfer Conf., St. Louis, MO, June 7-11, 1985.

${ }^{19}$ M. Bass, Physical Processes in Laser-Materials Interactions, edited by M. Beriolotte (Plenum, New York, 1983), pp. 77-115.

${ }^{20}$ Ya. Zeldovich and Yu.P. Raiẑer, Physics of Shock Waves and High Temperature Hydrodynamic Phenomena, Vol. I, p. 209.

${ }^{21}$ G. Gurtman (private communication, 1985).
${ }^{22}$ A. N. Pirri, "Key issues in impulse coupling efficiency of high intensity pulsed laser radiation," presented to APS Study Group, 1985.

${ }^{23}$ D. W. Gregg and S. J. Thomas, "Momentum Transfer Produced by Focused Laser Giant Pulses," J. Appl. Phys. 37, 2787 (1966).

${ }^{24}$ C. Woods, Western Research Corporation (private communication, 1985).

${ }^{25}$ V. A. Batanov, F. V. Bunkin, A. M. Prokhorov, and V. B. Fedorov, "Evaporation of Metallic Targets Caused by Intense Optical Radiation," Sov. Phys. JETP 36, 311 (1973).

${ }^{26}$ V. H. Shui, L. A. Young, and J. P. Reilly, "Impulse Transfer from Pulsed Laser Irradiation at Reduced Ambient Pressures," presented at AIAA 16th Aerospace Science Meeting, Huntsville, AL, Jan. 16-18, 1978.

${ }^{27}$ K. Keiriki and Y. Kitora, "Momentum Transfer to Target from Laser-Produced Plasma," Appl. Phys. Lett. 30, 443 (1977).

${ }^{28}$ J. A. Woodroffe, J. Hsia, and A. Ballantyne, "Thermal and Impulse Coupling to an Aluminum Surface by a Pulsed $\mathrm{KrF}$ Laser," Appl. Phys. Lett. 36, 14 (1980).

${ }^{29}$ P. K. Wu and P. E. Nebolsine, "Laser-Induced Impulse to a Phenolic Surface," AIAA Journal 18, 1101 (1978).

${ }^{30}$ C. Duzy, J. A. Woodroffe, J. C. Hsia, and A. Ballantyne, "Interaction of a Pulsed XeF Laser with an Aluminum Surface," Appl. Phys. Lett. 37 (6), 542 (1980).

${ }^{31}$ S. Marcus and J. E. Lowder, "Impulsive Loading of Targets by HF Laser Pulses," J. Appl. Phys. 46, 2293 (1975).

${ }^{32}$ C. T. Walters, "Single pulse laser effects on materials" (private communication, 1985).

${ }^{33}$ H. S. Janee, Science Applications International Technical Memorandum (December 1985).

${ }^{34}$ Papers presented at the AIAA Meeting on Laser Effects and Target Response, November 1985.

${ }^{35}$ R. S. Dingus, Los Alamos National Laboratory report, 1985 (unpublished).

${ }^{36}$ A. Hunter, Western Research Corporation (private communication, 1985).

${ }^{37}$ A. N. Pirri, "Analytic Solutions for Laser-Supported Combustion Wave Ignition Above Surfaces," AIAA Journal 15, 83 (1977).

${ }^{38}$ A. N. Pirri, "Theory for Laser Simulation of Hypervelocity Impact," Phys. Fluids 20, 221 (1977).

${ }^{39} \mathrm{R}$. Cunningham (private communication).

${ }^{40} \mathrm{G}$. Canavan (private communication).

${ }^{41}$ See, for example, P. Marmier and E. Sheldon, Physics of $\mathrm{Nu}$ clei and Particles (Academic, New York, 1969), p.165.

${ }^{42}$ Ashton Carter, Directed Energy Missile Defense in Space, OTA Report Background Paper (April 1984) gives estimates for lethality of particle beams:

\begin{tabular}{lr} 
Lethality mode & Dose $(\mathrm{J} / \mathrm{gm})$ \\
\hline Disruption of electronics & $0.1-1.0$ \\
Destruction of electronics & 10 \\
Detonation of $\mathrm{HE}$ and propellant & 200 \\
Softening of $\mathrm{V}$ or Pu & hundreds \\
Melting of $\mathrm{Al}$ & 1000
\end{tabular}

For ignition of high explosives by laser radiation, see A. I. Bykhalo, E. V. Zhuzhukalo, N. G. Koval'skii, A. N. Kolomiiski, V. V. Korobov, A. D. Rozhkov, and A. I. Yudin, "Ignition of PETN by High-Power Laser Radiation," Translated from Fizika Goreniya i Vzryva 21, No. 4, 110-113 (1985).

${ }^{43}$ M. A. Rose, "Nuclear Hardening of Weapons Systems," Defense Electronics, October 1979, pp. 71-79. 


\section{Chapter 7}

\section{ACQUISITION, TRACKING, AND DISCRIMINATION}

\section{CONTENTS}

7.1 Introductory Remarks

7.2 Boost Phase

7.2.1 IR Plume Sensing

7.2.2 IR Plume Imaging

7.2.3 Precision Tracking

7.3 Post-Boost Deployment Phase

7.3.1 Thermal Detection

7.3.2 Microwave and Optical Radars

7.3.2.1 Tracking with Microwave Radars

7.3.2.2 Discrimination by Microwave Radar

7.3.2.3 Tracking with Optical Radars

7.3.2.4 Discrimination by Optical Radars

7.4 Mid-Course: Decoy and Pen-Aid Philosophy

7.4.1 Passive Decoy Techniques

7.4.2 Active Penetration Aids

7.5 Mid-Course: LWIR Techniques

7.5.1 Tracking

7.5.2 Discrimination

7.5.3 LWIR Decoys and Pen Aids

7.6 Mid-Course: Radar Techniques

7.6.1 Tracking

7.6.2 Discrimination

7.7 Mid-Course: Interactive Discrimination Methods

7.7.1 Nuclear Explosions

7.7.1.1 Impulse Due to Nuclear Debris

7.7.1.2 Impulse Due to X-Ray Produced Ablation

7.7.1.3 Neutron Activation by a Nuclear Explosion

7.7.2 X-Ray Lasers

7.7.3 Directed Energy Beams

7.7.3.1 Neutral Particle Beams

7.7.3.2 Laser-Guided Electron Beams

7.7.3.3 Laser-Induced Impulse and Target Destruction

7.7.4 Systems Considerations

7.7.4.1 Basing Modes

7.7.4.2 Discrimination Signature, Energy, and Range

7.7.5 Interactive Discriminator Countermeasures

7.7.5.1 Shielding

7.7.5.2 Deception

7.7.6 Nuclear Precursor Bursts

7.7.6.1 Gamma Rays and Neutrons

7.7.6.2 Background Ionization and Heave

7.7.6.3 Nuclear Effects for Infrared Sensors

7.7.7 Interactive Discrimination Summary

7.8 Summary

7.9 Conclusions

References

\subsection{INTRODUCTORY REMARKS}

To function successfully, a missile defense system must have the capabilities of acquiring targets, generating accurate tracking data for targets once acquired, and discriminating lethal objects from decoys, penetration aids, and miscellaneous hardware. A fourth capability, vital for multilayered defenses, is that of kill assessment to ensure that defense assets are not wasted by repeated attack of targets already rendered inoperative.

In comparison with missile defense concepts which rely on ground-based nuclear interceptor kill, the introduction of directed energy weapons (DEWs) and smart kinetic energy weapons (KEWs) to missile defense creates new and heightened requirements for acquisition, tracking, discrimination, and kill assessment. In this section we discuss the first three of the above requirements. Kill assessment is not addressed in this report.

For defenses employing a DEW or KEW boost phase intercept layer, acquisition at the earliest possible moment after missile launch has high leverage since it allows the battle manager to efficiently assign tracking and kill platforms, thereby permitting precision tracking to be established early in the boost phase and weapon engagement time to be maximized. The requirement of early acquisition is critical since boost phase is already short in present missile systems $(2-3 \mathrm{~min})$ and can be reduced even further $(\approx 1 \mathrm{~min})$ in future systems. (See Section 2.3 where we also discuss the degree to which post-boost times can be reduced from current values.) The requirement for early and unambiguous acquisition capability is particularly clear in any defensive system which employs pop-up discrimination or kill components for use in boost phase, post-boost phase, or early midcourse.

The degree to which directed energy weapons require high precision track information is best illustrated by a simple calculation (which ignores trigonometric factors caused by the relative orientation of trajectory to line of sight). Suppose the upper stage of a booster is $1 \mathrm{~m}$ in diameter and $3 \mathrm{~m}$ in length and is to be destroyed by a DEW at a range of $2 \mathrm{Mm}$. Spot sizes of $1 \mathrm{~m}$ diameter or somewhat less* are desirable (otherwise energy is wasted and kill times drawn out). To be effective the beam spot must be held centered to an accuracy of, say, $20 \mathrm{~cm}$

\footnotetext{
* Booster structural analyses suggest minimum spot sizes are on the order of $0.60 \mathrm{~m}$ in diameter.
} 
during irradiation. This requires an ability to determine the absolute position in space of a moving target to an angular accuracy of $\delta \theta \approx 0.20 / 2 \times 10^{6}=0.1 \mu \mathrm{rad}$. If the booster is moving with a typical velocity of $6 \mathrm{~km} / \mathrm{s}$, and if the DEW must dwell on the booster for $1 \mathrm{~s}$, say, then the booster will move $6 \mathrm{~km}$ during the engagement, and thus through an angle as seen by the DEW of $\Delta \theta=6 \times 10^{3} / 2 \times 10^{6}=3000 \mu \mathrm{rad}$. Even for dwell times as short as $1 \mathrm{~ms}$, the booster motion is $3 \mu \mathrm{rad}$ which is large compared to the beam pointing requirement of $0.1 \mu \mathrm{rad}$.

In addition to the requirement for an absolute pointing accuracy of $0.1 \mu \mathrm{rad}$, the time of flight of energy from the DEW to the booster target must be taken into account. At a $2 \mathrm{Mm}$ range, the speed of light travel time is $\Delta \mathrm{t}=\mathrm{R} / \mathrm{c}=2 \times 10^{6} / 3 \times 10^{8}=6.7 \mathrm{~ms}$. During this time the booster moves an angle $\mathrm{D} \theta=6 \times 10^{3} \times 6.7 \times 10^{-3} /$ $2 \times 10^{6}=20 \mu \mathrm{rad}$. In order that $\delta(\mathrm{D} \theta)$, the error in $\mathrm{D} \theta$, be less than one-half of $\delta \theta$, one must have knowledge of the range accurate to $0.5 \%$. Calibration and verification of calibration are problems of enormous magnitude when parts per million ( $\mu \mathrm{rad})$ are required of any system, especially an operational system which has to function unattended for long periods of time.

In the stages of ICBM flight which follow boost phase, pointing and tracking accuracies are equally stressing. Target sizes are comparable or smaller and working ranges no less in most cases. As we discuss later, directed energy beams are also proposed as discrimination tools. These, too, will have submicroradian pointing accuracy requirements, and, similarly, precise lead-ahead and target motion corrections must be performed if $\mathrm{Mm}$ scale ranges are employed.

A key question in designing missile defenses in any phase is whether or not the required pointing and tracking performance can ever be met in an open loop system or whether a closed loop (feedback) system will have to be employed. The high angular precisions required indicate that closed loop tracking systems will most likely be required.

A significant question is whether the round-trip time delays inherent in such closed loop systems would permit the target to avoid destruction by acceleration during the round-trip time. A simple analysis shows that even if the sensor is at $15 \mathrm{Mm}$ range, the $0.1 \mathrm{~s}$ closed loop tracking time delay would not permit the target to maneuver more than $0.5 \mathrm{~m}$ from the DEW aim point even if the target were to accelerate at as much as $10 \mathrm{gs}$. Requirements for a return signal in a feedback system can severely limit sweep rate, maximum range, etc.

The requirements for good discrimination are best suggested by a simple threat estimate. (See Sections 2.2 and 2.3 for a detailed discussion of threat.) Consider that an attack by 1000 missiles might involve 10000 RVs using today's rocket technology. A modest increase in booster size or booster numbers could accommodate the same number of RVs but add a factor of 50 or more lightweight decoys for each RV. These decoys would move in similar ballistic trajectories once above the atmosphere.
Faced by perhaps a half million objects the defense cannot afford to attack indiscriminately; highly effective and reliable discrimination is a necessity. Of course, the defense would hope to reduce the numbers by effective defenses in boost and post-boost phases; even then good discrimination is required.

We now turn to a detailed discussion of acquisition, tracking, and discrimination. We discuss both the means which have been suggested and the limitations one can expect, and where possible the state of the technology.

\subsection{BOOST PHASE}

As already remarked in Chapter 2, the first warning of missile launch is currently obtained by infrared detection of the plume of the booster rocket from satellites at geosynchronous altitude. We now look more closely at the plume sensing and other methods of tracking in boost phase which might be employed in defensive systems.

\subsubsection{IR Plume Sensing}

During the boost portions of flight, large amounts of propellant are burned in short times; after exiting the rocket nozzle, the hot propulsion gases constitute an intense thermal radiator. A rough estimate of the total amount of energy emitted can be derived as follows.

The plume from a typical large first stage rocket at low altitude is of the order of $4 \mathrm{~m}$ in diameter at the rocket nozzle with a visible length ${ }^{1}$ of about $50 \mathrm{~m}$ or more. The temperature $e^{2}$ at the nozzle exit is about 1800 $\mathrm{K}$, and decreases to under $1000 \mathrm{~K}$ at the edges of the visible plume. The average temperature over the plume is on the order of $1400 \mathrm{~K}$.

At such a temperature the blackbody ${ }^{3}$ spectrum peaks at about $2 \mu \mathrm{m}$, with a radiation intensity of about $2 \times 10^{5} \mathrm{~W} / \mathrm{m}^{2}$ in a $0.8 \mu \mathrm{m}$ bandwidth. The total radiation in all directions is of the order of $100 \mathrm{MW}$, equivalent to $8 \mathrm{MW}$ per steradian. Since the earth background temperature is about $300 \mathrm{~K}$, earthshine radiation is negligible at $2 \mu \mathrm{m}$. The only significant background sources are solar reflections from water and clouds. In principle, a satellite boost phase surveillance and tracking sensor can be located in orbit at any altitude, provided that the infrared telescope aperture collects sufficient photons to overcome detector noise and, as we discuss below, has sufficient spatial resolution to keep solar-reflected energy less than the signal from the rocket plume.

At synchronous orbit ranges (altitude $40 \mathrm{Mm}$ ), the photon flux from a first stage of a large missile with the above intensity corresponds to about $50 \times 10^{9}$ photons $/\left(\mathrm{m}^{2} \mathrm{~s}\right)$. The detection of a signal this strong is not difficult provided solar reflection backgrounds are managed. As an extreme case consider an earth albedo of $100 \%$ and no atmospheric absorption of incident or reflected solar radiation. The reflected sunlight ${ }^{4}$ from the earth's surface between 2 and $3 \mu \mathrm{m}$ would be about $50 \mathrm{~W} / \mathrm{m}^{2}$. Sufficient spatial resolution in an IR sensor is therefore needed in order to limit this background 
radiation to less than that of the rocket plume given the large surface area of the earth. A mitigating factor in practice is atmospheric absorption which can reach values as high as $99 \%$ for one way vertical transmission at 1.9 and $2.7 \mu \mathrm{m} .{ }^{4}$ Two-way transmission of sunlight would be only $10^{-4}$, and the solar reflection background would be only $0.005 \mathrm{~W} / \mathrm{m}^{2}$ under such conditions. For reliable detection of signals of the order of $8 \mathrm{MW} / \mathrm{sr}$, the cell size of the detector field of view could be as large as $10^{8} \mathrm{~m}^{2}$ and still yield a 10:1 signal-to-background ratio. This is equivalent to a $10 \mathrm{~km} \times 10 \mathrm{~km}$ field of view.

The above estimates make it clear then that sensors can be built with optical apertures of reasonable size to detect first stage plumes at geosynchronous range. While it is probable that the ballistic missile defense mission may call for spatial resolution better than that now obtained from IR satellites at geosynchronous orbit, this is not likely to be stressing. Very important for defense is a low false alarm rate.

Surveillance and tracking of the second and third stages of ICBMs, or of first stages of SLBMs, will require significantly greater sensitivity, since the optical output of a plume is roughly proportional to thrust. In typical rockets, the second stage thrust is about one-fifth of the first stage and the third about one-fifth of that. Therefore, if the upper stages are to be tracked, the system should be capable of detecting signals of the order of $300 \mathrm{~kW} / \mathrm{sr}$ or equivalently, fluxes of the order of $2 \times 10^{9}$ photons $/\left(\mathrm{m}^{2} \mathrm{~s}\right)$ at $40 \mathrm{Mm}$ range. Reliable detection should be possible at synchronous orbit ranges with sensor optics of the order of $1 \mathrm{~m}$ in diameter. In this case, however, the field of view would have to be decreased by about a factor of 25 in area from that discussed above, i.e., about $2.0 \mathrm{~km}$ on a side.

Since rocket plume infrared radiation is so intense, it does not seem possible to conceal this radiation with shrouds, nor does it seem possible to simulate such intense sources, including spectrum and motion, without launching an actual rocket over a similar trajectory. Of course, decoy rockets need not have the precision, complex payload, nor survivable basing of ICBMs, and so could be made considerably cheaper; alternatively older generation missiles could be employed as decoys. (It seems implausible that decoy SLBMs would ever be attractive to the offense.) To be credible, decoy boosters need not closely resemble a particular operational booster, they need only appear to have the capability of launching a payload to intercontinental range. While it is plausible that the offense could increase the number of targets facing the boost phase defense by factors of two or three, an order of magnitude or greater increase seems unlikely given the complexity of even the simplest booster decoy.

\subsubsection{IR Plume Imaging}

When considered for aiming directed energy weapons, booster plume tracking does not provide sufficient precision. From geosynchronous distances, IR systems having optical apertures of $40 \mathrm{~m}$ would be required at $2 \mu \mathrm{m}$ wavelengths to give $1 \mathrm{~m}$ spatial accuracies; diffraction-limited, space-based optics on such a gigantic scale are clearly out of the question. Even though one can imagine constructing a constellation of lower altitude IR optical platforms $(4 \mathrm{~m}$ diffractionlimited optics would give a $1 \mathrm{~m}$ spatial precision up to sensor ranges of $2 \mathrm{Mm}$ ), the ultimate target of a defensive weapon is the booster, not its plume. It would be necessary therefore to image the plume and extrapolate forward by some amount to find the actual missile. The extrapolation distance is not easily determined since the geometry of a booster plume is a complex function of rocket motor design, motor operating characteristics, and other missile parameters. In addition, plume characteristics vary greatly with altitude and missile speed, and at high altitudes the plume can even envelop the booster itself. While all of this could be modeled and intelligence gathered for each missile type by surveillance, a considerable opportunity for plume modification exists. It seems likely therefore that a determined offense could defeat plans to locate and track the boosters to $1 \mathrm{~m}$ accuracy by plume imaging alone, even if the defense made the large investments required. Furthermore, as discussed in the introductory part of this section, spatial accuracies of better than $1 \mathrm{~m}$, say $20 \mathrm{~cm}$, are probably required. For these reasons some novel alternative acquisition techniques using shorter wavelength photons are now being examined. To the extent these look at signals from the plume, discussed above, similar problems will exist in locating the missile itself accurately.

\subsubsection{Precision Tracking}

Because of the limited spatial resolution which can be expected for IR systems, and especially because the extended intense plume source dominates the IR signature, some other means must be employed to achieve precision tracking if boost phase intercept is to be attempted. A likely arrangement would use IR plume tracking to determine a coarse boost phase trajectory, with this information then passed to the precision tracking system. Candidates for precision tracking are microwave radars, optical radars, and passive emissions at wavelengths shorter than IR.

Microwave and optical radars are discussed below in connection with post-boost and mid-course phases. If they are employed in these phases, platforms in range of booster fields could be used for boost phase tracking as well. We restrict our discussion here to those few items which are special to the boost case. The numerical examples considered here are illustrative and should not be considered definitive. The number of satellites required may be low by as much as an order of magnitude.

Because of their long wavelengths, microwave radars used alone do not have the cross range (angular) accuracy required for targeting directed energy weapons. In principle, however, a number of radars with accurate 
range capability could determine the position of a booster by triangulation with the obvious penalties of increased communication and signal processing loads. For the example cited in Section 7.3.2.1 below of 2000 boosters viewed by 200 on-station, space-based, microwave radars (the total number of radar platforms would be the number of on-station platforms times the absentee factor; see Appendix B) would require 6000 lines of site for triangulation to determine one accurate spatial fix of all boosters. Tracking would require a large number of such fixes. To accurately locate 2000 boosters with this approach, each radar would have to be steered to 30 different locations (given approximately to the radar by the passive IR sensor system). For example, if one needed updates on a given booster every $3 \mathrm{~s}$ then the total dwell and steering time per target for each radar would have to be no longer than $0.1 \mathrm{~s}$. As an example consider a set of 30 boosters spread out transversely over $100 \mathrm{~km} \times 100 \mathrm{~km}$ and viewed at $1 \mathrm{Mm}$ range-a field of view $6^{\circ} \times 6^{\circ}$. Large dish radars cannot be steered mechanically at the required rate over such angles (a few degrees per second in one dimension is the current norm), but for phased array radars the steering requirements could be met with existing technology. Obtaining the required accuracy and dwell times may be difficult.

In Section 7.3.2.3 below we discuss the application of optical radars to post-boost tracking with the assumption that such radars can be built with rapidly steerable beams. If this is the case, optical radars would be more attractive to the boost phase than microwave radars, even though the experience with the latter is so much greater. If nearUV wavelengths are chosen for the laser, one can contemplate cross range spatial accuracies from a single radar of less than a meter at ranges of $1 \mathrm{Mm}$ with meter aperture optics. The burden of triangulation between platforms, that is presented by microwave radars, would be lifted. It is important to note that a UV optical radar could provide precision tracking of the booster only in the upper part of the atmosphere where UV absorption would not pose a problem.

For the example cited in Section 7.3.2.3 one would have 10 optical radar platforms on-station over the launch complex. Each laser would have 200 boosters as its responsibility. If the radar can use a pulse as short as $10 \mu \mathrm{s}$ and the passive IR sensor tracking the booster plume has provided it with a field of view no bigger than $30 \times 30$ times the laser beam size, then a given optical radar will get one precision fix (scanning roughly to 900 pixels to locate each target) on each of its 200 boosters of responsibility after a total of $2 \mathrm{~s}$. This interval is less than the total duration of the boost phase (60 $\mathrm{s}$ for the fast burn case), but not by a large factor, especially if one is considering wavelengths for which atmospheric absorption is not negligible. In practice, booster track predictions can be made by extrapolating the results from the first few precision fixes, and this information can be used to reduce the number of pixels which have to be sequentially searched to make later fixes. Nevertheless, development of precision tracking concepts for use in boost phase, even using optical radars, will be challenging (especially if pulses longer than $10 \mu \mathrm{s}$ are required). An optical power output of order $20 \mathrm{~kW}$ in the UV would be required.

\subsection{POST-BOOST DEPLOYMENT PHASE}

At the end of the boost phase the characteristic rocket plume no longer exists and means other than short wavelength IR sensing must be used for surveillance and tracking. Two candidates are apparent: active radar (microwave or laser) and passive thermal detection. We first discuss thermal sensing and then radar.

\subsubsection{Thermal Detection}

The temperature of a post-boost deployment vehicle is likely to be that of the silo from which it came, typically $300 \mathrm{~K}$. If we take the surface area of a postboost vehicle (PBV) to be $10 \mathrm{~m}^{2}$ and its surface emissivity to be 0.1 , we find that the thermal power emitted is 30 $\mathrm{W} / \mathrm{sr}$. This signal is much weaker than that of the booster plume, and is comparable to the thermal background emitted by the earth. An additional complication is that the thermal radiation from the PBV is peaked at a wavelength of $10.0 \mu \mathrm{m}$, and so lies predominantly in the long wavelength infrared (LWIR) band, in contrast to emissions from the plume which are in the short wavelength infrared (SWIR) region. As a consequence, cooled detectors would have to be employed to avoid detector self-noise for thermal tracking of PBVs.

For the example numbers we have taken above, the flux at the aperture of a sensor at a range of $10 \mathrm{Mm}$ from a PBV would be $15 \times 10^{6}$ photons $/\left(\mathrm{m}^{2} \mathrm{~s}\right)$. Detection of this signal against an earth background may be impossible; detection against a space background would require sensitive detector arrays and large optics. Even if detection is feasible, imaging as a means to distinguish reentry vehicle (RV) deployments from decoy deployments seems unlikely.

Thermal detection of the weak PBV thruster plume is also conceivable, although these emissions could be reduced by using cold gas thrusters, or shrouds. Further, much additional information would be necessary to perform the precision tracking and discrimination that is required by the defense in the deployment phase than could be obtained from PBV plume sensing alone. At best, thermal detection can make only a partial contribution in the post-boost phase.

\subsubsection{Microwave and Optical Radars}

During the post-boost phase when the RVs and various decoys are being deployed, observation by active radars is most useful for tracking and discrimination. Two possible types of radar, microwave (or radio) and 
optical (near UV to near IR; $0.25-10 \mu \mathrm{m}$ ) are worthy of consideration. Microwave radars are vastly more developed and are discussed first.

\subsubsection{Tracking with Microwave Radars}

The number of on-station radars required depends both on the anticipated offensive threat and the percentage of missiles which survive the boost phase. For a minimal threat of less than 200 surviving missiles, say, one can contemplate a simple and well-understood system in which a separate radar is assigned to each PBV. For this purpose a single dish with relatively slow mechanical steering is sufficient to track the PBV. With existing busing methods, RVs and decoys separate from the PBV sufficiently slowly so that they remain in the radar dish's field of view for tens of seconds (long enough to watch the deployment) unless the dish is very large and the radar frequency very high.

Ground-based dishes with diameters of the order of $10 \mathrm{~m}$, operating at frequencies between 5 and $50 \mathrm{GHz}$, are fairly commonplace in radio astronomy; satellite deployment of similar dishes is feasible. Transmitter tubes for powerful ground-based radars are rather massive at the moment and it is not clear what degree of weight reduction for satellite deployment is possible. The tradeoff between radar range and satellite weight is therefore also not clear, but ranges between $1 \mathrm{Mm}$ and 5 $\mathrm{Mm}$ are presumably achievable. In this (one radar per PBV) system the number of active radar platforms does not increase with a decrease in radar range but the absentee factor goes up.

For purposes of simple estimates it is advantageous if the radar range is comparable to the size of the likely launch areas. For the numerical examples below we shall therefore assume a radar range $R_{r}$ of about $3 \mathrm{Mm}$.

For the simple radar system contemplated here the number $\mathrm{N}_{\mathrm{r}}$ of separate radars which have to be in orbit is simply the product of the number $\mathrm{N}_{\mathrm{PBV}}$ of surviving PBVs and the absentee ratio y. The latter is the ratio of the total number of radars in orbit to the number of sensors which are usefully employed (on station) at any instant of time, i.e., are within range of a launch zone so they can be assigned a PBV. For a given distribution of booster launchers and given distribution of satellite orbits for the sensors one can of course calculate y by numerical simulation. However, for illustrative purposes a very simple estimate is useful: Consider sensor orbits at low altitude which cover the earth's surface area $4 \pi R_{e}^{2}$ uniformly, each sensor with range $R_{r}$ and hence area of responsibility $\pi \mathbf{R}_{\mathrm{r}}^{2}$ as measured on the ground. Consider a simultaneous launch of the offensive boosters from $n$ independent launch zones with each zone having a dimension small compared with $R_{r}$ but with zone separations large compared with $\mathbf{R}_{r}$. In this case

$$
\mathrm{y}=\frac{4}{\mathrm{n}} \frac{\mathrm{R}_{\mathrm{e}}^{2}}{\mathrm{R}_{\mathrm{r}}^{2}} .
$$

For a given value of the range $R_{r}$ the offense can force up the value of $y$ by concentrating the launchers into smaller areas (decreasing $\mathrm{n}$ ), but the magnitude of such an increase depends on the numerical value of $R_{r}$. In the limit where $R_{r}$ is larger than the characteristic dimensions of the total region available for booster launch (land area for ICBMs, ocean operating area for SLBMs), one has $n=1$ effectively and so $y=4 R_{e}^{2} / R_{r}^{2}$. In this limit there is no advantage to the offense to further concentrate the launch sites.

A realistic calculation of $y$ is more complex because of the inherent discreteness of the problem, because of motion of the PBVs and satellite platforms during the engagement, and because one may want to use the same satellites in the mid-course and so would optimize on a system-wide basis. In almost no case would a satellite constellation approximating uniform coverage of the entire earth be optimal. If one only wants to cover launch areas, then orbital inclinations can be chosen to give an enhanced concentration of satellites over these areas and hence a reduced absentee factor. Namely, if $\lambda_{b}$ is the characteristic latitude of launch areas and $i$ is the satellite orbital inclination, then values $i=\lambda_{b}$ are near optimal. If the defense satellites used in the post-boost phase are to play a role in mid-course also, then values of $i$ close to $90^{\circ}$ are desirable (polar orbits).

As a simple example we take $R_{r}=3 \mathrm{Mm}$. Since this is comparable to the "radius" of the U.S. and Soviet Union, detailed dependence on launch configuration will be small. The above uniform-earth-coverage model gives $y=18$ for this case $(n=1)$. We adopt instead $y=10$ to allow for some constellation optimization (in principle $y$ could be as small as 5) weighed against the desire to have some redundancy to avoid the need for any handover of targets from one radar platform to another.

The number $\mathrm{N}_{\mathrm{PBV}}$ of surviving PBVs in an attack of course depends strongly on what choices the offense has made and the type of boost-phase defense in place. We consider three examples.

(a) As one extreme assume only current generation Soviet launch vehicles against an effective boost-phase defense, viz., 2000 separate launchers (see Section 3), each with boosters burning well above the atmosphere (above $120-150 \mathrm{~km})$. Suppose in the post-boost phase each missile deploys $5 \mathrm{RVs}$ and 50 decoys. In this case the boost phase defense has several tens of seconds of boostburn above the atmosphere before any deployments to kill boosters. A fashionable example is to assume that the boost phase layer of defense is $90 \%$ effective so that $N_{\text {PBV }}=200$ and the required number of orbiting simple radars is $N_{r}=N_{P B V} \cdot y=2000$.

(b) As an intermediate example assume that the offense has converted to fast burn boosters (burning stops before exiting from the atmosphere) but the post-boost vehicles are not modified from today's designs. Consequently we assume that boost phase kill is partially ineffective, so that 1000 (half of 2000) launch vehicles survive the boost phase. In this case we have $\mathrm{N}_{\mathrm{PBV}}=1000$ and $\mathrm{N}_{\mathrm{r}}=10000$. 
(c) As a third example assume the same fast-burn capability (and ineffectiveness of boost phase kill) as in (b), but assume in addition that the offense has abandoned the MIRV concept and switched instead to five times the number of current launchers, each with only one RV but with a similar proportion of decoys to RVs. This case requires $\mathrm{N}_{\mathrm{r}}=50000$, and opens up endless arguments about the worst case scenarios.

In connection with battle stations there has been considerable controversy over linear versus square-root scaling laws for the cost to the defense as the number $\mathrm{N}_{b}$ of offensive boosters is increased or decreased. (See Appendix B.) For our present problem of counting radars there is no controversy if the defense continues to assign one radar to each $\mathrm{PBV}$, no matter the value of $\mathrm{N}_{\mathrm{b}}$. In this case the required number of radars is simply proportional to $\mathrm{N}_{\mathrm{b}}$ (assuming constant boost phase kill efficiency). However, as $\mathrm{N}_{\mathrm{b}}$ increases, the distance from a radar to the nearest PBV (at any instant of time) decreases and the required range and cost per radar may decrease with increasing $\mathbf{N}_{\mathrm{b}}$. The total cost of the radars alone would thus increase less than linearly with increasing $\mathrm{N}_{\mathrm{b}}$. On the other hand, the portion of the command and control system which must interact with the $N_{r}$ radars has a task whose complexity and cost presumably increase more rapidly than linearly. It is thus not clear whether the portion of the total cost to the defense chargeable to radars increases with $\mathrm{N}_{\mathrm{b}}$ slightly slower or faster than linearly, but this uncertainty is relatively small. A greater uncertainty lies in the choice of defensive radar systems for various cases.

The 50000 separate radar satellites for case (c) above is so large an investment that the defense may well attempt a more complex system, which utilizes a much smaller number of phased-array radars in satellite orbits. With such orbiting phased-array radars it will still be necessary to employ triangulation for accurate transverse spatial precision; otherwise radar antenna apertures of several million wavelengths would be necessary to obtain $20 \mathrm{~cm}$ accuracy necessary for aiming of directed energy weapons. The use of phased-array radars with rapid electronic redirection of beam direction permits a triplet of such radars to track many PBVs. In theory, one such radar triplet could track very large numbers of PBVs or separate RVs. In that case it is possible that only $3 \times 10$ or 30 such radars might suffice. On the other hand, each of these radars would have a product of transmitter power times antenna area squared, which was 200 times that of the simple dish radars for case (a) above. Case (b) would require one 1000 times as great, and case (c) 5000 times as great. Such radars would require large antenna apertures with many thousands of radiating elements. The vulnerability of such large radars to attack by an enemy probably makes this tracking option unattractive. Such phased arrays, with many separate elements but electronic communication between all elements, have been built on the ground and used extensively. There are NASA and Air Force/Navy/DARPA plans for radar satellite deployment possibly in the early 1990s and NASA designs for detecting and correcting antenna deformations. This concept is sometimes called a floppy phased-array antenna with local station keeping. ${ }^{5,6}$ However, the feasibility of basing a complex phased-array radar system in space which would meet the transverse spatial precision required for strategic defense has yet to be attempted, let alone demonstrated.

\subsubsection{Discrimination by Microwave Radar}

A microwave radar operating at $1 \mathrm{~cm}$ wavelength with a 10-m-diameter dish or phased array has an angular resolution of order a milliradian, so that it illuminates an area of order $1 \mathrm{~km}^{2}$ at a typical $2 \mathrm{Mm}$ range. Of course this beam radius is not small enough to give good spatial resolution by traditional "angle-angle imaging," but it has the advantage that the various objects deployed by the PBV (for example, $5 \mathrm{RVs}$ and 50 decoys) are all illuminated at the same time (at least for many tens of seconds). Although cross-range imaging is not possible, one can measure the radar cross section of an object in the resolution cell and determine its Doppler velocity. If several objects are in the same range resolution cell at one instant of time, but have different velocities, the rangeDoppler method can still keep track of the different objects (given sufficient observing time and adequate computing power).

However, the ability of a given radar to discriminate RVs from decoys during deployment from the PBV is controversial, in part because it is not known what countermeasures the offense will adopt, and in part because a defense-conservative assessment is very different from an offense-conservative one. This difference is not only quantitative, but qualitative as well and is illustrated by two specific countermeasures against the discrimination radar during the PBV deployment phase namely, mainlobe radar jammers and PBV shrouds.

The current weight of a Soviet PBV is sufficiently large that an addition of a powerful "main-lobe jammer," a transmitter broadcasting at all expected radar frequencies and in almost all directions from the PBV, would not present a great weight penalty. From the point of view of the defense radar, this jammer is a very serious threat. At distances comparable to the maximum range for the radar, the jammer will "swamp" the radar and deny any range, Doppler and cross-section information during the vital phase when the RVs and decoys are being deployed from the PBV. It is not clear whether putting a separate jammer on each RV and each decoy package is practical or would result in too much of a weight penalty; without separate jammers the RVs and packages would become viewable after sufficient separation from the parent bus, but all data on the separation phase would be lost; this is precisely the most useful data for discrimination purposes.

As frightening as the PBV jammer might appear to the defense, it may not be a reassuring choice for the 
offense designer of PBVs because of many uncertainties outside of his control. For example, although a jammer could be powerful enough for typical distances from PBV to the defensive radar platforms to hide deployments, for some PBV-radar pairs the distance will be sufficiently small that the defense radar could do its job in spite of jamming. Furthermore, what distance is small enough for a given type of jammer depends strongly on radar techniques used by the defense but unknown to the PBV designer, not to mention the embarrassing possibility of the jammer design missing an important radar frequency. To summarize, designing a main-lobe jammer may not be a high confidence countermeasure choice for the PBV architect because the degree of success depends importantly on the enemy's radar; nevertheless, if done well, jamming would render the defense radar ineffective during the critical deployment phase.

A shroud or reflecting umbrella can, in principle, be put in place around part of the PBV during the deployment phase to deny the radar a look at the working face of the PBV as it releases objects. The RVs and decoys would become visible to the radar after moving away some distance from the PBV but the desirable data from the separation phase would be missing. The weight of the shroud itself could be quite small (thin metal sheets function well as radar reflectors). However, rechoreographing deployment scenarios to assure that the shroud would not interfere with offense deployment maneuvers would probably add a slight weight penalty and some deployment complexity to the PBV. Even then the shroud may be imperfect during short deployment segments. Nevertheless, the use of such a shroud is likely to be attractive to the countermeasure architect because its imperfections are understood without having to understand the design of the opponent's radar in detail.

It is not clear what strategy the defense would use if faced by a PBV equipped with a jammer and/or shroud. One discrimination strategy is fairly clear if there is neither a jammer nor a shroud and if the PBV is of the type currently in vogue, in which the PBV thrusts over an extended period while sequentially releasing RVs, the decoys and other penetration aids one at a time.

This kinematical discrimination technique for the post-boost phase will be much harder to utilize if the MIRVed missiles are abandoned in favor of single warhead missiles. In its simplest form, this means that each PBV will be relatively small and will contain only one RV and various associated decoys. Of course this PBV still has to engage in deployment maneuvers to release its $R V$ and decoys, but these have to separate from each other by only small distances in contrast to the large separations now desired to achieve larger footprints for MIRVed missiles. It seems unlikely that the kinematics associated with the very mild maneuvers of the PBV of a single warhead missile could be exploited for discrimination, but alternative discrimination schemes for post-boost phase are under consideration. In recent years the cost and size of inertial guidance systems as well as electronics and computer controls has dropped precipitously, so the former advantages of the MIRV system over single warhead missiles are gradually diminishing. The U.S. Midgetman and Soviet SS-25 are steps in this direction.

Between release from the booster and departing the atmosphere, the mini-buses described above still represent high value targets; each is known to contain an RV. It would be attractive therefore to the offense to be able to dispense decoys immediately after booster burnout and mini-bus separation. For booster burnout at $80 \mathrm{~km}$ altitude, the residual atmosphere is sufficient to cause differential acceleration between RVs and lightweight decoys. This differential acceleration could in principle allow the defense to discriminate RVs from decoys perhaps by range-Doppler radar. For an object of mass $m$ and effective area $A$ moving at velocity $v$, the deceleration a depends linearly on the density $\rho$ according to $\mathrm{a}=\rho \mathrm{Av} \mathrm{v}^{2} / \mathrm{m}$. This formula can be used to calculate the maximum altitude at which a known deceleration measurement capability can be used for a given decoy weight. (See Figure 2.9 for some typical values.)

\subsubsection{Tracking with Optical Radars}

Because of their shorter wavelengths, optical lasers employing mirrors of a few meters diameter can give much better angular resolution than microwave radars. Assuming a range of $1 \mathrm{Mm}$ as in the numerical example in Section 7.2.3, one can achieve a linear resolution of a few meters in the near infrared and a resolution of better than a meter in the near ultraviolet. The accuracy of range-Doppler measurements is also better in principle if an optical laser is used instead of microwave. However, the excellent angular resolution possible with optical radars also means that one is forced to scan over very many beam areas to detect and track after handover from the LWIR sensor systems. Standard mechanical slewing of an optical radar mirror is too slow; electronic beam steering has been considered and some unorthodox methods for rapid mechanical steering show promise. It is not clear whether rapid steering of optical radars can be achieved. If it can, each optical laser radar will be much more expensive than a single-dish microwave radar, but also should be able to track many more objects (in conjunction with passive LWIR sensors).

We shall explore here, as we did in earlier sections, a system employing 100 optical laser radars. For one of the most (defense) optimistic examples in Section 7.3.2.1, where only 200 PBVs survive boost phase (with an absentee ratio of 10), this means 20 PBVs per optical radar in contrast to one $\mathrm{PBV}$ per microwave radar. Each laser radar consists of a pair of satellites, one containing a multi-kw optical laser (but considerably less powerful than a DEW laser) and the other a sensor which collects reflected signals using an array with a very large number of detectors. The transmitter and receiver satellites have to be separated by a considerable distance $(10-20 \mathrm{~km})$, to avoid blinding of the sensors by corner reflectors which 
could easily be mounted on the PBV; this requirement for the defense should not present a serious station-keeping problem. We assume a laser beam-pulse of $\approx 100 \mu$ s and that, say, $30 \times 30$ pixels are assigned to each PBV. The laser thus has to be moved one beamwidth $10^{4}$ times a second and so one look at one PBV lasts about $0.1 \mathrm{~s}$. Standard mechanical steering of a mirror on such timescales is out of the question: Estimates for the shortest slewing time are of order $0.1-5 \mathrm{~s}$ for mechanical motion through an appreciable fraction of a radian. (The Astronomical Space Telescope being readied for deployment can slew about one beamwidth in $3 \mathrm{~s}$. However, for this system only a moderately small fraction of its design cost was allotted to improving slewing speed.) The agile beam required for a laser radar thus must be achieved with some kind of unorthodox mechanical steering, or by electronic means.

Some electronic steering of a laser beam has already been demonstrated in the laboratory in one dimension, but only at low laser power and for small angles. It is not clear whether it will be possible to invent a system which works at high power and employs an electronic mechanism to do large-angle steering in one dimension and an independent electronic scheme to do small-angle steering (at least) in the perpendicular direction. Although the total number of pixels in a full field of view is of order $\left(3 \times 10^{5}\right)^{2} \approx 10^{11}$, one might be able to use a combination of mechanical and electronic steering. One possible approach, for looking at $20 \mathrm{PBVs}$ would be the following: A mirror could probably be rotated with uniform angular velocity (hence no distortion) in such a way that one takes $2 \mathrm{~s}$ to rotate the mirror over a total angle $\theta$ which encompass in the $\mathrm{x}$ direction all $20 \mathrm{PBVs}$. One employs an electronic device (perhaps by writing a message on the laser end faces) which backsteers the whole laser beam through an angle of only $\theta / 20$ in the $x$ direction so as to dwell on a single PBV for $0.1 \mathrm{~s}$, say. In the perpendicular $y$ coordinate one employs another device to steer the full angle subtended by the 20 different PBVs. One possibility is to employ a laser whose frequency is swept by an appreciable factor and to put the laser beam through a diffraction grating. This step, for a high-power laser, would be particularly problematic. Upon approaching the mechanical stops, the mirror rotation would be slowed and then reversed so that the system after settling is ready for a second look, say $5 \mathrm{~s}$, after the start of the first look.

Assume an absentee factor $\mathrm{y}=10$, as in Section 7.3.2.1 and assume that only 200 of an initial 2000 boosters launched survive into the PBV phase. Assigning 20 PBVs to each on-station laser radar transmitterreceiver pair then corresponds to $(200 / 20) 10=100$ discrimination laser satellites and 100 associated detector satellites. Assuming a PBV deployment phase of $100 \mathrm{~s}$ duration and calling one look at a PBV a picture with $30 \times 30$ elements, the example above gives us 50 looks at each PBV body. More than 20 objects are released by each PBV, and so one probably needs 100 looks instead of 50; thus about 200 laser transmitter-receiver pairs would be required to build a discrimination scheme around this configuration of optical radars. If the offense used one PBV per RV, instead of current MIRV designs, one would need more laser radars even assuming that $90 \%$ of the boosters are killed before the post boost phase.

\subsubsection{Discrimination by Optical Radars}

Using a coherent laser radar allows the generation of range-Doppler images as an alternative to the angle-angle imaging described above. In this case the radar beam is made large enough to cover the whole PBV. Range resolution is accomplished through the use of short duration pulses. Resolution in the cross-range dimension is accomplished by measurement of the Doppler shift of the received pulses. To obtain the equivalent of $30 \times 30$ pixel resolution, 30 pulses are needed for Doppler resolution. Each pulse needs to be separated by 30 pulse intervals. An image can therefore be generated in as little as 900 pulse lengths or about $1 \mu \mathrm{s}$, providing sufficient signal-to-noise ratio is available.

With transmitter powers of a few tens of kilowatts, meter-class apertures, and a few megameter ranges, it is estimated that 50-100 looks per second can be achieved at considerably faster rates than for angle-angle imaging employing sequential pixel by pixel scanning. Imaging laser radars of the type described above can make a number of significant discrimination measurements on a PBV and the objects it releases. The use of a shroud to conceal deployments will make such measurements more difficult but several possible discriminants may still be visible. Considerable research will be needed before the practicality of such techniques is well understood. It should be noted that boost phase laser weapons could be used to good advantage at this phase to destroy fragile decoys and shrouds even though they might not be able to destroy a hardened PBV or the RVs.

\subsection{MID-COURSE: DECOY AND PEN-AID PHILOSOPHY}

After all warheads and other objects have been deployed from the post-boost vehicle, the relatively long mid-course phase begins. Without drag and thrusting, objects move only under the influence of gravity making it impossible, even in principle, to ascertain mass by metric tracking alone. Additional information must be gathered and exploited by the defense to distinguish RVs from decoys and penetration aids unless this information has been obtained with high confidence from observations during the post-boost phase. Of course, an offense could employ RVs which maneuver in the mid-course phase as well, but this would add considerable complexity to RV design and would provide additional signatures to the defense and might prove counterproductive.

Since the issue of decoys is so important in the midcourse, we begin with some general remarks about decoy 
and penetration aids from an offensive point of view. We then discuss the discrimination approaches which the defense might employ when faced by decoys. It is sometimes artificial to discuss decoy techniques for midcourse in isolation from reentry, so we will treat the latter as well when convenient. Although considerable theoretical attention has been given to decoys in the U.S., except for developing radar decoys, efforts at hardware development have been quite limited in comparison to the attention given offensive systems. Theory remains untested and unconstrained by fact.

Characteristics available for discrimination fall generally into two classes: (i) extrinsic characteristics, such as size, shape, surface temperature and surface emissivity, and (ii) intrinsic characteristics, such as mass, uranium, or plutonium content, the presence of high explosives, the presence of electronics, etc., all of which are required for a nuclear weapon. Accordingly, our discussion of discrimination divides naturally into two parts. In the first, discrimination by surface characteristics, we consider both passive LWIR and active radar methods. In the second, discrimination by intrinsic features, we survey a number of interactive, perturbing methods.

We address both active and passive decoy techniques.

\subsubsection{Passive Decoy Techniques}

The first passive approach is that of simulation, i.e., the use of replica decoys. In simulation one attempts to produce with a lightweight decoy responses in the sensors of the defense which are indistinguishable from those produced by an actual RV. Since the sensors of the defense might employ a variety of wavelengths (multiwavelength radars, multiband thermal radiation sensors, etc.) or might exploit two or more physical phenomena, the offense needs to have information about the defense's sensors to decoy with high confidence. The defense in turn needs to have confidence that $\mathrm{RV}$ (and decoy) signatures observed by monitoring testing or by other means are the signatures that will be present in an actual missile attack.

Typical examples of simulation decoys for exoatmospheric applications are lightweight objects erected or inflated in space (umbrellas or balloons, respectively) which have surface areas and shapes matching the radar scattering cross sections and the LWIR emissions of reentry vehicles. Typical masses for such decoys might be as small as a few kilograms including dispensing and erection hardware. Care must be taken in deployment to match the rigid body dynamics of the decoys to the dynamics of actual RVs; this can most easily be done if RV deployment techniques are adopted which result in simple motions of the RV about its center of mass (e.g., the angular momentum vector aligned with the RV symmetry axis).
Replica decoys capable of functioning throughout the mid-course and into the early parts of reentry require heavier designs since they must also duplicate (say by thrusting) the aerodynamic properties of real RVs in their interaction with the atmosphere. Below about $50 \mathrm{~km}$, a credible decoy must also duplicate the plasma wake that develops behind a reentry vehicle. Wake radar return replication by a decoy is possible at selected radar frequencies with on-board electronics. One can expect that decoys capable of functioning through early reentry will have masses heavier than those which are designed for only the mid-course. A reasonable estimate is $10 \mathrm{~kg}$ or more.

A second passive approach is that of traffic decoys. Here the strategy of the offensive is to saturate the processing and battle management capabilities of the defense by placing vast numbers of objects in the field of view. It is not required that the objects closely resemble actual RVs (the only requirement is that they cannot be eliminated by simple bulk filtering eliminating the requirement for sophisticated discrimination processing), so the number of traffic decoys that can be utilized greatly exceeds that which is possible with simulation or masking/antisimulation approaches.

Other passive decoy techniques were considered by the Study Group. These, however, are not discussed here because of classification restrictions.

A mixture of decoy/pen-aid techniques is a probable choice for the offense. Indeed, in a high traffic decoy environment discrimination time will be shrunk and sophisticated algorithms may become unusable and so the design requirements for simulation decoys will be relaxed.

\subsubsection{Active Penetration Aids}

There are many possible active pen-aid approaches. Generally the offense would select its response in accord with the sensors being used by the defense. Some offense knowledge of the defense is thus required. If not openly available such knowledge might be obtained by observing tests conducted by the defense or from the physical characteristics of defense sensor platforms and components. Active penetration methods, like passive ones, face an enhanced challenge if the defense employs multiple signatures for discrimination.

First consider jamming, i.e., transmitting a signal which overwhelms the signals created in the defense sensors by an RV. Jamming is a well-established radar technique. For microwave radars jamming can be done either in the main lobe or via side lobes. Although the latter requires considerably greater jammer power, directionality requirements are greatly reduced and so it may be the most attractive choice if the radar field of view is small.

In contrast to microwave radars, optical radars are considerably more difficult to jam because of the low side lobes of optical telescopes and because of the wide 
bandwidths available at optical frequencies for anti-jam waveforms. However, nuclear precursor bursts in the atmosphere could be a way of jamming optical sensors.

Another active penetration aid is that of maneuver. While probably not attractive for enhancing penetration of mid-course defenses, for reentry the picture changes. At least two different applications can be identified. The first, already mentioned above, involves the use of thrusted decoys so that the slowdown of light-weight decoys in the upper atmosphere can be reduced and matched to that of RVs down to some altitude.

A second application is to use maneuvering RVs (MaRVs) to compound defense tracking or to outmaneuver kinetic energy interceptors. Once within the atmosphere small, adjustable aerodynamic surfaces on an RV can be used to generate rapid velocity changes. A weight and cost penalty accrues to the offense, however, since maneuvering RVs must carry attitude control and some form of guidance if high accuracy is not to be lost.

\subsection{MID-COURSE: LWIR TECHNIQUES}

In the mid-seventies the U.S., in addition to continuing research on radar-based terminal ballistic missile defenses, began serious study of LWIR techniques for tracking and discrimination. While research has been largely confined to the laboratory, some experiments have been done in space. Enthusiasts argue that with passive LWIR sensing alone one can acquire all the information needed to operate missile defenses in the exoatmospheric or in the high endoatmospheric region; other analysts disagree and hold that the discrimination cannot be solved by passive LWIR means alone.

\subsubsection{Tracking}

A typical reentry vehicle has a surface area of $A=2 \mathrm{~m}^{2}$. For a blackbody (emissivity, $\epsilon=1$ ) the emitted power is $B_{s}=60 \mathrm{~W} / \mathrm{sr}$ for a typical temperature of $T$ $=300 \mathrm{~K}$. At these temperatures the radiation is predominantly in the long wavelength infrared region $2-20 \mu \mathrm{m}$; the peak occurs at $10 \mu \mathrm{m}$. Provided natural and induced backgrounds are low enough, this radiation may be sensed remotely by suitable detectors. Even if RVs are made with low emissivity coatings $(\epsilon<0.1)$, RVs may still be sensed passively by collecting LWIR radiation emitted by the earth (earthshine) and subsequently scattered from the RV; the reflection coefficient is $\rho=1-\epsilon$.

Whatever the source of signal, emission or reflection, LWIR sensing of RVs can only be done above the earth's limb, i.e., above the sensible atmosphere, because of intense LWIR emissions from the earth and lower atmosphere. Sensors could be space based or based aboard aircraft which operate at altitudes above $10 \mathrm{~km}$. This minimum altitude requirement is imposed by the large amount of water vapor present in the atmosphere at lower altitudes.

Consider the sensitivity required for a candidate sensor consisting of a telescope with a 50-cm-diameter aperture viewing an $R V$ at a range $R=2 \mathrm{Mm}$. The power incident on the sensor aperture is

$$
\mathbf{P}=\pi \frac{\mathrm{d}^{2}}{4}\left[\frac{1}{\mathrm{R}^{2}}\right] \mathbf{B}_{\mathrm{s}}
$$

which for $B_{s}=60 \mathrm{~W} / \mathrm{sr}$ corresponds to $\mathbf{P}=3 \times 10^{-12}$ W. A telescope with focal length $f=5 \mathrm{~m}$ could focus this to a diffraction limited spot of radius $0.1 \mathrm{~mm}$ yielding a focal plane intensity of $94 \times 10^{-6} \mathrm{~W} / \mathrm{m}^{2}$. This is detectable with cooled focal planes and cooled optical elements. To cover a large field of view one may employ staring sensors with large mosaic focal planes, or one may use scanning sensors which have simpler focal planes with fewer elements but give only intermittent looks at a given object.

Before considering issues of tracking, resolution, and discrimination in the LWIR region, we note that under conditions of solar illumination, the passive detection of RVs can be done much more simply in the visible region. Since the incident solar flux is $1400 \mathrm{~W} / \mathrm{m}^{2}$, the power reflected from an $\mathrm{RV}$-sized object is of the order of 45 $\mathrm{W} / \mathrm{sr}$ for a reflection coefficient of $\rho \approx 0.2$. Although slightly weaker than the power in the LWIR band, this visible radiation is more easily detectable because of its shorter wavelength. In particular, room temperature sensors and small diameter optical systems can be used.

Tracking is done by repeated observations. At $\lambda=10 \mu \mathrm{m}$, a range of $2 \mathrm{Mm}$, and a telescope aperture diameter of $0.5 \mathrm{~m}$, diffraction limits spatial resolution to $40 \mathrm{~m}$. From a single passive observation one cannot determine range if the emissivity area product of the object being viewed is unknown. However, one can determine range by a series of passive observations and the knowledge that an object is in a Keplerian (elliptical) orbit.

To specify the plane of a ballistic trajectory requires two parameters, two more are required to select a unique ellipse, and a fifth parameter is needed to specify the orientation of the ellipse in the plane. A sixth parameter giving position along the ellipse will uniquely specify the trajectory. Thus a minimum of three separate looks, each of which gives two angles, are required to fix a trajectory. In practice because of errors and finite resolution effects, many more observations are required. Typically, 10-20 looks, spread over 50-100 s interval, are necessary.

\subsubsection{Discrimination}

There are a number of LWIR techniques which may be considered to discriminate RVs from other objects in ballistic orbits. The simplest is the emissivity-area 
product $\epsilon \mathrm{A}$. Once a track has been achieved and range determined, this quantity can be determined. An RV candidate should have $\epsilon \mathrm{A}=(0.2-2) \mathrm{m}^{2}$, roughly.

Other specific potential discrimination techniques were considered by the Study Group. These, however, are not discussed here because of classification issues.

\subsubsection{LWIR Decoys and Pen Aids}

It is natural to ask whether or not LWIR techniques can do the whole discrimination job. We are persuaded the answer is no and base this conclusion on the following general considerations.

First, LWIR emission/reflections from an object are solely a function of surface characteristics (area, emissivity, temperature) and are independent of internal features. Although surface area is not easily modified, emissivity is readily varied-a coat of paint, surface contamination (intentional or otherwise), etc., can greatly affect the value of $\epsilon$ in the LWIR region. This means that RVs can be expected to have a broad intrinsic spread in $\epsilon \mathrm{A}$ values, a fact which limits the value of this discriminant.

There are other possible techniques which could confuse the LWIR signatures of an object and which would make the job of discrimination difficult. These were studied in some detail by the Study Group. No discussion is given here because of classification issues. However, References 7-13 give detailed descriptions which may be helpful to the reader.

If $1 \mathrm{~kg}$ is a reasonable estimate for a decoy which would match an RV containing a 200-300 kt (kiloton) warhead, 50-100 replica decoys could be carried for each RV offloaded. We estimate that adequate volume would be available on the PBV since the density of typical decoy constituents will be less than that of typical RV components. Thus, for a single booster with a $4000 \mathrm{~kg}$ payload, one has the capability of delivering up to 1000 LWIR indiscriminable replica decoys into the mid-course battle for each booster which penetrates the boost phase defenses.

Further, one could use spherical balloons which have the same $\epsilon \mathrm{A}$ product as RVs. Balloon decoys are simple to deploy and could be made even lighter than the inflatable, replica decoys mentioned above, namely about $0.5 \mathrm{~kg}$. Balloons would be effective traffic decoys and would stress the LWIR data processing of the defense.

To utilize a large number of decoys, the offense may have to use a more complex deployment mode than sequential dispensing from a single bus. A minibusstarburst approach is one possibility. Here some large number of minibuses, say 30, are deployed early in the post-boost phase. After exiting the atmosphere each minibus subsequently deploys 30 or so objects in rapid sequence with 0 or 1 of these objects being an RV. While this may be considerably more complicated than current deployments, it must be compared with the many difficult tasks which must be solved in building an effective defense. In addition, the offense has real opportunities for testing and perfecting decoy deployment mechanisms; the defense must rely upon simulation for much of its performance evaluation.

Aerosol clouds are another possible tool for the LWIR countermeasure engineer. Aerosols are particle clouds with the particle diameters chosen to be about that of the IR radiation of interest. Such particles have large Mie scattering cross sections and so scatter earthshine very effectively. If one embeds RVs and crude balloon decoys inside aerosol clouds, the defense works against a greatly reduced signature difference provided the net scattered radiation from the cloud is much stronger than that from the $\mathrm{RV} /$ decoy. The deployment of aerosol clouds must be done with precision. If the initial dispersal velocity is too large, the cloud will separate too early; if the cloud particles clump and do not separate at all, the effective LWIR scattering cross section will be small.

Given the poor prognosis for LWIR discrimination in the face of even simple decoy/pen-aid choices, passive thermal sensing is likely to be limited to the establishment of coarse tracks of all objects in mid-course, followed by simple bulk filtering and then hand over to sophisticated discrimination methods. Even coarse track information is valuable in reducing the areas which must be swept by other sensors/discriminators. Nonetheless, data processing loads for the LWIR systems will be great because of the enormous total number of objects possible in a major attack and the fact that all objects must be tracked for the duration of mid-course in order to minimize ambiguities coming from crossing tracks, closely spaced objects, etc.

\subsection{MID-COURSE: RADAR TECHNIQUES}

The mid-course phase is very long compared with all other phases. In principle the defense radars should be able to exploit this fact, but in practice mid-course radars (which have to be in satellite orbits) may have a difficult time because the offense has a maximum number of objects deployed, including very light and unsophisticated traffic decoys and possibly additional metal chaff and streamers.

\subsubsection{Tracking}

We consider again the numerical example of Sections 7.2.3 and 7.3.2. Namely, assume that only $10 \%$ of 2000 boosters survive boost phase and release of the order of 1000 RVs, 10000 decoys of modest sophistication and 100000 low-grade traffic objects which can be easily 
distinguished from other objects, but have to be looked at nevertheless. We again assume 2000 simple single-dish microwave radars in orbit or 100 optical radar transmitter-receivers in space. Because the threat cloud covers a larger area than the offense's launch complex we assume here an absentee ratio y of only 5 instead of 10 in our calculations, i.e., 400 useful microwave and 20 optical radars.

In this example, each single-dish microwave radar has to track accurately only $2.5 \mathrm{RVs}$ and 25 potentially threatening objects, which would not be too difficult if the packaging were right. In reality the radar has to contend with two problems: (a) there are 250 traffic decoys per radar, and (b) the 2000 radars only cover a solid angle of order $2 \times 10^{-3} \mathrm{sr}$ at any one instant and single-dish radars can be steered only relatively slowly (a few degrees per second is typical). Given the long time available during mid-course, single dish microwave radars may be able to do the job, but a plan for doing so has not been demonstrated yet. For phased-array radars, the steering is no problem, but space basing is yet to be demonstrated. If space basing of complex phased array radars turns out to be too difficult, either because of complexity or survivability, a possible alternative would be to base the phased array radars on the ground. A number of very large radars of this type have been built by both the U.S. and the U.S.S.R. Such radars would, of course, have to be defended against attack and they would have to be located well towards the north to see the early mid-course portion of the trajectory.

As discussed in Section 7.3.2, it is not clear whether agile beams for optical radars can be developed. If they can, then the question of steering is less of a problem, but the small beam size, while very useful for precision tracking is a problem for the traffic decoys. Consider first a hypothetical scan of part of the whole sky. Even with a near IR laser, one laser beam has a solid angle of only about $10^{-11} \mathrm{sr}$. With 20 optical laser radars on station, a $100 \mu$ s pulselength and an observing time of $1000 \mathrm{~s}$, such a scan would only cover $\approx 10^{-3} \mathrm{sr}$, a tiny fraction of the sky. For this reason one would not use a laser radar for acquisition, but only for precision tracking (and discrimination) of objects acquired by other, less precise, means. Thus, we only have to deal with $10^{5}$ traffic objects, but in practice it is not clear if a plan can be devised to make use of this fact. For both microwave and optical radars during mid-course, any assessment of feasibility will have to await detailed system designs.

\subsubsection{Discrimination}

High resolution optical or microwave radars can be used to image decoys and RVs in order to provide discrimination. Such radars are capable of seeing small details of construction, as well as measuring with great precision, spinning and nutation motions of the objects.
Very likely the use of replica decoys would be unattractive given such forms of discrimination.

\subsection{MID-COURSE: INTERACTIVE DISCRIMINATION METHODS}

To overcome the problems faced by passive LWIR and active radar discrimination techniques, several directed energy technologies have been suggested for use in interactive discrimination roles to provide measurements of intrinsic target characteristics, such as mass, uranium or plutonium content, etc. In this section we examine two generic interactive discrimination approaches: (i) the use of interactive methods to generate an impulse to the target, and then observing the resulting velocity change, and (ii) the use of energetic primary particles to penetrate the target, and then determining the target composition (and/or mass) by measuring the total flux and/or spectrum of the scattered secondary radiation. Among interactive approaches, we consider the use of nuclear explosions, nuclear directed energy beams, and several non-nuclear directed energy beams.

\subsubsection{Nuclear Explosions}

Perhaps the simplest interactive concept for identifying reentry vehicles in a decoy-rich environment is to detonate a nuclear explosive in the midst of the threat cloud. A single Soviet SS-18 booster is generally assumed to be capable of deploying 10 or more RVs, tens of $20 \mathrm{~kg}$ decoys (thrusted replicas, for example), and perhaps hundreds of lightweight $(1 \mathrm{~kg})$ balloon decoys. ${ }^{10,11}$ Depending on targeting details, these objects may be dispersed over a volume approximately $1000 \mathrm{~km}$ in length and a few hundred kilometers cross range at the end of the mid-course phase. This dispersion grows linearly with time after release from the bus and so is proportionally less in early mid-course. Hence, to contribute important discrimination capability, the effective range of a defense nuclear explosion should be as large as possible, but certainly no less than about $100 \mathrm{~km}$. We will consider three possible effects that might provide discrimination signatures. These are (i) impulse generation due to the expanding debris cloud, (ii) impulse generation due to $x$-ray produced surface ablation, and (iii) generation of secondary radiation by neutronproduced activation of the target.

\subsubsection{Impulse Due to Nuclear Debris}

The mass of a target object can be determined by measuring the velocity change $\Delta v_{t}$ resulting from an impulse $I$ according to $m_{t}=I A_{t} / \Delta v_{t}$, where $A_{t}$ is the effective area of the target. At a distance $R$ from the explosion the impulse resulting from the interaction of the 


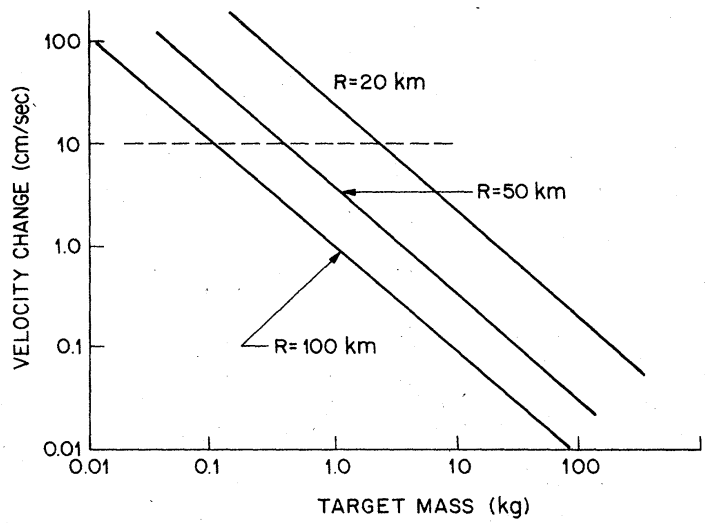

Figure 7.1. Velocity change induced by debris coupling from a $1 \mathrm{Mt}$ nuclear explosion as a function of target mass. Target cross sectional area has been taken to be $1 \mathrm{~m}^{2}$. Curves represent a gross upper bound since the debris kinetic energy has been set equal to the total yield.

energetic nuclear debris cloud with the target is given approximately by $I=m_{D}\left\langle v^{2}\right\rangle^{1 / 2} /\left(4 \pi R^{2}\right)$, where $m_{D}$ is the total debris mass and $\left\langle v^{2}\right\rangle^{1 / 2}$ is the rms velocity of the expanding debris cloud; $\left\langle v^{2}\right\rangle=2 E_{D} / m_{D}$ where $E_{D}$ is the total kinetic energy of the debris. Hence, the velocity change of the target object is given approximately by

$$
\Delta v_{t}=\frac{A_{t}}{m_{t}} \frac{\left(2 E_{D} m_{D}\right)^{1 / 2}}{4 \pi R^{2}} .
$$

In order to develop order-of-magnitude estimates, we will assume a one megaton (Mt) explosive yield, the energy of which is completely transferred into the expanding debris cloud of $300 \mathrm{~kg}$ total mass (a gross upper bound). We will also neglect any deceleration of the debris mass due to interactions with the atmosphere. (This effect will be important for altitudes of a few hundred kilometers or less.) The $\mathrm{RV} /$ decoy cross sectional area is assumed to be $1 \mathrm{~m}^{2}$. With these assumptions, the velocity change is graphed as a function of target mass in Figure 7.1 for $20 \mathrm{~km}, 50 \mathrm{~km}$, and 100 $\mathrm{km}$ ranges.

It is generally assumed that a velocity change of 10 $\mathrm{cm} / \mathrm{s}$ could provide some discrimination capability. For our assumptions, this velocity change is realized only at very short ranges $(20-30 \mathrm{~km})$ for light $(1 \mathrm{~kg})$ decoys. To achieve this velocity change at a range of $200 \mathrm{~km}$ would require an order-of-magnitude increase in both the explosive energy (10 Mton) and debris cloud mass (3000 $\mathrm{kg}$ ). Note also that for a relative velocity change of 10 $\mathrm{cm} / \mathrm{s}$, the maximum deviation of an object from a ballistic trajectory would be only $150 \mathrm{~m}$ over the total mid-course flight time of $25 \mathrm{~min}$, i.e., lightweight objects will not simply disappear from a sensor's field of view.

\subsubsection{Impulse Due to X-Ray Produced Ablation}

A large fraction of the energy of a nuclear explosion appears in the form of energetic $\mathrm{x}$ rays in a spectral range of 1-10 keV. Such photons are strongly absorbed by matter, and at large enough fluence, can cause explosive vaporization (ablation) at the surface of a target since the $\mathrm{x}$-ray pulse length is much shorter than thermal relaxation times. The resulting impulse can then be used to determine the target's mass.

From Chapter 6, the $\mathrm{x}$-ray-induced ablative impulse is given approximately by

$$
\mathrm{I}=\mathrm{m}_{\mathrm{a}}\left\langle\mathrm{v}^{2}\right\rangle^{1 / 2} / \mathrm{A}_{\mathrm{t}}=\left\{\frac{2 \mathrm{~F}_{0}}{\mu}\left[1-\frac{\epsilon}{\mathrm{F}_{0} \mu}\right) \ln \left(\mathrm{F}_{0} \frac{\mu}{\epsilon}\right)\right\}^{1 / 2},
$$

where $F_{0}$ is the incident $x$-ray fluence at the target surface, $\mu$ is the mass absorption coefficient, and $\epsilon$ is the energy per unit mass required for melting. Consequently, the minimum fluence required to produce ablative impulse is $\mathrm{F}_{\min }=\epsilon / \mu$. In general, the absorption coefficient lies in the range of $10^{3}-10^{4} \mathrm{~cm}^{2} / \mathrm{g}$, while the range of melt energies is $10^{3}-10^{4} \mathrm{~J} / \mathrm{g}$ for one kilovolt $\mathrm{x}$ ray. Using these parameter ranges, impulse is graphed as a function of incident fluence in Figure 7.2.

The ablative impulse will produce target motion according to $\Delta \mathrm{v}_{\mathrm{t}}=\mathrm{IA}_{\mathrm{t}} / \mathrm{m}_{\mathrm{t}}$. To provide a mass measurement that is reasonably material independent, the $\mathrm{x}$-ray fluence should exceed the maximum available material-dependent ablative threshold by a factor of several, i.e., a few tens of joules per square centimeter (see Figure 7.2). Assuming a one megaton explosion with all of the energy appearing as $\mathrm{x}$ rays, this fluence could be provided at ranges up to a few tens of kilometers.

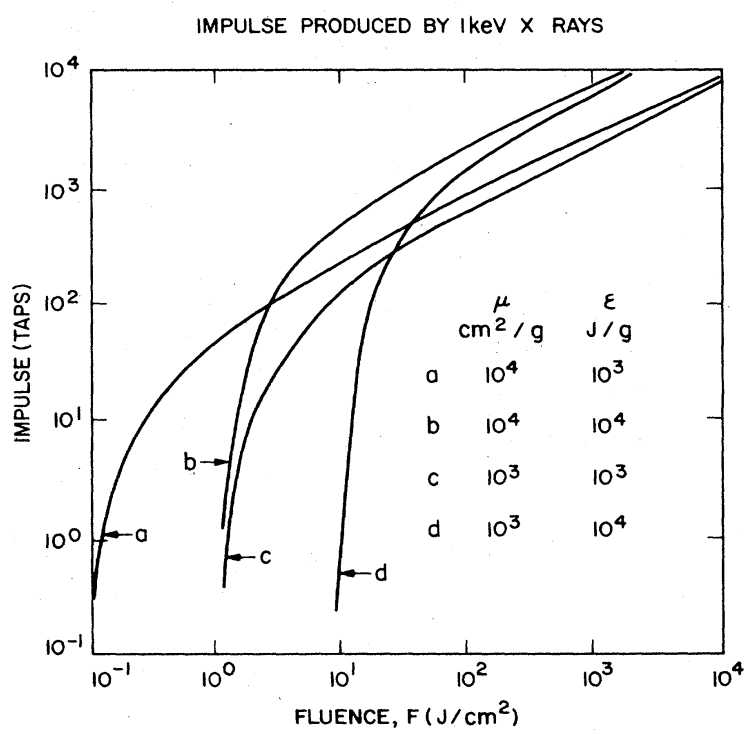

Figure 7.2. Impulse produced by $\mathrm{x}$-ray ablation caused by ablation as a function of fluence. A $1 \mathrm{~kg}$ target with cross sectional area $1 \mathrm{~m}^{2}$ is assumed. 


\subsubsection{Neutron Activation by a Nuclear Explosion}

In addition to explosive debris and the copious $x$-ray emission, a nuclear explosion generates a large neutron fluence which could be used to activate a target. The resulting decay radiation (secondary neutrons or gamma rays), if sufficiently intense, might be used to infer target mass, in addition to indicating the presence of fissionable material.

For a typical thermonuclear explosion the number of energetic neutrons (capable of producing fast fissions in ${ }^{238} \mathrm{U}$ ) is approximately $10^{26}$ multiplied by the explosive yield $\mathrm{Y}$ in megatons. Hence, the neutron fluence $\mathrm{F}$ at a target range $R_{t}$ assuming isotropic emission is given by

$$
\mathrm{F}=10^{26} \frac{\mathrm{Y}}{4 \pi \mathrm{R}_{\mathrm{t}}^{2}}
$$

and the total number of secondary particles or photons emitted by the target can be calculated according to

$$
S_{t}=F_{t} k f
$$

where $A_{t}$ is the effective target area, $k$ is the probability of a neutron interaction with the target, and $f$ denotes the number of secondary radiations produced per neutron interaction. Finally, the number of secondaries detected at a distance $R_{D}$ from the target is given by

$$
\mathrm{N}_{\mathrm{D}}=\mathrm{S}_{\mathrm{t}} \mathrm{A}_{\mathrm{D}} \frac{\epsilon_{\mathrm{D}}}{4 \pi \mathrm{R}_{\mathrm{D}}^{2}}
$$

where $A_{D}$ is the effective detector area, $\epsilon_{D}$ is the detection probability, and $R_{D}$ is the distance between the target and the detector.

In general only a small number of neutrons or gamma rays are released per fission event; hence, we will assume that the number of secondaries per interaction which escape the target will be of order unity. We will also assume that the fissionable material in the target has an effective area of 0.5 square meters, an average thickness of a few centimeters, and thus weighs about 200 $\mathrm{kg}$. Assuming an average fast fission cross section of one barn $\left(10^{-24} \mathrm{~cm}^{2}\right)$, the interaction probability is approximately $10 \%$. Further assuming a one megaton yield, a detector area of one square meter, and a detection probability of unity, the number of secondary radiations detected is given by

$$
\mathrm{N}_{\mathrm{D}} \approx 3 \times \frac{10^{22}}{\mathrm{R}_{\mathrm{t}}^{2} \mathrm{R}_{\mathrm{D}}^{2}}
$$

To assure reasonable statistics we require at least 100 detected secondary particles; this implies that the product
$R_{t}^{2} R_{D}^{2}$ must be less than $3 \times 10^{20} \mathrm{~m}^{4}$. Assuming that $R_{t}=R_{D}=R$, then the maximum useful range is about 100 $\mathrm{km}$. However, simple geometric considerations indicate the practical difficulties of implementing such a technique. For example, if both the explosion-target distance and the target-detector distance are of the order of $100 \mathrm{~km}$, then the explosion-detector distance will also be approximately $100 \mathrm{~km}$, and the number of explosion-produced neutrons arriving directly at the detector will be approximately $10^{11} / \mathrm{m}^{2}$, compared to $10^{2} / \mathrm{m}^{2}$ arising from the target. Consequently, the detector must be capable of extremely high rejection ratios in order to identify the target signal out of the nuclear background. The detector problem is further complicated because all the targets in the range of the explosion are irradiated at approximately the same time, and there is very little opportunity to use detector time gating to assist the identification process. As a consequence, the angular resolution of the detector would have to be very good in order to distinguish individual targets. All factors considered, the use of neutron activation resulting from a nuclear explosion does not appear practical as a discrimination technique.

\subsubsection{X-Ray Lasers}

The results of the previous section indicate the difficulty of trying to use undirected beams for discrimination in the vastness of space. In this section we assume that nuclear directed energy techniques, specifically x-ray lasers, can eventually be developed, and we examine their potential for use in interactive discrimination.

Quick estimates indicate that the $x$-ray laser has a limited utility as a discrimination tool to sweep a large volume of space. For example, assuming that the crosssectional area of the threat cloud is nominally $100 \mathrm{~km}$ by $100 \mathrm{~km}$, the total amount of $\mathrm{x}$-ray fluence required to cause surface ablation (at $30 \mathrm{~J} / \mathrm{cm}^{2}$ ) is about $3 \times 10^{15} \mathrm{~J}$. Nuclear explosion yields necessary for this fluence can be calculated once the $\mathrm{x}$-ray lasing efficiency is known (see, for example, Figure 3.32).

A more promising possibility is to individually target many objects in the threat cloud using multiple beams from a single $x$-ray laser device. The single beam $x$-ray fluence produced by a nuclear yield $Y$ at a distance $R$ is given by

$$
\mathrm{F}=\frac{\eta \mathrm{Y}}{\mathrm{N} \pi(\mathrm{R} \Delta \theta)^{2}}
$$

where $\eta$ denotes the overall conversion efficiency of nuclear yield into directed x-ray energy (see Section 3.5 and Figures 3.31 and 3.32), $\Delta \theta$ is the divergence of a single $\mathrm{x}$-ray beam, and $\mathbf{N}$ is the total number of beams generated. Consider, for example, a range of divergence from $1 \mathrm{mrad}$ to $10 \mu \mathrm{rad}$, a nominal range of $500 \mathrm{~km}$, and 
200 target objects. The nuclear yield required to produce $30 \mathrm{~J} / \mathrm{cm}^{2}$ will be in excess of $10000 / \eta$ tons for the 1 mrad divergence case, and will be in excess of $1 / \eta$ tons for the $10 \mu \mathrm{rad}$ divergence case, where $\eta$ is the overall conversion efficiency as described above (see also Section 3.5 on $x$-ray lasers and Figures 3.31 and 3.32). With the same parameters as in the first example, if the range were to be increased from $500 \mathrm{~km}$ to $5000 \mathrm{~km}$, nuclear yield 100 times that in the first example would be necessary (for the two respective cases of divergences). Hence, if sufficiently high-conversion efficiencies and sufficiently narrow divergences can be achieved, it is conceivable that $\mathrm{x}$-ray lasers could be used in an interactive discrimination role; however, to be cost effective, their use would probably require at least a few hundred independently targetable laser rods per nuclear device (in order to interrogate all of the targets from one booster, for example), and as many available $\mathrm{x}$-ray laser platforms as there are missiles which survive the boost and post-boost phases.

\subsubsection{Directed Energy Beams}

While nuclear explosions afford at best limited discrimination capability, the collateral effects could adversely affect other defensive elements. Hence, there is considerable interest in examining non-nuclear directed energy technologies for their potential as target discriminators. In this section we consider three possibilities in some detail. These include target activation by neutral particle beams, bremsstrahlung generation resulting from electron beam irradiation, and laser-induced impulse generation and target destruction. Concepts based on target activation by acceleratorgenerated neutrons and charge-neutralized ion beams are other possibilities but these approaches are severely hampered by beam divergence limitations and will not be considered here.

\subsubsection{Neutral Particle Beams}

Neutral particle beam technologies have been discussed in detail in Chapter 4. Here, we note that 100 $\mathrm{mA}$ negative hydrogen ion sources exist, and that estimates of stripping using atomic collisions indicate that a lower bound for the beam divergence angle is given approximately by

$$
\Delta \theta \approx \frac{20}{\sqrt{\mathrm{EA}}} \times 10^{-6}
$$

where $\mathrm{E}$ is the particle kinetic energy in $\mathrm{MeV}$, and $\mathrm{A}$ is the atomic mass number.

Since the beam will not be a single short burst it is more appropriate to consider the secondary radiation detection rate $\dot{N}_{D}$ given by

$$
\dot{\mathrm{N}}_{\mathrm{D}}=\dot{\mathrm{S}} \mathrm{A}_{\mathrm{t}} \frac{\mathrm{kf}}{\pi\left(\mathrm{R}_{\mathrm{t}} \Delta \theta\right)^{2}} \cdot \mathrm{A}_{\mathrm{D}} \frac{\epsilon_{\mathrm{D}}}{4 \pi \mathrm{R}_{\mathrm{D}}^{2}}
$$

where $\dot{\mathrm{S}}$ is the neutral beam current, i.e., the number of beam atoms crossing a plane perpendicular to the beam path per unit time, and $k$ and $f$ now denote the probability of a proton interaction with the target and the number of secondary radiations produced per incident particle.

As an example of this technique we will assume a $100 \mathrm{MeV}$ beam of hydrogen atoms, $\Delta \theta \approx 2 \mu \mathrm{rad}$, with an effective dc current of $100 \mathrm{~mA}$. We assume that the stripping cell is $50 \%$ effective. Also, since $100 \mathrm{MeV}$ protons have a range of $\approx 10 \mathrm{~g} / \mathrm{cm}^{2}$ in almost any material, we assume that the protons (incident neutral hydrogen atoms will be quickly stripped in the outer layers of the heat shield) will penetrate into the high atomic number materials in the warhead and undergo nuclear collisions which result in the production of secondary neutrons and gamma rays. Although the cross sections and the number of secondaries produced per collision are both energy dependent, if the protons have energies of at least a few tens of $\mathrm{MeV}$ when they enter the high- $\mathrm{Z}$ material, the relevant nuclear cross sections are generally of order $0.1-1 \mathrm{~b}$ and the number of secondaries per collision is of order unity. Hence, we take the product of $\mathrm{kf}$ also to be of order unity. As before, we assume that $A_{D}=1 \mathrm{~m}^{2}$, and $\epsilon_{D}=1$. Then

$$
\dot{\mathrm{N}}_{\mathrm{D}} \approx 10^{27} \frac{\mathrm{A}_{\mathrm{t}}}{\mathrm{R}_{\mathrm{t}}^{2} \mathrm{R}_{\mathrm{D}}^{2}}
$$

Assuming that $\mathbf{R}_{t}=\mathbf{R}_{\mathrm{D}}=\mathrm{R}=100 \mathrm{~km}$, then the radius of the beam is $20 \mathrm{~cm}$, and the effective target area is $0.1 \mathrm{~m}^{2}$. Hence, the rate of detected secondary particles is approximately $2 \times 10^{6} / \mathrm{s}$, which is very much higher than the background rate due to cosmic particle interactions $(\approx 1 / \mathrm{s})$. On the basis of these simple estimates, the neutral particle beam appears to offer promise for discrimination applications.

\subsubsection{Laser-Guided Electron Beams}

Laser-created ionization channels may permit the use of intense electron beams for exoatmospheric applications. The physics of such propagation modes are examined in more detail in Chapter 4. Here, we estimate the bremsstrahlung produced when an electron beam interacts with an $\mathbf{R V}$.

Note that $20 \mathrm{MeV}$ and $100 \mathrm{MeV}$ electrons will penetrate 10 and $50 \mathrm{~g} / \mathrm{cm}^{2}$ of almost any material, respectively. For very thin targets, such as lightweight balloons, the bremsstrahlung will be almost entirely forward-directed; however, for thick targets the internal path of an energetic electron will quickly become random, and the bremsstrahlung-generating source can be modeled 
approximately as an isotropic $10 \mathrm{MeV}$ electron source at a depth of approximately $5 \mathrm{~g} / \mathrm{cm}^{2}$. The relative importance of ionization or radiation processes in the electron stopping power depends importantly on the atomic number of the material; the two processes are approximately equal when $\mathrm{EZ}=800$ with $\mathrm{E}$ in $\mathrm{MeV}$. Although the bremsstrahlung region dominates for high- $Z$ targets, to be conservative we assume that only one-tenth of the electron energy will emerge from the target in a single $1 \mathrm{MeV}$ photon. We further assume that this bremsstrahlung x-ray source is approximately isotropic in the backscatter direction, although it typically will be slightly peaked at $180^{\circ}$ because of target absorption.

Since the propagation mode is that of a self-pinched electron beam with a radius of a few centimeters depending on the beam emittance, there is no electron beam divergence as it propagates to the target; rather, there will be erosion processes that decrease the pulse length linearly with distance. The modeling indicates the loss of a few $\mu$ s to about $1 \mu \mathrm{s}$ of beam pulse per $100 \mathrm{~km}$ for a $20 \mathrm{MeV}$ to $100 \mathrm{MeV}$ beam energy, respectively. Under these conditions the secondary bremsstrahlung $\mathrm{x}$-ray detection rate is approximately given by

$$
\dot{\mathrm{N}}_{\mathrm{D}}=\dot{\mathrm{S}} \mathrm{A}_{\mathrm{D}} \frac{\epsilon_{\mathrm{D}}}{4 \pi \mathrm{R}_{\mathrm{D}}^{2}} \mathrm{H}\left(\mathrm{ct}_{\mathrm{p}} / \mathbf{R}_{\mathrm{t}}-\alpha\right)
$$

where $\mathbf{H}$ denotes the Heaviside step function, $\alpha$ is the (dimensionless) erosion rate, $c$ is the speed of light, and $t_{p}$ is the electron pulse width. Assuming $A_{D}=1 \mathrm{~m}^{2}, \epsilon_{D}=1$, $\alpha \approx 0.003$, and a $100 \mathrm{MeV}$ electron beam of current I (in $\mathrm{kA}$,

$$
\dot{\mathrm{N}}_{\mathrm{D}}=5 \times \frac{10^{20}}{\mathrm{R}_{\mathrm{D}}^{2}} \mathrm{H}\left(3 \times 10^{8} \mathrm{t}_{\mathrm{p}} / \mathrm{R}_{\mathrm{t}}-0.003\right) \times(\mathrm{I} / 1 \mathrm{kA})
$$

For $\mathbf{R}_{\mathbf{D}}=\mathbf{R}_{\mathbf{t}}=\mathbf{R}(\mathbf{R}$ in $100 \mathrm{~km})$, we have

$$
\begin{aligned}
\dot{\mathrm{N}}_{\mathrm{d}}= & 5 \times 10^{20} \mathrm{H}\left(3 \times 10^{8} \mathrm{t}_{\mathrm{p}} / \mathrm{R}-0.003\right) \\
& \times(\mathrm{I} / 1 \mathrm{kA}) \times(\mathrm{R} / 100 \mathrm{~km})^{-2} .
\end{aligned}
$$

Hence, if the pulse length is sufficient for the beam to reach the target, the $\mathrm{x}$-ray detection rate is enormous, namely $5 \times 10^{9}$ detected photons per second for $\mathbf{I}=100 \mathrm{~A}$ and $\mathbf{R}_{\mathrm{D}}=\mathbf{R}_{\mathrm{t}}=100 \mathrm{~km}$, and $5 \times 10^{8}$ detected photons per second for $I=1 \mathrm{kA}$ and $\mathbf{R}_{\mathrm{D}}=\mathbf{R}_{\mathrm{t}}=1000 \mathrm{kM}$.

\subsubsection{Laser-Induced Impulse and Target Destruction}

Photons from high energy chemical, excimer, or free electron lasers can be used to generate ablation at a target's surface thereby inducing target motion according to $\Delta \mathrm{v}_{\mathrm{t}}=\mathrm{IA}_{\mathrm{t}} / \mathrm{m}_{\mathrm{t}}$. The basic interaction phenomena depend on several factors, including laser wavelength, pulse duration, and intensity, and the target material and surface preparation; the details of these processes are discussed in Chapter 6. In general, impulse is most efficiently generated by short pulse $(\lesssim 1 \mu \mathrm{s})$, short wavelength ( $\leqslant 1 \mu \mathrm{m}$ ) lasers; at fluence levels of several tens of joules per square centimeter, the coupling coefficient is relatively material independent, as indicated in Figure 6.12, and is a few dyne $\mathrm{s} / \mathrm{J}$.

Since the resultant velocity change depends on the area undergoing ablation, the beam spot size on target is an important factor. For example, because of the ablative threshold effect, the largest impulses will be produced when the spot size is a minimum. However, since the velocity change depends on the product of the impulse and the area on target, once the impulse saturates, it is best to increase the beam spot size. Also, in order to produce a velocity change of the target as a whole (rather than exciting internal vibration modes) the spot size should be a significant fraction of the target cross sectional area. For these reasons it seems sensible to require a laser spot size at the target of the order of $10^{3} \mathrm{~cm}^{2}$. Consequently, it appears that several tens of $\mathrm{kJ}$ of laser energy will produce sufficient velocity changes to permit discrimination of lightweight $(\sim 1 \mathrm{~kg})$ balloon decoys $(\Delta \mathrm{v} \sim 10 \mathrm{~cm} / \mathrm{s})$. The required mirror diameter $\mathrm{D}_{\mathrm{m}}$, assuming a diffraction-limited beam, is given by

$$
\frac{\lambda}{\mathrm{D}_{\mathrm{m}}} \approx \frac{\mathrm{D}_{\mathrm{s}}}{\mathrm{R}}
$$

For a photon wavelength of $1 \mu \mathrm{m}$ and ranges of order $100 \mathrm{~km}, \mathrm{D}_{\mathrm{m}} \approx 0.5 \mathrm{~m}$.

Compared with impulse-induced motion, the destruction of lightweight decoys with high energy lasers will require substantially more energy per target. For example, vaporization generally requires $10^{3}-10^{4} \mathrm{~J} / \mathrm{g}$. Hence, 1-10 MJ will be needed to destroy a $1 \mathrm{~kg}$ decoy. The advantage, of course, is that the decoy disappears from the threat cloud, although the energy required to destroy a few hundred lightweight decoys substantially exceeds the energy required to destroy a booster.

\subsubsection{Systems Considerations}

The preceding section identified several potentially attractive interactive discrimination concepts which could be used in missile defense systems. In our previous discussion we attempted to provide meaningful comparisons by using only very simple systems considerations, e.g., nominal interrogator-target and target-detector ranges of $100 \mathrm{~km}$. In this section we will broaden our scope in order to evaluate how the various directed energy technologies might be used in a systems sense and to illustrate the key technical tradeoffs. 


\subsubsection{Basing Modes}

In general, discriminator platforms may be used in two important basing modes: a space-based constellation, and/or a rocket-borne pop-up mode. Each approach has a few important advantages and disadvantages. With a space-based system the entire mid-course is available for discrimination. Moreover, if sufficiently bright, directed energy beams might also be used for kill in the boost and post-boost phases. However, the directed energy platforms must be survivable, and the beams must have sufficient range to keep the constellation size to a manageable number of satellites. Pop-up platforms, on the other hand, offer enhanced survivability and permit shorter range beam performance, although the mid-course interrogation time will be reduced and the DE technology itself must be capable of lightweight, compact construction. Such a system would be better suited for use against a salvo attack of time-urgent military targets, and high density population centers.

Because of the relatively low beam kinetic energy requirement, as well as the upper altitude restriction, the laser-guided electron beam approach appears to be bestsuited for operating in a pop-up basing mode. For the case of laser interrogators, if pop-up operation were desired the laser itself could be ground based, with the engagement mirrors being the only pop-up elements.

For NPB energies of 100-200 MeV, the large accelerator platform weight probably requires orbital basing (as opposed to pop-up mode). While the lower altitude restriction (see Chapter 4 ) will limit the available engagement time against depressed trajectories, this fact may not be a serious consideration since lightweight decoys cannot be deployed at low altitudes because of atmospheric drag. ${ }^{14}$ (See also Sec. 2.3.4.)

If lasers are used for discrimination either spacebasing or ground-basing with orbiting mirrors are possible approaches.

\subsubsection{Discrimination Signature, Energy, and Range}

Each interactive discriminator candidate has a unique set of operational characteristics. In this section we will illustrate these differences by examining the variation of the required energy and the strength of the discrimination signal with device platform-target range, and target-detector range.

For the NPB the approximate signal strength is given by Equation (7.11). As before, we assume that $k f \epsilon_{D} A_{D}=1 \mathrm{~m}^{2}$, and that $R_{t}=R_{D}=R$ (i.e., the detector is located on the accelerator platform), in which case

$$
\dot{\mathrm{N}}_{\mathrm{D}}=\dot{\mathrm{S}} \frac{\mathrm{A}_{\mathrm{t}}}{4 \pi^{2}(\Delta \theta)^{2} \mathbf{R}^{4}}
$$

We now examine two particular cases: deuterium-E
$=200 \mathrm{MeV}, \mathrm{A}=2$, and $\Delta \theta=10^{-6} \mathrm{rad}$; and hydrogen-E $=100 \mathrm{MeV}, \mathrm{A}=1$, and $\Delta \theta=2 \times 10^{-6}$. In Figure 7.3 we graph the total NPB energy required to give a total detected secondary particle count of 1000 as a function of range. For ranges of order $1000 \mathrm{~km}$ or longer, each target interrogation would require in excess of $1 \mathrm{MJ}$, with the energy scaling as $R^{4}$; i.e., for a range of $2000 \mathrm{~km}$, approximately $20 \mathrm{MJ}$ per target would be required. Assuming 10 decoys per RV, interrogation of a single SS-18 booster load (100 objects) would require a total NPB energy of 2 GJ at this range. In order to reduce this energy requirement, we now assume that the NPB accelerator and the detector are located on different platforms, and that the target-detector range is fixed at $500 \mathrm{~km}$. As illustrated in Figure 7.3 this approach would limit the energy required per target to several hundred kilojoules at a range of $1000 \mathrm{~km}$, and the energy would scale only as $R_{t}^{2}$ at longer ranges, i.e., several megajoules at $3000 \mathrm{~km}$. Assuming an average negative ion current of $100 \mathrm{~mA}$ in the accelerator, and a $50 \%$ efficient stripper cell, the total interrogation time at $1000 \mathrm{~km}$ required to accumulate the 1000 detected secondaries is $30-60 \mathrm{~ms}$. This parameter also scales as $\mathbf{R}_{\mathbf{t}}^{2}$ for longer ranges.

As an example calculation for this NPB operating mode we imagine a 1000 booster attack, with each booster capable of off-loading 100 objects, for a total threat number of $10^{5}$. The available discrimination time will be about $1000 \mathrm{~s}$, implying a required discrimination rate of about 100 objects per second. For an average interrogation range of $1500 \mathrm{~km}$ the interrogation time per object is $100 \mathrm{~ms}$, implying an allowable retarget rate of 10 Hz. Hence, there must be about 10 NPB platforms in the discrimination battle space. These estimates suggest a necessary constellation size of about 100 accelerator

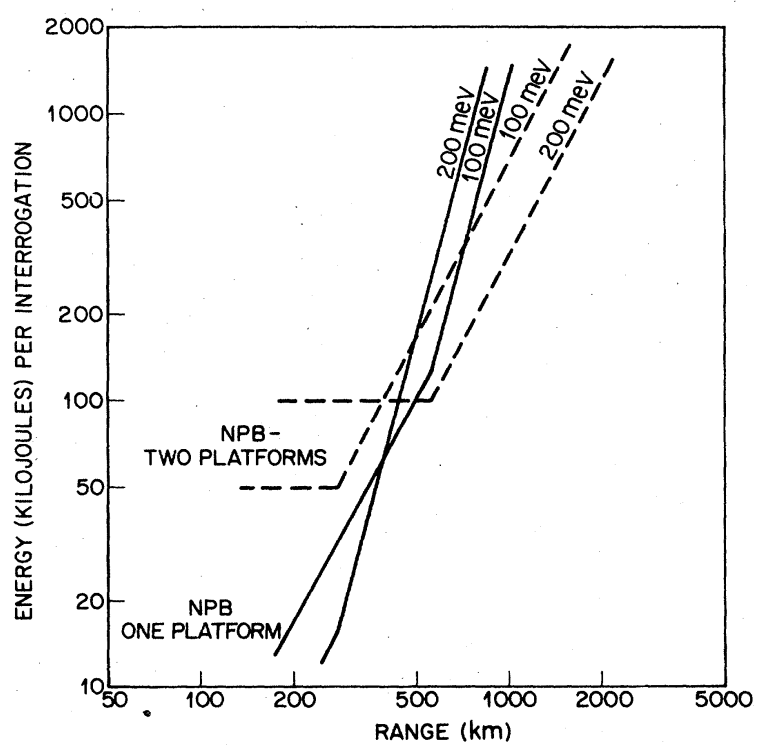

Figure 7.3. Energy per interrogation as function of range for a NPB discriminator. 
platforms at an orbital altitude of a few thousand kilometers, plus about 1000 detector platforms at an orbital altitude of about $1000 \mathrm{~km}$. The total amount of NPB energy required would be $100 \mathrm{GJ}$, with an average power consumption of $100 \mathrm{MW}$. The average beam power consumption for a platform in the battle would be $10 \mathrm{MW}$, with a maximum energy consumption per NPB platform of $10 \mathrm{GJ}$.

For a case of the laser-guided electron beam discriminator the situation is considerably different. We again consider two illustrative cases: (1) a $20 \mathrm{MeV}$ electron beam, and (2) a $100 \mathrm{MeV}$ electron beam system. We assume in both cases that the maximum available pulselength (due to possible instability growth, gross channel motion, etc.) is limited to $10 \mu \mathrm{s}$. From the analyses of the previous section, in the first case the total beam erosion rate is minimized at about $3 \mu$ s per $100 \mathrm{~km}$ of propagation for a beam current of less than $1 \mathrm{kA}$. At $100 \mathrm{MeV}$, the minimum erosion rate is approximately 1 $\mu$ s per $100 \mathrm{~km}$ at $1 \mathrm{kA}$. Hence, the maximum interrogation ranges for the two cases are about $300 \mathrm{~km}$ and $1000 \mathrm{~km}$, respectively.

The secondary bremsstrahlung $\mathrm{x}$-ray detection rate is approximately given by Equation (7.13). Assuming that $\epsilon_{\mathrm{D}} \mathrm{A}_{\mathrm{D}}=1 \mathrm{~m}^{2}$, and that the detector is co-located on the accelerator platform,

$\dot{\mathrm{N}}_{\mathrm{D}}=2.5 \times \frac{10^{20}}{\mathrm{R}^{2}} \mathrm{H}\left(3 \times 10^{8} \mathrm{t}_{\mathrm{p}} / \mathrm{R}-0.01\right) \quad(20 \mathrm{MeV})$

and

$\dot{\mathrm{N}}_{\mathrm{D}}=5 \times \frac{10^{20}}{\mathrm{R}^{2}} \mathrm{H}\left(3 \times 10^{8} \mathrm{t}_{\mathrm{p}} / \mathrm{R}-0.0024\right) \quad(100 \mathrm{MeV})$

If the beam reaches the target, the secondary detection rate is very high $\left(10^{3}-10^{4}\right.$ counts per microsecond), implying that the time required to accumulate 1000 counts is usually insignificant compared to the pulse length lost in propagating to the target. Hence, both the interrogation pulse time and the energy per interrogation are essentially determined by the beam erosion rate, and must scale linearly with range, as shown in Figure 7.4. For ranges of a few hundred kilometers, associated with a nominal $20 \mathrm{MeV}$ pop-up discriminator used in late midcourse, the required energy per discrimination is about $100 \mathrm{~kJ}$, implying a total energy of $10 \mathrm{MJ}$ for one SS-18 booster load (assuming 10 decoys per RV). Since the system is pop-up and the propagation physics limit the operational altitudes, the available engagement time is limited to the order of $100 \mathrm{~s}$, and the required repetition

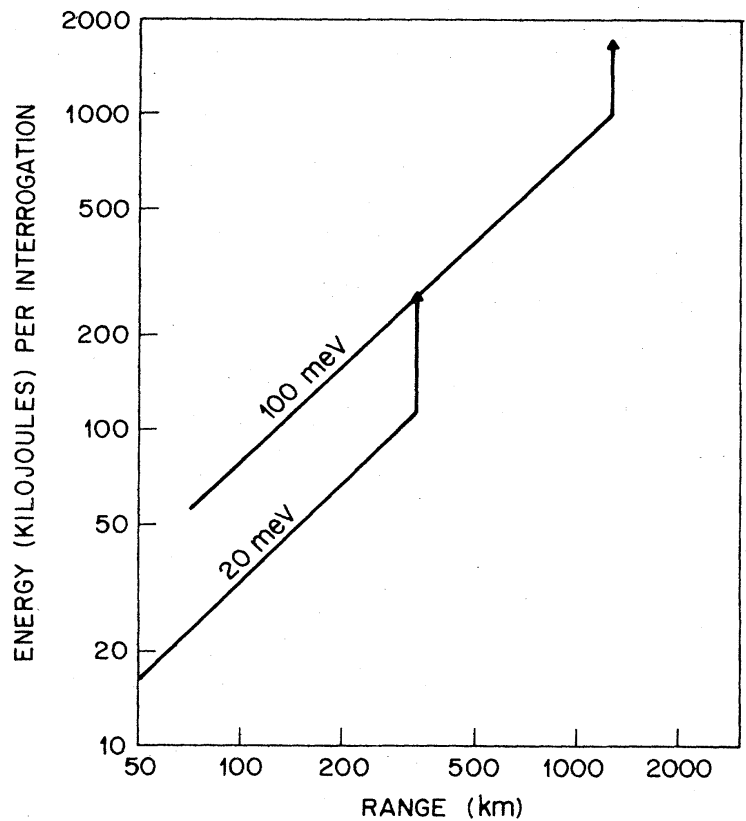

Figure 7.4. Energy per interrogation as a function of range for a laser-guided electron beam discriminator.

rate for interrogating the 100 objects of a single booster would be about $1 \mathrm{~Hz}$. The total time in which the pop-up discriminator is in the correct altitude band is probably several minutes, so that a single platform might be used to interrogate the objects from more than one booster; however, the total number of shots per platform as well as the repetition rate, would necessarily increase. Approximately 1000 such pop-up platforms would be needed to interrogate the objects of 1000 boosters, assuming $200 \mathrm{~km}$ propagation range to provide sufficient area coverage.

For the second case, which might be used as a space-based discriminator, the total energy required for interrogation of a single object at a range of $1000 \mathrm{~km}$ is almost $1 \mathrm{MJ}$. To achieve longer propagation ranges would require somewhat higher kinetic energies, and the total energy requirement would increase almost proportionally. For these ranges a few hundred discriminators at an altitude of about $400 \mathrm{~km}$ would be required. The major limitation is that the available engagement time is relatively short (a few hundred seconds out of $\approx 1500 \mathrm{~s}$ ) because of the upper altitude restriction, especially for lofted trajectories. For only modest increases in beam energy and pulse length, lethal fluences could be delivered to those targets identified as reentry vehicles.

For high energy lasers used as discriminators, fluences of several tens of joules per $\mathrm{cm}^{2}$ over areas of $1000 \mathrm{~cm}^{2}$, or several tens of kilojoules per target, are probably required. Assuming a diffraction-limited beam, the relationship between the required mirror diameter, the range, the beam spot size at the target, and the laser wavelength, is given by Equation (7.16). Hence, 
depending on wavelength the discriminating fluence need be no greater than several tens of kilojoules, regardless of range, with the restrictions being due to practical mirror considerations. For example, at $0.35 \mu \mathrm{m}$ the mirror diameter required to deliver $50 \mathrm{~kJ}$ to a $1000 \mathrm{~cm}^{2}$ area at a range of $100 \mathrm{~km}$ is only $20 \mathrm{~cm}$; however, the energy loading at the mirror would exceed $150 \mathrm{~J} / \mathrm{cm}^{2}$. At present, the criterion for mirror damage at these wavelengths is of the order of magnitude of $10 \mathrm{~J} / \mathrm{cm}^{2}$. At the other extreme, for a $10 \mu \mathrm{m}$ wavelength and an engagement range of $3000 \mathrm{~km}$, the required mirror diameter is almost $180 \mathrm{~m}$ !

We now illustrate two more practical examples, assuming fixed mirrors without adaptive optics. In the first case, we hypothesize a ground-based laser operating at $1 \mu \mathrm{m}$ in conjunction with pop-up mirrors. The desired range of operation is assumed to be $500 \mathrm{~km}$, and we require sufficient laser energy to discriminate at all shorter ranges. Accordingly, the focal length should be chosen to provide a $1000 \mathrm{~cm}^{2}$ beam cross-section area (waist) at 250 $\mathrm{km}$. The required mirror size is then about $1.5 \mathrm{~m}$. The area of the beam at $500 \mathrm{~km}$ range will be about $4000 \mathrm{~cm}^{2}$ and will scale quadratically with increased range. The total required beam energy will scale linearly with the beam cross-sectional area at the target range, as illustrated in Figure 7.5. Assuming a mirror damage threshold criterion of $10 \mathrm{~J} / \mathrm{cm}^{2}$, the maximum beam energy is about $180 \mathrm{~kJ}$ which would allow discrimination at the nominal $500 \mathrm{~km}$ range. Hence, a few hundred kilojoules per target would be required over the few hundred kilometer range of interest, and several megajoules would be necessary for interrogating one booster load of objects. Assuming $500 \mathrm{~s}$ for the available engagement time and a retarget time of $1 \mathrm{~s}$, approximately 500 objects ( 5 booster payloads) could be interrogated by one platform. The energy consumption would correspond to a few hundred megajoules in $500 \mathrm{~s}$ or $0.5-1 \mathrm{MW}$. Assuming an atmospheric loss factor of 10 , a $10 \mathrm{MW}$ ground-based laser would be required. For a 1000 booster

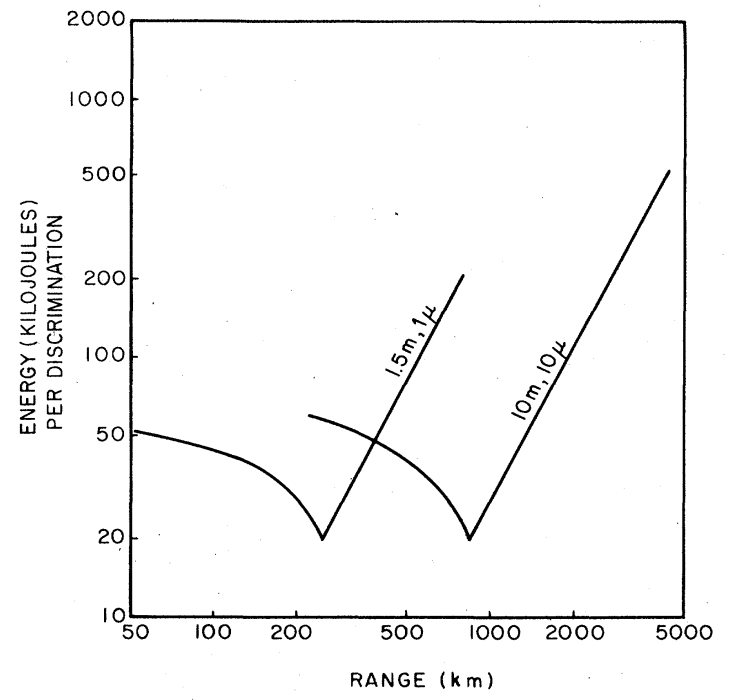

Figure 7.5. Energy per discrimination for a high energy laser discriminator.

attack approximately twenty $100 \mathrm{MW}$ lasers would be required at five different sites (a factor of 5 redundancy assured to account for adverse weather conditions). In addition to the GBLs and the pop-up mirrors, system effectiveness would require sensor platforms perhaps colocated on the mirror platform, with sufficient resolution to quickly detect the $10-100 \mathrm{~cm} / \mathrm{s}$ ablation-produced motion of lightweight decoys.

As a second example, we consider a space-based chemical laser system operating at $2 \mu \mathrm{m}$, with a desired interrogation range of $3000 \mathrm{~km}$, and sufficient laser energy to discriminate at all shorter ranges. The discrimination mechanism is assumed to be destruction of at least one-quarter of a decoy's surface area with an energy deposition requirement of about $10 \mathrm{~kJ} / \mathrm{g}$ for carbon cloth decoys. Selecting a focal spot size of

TABLE 7.1. Comparison on a per-target basis.

\begin{tabular}{|c|c|c|c|c|c|c|c|}
\hline System & $\begin{array}{l}\text { Assumed } \\
\text { platform } \\
\text { performance }\end{array}$ & ID mechanism & Criterion & \multicolumn{2}{|c|}{ Range $(\mathrm{km})$} & $\begin{array}{c}\text { Energy/ } \\
\text { interrogation }\end{array}$ & $\begin{array}{l}\text { Beam time/ } \\
\text { interrogation }\end{array}$ \\
\hline $\begin{array}{l}\text { NPB } \\
\text { (orbital) }\end{array}$ & $\begin{array}{l}200 \mathrm{MeV} \\
50 \mathrm{~mA} \text { (avg) } \\
1 \mu \mathrm{rad}\end{array}$ & $\begin{array}{l}\text { neutron } \\
\text { production }\end{array}$ & $\begin{array}{l}1000 \text { neuts } \\
\text { detected } \\
\text { per } R V\end{array}$ & 1500 & 500 & $1 \mathrm{MJ}$ & $0.1 \mathrm{~s}$ \\
\hline $\begin{array}{l}\text { LGEB } \\
\text { (pop-up) }\end{array}$ & $\begin{array}{l}20 \mathrm{MeV} \\
1 \mathrm{kA}\end{array}$ & $\begin{array}{l}\text { x-ray } \\
\text { production }\end{array}$ & $\begin{array}{l}1000 \times \text { rays } \\
\text { detected } \\
\text { per } R V\end{array}$ & 200 & 200 & $140 \mathrm{~kJ}$ & $7 \mu \mathrm{s}$ \\
\hline $\begin{array}{l}\text { SBL } \\
\text { (orbital) }\end{array}$ & $\begin{array}{l}50 \mathrm{MW} \\
2 \mu\end{array}$ & $\begin{array}{l}\text { thermal } \\
\text { destruction }\end{array}$ & $10^{4} \mathrm{~J} / \mathrm{g}$ & 2000 & & $5 \mathrm{MJ}$ & $0.1 \mathrm{~s}$ \\
\hline $\begin{array}{l}\text { GBL } \\
\text { (pop-up } \\
\text { mirrors) }\end{array}$ & $\begin{array}{l}100 \mathrm{MW} \\
1 \mu\end{array}$ & $\begin{array}{l}\text { ablative } \\
\text { impulse }\end{array}$ & $50 \mathrm{~J} / \mathrm{cm}^{2}$ & 500 & 3000 & $200 \mathrm{~kJ}$ & $2 \mathrm{~ms}$ \\
\hline
\end{tabular}


$5000 \mathrm{~cm}^{2}$ at $1500 \mathrm{~km}$ leads to a mirror diameter of about $7.5 \mathrm{~m}$. For $1 \mathrm{~kg}$ decoys with a total surface area of $2 \mathrm{~m}^{2}$, the minimum energy per target is about $2.5 \mathrm{MJ}$ at 1500 $\mathrm{km}$, increasing to $10 \mathrm{MJ}$ at $3000 \mathrm{~km}$. This parameter set leads to the second set of energy requirements shown in Figure 7.5. Assuming an average of 5 MJ per discrimination implies a total energy requirement of about $500 \mathrm{MJ}$ for destroying all the lightweight objects carried by a single booster.

Assuming 1000 boosters and an engagement time of $1000 \mathrm{~s}$ leads to a total energy requirement of $500 \mathrm{GJ}$ and an average power requirement of $500 \mathrm{MW}$. The required average discrimination rate is 100 objects per second. These requirements could be met by an average of ten 50 MW platforms operating in the battle space, with a nominal retarget time of $0.1 \mathrm{~s}$. Nominal constellation sizes would be $\sim 100$ platforms at an altitude of a few thousand kilometers.

The results of this section are summarized in Tables 7.1 and 7.2, in terms of required platform numbers for the various ranges discussed in the text. All systems are sized to handle a threat consisting of 1000 boosters each carrying approximately 100 credible objects $(9 \mathrm{RVs}$ and 90 decoys, for example).

\subsubsection{Interactive Discriminator Countermeasures}

At the present time we have little or no knowledge of how an adversary might approach the problem of devising countermeasures for interactive discriminators. However, since U.S. deployment of such systems could not occur for many years, it is important to understand the effects of various possible approaches. As two examples we consider the effects of countermeasure approaches to interactive discrimination based on shielding or deception (e.g., large balloons). In the next section we also examine potential problems associated with operating in a nuclear-disturbed environment.

\subsubsection{Shielding}

For particle beam interrogators we have already required beam kinetic energies sufficient to penetrate approximately $10 \mathrm{~g} / \mathrm{cm}^{2}$ of any material $(10-20 \mathrm{MeV}$ electrons and $100-200 \mathrm{MeV}$ protons). As a result, simple mass shielding techniques will likely carry severe weight penalties, $200 \mathrm{~kg}$ for a $2 \mathrm{~m}^{2}$ target. More sophisticated approaches involving a combination of low-atomicnumber materials are possible. Simple estimates for such approaches indicate that interrogation count rates from RVs with approximately $100 \mathrm{~kg}$ of low-Z shielding will be comparable to the count rates produced by interrogating high- $Z$ decoys weighing several tens of kilograms. Hence, a low-Z shielded $R V /$ high-Z decoy pair would impose a weight penalty of approximately $50 \%$ above that of a single unshielded RV.

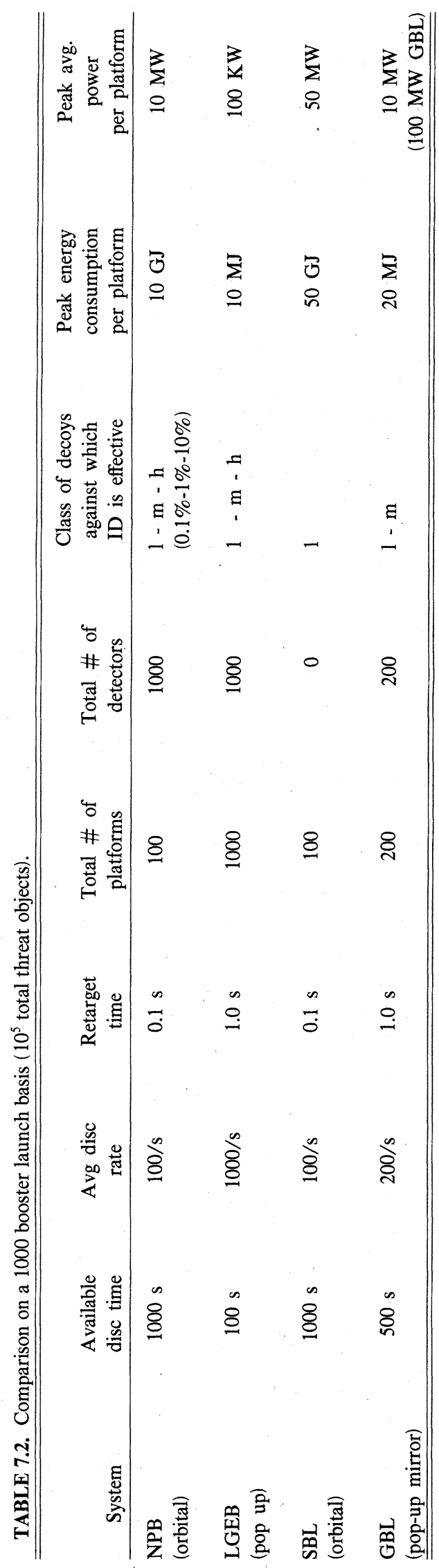


For laser systems, we required sufficient laser fluence to vaporize the target's surface, regardless of its composition. Since the velocity change produced by ablative impulse depends inversely on the target mass, in comparison with the $1 \mathrm{~kg}$ decoy previously considered, a $10 \mathrm{~kg}$ decoy will require a factor of ten increase in the product of the beam spot area (on the target) and the impulse to produce the same velocity change. Similarly, the destruction of a $10 \mathrm{~kg}$ decoy will require ten times as much energy as will the $1 \mathrm{~kg}$ decoy.

\subsubsection{Deception}

In order to keep energy requirements to a minimum, the directed energy approaches for interactive discrimination rely on achieving a small beam spot size at the target, i.e., small beam divergence angle. Hence, countermeasure techniques which cause aimpoint uncertainty, such as large balloons which may or may not contain RVs, will likely create difficulties for interactive discrimination based on DE technologies. Nominal approaches for interrogating the interior volume of a large balloon are to use an expanded beam, to probe many times, or to use some combination of these techniques. For neutral particle beams and lasers, expansion is probably relatively easy to accomplish. Techniques for expanding charged particle beams have been suggested (e.g., emittance variation late in the pulse), but have not been demonstrated.

For particle beam interrogators, as a first example we assume that the beam spot size is much less than a minimal RV cross-sectional area. We also assume that the probing pattern is that of hexagonal close-pack lattice, so that the effective probing area is approximately $1^{2}$ where 1 is a typical small RV length dimension. The total balloon area that must be probed is $\pi R^{2}$ where $R$ is the balloon radius. For $1=0.5 \mathrm{~m}$ and $\mathrm{R}=2.5 \mathrm{~m}$, the number of probings required is about 75-80, while the mass penalty to the defense is only $35-40$; i.e., assuming the balloon weight is determined by the balloon surface area. Note that for the same weight penalty, a single large balloon would require 80 probings while using many small balloons (of equal total weight) would require about 40 probings.

If the beam spot size at the target is increased to be roughly comparable to the cross-sectional area of the $R V$, then use of large balloons is probably of limited value. On the average, a raster scan of a single large balloon will find the RVs with less energy consumption than that required to interrogate a large number of small balloons whose collective weight equals that of the single large balloon. Finally, if the beam size is larger than the RV, fewer total probings will be required, but the discrimination signal strength for a given probing current will be reduced by the ratio of the beam area to the RV area.

For the case of lasers it is probably still necessary to interrogate approximately $10 \%$ of the cross-sectional area of the large balloon, implying that the laser energy requirements will be increased roughly in proportion to the balloon area (and mass). This energy, or area coverage, could be achieved by either expanding the beam, or more likely, by probing several times. As a final point, if the balloon is not rigidly attached to an enclosed $R V$, the observation time required to detect the RV could be several tens of seconds.

There are several limitations to the deployment of large balloons, including the mass penalty, the time required for credible deployment, and the need for screening the deployment. A large balloon, like a small balloon, must be adequately insulated against heat transfer and radar transmission to prevent detection of an enclosed $R V$ by passive optical or radar means. If the material thickness of a large balloon is required to be the same as for a small balloon, then the mass of a large balloon will, in fact, vary linearly with the balloon surface area. Further, if the enveloping balloons are radar reflecting, then they could be acquired and tracked more easily than bare RVs, and hence they would make certain tasks of the defense easier.

In summary, the use of large balloons as a countermeasure will require more attention to probing methodology for directed energy discriminators, but will also impose some penalties on the offense, and may not be the most attractive countermeasure option. Perhaps more worrisome from the defense viewpoint would be the development of stealth approaches suitable for use with both RVs and small balloons, which would greatly complicate the initial acquisition task.

\subsubsection{Nuclear Precursor Bursts}

We summarize here the effects of nuclear explosions on the various interactive discrimination concepts discussed above. Of most importance are problems of background neutron and gamma radiation for the particle beam detectors, striated optical and infrared backgrounds to acquisition and impulse sensors, electron beam propagation through the UV fireball, and neutral particle beam stripping in a "heaved" environment.

\subsubsection{Gamma Rays and Neutrons}

For particle beam discrimination concepts which rely on the detection of secondary particles, the effects of radioactive emissions resulting from a nuclear explosion are potentially serious. The prompt emission from a nuclear explosion lasts for only a few tens of nanoseconds during which a detector could probably be gated off. On the other hand, the delayed emissions from long-lived fission fragments may be important background sources for hundreds of seconds after the burst.

Nuclear fission fragments emit gamma rays with energies ranging up to $10 \mathrm{MeV}$ at a rate that decreases with time approximately as 


$$
\dot{\mathrm{N}}_{\gamma} \approx 2.5 \times 10^{16} \frac{\mathrm{fY}}{(1+\mathrm{t})^{1.2}}
$$

where $\dot{\mathbf{N}}_{\gamma}$ is the number of gamma/s, Y is the nuclear yield in megatons, $f$ is the fraction of the yield due to fission, and $t$ is the time in seconds after the burst. For scoping purposes we ignore the detailed motion of the fission debris and simply model the debris cloud as a point source, in which case the gamma-ray flux at a distance $\mathrm{r}$ is $\dot{\mathrm{N}}_{\gamma} / 4 \pi \mathrm{r}^{2}$. At $1000 \mathrm{~km}$ and $100 \mathrm{~s}$, the gamma flux from a one megaton fission yield will be about $10^{7} \gamma /\left(\mathrm{cm}^{2} \mathrm{~s}\right)$, which can be compared to the estimated secondary rate of $(0.1-1.0) \gamma /\left(\mathrm{cm}^{2} \mathrm{~s}\right)$ for a NPB-irradiated target at a range of $1000 \mathrm{~km}$.

At a minimum, the target return must be larger than the statistical variation in the nuclear background signal. If the time gate is $\tau$, then

$$
\frac{\Delta \mathrm{Bkg}}{\mathrm{N}_{\mathrm{RV}}}=\frac{1}{\dot{\mathrm{N}}_{\mathrm{D}}}\left(\frac{\dot{\mathrm{N}}_{\gamma}}{\mathrm{A}_{\mathrm{d}}}\right)^{1 / 2}
$$

We assume that $\tau$ must be sufficiently long to accumulate 1000 detected neutrons from an RV. For $A_{d}=1 \mathrm{~m}^{2}$ and $\dot{\mathrm{N}}_{\mathrm{D}}=1 \gamma /\left(\mathrm{cm}^{2} \mathrm{~s}\right), \quad \tau=0.1 \mathrm{~s}, \quad$ and $\quad \Delta \mathrm{Bkg} / \mathrm{N}_{\mathrm{RV}}=100$. Hence, an NPB discriminator based on detecting total gammas has serious limitations in a nuclear background environment. Further, since the gamma rays produced via the NPB interaction with the RV will have approximately the same spectrum as those produced by the fission debris, there appears to be little possibility of using a spectrum filter to separate gamma-ray signal from background.

For a pop-up laser-guided electron beam system the reduced target-detector range $(100 \mathrm{~km})$, and the much higher beam current $(\approx 1 \mathrm{kA})$ contribute to a much higher bremsstrahlung flux, approximately $5 \times 10^{6} \gamma /\left(\mathrm{cm}^{2} \mathrm{~s}\right)$. In this case $\Delta \mathrm{Bkg} / \mathrm{N}_{\mathrm{RV}}=1$ occurs $4-5$ $s$ after the burst, assuming the burst distance $r$ is also decreased to $100 \mathrm{~km}$. Hence, with minimal detector collimation it appears that the laser-guided beam approach might be functional within a few seconds following a nuclear explosion from the standpoint of the gamma background.

The Study Group considered the issue of the operation of particle beams in a discrimination mode in nuclear disturbed environments. Security considerations, however, prevent any discussion of our findings in this report.

\subsubsection{Background Ionization and Heave}

Depending on yield and altitude a nuclear burst can ionize a significant volume of atmosphere surrounding the point of the explosion. Very crudely, the radius $R_{i}$ of the ionized volume will be given by

$$
\mathbf{R}_{\mathrm{i}}(\mathrm{km}) \approx 10^{5}\left[\frac{\mathrm{Y}}{\mathrm{I}_{\mathrm{e}} \mathrm{n}_{0}}\right]^{1 / 3},
$$

where $\mathrm{n}_{0}$ is the neutral atom density (per cubic centimeter), $\mathrm{Y}$ is the explosive yield in megatons, and $I_{e}$ is the average energy (in $\mathrm{keV}$ ) absorbed per atom through excitation and ionization processes. Assuming a one megaton yield, and $I_{e}$ approximately $1 \mathrm{keV}, R_{i}$ varies from $1000 \mathrm{~km}$ to about $10 \mathrm{~km}$ as $\mathrm{n}_{0}$ varies from $10^{6} / \mathrm{cm}^{3}$ to $10^{12} / \mathrm{cm}^{3}(1000 \mathrm{~km}$ altitude to $100 \mathrm{~km}$ altitude $)$. Although the simple analysis neglects the layering effect of the exponential decrease in density with altitude, it is clear that the ionization volume can be quite significant. Moreover, the ionization level is expected to persist at $\left(10^{5}-10^{6}\right) / \mathrm{cm}^{3}$ for hundreds of seconds above several hundred kilometers altitude due to the absence of molecules and the attendant dissociative recombination processes.

About $10 \mathrm{~s}$ after the burst the hot ionized matter in the UV fireball will begin to rise at a rate of a few kilometers per second. The resulting vacuum can cause significant heave of the lower-lying atmosphere producing neutral densities of $10^{8}$ atoms per cubic centimeter at altitudes exceeding $1000 \mathrm{~km}$ at $100-200 \mathrm{~s}$ after the explosion. These neutral densities will persist at high altitudes for many hundreds of seconds (typically 10 $\min$.

Although the detailed results will depend somewhat on the magnitude of the burst and the burst altitude, it is apparent that ionization and heave can significantly affect the propagation of particle beams. For example, the analysis of Chapter 4 indicates that laser-created ion channel densities of $10^{7}-10^{8} / \mathrm{cm}^{3}$ would be used to guide the electron beam. Consequently, it is to be expected that nuclear explosion-produced background ion densities of the same magnitude will disrupt straight-line electron beam propagation. This implies that the electron beam could not propagate through the fireball.

A neutral particle beam will suffer significant ionization (tens of percent) after propagating through a few micrograms per square centimeter of matter. In a heaved environment significant neutral atom densities $\left(10^{10} / \mathrm{cm}^{3}\right)$ can exist for hundreds of seconds at altitudes of several hundred kilometers. Under these conditions the effective NPB range may be reduced.

\subsubsection{Nuclear Effects for Infrared Sensors}

Infrared sensors are required for several tasks in interactive discrimination, including tracking of targets, and possibly the measurement of impulse-generated velocity changes (the receiver of a coherent IR radar). The nature of the thermal emissions resulting from a nuclear explosion, and consequently, the effects on IR sensors, depends strongly on the altitude of the explosion. Low altitude bursts $(0-10 \mathrm{~km})$ are currently of lesser 
interest because IR sensors for interactive discrimination must operate at considerably higher altitudes.

A middle altitude burst $(10-100 \mathrm{~km})$ forms a welldefined fireball which radiates brightly in the IR for hundreds of seconds. The fireball rises (at nominally 1 $\mathrm{km} / \mathrm{s}$ ) and forms a torus after a few tens of seconds. The fireball emissions are strongest in the molecular bands of nitrogen oxides, ozone, water, and carbon dioxide. Even between these molecular bands, the fireball emission can be tens of $\mathrm{W} /\left(\mathrm{cm}^{2} \mu \mathrm{m}\right)$ for a few seconds. The IR emissions from the fireball are structured due to the turbulent flocculence and hot spots. After several tens of seconds for bursts in the upper end of this altitude range, the structure becomes striated with the striations aligned along the earth's magnetic field. These structures are commonly modeled by power spectral densities (PSDs) of the form $1 / \mathrm{k}^{3}$, where $\mathrm{k}$ is the spatial frequency of the electron density in cycles $/ \mathrm{km}$. There is considerable uncertainty in the PSDs of IR radiances, since they must be inferred from visible light photos and radar data from the limited number of old atmospheric tests.

The brightness and structure noise from middle altitude fireballs essentially prevents endoatmospheric sensors from viewing targets in front or behind these fireballs, unless the sensor is close to the target $(100 \mathrm{~km})$, and the target is bright (such as a reentering $R V$ ). Fortunately, middle altitude fireballs would occupy only relatively small volumes of the battle space $\left(100 \mathrm{~km}^{3}\right)$. Since the debris remains within the fireball, the decay gamma radiation can create additional noise in the focal planes of nearby endoatmospheric IR detectors.

For high altitude bursts in the range of $100-300 \mathrm{~km}$, the most important phenomena for IR sensors are probably the x-ray heated air (the x-ray patch), and the plasma formed by UV and debris heating. The x-ray patch is near $80 \mathrm{~km}$, and the brightest UV/debris plasma is between 100 and $180 \mathrm{~km}$. The $\mathrm{x}$-ray patch radiance typically lasts for hundreds of seconds, while the plasma radiance lasts for tens of seconds. The structure of the $\mathrm{x}$ ray patch results from the natural atmospheric density fluctuations being illuminated by the $x$ rays, while approximately a minute after the burst the plasma becomes striated along the earth's magnetic field. A PSD representation is also typically used to describe these structures.

For higher altitude bursts, the most important phenomena are the beta radiation, debris, and x-ray patches. A typical emission spectrum seen by a limb viewing sensor after a large yield burst (1 Mt), or several smaller bursts, is shown in Figure 7.6 for three times after the burst. The nitrogen oxides, ozone, and carbon dioxide emission bands are apparent. The structured nuclear IR radiance can convert a detector- or mirror-noise-limited limb viewing sensor into a background-limited sensor for lines of sight which pass near the burst point.

Debris patches occur at both conjugate regions where magnetic field lines, which pass near the burst point, enter the atmosphere. Approximately, one-third of the debris goes to each of the conjugate regions, while the remainder

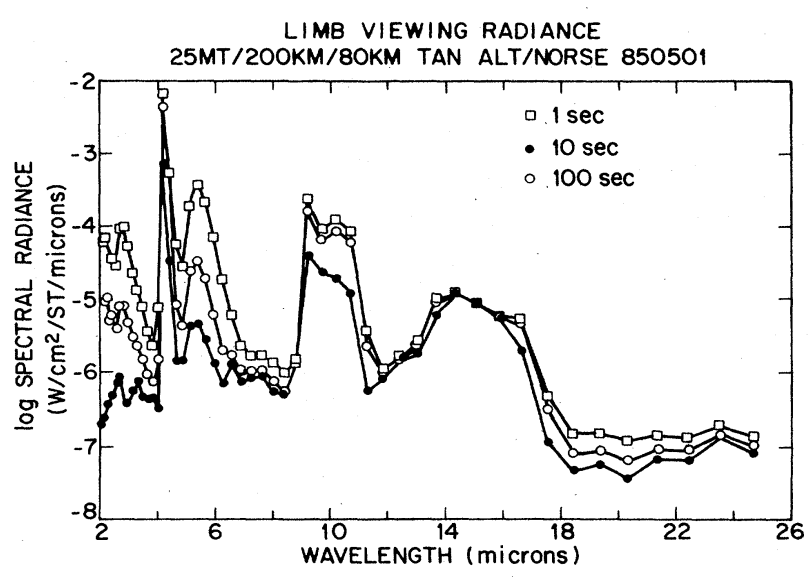

Figure 7.6. Computer predictions for the emission spectrum seen by a limb-viewing sensor after a $1 \mathrm{Mt}$ nuclear burst.

rises to higher altitudes. Both decay gammas and beta rays produced by the debris increase noise levels in IR sensors. Gamma fluxes typically begin at $10^{10}$ gammas $/ \mathrm{cm}^{2}$ and decay as $t^{-1.2}$, where $t$ is time in seconds. If the sensor is near the same magnetic field line as the burst, beta fluxes of the order of $10^{11} \mathrm{e} /\left(\mathrm{cm}^{2} \mathrm{~s}\right)$ can occur for a few seconds. The gamma noise is best reduced by circumvention, and the beta noise is best handled by shielding. The debris moving along magnetic field lines travels at approximately $500 \mathrm{~km} / \mathrm{s}$ so it can implant into the surfaces of space platforms. This implanted debris then gamma decays increasing the sensor noise. Prompt neutrons, both directly from the burst and reflected from the upper atmosphere, may also degrade IR sensors by neutron-gamma reactions and by activating materials around the focal plane.

Another phenomenon, which can affect IR sensors, is the air mass lofted to high altitudes by $\mathrm{X}$-ray deposition at lower altitudes. For reasonable scenarios, the air densities found initially near $100 \mathrm{~km}$ can be achieved near $200 \mathrm{~km}$ a few minutes later. Heaved air can then be illuminated by subsequent bursts effectively increasing the minimum tangent altitude for limb sensors.

To summarize briefly, the effects associated with nuclear bursts, which can significantly degrade IR sensor performance, include structured IR radiance, gammas, betas, neutrons, debris particles, and $\mathrm{x}$ rays. When designing IR sensors, care must be taken to incorporate shielding and circumvention techniques. Methods for reducing background clutter due to striations have been suggested, but further development efforts are necessary.

\subsubsection{Interactive Discrimination Summary}

From the relatively simple analyses of this section it appears that for pop-up discriminators the most reasonable candidates are a ground-based laser with pop- 
up mirrors or the laser-guided electron beam. For both types of systems the energy required per interrogation is of the order of several tens of kilojoules. For space-based discriminators, all candidates offer some potential, although the upper altitude restriction reduces the available engagement time for the electron beam concept. Typical energies required for a single interrogation are of the order of one megajoule for nominal ranges of a few thousand kilometers for the particle beam systems. On an energy cost basis alone, there does not appear to be an obvious best choice although thermal destruction can probably be dismissed. Hence, the most promising technology will likely depend on other factors, including technological maturity, system complexity, susceptibility to countermeasures, etc.

We have briefly considered some simple countermeasure approaches based on shielding, deception, and the effects of a nuclear precursor explosion. The mass penalty associated with attempts to shield against particle beam probes appears to be very high; increasing the target mass will decrease the resultant velocity created by laser-induced impulse, unless the impulse loading is increased or the target area undergoing loading is increased. For large balloons, expanding the beam to the size of an RV and then probing several times is probably the optimal technique for all interactive discrimination approaches. A nuclear explosion will create a number of adverse conditions for interactive discrimination, including IR red-out for laser sensors, large gamma and neutron backgrounds for NPBs, and volumetric ionization for laser-guided electron beams. In all cases precursor bursts may deny discrimination for a few seconds to a few tens of seconds in a volume with a typical scale length of $100 \mathrm{~km}$.

\subsection{CONCLUSIONS}

1. Detection and acquisition of ICBM launches will pose stringent requirements for high detection probability and low false alarm rates.

2. For boost phase, infrared tracking of missile plumes will have to be supplemented by other means to support sub-microradian aiming requirements of DEWs.

3. For post-boost and mid-course, precision tracking will require active sensor systems.
4. For mid-course, when the RVs are interspersed with penetration aids, interactive discrimination may be required. At present the application of DEW technologies to this task is only in the conceptual stage.

5. The development of an effective boost phase defense is highly desirable, perhaps essential for limiting the number of objects with which the mid-course and terminal defense elements must cope.

\section{REFERENCES}

${ }^{1}$ Marcel Barrere, Andre Jaumotte, Baudoin Fraeijs de Veubeke, and Jean Vandenkerckhove, Rocket Propulsion (Elsevier, New York, 1960), p. 12.

${ }^{2}$ Ibid., p. 171.

${ }^{3}$ Handbook of Chemistry and Physics, 55th Edition, edited by Robert C. Weast (CRC Press, Cleveland, 1974), p. E-226.

${ }^{4}$ Ibid., p. F-189.

${ }^{5}$ E. Brookner and T. F. Mahony, "Derivation of a Satellite Architecture for Air Surveillance," Proceedings EASCON '83: 16th Annual IEEE Electronics and Aerospace Systems Conference and Exposition (September 19-21, 1983) (IEEE, New York, 1983), pp. 465-475.

${ }^{6}$ E. Brookner, "Phased Array Radars," Sci. Am. 252 (No. 2), 94-102 (1985).

${ }^{7}$ Space Based Missile Defense, A Report of the Union of Concerned Scientists (March 1984), pp. 58-60.

${ }^{8}$ Ashton Carter and David Schwartz, Ballistic Missile Defense, Brookings Institution Report (1984), pp. 60-62.

${ }^{9}$ H. A. Bethe, J. Boutwell, and R. L. Garwin, "BMD Technologies and Concepts in the 1980s," in "Weapons in Space," Daedalus 114 (No. 2), 53-71 (1985).

${ }^{10}$ H. A. Bethe, R. L. Garwin, K. Gottfried, and H. W. Kendall, "Space Based Ballistic Missile Defense," Sci. Am. N251 (No. 4), 39-49 (1984).

${ }^{11}$ Ashton Carter, Directed Energy Missile Defense in Space, Background Paper for OTA Report (April 1984).

${ }^{12}$ U.S. Congress, Office of Technology Assessment, Ballistic Missile Defense Technologies, OTA-ISC-254 (U.S. Government Printing Office, Washington, D.C., September 1985), pp. 170-178.

${ }^{13}$ Weaponry in Space: The Dilemma of Security, edited by Y. Velikhov, R. Sagdeev, and A. Kokoshin, (Mir, Moscow, 1986), pp. 102-103.

14.J. McDowell, Sandia National Laboratory Memorandum (November 1985). 


\section{Chapter 8}

\section{SPACE-BASED PRIME POWER AND POWER CONDITIONING}

\section{CONTENTS}

8.1 Introduction

8.2 Station-Keeping and Alert Mode Operation

8.3 Engagement Mode Operation

8.4 Power Conditioning Systems

8.4.1 cw rf Approaches

8.4.2 Pulsed Power Induction Linac Approaches

8.5 Summary

8.6 Conclusions

References

\subsection{INTRODUCTION}

Various elements of a strategic defense system utilizing directed energy weapon components will require significant amounts of power for proper functioning, with the particular power levels depending on the operating mode. For example, relay and fighting mirror platforms will need "housekeeping" power to monitor their operational status, to control attitude and phase arrays, and to transmit and receive information; radar platforms will likely have similar station-keeping requirements. This minimum power level is estimated to lie in the range of $100 \mathrm{~kW}-700 \mathrm{~kW}$. In the "alert" mode, when the mirrors and radars are actively engaged in pointing and tracking, the power requirements may increase to 1-10 MW. Finally, during actual engagements (burst or sprint mode operation), the DEW platform itself may consume energy at the rate of $100 \mathrm{MW}-1 \mathrm{GW}$ for engagement times of several hundred seconds.

The basic elements of space power systems are schematically identified in Figure 8.1. In most cases, thermal energy will be converted into electrical energy which is then supplied to a conditioning system that delivers power in an appropriate form for the particular load application. Electrical power requirements for the various operating modes are summarized in Figure 8.2, which also compares the various primary energy sources in terms of power levels and duration times. For the stressing demands of strategic defense missions, it appears that nuclear reactors will be required for the stationkeeping and alert modes, while either nuclear reactors or chemical combustors (essentially rocket engines) could supply the very high power levels required during burst engagements. At present, very powerful, space-qualified, energy supplies based on these sources do not exist; for example, present military sensor platforms require about
$10 \mathrm{~kW}$ of $\mathrm{cw}$ power which is provided by solar cell arrays. For larger power requirements, the size of the solar cell arrays become a serious problem from the point of view of survivability and maneuverability in face of an attack. In this section, we will briefly summarize the state of the art and discuss the implication of strategic defense requirements on several prime power and power conditioning areas.

\subsection{STATION-KEEPING AND ALERT MODE OPERATION}

A consideration of Figure 8.2 indicates that the power levels over the operating times required for station-keeping and alert operating modes will have to be provided by nuclear reactors. (The only exceptions are those missions in which the space platform is rocketlaunched following determination that an offensive attack has been initiated. In these instances, for which the entire battle time may be only a few hundred seconds, battery power supplies are a reasonable approach. This technology is discussed in Appendix 8.A.)

Recognizing this necessity for space-based nuclear power, SDIO has accelerated the secular SP-100 (Space Power, $100 \mathrm{~kW}$ ) program in cooperation with DOE and NASA. This program is an outgrowth of early work at Los Alamos National Laboratories and in many ways resembles proposals advanced more than a decade ago for a space nuclear power system. The present concept uses a fuel core element consisting of highly enriched uranium cooled by electromagnetically-pumped liquid lithium to extract heat from the reactor when in operation. The

MULTI-MEGAWATT SPACE POWER SYSTEM

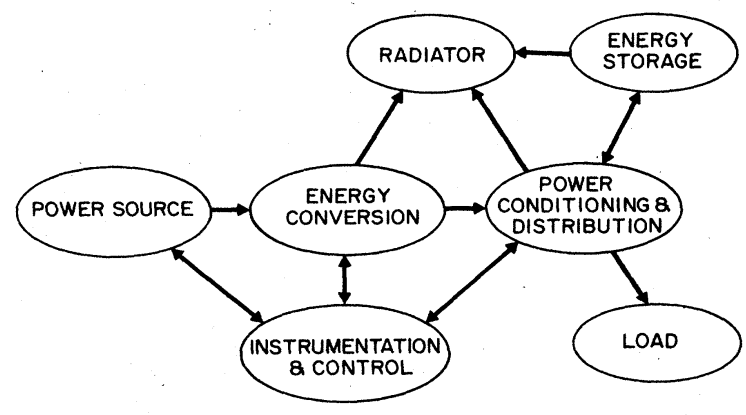

Figure 8.1. A schematic diagram illustrating the basic elements of a space power system. 


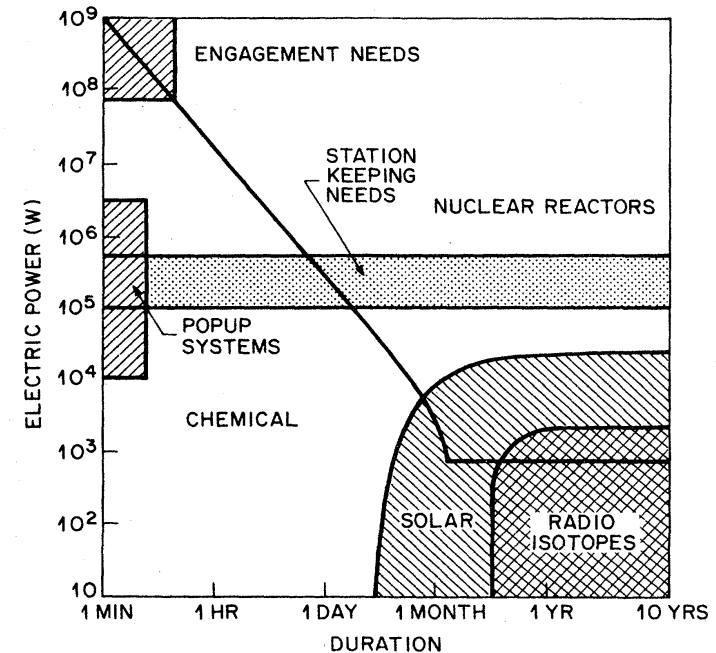

Figure 8.2. A comparison of the electrical power requirements of various operating modes, as well as the various primary energy sources.

power level is controlled by a system of external reflectors and absorbers on rotary drums at the periphery of the cylindrical core element. Complete shielding of the reactor is not possible due to the great mass of lithium and tungsten which would be needed to stop both the neutrons and gamma rays. Therefore, a shadow shield is employed so that the direct thermoelectric energy conversion system is radiatively connected to the high temperature loop. A lower temperature set of heat pipes dissipates the waste heat. If background neutron and gamma radiation is harmful to mirrors, sensors, or radar components, the power station would have to be positioned at an appropriate distance from the platform it serves with the power being transmitted via microwaves or power cables.

The SP-100 system has been described in the literature and is designed to be completely assembled on earth and fitted into the shuttle bay. The estimates of a maximum ultimate capability for $100 \mathrm{~kW}$ is based on shuttle volume limits rather than mass limits, permitting a chemical upper stage to lift the system to high earth orbit.

As with any new nuclear power plant, SP-100 development involves a complex, laborious testing procedure. The program is proceeding in an orderly fashion to obtain full component data in the coming five years. For example, fuel elements will be required to operate between 1300 and $1700 \mathrm{~K}$, although elements in normal reactors operate at about $900 \mathrm{~K}$. The component development phase will be followed by an all-up ground test in one of the many available space chambers before an anticipated launch.

While problems of launch safety are not trivial, the reactors would not be activated until they were stationed in safe orbits, the natural decay time of which would be sufficient to ensure fission product burnout. The only danger that might be anticipated would be a launch failure which might cause the reactor to become critical; however, this problem has been recognized and the core elements are designed with additional damping during launch to make this a very remote possibility.

The requirement for a relatively large number of space nuclear power plants is an indispensable element of a defensive shield complex requiring special considerations and operational constraints for space nuclear power plants. Space-based nuclear power plants are operated at a variety of altitudes. In many cases the altitudes are relatively low, particularly those used by the U.S.S.R. Therefore, an agreed upon set of guidelines for the use of space-based nuclear power plants has been accepted by the U.N. requiring that each nuclear power system be equipped with a boost or a reboost system which can move these satellites to a safe parking orbit, once its mission is terminated. The currently accepted safe parking orbit (provided that the ballistic coefficient is high enough, see Appendix 8.A) is set at at least $700 \mathrm{~km}$, which allows for an approximate decay time of about 300 years. ${ }^{1-3}$

These requirements are based upon the expected decay life of most intense radioactive components created in the reactor during its lifecycle. ${ }^{4}$ The most important of these with repect to health are cesium 137 and strontium 90. Fortunately, these components decay relatively rapidly so that after 300 years the health hazard is diminished by about five orders of magnitude. Nonetheless, other fission fragments and trans-uranic elements will create increases in background radiation for periods of more than $10^{6}$ years.

Another consideration for space parking orbits is implied by the choice of the ballistic coefficient of the reactor after reboost. This is a specially important parameter to the use of space-based nuclear power plants since we may expect, in many cases, that the power plant will be attached to a relatively large and complex space platform whose drag coefficient may be high. This implies that upon mission termination provisions must be made for the detachment of the nuclear core from the main structure for reboost into higher orbits. This stresses the requirement for highly reliable reboost capability or its redundancy to assure a reasonable level of safety when operating space-based nuclear power plants. The two nuclear powered satellites of the U.S.S.R. reentered due to failure of their reboost systems at a time when the radioactivity was at its maximum.

Continuously-operating, multimegawatt power systems are needed in the future. Providing this advanced technology will require the development of reactor concepts which are currently either in the laboratory stage or in the idea stage, and it is not believed that the present SP-100 technology can be easily scaled to these power levels.

While the main thrust of research about housekeeping power remains in the nuclear area, the growing realization of technical difficulties and long lead times for the development of nuclear power space systems 
in the $100 \mathrm{~kW}-1 \mathrm{MW}$ range has generated renewed interest in alternative approaches. These range from beaming microwave power transmission from the earth up through a system of relays to the satellite platform ${ }^{5}$ to new ultraefficient energy storage systems. These alternative concepts are all in the conceptual stage.

\subsection{ENGAGEMENT MODE OPERATION}

The energy required to kill a single target will generally be of the order of tens to thousands of megajoules for engagement times of the order of one second. Also, it may be necessary to engage tens to hundreds of targets during an engagement time window of a few hundred seconds. Hence, the prime power system may have to supply $10-1000 \mathrm{GJ}$ with average power levels of perhaps $0.1-10 \mathrm{GW}$, assuming efficiencies of order $10 \%$. For these very high demands, a consideration of the various options (Figure 8.3) indicates that the primary energy sources must be chemical combustory (rocket engines) and nuclear reactors. Chemical systems represent known technology, and there is a simple relationship between the amount of fuel required and the power levels and run times. As an example, for $\mathrm{cw}$ operation at $30 \mathrm{MW}$ for $200 \mathrm{~s}$, the required fuel weight (liquified $\mathrm{H}_{2}$ and $\mathrm{O}_{2}$ ) will be several thousand kilograms.

At the present time, several rocket systems exist which are capable of more than a gigawatt of power generation for several minutes, and the engineering design principles are well understood. Nonetheless, the engineering development problems are expected to be formidable.

While it is assumed that two opposing rocket systems would be utilized to balance the resultant thrusts, it is beyond the capability of present thrust controls to assure that unacceptable torques will not be applied to the space weapon platform because of the very high precision pointing and tracking required for DEWs. Alternatively, this power system may have to be separated from the space platform. Power transmission utilizing microwaves, or even cables, has been suggested. No acceptable solution has yet been developed.

Another serious problem which is also under study is the effluent from a rocket power system. In a matter of seconds, exhaust gases may engulf the entire space platform, perhaps blinding sensors and even rendering the various proposed weapons inoperative. Again, separation of the power station from the weapon platform may be necessary.

The current limits of nuclear reactor technology have

\section{ELECTRIC POWER REQUIREMENTS}
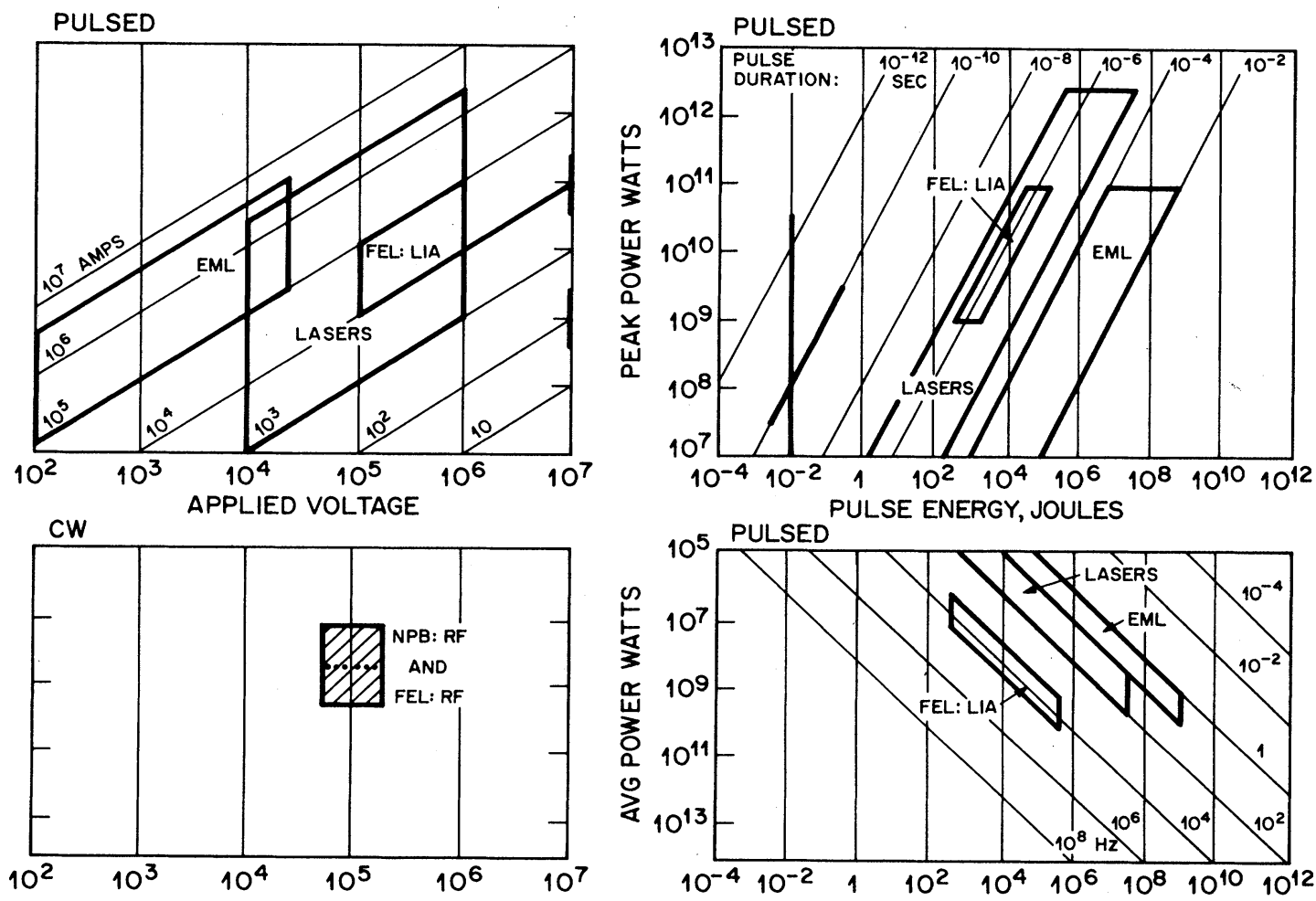

Figure 8.3. Specific electric power requirements for several DEW approaches (EML: electromagnetic launchers; FEL: free electron lasers; NPB: neutral particle beams). 
TABLE 8.1. Prime power technology summary.

\begin{tabular}{|c|c|c|c|}
\hline & $\begin{array}{l}\text { State of } \\
\text { the art }\end{array}$ & $\begin{array}{l}\text { SDI weapons mode } \\
\text { requirements } \\
\text { (derived) }\end{array}$ & Critical issues \\
\hline Nuclear & $\begin{array}{l}\text { A few } \\
\mathrm{kW}\end{array}$ & $100-1000 \mathrm{MWe}$ & $\begin{array}{l}\text { - Many technologies must be invented } \\
\text { - High temperature reactor/turbine } \\
\text { - Selection, design, development and test }\end{array}$ \\
\hline $\begin{array}{l}\text { Combustion } \\
\text { turbine }\end{array}$ & $\begin{array}{l}4 \mathrm{MWe} \\
100 \mathrm{~s}(\mathrm{~s}) \\
1600 \mathrm{deg} \mathrm{F}\end{array}$ & $100-1000 \mathrm{MWe}$ & $\begin{array}{l}\text { - Effluent and thrust management } \\
\text { - Restartable gas generator/turbine } \\
\text { - High internal temperatures }\end{array}$ \\
\hline ac generator & $\begin{array}{l}20 \mathrm{MWe} \\
30 \mathrm{kV} \\
400 \mathrm{~Hz}\end{array}$ & $\begin{array}{l}100-1000 \mathrm{MWe} \\
>100 \mathrm{kV} \\
1000-3000 \mathrm{~Hz}\end{array}$ & $\begin{array}{l}\text { - Structural materials } \\
\text { - Vibration and torque }\end{array}$ \\
\hline dc generator & $\begin{array}{l}4 \mathrm{MWe} \\
1 \mathrm{MA}\end{array}$ & $\begin{array}{l}\geq 5 \mathrm{MA} \\
100-1000 \mathrm{MWe}\end{array}$ & $\begin{array}{l}\text { - High current collectors } \\
\text { - Vibration and torque }\end{array}$ \\
\hline Pulsed generator & $\begin{array}{l}0.3 \mathrm{MJ} \\
0.5 \mathrm{MA} \\
4 \mathrm{~Hz} \\
0.5 \mathrm{~s}\end{array}$ & $\begin{array}{l}100 \mathrm{MJ} \\
5 \mathrm{MA} \\
5 \mathrm{~Hz}\end{array}$ & Same \\
\hline
\end{tabular}

been previously discussed. Newer technologies, involving composite reactors using new fuel materials and geometries operating at higher temperatures will be needed to meet the high power demands, yet satisfy the requirements for safety and reliability. A potential advantage of the reactor approach is that it should be able to satisfy the power requirements for station-keeping and alert operating modes, as well as the burst engagement mode. A potential disadvantage, however, is the need for adequate shielding to prevent component degradation due to nuclear radiation dose effects. Open cycle reactors will likely be competitive with chemical energy sources in terms of weight per unit energy output, although closed cycle reactors will probably be 5-10 times heavier.

Possible energy converter systems for both chemical and nuclear energy sources include turbo-alternators and motor generators, as well as magnetodynamic (MHD) cells which use the motion of ionized gases through magnetic fields to generate electrical power. A summary of the present state-of-the-art parameters and strategic defense burst mode requirements for the various power technologies, along with key technical issues, is presented in Tables 8.1 and 8.2.

TABLE 8.2. Prime power technology summary.

\begin{tabular}{|c|c|c|c|}
\hline & $\begin{array}{l}\text { State of } \\
\text { the art }\end{array}$ & $\begin{array}{l}\text { SDI weapons mode } \\
\text { requirements } \\
\text { (derived) }\end{array}$ & Critical issues \\
\hline \multicolumn{4}{|l|}{ MHD generators } \\
\hline - Combustion & $\begin{array}{l}100 \mathrm{MW} \\
10 \mathrm{~s}(\mathrm{~s}) \\
1-10 \mathrm{kV}\end{array}$ & $\begin{array}{l}100-1000 \mathrm{MWe} \\
10-100 \mathrm{kV}\end{array}$ & $\begin{array}{l}\text { - High temperatures } \\
\text { - Effluent management } \\
\text { - Vibration } \\
\text { - Thrust }\end{array}$ \\
\hline - Explosive & $\begin{array}{l}37 \mathrm{~kJ} \\
1 \mu \mathrm{s} \\
\text { single shot }\end{array}$ & $\begin{array}{l}100 \mathrm{KJ}-10 \mathrm{~s} \mathrm{MJ} \\
1 \mu \mathrm{s}\end{array}$ & $\begin{array}{l}\text { - Rep rate } \\
\text { - Debris management } \\
\text { - Shock/vibration } \\
\text { - Thrust }\end{array}$ \\
\hline - MCG & $\begin{array}{l}20 \mathrm{MJ} \\
\text { Single shot }\end{array}$ & $\begin{array}{l}100 \mathrm{MJ} \\
1-5 \mathrm{~Hz}\end{array}$ & $\begin{array}{l}\text { - Rep rate } \\
\text { - Shock/vibration } \\
\text { - Thrust } \\
\text { - Impedance matching }\end{array}$ \\
\hline
\end{tabular}




\subsection{POWER CONDITIONING SYSTEMS}

Space-based chemical lasers have the advantage that chemical energy stored in the fuel is directly converted into useful laser output with an efficiency of about $10 \%$. Other DEW approaches, including excimer and free electron lasers, and particle beam concepts, will require electric power at high voltages and currents. The specific electric power requirements are summarized in Figure 8.3.

The power conditioning approach for the several DEW concepts generally fall into two categories: (1) continuous $\mathrm{rf}$ power output for $\mathrm{rf}$ linacs (NPBs and $\mathrm{cw}$ FELs), and (2) high voltage pulsed power for induction linac/pulsed diode technologies (pulsed FELs, excimer lasers, and laser-guided electron beams). We will consider these approaches separately.

\subsection{1 cw rf Approaches}

Both the space-based NPB and FEL concepts would use rf linear accelerators requiring tens to hundreds of megawatts of $\mathrm{rf}$ power. Since the load of the $\mathrm{rf}$ power system is a resonant accelerator cavity, the power supply must be capable of tolerating a certain amount of reflected power. For the NPB, the operating frequency range must be consistent with the particular light ion and is about $200 \mathrm{MHz}-1 \mathrm{GHz}$. For FEL applications, frequencies of $1-3 \mathrm{GHz}$ are about optimum. The power per device or module should be about $1 \mathrm{MW}$. The pulse lengths could be as short as $1 \mathrm{~ms}$, although $\mathrm{cw}$ operation is a useful goal, as is a $100 \%$ duty cycle. For the NPB, the space environment may play an important role in power system selection when considerations of quiet, vibration-free, effluent-free power are important. The overall system specific power goal should be a few $\mathrm{kW} / \mathrm{kg}$.

There are several potential candidate rf power sources, including both tubes and solid state devices. Table 8.3 indicates what is presently available either in actual devices or demonstrated performance that could be duplicated.

Klystrons are the present tube of choice for high powers and high frequencies; they typically operate with $100 \mathrm{kV} \mathrm{dc}$ anode potentials with $20 \mathrm{~A}$ of electron beam current. They operate at room temperature, are watercooled, and are rated for long lifetimes. While they could deliver the requisite power levels, they would dominate the DEW platform in size, mass, and power consumption. For example, one tube being built by Thomson-CSF (French) operates at $1 \mathrm{MW} \mathrm{cw}$ with $65-70 \%$ efficiency at $350 \mathrm{MHz}$; however, it is $4.5 \mathrm{~m}$ long and weighs $2200 \mathrm{~kg}$.

Gridded tubes are used in low frequency accelerator applications. The magnetron or crossed-field amplifier (CFA) is used on small electron accelerators, but suffers from too much reflected power on large, ion accelerators. Alternative conventional tube concepts that are potential candidates include the klystrode, the planar triode, and
TABLE 8.3. Currently available rf power sources.

\begin{tabular}{lccc}
\hline Device & Power & $\begin{array}{c}\text { Frequency } \\
(\mathrm{GHz})\end{array}$ & $\begin{array}{c}\text { Power density } \\
(\mathrm{W} / \mathrm{kg})\end{array}$ \\
\hline Klystrons & Multi-MW & $\leqslant 10 \mathrm{GHz}$ & 500 \\
Klystrodes & $100 \mathrm{~kW}$ & $\leqslant 10 \mathrm{GHz}$ & 2000 \\
TWT & $250 \mathrm{~kW}$ & $1-3 \mathrm{GHz}$ & \\
& $100 \mathrm{~kW}$ & $10 \mathrm{GHz}$ &
\end{tabular}

CFA Multi-MW

Gridded Multi-MW VHF - 1-band

tubes

Transistors $\quad 100-400 \mathrm{~W} \quad 400 \mathrm{MHz} \quad 400$

the relativistic electron cyclotron mode device known as the gyro-klystron. Of the three, the klystrode appears to offer considerable potential. In this device, bunching is achieved by gating the beam; i.e., a grid replaces a klystron cavity. The klystrode could be significantly shorter than a klystron and could possibly operate with electrostatic focusing, eliminating the very heavy klystron magnets. The planar triode may also be an alternative for less demanding missions. These tubes are compact, robust, and operate at lower anode potentials, 5-100 kV. They exhibit only moderate gains $(30 \mathrm{db})$ and can produce about $5-10 \mathrm{~kW}$ of $\mathrm{rf}$. The gyro-klystron will probably have limited utility for frequencies much below about $1 \mathrm{GHz}$. Candidates requiring more basic research include $\mathrm{rf}$ production using self-modulated relativistic electron beams, so-called direct-drive linac cavities, and deflection-modulated rf amplifiers such as the gyrocon.

The recent development of solid state amplifiers for high power $(200 \mathrm{~kW}$ pulsed) systems for Navy radar transmitters has afforded another approach for $\mathrm{rf}$ accelerator drivers. Individual devices are typically of the 200-500 W power class necessitating combinations of many devices in a module. Consequently, amplitude and phase control, interfacing cell and carrier architecture, and thermal management are crucial problems. The state of the art is probably represented by the SPS-40 radar system that operates at $400-450 \mathrm{MHz}$, and produces $200 \mathrm{~kW}$ peak power in 3 or $60 \mu$ s pulses. The transmitter weighs about $1100 \mathrm{~kg}$. The devices themselves are bipolar transistors that exhibit efficiencies of about 40-50\% (pulsed), gains of $10-15 \mathrm{db}$, and specific powers of $200-400 \mathrm{~W} / \mathrm{kg}$. Including structural framework, rf drive systems, and cooling, the specific powers for this solid state approach are about $20-40 \mathrm{~W} / \mathrm{kg}$. Some recent system design studies claim efficiencies comparable to klystrons; however, device gains do not yet compare favorably with klystrons and other tubes.

When examined as an integral element of a total system that encompasses the prime power and power 
conditioning as well as the distributed system of resonant cavities of the accelerator, solid state rf systems may offer new approaches with potential advantages. For example, solid state devices that operate at low voltages of about 25-40 V are an attractive match to batteries, fuel cells, or homopolar generators which can produce power at these voltages.

\subsubsection{Pulsed Power Induction Linac Approaches}

A generic power conditioning diagram for both a linear induction accelerator and an excimer laser is shown in Figure 8.4. There are several common elements, including a pulsed power unit that stores energy from a dc or ac supply, a switching element (closing or opening), a transformer stage that generates a voltage pulse of the correct amplitude, a pulse-forming and shaping network, usually an output switching stage, and the beam generating diode or acceleration cell. In the case of an induction linac, the pulse power modules are effectively added in series by using a particle beam to link multiple accelerator cells. In the case of a high power excimer laser, the pulse power modules are effectively used in parallel, generating multiple electron beams which excite the laser volume. For pulsed FELs and laserguided electron beam applications, the output pulses delivered to the accelerating cells are typically $100 \mathrm{kV}-1 \mathrm{MV}$ and a few kiloamperes with pulse duration of the order of tens of nanoseconds to a few microseconds. The output pulses for an excimer laser diode will typically be $1 \mathrm{MV}$ and $100 \mathrm{kA}$ for pulse durations of the order of $1 \mu \mathrm{s}$.

Inverter/converter elements are required to condition energy from primary sources into electrical pulses suitable for use in the energy storage system. Critical components included in such elements are fast switches, rectifier diodes, high frequency ac capacitors, and transformers and other magnetic components. The present state of the art is about $200 \mathrm{~W} / \mathrm{kg}$; i.e., the inverter/converter required to produce an average power of $100 \mathrm{MW}$ would weigh about 500 metric tonnes. A clear goal is to develop standard lightweight I/C units $(2 \mathrm{~kW} / \mathrm{kg})$ that could be used in combinations to satisfy strategic defense requirement for several different DEW approaches.

In order to deliver the very high peak power pulses (terawatts) required for some applications, the energy from the I/C unit must be stored in capacitors or inductors, or possibly as rotational energy. The advantages of capacitative storage units (Marx generators, for example) include a highly-developed technology base, use of closing switches, and relatively low internal losses over long storage times. On the other hand, the energy storage density is relatively low $\left(1 \mathrm{MJ} / \mathrm{m}^{3}\right)$, and the energy must be stored at high voltages with large electrical insulation stresses. The potential advantages of inductive storage include higher energy storage density $\left(10 \mathrm{MJ} / \mathrm{m}^{3}\right)$, and storage at low voltages with only mechanical stresses; however, inductive storage has been largely ignored because of the lack of good, repetitive opening switches. Large inertial rotors (flywheels) energized directly by a turbine may be very useful storage devices for land-based applications, but space-based platforms may require specially-constructed counterrotating designs. The mechanical energy can be converted to an electrical pulse by applying a magnetic field perpendicular to coils attached to the rotor, for example. A comparison of various storage methods in terms of discharge time and energy density is given in Appendix 8.A.

There are several closing switch candidates for repetitive operation, including thyratrons, solid state switches, high pressure plasma switches, gas spark gaps, and magnetic switches (saturable-core inductors). The latter have demonstrated good operation at $0.25 \mathrm{MW}$, $100 \mathrm{~ns}$, and $5 \mathrm{kHz}$, but can be quite heavy. At present, the voltage and current capabilities of solid state closing switches are too low for strategic defense applications. Thyratrons have a demonstrated capability at $800 \mathrm{~kW}$ continuous with $1 \mathrm{MW}$ bursts in $10 \mu \mathrm{s}$ pulses at $120 \mathrm{~Hz}$. High voltage breakdown generally restricts peak voltages to less than $50 \mathrm{kV}$, while current density limitations at the cathode restrict peak currents to $150 \mathrm{kA}$. High voltage spark gaps have demonstrated $20 \mathrm{~Hz}$ switching at 1. $5 \mathrm{MV}, 5 \mathrm{~kJ} / 60 \mathrm{~ns}$ pulses for over one hundred thousand total shots, and $1 \mathrm{kHz}$ for a 10 pulse burst at $25 \mathrm{kV}$, and $300 \mathrm{~J} / 50 \mathrm{~ns}$ pulses. At the latter parameters, the same switches have operated for over ten million shots at a few $\mathrm{Hz}$. Improvements are necessary to minimize gas flow and electrode erosion, as well as to decrease the size and weight. For all applications and switches, low switch jitter (ns) is essential.

For opening switch technologies, a key problem is the dissipative loss during the conductive phase; primarily because of this difficulty, there are no reasonable high power opening switches for pulse compression from

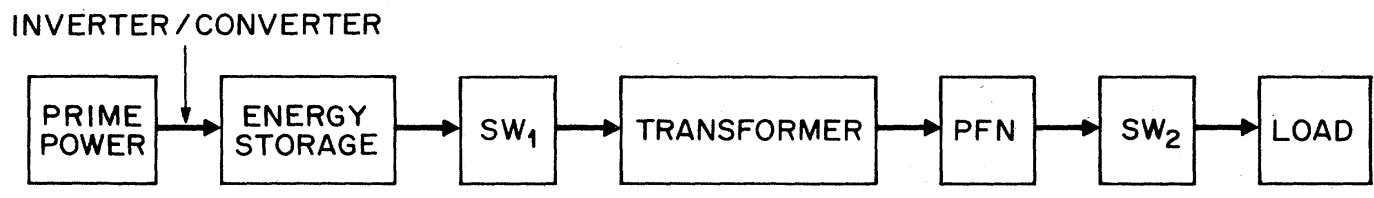

Figure 8.4. A generic power conditioning diagram for both linear induction accelerators and pulsed excimer lasers. 
milliseconds to microseconds presently available. However, there are two general approaches which offer promise. The first depends on the use of controlled plasmas, and includes plasma erosion switches and electron-beam-controlled switches. The characteristic conduction time is fairly short, suggesting that their main usage may be in the second or third stage in systems requiring very fast opening times. The second approach utilizes solids with nonlinear resistivity controlled by external means, including laser illumination and heating. The laser illumination switches also conduct for only short times, although the conduction times for low resistance ceramics such as doped $\mathrm{BaTiO}_{3}$, can be quite long (seconds), and it appears that these devices may be able to switch $1 \mathrm{MJ}$ per $\mathrm{kg}$ of material.

The current state of the art in transformers is about $25 \mathrm{~kW}, 20 \mathrm{kHz}$, and $20 \mathrm{kV}$ with an associated specific weight of about $8 \mathrm{~kW} / \mathrm{kg}$ with conduction and radiation cooling. For strategic defense applications, nominal goals are to develop transformer designs capable of hundreds of kilowatts continuous and up to a few megawatts in burst mode operations. In order to achieve these power levels, it will be necessary to improve insulation, improve cooling techniques to reduce heating of the primary winding, and increase magnetic flux density in the transformer core from $1 \mathrm{~T}$ to $5 \mathrm{~T}$.

\subsection{SUMMARY}

In this chapter, we have summarized the power requirements for the various operating modes of a DEW system with space-based elements, and we have viewed the several technological approaches which might satisfy these power requirements. For the station-keeping mode, only 100-700 $\mathrm{kW}$ may be required, but this power level will have to be continuously supplied over very long times (years) for a total energy consumption of perhaps $10^{13} \mathrm{~J}$. For the alert mode, the required power level will likely be $1-10 \mathrm{MW}$ over perhaps several periods (over a 10-year lifetime) of a few hours to a few days in duration, for a total energy requirement of $10^{12} \mathrm{~J}$. Finally, for actual engagements, the DEW platform may require $1 \mathrm{GW}$ for approximately $100 \mathrm{~s}$ for a total energy consumption of $10^{11} \mathrm{~J}$. Since chemical energy sources have a specific energy density of a few megajoules per kilogram, the weight of chemicals consumed during an engagement might be only a few tens of tons; however, using chemical energy for the alert operating mode (hundreds of tons of fuel) and station-keeping modes (thousands of tons of fuel) does not appear to be sensible. Hence, it may be necessary to have a nuclear reactor service every space platform or else devise alternate power transmission strategies.

There is an existing program (the SP-100) to develop space-based nuclear power systems capable of delivering $100 \mathrm{~kW}$ power levels, and conceptual studies of higher power approaches have been initiated. Since background nuclear radiation levels may damage mirrors, sensors, radar components, etc., it may be necessary to locate the nuclear power stations at an appropriate distance from the platform that it serves.

The prime power and power conditioning system of the several directed energy concepts may have to provide 100-1000 MW for burst engagement times of several hundred seconds. At present, such supplies do not exist although much progress has been made. In most cases, the thermal energy supplied by a chemical or nuclear energy source will be converted into electrical energy (for example, using turbo-alternators or MHD cells), which is then supplied to a power conditioning system. For DEW concepts based on cw rf linac technology (NPBs and cw FELs), the electrical power is used to drive $\mathrm{rf}$ sources which power the accelerating cavities of a particle accelerator. At present the only suitable rf tube is the klystron, which is somewhat bulky and quite heavy for space platforms. Other devices, such as the klystrode and solid state bipolar transistors, offer promise for this application but require additional development.

For DEW concepts which produce repetitive, very high power output pulses, such as pulsed excimer and free electron lasers, the high average power must first be stored and then switched into the load. Capacitive storage has a solid technology base and there are many types of closing switch technologies available, although the energy storage density is probably limited to $1 \mathrm{MJ} / \mathrm{m}^{3}$. Inductive stores promise higher specific energy density, but require the development of low-loss, repetitive opening switches. Rotating machines have considerable promise for land-based systems which do not require extremely rapid discharge rates.

In summary, several approaches exist for providing the demanding power requirements of strategic defense burst engagements, but all require development, especially those which demand space basing. System sizes and weights must be reduced, and space qualification of the hardware components is essential.

\subsection{CONCLUSIONS}

1. Housekeeping power requirements for operational maintenance of many space platforms for strategic defense applications necessitate nuclear reactor driven power plants on each of these platforms.

2. During engagements prime power requirements for electrically, driven space-based DEW present significant technical obstacles.

\section{REFERENCES}

${ }^{1}$ Gary L. Bennett, "Overview of the U.S. Flight Safety Process for Space Nuclear Power," J. Nucl. Safety 22 (No. 4), 1981. 
2"Report of the Working Group on the Use of Nuclear Power Sources in Outer Space on the Work of its Second Session," U.N. General Assembly Report by the Committee on Peaceful Uses of Outer Space, February 1980.

3"Nuclear Safety Criteria and Specifications for Space Nuclear Reactors," Department of Energy Document, Office of Space Nuclear Projects, OSNP-1, Rev 0, August 1982.
${ }^{4}$ Bernard L. Cohen, "The Disposal of Wastes from Fission Reactors," Sci. Am. June, p. 21 (1977).

${ }^{5}$ For an example of transmission of large amounts of electrical power via microwave beaming, see Electrical Power from Orbit-A critique of a satellite power system (National Academy Press, Washington, D.C., 1981). 


\section{APPENDIX 8.A NEW DEVELOPMENTS IN BATTERY TECHNOLOGY}

Current battery technologies are capable of energy storage densities of hundreds of kilojoules per kilogram, i.e., about a factor of ten less than that of chemical fuels. Hence, batteries would appear to be unsuitable for providing the very large amounts of energy necessary for the various strategic defense operating modes. However, for certain time-limited missions, including rocketlaunched probes or discriminators, batteries may offer an attractive approach. As an example, consider the laserguided electron beam as a pop-up discriminator. For this application, the reduced operating ranges imply beam kinetic energies of a few tens of $\mathrm{MeV}$, a beam current of about a kiloampere, and a beam pulse length of several microseconds. Hence, the total pulse energy will be several tens of kilojoules. Assuming 1000 such pulses as a mission requirement, the total energy storage would be several tens of megajoules. Assuming this energy would be delivered in a few hundred seconds, the average power requirement would be hundreds of kilowatts.

A summary of ambient-temperature battery systems is given in Figure 8.A.1. Lithium-based battery approaches are estimated to be capable of several hundred kilojoules per kilogram with instantaneous power densities of several hundred watts per kilogram. Hence, a battery supply weighing at most a few hundred kilograms would be capable of supplying the required energy at the rate required for the suggested discrimination mission. Other examples, such as rocket-launched radar probes, or mirror platforms, would probably require even less total battery weight. In the remainder of this appendix, we summarize the present state of battery technology and review several new approaches.

In general, all electrochemical systems have a well-

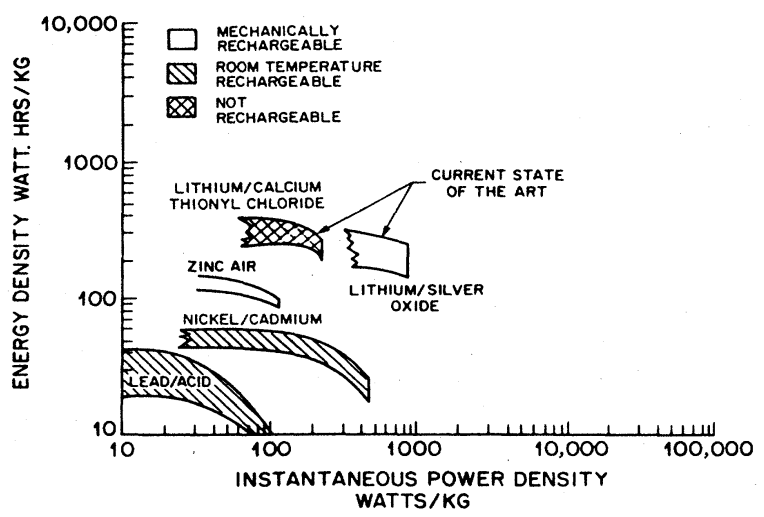

Figure 8.A.1. A comparison of presently available battery systems in terms of specific energy density and instantaneous power density. defined maximum energy density and open circuit voltage given by the following relation:

$$
\mathrm{V}=\frac{-\Delta \mathrm{G}^{0}}{\mathrm{nF}}
$$

where $\Delta G^{0}$ is the Gibbs free energy of the reaction, $V$ is the maximum open circuit voltage or standard cell potential, $\mathbf{n}$ is the number of electrons involved in the stoichiometric reaction, and $F$ is a constant known as the Faraday. The maximum useful work $\mathrm{W}_{\max }$ is obtained by dividing $\left(\Delta G^{0}\right)$ by the molecular weight $M$ of the stoichiometric reactants

$$
\mathbf{W}_{\max }=\frac{-\Delta \mathbf{G}^{0}}{\mathbf{M}}
$$

Therefore, the maximum energy density of a battery is well defined by thermodynamics. Battery system designers are then left to design systems that approach this maximum as closely as possible by reducing internal losses and maximizing current, by packaging for maximum power densities with minimum polarization losses.

Batteries may be divided into primary and secondary cells. Primary cells generate electricity through an irreversible reaction. Among these, lithium/air and aluminum/air systems can be recharged mechanically, by replacing the depleted metal plates and adding electrolyte. Other primary cells, such as lithium/thionyl chloride are only usable until the energy is consumed.

Secondary, or storage, batteries are electrically rechargeable. Lead/acid and nickel/cadmium batteries operate at room temperature, but higher performance in terms of both energy and power density is usually achieved with operation at high temperatures (over $200^{\circ} \mathrm{C}$ ) where the ion mobility is high.

In general, it is helpful to examine the trade-off between energy and power density. In Figure 8.A.2, the presently available battery systems are compared in this manner. Clearly, lithium-based systems offer the greatest opportunity because of the high reactivity of $\mathrm{Li}$, the heat of reaction of lithium with water, $\mathrm{H}_{2} \mathrm{O}_{2}$, or $\mathrm{O}_{2}$, etc., and the low molecular weight of $\mathrm{Li}$. However, present systems for $\mathrm{Li}$ are not rechargeable except mechanically. If we examine the batteries currently in the early research and development phases as shown in Figure 8.A.3, we clearly see that there are new primary lithium systems that may increase power and energy density by nearly an order of magnitude each.

A novel concept is the dynamic cell which uses a 


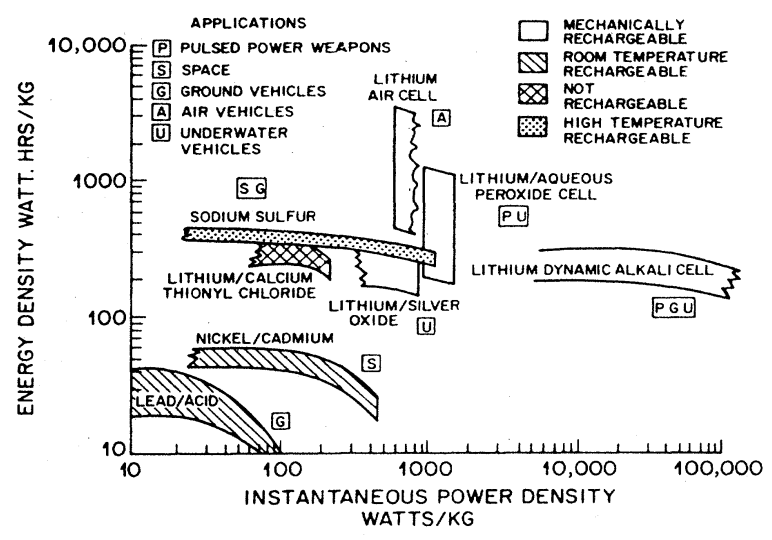

Figure 8.A.2. A comparison of potentially available battery systems in terms of specific energy density and instantaneous power density.

rotating cathode to control the waste heat from a lithium-based primary battery. The battery has achieved over $60 \mathrm{~A} / \mathrm{cm}^{2}$ for many minutes using a $\mathrm{Li} / \mathrm{HNO}_{3}$ couple. If the dynamic cathode concept were not employed, the system would experience thermal "run away" at these current densities. Electrolyte flow alone would not prevent this problem.

The most promising rechargeable system under investigation at the present time is the $\mathrm{Na} / \mathrm{S}$ system. The system uses molten sodium as the anode through the reaction

$$
\mathrm{Na} \rightarrow \mathrm{Na}^{+}+\mathrm{e}^{-}
$$

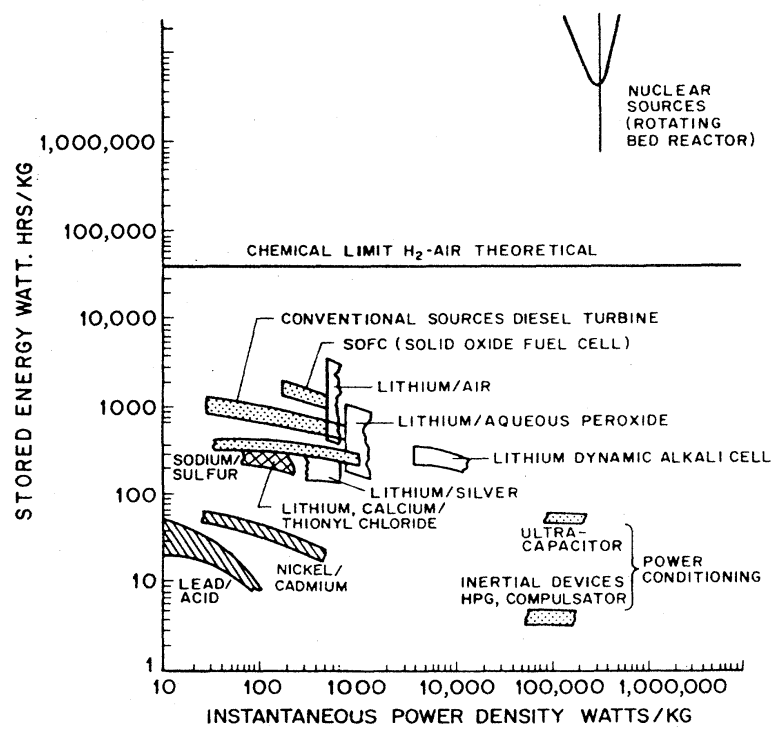

Figure 8.A.3. A comparison of presently available battery systems with newer systems in the research and development phase. and a sulfur-sulfide mix for the cathode that is given by

$$
\begin{aligned}
2 \mathrm{e}^{-}+\mathrm{XS} & \rightarrow \mathrm{S}_{\mathrm{x}}, \\
2 \mathrm{Na}+\mathrm{XS} & \rightarrow \mathrm{Na}_{2} \mathrm{~S}_{\mathrm{x}} \text { with } \mathrm{E}_{\text {reversible }}=2.08 \mathrm{~V}, \\
\mathrm{Na}+\mathrm{Na}_{2} \mathrm{~S}_{5} \rightarrow \mathrm{Na}_{3} \mathrm{~S}_{5} & \\
& \text { with } \mathrm{E}_{\text {reversible }}=1.79-2.08 \mathrm{~V} .
\end{aligned}
$$

For the Dow $\mathrm{Na} / \mathrm{S}$ system, high power density is achieved by placing the sodium in hollow $\mathrm{Na}^{+}$ conducting glass fibers with approximately a $80 \mu \mathrm{m}$ O.D. and a $50 \mu \mathrm{m}$ I.D. The sodium ion moves through this glass into a sulfur bath at approximately $300^{\circ} \mathrm{C}$. The high power density is achieved by placing hundreds or thousands of these fibers in a bundle which results in a very large surface area for ion migration and, therefore, potentially high current densities. The range of performance is also shown in Figure 8.A.3.

Another novel concept is the "super capacitor" that develops a chemically assisted storage capacity orders of magnitude higher than a flat plate capacitor of the same geometry. Such systems offer great promise for power conditioning and energy storage, although they may not always be capable of deep discharge.

Finally, the development of solid state batteries and electrolytes offers long range potential for high energy density storage. Using insertion reaction mixedconducting solid-solution electrodes, in which the charge is stored throughout the bulk of the solid electrode structure, one can make a conservative performance estimate based on classical assumptions for the energy density. Assuming a solubility of one electroactive ion per host unit cell, or one equivalent per mole, the electrode charge capacity with $10^{5} \mathrm{C} / \mathrm{mole}$, with a molar volume of $33 \mathrm{~cm}^{3} /$ mole. Such an electrode can have a charge density of $3 \times 10^{3} \mathrm{C} / \mathrm{cm}^{3}$. At $1 \mathrm{~V}$, this gives a capacity density of $3 \times 10^{3} \mathrm{~F} / \mathrm{cm}^{3}$ or an energy density of $3 \times 10^{3} \mathrm{~J} / \mathrm{cm}^{3}$. One can also get an order of magnitude estimate considering the chemical diffusion constant and the known crystal structure of a compound such as $\mathrm{TiSi}_{2}$, where the energy density will be of the order $2 \mathrm{eV} / 100 \mathrm{~A}^{3}$ which also give $4 \times 10^{3} \mathrm{~J} / \mathrm{cm}^{3}$.

If one compares these values to those which are typical of the common conventional dielectric/metal capacitors in the best practical cases, one typically gets $1-2 \times 10^{-3} \mathrm{C} / \mathrm{cm}^{2}$. If operating at $5 \mathrm{~V}$, this is equivalent to a specific capacity of $2-4 \times 10^{-4} \mathrm{~F} / \mathrm{cm}^{2}$ and $2.5-5 \times 10^{-3} \mathrm{~J} / \mathrm{cm}^{3}$. Hence, using very conservative estimates the difference between the two systems is a factor of $10^{6}$. Fabricated intercalated compounds (FIC) of lithium borate glass and silver borate glass have been studied. Work is also proceeding on other materials such as $\mathrm{In}_{2} \mathrm{Se}_{3}, \mathrm{PNb}_{2} \mathrm{~S}_{10}$, and $\mathrm{P}_{2} \mathrm{Nb}_{4} \mathrm{~S}_{21}$. These materials are prime candidates for electrodes, since their layered structure is similar to FIC. Thus, migrating 
ions can readily move across the electrolyte/electrode interface for compatible materials.

In summary, it is apparent that lithium and aluminum systems have the most promise as high energy density primary cells. It must be noted that these systems require a flowing electrolyte management sub-system, and may require a slightly higher than ambient temperature of the fluid (about $60^{\circ} \mathrm{C}$ for the lithium systems). The aluminum/air battery encounters problems with maintaining the voltage of the full cell and passivation of the aluminum surface due to oxidation.

In terms of projected power and energy density, the sodium/sulfur system has the most potential as a rechargeable system. This system must be operated at approximately $300 \mathrm{~K}$, which may not be a significant design problem for SDI since waste heat management is normally required for all batteries. The sodium/sulfur system with sodium-permeable glass appears to have the best projected power density.

\section{REFERENCES}

${ }^{1}$ R. Kerber, "The DARPA Battery Program" (1984).

${ }^{2}$ T. Goddard and G. B. Taggart, "Analysis of Electrochemical Power Sources," The BDM Corp., Report No. BDM/M-84-0041-TR (1984).

${ }^{3}$ W. S. Bishop, "Batteries and Fuel Cells for Pulsed Power Applications," Pulsed Power Lecture Series, Texas Tech University, 1985.

4“Assessment of Research Needed for Advanced Battery Systems," National Materials Advisory Board, Commission on Engineering and Technical Systems, National Research Council, Publication NMAB-390 (National Press, Washington, D.C., 1982).

${ }^{5}$ M. N. Golein, A Survey of Soviet Research on Electric Guns and High Power Batteries (Battelle Columbus Laboratories, Columbus, OH, 1982).

${ }^{6}$ Handbook of Batteries and Fuel Cells, edited by David Linden (McGraw Hill, New York, 1984).

${ }^{7}$ R. F. Wallis (private communication, 1984).

${ }^{8} \mathrm{M}$. Balkanski (private communication, 1985). 


\section{Chapter 9}

\section{SURVIVABILITY}

\section{CONTENTS}

9.1 Introduction

9.2 Survival in Peacetime

9.2.1 Ground-Based Assets

9.2.2 Space-Based Assets

9.3 Survival in Wartime

9.3.1 Survivability of Space-Based Components

9.3.1.1 Pellet Clouds

9.3.1.2 Direct Ascent Nuclear ASAT

9.3.1.3 Kinetic Energy Weapons (KEW)

9.3.1.4 Directed Energy Weapons

9.3.1.5 X-Ray Lasers

9.3.2 Survivability of Ground-Based Assets

9.4 Defense Survivability Tactics

9.4.1 Decoys, Shrouds, and Stealth

9.4.2 Maneuver

9.4.3 Proliferation

9.4.4 Hardening

9.5 Space Mines

9.6 Survivability of Sensor Platforms

9.6.1 Infrared, Longwave Infrared, and Optical Sensor Platforms

9.6.2 Survivability of Radar Platforms

9.7 Conclusions

References

In Chapter 2 we described the Soviet offensive threat and a number of countermeasures the Soviets might take if the U.S. deployed ballistic missile defenses. In Chapter 6 we discussed the interaction of DEW with targets, and the requirements imposed on defensive DEW so that they can make the intended targets of the offense inoperative (lethality). In this chapter the roles are reversed. The DEW system of the defense is now considered a target for the offense, and it must meet the requirement of survivability.

\subsection{INTRODUCTION}

Survivability of a ballistic missile defense system encompasses two broad issues: (i) survivability of system components and (ii) survivability of system function. The former need not be perfect; the latter must be so. A system is defined as "survivable" if it remains functional long enough to carry out its mission, that is, to prevent nuclear warheads from hitting their targets in the U.S.
Numerous concepts have been proposed for enhancing survivability, but little evaluation has actually been done to see which, if any, might be cost effective while retaining system performance. The primary reason for the current dearth of knowledge should be clear to the reader of the preceding chapters: component technologies are in very early development stages, few if any technology selections have been made, and architectures are as yet undefined.

Even so, it seems likely that survivability will be a major, if not the major, driver of system architecture. Survivability will make additional demands on performance requirements for sensors, weapons, and $\mathrm{C}^{3} \mathrm{I}$; it is possible that for any particular architecture these additional demands will be too great to be satisfied at any reasonable cost.

Survivability has been recognized from the beginning to be a major issue for ballistic missile defenses, whether land based or space based. It was a central issue in the BMD debate in the late $60 \mathrm{~s}$ and early $70 \mathrm{~s}$, and was one of the reasons the U.S. decided in 1976 to deactivate its BMD system at Grand Forks after a brief period of operation. The importance of survivability in the current era is clearly indicated by the fact that it is listed as one of the "Nitze Criteria" which the administration asserts must be assured before BMD deployments could be considered. Our remarks are necessarily qualitative because of uncertainties in defense system architectures and offensive threats (particularly responsive threats).

In discussing survivability, it is useful to distinguish two very different contexts, peacetime and wartime. By the former we refer to times long before the outbreak of strategic nuclear war; wartime includes the period just prior to the outbreak of strategic nuclear war, and of course the conflict itself.

\subsection{SURVIVAL IN PEACETIME}

\subsubsection{Ground-Based Assets}

Ground-based assets do not raise serious survivability issues in peacetime. They can be tested, serviced, and repaired to maintain full capabilities in spite of component deterioration or damage by natural causes, or of isolated acts of sabotage. Any coordinated attack on them would be considered an act of war. The survivability of ground-based assets in wartime will be discussed in Section 9.3.2. 


\subsubsection{Space-Based Assets}

An adversary may feel sufficiently threatened by deployment of space-based DEW defensive assets that he may decide to attack such assets in peacetime. During the stages of assembly, a defensive system is unlikely to have the capability of fully defending itself.

In the case of assembled assets, the adversary has the option, besides attack, of placing space mines in the vicinity of each high-value defensive space platform. A "space mine" is a small satellite that accompanies a larger enemy satellite in almost the same orbit and has the capability of destroying its larger and more expensive adversary at whatever time it chooses. A space mine is only one of several possible antisatellite weapons, but there is a qualitative difference because it is put in place in peacetime, long before any military engagement.

Space mines threaten cost-effective survivability of defensive platforms. It has been suggested that wartime survivability against space mines can be enhanced by enforcing during peacetime a "keep-out" zone around each defense platform. Thus, the survivability of defensive DEW space platforms is immediately tied in with national and international policy issues. The extent to which such policy or treaties can be relied upon is problematic. For a defense whose survivability depends upon such agreements or policies, it is unclear that adequate means exist to deal with violations without endangering the defensive system itself, and without precipitating escalation and attack.

Attacks on partially or completely deployed defense could be overt and prompt, as with an ASAT weapon; overt and slow, as for a laser attack on a satellite during its repeated passage over an adversary's ground station; or covert, as in the case of pellet clouds clandestinely introduced into counter-orbits and allowed to intercept defense satellites by random drift. The question of whether a prompt attack on a defensive space platform by an ASAT weapon in space above Soviet territory, without loss of life, will be considered an act of war leads again to an issue of policy. Slow, overt attacks and, especially, covert attacks, pose even more delicate issues.

Sensors, which must actively survey offensive territory in peacetime, cannot be directly shielded from laser radiation originating from that same territory. Counter-countermeasures which have been discussed but not yet studied for practicality include self-darkening windows and TR (transmit/receive) plasma switches to protect focal-plane arrays of detectors. Overt offensive irradiation, over extended periods or in sudden bursts, may cause blinding of sensor platforms.

Covert attacks present more intractable problems. Initially such attacks might appear merely as high failure rates of defense components. The defense should eventually be able to discover that its space components were failing through unnatural causes. The agent (nation) responsible for the failures would still have to be discovered and proven responsible. Even then, if only a few space objects were involved, and especially if the evidence pointing to the perpetrator came long after the event and were ambiguous, it would be difficult to respond with military force.

One should wish to lessen the chance that such judgments would have to be made, and so to take all practical measures to enhance defense survivability against covert attack in peacetime. To a considerable extent, many such steps could be covered by survivability measures designed to handle an overt wartime attack on the defense.

An important point is that peacetime survivability issues, and some of their extensions to wartime survivability, are inextricably linked to policy issues and to international agreements. These are, in turn, connected with issues of strategic stability and arms control. Since this study is focused on technical issues of DEW, the Study Group refrains from further comment.

\subsection{SURVIVAL IN WARTIME}

Consider now a direct attack by the offense without the constraint that the attack be covert or any concern of its leading to war. The attack by the offense on the defensive system might be a prelude to subsequent launch of ballistic missiles, or it might occur simultaneously.

No defensive system could survive an unconstrained attack. Fuel and other defense expendables would eventually be exhausted, even if the defense were able to avoid being overwhelmed sooner. In wartime the only constraint on the defense-suppression attacks by the offense will be the relative cost of attacking the defense versus offensive proliferation. Survivability comes down to a cost exchange calculus. One trades the marginal cost to the offense for destroying a unit of defense capability against the marginal cost for the defense of protecting against this incremental threat. These marginal costs will reflect the relative technical and system complexity of offense versus defense. It is clear that survivability is not a quantity which will ever be known with precision; systems architectures must provide significant costeffectiveness techniques in solving survivability problems.

The survival of components based in space (satellites) is a special concern. Satellites move along predictable paths in known orbits, can be studied in detail before attack, and will be expensive to harden, emplace, and proliferate. Defense satellites will generally be more vulnerable than RVs. Directed energy weapons platforms are quite large, and therefore difficult and expensive to harden; sensor satellites, although compact, are limited by the essential fragility of the sensors themselves. Consequently, the same type of weapons which the defense may seek to use against RVs could prove to be serious offensive threats against the space-based platforms of the defense itself. 


\subsubsection{Survivability of Space-Based Components}

The range of possible defense suppression attacks on space-based components is large, including the examples of offensive weapons discussed in Chapter 2. In practice a combination of these means must be expected, but because the precise combination will be unpredictable, ballistic missile defenses must have the capability to handle a wide ensemble of defense suppression attacks. Direct attack possibilities which will have to be taken into account include the following.

- Attrition attacks employing large clouds of pellets placed in orbit to destroy defensive space platforms. Such clouds are not really an attractive option to the offense but they are addressed here because of their simplicity and because they have been widely discussed.

- Attack of key space-based elements such as sensor platforms during the early construction of the defense system before the system can fully defend itself well. Non-nuclear or nuclear ASATs could be employed.

- Deployment of trailing space platforms, "space mines," during the early phases of defense deployment.

- Attack with nuclear weapons delivered by the ICBM force itself or by dedicated direct-ascent rockets.

- Direct attacks with kinetic energy weapons (ground based or space based).

- Attack with directed energy weapons, either ground based, delivered by pop-up missiles, or based on space platforms.

\subsubsection{Pellet Clouds}

A potential long-term attack on a space-based system could be a cloud of counterorbiting pellets. Such attacks would be initiated by placing a large number of relatively small pellets at the orbital altitudes of the space defense system. By calculating the probability of random interactions between the pellets and the space defense constellation, the effectiveness of the pellet cloud can be computed.

A counterorbiting pellet attack is unpredictable in terms of the individual platform hit and the time of the interaction between pellets and platform. This lack of predictability forces the attacker to put up much extra pellet mass or be patient and let the pellets and the constellation interact longer than would be the case with direct attack weapons. Because of randomness, such attacks cannot be depended upon to cut a well-defined hole in the defense constellation for permitting a sure safe launch of boosters. However, a significant degradation to the performance of the defense might well be caused and might lead to the destruction of the constellation over time.

Figure 9.1 illustrates the effectiveness of space pellets on a constellation of 81 defensive platforms in a $9 \times 9$ configuration ( 9 satellites in each of 9 orbits). In this example, it is assumed that a $100 \mathrm{~m}^{2}$ area per defensive satellite is vulnerable to attack by counterorbiting pellets. The pellets are chunks of material with a mass of $100 \mathrm{~g}$ each. At counterorbiting velocities, an individual pellet delivers about $10 \mathrm{MJ}$ of energy on impact. This figure shows the number of hits or collisions between the constellation and the pellet cloud in a year. The number of hits is not equal to the number of individual satellites damaged in a year since some might be hit more than once. The number of individual satellites having suffered at least a collision is roughly $90 \%$ of the total number of hits because of multiple hits on the same platform.

In Figure 9.1, it can be seen that the greater the altitude of the defensive satellites the larger the mass of the pellets required to achieve a particular effectiveness. This is due to the smaller percentage of space swept by a fixed mass of pellets at the higher altitudes.

There are three obvious responses to pellet attacksmaneuver, shields, and counterattack. Maneuver must be an altitude change as opposed to a plane change. Only a change of altitude avoids the clouds of pellets completely. Shields offer a means of remaining at a given orbit altitude and still being able to deal with a pellet attack. However, shields capable of handling counterorbiting pellets with masses of $100 \mathrm{~g}$ will tend to be very massive themselves. Counterattack could involve an attack of the launch facilities used to place the pellets in space, assuming the facility could be identified and the intention to dispense pellets known in advance. Such a policy stand might require the direct attack of launch facilities in the Soviet Union-clearly a provocative act.

Because of the large masses of pellets which must be put on orbit, because pellet clouds could be defeated by small maneuvers, and because the clouds would also present risk to the attacker's own systems (launch vehicles, ICBMs, etc.) the use of such clouds is questionable as a countermeasure.

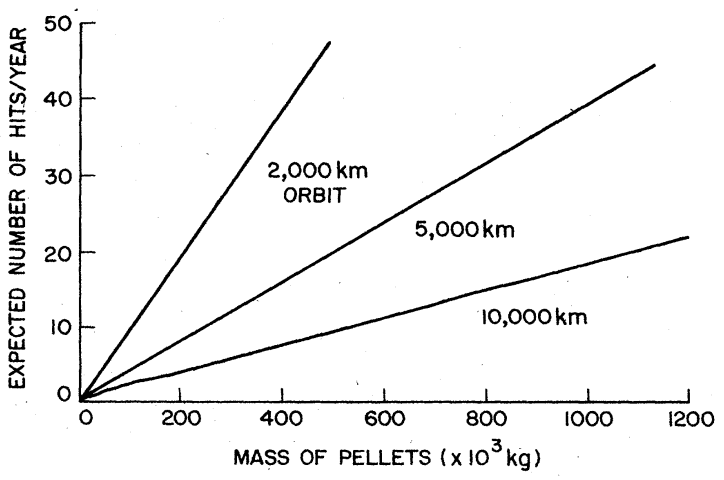

Figure 9.1. Effectiveness of a space pellet cloud on 81 space platform constellation ( 9 satellites in each of 9 orbits). 


\subsubsection{Direct Ascent Nuclear ASAT}

Probably the most straightforward approach to attacking a defense's low altitude platform is a reasonably high-performance rocket carrying a well-hardened nuclear warhead, and probably a number of decoys as well. The hardening of such an ASAT weapon could be tailored to the particular type of defense platform under attack and any protective satellites accompanying it. Large amounts of ablator material could be applied, for example, if attacking a laser battle station.

The most obvious such ASAT attack could come from the offense's ICBMs themselves. The attacker could devote some fraction of his force to defense suppression, or produce additional ICBMs to serve as ASATs. In either case, the ASATs would be indistinguishable from other ICBMs during the first phase of their attack.

In this connection, it is important to consider the battle time available. If one credits the offense with a nuclear ASAT attack capable of flying to $1000 \mathrm{~km}$ altitude in $300 \mathrm{~s}$, launching 10 offensive interceptors against each platform under attack and deploying 1000 decoys and 2 hardened warheads from each interceptor, the defense is faced with a high traffic rate. It needs to address approximately 30 targets per second and a total of approximately 10000 targets per battle station.

Also worth noting is the nature of the attacker's decoys. Decoy requirements here appear to be much less demanding than those for RVs traveling on ICBM trajectories. A nuclear ASAT threat (warheads and decoys) need not be delivered to a precise point (as in the case of RVs targeted against hardened sites); any object projected to come within several tens of $\mathrm{km}$ of the battle station must be considered a threat. Further, the time for discrimination of warheads and decoys is very short compared to the missile defense case (approximately $250 \mathrm{~s}$ vs $1000 \mathrm{~s})$.

The offense may alternatively attack space-based DEW platforms with a large number of dedicated, highperformance interceptor rockets carrying nuclear warheads (and decoys). These might be used in conjunction with ICBM ASATs. The seriousness of this threat is evident from its numerical size alone. For an investment of about $\$ 100$ billion (which is a canonical first-estimate price for the deployed directed energy platforms themselves, without associated surveillance and tracking), the offense could afford to deploy 10000-20000 interceptors. Suppose these interceptors are capable of $6 \mathrm{~km} / \mathrm{s}$ and capable of reaching orbiting platforms at altitudes of about $500 \mathrm{~km}$. Thus, the flight time of an interceptor to land its nuclear weapon precisely on a platform is $83.3 \mathrm{~s}$. If the retarget time for the space laser is $0.1 \mathrm{~s}$, then 850 interceptors will eliminate one laser platform with probability essentially one, even if the laser is infinitely bright. Since only 5-6 laser stations are actually in the battle space in many defense architectures, an arsenal of only 10000 interceptors is multiply redundant to take out such a boost phase defense.
The example above highlights an important generality: the retarget time of a space-based DEW is a dominant parameter not only in its systems performance, but in its survivability as well.

Direct-ascent nuclear attacks are so threatening that no directed energy battle stations in low earth orbit can be presumed to be survivable on their own. In recognition of this fact, systems architecture studies currently concentrate on protecting directed energy platforms with complementary constellations of kinetic energy platform satellites. The survivability of these platforms is supported by the relative cheapness and hence potentially larger numbers. Analysis of the system effectiveness of such kinetic energy constellation is beyond the scope of the present study. The dominant point here is that survivability of low altitude space-based DEW battle stations must be guaranteed by means beyond selfdefense.

The foregoing arguments appear to discourage the deployment of DEW assets at low altitudes, and to favor deployment at altitudes well above $500 \mathrm{~km}$. Going to much higher altitudes would certainly enhance the survivability, but would exact a severe penalty in brightness requirement and accuracy of beam pointing. The brightness required is proportional to the square of the range. In Chapter 3 an example of a range of $3000 \mathrm{~km}$ was chosen as a reasonable approximation for a system architecture with DEW lasers in which the fighting mirrors are deployed at an altitude around $1000 \mathrm{~km}$. Since a range of $3000 \mathrm{~km}$ already severely stresses the requirements on any type of laser or particle beam weapon, deployment of DEW platforms at higher altitudes would increase the shortfall between required technologies and existing state of the art by additional orders of magnitude.

\subsubsection{Kinetic Energy Weapons (KEW)}

One form of kinetic energy weapons (KEW) are small rockets which home in on their targets and destroy them by direct impact, which can transfer many megajoules of energy. Development programs are currently aimed at producing KEW vehicles with gross weights around one hundred pounds and payloads on the order of ten pounds.

A scenario very similar to the one just presented could be described for the use of such small hit-to-kill vehicles plus decoys. As with direct-ascent nuclear rockets, large numbers of interceptors are easily possible and affordable. It is worth noting, however, that kinetic energy weapon technology developments that may make it possible to deploy very light KEW interceptors in defense of DEW platforms also would make it possible to loft large numbers of such interceptors in boosters to act as defense suppression weapons. If a DEW cannot defend itself successfully by attacking boosters, then a stratagem of carrying $\mathrm{KEW}$ interceptors into space to 
defend it can be neutralized by lofting similar interceptors into space to attack it.

Defense against kinetic energy weapons used by the offense presents another problem. It would not be practical to attempt to vaporize an incoming mass of $10 \mathrm{~kg}$, with a thickness of at least several $\mathrm{cm}$, with a DEW. Nor is it likely that one can economically shield against the impact of such projectiles. Survivability against attack by kinetic energy weapons would probably require the possibility of maneuvering. The fuel penalty for maneuverability is discussed below in Section 9.4.2.

\subsubsection{Directed Energy Weapons}

Chapters 3-8 effectively summarize the capabilities of DEWs to attack objects in space. If one credits the offense with DEW technologies comparable with those posited for the defense, the handling of a direct attack becomes an especially demanding task. The hardness levels of critical elements of the space-based platforms are not all likely to be as high as those of the boosters or RVs the DEW was designed to destroy. Thus any effective BMD DEW also becomes a potent threat to space-based components of an opponent's defense.

\subsubsection{X-Ray Lasers}

Pop-up x-ray lasers pose such a serious potential threat as ASATs that slightly more detailed discussion is warranted. A high intensity $x$-ray beam can bleach through the atmosphere from altitudes of $80 \mathrm{~km}$ on up. Therefore, the pop-up x-ray laser is an attractive response of the offense against space-based defensive platforms, since most of these platforms would be large, valuable targets, reasonably few in number (with high absentee ratios).

If $\mathrm{x}$-ray lasers with brightness higher than that of other directed energy weapons could be developed, $x$-ray lasers would surpass other space-based directed energy weapons both in their lethality and in survivability.

Of course, hardening of the battle station must be considered. But against an $\mathrm{x}$-ray laser the hardness levels required for survival are potentially very high. The discusssion in Section 3.5 and Figures 3.31 and 3.32 can be used to derive values for the necessary nuclear explosion yield for producing a $12 \mathrm{~kJ} / \mathrm{cm}^{2}$ x-ray laser fluence on a target at $100 \mathrm{~km}$. As shown in Chapter 6, this fluence is above the threshold for impulse damage to a target hardened with $1 \mathrm{~cm}$ of structural element. For multiple targets, multiple beams may be used. Again, the discussion in Section 3.5 can be used to arrive at the necessary nuclear explosion yield in order to illuminate the multiple targets with the desired $\mathrm{x}$-ray laser fluence. While expendable shields might be devised for protection against one attack, as discussed in Chapter 6, it is doubtful that affordable protective shields can be designed against multiple attacks closely spaced in time.

\subsubsection{Survivability of Ground-Based Assets}

Based on the brightness calculations in Section 3.1, the two principal classes of ground-based DEW devices, excimer lasers and FELs, will need extremely large facilities to meet the energy and/or power requirements of greater than $100 \mathrm{MJ} / 1 \mathrm{GW}$ device output. The lasers themselves, with their associated optics (and uplink compensation), will occupy well over 100 acres; the power supplies will also have to be reasonably extensive. In addition, the great expense associated with these facilities will militate strongly against building extra facilities beyond those necessary to provide adequate mission brightness (see Section 5.7).

The large size of the facilities makes them difficult to hide and very difficult to harden against direct attack. Their high cost makes it difficult to make them survivable through proliferation. Taken together, then, these characteristics raise serious questions of survivability for the ground-based lasers. Attacks by moderate-yield (hundreds of kilotons) nuclear weapons could very effectively prevent the lasers from performing strategic defense missions.

Such nuclear attacks could obviously be delivered by present submarine-launched ballistic missiles (SLBMs), since hard-target accuracy would not be necessary. Thus, a strategic defense in which ground-based lasers played a role would have to be extremely effective against SLBMs as well. Even fairly low leakage rates could be lethal to the laser system.*

The small number of laser sites also raises a question concerning the effectiveness of sabotage attack. A nuclear weapon of sufficient yield could easily be packed in an automobile, or carried by one or two men. Thus extraordinarily effective security measures would have to be developed to protect these key facilities against such straightforward attacks by foreign agents. Although no one doubts the presence of numerous Soviet agents in this country, or the ease with which nuclear weapons could be smuggled in by a determined adversary, this threat does not seem to have been given serious study.

Other nuclear weapon delivery means could be equally effective. Stealth cruise missiles could penetrate to ground-based laser targets with far better accuracy than would be needed. Sea-launched cruise missiles might be able to attack with little or no advanced warning, despite their long flight times. Thus, this defense would also have to succeed against cruise missile attack.

\footnotetext{
*Only a single warhead would be needed to put the laser out of action. Thus, ten SLBMs, each carrying ten MIRVed warheads, could destroy a laser in a defense that was $99 \%$ effective against SLBMs. Such an attack price would hardly be exorbitant if it disarmed the boost phase defense.
} 


\subsection{DEFENSE SURVIVABILITY TACTICS}

\subsubsection{Decoys, Shrouds, and Stealth}

Many of the measures an offense might use to counter defenses can be considered as countercountermeasures for application to the problem of defense survivability. Decoying of defense satellites is one suggestion of this type. But decoying of large space platforms, such as ones carrying lasers, particle beam accelerators, or large optical components, is likely to be quite difficult, much more so than decoying RVs. Passive discrimination of actual defense components from decoy components would likely be easier than discrimination of RVs from RV decoys because longer times would be available (months, even years) and because defense components will almost certainly require active decoys to match various emissions from real defense components. For example, defense platforms must have communication links, and these must be used even in peacetime, at the least to report the platform's status of repair and activity. Convincing decoys would need to do the same. In addition, platforms with DEWs (with the exception of $\mathrm{x}$-ray lasers) must be accompanied by large electrical power sources (nuclear or chemical) which will have large thermal signatures and so are not easily masked or decoyed. Relay components such as spacebased mirrors may be more realistically decoyed. But even these would have to be exercised from time to time, and would have to communicate with other parts of the defense system. Simple, lightweight decoys for DEW platforms may not be feasible.

Even if such decoys can be developed and deployed, the offense could respond by deploying inspector satellites which in peacetime would examine defense satellites close up and from many aspects to aid discrimination. Such an eventuality would then require the defense to deploy its decoys immediately before or during an engagement. This prospect raises questions of practicality which remain, at the currently limited level of study, entirely problematic.

Shrouds which hide defense components from earth or inspector satellite view might be used. These could make it difficult to aim ground-based directed energy weapons at defense satellites and could frustrate homing kinetic energy weapons as well. However, since shrouds would likely interfere with normal defense operations, their ultimate value in wartime is unclear. For protection against attack from all directions (possibly by groundbased lasers with space-based relay mirrors), the shrouds would have to be enveloping balloons; these surely would interfere with defense operations.

Stealthlike measures which reduce the observables of satellites (radar cross sections, LWIR signatures, visible cross sections) are sometimes proposed for survivability enhancement. A few general remarks can be made. The chances that the large platform with nuclear power generators can be made invisible to an offense which has unlimited time of observation before attack appear remote. Defense components must be in frequent communication to report status, be alert for commands from the battle-manager, etc. Any satellite which can receive communications, and which must also radiate information about itself, cannot truly be invisible over very long periods of time. Additional discussion of decoys is found in Chapter 7.

\subsubsection{Maneuver}

Maneuver is another way in which space-based elements of the defense might enhance their survivability against attack. The distance the satellite must move depends on the kill range of the attacking weapon and its maneuver capability, if any. Figure 9.2 shows maneuver fuel requirements for a $100000 \mathrm{~kg}$ satellite as a function of offset, assuming $200 \mathrm{~s}$ of maneuver time. Fuel, both to accelerate and decelerate (i.e., actually regain original orbital velocity), is considered in the quantities shown. Further, it is unlikely that satellites could fire to microradian accuracies while undergoing rapid acceleration. As shown, pellet cloud avoidance $(1 \mathrm{~km}$ offset) requires about one quarter of a tonne of fuel per maneuver and nuclear attack avoidance necessitates almost 9 tonnes of fuel for a $50 \mathrm{~km}$ keep-out requirement. Clearly maneuver cannot handle multiple nuclear attack for heavy DEW platforms in a practical way, and even small offsets are fuel intensive. Maneuverability, while an important capability (especially for smaller satellites), cannot in itself guarantee survivability.

\subsubsection{Proliferation}

Survivability can be enhanced by proliferation of the components of the defensive system. The issue here is cost. Defense proliferation is almost certain to be costineffective for very expensive space-based components.

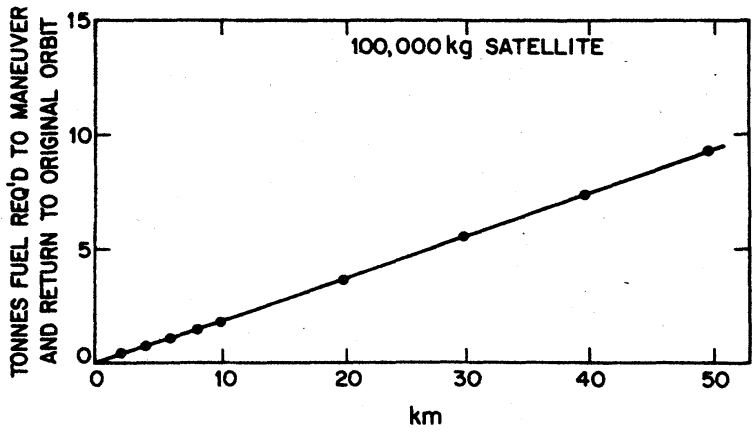

Figure 9.2. Fuel necessary for orbital maneuvering of a 100000 $\mathrm{kg}$ satellite as a function of offset. Fuel values include quantities needed for both acceleration and deceleration. A $200 \mathrm{~s}$ maneuver time is assumed. 
None of the directed energy weapons discussed in earlier chapters of this report are likely to be cheaper than direct ascent nuclear or kinetic energy ASAT weapons, excepting possibly the $\mathrm{x}$-ray laser which is basically more survivable anyway, being deployable in a pop-up mode. The same will be true for sensor platforms, and probably space-based relay mirrors as well. Survivability through proliferation may be most attractive when small spacebased kinetic energy weapons (described above) are employed by the defense. Generally, proliferation will be expensive; that expense will dictate small numbers. As a result, proliferation alone is an unattractive means of achieving survivability.

\subsubsection{Hardening}

Like RVs and boosters, defense satellites could be hardened against attack by kinetic and directed energy weapons. Protection against thermal kill or impulse kill lasers requires hardening in accordance with the lethality discussion of the preceding chapters. Sacrificial shields surrounding defense satellites were considered in Chapter 6 (Figure 6.24). Such shields would probably need to be at least partially jettisoned during the operation of the satellite, for example, on command from the early warning sensors platforms.

\subsection{SPACE MINES}

Although operational space mines do not yet exist, their practical development does not require new technologies. Space mines could be put into orbit in peacetime to trail satellites of the defense. These mines would need to have tracking systems and thrusters to enable them to follow a satellite as it conducted routine maneuvers or "delousing" operations. Upon command in wartime, space mines would move close to their prey and explode or release small homing rockets. Kill radii might extend upward to $1 \mathrm{~km}$ for conventional explosive mines and $10-100 \mathrm{~km}$ for nuclear mines, depending on the yield of the mine and the nuclear hardness of the platform. For self-defense, space mines could be booby-trapped by presetting them to explode if perturbed by outside nongravitational forces.

It has often been suggested that, to cope with space mines, nations could establish, either by unilateral declaration or by bilateral agreement, "keep-out" zones. That is, announce "rules of the road" according to which satellites must maintain substantial distances from one another. These zones could be defined for unconditional safety-say, radii of $1000 \mathrm{~km}$. The rules might be enforced by international agreement or by unilateral declaration that foreign satellites which approached closer than the specified keep-out distance would be destroyed, either in peacetime or in wartime. The effectiveness of this measure in wartime as in peacetime, is highly questionable. In particular, if the space mine contains an $\mathrm{x}$-ray laser, keep-out zones are of no use.

One of the technical uncertainties associated with policy solutions to the space mine problem is the possibility of covert space mines. We have not studied this issue. The space mine must make orbital maneuver to maintain trail, and these maneuvers could provide signatures for the defense. Despite this difficulty, space mines with small observable signatures could be taxing to the defense since the defense must watch all directions and would prefer as long a range of observation as possible. Such space mines would limit defense reaction time and maneuver options.

\subsection{SURVIVABILITY OF SENSOR PLATFORMS}

\subsubsection{Infrared, Longwave Infrared, and Optical Sensor Platforms}

As discussed earlier, these assets may be subject to attack by direct ascent nuclear and non-nuclear vehicles, possibly with accompanying decoys. Attack warning sensors which must continuously survey territory of the offense may be blinded by laser or nuclear blast radiation at relatively low levels.

Clearly sensor protection is essential, but is peripheral to this study. Much effort to increase hardening of satellite-based sensors beyond their current state is certainly necessary. Complex cost and systems issues will also be involved. For example, one might protect most sensors with shields during the early stages of conflict, then bring new groups of sensors into operation (by removing the shields) in phases as the earlier groups are destroyed or blinded. This simple strategy might or might not prove practical, but it would certainly require a large proliferation of sensor assets, with consequent effects on system cost and complexity.

Nuclear precursor or salvage fused bursts are a response option easily available to the offense. The feasibility of salvage fusing is well established. The precise effects of atmospheric nuclear detonations still contain significant uncertainties, but their general effects are understood and current predictive capability indicates that their effects upon sensor performances can be essential. Thus, while such tactics are unlikely to be seen as offering the attacker high confidence of specific rates of penetrating various defenses, they are very likely to be seen as greatly increasing the complexity, or lowering the effectiveness, of the defense. High yield (megaton class) nuclear precursors detonated at high altitude will decrease the signal/noise ratio for space-based DEW sensors for significant periods of time-seconds to minutesdepending on yield, detonation altitude, and sensor characteristics. While we expect that precursor bursts would not be depended on as the primary means of defense suppression, their use seems likely as a means of degrading IR, radar, and nuclear signature sensors 
associated with pointing and tracking, homing, and discrimination.

Offensive nuclear bursts perhaps will take on special significance if the defense uses nuclear signatures (such as $\gamma$ or neutron emanation from targets interrogated by NPBs) for interactive discrimination. There, the nuclear noise created by salvage or precursor bursts could greatly decrease $\mathbf{S} / \mathbf{N}$. Whether the consequence is reasonable for the defense to cope with depends on the details of the defense system (power of NPB, range, number of sensors, etc.), as discussed in Chapters 4 and 7.

\subsubsection{Survivability of Radar Platforms}

Space-based radars, either microwave or optical, are essential to acquire and track offensive missiles in boost and post-boost phases. Space-based microwave radars either of the phased array or reflector antenna variety, require very large antenna areas (hundreds of square meters) to achieve the necessary range. With such large antenna areas, it is likely to be very difficult to protect such structures from attack by directed energy weapons. In contrast, space-based optical radars have smaller areas which need to be protected. This advantage may be offset, however, by the greater difficulty of maintaining surface integrity at optical wavelengths.

\subsection{CONCLUSIONS}

1. Survivability is an essential feature of a BMD system employing space-based assets; such survivability is highly questionable at present. Evaluation of these issues requires a systems approach that includes hardening, active defense, and operational tactics. During the deployment phase, the space-based assets are especially vulnerable.

2. Survivability of ground-based facilities also raises serious issues. The relatively small number of large facilities associated with ground-based laser sites makes these facilities high-value targets.

3. Directed energy weapons with capabilities below those needed for many ballistic missile defense applications can threaten space-based assets of a defensive system.

4. X-ray lasers driven by nuclear explosions would constitute a special threat to space-based sensors, electronics, and optics.

5. Since a long time will be required to develop and deploy an effective ballistic missile defense, it follows that a considerable time will be available for responses by the offense. Any defense will have to be designed to handle a variety of responses since a specific threat cannot be predicted accurately in advance of deployment.

\section{REFERENCES}

${ }^{1}$ Ballistic Missile Defense, edited by A. B. Carter and D. N. Schwartz (The Brookings Institution, Washington, D.C., 1984). 


\section{APPENDIX A}

\section{ISSUES IN SYSTEMS INTEGRATION*}

\section{CONTENTS}

A.1 Introduction

A.2 Architecture and System Complexity

A.3 Computing and Communications

A.4 Simulation and Testing

A.5 Effectiveness

A.6 Deployment

References

\section{A.1 INTRODUCTION}

The development of a defense against ballistic missiles requires that individual components and supporting technologies be integrated into a coherent whole, a system in which the capabilities and limitations of the various subsystems are balanced, communication is provided, and a suitable command network is established to coordinate operations. The integration of such a system raises many complex considerations, e.g., the specification of system goals and technical requirements, the choice of a system architecture, the issues of computability, testability, maintainability, and the evaluation of system effectiveness against an evolving offensive threat that would be the result of BMD deployment. Of course, a decision to deploy a BMD system would depend not merely on satisfactory resolution of system integration issues but also on political and economic considerations.

The process of logically organizing the system functions and components, known as systems engineering, is a complex one involving tradeoffs, judgments, and cost analyses. In practice, systems engineering is not necessarily an orderly process but rather a dynamic, iterative one well seasoned with intuition and experience. Appropriate goals and technical requirements for BMD are themselves subjects of controversy. Depending on the goals chosen-e.g., to render nuclear weapons impotent and obsolete, to complicate Soviet attacks, to improve strategic and arms control stability, to limit damage to military and civilian targets, or to hedge against Soviet breakthroughs in BMD research and development-the system structure and requirements will vary greatly. Of particular

\footnotetext{
${ }^{*}$ For help in preparing this appendix, the Study Group acknowledges valuable consultation with Dr. Edward Cornet, Dr. Curtis Hines, Dr. Brockway McMillan, and Dr. Charles Zraket.
}

relevance is the scale of offensive threat to be countered as well as whether the threat is constrained by arms control agreements or only by economic and policy considerations. In any case, compromises between systems goals and technical realities would be forged in the process of systems engineering.

\section{A.2 ARCHITECTURE AND SYSTEM COMPLEXITY}

The goals of a system typically are expressed by numerous objectives which must be accomplished within the limits of externally and internally imposed constraints. The particular concept chosen for the system configuration is referred to as its architecture. An architecture is an expression of two related hierarchies, one of structure and the other of function, which together prescribe the system components and their modes of interaction. There may be an ensemble of architectures which satisfy system goals, wherein various members of the ensemble may employ different components and technologies. Not all of the architectures will prove technically feasible; not all of the technically feasible architectures will be deemed desirable when judged against performance, cost, and other criteria.

Complexity in the architecture of a large system is expressed by the multiplicity of its levels, and by the diversity and interconnectivity of its components. In large systems these factors result in complex and often unanticipated behavior that one cannot predict simply from the properties of individual components. A system is more than the sum of its parts.

The context in which the system must function is another important source of complexity. In addition to natural limitations, architectures must accommodate many man-made constraints. Examples of natural limitations relevant to missile defenses are the backgrounds produced by particle fluxes in space, cloud cover obscuration of ground-based laser sites, and the finite lifetimes of components and subsystems in the space environment. Man-made constraints of a technical nature are, for example, infrared and gamma-ray backgrounds and other electromagnetic disturbances generated by nuclear explosions. Other examples of man-made constraints are political ones, e.g., those imposed by treaties or policy decisions that forbid the use of certain technologies or those which constrain testing, or economic constraints. Although some constraints, either natural or man-made, can be specified at the 
outset, others cannot. To deal with uncertainty, systems engineering has to rely on theoretical modeling, expert judgments, and best estimates.

\section{A.3 COMPUTING AND COMMUNICATIONS}

Computing in a strategic defense system includes processing of inter- and intra-level subsystem communication as well as computational tasks such as the processing of sensor signals, establishing and maintaining track files, and the comparison of processed signals to preprogrammed references. The software for many tasks will entail assumptions that impact system behavior in real engagements that are difficult and perhaps impossible to anticipate fully in advance. The necessity for such assumptions and their effects are major factors in the present controversy ${ }^{1}$ about computability in BMD systems.

A key tradeoff in any large system is the organization of computing resources. In some cases computation is centrally organized and managed. However, for most large systems, a decentralized computation regime is more typical. That is, subsystems or groups of subsystems of the overall system are configured to be operationally and computationally autonomous to a large extent. Highly processed information ("traffic") is passed between groups; this intergroup communication affects a high level of overall control without requiring extensive central computation and corresponding large communication load.

For BMD systems the optimum balance between centralized control and decentralized computational autonomy presents challenging issues: e.g., the development of appropriate computing algorithms, given the likelihood of errors; methods to measure and maintain the required level of system reliability; means of maintaining overall coherent command and control. In addition, system design must minimize potential vulnerability to loss of function through attacks on a few system nodes. To mitigate against such a possibility, one option is to highly decentralize BMD system control. But then autonomous action by a part of the system becomes potentially troublesome; namely, if computation and decision-making is highly decentralized and predelegated, the danger that a part of the subsystem will respond inappropriately becomes serious. Thus, decentralization may increase the problems of command and control, while more centralized organization may entail increased vulnerability.

In addition, a BMD system must deal with the large number of potentially threatening objects that might appear in a strategic exchange and the limited engagement times available. In architectures that require "birth-to-death" tracking, adequate and redundant capability must be present to pass track files of the large number of objects from station to station during midcourse and early reentry and to deal with local saturation, crossing tracks, closely spaced objects, and changes in the number of objects.

It is important to recognize that some of the computation must be error-free, while some of it may be in a category known as "fault-tolerant." For instance, the coding which allocates functions to various system subelements must be free of errors. But the coding which processes information from the various sensors can in principle be designed to accomplish its objectives in spite of occasional errors.

Software verification and reliability also raise major systems integration issues, in view of the large amount of code (many millions of lines by various estimates) required to implement the many system functions already anticipated. The extent to which the methods of structured programming can deal with these issues remains a subject of considerable controversy. 1 .

\section{A.4 SIMULATION AND TESTING}

Simulation is a major tool in the design phase of a large system and in early verification of subsystem performance and behavior. But as subsystems are combined and interconnected, especially as multiple layers develop, the system becomes more complex and its behavior becomes harder to predict and simulate with confidence. The systems engineer cannot be sure that the models used for simulation have not left out some critical ingredient. Thus, the desirability of field testing arises.

To have confidence in its performance, a BMD system would have to be tested under simulated combat conditions. This poses serious conceptual and practical problems. First of all, the field testing of any large and complex system is problematic because of the number and range of possible parameters for the full system. At best one could select parameters and conditions statistically and settle for verifying a limited set of operational possibilities. For BMD, field testing must deal with possible treaty restrictions. Moreover, the unpredict- ability of the evolving threat complicates testing enormously. A further complication arises from the difficulty of accurately simulating the system's operation in nuclear environments. Indeed, the interactions of multiple nuclear explosions with the upper atmosphere, and the effect of these interactions upon weapons and sensors, are only understood (and hence simulated) on the basis of theory that is grounded on a very sparse, pre-1963 data base. Thus, the uncertainty in understanding and verifying BMD system performance would pose a major predicament to defense system designers (as well as to designers of offensive systems).

In reality, large complex systems typically go through an iterative process-simulation, test, and modification combined with periods of actual operation-to achieve well understood, verifiable performance. 


\section{A.5 EFFECTIVENESS}

BMD system effectiveness could be addressed once system goals and objectives are delineated, candidate architectures have been specified, and information on component capability is available. That is, the effectiveness of each architecture and its components could be estimated with computer-aided modeling against an ensemble of offensive threats including a variety of direct attacks on BMD components. Only when this has been done can offense/defense cost exchange ratios be estimated, for example, to evaluate measures such as the Nitze criteria that are described in Section 1.5.

In practice, estimations of effectiveness and cost exchange ratios are complicated by many uncertainties, some involving the offense and others involving the defense. Therefore, a range of values would be obtained even for a simple measure of system effectiveness like the fraction of offensive RVs negated by the defense. One also must recognize that some of the goals for a BMD system may be hard to quantify, e.g., deterring first strike by lowering the confidence of the offense. Thus, effectiveness estimates would entail many qualitative judgements together with quantitative assessments.

\section{A.6 DEPLOYMENT}

Given a go-ahead as a result of the politicaltechnical-economic decision to deploy BMD, a whole system would ultimately come into existence, and limited tests of its overall performance could then be conducted.
As noted above, the evaluation could not be carried out with real signals or operational conditions, so that a comprehensive performance evaluation would remain elusive. Nevertheless, it is likely that much would be learned; design mistakes and incompatibilities that were previously overlooked would become apparent, and cost estimates would be replaced by costs.

For a deployed BMD system, maintenance of spacebased components poses special requirements and problems. While the past U.S. experience with satellites is extremely valuable in designing space-based components of a defense system, one cannot assume that space-based directed energy weapons and related elements would automatically enjoy the low maintenance and replacement schedules associated with many U.S. satellite systems.

Finally, one cannot expect a BMD system to be static either in design or hardware. One major factor in BMD system evolution would be the changes in offensive threat. Another major factor would be the evolution in scientific and engineering knowledge. New components of greater capability would come within reach. One would expect BMD system evolution to occur incrementally as have other large systems in the past.

\section{REFERENCES}

${ }^{1}$ See, for example, D. L. Parnas, Comm. ACM 28, 1326-1335 (1985). For a different point of view, see the Eastport Study Group, Summer Study (1985): A Report to the Director for the Strategic Defense Initiative Organization, December 1985. 


\section{APPENDIX B}

\section{SATELLITE CONSTELLATIONS}

\section{CONTENTS}

B.1 Satellite Number Requirements

B.1.1 Distributed Boosters - Flat Earth

B.1.2 Concentrated Boosters - Flat Earth

B.2 Satellite Constellation Details

B.2.1 Distributed Boosters - Spherical Earth

B.2.2 Concentrated Boosters - Spherical Earth

B.3 Orbit Choices and Numbers

References

\section{B.1 SATELLITE NUMBER REQUIREMENTS}

Ballistic missile defenses operating in boost phase, post-boost phase, or mid-course necessarily require satellite elements. For example, sensors for acquisition, tracking, and discrimination as well as components for data communication and relay necessarily must be space-based given the curvature of the earth and the need to view missiles over the Soviet Union soon after launch. Moreover, most of the proposed directed energy weapons for attacking missiles and RVs must also be based in space or have space-based components. Only terminal defenses can in principle do all tracking from the ground or from airborne platforms and thus escape the need for space-based elements. But even in this case some space elements are likely.

The number of satellites required for sensing or kill is a strong function of the maximum range at which a system can function and the kill time per event. It is also strongly affected by the spatial and temporal distribution of the missiles, RVs, and decoys which must be addressed. Two other factors which have great impact on satellite number requirements are defense survivability and cost. Survivability is discussed elsewhere in this report; costs are not.

The problem of determining satellite numbers is best separated into two cases: boost and mid-course. Post-boost falls in between these but may be treated with the same arguments used for boost phase if the total post-boost time is short enough that deployments are localized in a small region of space. If, on the other hand, post-boost activities are long and an extended spatial regime is involved, post-boost requirements must be estimated in the manner of mid-course requirements.

We consider only the boost phase since it is easiest and has received the greatest attention to date. ${ }^{1-10}$ The same principles apply to the mid-course case, but the kinematics are more complex.

Satellite requirements for boost phase intercept have been the subject of great controversy. This is perhaps surprising since, given a complete set of parameters describing the performance of a satellite battle station and the distribution of boosters, the number of satellites required is a problem with a demonstratively correct answer (in contrast to most other issues discussed in this report). Most of the early controversies over minimum satellite requirements for boost phase defense have been resolved in principle. Those which remain concern values of parameters which are likely to be achieved by actual hardware. As interesting as these remaining controversies may be, it seems likely that in the end that survivability considerations be overarching and that the resulting satellite constellations will bear little resemblance to those optimized in the absence of a survivability requirement.

Any actual system will have a discrete distribution of satellites and boosters and a resulting coverage which shows variability due to this discreteness. Under such conditions a complete study is done best by computer simulation. However, to gain insight into systems issues it is advantageous to smooth out this variability by either considering continuous spatial distributions of boosters or of satellites, or both. Once smoothing has been done, many aspects of the problem can be handled with simple equations. We adopt such an approach here.

Even after smoothing, two factors remain to complicate the analysis: (i) the earth is not flat and (ii) the earth rotates. We begin by ignoring the earth's rotation; we treat the flat earth case here and give in Section B.2.1 the corrections appropriate for the spherical earth. We assume that the motion of the booster may be ignored during the boost phase engagement time. In doing so one should regard the booster as fixed at some mean location along its trajectory; in general this will not be at the earth's surface.

Let $\mathrm{H}$ be the hardness in $\mathrm{J} / \mathrm{m}^{2}$ of the booster and $\mathrm{B}$ the brightness in $\mathrm{W} / \mathrm{sr}$ of the weapon. Since it appears frequently we define the auxiliary quantity $k=H / B$. In the case of directed energy discrimination rather than directed energy kill, $H$ should be interpreted as the amount of energy required per interrogation. 


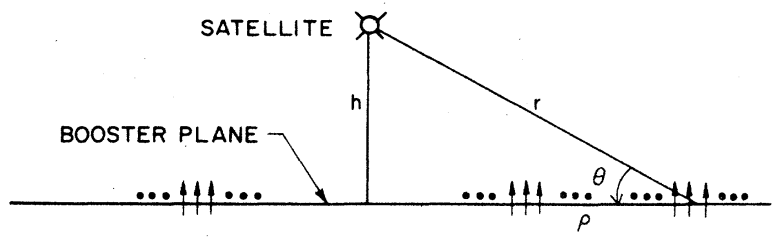

Figure B.1. Geometry for the distributed booster case in the flat earth approximation. The slant range from the satellite to the booster is $r$ and the satellite height above the booster plane is $h$. The distance $\rho$ is measured in the booster plane.

\section{B.1.1 Distributed Boosters-Flat Earth}

At slant range $r$ (Figure B.1) the time required to kill one booster is

$$
t(r)=\frac{H r^{2}}{B}=k r^{2}
$$

If boosters are uniformly distributed in a plane with a density of $b$ boosters per square meter and the slew and settle time between individual booster kills is $T_{s}$, then in time $T$ a single satellite at height $h$ above the booster plane can destroy all boosters out to a radius $\rho_{\mathrm{c}}$ (measured in the booster plane) where

$$
\begin{aligned}
\mathrm{T} & =2 \pi \mathrm{b} \int_{0}^{\rho_{\mathrm{c}}}\left[\mathrm{k}\left(\rho^{2}+\mathrm{h}^{2}\right)+\mathrm{T}_{\mathrm{s}}\right] \rho \mathrm{d} \rho \\
& =2 \pi \mathrm{bk} \int_{0}^{\rho_{\mathrm{c}}}\left[\rho^{2}+\frac{\mathrm{T}_{\mathrm{s}}^{\prime}}{\mathrm{k}}\right] \rho \mathrm{d} \rho,
\end{aligned}
$$

where $\mathbf{T}_{\mathrm{s}}^{\prime}=\mathrm{T}_{\mathrm{s}}+\mathrm{kh}^{2}$ is an effective slew time which includes the effect of satellite altitude above the booster plane. As written, expression (B.2) assumes normal incidence independent of satellite position, i.e., spherical boosters. If the assumption of normal incidence is not a good approximation, kill times are increased over those given above by an inverse power of the cosine of the angle of incidence. Some authors attempt to model this by taking the angle $\theta$ in Figure B.1 as the angle of incidence. The real world is likely to be more complicated.

We set the left-hand side of Equation (B.2) equal to $T_{0}$, the total boost time available for kill (i.e., actual boost time reduced by the sum of the following: the time consumed by the early warning and tracking systems, the time for the battle management to make decisions and communicate orders, and the time it takes for a booster to rise above the atmosphere in the case of nonpenetrating weapons). We have then upon carrying out the integral in Equation (B.2):

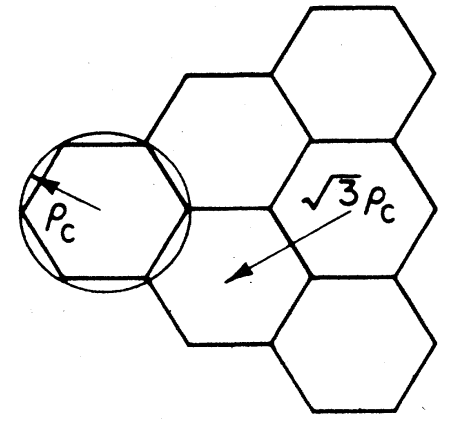

Figure B.2. A hexagonal closed packed arrangement of satellites which provides coverage with no gaps for a uniform booster field.

$$
\frac{\mathrm{T}_{0}}{\pi \mathrm{bk}}=\frac{1}{2} \rho_{\mathrm{c}}^{4}+\frac{\mathrm{T}_{\mathrm{s}}^{\prime}}{\mathrm{k}} \rho_{\mathrm{c}}^{2}
$$

Since one satellite covers an area $\pi \rho_{\mathrm{c}}^{2}$ in the booster plane, we obtain that the required satellite area density $s$ satisfies

$$
\frac{\mathrm{T}_{0}}{\mathrm{bk}}=\frac{1}{2 \pi}\left(\frac{1}{\mathrm{~s}}\right)^{2}+\left(\frac{\mathrm{T}_{\mathrm{s}}^{\prime}}{\mathrm{k}}\right) \frac{1}{\mathrm{~s}} .
$$

There are edge effects since circles do not cover the plane uniformly. To take care of this consider hexagons inscribed in circles of radius $\rho_{\mathrm{c}}$. We may tile the plane by arranging the hexagon centers on a triangular lattice (Figure B.2) with a nearest neighbor spacing of $\sqrt{3} \rho_{\mathrm{c}}$. The area of each hexagon is $(3 \sqrt{3}) / 2\left(\rho_{\mathrm{c}}\right)^{2}=2.60 \rho_{\mathrm{c}}^{2}$, which is $17 \%$ less than that of the exscribed circle of area $\pi \rho_{\mathrm{c}}^{2}$. Thus if we want coverage with no gaps, we should increase the value of $\mathrm{s}$ implied by Equation (B.4) by $17 \%$. We will not bother with this latter refinement here.

The solution of Equation (B.4) is

$$
\left.\mathrm{s}=\frac{\mathrm{b}}{2} \cdot \frac{\mathrm{T}_{\mathrm{s}}^{\prime}}{\mathrm{T}_{0}}\right]\left\{1+\sqrt{1+\left(\mathrm{b}^{*} / \mathrm{b}\right)}\right\},
$$

where

$$
\mathrm{b}^{*}=\frac{2}{\pi}\left(\frac{\mathrm{T}_{0}}{\mathrm{~T}_{\mathrm{s}}^{\prime}}\right)\left(\frac{\mathrm{k}}{\mathrm{T}_{\mathrm{s}}^{\prime}}\right)
$$

The minimum value of $s$ clearly occurs for $h=0$, i.e., satellite in the booster plane so $T_{s}^{\prime}=T_{s}$. In practice this will not be achievable even for boosters above the atmosphere for a variety of reasons among which is the motion of the boosters during the engagement. For example, at a velocity of $7 \mathrm{~km} / \mathrm{s}$, a 
booster will move $700 \mathrm{~km}$ in $100 \mathrm{~s}$, a distance which is not negligible compared to typical kill ranges. Setting $\mathrm{h}=0$ therefore underestimates the required satellite density. We will carry $h$ as a parameter and choose some typical values for numerical illustrations. We will find that generally values of $\mathrm{h}$ less than $200 \mathrm{~km}$ change numerical results little for even the most optimistic slew times.

If $b \gg b^{*}$ (slew time dominant) Equation (B.5) reduces to a linear relation between satellite density and booster density

$$
\mathrm{s}=\mathrm{b}\left(\frac{\mathrm{T}_{\mathrm{s}}^{\prime}}{\mathrm{T}_{0}}\right)
$$

The physical interpretation of this last expression is obvious; independent of range, each satellite destroys one booster each retarget time until the boost phase is over.

On the other hand, if effective slew time is negligible, $b \ll b^{*}$, we have a square root relationship between satellite and booster densities

$$
\mathrm{s}=\frac{1}{2}\left(\frac{\mathrm{T}_{\mathrm{s}}^{\prime}}{\mathrm{T}_{0}}\right) \sqrt{\mathrm{bb}^{*}}=\sqrt{\mathrm{bk} / 2 \pi \mathrm{T}_{0}}
$$

A combination of small spacing between the satellite and booster planes, short slew time, long boost time, high booster hardness, and low weapon brightness is required to be in the square root regime.

As a numerical example take $T_{0}=100 \mathrm{~s}$, $\mathbf{H}=200 \mathrm{MJ} / \mathrm{m}^{2}, \quad \mathbf{B}=2.69 \times 10^{20} \mathrm{~W} / \mathrm{sr}, \quad$ and $\mathrm{h}=200 \mathrm{~km}$. We have $\Delta \mathrm{T}_{\mathrm{s}} \equiv \mathrm{kh}^{2}=0.030 \mathrm{~s}$ and so the altitude effect makes a negligible correction for slew times greater than $0.1 \mathrm{~s}$. (Shorter slew times appear unlikely.) If instead, $\mathrm{h}=500 \mathrm{~km}$, the altitude contribution to effective slew time is $0.19 \mathrm{~s}$ and thus not negligible for very short slew times. For $\mathrm{h}=1000 \mathrm{~km}, \Delta \mathrm{T}_{\mathrm{s}}=0.74 \mathrm{~s}$, a number which is likely to dominate other contributions to slew time.

If we use the first set of parameters above and choose $T_{s}=1 \mathrm{~s}$, we have $b^{*}=47 /(\mathrm{Mm})^{2}$, whereas for a slew time an order of magnitude shorter, $T_{s}=0.1 \mathrm{~s}$, we have instead $b^{*}=4710 /(\mathrm{Mm})^{2}$. To put this in context, imagine distributing 1400 boosters (current inventory) uniformly over the current Soviet deployment area of $10(\mathrm{Mm})^{2}$. This gives $b=140 /(\mathrm{Mm})^{2}$. We see that for the above parameters, the conditions of square root scaling are not satisfied for $1 \mathrm{~s}$ slew times but are met for $0.1 \mathrm{~s}$ slew times. The transition point for the above choice of parameters is $T_{s}=0.58 \mathrm{~s}$. If instead we choose $h=1000 \mathrm{~km}$, we find $b^{*}=67 /(\mathrm{Mm})^{2}$ for the short slew time $(0.1 \mathrm{~s})$ and so conditions are not in the square root regime for even current Soviet booster deployments. Since survivability requirements are likely

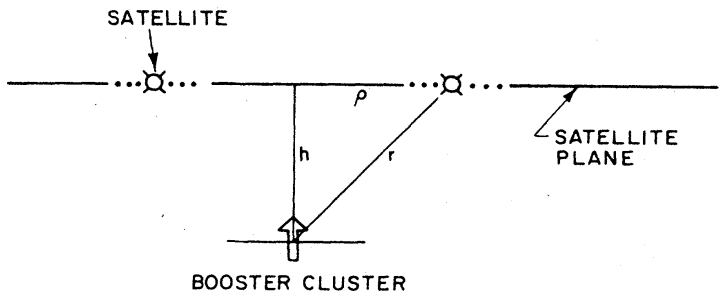

Figure B.3. Geometry for the concentrated booster case in the flat earth approximation. The slant range is $r$ and the satellite plane is a height $h$ above the booster cluster. The distance $\rho$ is measured in the satellite plane.

to require architectures in which space platforms are deployed at altitudes of $500 \mathrm{~km}$ or higher, the linear relation will usually be operative. Note that Equation (B.7), the linear, asymptotic result, is a lower bound to the exact nonlinear result. The square root law in Equation (B.8) applies near the origin (small b) not large $b$.

\section{B.1.2 Concentrated Boosters-Flat Earth}

Now suppose all boosters are concentrated at a single point while satellites are uniformly distributed in a plane with density $s$. In the available boost time $\mathbf{T}_{0}$, the annulus of satellites (Figure B.3) lying between $\rho$ and $\rho+\mathrm{d} \rho$ as measured in the plane of satellites can kill

$$
\mathrm{dB}=\mathrm{s} 2 \pi \rho \mathrm{d} \rho \mathrm{b}(\rho)
$$

boosters where

$$
\mathrm{b}(\rho)=\frac{\mathrm{T}_{0}}{\mathrm{k}\left(\rho^{2}+\mathrm{h}^{2}\right)+\mathrm{T}_{\mathrm{s}}} .
$$

The kill potential of the satellite constellation is therefore

$$
\begin{aligned}
\mathbf{B} & =\frac{\mathrm{T}_{0} \pi \mathrm{s}}{\mathrm{k}} \int_{0}^{\rho_{\mathrm{m}}} 2 \rho \mathrm{d} \rho \frac{1}{\rho^{2}+\mathrm{T}_{\mathrm{s}}^{\prime} / \mathrm{k}} \\
& =\frac{\pi \mathrm{s}}{\mathrm{k}} \mathrm{T}_{0} \ln \left\{\frac{\mathrm{k}\left(\rho_{\mathrm{m}}^{2}+\mathrm{h}^{2}\right)+\mathrm{T}_{\mathrm{s}}}{\mathrm{kh}^{2}+\mathrm{T}_{\mathrm{s}}}\right\},
\end{aligned}
$$

where the integral has been cut off at an upper limit $\rho_{\mathrm{m}}$ which we now discuss.

In the flat earth case the cutoff is fixed by the maximum range beyond which a satellite can no longer 
kill even a single booster in time $T_{0}: b\left(\rho_{\mathrm{m}}\right)=1$. This gives

$$
\rho_{\mathrm{m}}^{2}+\mathrm{h}^{2}=\frac{\mathrm{T}_{0}-\mathrm{T}_{\mathrm{s}}}{\mathrm{k}}
$$

and so

$$
\mathrm{B}=\frac{\pi \mathrm{s}}{\mathrm{k}} \mathrm{T}_{0} \ln \left[\frac{\mathrm{T}_{0}}{\mathrm{~T}_{\mathrm{s}}^{\prime}}\right]
$$

We note that the required satellite density is linear in the number of boosters; there exists no square root regime in this case.

In Section B.2.1 we rederive Equations (B.5) and (B.13) taking into account the curvature of the earth. As expected, the corrections are small for cases of practical interest. In Section B.3 we take up the question of relating the satellite density $s$ discussed here to an actual configuration of satellites taking into account satellite and earth rotation.

\section{B.2 SATELLITE CONSTELLATION DETAILS}

We consider here the generalizations to Equations (B.5) and (B.13) to the case of the curved earth and find that the changes are minor for satellite altitudes $h$ small compared to the radius of the earth. The one exception is the case where the maximum kill range is set by line-of-sight to the earth's limb rather than by total available boost time. This, however, can be easily handled in Equation (B.5) by introducing an appropriate cutoff.

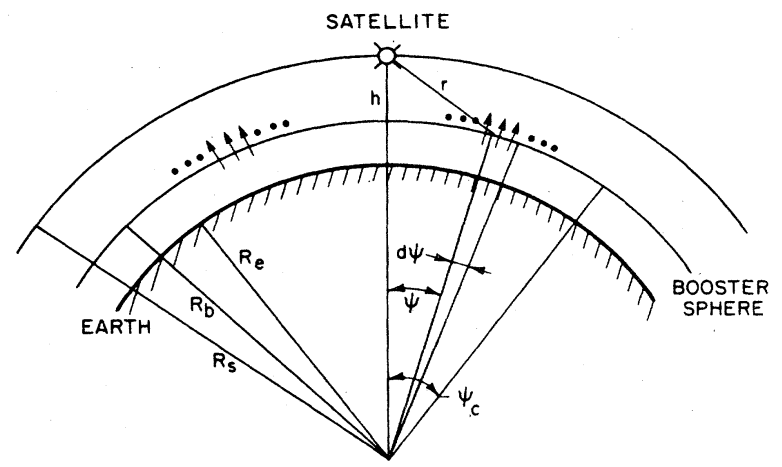

Figure B.4. Geometry for the distributed booster case when the earth's curvature is included. Boosters are uniformly distributed on a spherical shell of radius $\mathbf{R}_{\mathrm{b}}$; discrete satellites are located at radius $\mathbf{R}_{\mathrm{s}}$. The cutoff angle $\psi_{\mathrm{c}}$ is discussed in the text.

\section{B.2.1 Distributed Boosters-Spherical Earth}

The above calculation can be repeated including the curvature of the earth; see Figure B.4. Kill time may be expressed

$t(r)=T_{s}+k r^{2}=T_{s}+k\left(R_{s}^{2}+R_{b}^{2}\right)-2 k R_{s} R_{b} \cos \psi$,

where $\psi$ is an angle measured at the earth's center. The number of boosters in an annulus of angular width $\mathrm{d} \psi$ is

$$
\mathrm{dn}=2 \pi b R_{b}^{2} \sin \psi d \psi
$$

We have then for the relation between boost time $T_{0}$ and the maximum angle $\psi_{\mathrm{c}}$ which can be covered by a single satellite

$$
\mathrm{T}_{0}=2 \pi \mathrm{R}_{\mathrm{b}}^{2} \mathrm{~b} \int_{0}^{\psi_{\mathrm{c}}} \mathrm{d} \psi \sin \psi[\alpha-\beta \cos \psi]
$$

where

$$
\alpha=\mathrm{T}_{\mathrm{s}}+\mathrm{k}\left(\mathrm{R}_{\mathrm{s}}^{2}+\mathrm{R}_{\mathrm{b}}^{2}\right)
$$

and

$$
\beta=2 \mathbf{k R}_{\mathrm{s}} \mathbf{R}_{\mathrm{b}} \text {. }
$$

If the maximum angle is determined by a horizon

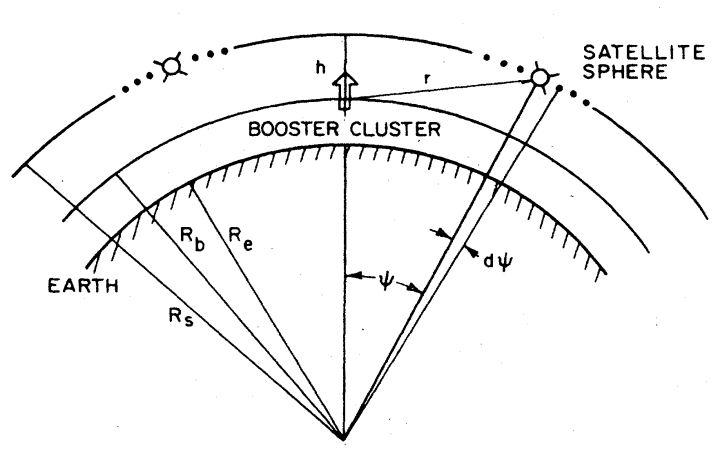

Figure B.5. Geometry for the concentrated booster case when the earth's curvature is included. Satellites are uniformly distributed on a spherical shell of radius $R_{s}$; the booster cluster is at radius $R_{b}$. Cutoff angles are discussed in the text. 
cutoff and not time, the required satellite density is independent of $b$ and is determined entirely by geometry once a satellite altitude has been chosen. In this case each satellite covers an area out to an angle $\psi_{\mathrm{c}^{\prime}}$ (Figure B.5):

$$
\psi_{\mathrm{c}}^{\prime}=\cos ^{-1}\left[\frac{\mathbf{R}_{\mathrm{b}}}{\mathbf{R}_{\mathrm{s}}}\right) \text {. }
$$

(This is the "overkill" case; the weapons are oversized for the job required.) The above expression assumes that atmospheric or other considerations makes it impossible to shoot below the altitude of the booster shell to reach more distant satellites. If this is not the case and only the earth's surface provides a hard cutoff, the maximum angle becomes instead

$$
\psi_{\mathrm{c}}^{\prime \prime}=\cos ^{-1}\left(\frac{\mathbf{R}_{\mathrm{e}}}{\mathbf{R}_{\mathrm{s}}}\right)+\cos ^{-1}\left(\frac{\mathbf{R}_{\mathrm{e}}}{\mathbf{R}_{\mathrm{b}}}\right) .
$$

More likely is the case in which the cutoff is total boost time. In this case each satellite handles an area

$$
\mathbf{A}_{\mathrm{s}}=2 \pi \mathbf{R}_{\mathrm{b}}^{2}\left(1-\cos \psi_{\mathrm{c}}\right)
$$

where $\psi_{c}$ is related to $T_{0}$ by Equation (B.14). The satellite density satisfies therefore

$$
\frac{T_{0}}{b}=\left\{(\alpha-\beta) \frac{1}{s}+\frac{\beta}{4 \pi} \frac{1}{R_{b}^{2} s^{2}}\right\}
$$

The solution of Equation (B.22) has a form that is identical to the flat earth case except that the quantity $b^{*}$ in Equation (B.5) is replaced by $b^{* *}$. where

$$
\mathrm{b}^{* *}=\mathrm{b}^{*}\left(\frac{\mathbf{R}_{\mathrm{b}}+\mathbf{h}}{\mathbf{R}_{\mathrm{b}}}\right) \text {. }
$$

As before one has the special limits of a slew-timedominated linear region and a square root region for negligible slew times and low booster densities. We see from Equation (B.23) that, except for very high satellite altitudes, the corrections due to earth curvature are negligible except for those cases in which the earth's curvature provides the cutoff.

\section{B.2.2 Concentrated Boosters-Spherical Earth}

In the spherical earth case the satellites are supposed instead to be distributed on a spherical shell of radius $R_{s}$ and the boosters are clustered at a point on a shell of radius $\mathbf{R}_{\mathrm{b}}$; Figure B.5. The total number of boosters which can be killed is

$$
\begin{aligned}
\mathbf{B} & =\pi \mathbf{R}_{\mathrm{s}}^{2} \mathrm{~s} \mathbf{T}_{0} \int_{0}^{\psi_{\mathrm{m}}} \mathrm{d} \psi \sin \psi \frac{1}{(\alpha-\beta \cos \psi)} \\
& =\frac{\pi \mathbf{R}_{\mathrm{s}}^{2} \mathrm{~s} \mathrm{~T}_{0}}{\beta} \ln \left(\frac{\alpha-\beta \cos \psi_{\mathrm{m}}}{\alpha-\beta}\right),
\end{aligned}
$$

where $\alpha$ and $\beta$ are as before. The upper limit to the integration is set either by the earth's horizon or by the condition that the marginal satellite can kill just one booster in the total boost time $\mathrm{T}_{0}$.

For the time limited case, we have

$$
\cos \psi_{\mathrm{m}}=\frac{\alpha-\mathrm{T}_{0}}{\beta}
$$

and thus

$$
\mathbf{B}=\frac{\pi \mathrm{s}}{\mathrm{k}} \mathrm{T}_{0} \frac{\mathrm{R}_{\mathrm{s}}}{\mathrm{R}_{\mathrm{b}}} \ln \left\{\frac{\mathrm{T}_{0}}{\mathrm{kh}^{2}+\mathrm{T}_{\mathrm{s}}}\right\}
$$

as the generalization of Equation (B.13). For the horizon-limited case we have instead

$$
\begin{aligned}
& \cos \psi_{\mathrm{m}}^{\prime}=\frac{\mathbf{R}_{\mathrm{b}}}{\mathbf{R}_{\mathrm{s}}} \\
& \mathbf{B}^{\prime}=\frac{\pi \mathrm{s} \mathbf{T}_{0}}{\mathrm{k}}\left(\frac{\mathbf{R}_{\mathrm{s}}}{\mathbf{R}_{\mathrm{b}}}\right) \ln \left\{\frac{\mathbf{T}_{\mathrm{s}}+\mathrm{kh}\left(2 \mathbf{R}_{\mathrm{b}}+\mathrm{h}\right)}{\mathrm{T}_{\mathrm{s}}+\mathbf{k h}^{2}}\right\} .
\end{aligned}
$$

We note in all cases for point launch the satellite density is linear in the number of boosters just as it was in the corresponding flat earth case.

The above expressions can be minimized with respect to altitude. For the time-limited case the optimal altitude is obviously $h=0$ and so

$$
\mathrm{B}_{\max }=\left(\frac{\pi \mathrm{s}}{\mathrm{k}} \mathrm{T}_{0}\right) \ln \left(\frac{\mathrm{T}_{0}}{\mathrm{~T}_{\mathrm{s}}}\right)
$$

for either a flat or spherical earth. For the horizonlimited spherical earth case we have instead as an optimal altitude (assuming $\mathrm{h} \ll \mathrm{R}_{\mathrm{b}}$ ) 
$h=\sqrt{\left(T_{s} / k\right)}$

and as a consequence

$\mathbf{B}_{\max }^{\prime}=\frac{\pi \mathrm{s}}{\mathrm{k}} \mathbf{T}_{0}\left\{1+\frac{\sqrt{\mathrm{T}_{\mathrm{s}}}}{\sqrt{\mathrm{kR}_{\mathrm{b}}^{2}}}\right\} \ln \left\{\frac{\mathrm{T}_{\mathrm{s}}+\mathrm{R}_{\mathrm{b}} \sqrt{\mathrm{kT}_{\mathrm{s}}}}{\mathrm{T}_{\mathrm{s}}}\right\}$.

\section{B.3 ORBIT CHOICES AND NUMBERS}

The above equations give the satellite density $s$ in satellites $/ \mathrm{m}^{2}$ required to defeat a given booster threat in either the distributed launch case (b boosters $/ \mathrm{m}^{2}$ ) or point launch case (B boosters total). We now turn to the calculation of the total number $S$ of satellites required. Two effects must be dealt with: (i) rotation of the earth about its axis, and (ii) motion of the satellite along its trajectory.

The crudest calculation assumes that the entire earth is covered with satellites with uniform density s. In this case the total number of satellites required is

$$
\mathrm{S}=4 \pi \mathrm{R}_{\mathrm{s}}^{2} \mathrm{~s}
$$

where $R_{s}$ is the radius of the sphere over which the satellites are deployed. Let a denote the radius of a

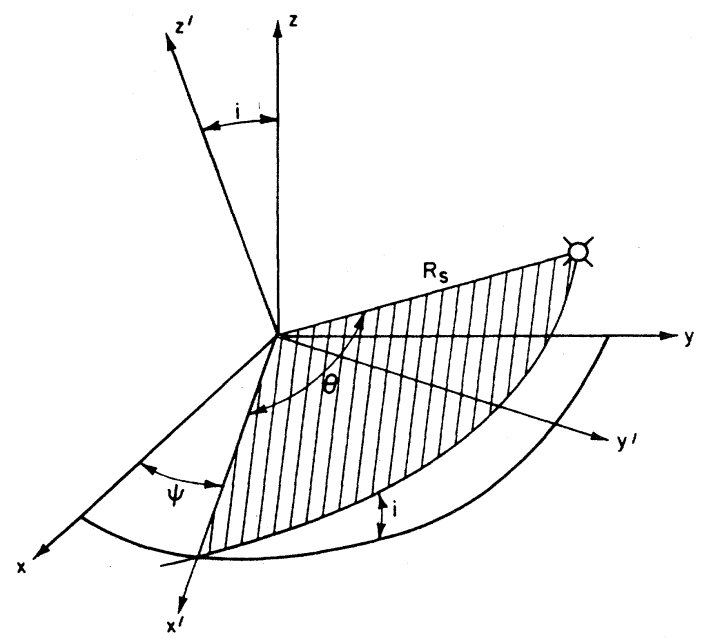

Figure B.6. Coordinate system $x y z$ is space fixed with its origin at the earth's center and $z$ axis along the earth's spin axis. Satellite motion takes place in the $x^{\prime} y^{\prime}$ plane of the $x^{\prime} y^{\prime} z^{\prime}$ coordinate system. The angles $\psi$ and $i$ specify the orientation of the satellite plane; the angle $\theta$ the position of the satellite in the plane. circle in the satellite plane which encompasses all the satellites active in a particular engagement. The quantity

$$
\mathrm{AR} \equiv \frac{4 \pi \mathrm{R}_{\mathrm{s}}^{2}}{\pi \mathrm{a}^{2}}=\frac{4 \mathrm{R}_{\mathrm{s}}^{2}}{\mathrm{a}^{2}}
$$

is called the absentee ratio. Although one can choose satellite constellations which give uniform density over the sphere in the continuum limit, such a choice is a poor one in any case of interest.

To see how one can do better than a uniform distribution, consider a single plane of satellites in circular orbit of radius $\mathbf{R}_{\mathrm{s}}$ about the earth. Relative to a space fixed coordinate system xyz whose $\mathrm{z}$ axis coincides with the earth's 'spin axis, we may describe the position of a given satellite by angles $\psi, \mathrm{i}$, and $\theta$ as shown in Figure B.6. The angle $\psi$, called the ascending node, specifies the intersection ( $\mathrm{x}^{\prime}$ axis) of the plane containing the satellite orbit and equitorial (xy) plane; the angle $i$ specifies the inclination of the satellite plane relative to the equitorial plane; and the angle $\theta$ the phase of the satellite relative to the $x^{\prime}$ axis. The ranges are $0 \leq \psi<2 \pi, \quad 0 \leq \mathrm{i}<\pi$, and $0 \leq \theta<2 \pi$. In circular orbits the motion of a satellite corresponds to uniform angular motion in $\theta$ at fixed $\psi$ and $i$. (We neglect the precision of the satellite orbit plane in $\psi$ caused by the oblateness of the earth.) If we have a total of $\mathbf{N}$ satellites in the plane distributed uniformly on a circle, then in a sector $\mathrm{d} \theta$ the number of satellites is

$$
\mathrm{dN}=\frac{\mathrm{N}}{2 \pi} \mathrm{d} \theta
$$

Points on the earth are specified by a longitude $\phi$ and a latitude $\lambda ; 0 \leq \phi<2 \pi,-\pi / 2<\lambda<\pi / 2$. Rotation of the earth corresponds to uniform angular

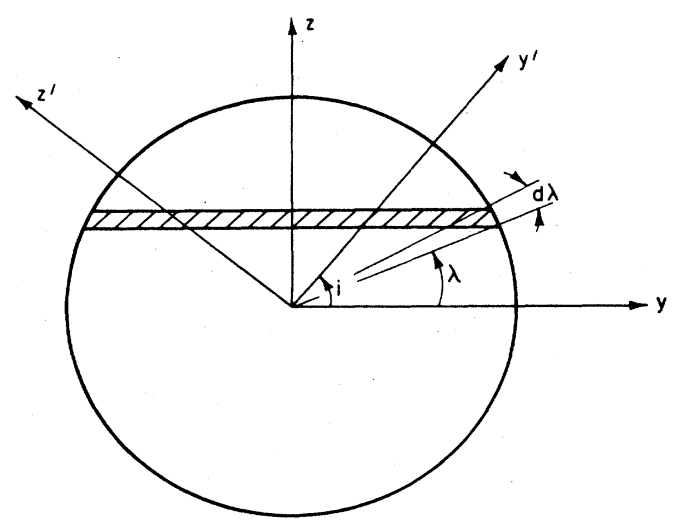

Figure B.7. A view along the $\mathrm{x}^{\prime}$ axis illustrating the region covered by satellites in a latitude band $\mathrm{d} \lambda$ when the earth's rotation is taken into effect. 
velocity in $\phi$ at constant $\lambda$; namely, $\dot{\phi}=2 \pi / \mathrm{D}$ where D is the sidereal day. Now consider an interval of latitude $\mathrm{d} \lambda$ on the earth, Figure B.7. Using

$$
\sin \theta \sin i=\sin \lambda
$$

we have

$$
\cos \theta \sin i d \theta=\cos \lambda d \lambda
$$

and find that there are

$$
\mathrm{dN}=[2] \frac{\mathrm{N}}{2 \pi} \theta(\mathrm{i}-|\lambda|) \frac{\cos \lambda \mathrm{d} \lambda}{\sqrt{\sin ^{2} \mathrm{i}-\sin ^{2} \lambda}}
$$

satellites in the interval $\mathrm{d} \lambda$ where [2] in Equation (B.37) is a counting factor which recognizes that a given satellite orbit crosses the internal $\mathrm{d} \lambda$ twice.

The rotation of the earth smears these $\mathrm{dN}$ satellites over a band which has an area

$$
\mathrm{dA}=\mathrm{R}_{\mathrm{s}}^{2} 2 \pi \cos \lambda \mathrm{d} \lambda
$$

Hence the earth spin averaged satellite density for one plane of $\mathbf{N}$ satellites is

$$
\frac{\mathrm{dN}}{\mathrm{dA}}=\frac{\mathrm{N}}{2 \pi^{2} \mathbf{R}_{\mathrm{s}}^{2}} \frac{1}{\sqrt{\sin ^{2} \mathrm{i}-\sin ^{2} \lambda}} \theta(\mathrm{i}-|\lambda|) .
$$

We may rewrite the above as

$$
\frac{\mathrm{dN}}{\mathrm{dA}}=\left[\frac{\mathrm{N}}{4 \pi \mathrm{R}_{\mathrm{s}}^{2}}\right] \mathrm{y}(\lambda, \mathrm{i})
$$

where the factor in parentheses corresponds to a uniform satellite density and $y$ is the "satellite enhancement" factor

$$
y(\lambda, i)=\frac{2}{\pi} \frac{1}{\sqrt{\sin ^{2} i-\sin ^{2} \lambda}} \theta(i-|\lambda|) .
$$

We see that if all boosters are located near a common latitude $\lambda_{b}$, then choosing an orbit inclination $i \simeq \lambda_{b}$ gives a coverage which is substantially improved over that for the uniform case. Strictly speaking, for a point concentration of boosters at $\lambda_{b}$, the choice $i=\lambda_{b}$ gives an infinite enhancement factor. This is an integrable singularity, however, and so is washed out by lattice effects in any discrete configuration of satellites and also by finite size effects of the booster distribution if these are comparable to or larger than nearest neighbor satellite spacings.

It is easy to generalize to the case where a range of orbit inclinations are chosen. Let

$$
\mathrm{df}=\rho(\mathrm{i}) \mathrm{di}
$$

be the fraction of the total number of orbital planes in internal di. The normalization is

$$
\int_{0}^{\pi} \rho(\mathrm{i}) \mathrm{di}=1
$$

The generalization of Equation (B.40) becomes

$$
\frac{\mathrm{dN}}{\mathrm{dA}}=\left[\frac{\mathrm{N}}{4 \pi \mathrm{R}_{\mathrm{s}}^{2}}\right] \int \rho(\mathrm{i}) \mathrm{y}(\lambda, \mathrm{i}) \mathrm{di},
$$

where $\mathrm{y}$ is the same as before and $\mathrm{N}$ is the total number of satellites in the constellation. We see that the presence of any smooth $\rho(i)$ adds additional tempering of the square root singularity of $y(\lambda, i)$ in the pointlaunch case. One may check by direct integration that the choice

$$
\rho(\mathrm{i})=\frac{1}{2} \sin (\mathrm{i})
$$

corresponds to an earth-spin averaged uniform density.

A few features of the satellite enhancement factor in Equation (B.41) deserve comment. The case $\mathrm{i}=0$ is singular and represents the fact that the band of coverage collapses to line the equator. (For the further special case of geosynchronous altitude, earth-spin averaging is absent and the coverage collapses to a single point.)

The case of polar orbits, $\mathrm{i}=\pi / 2$, where

$$
y(\lambda, \pi / 2)=\frac{2}{\pi} \frac{1}{\cos \lambda}
$$

is of special interest to space-based defense systems. One has some enhancement over uniform distributions since the Soviet land mass and existing missile fields are at high latitudes $\left(\lambda_{\mathrm{b}} \simeq 57^{\circ}\right.$ is a typical value) but more importantly one has a very strong enhancement at the poles since the denominator in Equation (B.45) vanishes there. The fact that the enhancement is stronger in the polar case is obvious on physical grounds since every satellite passes over the poles on every orbit. Mathematically it corresponds to the collapse of the ring of square root singularities at $\lambda=i$ (and $\lambda=-i$ ) to a point linear singularity in the case $i=\pi / 2$. Since 
missile trajectories from the Soviet Union to the continental U.S. all pass near the north pole, one has in a polar constellation of satellites an enhancement which is particularly efficient for mid-course defense actions.

\section{REFERENCES}

${ }^{1}$ A. B. Carter, Directed Energy Missile Defense in Space, OTA Background Paper, April 1984.

${ }^{2}$ Union of Concerned Scientists Report: Space Based Missile Defense, March 1984 and Erratum, April 1984; Book: The
Fallacy of Star Wars, edited by J. Tirnman (Vintage Books, Random House, New York, 1984).

${ }^{3}$ R. L. Garwin, Nature 315, 286-290 (1985).

${ }^{4}$ G. L. Canavan, Comments on the OTA Paper on Directed Energy Missile Defense in Space, LASL Document P/AC:84:43, May 1984 (unpublished).

${ }^{5}$ G. L. Canavan, Simple Estimates of Satellite Constellation Sizing, LASL Document 0903B, Aug. 1984 (unpublished).

${ }^{6}$ G. L. Canavan (private communication).

${ }^{7}$ C. T. Cunningham, LLNL Document, Critique of Systems Analyses in the OTA Study, Directed Energy Missile Defense in Space, No. DDV-84-0007 (1984).

${ }^{8}$ C. T. Cunningham (private communication).

${ }^{9}$ G. Field and D. Speigel, "Cost of Space-Based Laser Ballistic Missile Defense," Science 231, 1387-1393 (1986).

${ }^{10} \mathrm{M}$. LeCar, Harvard University, unpublished calculations. 


\section{APS COMMITTEE ON THE STUDY OF DIRECTED ENERGY WEAPONS}

\author{
Professor N. Bloembergen (Co-chairman) \\ Pierce Hall \\ Harvard University \\ Cambridge, MA 02138 \\ Dr. C. K. N. Patel (Co-chairman) \\ AT\&T Bell Laboratories \\ 600 Mountain Avenue \\ Murray Hill, NJ 07974 \\ Dr. Petras Avizonis \\ Air Force Weapons Laboratory \\ Kirtland A.F.B. \\ Albuquerque, NM 87117-6008 \\ Dr. Robert G. Clem \\ Sandia National Laboratories \\ P.O. Box 5800, Org. 1600 \\ Albuquerque, NM 87185 \\ Professor Abraham Hertzberg \\ Aerospace \& Energetics \\ Research Program FL-10 \\ University of Washington \\ Seattle, WA 98195 \\ Lt. Colonel Thomas H. Johnson \\ Dept. of the Army/MADN-5 \\ U.S. Military Academy \\ West Point, NY 10996 \\ Professor Thomas Marshall \\ 213 Mudd Building \\ Columbia University \\ New York, NY 10027 \\ Dr. R. Bruce Miller \\ Sandia National Laboratories \\ P.O. Box 5800, Org. 1270 \\ Albuquerque, NM 87185 \\ Dr. Walter E. Morrow \\ Lincoln Laboratories \\ Massachusetts Institute of Technology \\ P.O. Box 73 \\ Lexington, MA 02139
}

Dr. Edwin E. Salpeter

Newman Laboratory for Nuclear Studies Cornell University

Ithaca, NY 14853

Dr. Andrew M. Sessler

MSE Building 4

Lawrence Berkeley Laboratory

1 Cyclotron Road

Berkeley, CA 94720

Professor Jeremiah D. Sullivan

Physics Department

University of Illinois

1110 West Green Street

Urbana, IL 61801

Dr. James C. Wyant

Wyko Corp.

2990 E. Fort Lowell

Tucson, AZ 85716

Professor Amnon Yariv

Department of Applied Physics

California Institute of Technology

Pasadena, CA 91125

Professor Richard N. Zare

Department of Chemistry

Stanford University

Stanford, CA 94305

Dr. A. J. Glass (Principal Consultant)

KMS Fusion, Inc.

3621 So. State Road

P.O. Box 1567

Ann Arbor, MI 48106-1567

Dr. L. Charles Hebel (Executive Officer)

Palo Alto Research Center

3333 Coyote Hill Road

Palo Alto, CA 94304 


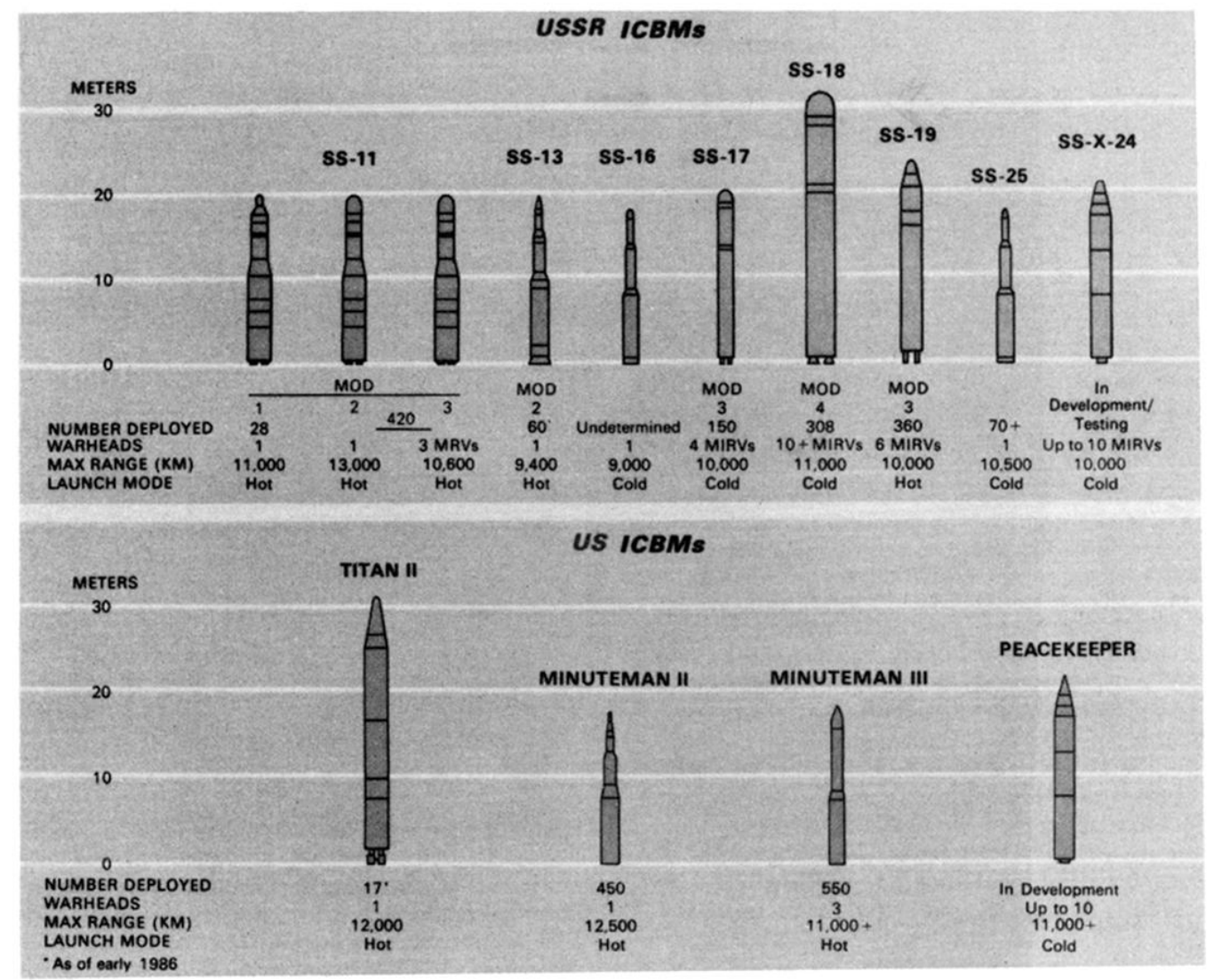

Figure 2.4 U.S. and Soviet ICBM deployments. 


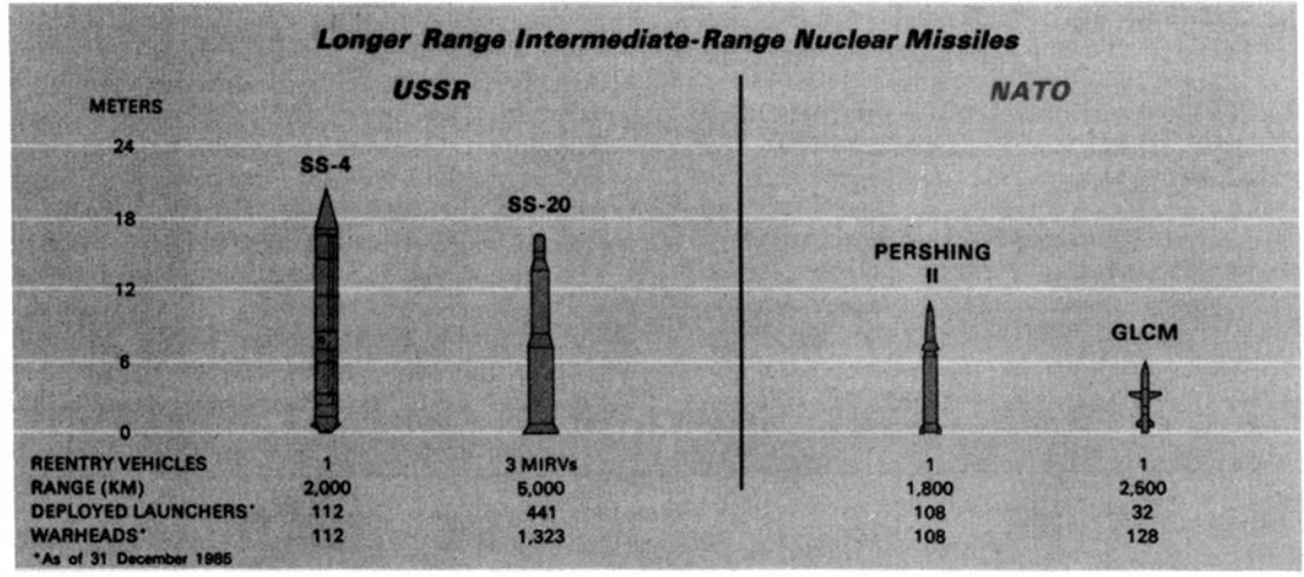

Figure 2.5 U.S. and Soviet LR-IRBM deployments. 


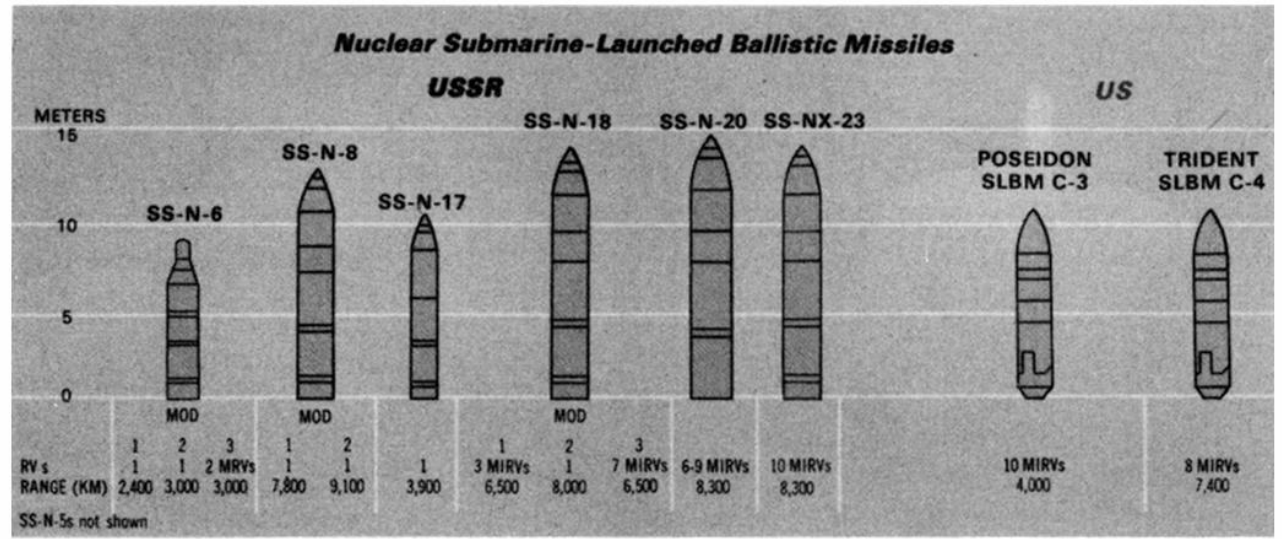

Figure 2.6 U.S. and Soviet SLBM deployments. 


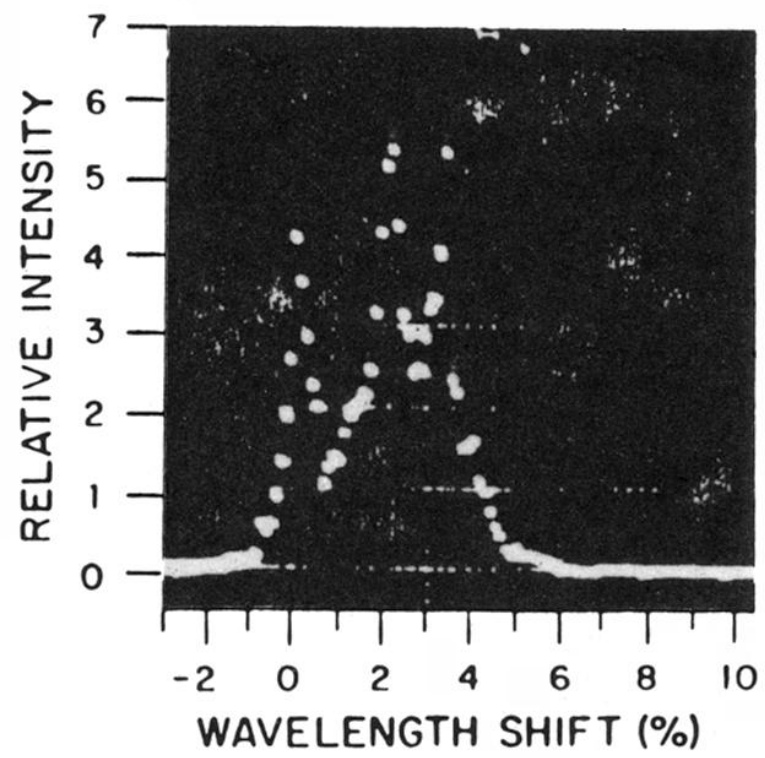

Figure 3.30. Sidebands in the LANL free-electron laser (Reference 80). 


\section{ALPHA RESONATOR HIGH EXTRACTION DECENTERED ANNULAR RING RESONATOR (HEX-DARR)}

\section{IT}

TRW Space 8 Technology Group

WAXICON/REFLAXICON

BEAM COMPACTOR

- laRge phase SHIfT COATINGS NOT REQUIRED

RETRO-REFLECTING

POWERED REAR CONE

- INSENSITIVE TO TILT

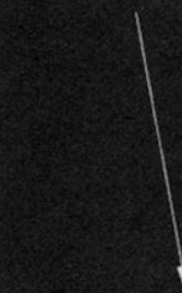

1
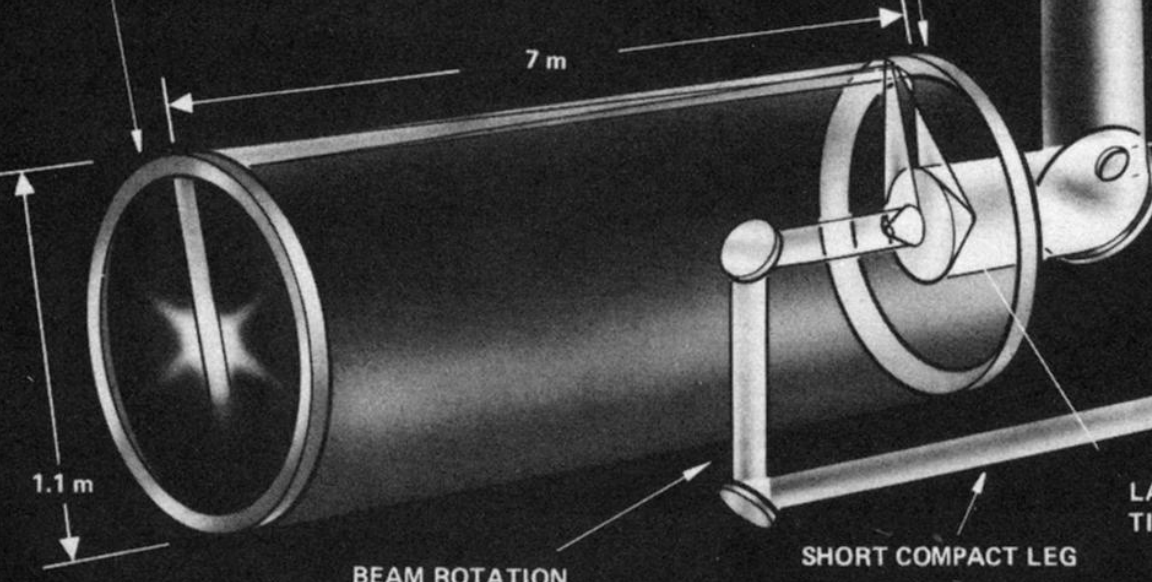

BEAM ROTATION

\section{DECENTERED}

- UNIFORM FLUX LOADINGS

- NO TIP LOADING PROBLEM

- ABERRATION SENSITIVITY REDUCED

- OPTIC AXIS AWAY FROM AXICON TIPS AND NOT SPLIT

- reverse waVe Reduced

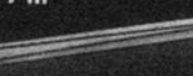

SHORT COMPACT LEG

- $7 \mathrm{~m}$

\section{.}

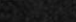

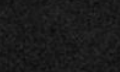

.
LARGE AXICON

TIP TRUNCATION $(1-3 \mathrm{~cm})$

- heX FAB EASED

- coATING EASED

Figure 3.8. High extraction decentered annular ring resonator (HEX-DARR) design. 


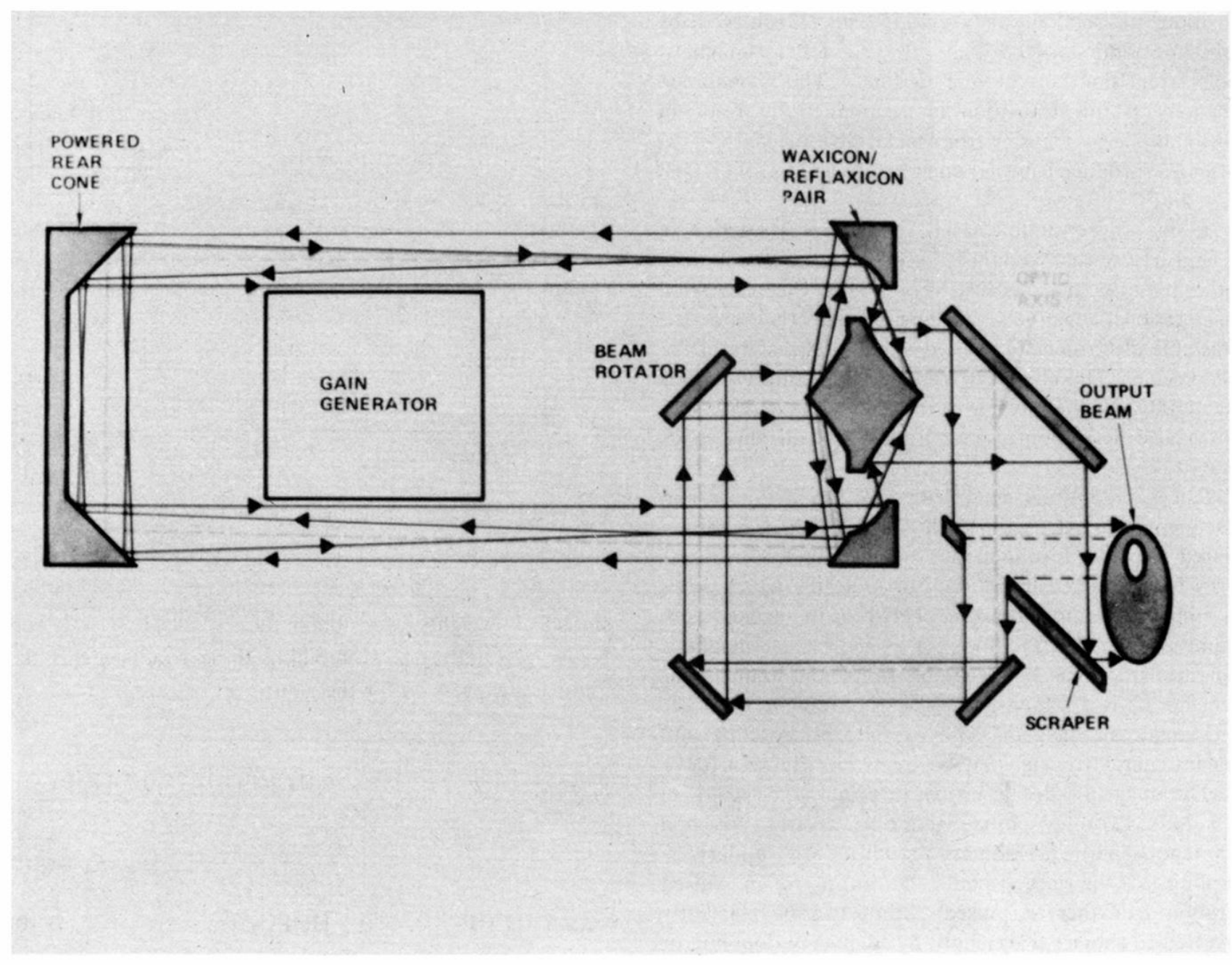

Figure 3.9. Optical ray trace of HEX-DARR design shown in Figure 3.8. 


\section{EXPERIMENT}

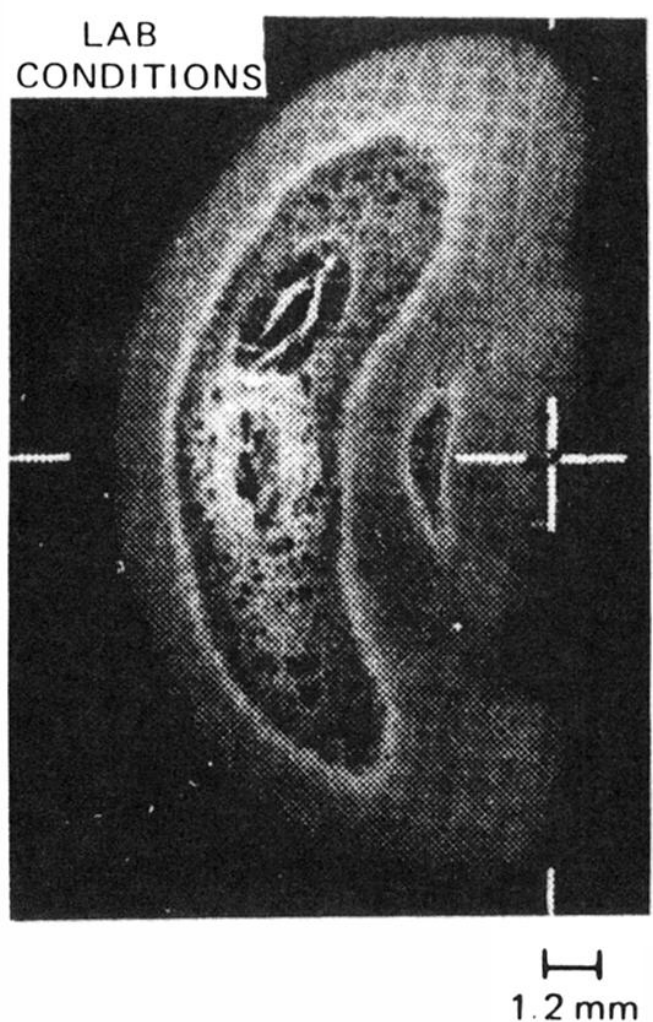

THEORY

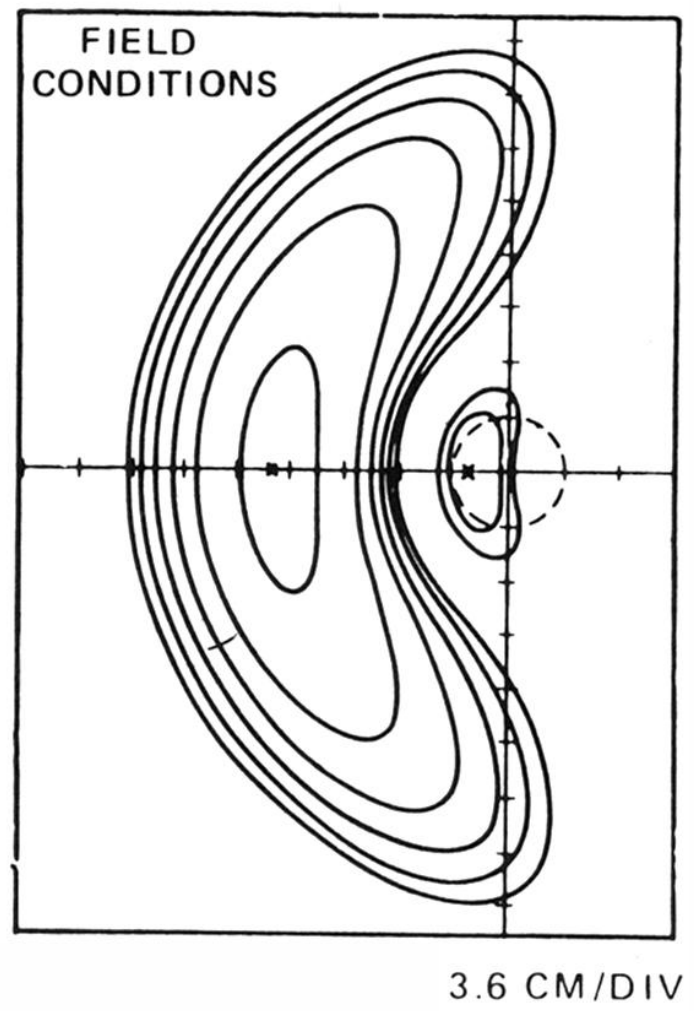

Figure 5.20. Photograph of $\mathrm{cw} \mathrm{CO}_{2}$ laser beam profile from laboratory simulation experiments of thermal blooming in the atmosphere and numerically calculated intensity contours from a theoretical simulation. Dimensionless parameters characterizing thermal blooming were the same in both cases; the results show good correlation and therefore understanding of $\mathrm{cw}$ thermal blooming. For further information see Reference 17. 


\section{(a)}

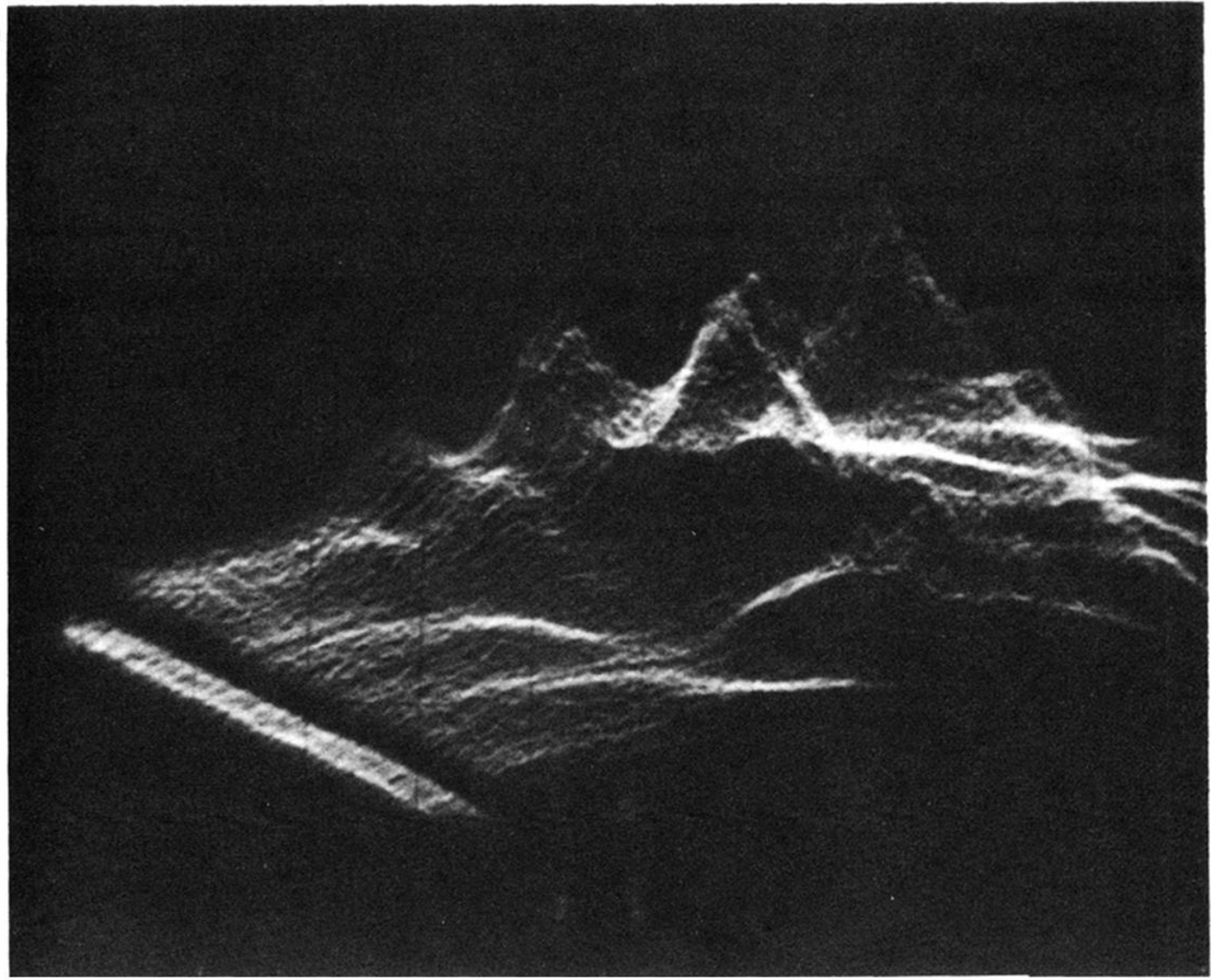

(b)

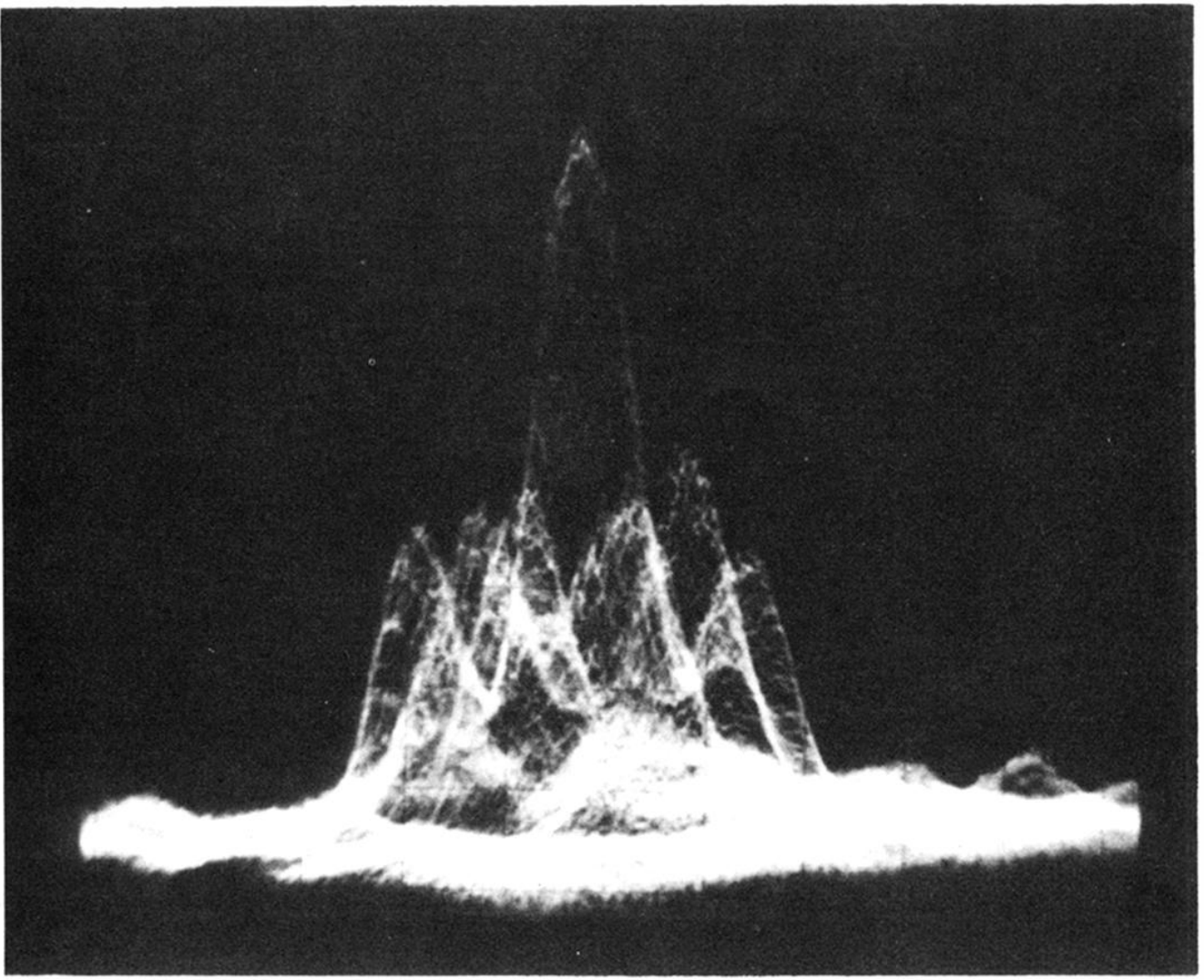

Figure 5.7. Measured far field intensity distribution of a three telescope array (from References 3 and 4). (a) Optically aligned but not phased (argon ion laser wavelength). (b) Dynamically phased for piston and tilt. Overall achieved phased error is $\lambda / 50$. (Argon ion laser wavelength.) 